US Army Corps

of Engineers $S_{\circledast}$

Engineer Research and

Development Center

Flood and Coastal Storm Damage Reduction Program

\title{
Analysis of a Floodplain I-Wall Embedded in Horizontally Stratified Soil Layers During Flood Events Using Corps_I-Wall Software Version 1.0
}

Robert M. Ebeling, Moira T. Fong, and Barry C. White

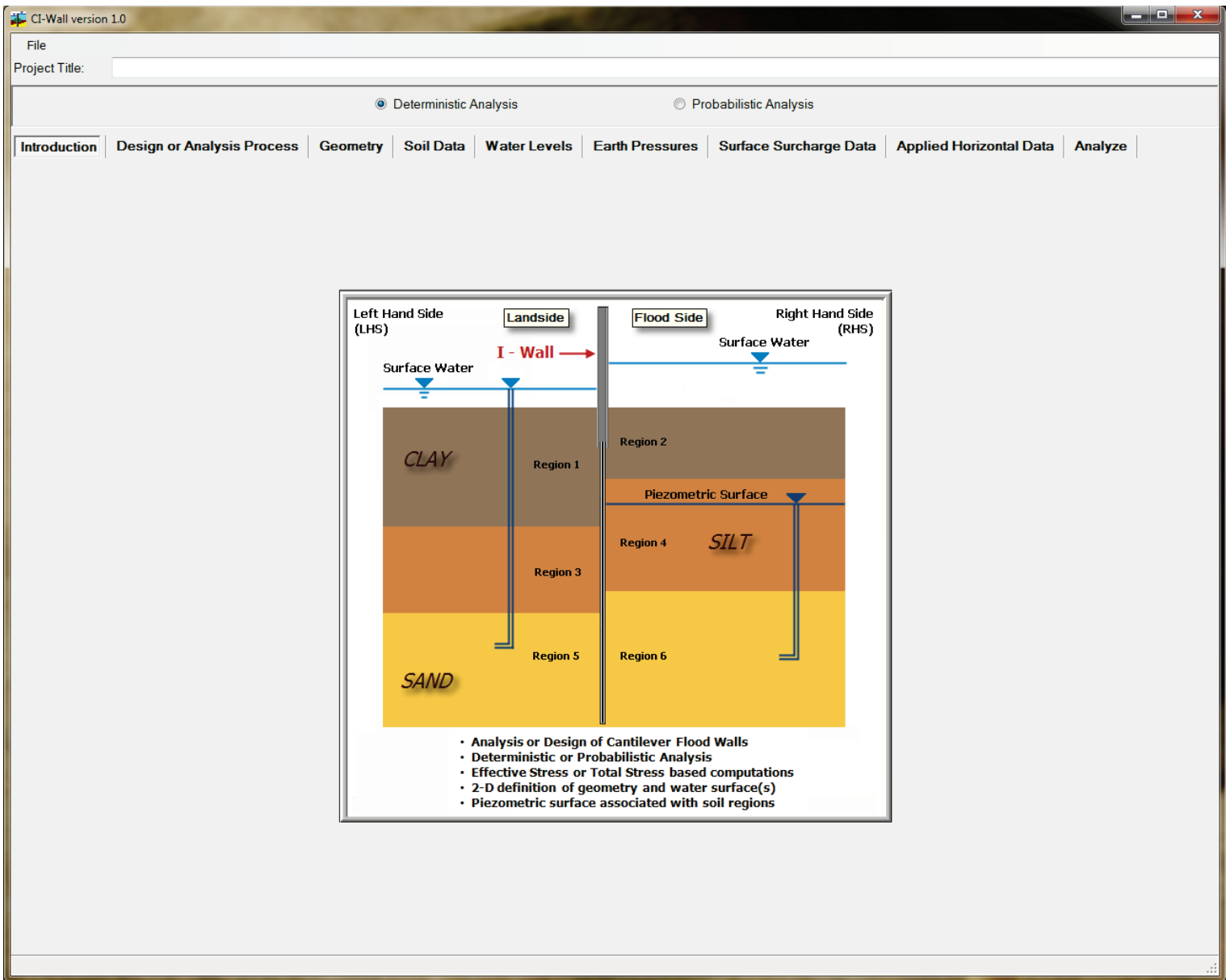


The U.S. Army Engineer Research and Development Center (ERDC) solves the nation's toughest engineering and environmental challenges. ERDC develops innovative solutions in civil and military engineering, geospatial sciences, water resources, and environmental sciences for the Army, the Department of Defense, civilian agencies, and our nation's public good. Find out more at www.erdc.usace.army.mil.

To search for other technical reports published by ERDC, visit the ERDC online library at http://acwc.sdp.sirsi.net/client/default. 


\section{Analysis of a Floodplain I-Wall Embedded in Horizontally Stratified Soil Layers During Flood Events Using Corps_I-Wall Software Version 1.0}

Robert M. Ebeling, Moira T. Fong, and Barry C. White

Information Technology Laboratory

U.S. Army Engineer Research and Development Center

3909 Halls Ferry Road

Vicksburg, MS 39180-6199

Final report

Approved for public release; distribution is unlimited.

Prepared for Headquarters, U.S. Army Corps of Engineers

441 G Street NW

Washington, DC 20314-1000

Under Work Unit CL618F 


\section{Abstract}

The purpose of the I-Wall Analysis R\&D effort is to provide the U.S. Army Corps of Engineers (USACE) districts with essential tools in analyzing and evaluating I-Wall performance in riverine, fluvial, and coastal environments. This report summarizes an engineering method and its implementation in the software Corps_I-Wall Version 1.o. The executable program is referred to as CI-Wall. This initial version of Corps_I-Wall is used for the design of new I-Walls or the analysis of existing I-Walls in floodplains (i.e., I-Walls embedded in level ground) during flood loading. Horizontally stratified soil layers are assumed in Version 1.0.

Other Corps_I-Wall software capabilities for engineering analysis include: total stress and/or effective stress earth pressure calculations; Rankine, Coulomb, or logarithmic spiral-based earth pressure coefficients; hydrostatic pore water pressure computed from surface water(s) and/or piezometric surface(s) within each of the layered soil regions; pore water pressures computed from line of seepage, steady-state seepage in soil regions with different hydraulic conductivities; gap initiation and propagation using hydraulic fracturing criteria; and boundary pressures (e.g., for wave loading of coastal structures) and/or surface surcharge.

Probabilistic analysis is a primary component of Corps_I-Wall for new and existing I-Wall analysis. The probabilistic analysis capabilities for the design of new I-Walls results in a statistical characterization of sheet-pile tip embedment. The probabilistic analysis capabilities for analyzing existing I-Walls include the construction of a system response curve (a.k.a., fragility curve), which gives the probability of rotational instability as a function of flood elevation.

Examples are provided to highlight each of these capabilities and a user's manual for using the Graphical User Interface for CI-Wall is included.

DISCLAIMER: The contents of this report are not to be used for advertising, publication, or promotional purposes. Citation of trade names does not constitute an official endorsement or approval of the use of such commercial products. All product names and trademarks cited are the property of their respective owners. The findings of this report are not to be construed as an official Department of the Army position unless so designated by other authorized documents. 


\section{Addendum 1}

ERDC/ITL TR-16-3

April 2017

Analysis of a Floodplain I-Wall Embedded in Horizontally Stratified Soil Layers during Flood Events Using Corps_I-Wall Software Version 1.0

\section{Summary}

This addendum sheet has been assembled using the results of the updated code for Corps_I-Wall version 1.0, which has been modified to more correctly handle gap propagation due to hydraulic fracturing and seepage effects.

\section{Observation}

Software (CI-Wall) updates have had the most significant effect on Example problem 4.4, found in Chapter 4 of the cited ERDC technical report (TR) (ERDC/ITL TR-16-3).

\section{Updated Results for the TR's Example Problems}

1. Page 143, change Figure 4.7 with

Figure 4.7 Pore water pressures. (a) Left- and right-hand side of wall. (b) Net water pressures.

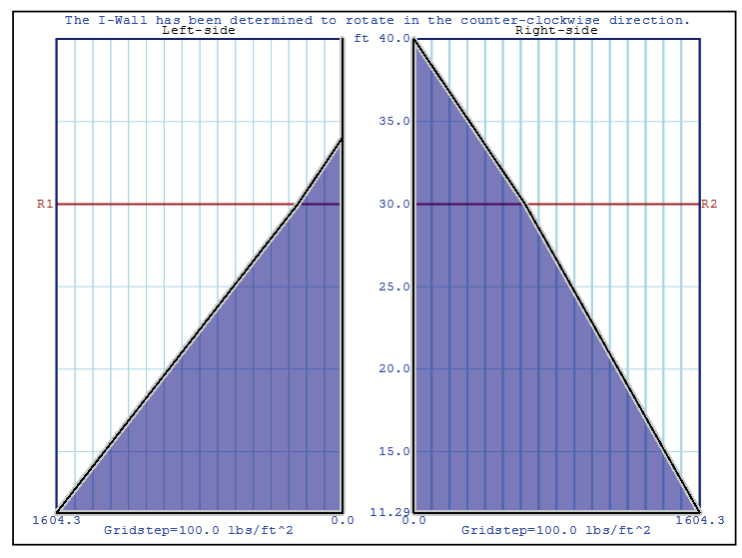

(a)

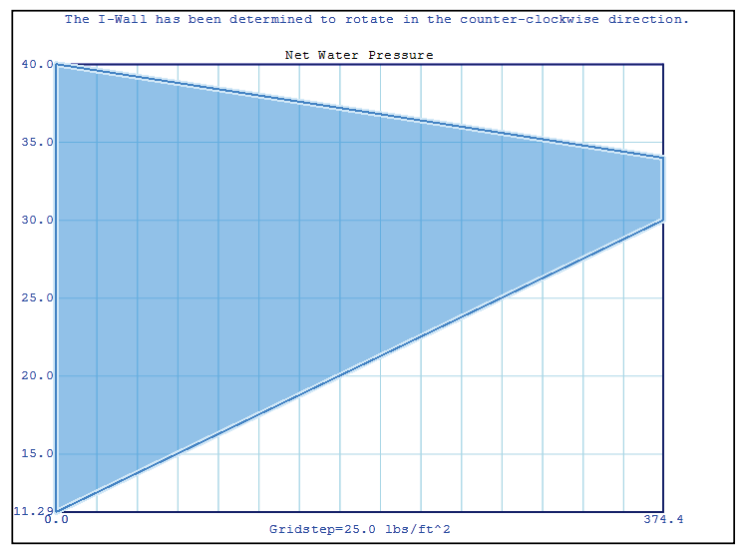

(b) 
2. Pages 162-166, reanalysis of Example 4.4 with replacement figures starting after Figure 4-28.

The active and passive earth pressures for both sides of the sheet-pile wall are illustrated in Figure 4.29. Note the change in earth pressures at the region interfaces at el -5 , el -10, and el -13 ft of Figure 4.29a, illustrating active and passive earth pressures on the LHS, as well as the change in earth pressures at the region interfaces at el 5, el o, el -4, el -10, and el -12 ft as shown in Figure 4.29b.

Figure 4.29. Active and passive earth pressures. (a) Left-hand side of wall. (b) Right-hand side of wall.

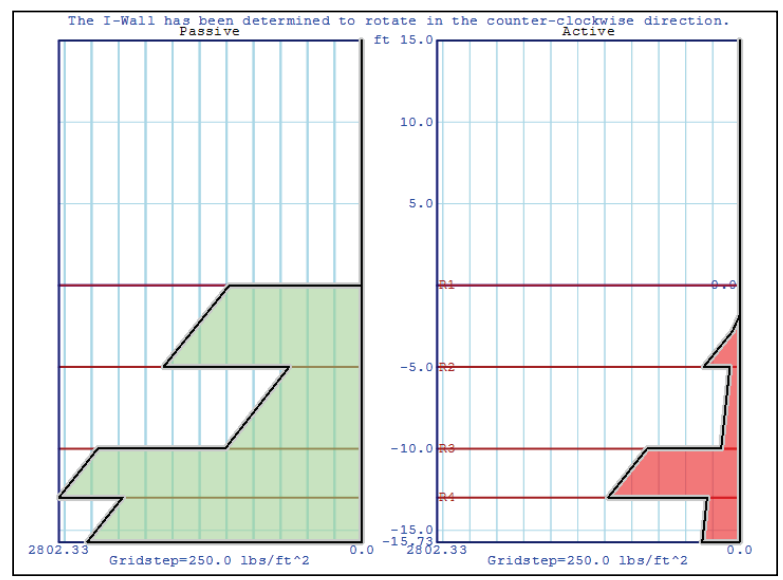

(a)

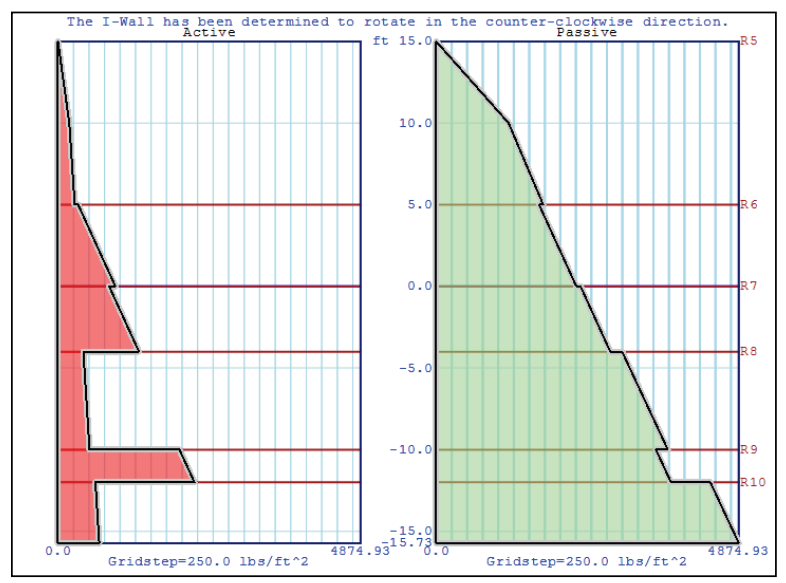

(b)

The hydrostatic water pressures acting on both sides of the sheet-pile wall are shown in Figure 4.30a with the resultant net water pressure calculated by taking the difference between the water pressures acting on both sides of the wall (Figure 4.30a) and shown in Figure 4.30b.

Figure 4.30. Pore water pressures. (a) Left- and right-hand side of wall. (b) Net water pressures.

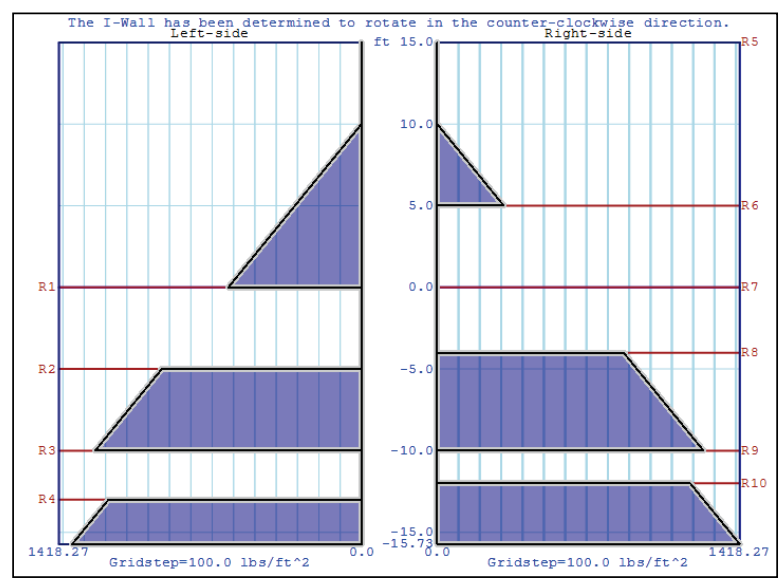

(a)

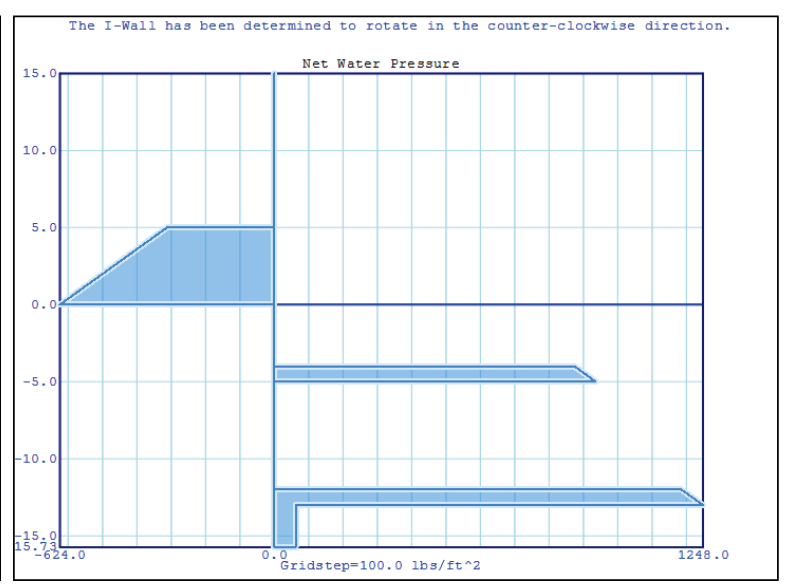

(b) 
From the calculated pressures of Figures 4.29 and 4.30, the net active and net passive pressures are constructed and presented in Figure 4.31a. For the prescribed LHS flood loading, the I-Wall will rotate in a counterclockwise direction. The net active pressure is determined from the difference between the LHS-mobilized active earth pressure and the RHS-mobilized passive earth pressure (i.e., acting on the other side of the wall) with the addition of the net water pressures and any external horizontal net pressures and overburden net pressures (if any). The net passive pressure is determined by the difference between the LHS-mobilized passive earth pressure and the RHS-mobilized active earth pressure acting on the other side of the sheet-pile wall with the addition of any net horizontal water pressures and any external horizontal net pressures (if any). The net pressure diagram of Figure $4.31 \mathrm{~b}$ is derived from the replication of all values of the net active pressures, from the top of the wall until the elevation at the point of rotation (at el -13.04 ft), at which instant the remaining values of the net pressure diagram are assumed to be linear with elevation between (1) the net active pressure value at the elevation of the point of rotation and (2) the net mobilized passive pressure at the elevation of the approximated sheet-pile tip (i.e., el -15.73). ${ }^{1}$ Figure $4.31 \mathrm{~b}$ is an illustration of how the net pressure diagram was formed. Because the I-Wall has been determined to rotate in a counterclockwise direction, the net pressure diagram of Figure 4.31b shows that the upper zone of net active pressures is plotted on the RHS, and the lower zone is plotted on the LHS. The net passive pressures are plotted on the RHS of the sheet-pile wall. The final results are illustrated in Figure 4.32 by the presentation of the net pressure diagram Figures 4.32a (replica of Figure 4.31b) and 4.32b, the shear and moment diagrams. Figure $4.32 \mathrm{~b}$ shows that the maximum bending moment occurs at the zero crossing of the shear diagram.

\footnotetext{
1 Only one mobilized net passive pressure value in Figure 4.31a is used to construct the Figure 4.31b net pressure diagram corresponding to the sheet-pile tip elevation at el $-15.73 \mathrm{ft}$. The majority of the net pressure diagram is derived from the Figure 4.31a net active pressure diagram.
} 
Figure 4.31. Net pressures. (a) Net active and net passive. (b) Net pressure diagram.

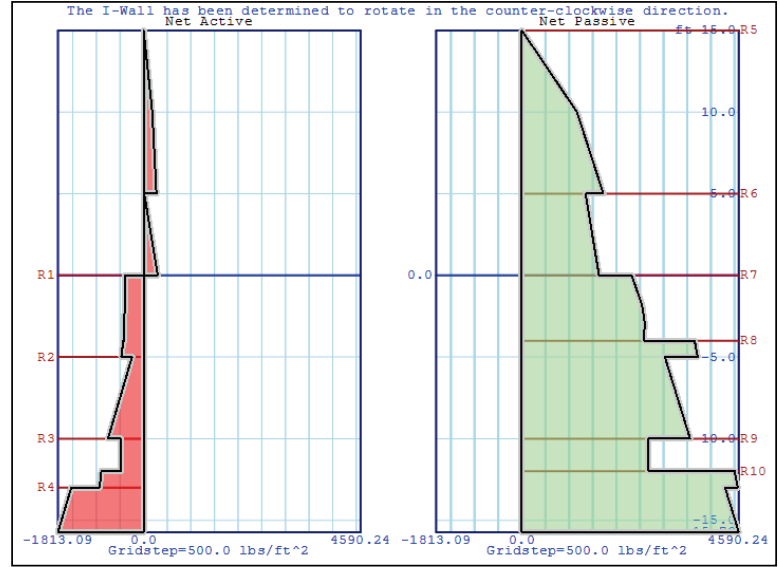

(a)

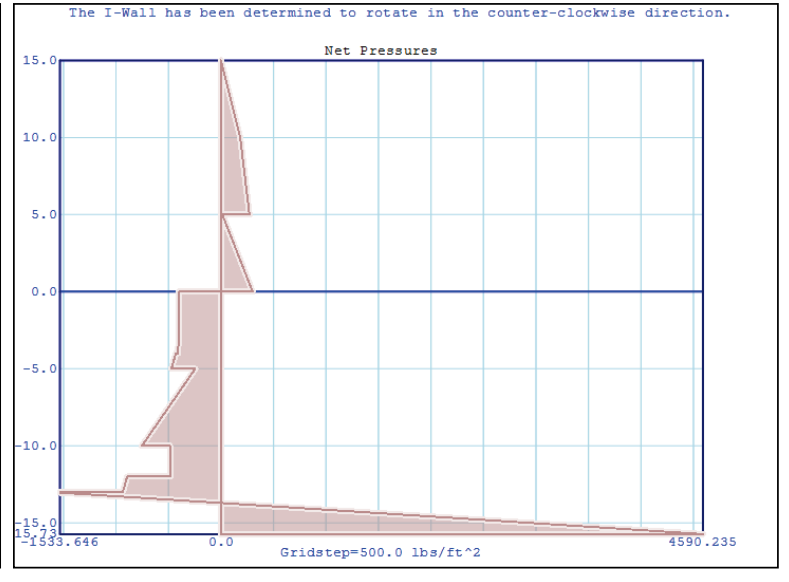

(b)

Figure 4.32. (a) Net pressure diagram. (b) Shear and moment.

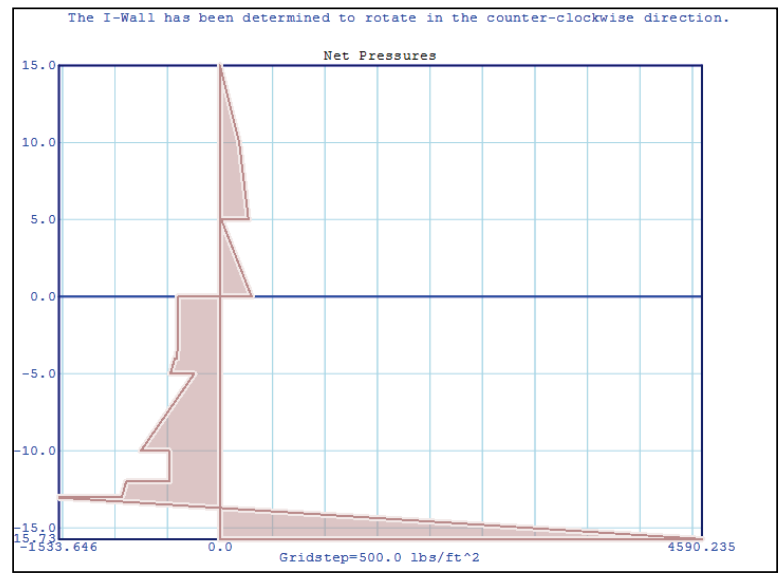

(a)

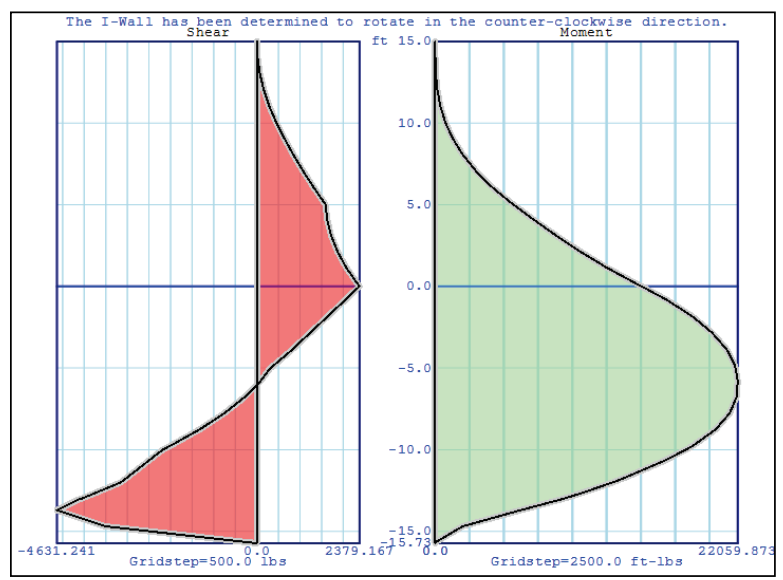

(b)

The various input conditions for the 10 layers of soil and final results for Example 4.4 are tabulated in Table 4.4. 
Table 4.4. Multilayered soils with effective stress strength definitions (sands with $c^{\prime}=0$ ) and total stress strength definitions (clays with $\varphi=0$ ).

\begin{tabular}{|c|c|c|c|c|c|c|c|c|c|c|c|}
\hline $\begin{array}{l}\text { Region } \\
\text { ID }\end{array}$ & $\begin{array}{l}\text { Material } \\
\text { Layer ID }\end{array}$ & Soil Type & $\begin{array}{l}\text { Piezometric } \\
\text { Surface ID }\end{array}$ & $\begin{array}{l}\varphi^{\prime} \\
\text { (deg) }\end{array}$ & $\begin{array}{l}c \\
\text { (psf) }\end{array}$ & $\begin{array}{l}\text { Y_moist } \\
\text { (pcf) }\end{array}$ & $\begin{array}{c}\text { Y_sat } \\
\text { (pcf) }\end{array}$ & $\mathrm{K}_{\mathrm{a}} m o b$ & $\mathrm{~K}_{\mathrm{p}} m o b$ & $\begin{array}{l}\text { Elevation at } \\
\text { Point of Rotation }\end{array}$ & $\begin{array}{l}\text { Elevation at Tip } \\
\text { of Pile }\end{array}$ \\
\hline 1 & 9 & Clay & 1 & 0 & 450 & 0 & 122 & 1.0000 & 1.0000 & \multirow{10}{*}{-13.04} & \multirow{10}{*}{-15.73} \\
\hline 2 & 4 & Sand & 1 & 32 & 0 & 0 & 115 & 0.3073 & 2.2496 & & \\
\hline 3 & 10 & Clay & 1 & 0 & 475 & 0 & 120 & 1.0000 & 1.0000 & & \\
\hline 4 & 5 & Sand & 3 & 32 & 0 & 0 & 116 & 0.3073 & 2.2496 & & \\
\hline 5 & 1 & Sand & 2 & 30 & 0 & 110 & 115 & 0.3333 & 2.1212 & & \\
\hline 6 & 6 & Clay & 2 & 0 & 400 & 0 & 120 & 1.0000 & 1.0000 & & \\
\hline 7 & 7 & Clay & 2 & 0 & 450 & 0 & 120 & 1.0000 & 1.0000 & & \\
\hline 8 & 2 & Sand & 2 & 32 & 0 & 0 & 117 & 0.3073 & 2.2496 & & \\
\hline 9 & 8 & Clay & 2 & 0 & 475 & 0 & 121 & 1.0000 & 1.0000 & & \\
\hline 10 & 3 & Sand & 4 & 32 & 0 & 0 & 117 & 0.3073 & 2.2496 & & \\
\hline
\end{tabular}

The angle of wall friction $(\delta)$ and the adhesion $(\mathrm{Ca})$ for all soil layers are zero. The Coulomb earth pressure coefficient method is being used to compute the earth pressures acting on the faces of the sheet-pile wall, and hydrostatic pore water pressures are being used in the effective stress calculations. The design Factor of Safety is 1.0 for active earth pressures, and for passive earth pressures the design Factor of Safety is 1.5. Elevations and depths are reported in units of feet. 
The sheet-pile tip elevation is computed at $-15.73 \mathrm{ft}$ with a calculated depth of embedment of approximately $15.73 \mathrm{ft}$.

3. Page 217 , change Figure 4.88 with

Figure 4.88. Corps_I-Wall output information.

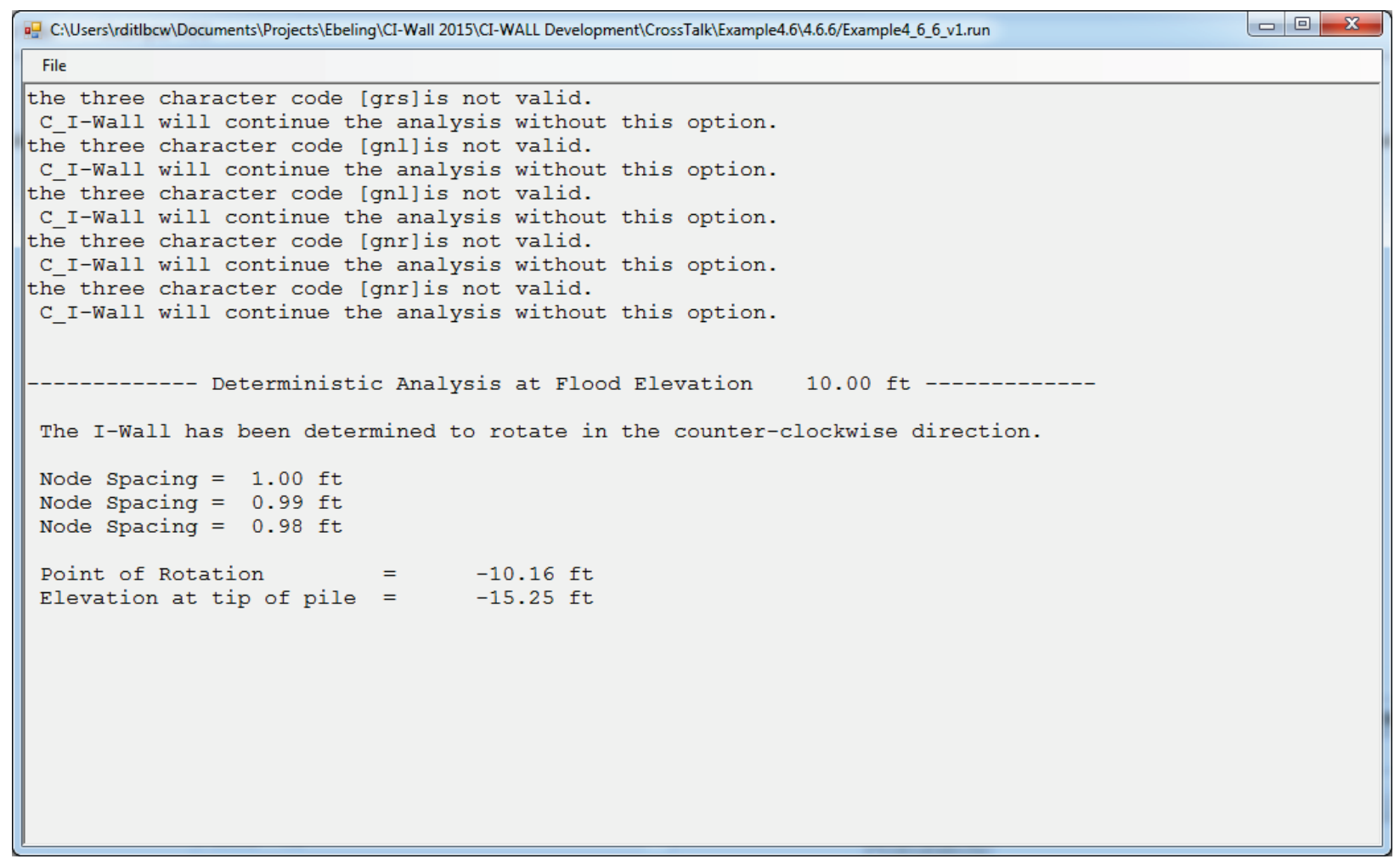

4. Pages 217-223 subsection 4.6.5 replacement figures with expanded modeling discussion.

\section{Model Setup Tips for Calculating a Depth of Hydraulic}

Fracturing: Corps_I-Wall Version 1.0 has the capability to assess the depth that a zone of separation (i.e., a gap) forms along the flood side of the soil to sheet pile I-Wall interface during flood loading. The devised gap initiation and gap propagation engineering methodology implemented within the software (CI-Wall) makes use of a hydraulic fracturing criterion, as discussed in Appendix B of this ERDC/ITL TR-16-3. There are two prerequisites for gap development by CI-Wall: (1) The soil that may potentially develop a gap must be assigned a nonzero value for its cohesive strength (either total or effective stress shear strength parameters); (2) On the side of the I-Wall with flood loads, the surface flood water (elevation, designated as "el") must be associated with all soil regions that may 
develop a gap within or through the region. Additionally, gap propagation proceeds from the ground surface down on the flood side of the I-Wall as the I-Wall rotates away from the flood pool loading side. Note that if during the course of the hydraulic fracturing analysis the gap propagation algorithm detects that the gap is about to enter into a zero cohesion soil region, then gap propagation will terminate at the top of the cohesionless region.

Model setup tips for a gap initiation and gap propagation can be explained using an example sheet-pile wall penetrating clay problem similar to the Example 4.6.7 problem, as discussed in subsection 4.6.5 on pages 217-223 of the ERDC/ITL TR-16-3. This example's unique feature is a partially saturated (i.e., moist) clay top layer (versus a saturated layer) in a total stress site response analysis to a left-hand side (LHS) that is flood loaded. The model setup would follow the updated Figure 4.89.

Figure 4.89. Sheet-pile wall penetrating clay, the errata sheet's expanded version with two soil layers.

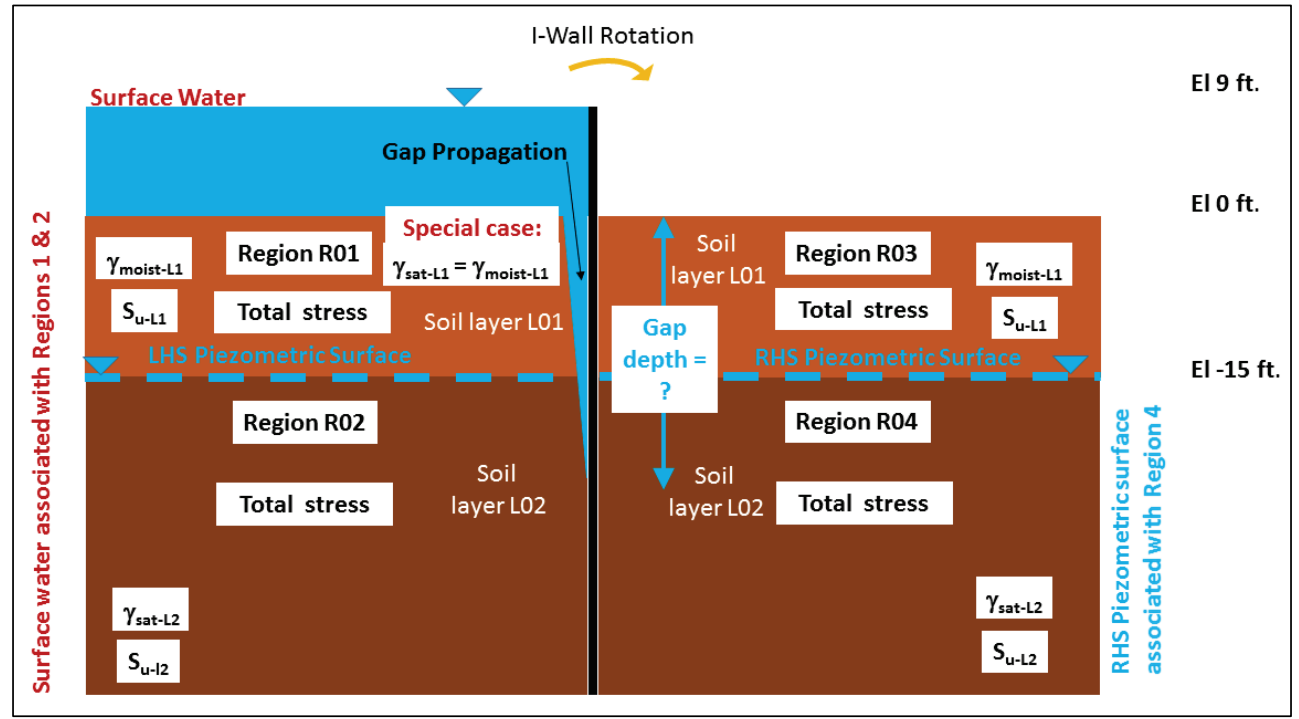

For this expanded version, the site consists of two layers of homogenous clay. Each soil layer, designated L01 and L02, possesses values of moist unit weight, saturated unit weight, and constant undrained shear strength, $\mathrm{S}_{\mathrm{u}}$. The total angle of internal friction, $\phi$, is equal to zero. Table 1 summarizes this soil material information for each of the layers. 
Table 1. Geotechnical properties for the two soil layers contained in Example 4.6.7.

\begin{tabular}{|l|l|l|l|l|l|}
\hline Soil layer no. & $\begin{array}{l}\text { Total or effective } \\
\text { stress analysis }\end{array}$ & $\begin{array}{l}\text { Moist Unit } \\
\text { weight, } \gamma_{\text {moist }} \\
\left(\mathbf{p c f}^{1}\right)\end{array}$ & $\begin{array}{l}\text { Saturated Unit } \\
\text { weight, } \gamma_{\text {sat }} \\
(\mathbf{p c f})\end{array}$ & $\begin{array}{l}\text { Cohesion, } \\
\left(\mathbf{p s f}^{2}\right)\end{array}$ & $\begin{array}{l}\text { Angle of internal } \\
\text { friction, } \phi \\
\text { (degrees) }\end{array}$ \\
\hline L01 & Total & $\gamma_{\text {moist-L1 }}$ & $\gamma_{\text {sat-L1 }}$ & S & 0.0 \\
\hline L02 & Total & $\gamma_{\text {moist-L2 }}$ & $\gamma_{\text {sat-L2 }}$ & Su-L2 & 0.0 \\
\hline
\end{tabular}

${ }^{1}$ pounds/cubic foot; ${ }^{2}$ pounds/square foot

A total stress analysis is being specified in the CI-Wall material properties input for this problem because a soil shear strength definition is given in terms of $\mathrm{Su}$ values. The top of moist clay layer Lo1 is at ground surface el $0.0 \mathrm{ft}$. The upper region of this clay layer is partially saturated while below a piezometric surface of el -15.0, the second clay layer Lo2 is saturated.

Insight: In a total stress analysis, without gapping, using CI-Wall, the only purpose a piezometric surface specification serves is to distinguish between the assignments of moist or saturated unit weights within that soil region. A piezometric surface is associated with this region.

Specifically, for the soil within the region located above the piezometric surface (that is assigned to that region), a moist unit weight is assigned by the software while a saturated unit weight is assigned to the soil below the piezometric surface elevation for use in overburden computations. With CI-Wall, an alternative modeling procedure for piezometric boundaries is to divide a total stress soil layer into two regions at the elevation of the piezometric surface. In this case, no piezometric surface is associated with the upper region where a moist unit will be used by the software. But the piezometric surface must be associated with the lower region so that the saturated unit weight is assigned to this lower soil region for the overburden computations.

When conducting a gap initiation and propagation analysis using CI-Wall, the surface water must be associated with the potential gapping regions of the cohesive soil(s). This also means that the saturated unit weight input data will be used exclusively for total overburden computations. Thus, unsaturated soil layers (above the piezometric surface) that may experience gapping need to have their actual moist unit weight input for the modeled saturated unit weight. Again, the alternative modeling procedure of dividing this total stress layer into two regions at the elevation of the piezometric surface should be considered. 
Four regions: The two soil layers are subdivided into four regions as shown in the Figure 4.89 CI-Wall model. This results in two soil layer regions on each side of the wall. Three material properties are specified for this model and are listed in Table 2. Table 3 associates the material numbers with the appropriate Figure 4.89 region numbers.

Table 2. Material property information for the four-region model of Example 4.6.7.

\begin{tabular}{|l|l|l|l|l|l|}
\hline Material no. & $\begin{array}{l}\text { Total or effective } \\
\text { stress analysis }\end{array}$ & $\begin{array}{l}\text { Moist Unit } \\
\text { weight, } \gamma_{\text {moist }} \\
\text { (pcf) }\end{array}$ & $\begin{array}{l}\text { Saturated Unit } \\
\text { weight, } \gamma_{\text {sat }} \\
\text { (pcf) }\end{array}$ & $\begin{array}{l}\text { Cohesion, c } \\
\text { (psf) }\end{array}$ & $\begin{array}{l}\text { Angle of internal } \\
\text { friction, } \phi \\
\text { (degrees) }\end{array}$ \\
\hline M01 & Total & $\gamma_{\text {moist-L1 }}$ & $\begin{array}{l}\text { set equal to } \\
\gamma_{\text {moist-L1 }}\end{array}$ & Su-L1 & 0 \\
\hline M02 & Total & $\gamma_{\text {moist-L2 }}$ & $\gamma_{\text {sat-L2 }}$ & Su-L2 & 0 \\
\hline M03 & Total & $\gamma_{\text {moist-L1 }}$ & $\gamma_{\text {sat-L1 }}$ & Su-L1 & 0 \\
\hline
\end{tabular}

Table 3. Material property assignments to the four-region model of Example 4.6.7.

\begin{tabular}{|l|l|}
\hline Region no. & Material no. \\
\hline R01 & M01 \\
\hline R02 & M02 \\
\hline R03 & Mo3 \\
\hline R04 & M02 \\
\hline
\end{tabular}

Surface water at el 9ft: A flood el of $9 \mathrm{ft}$ is specified on the LHS of the IWall in this total stress analysis. Since c ( $\mathrm{S}_{\mathrm{u}}$ for total stress analysis) is nonzero, the CI-Wall hydraulic fracturing criteria will be engaged to calculate the depth of gapping on the LHS of the I-Wall. Prior to CI-Wall analysis, it is not known if the gap propagates partially into region Ro1 or fully through region Ro1 and into region Ro2. In order to allow the CI-Wall hydraulic fracturing criteria to compute the depth of gapping on the LHS of the soil-to-sheet pile interface, it is necessary to associate the LHS surface water (at el $9.0 \mathrm{ft}$ ) with both region Ro1 and region Ro2 (refer to Figure 4.89) in the CI-Wall model input. Because this is a total stress analysis, associating these two regions with the surface water means that overburden computations are made using the saturated unit weight inputs.

Left-Hand Side (LHS) piezometric surface at el -15ft: Since there is a possibility in this total stress analysis that the gap may propagate through region Ro1 and into region Ro2, the LHS piezometric surface is 
not associated with region Ro2. This allows for the surface water to be associated with region Ro2 for gap propagation considerations. Recall that since the surface water is associated with region Ro2, a saturated unit weight is used in CI-Wall overburden computations for this region (versus using the moist unit weight input).

Right-Hand Side (RHS) piezometric surface at el -15ft: The RHS piezometric surface at el -15.o ft is specified at the top of soil region Ro4. Since the RHS piezometric surface is associated with region Ro4, a saturated unit weight is used in CI-Wall overburden computations for this region (versus using a moist unit weight).

Soil unit weights: Because of the presence of the upper moist (i.e, nonsaturated) region Ro1 clay layer that is immediately below the flood surface water, three different material types are specified for the model. The lower clay regions Ro2 and Ro4 are assigned a saturated unit weight, and soil region Ro3 is assigned a moist unit weight in total stress, overburden computations. This is consistent with conventional geotechnical criteria. For soil region Ro1, a moist unit weight value is assigned to the saturated unit weight input in CI-Wall. A special material number Mo1 shows this special input data in Table 2. This data input is required because, in a total stress soil region for which the LHS surface water is associated, the CI-Wall hydraulic fracturing algorithm makes exclusive use of the saturated unit weight input value in its total stress, overburden calculations.

Example 4.6.7 consists of a homogenous soil layer of constant undrained shear strength $S_{u}$ equal to 300 psf (check problem 2 in ERDC/ITL TR-16-3, Table 4.15) and an assignment of the same value to the moist and saturated unit weights ( $=110 \mathrm{pcf})$. Because of the way gap propagation is enabled in Corps_I-Wall version 1.o, any soil region that may gap needs to be associated with the Surface Water (flood el $9 \mathrm{ft}$ ) on the side where gapping is expected to occur (i.e., LHS). This means that the saturated unit weight material input variable value will be used within potential gapping cohesive soil regions for CI-Wall software overburden computations. In Example 4.6.7, the soil region on the LHS of the structure has a surface water that acts to not only load the I-Wall laterally but also acts as overburden pressure to the ground surface. Between el o and $-15 \mathrm{ft}$, this cohesive soil zone is unsaturated, so a moist unit weight should be used in the overburden pressure computations analysis. The lower zone (i.e., below el - 
$15 \mathrm{ft}$ ) is saturated because of the presence of its piezometric surface. To model this situation correctly in CI-Wall so that gapping can occur, the upper moist and lower saturated LHS soil layer is divided into two regions along the piezometric boundary line. Each of these divided regions should then be associated with the surface water, which will enable gapping. The key to accounting for the effect of the LHS piezometric surface comes in CIWall's use of saturated unit weights for each LHS region because the (association of the) surface water will cause both regions to be considered saturated in the analysis. By setting the upper region material's saturated unit weight to the value of the moist (i.e., unsaturated) unit weight, and the bottom region material's saturated unit weight to the value of the saturated unit weight (as usual), the CI-Wall model captures the effect of the piezometric surface. The simplifying assumption of the same moist and saturated unit weight values allows for the use of only two material regions in the model. Thus, Figure 4.90 should be as follows:

Figure 4.90. Corps_I-Wall schematic of sheet-pile wall in clay site.

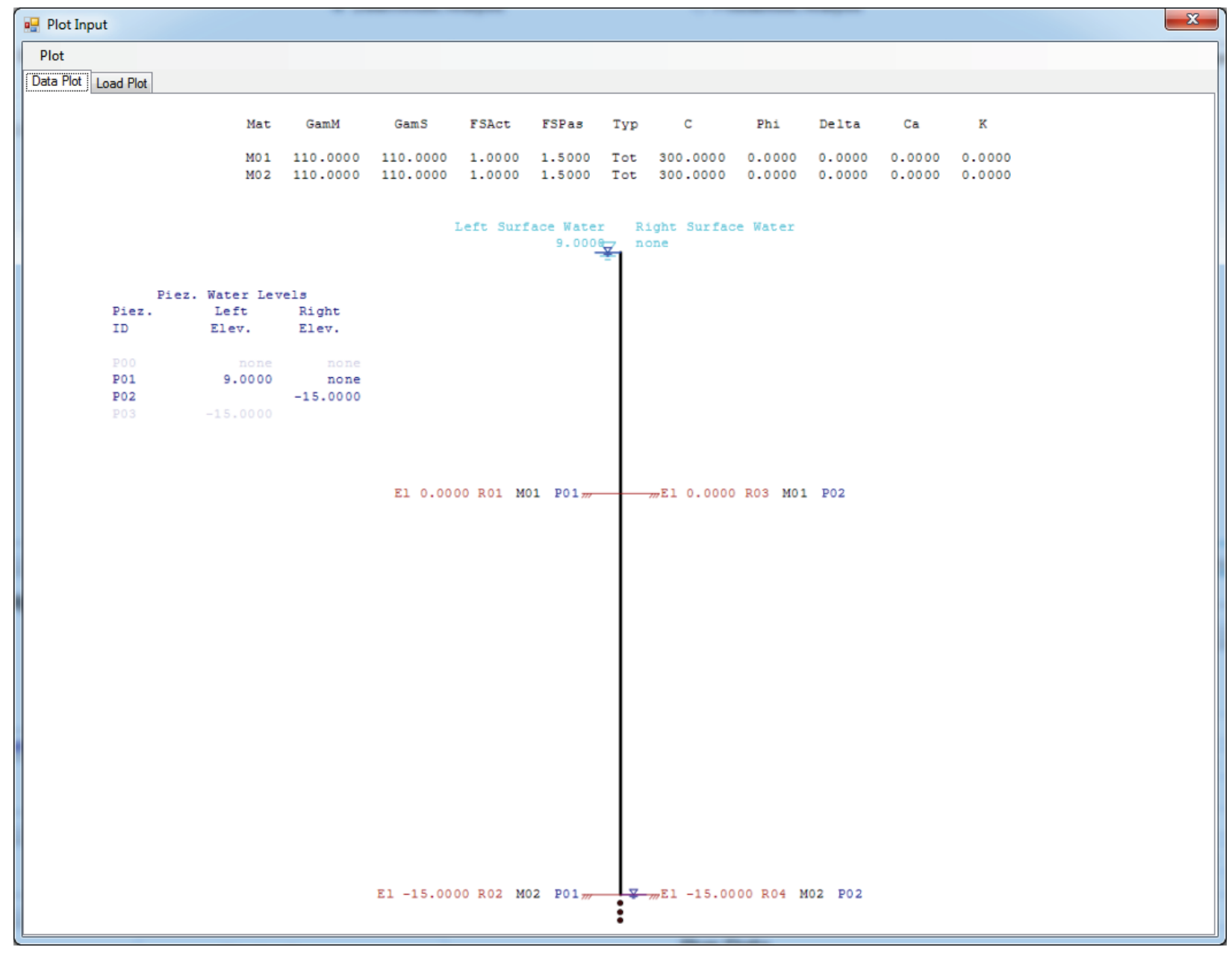


When analyzing a partially saturated, cohesive site with layered soils of different moist and saturated unit weights and/or different cohesion values, refer to the modeling tips centered around Figure 4.89 that were presented earlier in this errata sheet.

\section{Page 227, expanded conclusion.}

Chapter 4 contains several example problems. Because there were minor changes made to the Corps_I-Wall version 1.o engineering code to more accurately handle gap propagation and seepage effects, the results of these example problems have changed minutely. In all of the cases, except Example 4.4, the remaining curves have retained the same shape trends, so each image in ERDC/ITL TR-16-3 will not be changed. For verification purposes, the following table gives the resulting run values of point of rotation, pile tip depth, and gap depth for each of the example problems as compared to the original TR results. These numbers should match the runs for the example problems if they were built according to the designated example input parameters.

Table 4. Corrections to results of the Chapter 4 examples.

\begin{tabular}{|c|c|c|c|c|c|c|}
\hline \multirow[b]{2}{*}{$\begin{array}{l}\text { Example } \\
\text { \# }\end{array}$} & \multicolumn{3}{|c|}{ Corps_I-Wall Version 1.0 TR-16-3 } & \multicolumn{3}{|c|}{ Corrected Values } \\
\hline & \begin{tabular}{l|} 
Point of \\
Rotation
\end{tabular} & \begin{tabular}{|l} 
Pile Tip \\
Elevation
\end{tabular} & $\begin{array}{l}\text { Gap } \\
\text { Depth }\end{array}$ & \begin{tabular}{l|} 
Point of \\
Rotation
\end{tabular} & \begin{tabular}{|l|} 
Pile Tip \\
Elevation
\end{tabular} & $\begin{array}{l}\text { Gap } \\
\text { Depth }\end{array}$ \\
\hline 4.1 .1 & 14.41 & 8.02 & & 14.48 & 8.12 & N/A \\
\hline 4.1 .2 & 17.35 & 11.80 & & 17.43 & 11.92 & N/A \\
\hline 4.1.3_1 & 17.34 & 11.78 & & 17.42 & 11.91 & $\mathrm{~N} / \mathrm{A}$ \\
\hline 4.1.3_2 & 18.21 & 12.92 & & 18.30 & 13.05 & N/A \\
\hline 4.1.3_3 & 18.47 & 13.25 & & 18.56 & 13.38 & N/A \\
\hline 4.1 .4 & 17.34 & 11.78 & & 17.42 & 11.91 & $\mathrm{~N} / \mathrm{A}$ \\
\hline 4.1 .5 & 16.24 & 11.29 & & 16.24 & 11.29 & N/A \\
\hline 4.2 .1 & 20.68 & 15.87 & 7.59 & 20.91 & 16.22 & N/A \\
\hline 4.2 .2 & 19.12 & 14.29 & 5.00 & 19.41 & 14.71 & N/A \\
\hline 4.2 .3 & 19.00 & 15.00 & 8.26 & 19.39 & 14.14 & 8.2 \\
\hline 4.3 & -20.59 & -28.27 & 13.31 & -20.52 & -28.17 & 13.31 \\
\hline 4.4 & -13.71 & -15.69 & 0.0 & -13.04 & -15.73 & 0.0 \\
\hline 4.5 .1 & -12.06 & -15.79 & 0.0 & -12.06 & -15.79 & 0.0 \\
\hline 4.5 .2 & -25.41 & -36.37 & & -23.00 & -33.00 & N/A \\
\hline 4.5 .3 & -9.77 & -11.55 & 3.23 & -9.75 & -11.51 & 3.23 \\
\hline
\end{tabular}




\begin{tabular}{|l|l|l|l|l|l|l|}
\hline \multirow{2}{*}{$\begin{array}{l}\text { Example } \\
\#\end{array}$} & \multicolumn{2}{|c|}{ Corps_I-Wall Version 1.0 TR-16-3 } & \multicolumn{3}{c|}{ Corrected Values } \\
\cline { 2 - 7 } & $\begin{array}{l}\text { Point of } \\
\text { Rotation }\end{array}$ & $\begin{array}{l}\text { Pile Tip } \\
\text { Elevation }\end{array}$ & $\begin{array}{l}\text { Gap } \\
\text { Depth }\end{array}$ & $\begin{array}{l}\text { Point of } \\
\text { Rotation }\end{array}$ & $\begin{array}{l}\text { Pile Tip } \\
\text { Elevation }\end{array}$ & $\begin{array}{l}\text { Gap } \\
\text { Depth }\end{array}$ \\
\hline 4.5 .4 & Balanced & & & Balanced & & N/A \\
\hline 4.6 .1 & -17.71 & -21.71 & & -17.65 & -21.64 & N/A \\
\hline 4.6 .2 & -0.91 & -2.19 & & -0.87 & -2.14 & N/A \\
\hline 4.6 .3 & -20.73 & -25.72 & & -20.68 & -25.65 & N/A \\
\hline 4.6 .4 & -27.62 & -34.50 & & -27.58 & -34.44 & N/A \\
\hline 4.6 .5 & -4.61 & -10.04 & & -4.61 & -10.04 & N/A \\
\hline 4.6 .6 & -10.07 & -15.14 & & -10.16 & -15.25 & N/A \\
\hline $4.6 .7 \_1$ & -76.32 & -83.10 & 8.40 & -75.99 & -82.75 & 8.40 \\
\hline $4.6 .7 \_2$ & -23.07 & -29.85 & 12.61 & -22.99 & -29.74 & 12.61 \\
\hline $4.6 .7 \_3$ & -16.31 & -22.20 & 16.31 & -15.70 & -21.15 & 15.70 \\
\hline $4.6 .7 \_4$ & -22.21 & -28.96 & 12.92 & -22.13 & -28.85 & 12.92 \\
\hline $4.6 .7 \_5$ & -16.38 & -22.33 & 16.38 & -15.77 & -21.26 & 15.77 \\
\hline 4.7 & -20.59 & -28.27 & 13.31 & -20.52 & -28.17 & 13.31 \\
\hline
\end{tabular}




\section{Contents}
Abstract ii
Figures and Tables. .. $\mathbf{i}$
Preface ..$x v i$

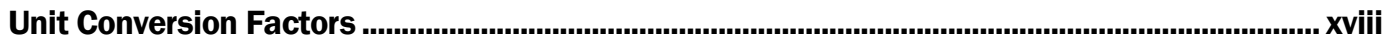
1 Analysis of an I-Wall in Fluvial and Coastal Environments ................................................. 1

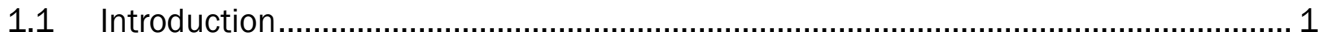

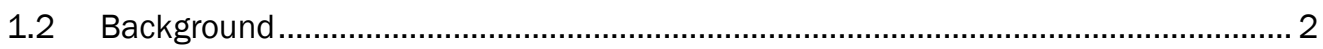
1.3 Overview of Corps_I-Wall .............................................................................. 5
1.4 Overview of Design and Analysis modes.......................................................... 11

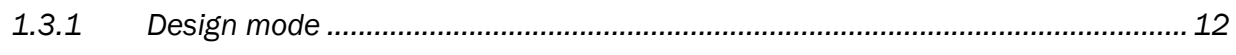

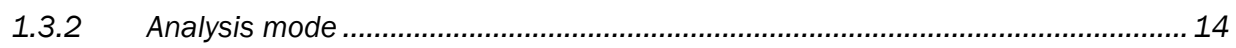

2 Engineering Formulation ....................................................................................................15

2.1 Layered soils site model................................................................................... 15

2.2 Types of analysis used to define earth pressures ................................................ 18

2.2.1 Total stress - mobilized active earth pressure .................................................. 21

2.2.2 Total stress - mobilized passive earth pressure.................................................. 23

2.2.3 Effective stress.............................................................................................. 24

2.2.4 Effective stress - mobilized active earth pressure .............................................. 25

2.2.5 Effective stress - mobilized passive earth pressure................................................ 26

2.3 Earth pressure coefficient methods used for computing earth pressures .............. 27

2.3.1 Rankine's active earth pressure coefficients ..................................................... 27

2.3.2 Rankine's passive earth pressure coefficients .................................................... 28

2.3.3 Coulomb's active earth pressure coefficients ........................................................ 29

2.3.4 Coulomb's passive earth pressure coefficients ..................................................... 30

2.3.5 Logarithmic-spiral method for the determination of a passive earth pressure coefficients ...................................................................................................... 31

2.4 Converting a trapezoidal pressure distribution acting on a discrete segment of the I-Wall face into equivalent end point forces ............................................33

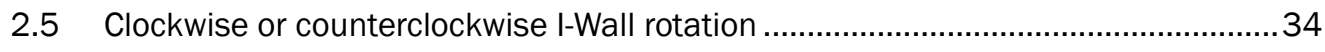

2.6 Net active pressure and net passive pressure .....................................................35

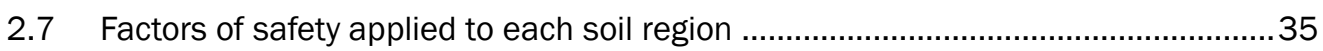

2.8 Distributions of water pressures acting on the I_Wall and sheet-pile wall due to surface water and piezometric surfaces ...............................................................36

2.9 Surcharge pressures, horizontal pressures and line loads......................................43

2.9.1 Externally applied horizontal pressure distribution and line loading....................43

2.9.2 Externally applied vertical pressure distribution and line loading .........................44

2.10 Shear force and moment distribution internal to the I-Wall and sheet pile .............46 


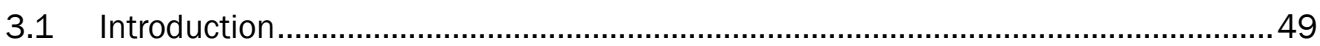

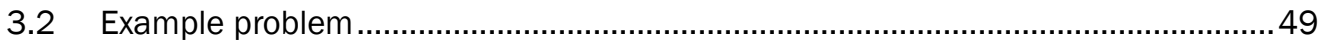

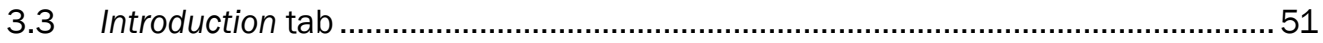

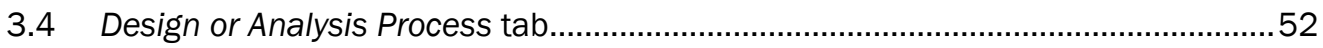

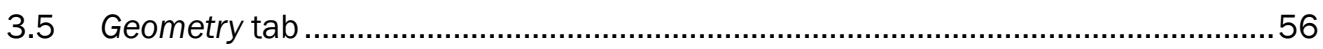

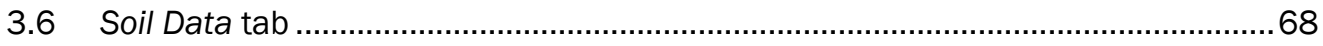

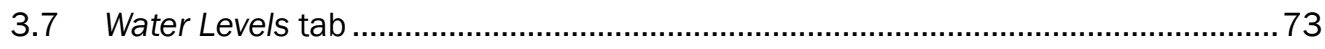

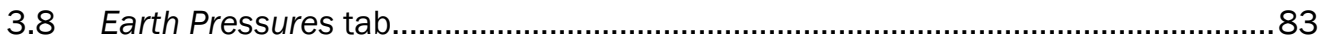

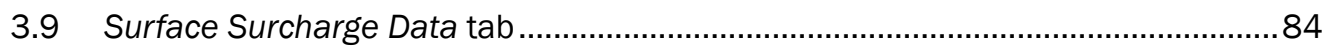

A.10 Applied Horizontal Loads tab........................................................................90

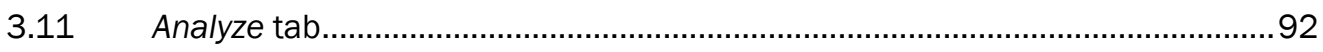

4 Deterministic Example Problems ........................................................................................133

4.1 Example 4.1 sand site, effective stress strength definition with $\varphi^{\prime}=30$, $c^{\prime}=0$, with either hydrostatic pore water pressures or homogeneous seepage ...............133

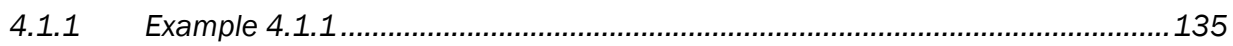

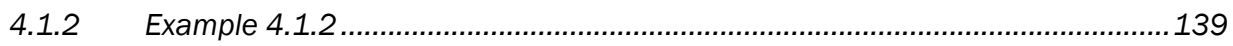

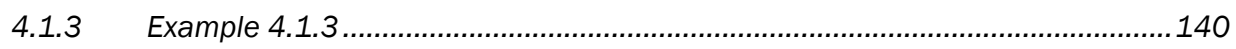

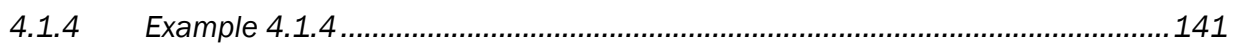

Example 4.1.5 ................................................................................................. 142

4.2 Example 4.1 sand site, effective stress strength definition with $\varphi^{\prime}=30 \mathrm{deg}$ and $c^{\prime}=125$ psf, with either hydrostatic pore water pressures or homogeneous

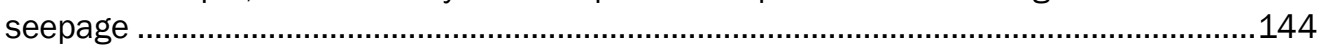

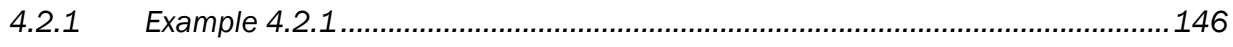

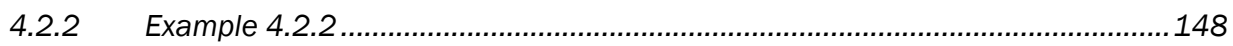

4.2.3 Example 4.2.3 ................................................................................................... 152

4.3 Example 4.3 clay site, total stress strength definition with $\phi=0 \ldots \ldots \ldots \ldots \ldots \ldots \ldots . . . . . . . .156$

4.4 Example 4.4 mixed total and effective stress based shear strength definitions in layered soils site ..............................................................................160

4.5 Coastal site evaluation, designing for wave loads .............................................166

4.5.1 Example 4.5.1: Coastal site, effective stress strength definition with $\varphi^{\prime}$

$=35^{\circ} \quad 167$

4.5.2 Example 4.5.2 Coastal site with surge loading .....................................................171

4.5.3 Example 4.5.3 Coastal site including backfill.......................................................179

4.5.5 Example 4.5.4 Coastal site including backfill with surge loading.........................185

4.5.6 Comparison and interpretation of backfill with surge loading .............................188

4.6 Example 4.6 comparison to Corps_ I-Wall results contained within other technical publications (Das; Bowles; Pace) ................................................................194

4.6.1 Example 4.6.1 sheet-pile wall penetrating sand (Das) .......................................195

4.6.2 Example 4.6.2 sheet-pile wall penetrating saturated clay (Das)..........................200

4.6.3 Sheet-pile wall penetrating sand (Bowles) .........................................................205

4.6.4 Sheet-pile wall penetrating saturated clay (Bowles)............................................211

4.6.5 Example 4.6.7 sheet-pile wall penetrating clay (Pace et al. 2012) ....................217

4.7 Example 4.7 Analysis mode of operation for a clay site, an extension of

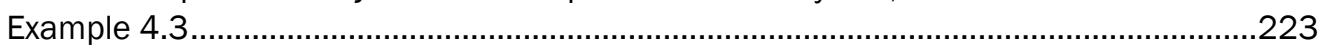


5.1 Fragility curve (i.e., system response curve)

5.2 Random variables for each soil layer $(\varphi, c, \delta, C a)$ and correlation between variables

5.3 Finding the system response curve for an analysis of an existing I-Wall ..............232

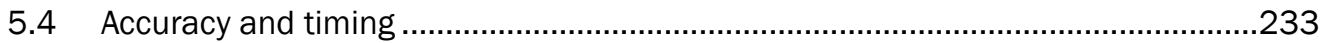

5.5 Example 5.1 sand site with hydraulic fracturing criteria and seepage, an extension of design problem 4.1.5.

5.5.1 Evaluation of results from the deterministic solution with flood elevation

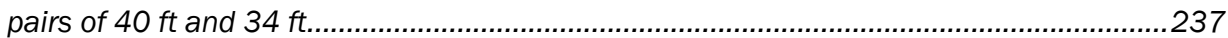

5.5.2 Evaluation of results for 1,000 simulations with seepage ................................241

5.5.3 Comparisons of results for 100, 300, 600 and 1,000 simulations.....................242

5.6 Example 5.2 system response curve (clay site with hydraulic fracturing, an extension of Example 4.7)

5.7 Comparisons of results for 100, 300, 1,000 and 2,000 simulations..... 248

5.8 Example 5.3 system response curve (two-layer sand site with hydraulic fracturing criteria and seepage) ......................................................................... 251

5.9 Comparisons of results for $100,300,500$ and 1,000 simulations .....................253

6 Summary and Conclusions ................................................................................................ 256

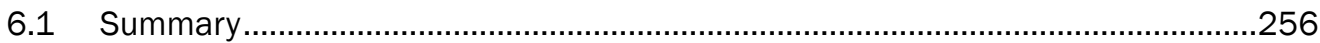

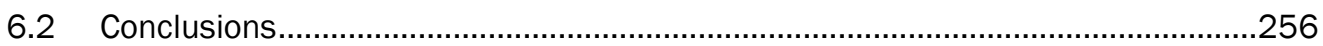

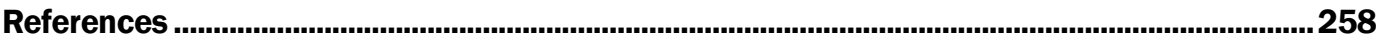

Appendix A: Iterative Solution to Compute the Design Depth Using a Force and a Moment Imbalance Based Computational Procedure.

Appendix B: Gap Initiation and Propagation Criteria and Formulation ....................................269

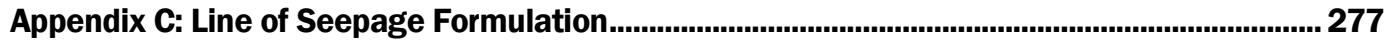

Appendix D: Description of Corps_I-Wall ASCII Input Data File (project.in).................................. 281

Appendix E: Description of Corps_I-Wall ASCII Output Data Files................................................. 292

Appendix F: Calculation of Coastal Surge Pressures Acting on an I-Wall for a Breaking Wave

\section{Report Documentation Page}




\section{Figures and Tables}

\section{Figures}

Figure 1.1. Idealization of I-Wall rotation about a PR within proximity of the sheet-pile tip during flood loading.

Figure 1.2. Schematic idealization of the consideration of stresses imposed on a soil element for a gap originating and propagating in an overconsolidated clay layer and stopping within the normally consolidated clay below, according to the method implemented within Corps_I-Wall.

Figure 1.3. Idealization of a system response curve for an I-Wall during variable flood elevation loading from 0 to $10 \mathrm{ft}$. 5

Figure 1.4. Corps_I-Wall Version 1.0 for level ground GUI.

Figure 2.1. I-Wall site model configuration for Corps_I-Wall Version 1.0 absent any flood loading......

Figure 2.2. I-Wall flood load problem model setup for a Corps_I-Wall Version 1.0 analysis, including wave loading.

Figure 2.3. Location of four select nodes identified along the RHS of the I-Wall for total vertical stress computations calculated during flood loading.

Figure 2.4. Distribution of stress acting normal to a discrete segment $k$ of the wall face.

(a) Trapezoidal pressure distribution. (b) Equivalent nodal point forces.

Figure 2.5. I-Wall rotation. (a) Counterclockwise rotation about a point of rotation PR due to flood loading applied to the right-hand side of the I-Wall. (b) The net water and earth

pressures acting on the I-Wall during its rotation.

Figure 2.6. Distributions of water pressures acting normal to the I-Wall and sheet-pile wall - hydrostatic water pressures. (a) Hydrostatic water and pore-water pressure distributions on the LHS and RHS. (b) The net water pressure distribution.

Figure 2.7. Distributions of hydrostatic water pressures acting normal to the I-Wall and sheet-pile wall - hydrostatic water pressures. (a) Hydrostatic water and pore water pressure distributions on the LHS and RHS. (b) The net water pressure distribution

Figure 2.8. Distributions of water pressures acting normal to the I-Wall and sheet-pile wall - homogenous seepage. (a) Water and pore-water pressure distributions on the LHS and RHS. (b) The net water pressure distribution.

Figure 2.9. Distributions of water pressures acting normal to the I-Wall and sheet-pile wall - hetergenous seepage for two soil layers, (a) Water and pore-water pressure distributions on the LHS and RHS (b) The net water pressure distribution.

Figure 2.10. External horizontal line load and pressures. (a) Line load. (b) Distributed pressures

Figure 2.11. Vertical line load.

Figure 2.12. Vertical pressure distributions. (a) Strip pressure. (b) Ramp pressure. (c) Triangular pressure. (d) Trapezoidal pressure.

Figure 2.13. Internal shear and moment at node $i+1$ for wall segment $k$. (a) Trapezoidal pressure distribution. (b) Equivalent nodal point forces.

Figure 2.14. Internal shear and moment at node $i+2$ for wall segment $k+1$. (a) Trapezoidal pressure distributions. (b) Equivalent nodal point forces. 
Figure 3.1. Example problem for an I-Wall section used to illustrate features of the GUI..................50

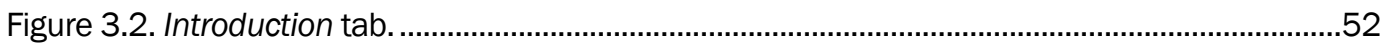

Figure 3.3. Design or Analysis Process - Design mode. .................................................................53

Figure 3.4. Design or Analysis process - Analysis mode. ................................................................55

Figure 3.5. Creation of geometry is done with the Geometry tab. ..................................................56

Figure 3.6 The procedure to input a node into the I-Wall geometry...................................................58

Figure 3.7a-d. The procedure for creating a new edge in the I-Wall geometry.................................60

Figure 3.8. The procedures for selecting a node, and for deleting the node (and its

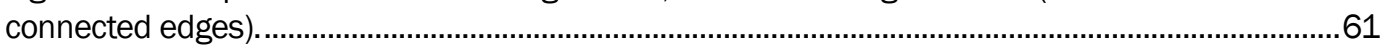

Figure 3.9. The procedure for selecting an edge. ......................................................................61

Figure 3.10a-b. The procedure for zooming with the right mouse. ..................................................63

Figure 3.11. Using the Zoom Extents button...............................................................................64

Figure 3.12. Modifying the extents of the soil regions to the sides of the I-Wall................................65

Figure 3.13a-c. Usage of the Undo button...................................................................................6

Figure 3.14. Using the Swap Landside button to reverse the Flood and Landsides of the

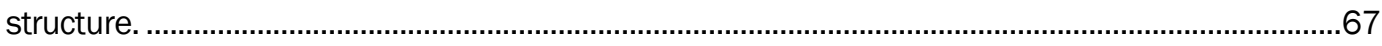

Figure 3.15 Generating regions adds new nodes and edges (to account for intersections with the I-Wall and other edges, and the full I-Wall area). Each area enclosed by edges is a region.

Figure 3.16 Choosing the method of gap creation......................................................................68

Figure 3.17. Entering region attributes with the Soil Data tab. ........................................................69

Figure 3.18. Reference values for soil types. .............................................................................70

Figure 3.19. Soil material probabilistic input................................................................................... 71

Figure 3.20. Selecting/changing a distribution. ………….........................................................

Figure 3.21. Water levels are defined either as level surface waters or piezometric

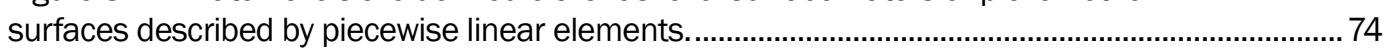

Figure 3.22. Choosing the appropriate seepage method. .................................................................75

Figure 3.23. Entering a homogeneous seepage parameter (and the warnings associated with this method)

Figure 3.24 Creating a piezometric surface highlighting the selector and region creation

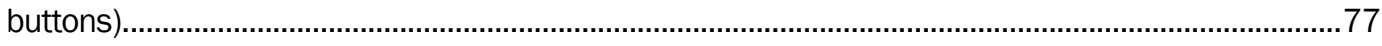

Figure 3.25. Assigning soil regions to a user-defined piezometric surface (whose point coordinates are in the piezometric area)....................................................................................

Figure 3.26. Assigning soil regions to the flood side surface water....................................................79

Figure 3.27. The piezometric surface immediately after the Add Landside Piezometric

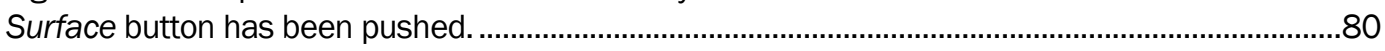

Figure 3.28. The points of the piezometric are in the grid below the point definition area................81

Figure 3.29. If an input point has an $X$ value that is already on the curve, then that point is moved to the new $Y$ location on confirmation....................................................................................82

Figure 3.30. After both ends of the piezometric surface have been moved. .....................................82

Figure 3.31. Choosing the coefficient method using the Earth Pressures tab. .................................83

Figure 3.32. Surface Surcharge Data tab. ..................................................................................84

Figure 3.33. The input window for line loads. ………………......................................................8 
Figure 3.34. The input window for uniform loads......................................................................

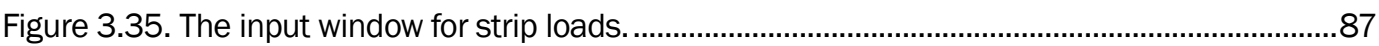

Figure 3.36. The input window for ramp loads. ......................................................................... 88

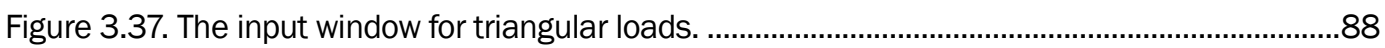

Figure 3.38. The input window for trapezoid/distributed loads. …….............................................89

Figure 3.39. Surface Surcharge Data tab with highlighted loads.....................................................90

Figure 3.40. The Applied Horizontal Loads tab allows input of point loads (on the right) and linearly distributed loads (on the left).....................................................................................

Figure 3.41. The Analyze tab at the beginning of a deterministic analysis.....................................93

Figure 3.42. The Analyze tab for a probabilistic analysis without landside water levels....................94

Figure 3.43. The Analyze tab for a probabilistic analysis with constant landside water level.

Figure 3.44. (a) The Analyze tab. (b) Its procedure for a probabilistic analysis with interpolated landside water levels. ......................................................................................................96

Figure 3.45. Plotting the input model for a deterministic analysis...................................................98

Figure 3.46. Plotting the input model for a probabilistic range of both flood and

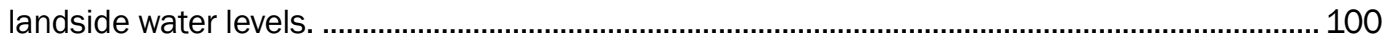

Figure 3.47. View of the pile without horizontal and vertical loads. .............................................. 100

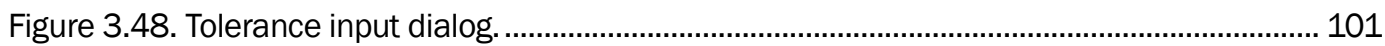

Figure 3.49. The Corps_I-Wall processor in action................................................................... 102

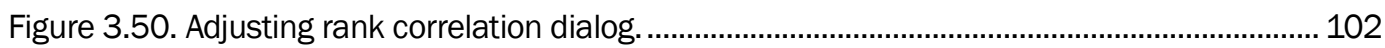

Figure 3.51. If problems occur during adjustment of rank correlation, this dialog appears......... 103

Figure 3.52. After the processor has finished, output options are made available. ...................... 104

Figure 3.53. The Run Data subsection allows the user to see processor input, results, and

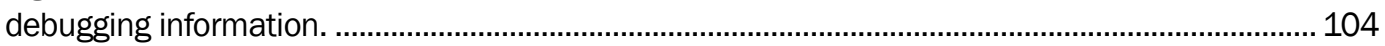

Figure 3.54. The display run window shows the output and debugging information from

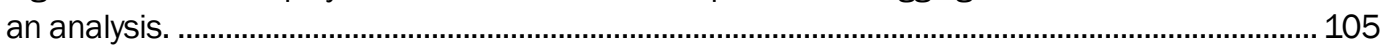

Figure 3.55. The display input data button allows the user to see the processor input file........... 106

Figure 3.56. The output plot subsection allows the user to see data based on a flood

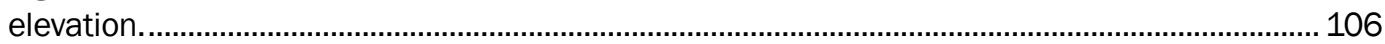

Figure 3.57. Pressure data for both the left and right sides of the I-Wall.........................................107

Figure 3.58. Left-side horizontal mobilized passive and active pressures. .................................... 108

Figure 3.59. Right-side horizontal mobilized active and passive pressures.................................. 108

Figure 3.60. Left- and right-side water pressures...................................................................... 109

Figure 3.61. Displaying the net pressure data....................................................................... 110

Figure 3.62. Active and passive net pressures....................................................................... 110

Figure 3.63. Displaying the net water pressures..................................................................... 111

Figure 3.64. Shear, moment, and combined net pressures........................................................ 111

Figure 3.65. Combined net pressures with soil and water pressures........................................... 112

Figure 3.66. Shear and moment diagrams for the I-Wall. .......................................................... 112

Figure 3.67. Display of seepage data for both the left and right sides of the I-Wall. ...................... 113

Figure 3.68. Seepage plot of total head loss in actual (untransformed) space............................. 115 


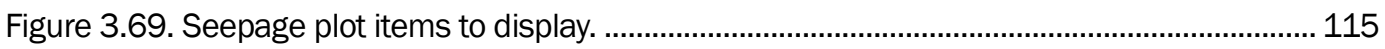

Figure 3.70. Seepage plots selector for untransformed or transformed space. ......................... 116

Figure 3.71. Seepage plot of total head loss in transformed space. ............................................. 116

Figure 3.72. Seepage plot of seepage gradient due to soil region properties (untransformed).

Figure 3.73. Defining internal and external material correlations for probabilistic analysis for Example 5.2 input.

Figure 3.74. Probabilistic analysis options for the Analysis option run for Example 5.2 from Chapter 5.

Figure 3.75. Warning that the correlation matrix between variables may change.

Figure 3.76. Error dialog that occurs when the software altered correlations cannot match the user input correlations.

Figure 3.77. Active probabilistic output options in the Analyze tab after execution of a Design mode.

Figure 3.78. Displayed sheet-pile tip elevation histogram data after execution of a Design mode.

Figure 3.79. Plotted sheet-pile tip elevation histogram data after execution of a Design mode.

Figure 3.80. Plotting the sheet-pile tip elevation histogram data as a set of linear segments passing through the center of each bin after execution of a Design mode.

Figure 3.81. The combined plot of bins and linear interpolation of sheet-pile tip elevation histogram data after execution of a Design mode.

Figure 3.82. Displayed cumulative sheet-pile tip elevation distribution data after execution of a Design mode.

Figure 3.83. Plotted cumulative sheet-pile tip elevation distribution data after execution of a Design mode.

Figure 3.84. Displayed sheet-pile tip depth histogram data after execution of a Design mode.

Figure 3.85. Plotted sheet-pile tip depth histogram data after execution of a Design mode. ....... 127

Figure 3.86. Displayed cumulative sheet-pile tip depth data after execution of a Design mode.

Figure 3.87. Plotted cumulative sheet-pile tip depth data after execution of a Design mode.

Figure 3.88. The selection of probabilistic output for an Analysis mode problem

Figure 3.89. Histogram data of passive Factor of Safety probabilities for flood elevation $4 \mathrm{ft}$ after execution of an Analysis mode.

Figure 3.90. Histogram plot of passive Factor of Safety probabilities for flood elevation $4 \mathrm{ft}$ after execution of an Analysis mode.

Figure 3.91. Cumulative distribution data of probabilities given a passive Factor of Safety for flood elevation $4 \mathrm{ft}$ after execution of an Analysis mode.

Figure 3.92. Cumulative distribution plot of probabilities given a passive Factor of Safety for flood elevation $4 \mathrm{ft}$ after execution of an Analysis mode.

Figure 3.93 Displayed system response curve for 1,000 simulations per flood elevation with 15 bins 
Figure 3.94. Plotted system response curve for 1,000 simulations per flood elevation with 15 bins

Figure 4.1. Sheet-pile wall in sand site. 134

Figure 4.2. Corps_I-Wall schematic of sheet-pile wall in sand site. 134

Figure 4.3. Active and passive earth pressures. (a) LHS of wall. (b) RHS of wall. 136

Figure 4.4. (a) Pore water pressures for the left- and right-hand side of the wall. (b) Net water pressures. 136

Figure 4.5. Net pressures. (a) Net active and net passive. (b) Net pressure diagram. 137

Figure 4.6. (a) Net pressure diagram. (b) Shear and moment. 137

Figure 4.7. Pore water pressures. (a) Left- and right-hand side of wall. (b) Net water pressures. 143

Figure 4.8. Line of seepage. (a) Direction of flow. (b) Total head along distance of flow. 144

Figure 4.9. Corps_I-Wall schematic of sheet-pile wall in sand site. 145

Figure 4.10. Active and passive earth pressures. (a) Left-hand side of wall, (b) Right-hand side of wall.

Figure 4.11. Pore water pressures. (a) Left- and right-hand side of wall (b) Net water pressures.

Figure 4.12. Net pressures. (a) Net active and net passive. (b) Net pressure diagram..................147

Figure 4.13. (a) Net pressure diagram. (b) Shear and moment..................................................... 148

Figure 4.14. Corps_I-Wall schematic of sheet-pile wall in sand site with two layers........................ 149

Figure 4.15. Active and passive earth pressures. (a) Left-hand side of wall (b) Right-hand side of wall.

Figure 4.16. Net pressures. (a) Net active and net passive. (b) Net pressure diagram................. 151

Figure 4.17. (a) Net pressure diagram. (b) Shear and moment. 152

Figure 4.18. Active and passive earth pressures. (a) Left-hand side of wall, (b) Right-hand side of wall.

Figure 4.19. Pore water pressures. (a) Left- and right-hand side of wall. (b) Net water pressures

Figure 4.20. Net pressures. (a) Net active and net passive. (b) Net pressure diagram................. 154

Figure 4.21. (a) Net pressure diagram. (b) Shear and moment..................................................... 154

Figure 4.22. Line of seepage. (a) Direction of flow. (b) Total head along distance of flow.............. 155

Figure 4.23. Corps_I-Wall schematic of sheet-pile wall in clay site.................................................. 157

Figure 4.24. Active and passive earth pressures for undrained shear strength. (a) Lefthand side of wall. (b) Right-hand side of wall.

Figure 4.25. Pore water pressures. (a) Left- and right-hand side of wall. (b) Net water pressures

Figure 4.26. Net pressures. (a) Net active and net passive. (b) Net pressure diagram.................. 159

Figure 4.27. (a) Net pressure diagram. (b) Shear and moment...................................................... 160

Figure 4.28. Corps_I-Wall schematic of sheet-pile wall in clay site.................................................... 162

Figure 4.29. Active and passive earth pressures. (a) Left-hand side of wall. (b) Right-hand side of wall

Figure 4.30. Pore water pressures. (a) Left- and right-hand side of wall. (b) Net water pressures. 
Figure 4.31. Net pressures. (a) Net active and net passive. (b) Net pressure diagram................. 164

Figure 4.32. (a) Net pressure diagram. (b) Shear and moment................................................... 164

Figure 4.33. Corps_I-Wall schematic of sheet-pile wall in sand site................................................. 168

Figure 4.34. Corps_I-Wall run file for the specified input. ............................................................ 168

Figure 4.35. Corps_I-Wall run file for the specified input. ............................................................ 169

Figure 4.36. Pore water pressures. (a) Left- and right- hand side of wall. (b) Net water

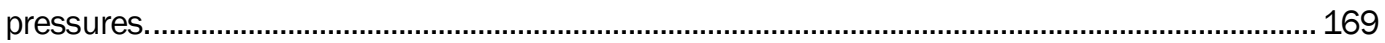

Figure 4.37. Net pressures. (a) Net active and net passive. (b) Net pressure diagram. ................. 170

Figure 4.38. (a) Net pressure diagram, (b) Shear and moment....................................................171

Figure 4.39. Drop in total heads due to seepage diagram. ........................................................ 172

Figure 4.40. The components of Minikin wave pressure diagram................................................174

Figure 4.41. Corps_I-Wall. (a) Schematic of sheet-pile wall in sand site. (b) Wave load applied to flood side (LHS) of sheet-pile wall. ………………………………………............... 175

Figure 4.42. Corps_I-Wall run file for the specified input. ...............................................................176

Figure 4.43. Active and passive earth pressures for drained shear strength. (a) Left-hand

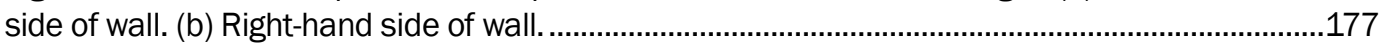

Figure 4.44. Pore water pressures. (a) Left- and right-hand side of wall. (b) Net water

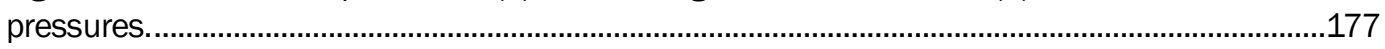

Figure 4.45. Net pressures. (a) Net active and net passive. (b) Net pressure diagram...................177

Figure 4.46. (a) Net pressure diagram. (b) Shear and moment.................................................. 178

Figure 4.47. Drop in total heads due to seepage diagram. ........................................................ 179

Figure 4.48. Sheet-pile wall with berm on landside (RHS). (a) Schematic of sheet-pile wall in sand site. (b) Plot of external loads. ............................................................................................ 181

Figure 4.49. Corps_I-Wall run file for the specified input. ........................................................... 182

Figure 4.50. Active and passive earth pressures for drained shear strength. (a) Left-hand side of wall. (b) Right-hand side of wall. ....................................................................................... 183

Figure 4.51. Pore water pressures. (a) Left- and right-hand side of wall. (b) Net water

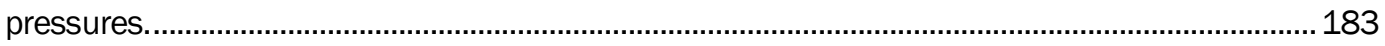

Figure 4.52. Net pressures. (a) Net active and net passive. (b) Net pressure diagram.................. 184

Figure 4.53. (a) Net pressure diagram. (b) Shear and moment..................................................... 184

Figure 4.54. Sheet-pile wall with berm on landside (RHS). (a) Schematic of sheet-pile wall in sand site. (b) Wave load applied to flood side (LHS) of sheet-pile wall. ....................................... 186

Figure 4.55. Corps_I-Wall run file for the specified input (with seepage enabled). ......................... 187

Figure 4.56. Corps_I-Wall run file for the specified input (without seepage)................................... 188

Figure 4.57. Representation (not to scale) of both the water and earth pressures acting on

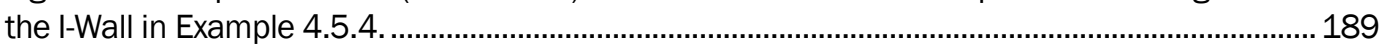

Figure 4.58. Results of lateral berm extent calculations for a 15-ft-high berm and a Factor

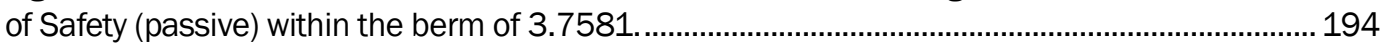

Figure 4.59. Sheet-pile wall penetrating sand. ……………………………………………….... 196

Figure 4.60. Corps_I-Wall schematic of sheet-pile wall in sand site................................................ 196

Figure 4.61. Corps_I-Wall output information. ........................................................................... 197

Figure 4.62. Active and passive earth pressures for drained shear strength. (a) Left-hand side. (b) Right-hand side. 
Figure 4.63. Hydrostatic water pressures. (a) Left- and right-hand side. (b) Net water

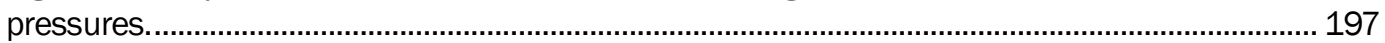

Figure 4.64. (a) Net pressure diagram. (b) Shear and moment diagram..................................... 199

Figure 4.65. (a) Net pressure diagram. (b) Shear and moment diagram......................................200

Figure 4.66. Sheet-pile wall penetrating saturated clay. ............................................................. 201

Figure 4.67. Corps_I-Wall schematic of sheet-pile wall in sand and saturated clay site................. 201

Figure 4.68. Corps_I-Wall output information.......................................................................... 202

Figure 4.69. Active and passive earth pressures. (a) Left-hand side. (b) Right-hand side............. 203

Figure 4.70. Hydrostatic water pressures. (a) Left- and right-hand side. (b) Net water pressures.

Figure 4.71. Net pressures. (a) Net active and net passive pressures. (b) Net pressure

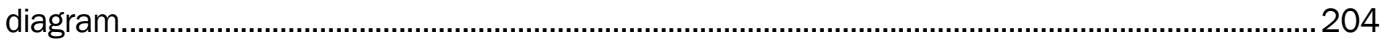

Figure 4.72. (a) Net pressure diagram. (b) Shear and moment diagram. ..................................... 204

Figure 4.73. Sheet-pile wall penetrating sand. …………................................................................ 207

Figure 4.74. Corps_I-Wall schematic of sheet-pile wall in sand site. ............................................. 207

Figure 4.75. Corps_I-Wall output information.......................................................................... 208

Figure 4.76. Active and passive earth pressures for drained shear strength. (a) Left-hand

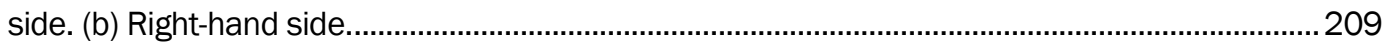

Figure 4.77. Hydrostatic water pressures. (a) Left- and right-hand side. (b) Net water pressures. 209

Figure 4.78. Net pressures. (a) Net active and net passive pressures. (b) Net pressure

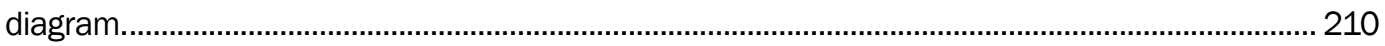

Figure 4.79. (a) Net pressure diagram. (b) Shear and moment diagram..................................... 210

Figure 4.80. Corps_I-Wall output information............................................................................... 211

Figure 4.81. Sheet-pile wall penetrating saturated clay.................................................................. 213

Figure 4.82. Corps_I-Wall schematic of sheet-pile wall in sand and saturated clay site. ............... 213

Figure 4.83. Corps_I-Wall output information. ......................................................................... 214

Figure 4.84. Active and passive earth pressures. (a) Left-hand side. (b) Right-hand side............. 214

Figure 4.85. Hydrostatic water pressures. (a) Left- and right-hand side. (b) Net water pressures.

Figure 4.86. Net pressures. (a) Net active and net passive pressures. (b) Net pressure

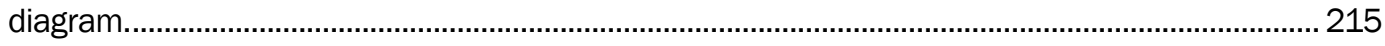

Figure 4.87. (a) Net pressure diagram. (b) Shear and moment diagram..................................... 216

Figure 4.88. Corps_I-Wall output information. ...............................................................................217

Figure 4.89. Sheet-pile wall penetrating clay. …………………………………………….... 218

Figure 4.90. Corps_I-Wall schematic of sheet-pile wall in clay site................................................. 219

Figure 4.91. Corps_I-Wall output information. .......................................................................... 219

Figure 4.92. Active and passive earth pressures. (a) Left-hand side. (b) Right-hand side.............. 220

Figure 4.93. Hydrostatic water pressures. (a) Left- and right-hand side.(b) Net water pressures

Figure 4.94. Net pressures (a) Net active and net passive pressures. (b) Net pressure diagram.

Figure 4.95. (a) Net pressure diagram. (b) Shear and moment diagram. ...................................... 221 
Figure 4.96. Corps_I-Wall schematic of sheet-pile wall in clay site.

Figure 4.97. Active and passive earth pressures for undrained shear strength. (a) Lefthand side of wall, (b) Right-hand side of wall.

Figure 4.98. Pore water pressures. (a) Left- and right-hand side of wall. (b) Net water pressures.

Figure 4.99. Net pressures. (a) Net active and net passive. (b) Net pressure diagram. 226

Figure 4.100. (a) Net pressure diagram. (b) Shear and moment.

Figure 5.1. System response curve for rotational limit state. 229

Figure 5.2. System response curve. (a) underestimated flood elevations for SRC,

(b) overestimated flood elevations for SRC, (c) located region of SRC curve................................... 233

Figure 5.3. Corps_I-Wall schematic of sheet-pile wall in sand site. ............................................... 235

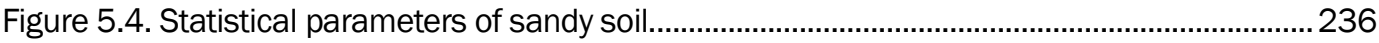

Figure 5.5. Active and passive earth pressures. (a) left hand side of wall, (b) right hand side of wall.

Figure 5.6. Pore water pressures. (a) left and right hand side of wall, (b) net water pressures.

Figure 5.7. Net pressures. (a) Net active and net passive. (b) Net pressure diagram. 239

Figure 5.8. (a) Net pressure diagram. (b) Shear and moment. ........................................................ 240

Figure 5.9. Line of seepage. (a) Direction of flow. (b) Head loss along distance of flow..................241

Figure 5.10. Output runtime file.

Figure 5.11. Design probabilistic analysis of sheet-pile tip with 1,000 simulations

(a) Histogram. (b) Cumulative distribution function.

Figure 5.12. Design probabilistic analysis of depth of embedment with 1,000 simulations

(a) Histogram. (b) Cumulative distribution function. 244

Figure 5.13. Cumulative distribution function for sheet-pile tip elevation for $100,300,600$ and 1,000 simulations.

Figure 5.14. Schematic of sheet-pile wall in clay site for various water levels. 246

Figure 5.15. Statistical parameters for the clayey soil of material M01.

Figure 5.16. System response curve for 1,000 simulations at 0.25-ft and $1-\mathrm{ft}$ flood increments.

Figure 5.17. System response curve for $100,300,1,000$ and 2,000 simulations at 0.25-ft

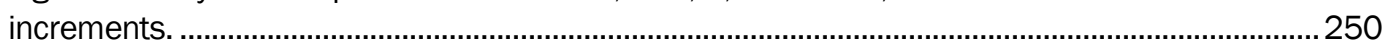

Figure 5.18. Schematic of sheet-pile wall in sandy site for 12 flood levels. .................................. 252

Figure 5.19. Statistical parameters for the sandy soils. ................................................................ 253

Figure 5.20. System response curve for $100,300,500$ and 1,000 simulations.......................... 255

Figure A1. Cantilever retaining wall. (a) Two layered soil site. (b) Counterclockwise rotation of the cantilever sheet-pile wall about its point of rotation (PR).

Figure A2. Schematic of summation of horizontal forces versus trial points of rotation. 265

Figure A3. Schematic of summation of moments versus trial points of rotation. 266

Figure B1. Schematic of gap propagation for a soil element above the point of rotation with a mobilized total horizontal earth pressure definition.

Figure B2. Schematic of gap propagation for a soil element above the point of rotation with a mobilized effective horizontal earth pressure definition. 
Figure B3. Schematic of gap propagation for a soil element located at the point of rotation with a mobilized total horizontal earth pressure definition.

Figure B4. Schematic of gap propagation for a soil element located above the point of rotation with a mobilized effective horizontal earth pressure definition.

Figure C1. I-Wall. (a) Potential slip plane within soil regions with different saturated hydraulic conductivities $(K)$ (lengths not to scale). (b) Constant slope in total head with distance along the transformed length of line of seepage. (c) Variation of slope in total head with distance along the three soil regions of the line of seepage.

Figure F1. Profile used in the initial I-Wall design calculations, including a soil buttress on the landside using an overly simplified and incomplete representation of wave loadinduced, pseudo-static pressures.

Figure F2. Minikin breaking wave pressure diagram (after Figure 7-99; Shore protection manual 1984).

Figure F3. Dimensionless design breaker height versus relative depth at the wall (after Figure 7-4 Shore protection manual 1984 and Weggel 1972).

Figure F4. Dimensionless Minikin wave pressure and force (after Figure 7-100; Shore protection manual 1984).

\section{Tables}

Table 1.1. Method appropriate for computing the passive earth pressure coefficient,

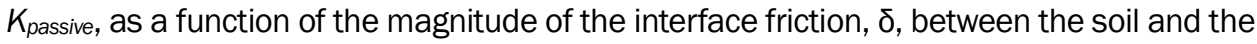
sheet pile and in comparison to the magnitude of the soil's angle of internal friction, $\varphi$

Table 2.1. I-Wall flood loading problem soil layering, piezometric surface definitions, and material property specifications for a Corps_I-Wall Version 1.0 model setup.

Table 2.2. Passive earth pressure coefficient $K$ Passive $(K p)$ values for $-\delta$ equal to $\varphi$ and a solution based on assuming a failure surface composed of logarithmic spiral portion and a planar surface portion.

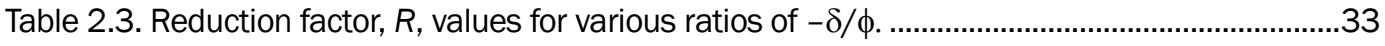

Table 3.1. Input soil properties for the example problem................................................................51

Table 4.1. Examples of effective stress strength definitions (sands with $c^{\prime}=0$ ). ...........................138

Table 4.2. Results from wall friction angles with $K_{p}$ coefficients from logarithmic spiral procedure.

Table 4.3. Examples of effective stress strength definitions (sands with $c^{\prime}=125$ ).

Table 4.4. Multilayered soils with effective stress strength definitions (sands with $c^{\prime}=0$ ) and total stress strength definitions (clays with $\varphi=0$ ).

Table 4.5. Hydrostatic pressures with elevations from the site.

Table 4.6. Combining the left-hand side and right-hand side hydrostatic pressures to get the net pressure.

Table 4.7. Dynamic water pressures with elevations calculated with the Minikin method.

Table 4.8. Forces and moments calculated from water pressure distributions at the datum elevation.

Table 4.9. Determining earth pressures on the right-hand side of the wall with Factor of Safety 1.5 .

Table 4.10. Forces and moments acting on the right-hand side of the wall with Factor of Safety 1.5. 
Table 4.11. Determining earth pressures on the right-hand side of the wall with Factor of Safety 3.7581

Table 4.12. Forces and moments acting on the right-hand side of the wall with Factor of Safety 3.7581 193

Table 4.13. Comparisons of I-Wall results contained within Das (2007). 198

Table 4.14. Comparisons of I-Wall results contained within Bowles (1968). 206

Table 4.15. Comparisons of I-Wall results contained within Pace (2012). 222

Table 5.1. Correlations used among variables in some example problems. 231

Table 5.2. Ranges of the most frequent values of coefficients of variation. 232

Table 5.3. Execution times for 100, 300, 600 and 1,000 simulations. 245

Table 5.4. Probabilities of a rotational limit state for 100, 300, 1,000 and 2,000 number of simulations. 249

Table 5.5. Execution times for 100, 300, 1,000 and 2,000 number of simulations. 251

Table 5.6. Probabilities of a rotational stability for $100,300,500$ and 1,000 simulations. .......... 254

Table 5.7. Execution times for 100, 300, 500 and 1,000 simulations. 255

Table A1. Factors of Safety assigned to the sand and clay layers. 262

Table A2. PR elevations bounding the zero crossings for summation of forces. 265

Table A3. PR elevations bounding the zero crossings for summation of moments. 266

Table F1. Summary of 2008 preliminary I-Wall analysis results for varied heights of soil surface on the landside for an overly simplified and incomplete representation of wave load-induced pressures. 


\section{Preface}

The response of I-Walls in New Orleans, LA, during Hurricane Katrina in August 2005 brought to light the possible development of a zone of separation along the flood side of the soil-to-I-Wall interface as flood loading occurs. Both the field observations of the New Orleans I-Walls and the results from two series of complete soil-structure interaction (SSI) nonlinear finite element studies for I-Walls at New Orleans and other locations demonstrated the need for the engineering analysis of an I-Wall to account for gap initiation and gap propagation on the flood side of an I-Wall. The large number of existing and planned Corps I-Walls in riverine, fluvial, and coastal environments reveals the importance of having methods to predict and plan for this condition with readily available PC software tools.

The purpose of the I-Wall Analysis research and development effort is to provide the U.S. Army Corps of Engineers (USACE) districts with essential tools in analyzing and evaluating I-Wall performance in riverine, fluvial, and coastal environments. Accurate, easy-to-use, PC-based software is required for day-to-day District deterministic- or probabilistic-based designs/evaluations of I-Walls.

This technical report summarizes an engineering method addressing these needs and its implementation in the software Corps_I-Wall, Version 1.o. This initial version of Corps_I-Wall is used for the design of new I-Walls or the analysis of existing I-Walls in floodplains (i.e., I-Walls embedded in level ground) during flood loading. Horizontally stratified soil layers are assumed for Version 1.0.

The engineering formulation and software programming reported herein was authorized by Headquarters, U.S. Army Corps of Engineers (HQUSACE), and was conducted under the Infrastructure Resiliency and Reliability Research Program. The Technical Director for Infrastructure Resiliency and Reliability Research Program was Dr. Michael K. Sharp, U.S. Army Engineer Research and Development Center (ERDC), Geotechnical and Structures Laboratory (GSL). Dr. Maureen Corcoran, GSL Associate Technical Director, was the Program Manager for the Infrastructure Resiliency and Reliability Research Program. Funding was provided by the Infrastructure Resiliency and Reliability Research 
Program under the Flood and Coastal Storm Damage (FCSDR) Reduction Research and Development Program in the focus area title I-Wall Analysis. William R. Curtis was the Technical Director of the Coastal and Hydraulics Laboratory (CHL) for the FCSDR Program. The Program Manager for FCSDR was Dr. Cary A. Talbot, CHL.

The research discussed in this report was led by Dr. Robert M. Ebeling, Information Technology Laboratory (ITL), under the general supervision of Dr. Reed L. Mosher, Director, ITL; Patti S. Duett, Deputy Director, ITL. This work effort was also done under the general supervision of Dr. Robert M. Wallace, Chief, Computational Science and Engineering Division (CSED), ITL, during software development. During a majority of the report publication process, Elias Arredondo, Dr. Kevin Abraham and Dr. Jerrell R. Ballard were Acting Division Chiefs. Dr. Ballard is the CSED Chief for the final stage of the publication process. Dr. Ebeling was Principal Investigator of the I-Wall Analysis work unit. This report was written and the software was developed by Dr. Ebeling, Moira T. Fong (retired), and Barry C. White of ITL. Fong and White are with the Computational Analysis Branch (CAB), of which Elias Arredondo was Chief.

Anjana Chudgar (HQ-retired), Kent D. Hokens, and Neil T. Schwanz, both of the USACE St. Paul District, were team members providing input and guidance on District needs during the course of this research.

COL Brian S. Green was the Commander of ERDC, and Dr. Jeffery P. Holland was the Director. 


\section{Unit Conversion Factors}

\begin{tabular}{|l|l|l|}
\hline Multiply & By & To Obtain \\
\hline cubic feet & 0.02831685 & cubic meters \\
\hline cubic inches & $1.6387064 \mathrm{E}-05$ & cubic meters \\
\hline degrees (angle) & 0.01745329 & radians \\
\hline feet & 0.3048 & meters \\
\hline inches & 0.0254 & meters \\
\hline pounds (force) & 4.448222 & newtons \\
\hline pounds (force) per square foot & 47.88026 & pascals \\
\hline pounds (force) per square inch & 6.894757 & kilopascals \\
\hline square feet & 0.09290304 & square meters \\
\hline square inches & $6.4516 \mathrm{E}-04$ & square meters \\
\hline square miles & $2.589998 \mathrm{E}+06$ & square meters \\
\hline
\end{tabular}




\section{Analysis of an I-Wall in Fluvial and Coastal Environments}

\subsection{Introduction}

Hurricane Katrina produced unparalleled wave and storm surge conditions for the New Orleans vicinity when it passed the New Orleans area on the morning of 29 August 2005 (IPET 2007). To provide an understanding of the impact of Hurricane Katrina on the New Orleans Hurricane Protection System in New Orleans, LA, the Interagency Performance Evaluation Task Force (IPET) was established by the Chief of Engineers. As a result of this study, a possible development of a zone of separation was identified that formed along the flood side of the soil-to-I-Wall interface as flood loading occurred (IPET 2007). In response to this discovery, Headquarters, U.S. Army Corps of Engineers (HQUSACE), recognized the lack of a method to fully evaluate I-Walls and thereby tasked the U.S. Army Engineer Research and Development Center (ERDC) with providing the USACE districts with the engineering capability to analyze and evaluate I-Wall performance in all geomorphic environments, fluvial, riverine, and coastal, in which I-Walls exist. To meet this objective, ERDC developed and designed the Corps_I-Wall software Version 1.o. Corps_I-Wall includes a PC-based FORTRAN (Formula Translating System) program, a Graphical User Interface (GUI), engineering processor, and visual postprocessor. The Corps_I-Wall software is accessed using the CI-Wall executable program contained on ERDC's Knowledge Hub (https://knowledge usace.army.mil) within the Computer-Aided Structural Engineering (CASE) community. This version of Corps_I-Wall is used for the design of new I-Walls or the analysis of existing I-Walls in a fluvial or coastal environment (i.e., I-Walls embedded in level ground or floodplain) during flood loading. In coastal regions, the capability to analyze I-Wall performance to storm surge is of particular importance because of the potential destructive force of storm surge. Horizontally-stratified soil layers are assumed for Version 1.0. As the development of this program continues, the remaining environments will be included in Corps_I-Wall Version 2.0. This technical report summarizes Corps_I-Wall, Version 1.o. 


\subsection{Background}

Two previous numerical studies (IPET 2007; Pace et al. 2012) of complete soil-structure interaction (SSI) using nonlinear finite element analyses were conducted by ERDC that directly apply to developing Corps_I-Wall. The studies used different soils and cross sections. Dr. Robert Ebeling, of the ERDC-Information Technology Laboratory, was the technical lead on both complete SSI studies and principal investigator on the second (2012) study.

As part of the IPET evaluation, the first study devised a method to assess the implementation of a zone of separation in the complete SSI analyses of four different New Orleans I-Wall cross sections as floodwaters were raised (in 0.5 to 1 -ft increments). The second study followed the same approach. The assessment included when and to what depth the zone of separation was expected to occur. Hydrostatic floodwater loading of the I-Wall due to floodwater penetration was then applied to understand the effect of loading on the zone of separation.

The second complete SSI numerical effort investigated I-Walls embedded in level ground consisting of four different soils that were stronger and stiffer than the fine-grained New Orleans soils (Pace et al. 2012). The different soils considered I-Walls founded in overconsolidated lean clay, stratified clays of high plasticity, homogenous clay, and silt of low plasticity.

Both the field observations of the New Orleans I-Walls made during the IPET study and the results from the two complete SSI nonlinear finite element studies demonstrated the need for the analysis to account for gap initiation and gap propagation on the flood side of an I-Wall as floodwaters rise. An important observation is revealed by these two complete SSI numerical studies of I-Walls designed according to Corps guidance, EM 1110-2-2504 (HQUSACE 1994): When an I-Wall posesses global stability and local stability of the I-Wall dominates the soil pressures acting on the I-Wall, there is a tendency for the I-Wall to rotate about a point (designated as point of rotation (PR) in Figure 1.1) that is often within the proximity of the sheet-pile tip. This deformation behavior results in the development of a passive soil zone on the flood side of the I-Wall, located between the point of I-Wall rotation and the sheet-pile tip (i.e., within Quadrant IV of Figure 1.1). In Figure 1.1, the quadrants are identified as zones of either (mobilized) active or (mobilized) passive earth pressures and also include the side of the I-Wall where the soil zone exists. Quadrant I is the soil zone labeled as the 
active zone on the flood side of the I-Wall; Quadrant II is the soil zone labeled as the passive zone on the landside of the I-Wall; Quadrant III is the soil zone labeled as the active zone on the landside of the I-Wall; and Quadrant IV is the soil zone labeled as the passive zone on the flood side of the I-Wall.

Figure 1.1. Idealization of I-Wall rotation about a PR within proximity of the sheet-pile tip during flood loading.

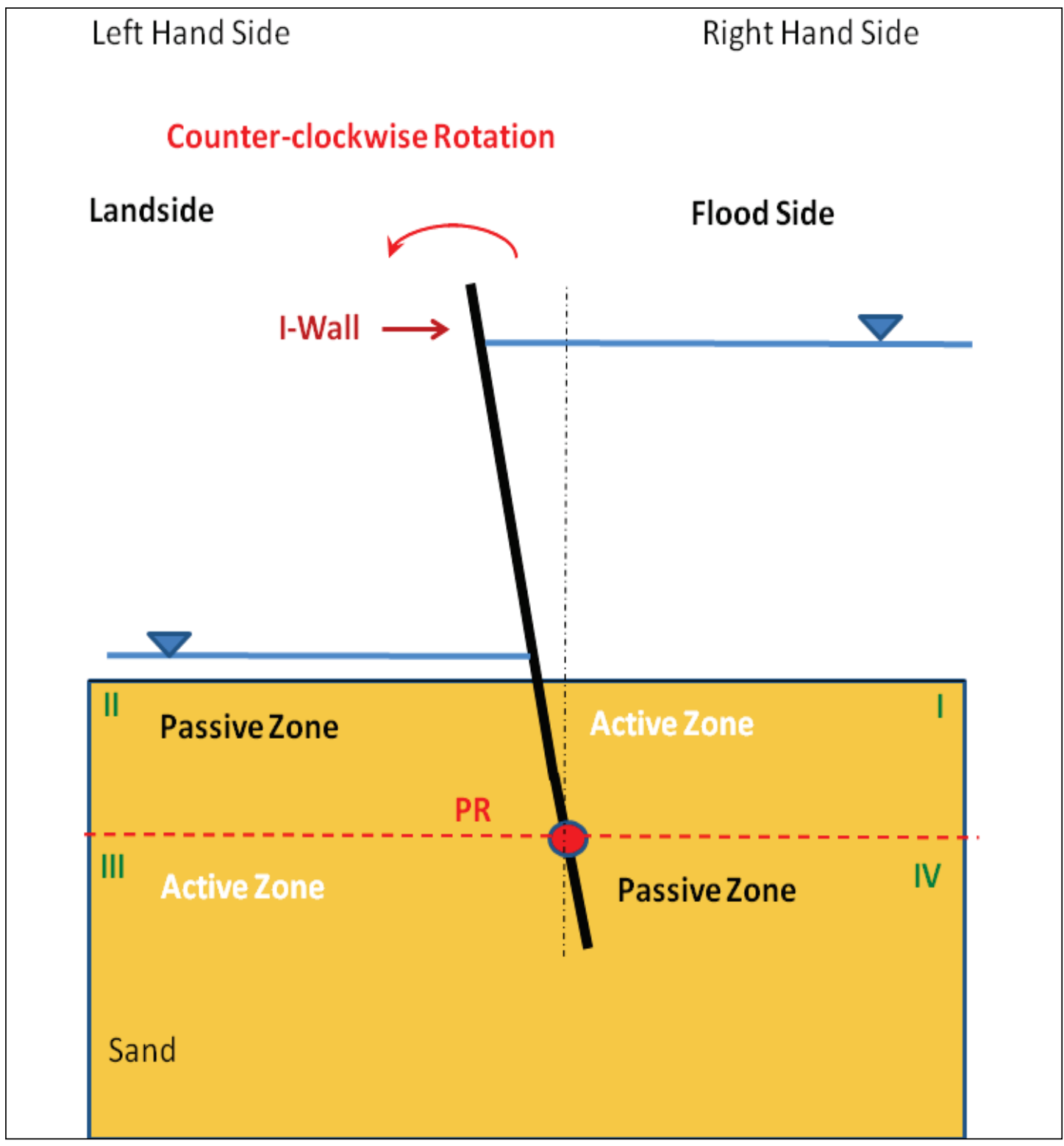

The complete SSI numerical studies have also shown that a sufficiently large magnitude (mobilized) passive pressure within this zone (Quadrant IV, Figure 1.1) below the point of rotation can terminate gap propagation in a cohesive soil on the flood side of the I-Wall so long as 
global instability and seepage along the sheet pile or an underseepageinduced piping failure are not an issue.

Thus, the ability to identify gap initiation and to propagate this gap on the flood side of an I-Wall is an important capability that has been implemented within the Corps_I-Wall engineering computational procedure of analysis (Figure 1.2). ${ }^{1}$ This capability is especially needed for analyses in which the flood elevation is increased for the different loading cases being considered, as is the case when constructing a system response curve (SRC) (Figure 1.3) in a probabilistic fragility analysis. An SRC is also known as a fragility curve. The SRC in Figure 1.3 shows the probability of a rotational limit state range in magnitude between a value of $o$ and 1.o, with a higher probability of failure occurring for higher flood elevations.

Figure 1.2. Schematic idealization of the consideration of stresses imposed on a soil element for a gap originating and propagating in an overconsolidated clay layer and stopping within the normally consolidated clay below, according to the method implemented within Corps_I-Wall.

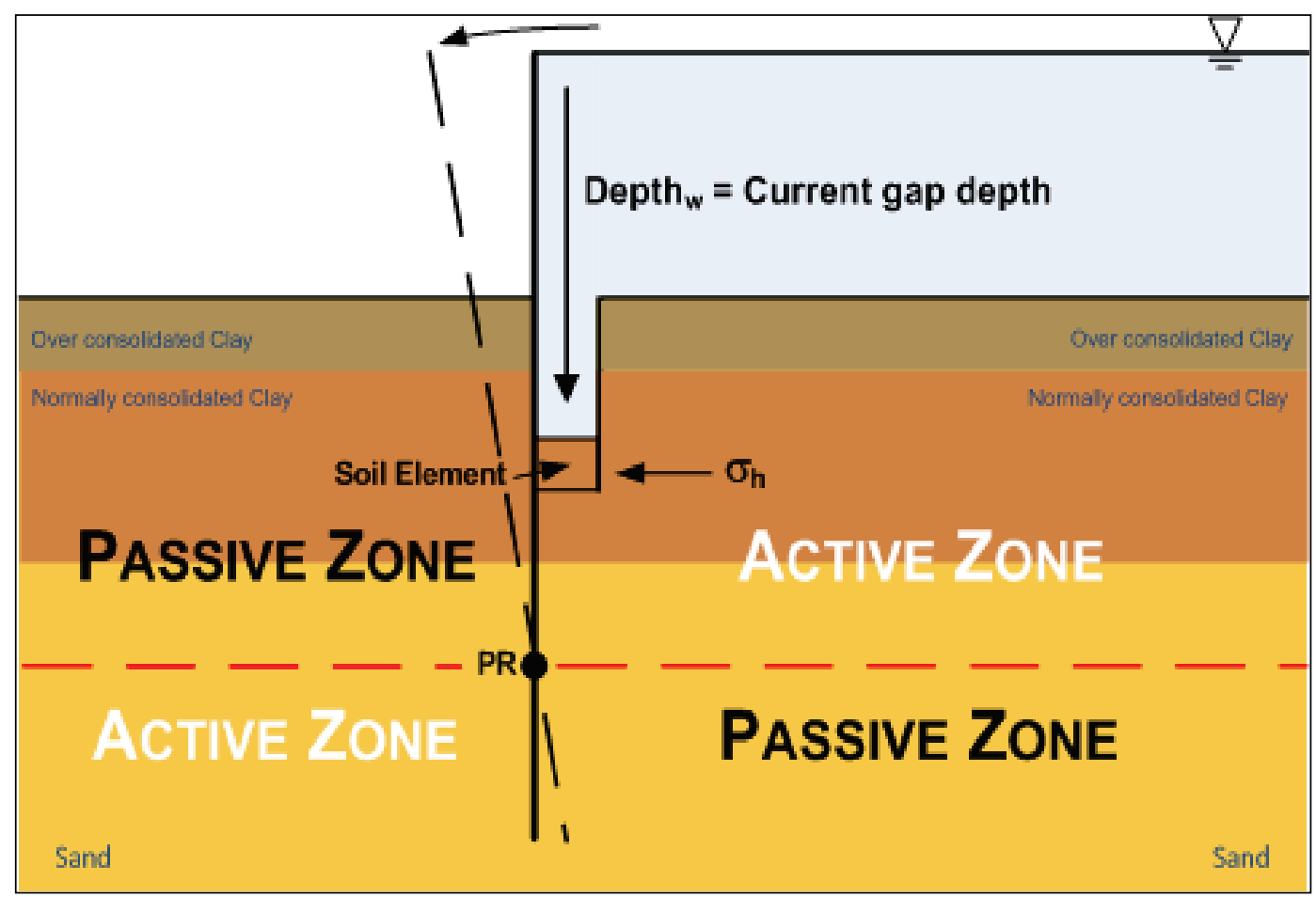

\footnotetext{
${ }_{1}$ Appendix B describes the hydraulic fracture phenomenon applied in Corps-I-Wall Version 1.0 to gap
} initiation/propagation. 
Figure 1.3. Idealization of a system response curve for an I-Wall during variable flood elevation loading from 0 to $10 \mathrm{ft}$.

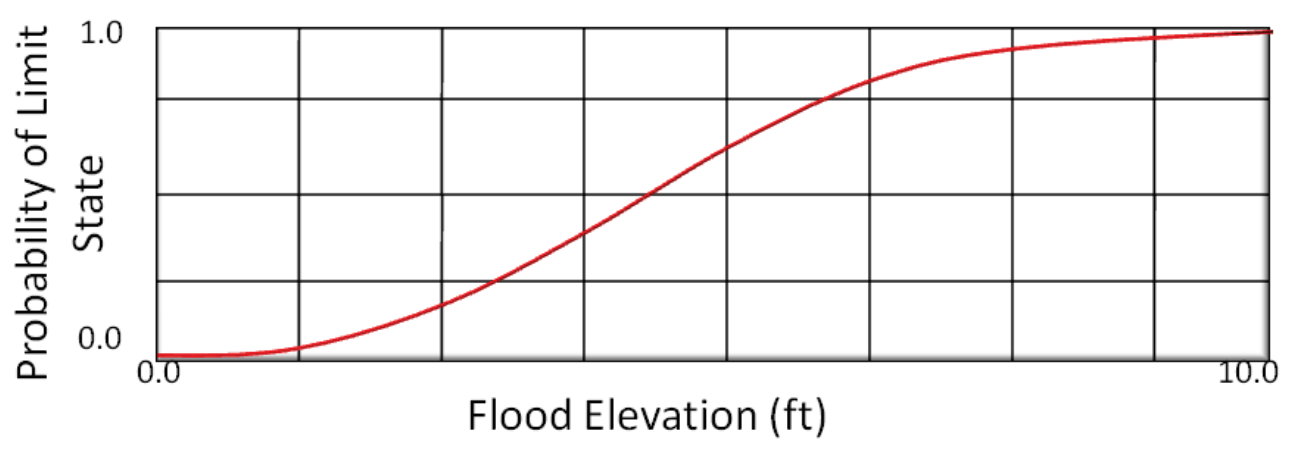

\subsection{Overview of Corps_I-Wall}

This section briefly describes selecting a method for analysis, Deterministic or Probabilistic, and includes the problem-solving capabilities within Corps_I-Wall. The purpose of this discussion is to provide the user with an understanding of engineering features involved in I-Wall analysis: layered soil strata, factors of safety, total or effective stress analysis, piezometric surface or surface water, gap initiation/propagation, clockwise or counterclockwise, I-Wall rotation, boundary pressures, point loads, and methods for computing the earth pressure coefficient. For a visual representation of the features involved in processes, Figure 1.4 depicts the engineering features relevant to an analysis within Corps_I-Wall. The capability of Corps_I-Wall to evaluate a coastal problem is also described.

The visual modeler for Corps_I-Wall is tab-based as shown in Figure 1.4. Nine tabs define the I-Wall problem configuration as well as the input of the soils properties: Introduction, Design or Analysis Process, Geometry, Soil Data, Water Levels, Earth Pressures, Surface Surcharge Data, Applied Horizontal Data, and Analyze. For a detailed discussion of these tabs, refer to Chapter 3.

Deterministic or Probabilistic: When selecting a method for analysis, either Deterministic or Probabilistic ${ }^{1}$, the user must consider the inherent aleatoric uncertainty of soil properties. In Corps_I-Wall, the floodwall loading conditions on I-Walls can be analyzed for a deterministic problem as well as for a probabilistic method of analysis based on consideration of

\footnotetext{
1 Refer to the user's selection option for selection of either probabilistic or deterministic analysis located above the labels for the nine tabs shown in Figure 1.4.
} 
Figure 1.4. Corps_I-Wall Version 1.0 for level ground GUI.

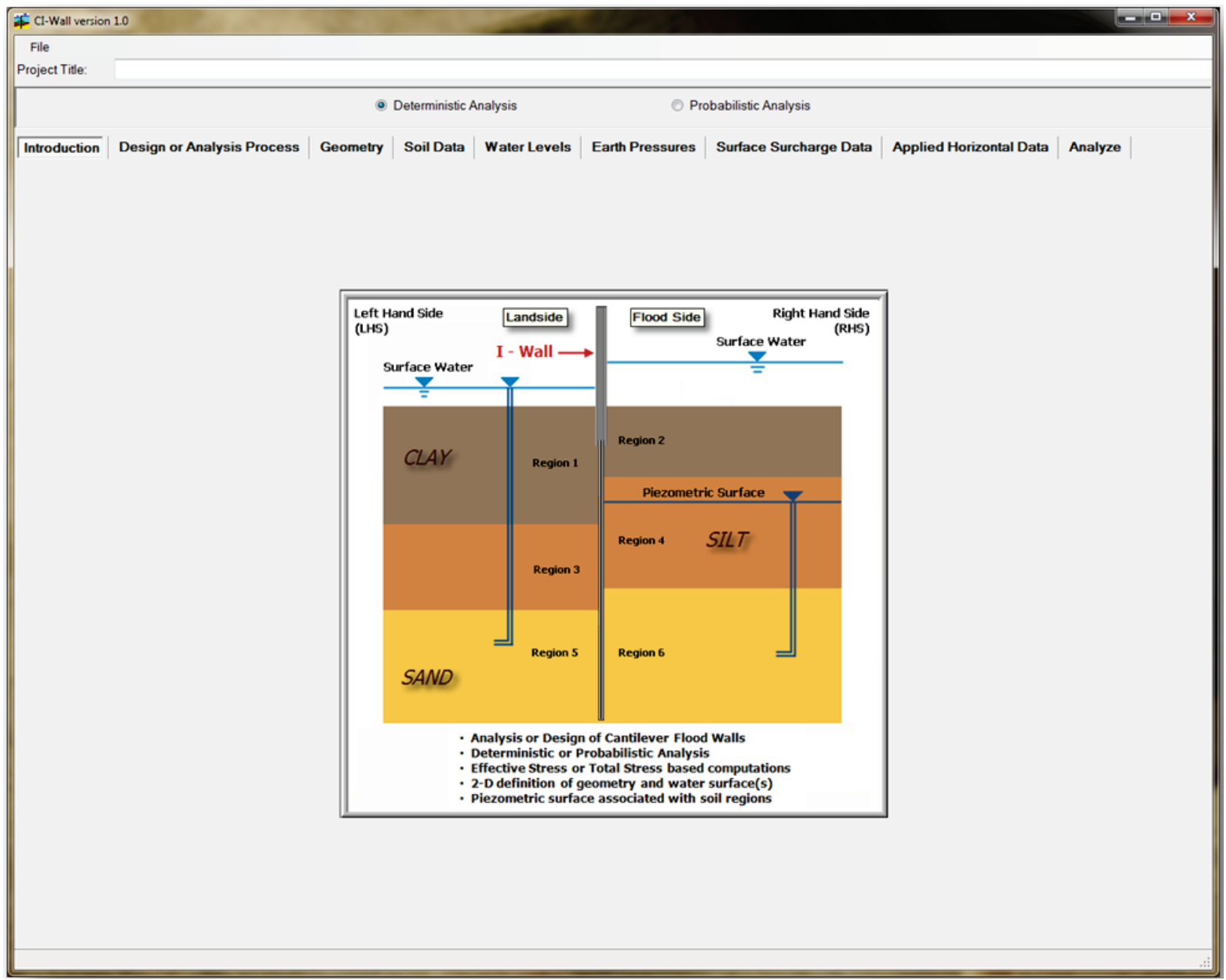

the inherent aleatoric uncertainty of soil properties. In a deterministic I-Wall design or analysis, all user-specified input for the shear strength parameters of the soils is made with absolute certainty in their assigned values. In a probabilistic analysis, the user has the ability to account for uncertainty in the soil's shear strength parameters by specifying for each variable in each soil region, a mean value, its standard deviation, and its distribution type (e.g., truncated normal, logarithmic normal, or uniform). When the user selects both the Probabilistic feature and conducts an Analysis of an existing I-Wall for a user-specified sheet-pile tip elevation, a Fragility Analysis is performed. The computations result in construction of a SRC (Figure 1.3). This SRC depicts the probability of a rotation about a point along the embedded portion of the sheet-pile wall as a function of flood elevation. In a safety or risk assessment of I-Walls, the rotational limit state or probability of rotational failure of the I-Wall about a point along the embedded portion of the sheet-pile wall serves as a "yardstick" of 
floodwall system performance. As stated previously, the SRC is used to predict the probability of failure, given the flood hazard (Figure 1.3).

When the user selects both the Probabilistic feature and conducts a Design of a new I-Wall, calculations are made to determine the sheet-pile tip elevation. With appropriate consideration of uncertainty in the shear strength parameters for the soils, both the force and moment equilibrium computations result in a statistical distribution for sheet-pile tip elevation. This distribution provides the mean and variance of sheet-pile tip depths for interpretation by the designing engineer. The engineer can see this distribution in a histogram plot, as in Figure 3.79 (mentioned here for reference but included in Chapter 3 for detailed discussion).

Layered Soil Strata: This first version of Corps_I-Wall has the ability to analyze level ground soil sites (e.g., floodplains) with layered soil strata. The boundary between each of the user-specified soil layers is assumed to be horizontal. The soil strata are referred to as soil regions within the Corps_I-Wall formulation. Although the I-Wall may be embedded within a single soil stratum, two different soil regions are specified: one on the flood side of the I-Wall and a second on the landside of the I-Wall. For multiple soil strata, the number of soil regions is generally twice the number of soil strata.

Factors of Safety: In a Design, Corps_I-Wall allows the user to specify a value for the Factor of Safety to be used in active earth pressure computations ( $F S_{\text {active }}$ ) and a value for the Factor of Safety to be used in passive earth pressure computations ( $\left.F S_{\text {passive }}\right)$. The value for $F S_{\text {active }}$ is typically set equal to 1.0 in a Corps I-Wall Design according to EM 1110-22504 (HQUSACE 1994). In an Analysis, the user specifies the elevation of the sheet-pile tip and then the corresponding value of Factor of Safety for the two passive earth pressure zones ( $\left.F S_{\text {passive }}\right)$, identified in Figure 1.1, is computed. The user is allowed to specify the value for the active earth pressure computations ( $F S_{\text {active }}$ ) in this case, but the value is typically set to 1.0 according to EM 1110-2-2504.

These Factors of Safety are applied to the Mohr-Coulomb total stress-based shear strength parameters $c$ and $\varphi$ (or $c^{\prime}$ and $\varphi^{\prime}$ in an effective stress based strength definition), which define the mobilized shear strength parameters $c_{m o b}$ and $\varphi_{m o b}$ (or $c^{\prime}$ mob and $\varphi^{\prime}$ mob) used to compute the mobilized active and passive earth pressures action on the I-Wall/sheet-pile wall. For each soil 
region type, the mobilized active and passive earth pressure coefficients, (Kactive $)_{m o b}$ and $\left(K_{\text {passive }}\right)_{m o b}$ are defined by the value of $\varphi m o b$ for a total stress material type assigned to the soil region (or by $\varphi^{\prime}$ mob for a effective stress material type).

Total or Effective Stress Analysis: Total stress or effective stressbased soil strength properties used for computing earth pressure coefficients are specified for each material type. Moist and saturated soil unit weights are specified by the user for each soil material type. A specific material type number is assigned to each soil region. Different soil regions may be assigned the same material type number designation. The total and/or effective stress-based soil regions can be intermixed in the I-Wall/ sheet-pile wall model.

Within an Effective Stress-designated material type that is assigned to a particular soil region, the total vertical overburden pressures are combined with pore water pressures, a cohesion value (if any), a sheet-pile-to-soil interface friction value (if any), and a horizontal earth pressure coefficient to obtain the distribution of horizontal earth pressures acting normal to the face of the sheet-pile wall within each soil region. Within a soil region containing a Total Stress designated material type, pore-water pressures are excluded from these computations.

Piezometric Surface or Surface Water: Either a Piezometric Surface or a Surface Water elevation may be assigned to each region. During the vertical earth pressure computations, saturated unit weights are assigned to soil elevations that lie below the Piezometric Surface (or Surface Water) in the computations for each soil region. If the Piezometric Surface (or Surface Water) lie within the soil region, then moist soil unit weights are used in the vertical total overburden stress computation for soil elevations above the Piezometric Surface (or Surface Water) elevation, with saturated soil unit weights assigned below this elevation and down to the bottom of this region. If no Piezometric Surface (or Surface Water) is assigned to a soil region, then the moist unit weight of the soil is used in the vertical total overburden stress computations made within this region.

Pore-water Pressures: Corps_I-Wall has a feature to compute porewater pressures acting on the riverside and flood side faces of the I-Wall assuming either (1) the hydrostatic water pressures of a "no flow" condition within various soil regions, or (2) water pressures computed by means 
of steady-state seepage analysis within a homogenous, or a heterogeneous, multilayered soil regions system.

- Hydrostatic: In the case of hydrostatic water pressure, a Piezometric Surface is assigned to the soil region, and the pore-water pressures at any point within the region are computed as equal to the unit weight of water, $\gamma_{\mathrm{w}}$, times the depth from the Piezometric Surface to that point in the soil at which the computation is being made. A Surface Water elevation may be assigned in lieu of a Piezometric Surface elevation to the strata.

- Seepage: Assuming steady-state flow, pore-water pressures along the entire wetted perimeter of the I-Wall (i.e., on both faces), total heads are computed from the results of a simplified steady-state flow analysis using the line of seepage (a.k.a., line of creep) method of analysis. Use of Bernoulli's equation allows for the conversion of total heads into pore-water pressures. To conduct this analysis, values for hydraulic conductivity are assigned to every soil strata, including any stratum defined as a Total Stress material zone. ${ }^{1}$ The user specified value for hydraulic conductivity is not required to be the same for the material types assigned to the different soil regions. In the line of seepage analysis, no more than one Surface Water elevation (or Piezometric Surface elevation) is assigned to the right-hand side (RHS) of the I-Wall and no more than one is assigned to the left-hand side (LHS). The procedure of steady-state, line of seepage analysis implemented in Corps_I-Wall is described in Appendix C.

Gap Initiation/Propagation: Corps_I-Wall accommodates gap initiation/propagation emanating at the ground surface on the flood side of the I-Wall. A surface water elevation at or above the ground surface on the flood side of the I-Wall is required for a gaping to initiate. Cohesive soil properties (either effective or total stress based) are required on the flood side of the wall to allow for the potential for gap development. It is a top down method of analysis; that is, the gap starts at the ground surface and proceeds downward. A gap moves progressively deeper from the uppermost stratum down to the next (e.g., intermediate) stratum down, and so forth. It

\footnotetext{
1 For those stratum designated as a Total Stress material zone, pore-water pressures are computed during a steady-state line of seepage analysis, but they are not assigned during the earth pressure computations within the Total Stress zone(s). For a mixed soil layers of Total Stress and Effective Stress zones, computed pore-water pressures are only assigned to the soil regions designated as an Effective Stress zone.
} 
does not skip by this intermediate strata on to the lower strata. A gap can be engaged in either a hydrostatic or line of seepage type of analysis.

Clockwise or Counterclockwise I-Wall Rotation: Corps_I-Wall has the ability to accommodate either a clockwise or a counterclockwise I-Wall rotation. The direction of I-Wall rotation is dictated by the direction of net loading and its corresponding moment applied to the I-Wall. ${ }^{1}$ Logic was developed to ascertain the direction of I-Wall rotation based upon both the flood and earth loading specified for the problem. This capability was specifically developed to accommodate the coastal problem for which the magnitude of user-specified surge water pressure (i.e., height of design storm surge wave) can alter the direction of I-Wall rotation, relative to the calm water design case. The direction of rotation is determined internally by Corps_I-Wall and reported in the output information contained within the Analysis tab.

Boundary Pressures: Horizontal and/or vertical boundary pressure distributions may be specified. For coastal problems, a horizontal boundary pressure distribution is used to represent the storm surge in a Corps_I-Wall analysis. Vertical boundary pressures are typically used to represent surcharge(s).

Point Loads: Horizontal and/or vertical point loads per foot run of wall (often referred to as line loads in this type of two-dimensional (2-D) analysis) may be specified.

Methods for Computing the Earth Pressure Coefficient: Within each soil region, the horizontal earth pressure acting normal to the sheetpile wall is computed using (1) the vertical, overburden pressure, (2) the earth pressure coefficient for the soil, (3) the soil's cohesion value, (4) the sheet-pile-to-soil interface friction value, and when conducting an Effective Stress analysis, and (5) the pore-water pressure. ${ }^{2}$ Corps_I-Wall allows the user to request that the passive earth pressure coefficient ( $\left.K_{\text {passive }}\right)$, which is used in the horizontal earth pressure computations for the two mobilized passive zones identified in Figure 1.1, be computed by (1) Rankine theory, (2) Coulomb theory, or (3) a logarithmic spiral procedure. The appro-

\footnotetext{
1 To determine the direction of I-Wall rotation, the moment for the net loading is computed about the lower of the right-hand side or left-hand side ground surfaces.

2 The complete equations used to compute the horizontal earth pressure acting normal to the sheet-pileto-soil interface in a Total Stress or an Effective Stress analysis are given in Chapter 2.
} 
priateness for selecting the computational method to use depends upon the value of interface friction between the soil and the sheet-pile relative to the value for the angle of internal friction, $\varphi$. Table 1.1 provides general guidance using information from Dr. Ebeling's lecture on "Earth Pressure Theory" that is given annually in the Proponent-Sponsored Engineering Corps Training (PROSPECT) SSI course taught at ERDC in Vicksburg, MS.

Table 1.1. Method appropriate for computing the passive earth pressure coefficient, $K_{\text {passive, }}$ as a function of the magnitude of the interface friction, $\delta$, between the soil and the sheet pile and in comparison to the magnitude of the soil's angle of internal friction, $\varphi$.

\begin{tabular}{|l|l|}
\hline$\delta$ greater than $\varphi / 2$ & Use coefficients from logarithmic spiral procedure \\
\hline$\delta$ less than or equal to $\varphi / 2$ & $\begin{array}{l}\text { Use coefficients from logarithmic spiral procedure, or } \\
\text { Coulomb theory, or trial wedge procedure }\end{array}$ \\
\hline$\delta$ equal to 0 & $\begin{array}{l}\text { Use coefficients from logarithmic spiral procedure, or } \\
\text { Coulomb theory, or trial wedge procedure, or Rankine } \\
\text { theory }\end{array}$ \\
\hline
\end{tabular}

It is important that the Table 1.1 guidelines are adhered to so that an unconservative, overestimate of the mobilized passive earth pressures is not made within the two passive zones identified in Figure 1.1. An overestimate for the $K_{\text {passive }}$ value(s) would result in a calculated depth of sheetpile embedment shallower than is actually required.

Coastal Problem: The PC-based Corps_I-Wall Visual Modeler package includes the capability to analyze coastal problems. The capability to model storm surge on I-Wall performance is of particular importance because of the destructive force of storm surge. The calculation of coastal surge pressures acting on an I-Wall is discussed in Appendix $\mathrm{F}$ for a breaking wave example. Breaking waves or nonbreaking waves may be specified in the I-Wall rotational stability analysis. Wave loading is specified as a pseudo-static water pressure force along the vertical exposed face of the I-Wall being analyzed. This software program allows the engineer to rapidly make an accurate assessment of the effects of storm surge on I-Walls and to add a protective berm, when deemed necessary.

\subsection{Overview of Design and Analysis modes}

This section provides background on the Design or Analysis mode. For information on the other tabs, refer to Chapter 3. 
Corps_I-Wall can be used to either design a new I-Wall or analyze an existing I-Wall. The functionality of Corps_I-Wall includes a Design or Analysis mode of stability analysis. The Design mode is used to design new I-Walls. This mode computes the required depth of embedment and the shear and moment demands internal to the I-Wall/sheet-pile structural system. These computed results are then used to size the sheetpile for the user-prescribed flood loading. The Analysis mode is used to evaluate existing I-Walls with a known depth of embedment. The primary unknown is the Factor of Safety contained within the two mobilized passive zones identified in Figure 1.1.

This subsection provides an overview of the iterative procedures used in both the Design mode and the Analysis mode solution formulations of Corps_I-Wall.

\subsubsection{Design mode}

Corps_I-Wall can be used to design a new I-Wall. The primary series of computations are used to determine the required depth of sheet-pile embedment for the user-prescribed flood loading. An iterative procedure computes the required depth of penetration in a Design mode. Summarizing, this calculation procedure proceeds as follows:

1. A trial depth of penetration is established by Corps_I-Wall. For this trial depth of penetration:

a. Distributions of surface water pressures, gap water pressures, and pore-water pressures (for the effective stress designated soil regions) are established along both the left-hand side and the right-hand side of the I-Wall shown in Figure 1.1.

b. The net water pressure distribution is calculated by taking the difference between the water pressures acting along the two sides of the I-Wall.

c. Active and mobilized passive earth pressures distributions are established along both sides of the I-Wall.

d. A calculation is made (internal to Corps_I-Wall) using the applied water and (active and factored passive) earth pressure distributions, to determine if the I-Wall will rotate clockwise or counterclockwise. 
2. An initial trial point of rotation is assigned (for the first computation) to a point just below the ground surface. ${ }^{1}$ For this trial point of rotation:

a. The net active pressure distribution is formed on the side that the I-Wall rotates. In Figure 1.1, this is labeled the Flood Side (i.e., the right-hand side). It consists of the active pressure on the side the I-Wall rotates away from (Quadrant I labeled Active Zone in Figure 1.1 on the flood side) minus the mobilized passive pressure on the side the I-Wall rotates (the Quadrant II labeled Passive Zone in Figure 1.1 on the Landside). The net water pressure distribution (along with any external horizontal net pressures and/or overburden induced horizontal pressures, if present) is added on this diagram.

b. The net passive pressure distribution is also formed on the side that the I-Wall rotates. In Figure 1.1, this is labeled the Flood Side (i.e., the right-hand side). It consists of the mobilized passive pressure (the Quadrant IV labeled Passive Zone in Figure 1.1 on the Flood Side) below the point of rotation minus the active pressure (Quadrant III labeled Active Zone in Figure 1.1 on the Landside) below the point of rotation. The net water pressure distribution, along with any external horizontal net pressures and/or overburden induced horizontal pressures, if present, is added on this diagram.

c. The net active pressure distribution becomes the net pressure distribution down to the trial point of rotation (PR). At the sheet-pile tip, the value for the net passive pressure diagram at this elevation becomes the value for net pressure.

d. Between the trial point of rotation elevation and the sheet-pile tip, the net pressure diagram is assumed linear.

e. The net pressure diagram is then converted into equivalent nodal point forces. Horizontal equilibrium and moment equilibrium (about the trial point of rotation) is checked.

f. If there is a force imbalance and/or a moment imbalance, the trial PR is moved down the sheet-pile wall by a small increment, and Step (2) is repeated.

3. Should this trial point of rotation reach the elevation for the trial sheet-pile tip in this iterative process, then the program returns to Step (1). The trial sheet-pile tip elevation is moved downward by a small increment, and Step (2) is started again with a trial point of rotation established just below the ground surface.

\footnotetext{
${ }^{1}$ Note that the point of rotation cannot be at or below the trial sheet-pile tip elevation in a Corps_I-Wall
} rotational stability analysis. Corps_I-Wall Version 1.0 does not perform a global stability assessment. 
4. Convergence of the trial sheet-pile tip and trial point of rotation on their final values is achieved when the force and moment imbalances are within tolerances.

\subsubsection{Analysis mode}

The Analysis mode is used to evaluate existing I-Walls. Existing I-Walls possess a known depth of embedment. Therefore, the primary unknown is the Factor of Safety contained within the two mobilized passive zones $\left(F S_{\text {passive }}\right)$ identified in Figure 1.1. An iterative procedure is used to calculate the value for $F S_{\text {passive. }}$. This procedure is as follows:

1. Assume a trial value for $F S_{\text {passive }}$. The procedure usually starts with $F S_{\text {passive }}$ $=1.5$.

2. Use the Design mode to compute an imaginary sheet-pile tip elevation.

3. If the imaginary sheet-pile tip elevation computed via the Design mode is above the actual sheet-pile tip elevation of the existing I-Wall, reduce the trial $F S_{\text {passive }}$ value. If the imaginary sheet-pile tip elevation computed via the Design mode is below the actual sheet-pile tip elevation of the existing I-Wall, increase the trial $F S_{\text {passive }}$ value.

4. Repeat Step (2) until the imaginary sheet-pile tip elevation is within a small tolerance of the actual sheet-pile tip elevation. 


\section{Engineering Formulation}

The engineering formulation used in Corps_I-Wall Version 1.0 software for a floodplain I-Wall embedded in horizontally-stratified soil layers during flood events is discussed in this chapter. This discussion includes the relationships used to compute the horizontal water and earth pressures acting normal to the freestanding and embedded regions of the I-Wall. The earth pressure coefficient relationships used in computing earth pressures and how water pressures are included in the calculations are also included in the discussion. Key details regarding the procedure of rotational stability analysis are also described. Relationships for converting the different types of surcharge pressures and line loadings into horizontal loads acting on the I-Wall are summarized. Lastly, the procedure used to compute the shears and moments internal to the I-Wall/sheet-pile wall is provided.

\subsection{Layered soils site model}

Corps_I-Wall Version 1.0 is able to model and design I-Walls to be embedded within a horizontally-stratified soil site. Figure 2.1 depicts a typical site configuration for which software Version 1.0 would be used to design the I-Wall for rotational stability and compute the minimum depth of sheet-pile embedment. The soil stratum in which the I-Wall is to be embedded consists of three different types: silty clay, medium sand, and clay. The I-Wall is to be buttressed on the landside (i.e., left-hand side) by a shallow sand berm. The ground surface, interfaces between soil layering, surface water (when present) and piezometric surfaces are all modeled as horizontal interfaces in Version 1.0.

The upper soil layer at the site shown in Figure 2.1 consists of silty clay. The left-hand side of Figure 2.1, is depicted as Region Ro2, and the right-hand side is Region Ro5 for the silty clay. A piezometric surface exists about midelevation within this layer, which separates the moist and saturated zones with unit weights of $\gamma_{\text {moist-SC }}$ and $\gamma_{s a t-S C}$, respectively. Even though soil layer is the same for both regions, the software requires separate LHS and RHS region numbers as well as separate piezometric surface numbers (i.e., Po3 and Po4). The Corps_I-Wall GUI will automatically generate each of the pair of region and piezometric surface numbers. The horizontal earth pressures are computed using total stress analysis (to be discussed in the next subsection). 
Figure 2.1. I-Wall site model configuration for Corps_I-Wall Version 1.0 absent any flood loading.

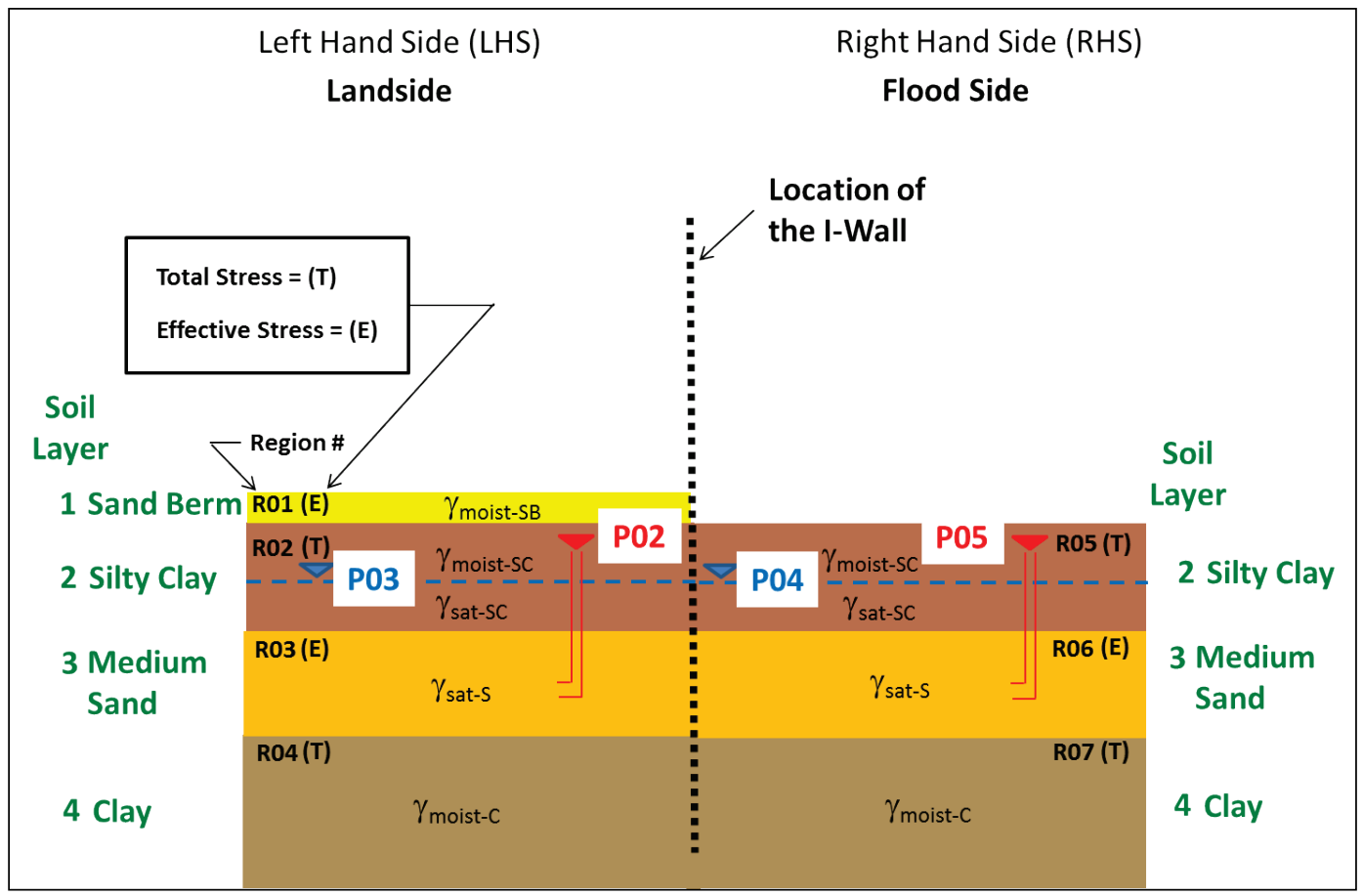

The middle soil layer consists of medium sand. On the LHS of Figure 2.1, it is depicted as Region Ro3 and on the RHS as Region Ro6. Due to access by this soil layer to a water source beyond the extent of this section, the piezometric surface within this soil layer rises to a head that is above the top of this layer. The LHS and RHS piezometric surface numbers are Po2 and Po4, respectively, and are at the same elevation. The saturated sand soil unit weight of $\gamma_{\text {sat-s }}$ is input as a material property by the user and assigned to the two respective soil regions. The horizontal earth pressures are computed using effective stress analysis (to be discussed in the next subsection).

The lowest soil layer consists of clay. On the LHS of Figure 2.1, the layer is depicted as Region Ro4 and on the RHS as Region Ro7. No piezometric surface is assigned to this soil layer. The moist clay soil unit weight of $\gamma$ moist- $C$ is input as a material property by the user and assigned to the two respective soil regions. The horizontal earth pressures are computed using total stress analysis.

A sand berm is to be added landside during construction of the I-Wall. On the LHS of Figure 2.1, it is depicted as Region R01. No piezometric surface is assigned to this soil layer. The moist sand unit weight of $\gamma_{\text {moist-SB }}$ is input as a 
material property by the user and assigned to the single berm soil region. The horizontal earth pressures are computed using effective stress analysis.

Figure 2.2 shows the I-Wall site from Figure 2.1 configured with temporary flood loading (i.e., short-term loading), including the wave action on the RHS of the I-Wall. The rotational stability analysis scenario will be evaluated using the Corps_I-Wall Version 1.0 software. The minimum depth of sheet-pile embedment will be computed during the design process by the software at this layered soil site.

Figure 2.2. I-Wall flood load problem model setup for a Corps_I-Wall Version 1.0 analysis, including wave loading.

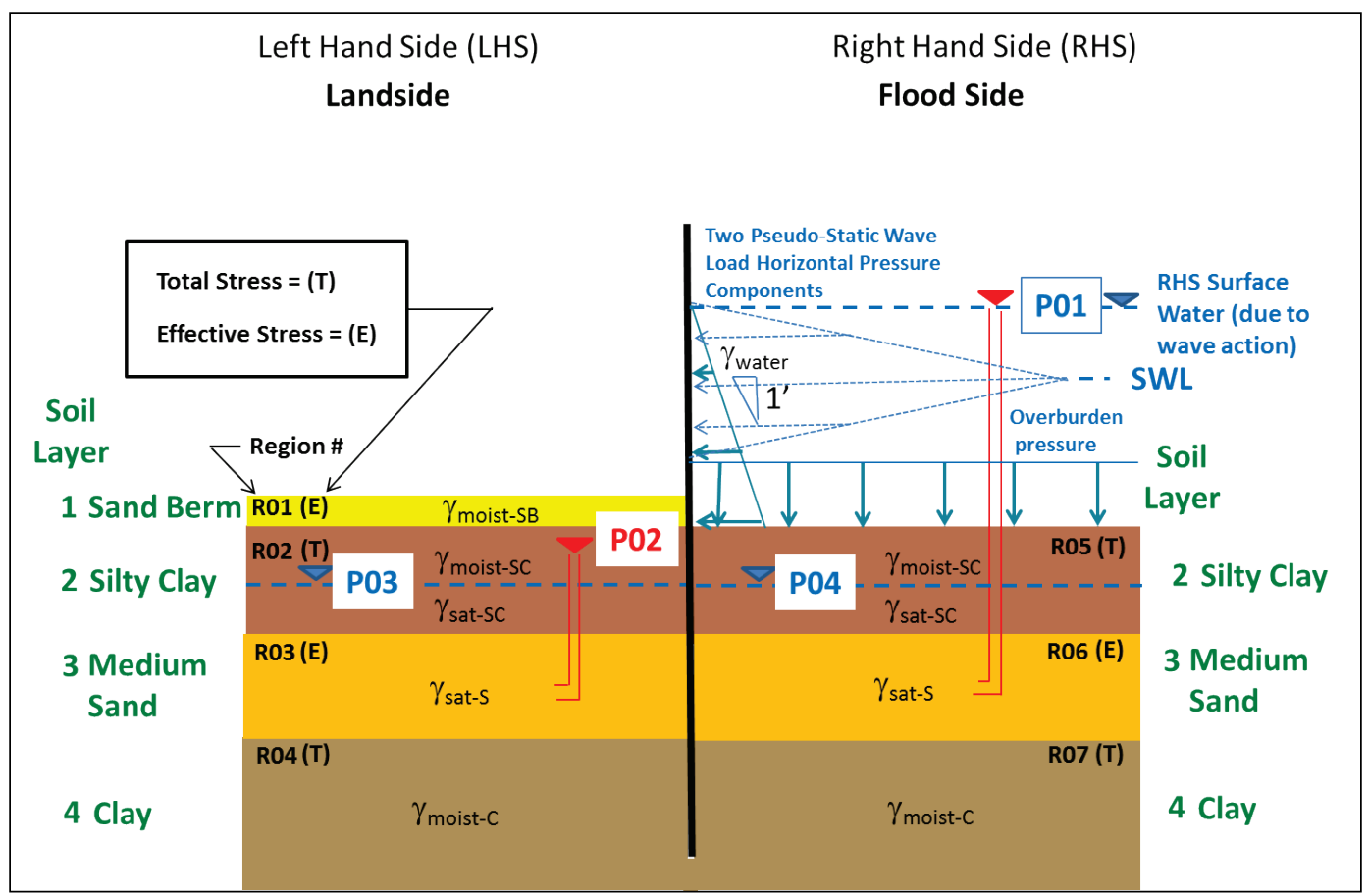

The Figure 2.2 site configuration expands on the Figure 2.1 site details to accommodate a flood of short duration (i.e., several days) to the RHS of the I-Wall. The changes from Figure 2.1 include:

1. The addition of a rise in still-water level (SWL) on the RHS of the I-Wall.

2. The application of two pseudo-static wave load horizontal pressure components to account for the effects of wave loading on the I-Wall. A pseudo-static procedure in Corps_I-Wall is used in a design to represent a dynamic event, such as either breaking or nonbreaking waves. This procedure is discussed in Appendix F. These two components of horizontal 
pressure distributions act normal to the RHS of the I-Wall, as shown in Figure 2.2.

3. Because the middle soil layer of medium sand responds to the water heads of the surface water and flood conditions, Region Ro6, on the RHS, is now subjected to a piezometric head corresponding to the surface water elevation. This piezometric surface is designated as number Po1 by the Corps_I-Wall GUI. ${ }^{1}$

Table 1.1 summarizes the soil layers, piezometric surface definitions, and material property specifications for a Corps_I-Wall Version 1.0 model setup for the Figure 2.2 I-Wall flood loading problem.

Table 2.1. I-Wall flood loading problem soil layering, piezometric surface definitions, and material property specifications for a Corps_I-Wall Version 1.0 model setup.

\begin{tabular}{|c|c|c|c|c|c|c|c|}
\hline \multirow[b]{2}{*}{$\begin{array}{l}\text { I-Wall } \\
\text { Side }\end{array}$} & \multirow[b]{2}{*}{$\begin{array}{l}\text { Region } \\
\text { No. }\end{array}$} & \multirow[b]{2}{*}{$\begin{array}{l}\text { Piezometric } \\
\text { Surface No. }\end{array}$} & \multicolumn{2}{|r|}{ Soil } & \multicolumn{2}{|c|}{ Soil Unit Weight } & \multirow[b]{2}{*}{$(T)$ or $(E)^{2}$} \\
\hline & & & $\begin{array}{l}\text { Layer } \\
\text { No. }\end{array}$ & Type & $\begin{array}{l}\text { Above the } \\
\text { Piezometric } \\
\text { Surface }\end{array}$ & $\begin{array}{l}\text { Below the } \\
\text { Piezometric } \\
\text { Surface }\end{array}$ & \\
\hline \multirow{4}{*}{ LHS } & R01 & None & 1 & Sand Berm & $\gamma_{\text {moist-SB }}$ & - & (E) \\
\hline & R02 & P03 & 2 & Silty Clay & Ymoist-sc & $\gamma_{\text {sat-SC }}$ & $(\mathrm{T})$ \\
\hline & R03 & P02 & 3 & Medium Sand & - & $\gamma_{\text {sat-S }}$ & $(\mathrm{E})$ \\
\hline & R04 & None & 4 & Clay & $\gamma_{\text {moist-C }}$ & - & $(\mathrm{T})$ \\
\hline \multirow{3}{*}{ RHS } & R05 & P04 & 2 & Silty Clay & $\gamma_{\text {moist-SC }}$ & - & $(\mathrm{T})$ \\
\hline & R06 & P01 ${ }^{b}$ & 3 & Medium Sand & - & $\gamma_{\text {sat-S }}$ & $(E)$ \\
\hline & R07 & None & 4 & Clay & $\gamma_{\text {moist-C }}$ & - & $(\mathrm{T})$ \\
\hline
\end{tabular}

a Total Stress (T) or Effective Stress (E) region.

${ }^{b}$ The elevation of P01 on the RHS of the I-Wall is set equal to the surface water elevation in this example. The piezometric surface is obtained from the water surface and is, therefore, labeled in blue. The RHS medium sand, Region R06, is in contact with the floodwater (not shown). Consequently, the pore-water pressures within this region are controlled by the floodwater.

\subsection{Types of analysis used to define earth pressures}

Total stress or effective stress based soil strength properties, used for computing earth pressure coefficients, are specified for each material type. Thus, two different methods are used to compute the magnitude of the earth pressures acting on the embedded sheet-pile portion of the I-Wall based on which is specified: total or effective stress.

\footnotetext{
1 Whenever surface water is specified, the Corps_I-Wall GUI always designates it with the label P01. P01 has values for the surface water levels on both the LHS and RHS of the I-Wall. Piezometric surfaces other than the surface water level only have values for the LHS or RHS, but not both.
} 
Each of the seven soil regions in Figure 2.2 is assigned a soil material type. Each material type is identified by a material type number that is assigned using the Corps_I-Wall GUI, as will be discussed in Chapter 3. At the discretion of the user, horizontal earth pressures acting normal to the embedded sheet-pile wall portion of the I-Wall are based on either total stress or effective stress. Total stress- or effective stress-based soil strength properties are used for computing earth pressure coefficients and are specified for each material type.

Points along the center line of the vertical Figure 2.2 I-Wall/sheet-pile wall are discretized in two phases. The first phase is to define an initial set of nodal points at elevations corresponding to top and bottom region elevations, surface water and/or piezometric surface elevations, points of horizontal traction pressures, and/or point/line loads. This occurs for both sides of the I-Wall. The second phase is to add additional nodes below each of the initial node segments at approximately every foot ${ }^{1}$ and then connect sequentially all of the nodes by a series of vertical I-Wall/sheetpile wall line elements. On each side of the I-Wall/sheet-pile wall and at each nodal elevation, computations are made of the (1) net water pressures and (2) net soil pressure distributions acting normal to the vertical I-Wall/ sheet-pile wall line model, along with (3) the net external horizontal forces (or pressures), and (4) horizontal forces due to external overburden pressures acting on an elevation behind the sheet-pile wall. Each is then converted into equivalent nodal point forces using the one-third/one-sixth relationship. ${ }^{2}$ Force and moment equilibrium are imposed on this I-Wall model in order to compute either $\{a\}$ the minimum depth of sheet-pile penetration (in Design mode) or $\{\mathrm{b}\}$ to compute the Factor of Safety applied to the mobilized shear strength(s) of the soil region(s) when computing passive earth pressures (in Analysis mode).

Total stress- or effective stress-based soil strength properties are used for computing the earth pressure coefficients. 3 These earth pressure coefficients are then used by the software to compute the horizontal earth pressures acting normal to the I-Wall/sheet-pile wall. The details of these two series of calculations are different, depending upon if the soil region is defined as either total stress or effective stress material type. But both

\footnotetext{
1 The nodal point discretization may be revised in subsequent versions.

2 The one-third/one-sixth force computation is discussed in Section 2.4.

3 The computation of earth pressure coefficients using Mohr-Coulomb soil shear strength parameters is discussed in Section 2.3.
} 
computation processes start with the calculation of the total vertical stress acting within the soil adjacent to each node. This evaluation process is made for each node and for each side of the I-Wall/sheet-pile wall.

For either a total stress or effective stress analysis, the computation of the horizontal earth pressure acting normal to the sheet-pile wall starts with a calculation of total vertical stress. An example of this computation will be made within the three soil regimes labeled Ro5, Ro6, and Ro7 located along the RHS of the I-Wall/sheet-pile wall at the four nodes labeled A, B, C, and $\mathrm{D}$ in Figure 2.3. Because of the possibility of intermixed layers, each node (labeled A, B, C, and D) is further designated with a plus and a minus, as shown in Figure 2.3. Considering Region Ro5 is a total stress layer and Region Ro6 is an effective stress layer, the earth pressure computations will be different immediately above and below this region interface boundary. Two separate earth pressure computations are calculated by the software at each node to accommodate this possibility; they are designated as $\mathrm{C}^{+}$and $\mathrm{C}^{-}$ on the upper and lower side of this identified interface node, respectively. This computational approach is followed at all nodes.

Figure 2.3. Location of four select nodes identified along the RHS of the I-Wall for total vertical stress computations calculated during flood loading.

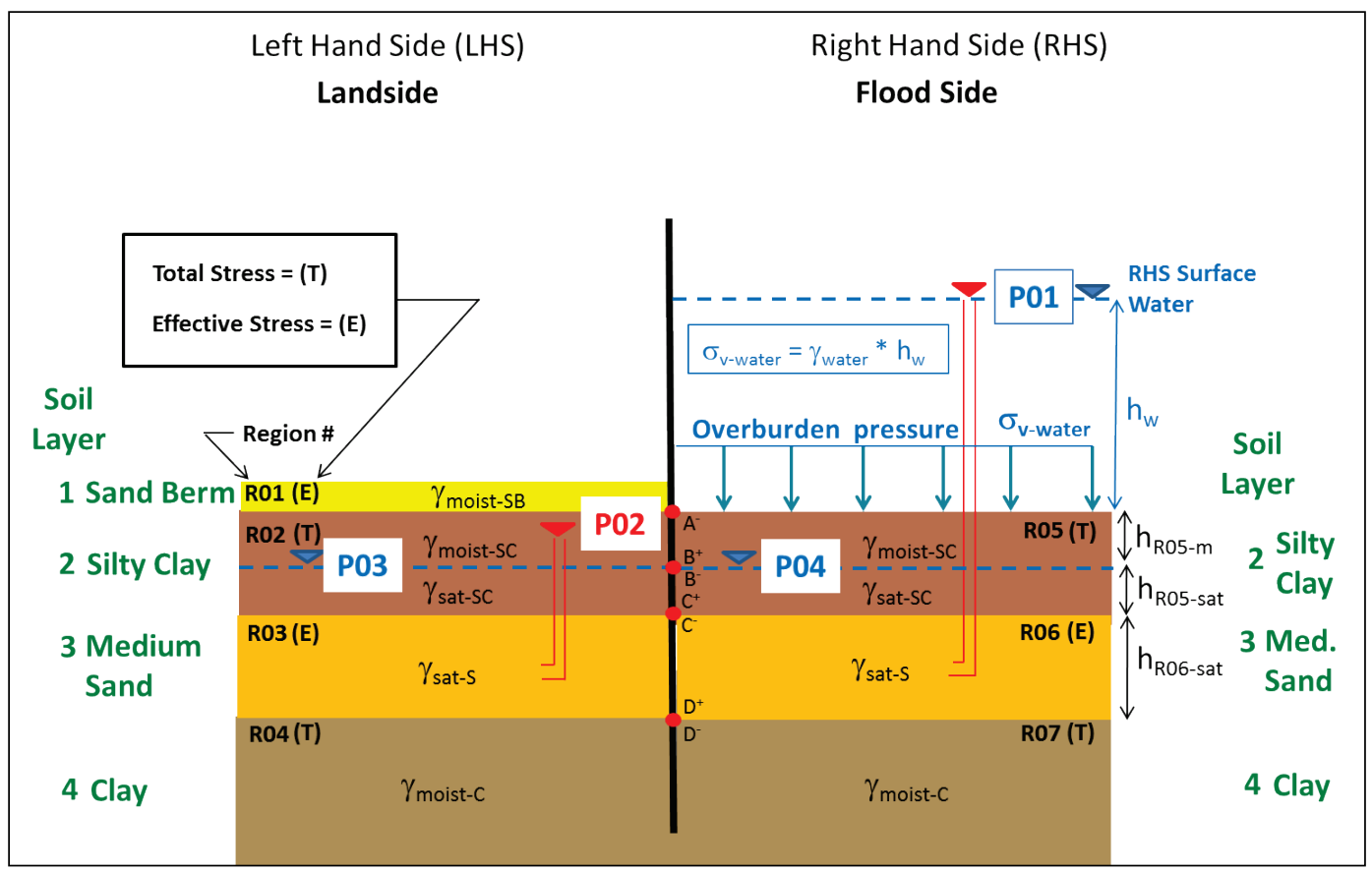

On the RHS of Figure 2.3, the total vertical stress, $\sigma_{v}$, is computed incrementally from the surface water node down from node-to-node by the software. This means that at the RHS ground surface, node A has a total 
vertical stress, $\sigma_{v-A}$, corresponding to the overburden pressure of water to a depth $h_{w}$,

$$
\sigma_{v-A}=\gamma_{w a t e r} \cdot h_{w}
$$

Similarly, at nodes B, C and D, the total vertical stresses are equal to

$$
\begin{gathered}
\sigma_{v-B}=\sigma_{v-A}+\gamma_{\text {moist }-S C} \cdot h_{R 05-m} \\
\sigma_{v-C}=\sigma_{v-B}+\gamma_{s a t-S C} \cdot h_{R 05-s a t}
\end{gathered}
$$

and

$$
\sigma_{v-D}=\sigma_{v-C}+\gamma_{s a t-S C} \cdot h_{R 06-s a t}
$$

The computation of the total vertical stress within the soil regime for all RHS nodes by the software follows this incremental node-to-node computational approach downward along the embedded sheet-pile wall. The value for total vertical stress is the same at above and below the regions boundary and on the plus and minus side of each Figure 2.3 node.

This same incremental top down, node-to-node computational approach is used for computing the total vertical stress within the soil regime for all LHS nodes as is used for the RHS nodes by the software.

\subsubsection{Total stress - mobilized active earth pressure}

For each side and within each soil region that is designated with a total stress material property (through its user provided material type input information), the mobilized active earth pressure is computed at each node using the relationship

$$
\sigma_{m o b-A c t i v e}=K_{m o b-A c t i v e} \cdot \sigma_{v}-2 \cdot c_{m o b-A c t i v e} \cdot \sqrt{K_{m o b-A c t i v e}}
$$

According to Jumikis (1984), consideration of stress condition in soil shows that cohesion of a cohesive-frictional $\{c, \varphi\}$ soil does not affect the position of the rupture surface. Because of this, Jumikis concludes that the earth pressure of a cohesive-frictional $\{c, \varphi\}$ soil can be approximately determined by the method used for cohesionless (i.e., noncohesive) soil as described in his book (on page 380). Jumikis extended this procedure to \{c, $\varphi\}$ soils for Coulomb active and passive earth pressure-based calculations. 
By this Jumikis approximation as well as by a similar procedure presented in EM-1110-2-2502 (HQUSACE 1989) of Coulomb wedge results, Coulomb active and passive earth pressure coefficients of a $\{c, \varphi\}$ soil (computed using the relationships given in Sections 2.3.3 and 2.3.4) may be used in Equations 2.5 and 2.9 to compute the active and passive soil pressure distributions. This same logic holds true for the effective stress-based earth pressure relationships given by Equations 2.19 and 2.23 for mobilized active and passive earth pressures, respectively.

The mobilized active earth pressure (by Equation 2.5) acts at an angle of $\delta$ mob-Active from horizontal. ${ }^{1}$ The horizontal component of $\sigma$ mob-Active given by

$$
\sigma_{\text {mob-Active-horizontal }}=\sigma_{\text {mob-Active }} \cdot \cos \left(\delta_{\text {mob-Active }}\right)
$$

The value of the active earth pressure coefficient $K_{\text {mob-Active }}$ is computed using one of two relationships given in a subsequent subsection. The computation of the total vertical stress $\sigma_{v}$ is made at each node down the I-Wall and follows the procedure outlined in the Section 2.2. The mobilized

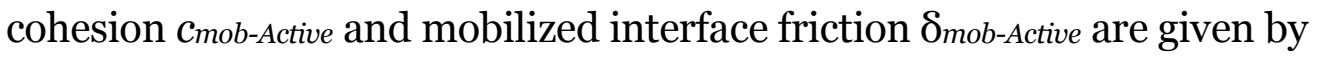

$$
\begin{gathered}
c_{\text {mob-Active }}=\frac{c}{F S_{\text {Active }}} \\
\tan \left(\delta_{\text {mob-Active }}\right)=\frac{\tan (\delta)}{F S_{\text {Active }}}
\end{gathered}
$$

with $c$ and $\delta$ being the user-specified values for cohesion and interface friction, respectively, assigned to the soil region. Because each soil region may be assigned different strength parameters, there can be a discontinuity in the active earth pressure at each interface between adjoining soil regions. Thus, the need for maintaining an upper and a lower earth pressure computation per node to accommodate this possibility (e.g., for node $\mathrm{C}$, designating it as $\mathrm{C}^{+}$and $\mathrm{C}^{-}$in Figure 2.3). FS Active is the user-specified Factor of Safety to be used in the active earth pressure computations and is usually assigned a value of 1.0 in a Corps_I-Wall design effort.

\footnotetext{
1 When the user selects $K_{m o b-A c t i v e ~}$ to be calculated by Rankine earth pressure theory in the software the mobilized interface friction $\delta_{\text {mob-Active }}$ is set equal to zero, as will be discussed in Section 2.3.1.
} 


\subsubsection{Total stress - mobilized passive earth pressure}

For each side and within each soil region that is designated with a total stress material property (through its user provided material type input information), the mobilized passive earth pressure is computed at each node using the relationship

$$
\sigma_{m o b-P a s s i v e}=K_{m o b-P a s s i v e} \cdot \sigma_{v}+2 \cdot c_{m o b-P a s s i v e} \cdot \sqrt{K_{m o b-P a s s i v e}}
$$

This mobilized passive earth pressure acts at an angle of $\boldsymbol{\delta}_{\text {mob-Passive }}$ from horizontal. ${ }^{1}$ The horizontal component of $\sigma_{\text {mob-Passive }}$ given by

$$
\sigma_{\text {mob-Passive-horizonal }}=\sigma_{\text {mob-Passive }} \cdot \cos \left(\delta_{\text {mob-Passive }}\right)
$$

The value of the passive earth pressure coefficient $K_{\text {mob-Passive }}$ is computed using one of three relationships given in a subsequent subsection. The computation of the total vertical stress $\sigma_{v}$ is made at each node down the I-Wall and follows the procedure outlined in the Section 2.2. The mobilized cohesion $c_{m o b}$-Passive and mobilized interface friction $\delta_{m o b-P a s s i v e}$ are given by

$$
\begin{gathered}
c_{\text {mob-passive }}=\frac{c}{F S_{\text {Passive }}} \\
\tan \left(\delta_{\text {mob-Passive }}\right)=\frac{\tan (\delta)}{F S_{\text {Passive }}}
\end{gathered}
$$

with $c$ and $\delta$ being the user-specified values for cohesion and interface friction, respectively, assigned to each soil region. Because each soil region may be assigned different strength parameters, there can be a discontinuity in the earth pressure at each interface between adjoining soil regions. Thus, the need for maintaining an upper and a lower earth pressure computation per node to accommodate this possibility (e.g., for node C, designating it as $\mathrm{C}^{+}$and $\mathrm{C}^{-}$in Figure 2.3). FS Passive is the user-specified Factor of Safety for the passive earth pressure computations and is assigned a value greater than 1.0 in a Corps_I-Wall design effort.

\footnotetext{
1 When the user selects $K_{\text {mob-Passive }}$ to be calculated by Rankine earth pressure theory in the software the mobilized interface friction $\delta_{\text {mob-Passive }}$ is set equal to zero, as will be discussed in Section 2.3.2.
} 


\subsubsection{Effective stress}

Within an effective stress designated material type assigned to a particular soil region, the total vertical stress $\sigma_{v}$ acting in the soil adjacent to each node is combined with the soil regions pore water pressure $u$ (if any) to compute the vertical effective stress $\sigma_{v}^{\prime}$. Terzaghi's principle of effective stress is expressed as

$$
\sigma_{v}=\sigma_{v}^{\prime}+u
$$

Rearranging terms,

$$
\sigma_{v}^{\prime}=\sigma_{v}-u
$$

Recall $\sigma_{v}$ is computed using the procedure outlined in Section 2.2. The effective vertical stress is then used to compute the mobilized active earth pressure acting on the node of the embedded sheet pile.

Example: The Figure 2.3 medium sand Region Ro6 is designated an effective stress material type. At the top of this medium sand region and at a point designated in Figure 2.3 as node $\mathrm{C}^{-}$, the vertical effective stress $\sigma^{\prime} v$ - $\mathrm{C}^{-}$ is computed at to be

$$
\sigma_{v-C^{-}}^{\prime}=\sigma_{v-C}-u_{C^{-}}
$$

where the pore water pressure $u$ at the top of this effective stress region and at node $C^{-}$, is computed as

$$
u_{C^{-}}=\gamma_{\text {water }} \cdot\left(h_{w}+h_{R 05-m}+h_{R 05-s a t}\right)
$$

and $\sigma_{v-C}$ is computed using Equation 2.3. Referring to Figure 2.3, the piezometric surface number P01 for this soil Region Ro6 has an elevation that corresponds to the RHS surface water.

At the bottom of this medium sand region and at a point designated in Figure 2.3 as node $D^{+}$, the vertical effective stress $\sigma_{v-D^{+}}^{\prime}$ is computed at to be

$$
\sigma_{v-D^{+}}^{\prime}=\sigma_{v-D}-u_{D^{+}}
$$

where the pore-water pressure $u$ at the bottom of this effective stress region and at node $D^{+}$, is computed as 


$$
u_{D^{+}}=\gamma_{\text {water }} \cdot\left(h_{w}+h_{R 05-m}+h_{R 05-s a t}+h_{R 06-s a t}\right)
$$

and $\sigma_{v-D}$ is computed using Equation 2.4.

Because soil Region Ro5 in Figure 2.3 is a total stress material and Region Ro6 is an effective stress material, there will be a discontinuity in the active earth pressures at node $\mathrm{C}$. The same is true for node $\mathrm{D}$, the interface of the effective stress, medium sand Region Ro6 with the total stress, clay Region R07. The same is true for passive earth pressures.

\subsubsection{Effective stress - mobilized active earth pressure}

For each side and within each soil region that is designated with an effective stress material property, the mobilized effective active earth pressure is computed at each node using the relationship

$$
\sigma_{\text {mob-Active }}^{\prime}=K_{\text {mob-Active }} \cdot \sigma_{v}^{\prime}-2 \cdot c_{\text {mob-Active }}^{\prime} \cdot \sqrt{K_{\text {mob-Active }}}
$$

This mobilized effective active earth pressure acts at an angle of $\delta^{\prime}$ mob-Active from horizontal. ${ }^{1}$ The computation of the effective vertical stress $\sigma^{\prime} v$ is made at each node down the I-Wall and follows the procedure outlined in the Section 2.2.3.The horizontal component of $\sigma^{\prime}$ mob-Active given by

$$
\sigma_{\text {mob-Active-horizontal }}^{\prime}=\sigma_{\text {mob-Active }}^{\prime} \cdot \cos \left(\delta_{\text {mob-Active }}^{\prime}\right)
$$

The value of the active earth pressure coefficient $K_{\text {mob-Active }}$ is computed using one of two relationships given in a subsequent subsection. The mobilized effective cohesion $c^{\prime}$ mob-Active and mobilized effective interface friction $\delta^{\prime}$ mob-Active are given by

$$
\begin{gathered}
c_{\text {mob-Active }}^{\prime}=\frac{c^{\prime}}{F S_{\text {Active }}} \\
\tan \left(\delta_{\text {mob-Active }}^{\prime}\right)=\frac{\tan \left(\delta^{\prime}\right)}{F S_{\text {Active }}}
\end{gathered}
$$

with $c^{\prime}$ and $\delta^{\prime}$ being the user-specified values for effective cohesion and effective interface friction, respectively, assigned to the soil region. FS Active is the user-specified Factor of Safety to be used in the active earth pressure

\footnotetext{
1 When the user selects $K_{\text {mob-Active }}$ to be calculated by Rankine earth pressure theory in the software the
} mobilized interface friction $\delta^{\prime}$ mob-Active is set equal to zero, as will be discussed in Section 2.3.1. 
computations and is usually assigned a value of 1.0 in a Corps_I-Wall design.

In an effective stress-based analysis, in addition to the mobilized active earth pressure, the pore-water pressure also acts normal to the node on the sheet-pile wall.

Lastly, there can be a discontinuity in the active earth pressure at each soil region because each soil region may be assigned different strength parameters.

\subsubsection{Effective stress - mobilized passive earth pressure}

For each side and within each soil region that is designated with an effective stress material property, the mobilized effective passive earth pressure is computed at each node using the relationship

$\sigma_{m o b-P a s s i v e}^{\prime}=K_{m o b-P a s s i v e} \cdot \sigma_{v}^{\prime}+2 \cdot c_{m o b-P a s s i v e}^{\prime} \cdot \sqrt{K_{m o b-P a s s i v e}}$

This mobilized effective passive earth pressure acts at an angle of $\delta^{\prime}$ mob-Passive from horizontal. ${ }^{1}$ The computation of the effective vertical stress $\sigma^{\prime} v$ is made at each node down the I-Wall and follows the procedure outlined in the Section 2.2.3. The horizontal component of $\sigma^{\prime}$ mob-Passive given by

$$
\sigma_{\text {mob-Passive-horizontal }}^{\prime}=\sigma_{m o b-P a s s i v e}^{\prime} \cdot \cos \left(\delta_{m o b-P a s s i v e}^{\prime}\right)
$$

The value of the passive earth pressure coefficient $K_{\text {mob-Passive }}$ is computed using one of three relationships given in a subsequent subsection. The mobilized effective cohesion $c^{\prime}$ mob-Passive and mobilized effective interface friction $\delta^{\prime}$ mob-Passive are given by

$$
\begin{gathered}
c_{\text {mob-Passive }}^{\prime}=\frac{c^{\prime}}{F S_{\text {Passive }}} \\
\tan \left(\delta_{\text {mob-Passive }}^{\prime}\right)=\frac{\tan \left(\delta^{\prime}\right)}{F S_{\text {Passive }}}
\end{gathered}
$$

\footnotetext{
1 When the user selects $K_{\text {mob-Passive }}$ to be calculated by Rankine earth pressure theory in the software the mobilized interface friction $\delta^{\prime}$ mob-Passive is set equal to zero, as will be discussed in Section 2.3.2.
} 
with $c^{\prime}$ and $\delta^{\prime}$ being the user-specified values for effective cohesion and effective interface friction, respectively, assigned to the soil region. FSPassive is the user-specified Factor of Safety in the passive earth pressure computations and is usually assigned a value greater than 1.0 in a Corps_I-Wall design effort.

In an effective stress based analysis, in addition to the mobilized passive earth pressure, the pore water pressure also acts normal to the node on the sheet-pile wall.

Lastly, there can be a discontinuity in the passive earth pressure at each soil region because each soil region may be assigned different strength parameters.

\subsection{Earth pressure coefficient methods used for computing earth pressures}

Earth pressure coefficient methods are used in Corps_I-Wall Version 1.0 to compute the mobilized active and passive horizontal pressures acting along the sheet-pile wall to soil interface, as described in Section 2. The user may select one of two methods to compute a value for the active earth pressure coefficient: Rankine or Coulomb. To compute a value for the passive earth pressure coefficient, there are three options available: Rankine, Coulomb or a passive earth pressure coefficient computed using a logarithmic spiral based method.

Rankine earth pressures always act normal to the (sheet-pile) wall. Consequently, $\delta$ in a total stress soil or $\delta^{\prime}$ in an effective stress soil is set equal to zero degrees for the active earth pressure relationships of Equations 2.6, 2.10, 2.20 and 2.24.

\subsubsection{Rankine's active earth pressure coefficients}

Total stress: For a horizontal ground surface, Rankine's active earth pres-

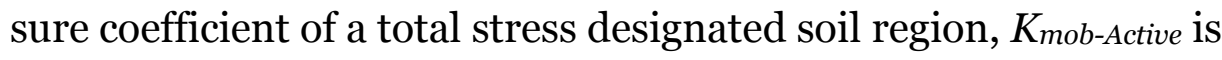

$$
K_{\text {mob-Active }}=\tan ^{2}\left(45^{\circ}-\frac{\varphi_{\text {mob-Active }}}{2}\right)
$$

where $\varphi_{m o b}$ is the mobilized angle of internal friction of the soil. The value for $\varphi_{m o b}$ is given by 


$$
\tan \left(\varphi_{\text {mob-Active }}\right)=\frac{\tan (\varphi)}{F S_{\text {Active }}}
$$

Rankine's earth pressure theory for a $\{c, \varphi\}$ soil assumes the earth pressure acts normal to the sheet-pile wall. When the user selects $K$-mobActive to be calculated by Rankine earth pressure theory, the interface friction $\delta$ is set equal to zero in Equation 2.8 and thus, Equation 2.5 describes the earth pressure normal to the sheet-pile wall.

Effective stress: For an effective stress soil region below a horizontal ground surface, Rankine's mobilized effective active earth pressure coefficient $K_{\text {mob-Active }}$ is

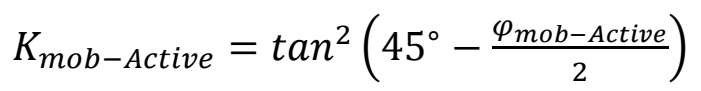

where $\varphi^{\prime}$ mob is the mobilized effective angle of internal friction of the soil. The value for $\delta^{\prime}$ mob is given by

$$
\tan \left(\varphi_{\text {mob-Active }}^{\prime}\right)=\frac{\tan \left(\varphi^{\prime}\right)}{F S_{\text {Active }}}
$$

Rankine's earth pressure theory for a $\left\{c^{\prime}, \varphi^{\prime}\right\}$ soil assumes the earth pressure acts normal to the sheet-pile wall. So when the user selects $K_{\text {-mob- }}$ Active to be calculated by Rankine earth pressure theory, the interface friction $\varphi^{\prime}$ is set equal to zero in Equation 2.22 and thus, Equation 2.19 describes the earth pressure normal to the sheet-pile wall.

\subsubsection{Rankine's passive earth pressure coefficients}

For a horizontal ground surface, Rankine's passive earth pressure

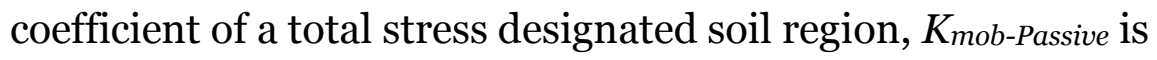

$$
K_{m o b-P a s s i v e}=\tan ^{2}\left(45^{\circ}-\frac{\varphi_{\text {mob-Passive }}}{2}\right)
$$

where $\varphi_{m o b}$ is the mobilized angle of internal friction of the soil. The value for $\varphi m o b$ is given by

$$
\tan \left(\varphi_{\text {mob-Passive }}\right)=\frac{\tan (\varphi)}{F S_{\text {Passive }}}
$$


Rankine's earth pressure theory for a $\{c, \varphi\}$ soil assumes the earth pressure acts normal to the sheet-pile wall. When the user selects $K$-mob-Passive to be calculated by Rankine earth pressure theory, the interface friction $\delta$ is set equal to zero in Equation 2.12 and thus, Equation 2.9 describes the earth pressure normal to the sheet-pile wall.

Effective stress: For an effective stress soil region, Rankine's mobilized effective passive earth pressure coefficient $K_{m o b-A c t i v e ~}$ is

$$
K_{\text {mob-Passive }}=\tan ^{2}\left(45^{\circ}-\frac{\varphi_{\text {mob-Passive }}}{2}\right)
$$

where $\varphi^{\prime}$ mob is the mobilized effective angle of internal friction of the soil. The value for $\varphi^{\prime}{ }_{m o b}$ is given by

$$
\tan \left(\varphi_{\text {mob-Passive }}^{\prime}\right)=\frac{\tan \left(\varphi^{\prime}\right)}{F S_{\text {Passive }}}
$$

Rankine's earth pressure theory for a $\left\{c^{\prime}, \varphi^{\prime}\right\}$ soil assumes the earth pressure acts normal to the sheet-pile wall. When the user selects $K$-mobPassive to be calculated by Rankine earth pressure theory, the interface friction $\delta^{\prime}$ is set equal to zero in Equation 2.26 and thus, Equation 2.23 describes the earth pressure normal to the sheet-pile wall.

\subsubsection{Coulomb's active earth pressure coefficients}

Total stress: For a horizontal ground surface, Coulomb's active earth pressure coefficient (Ebeling and Morrison 1992) of a total stress designated

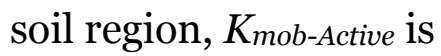

$$
K_{\text {mob-Active }}=\frac{\cos ^{2} \varphi_{\text {mob-Active }}}{\cos \left(\delta_{\text {mob-Active }}\right)\left[1+\sqrt{\frac{\sin \left(\varphi_{\text {mob-Active }}+\delta_{\text {mob-Active }}\right) \sin \left(\varphi_{\text {mob-Active }}\right)}{\cos \left(\varphi_{\text {mob-Active }}\right)}}\right]^{2}}
$$

where $\varphi m o b$-Active is the mobilized angle of internal friction of the soil and $\delta_{\text {mob-Active }}$ is the mobilized soil to sheet-pile interface angle of friction. The

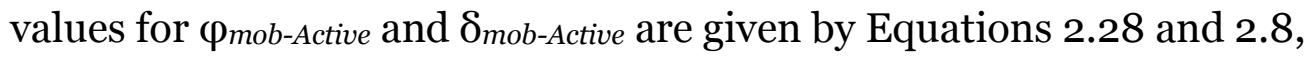
respectively.

Effective stress: For an effective stress soil region below a horizontal ground surface, Coulomb's mobilized effective active earth pressure coefficient $K_{\text {mob-Active }}$ is 


$$
K_{\text {mob-Active }}=\frac{\cos ^{2} \varphi_{\text {mob-Active }}^{\prime}}{\cos \left(\delta_{\text {mob-Active }}^{\prime}\right)\left[1+\sqrt{\frac{\sin \left(\varphi_{\text {mob-Active }}^{\prime}+\delta_{\text {mob-Active }}^{\prime}\right) \sin \left(\varphi_{\text {mob-Active }}^{\prime}\right)}{\cos \left(\delta_{\text {mob-Active }}^{\prime}\right)}}\right]^{2}}
$$

where $\varphi^{\prime}$ mob-Active is the mobilized effective angle of internal friction of the soil and $\delta^{\prime}$ mob-Active is the mobilized soil to sheet-pile interface angle of friction. The values for $\varphi^{\prime}$ mob-Active and $\delta^{\prime}$ mob-Active are given by Equations 2.30 and 2.22, respectively.

\subsubsection{Coulomb's passive earth pressure coefficients}

Total stress: For a horizontal ground surface, Coulomb's passive earth pressure coefficient (Ebeling and Morrison 1992) of a total stress designated soil region, $K_{\text {mob-Passive }}$ is

$$
K_{\text {mob-Passive }}=\frac{\cos ^{2} \varphi_{\text {mob-Passive }}}{\cos \left(\delta_{\text {mob-Passive }}\right)\left[1+\sqrt{\frac{\sin \left(\varphi_{\text {mob-Passive }}+\delta_{\text {mob-Passive }}\right) \sin \left(\varphi_{\text {mob-Passive }}\right)}{\cos \left(\varphi_{\text {mob-Passive }}\right)}}\right]^{2}}
$$

where $\varphi_{\text {mob-Passive }}$ is the mobilized angle of internal friction of the soil and $\delta_{\text {mob-Passive }}$ is the mobilized soil to sheet-pile interface angle of friction. The values for $\varphi$ mob-Passive and $\delta_{\text {mob-Passive }}$ are given by Equations 2.32 and 2.12, respectively.

The application of this Coulomb $K_{m o b}$-Passive relationship is to soils with

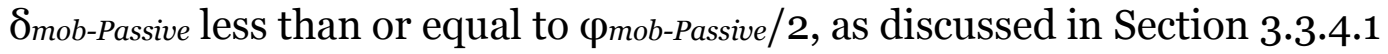
of Ebeling and Morrison (1992) or Section 4.6 of Chen and Liu (1990). This is due to the inappropriateness of a planar slip surface assumption used in defining the resultant passive earth pressure force relationship from which the Coulomb passive earth coefficient is derived. A curved slip surface is more appropriate when $\delta_{\text {mob-Passive }}$ is greater than $\varphi_{\text {mob-Passive }} / 2$.

Effective stress: For an effective stress soil region below a horizontal ground surface, Coulomb's mobilized effective passive earth pressure coefficient $K_{\text {mob-Passive }}$ is

$$
K_{\text {mob-Passive }}=\frac{\cos ^{2} \varphi_{\text {mob-Passive }}^{\prime}}{\cos \left(\delta_{\text {mob-Passive }}^{\prime}\right)\left[1-\sqrt{\frac{\sin \left(\varphi_{\text {mob-Passive }}^{\prime}+\delta_{\text {mob-Passive }}^{\prime}\right) \sin \left(\varphi_{\text {mob-Passive }}^{\prime}\right)}{\cos \left(\delta_{\text {mob-Passive }}^{\prime}\right)}}\right]^{2}}
$$


where $\varphi^{\prime}$ mob-Passive is the mobilized effective angle of internal friction of the soil and $\delta^{\prime}$ mob-Passive is the mobilized soil to sheet-pile interface angle of friction. The values for $\varphi^{\prime}$ mob-Passive and $\delta^{\prime}$ mob-Passive are given by Equations 2.34 and 2.26, respectively.

The application of this Coulomb $K_{\text {mob-Passive }}$ relationship is to soils with $\delta^{\prime}$ mob-Passive less than or equal to $\varphi^{\prime}$ mob-Passive/2, as discussed in

Section 3.3.4.1 of Ebeling and Morrison (1992) or Section 4.6 of Chen and Liu (1990). This is due to the inappropriateness of a planar slip surface assumption used in defining the resultant passive earth pressure force relationship from which the Coulomb passive earth coefficient is derived. A curved slip surface is more appropriate when $\delta^{\prime}$ mob-Passive is greater than $\varphi^{\prime}$ mob-Passive/2.

\subsubsection{Logarithmic-spiral method for the determination of a passive earth pressure coefficients}

The logarithmic spiral earth pressure theory (sometimes referred to as the logarithmic spiral method) is described by Terzaghi (1943), Chen and Liu (1990), and others. A significant portion of the slip surface in a retained soil regime is approximated by a logarithmic spiral. Under this condition and using different sets of engineering formulations, values for passive earth coefficients were produced as a function of internal friction values for cohesionless soils (e.g., tabulations given in Caquot 1948; Kerisel and Absi 1990; Chen and Liu 1990; Sokolovskii 1965; and in the Figure 5 chart in Chapter 3 of NAVFAC DM 7.2 (Department of the Navy 1982). The Figure 5 chart given in Chapter 3 in NAVFAC DM 7.2 (1982) provides values for $K_{\text {Passive }}$ for $\delta$ equal to $\varphi$ as a nonlinear curve that is a function of $\varphi$. Using this information, a tabulation of $K$ Passive values was assembled (Table 2.2). In order to determine an appropriate value for $K_{\text {Passive }}$ in those cases when $\delta$ is less then $\varphi$, a reduction factor, $R$, is applied to the Table 2.3 Kassive value.

$$
K_{\text {Passive }}(\delta)=K_{\text {Passive }} \cdot R(\delta, \phi)
$$

Values for $R$ are provided in Table 2.3 as a function of the value of $\varphi$ and the value of the ratio $\delta / \varphi$. For intermediate values, interpolation of the Tables 2.2 and 2.3 are appropriate. 
Table 2.2. Passive earth pressure coefficient $K_{\text {Passive }}$ (Kp) values for $-\delta$ equal to $\varphi$ and a solution based on assuming a failure surface composed of logarithmic spiral portion and a planar surface portion.

\begin{tabular}{|c|c|}
\hline$\phi$ & $\mathrm{Kp}(-\delta=\phi)$ \\
\hline 0 & 1 \\
\hline 5 & 1.28 \\
\hline 10 & 1.64 \\
\hline 15 & 2.19 \\
\hline 20 & 3.01 \\
\hline 25 & 4.29 \\
\hline 30 & 6.42 \\
\hline 35 & 10.2 \\
\hline 40 & 17.5 \\
\hline 45 & 33.5 \\
\hline 50 & 74.3 \\
\hline 51 & 90 \\
\hline 52 & 110 \\
\hline 53 & 130 \\
\hline 54 & 160 \\
\hline 55 & 204 \\
\hline 60 & 782 \\
\hline
\end{tabular}

To use the Tables 2.2 and 2.3 data in total stress designated materials, the value for mobilized interface friction, $\delta_{\text {mob-Passive }}$ is substituted for $\delta$ and the value for mobilized angle of internal friction $\varphi$ mob-Passive is substituted for $\varphi$. A similar substitution is made for effective stress designated materials. These tables of values may be used for soils designated as either total or effective stress materials. Lastly, due to the sign convention used to describe the passive earth pressure force based on the data contained in the Figure 5 chart in Chapter 3 of NAVFAC DM 7.2 (Department of the Navy 1982), $\delta$ is expressed as a negative value when using Tables 2.2 and 2.3.

Within the software, the $K_{\text {Passive }}$ and $R$ value data contained in Tables 2.2 and 2.3 are stored in separate arrays. When the user requests via the GUI that the value for $K_{m o b-P a s s i v e}$ be calculated using the logarithmic-spiral method, the values of both $K_{\text {mob-Passive }}$ and $R$ are obtained by linear inter-

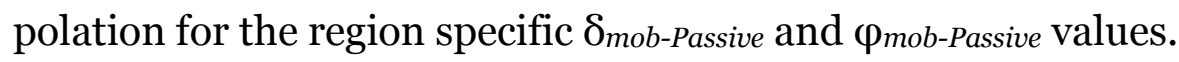


Table 2.3. Reduction factor, $R$, values for various ratios of $-\delta / \phi$.

\begin{tabular}{|c|c|c|c|c|c|c|c|c|c|c|c|}
\hline \multirow{2}{*}{$\phi \quad($ deg $)$} & \multicolumn{11}{|c|}{$\delta / \phi$} \\
\hline & -1 & -0.9 & -0.8 & -0.7 & -0.6 & -0.5 & -0.4 & -0.3 & -0.2 & -0.1 & 0 \\
\hline 0 & 1 & 1 & 1 & 1 & 1 & 1 & 1 & 1 & 1 & 1 & 1 \\
\hline 5 & 1 & 0.996 & 0.995 & 0.989 & 0.981 & 0.973 & 0.9645 & 0.956 & 0.949 & 0.9405 & 0.932 \\
\hline 10 & 1 & 0.991 & 0.989 & 0.978 & 0.962 & 0.946 & 0.929 & 0.912 & 0.898 & 0.881 & 0.864 \\
\hline 15 & 1 & 0.986 & 0.979 & 0.961 & 0.934 & 0.907 & 0.881 & 0.854 & 0.83 & 0.803 & 0.775 \\
\hline 20 & 1 & 0.983 & 0.968 & 0.939 & 0.901 & 0.862 & 0.824 & 0.787 & 0.752 & 0.716 & 0.678 \\
\hline 25 & 1 & 0.980 & 0.954 & 0.912 & 0.86 & 0.808 & 0.759 & 0.711 & 0.666 & 0.62 & 0.574 \\
\hline 30 & 1 & 0.980 & 0.937 & 0.878 & 0.811 & 0.746 & 0.686 & 0.627 & 0.574 & 0.52 & 0.467 \\
\hline 35 & 1 & 0.980 & 0.916 & 0.836 & 0.752 & 0.674 & 0.603 & 0.536 & 0.475 & 0.417 & 0.362 \\
\hline 40 & 1 & 0.980 & 0.886 & 0.783 & 0.682 & 0.592 & 0.512 & 0.439 & 0.375 & 0.316 & 0.262 \\
\hline 45 & 1 & 0.979 & 0.848 & 0.718 & 0.6 & 0.5 & 0.414 & 0.339 & 0.276 & 0.221 & 0.174 \\
\hline 50 & 1 & 0.975 & 0.797 & 0.638 & 0.506 & 0.399 & 0.313 & 0.242 & 0.185 & 0.138 & 0.102 \\
\hline 55 & 1 & 0.966 & 0.731 & 0.543 & 0.401 & 0.295 & 0.215 & 0.153 & 0.108 & 0.0737 & 0.0492 \\
\hline 60 & 1 & 0.948 & 0.647 & 0.434 & 0.29 & 0.193 & 0.127 & 0.0809 & 0.0505 & 0.0301 & 0.0178 \\
\hline
\end{tabular}

\subsection{Converting a trapezoidal pressure distribution acting on a discrete segment of the I-Wall face into equivalent end point forces}

Both the earth and water pressure distributions acting on the I-Wall are converted into equivalent forces. The Corps_I-Wall software uses the onethird/one-sixth force computational procedure to convert a trapezoidal distribution of a segment of stress between the closest nodes into equivalent nodal forces. This computational procedure is summarized using the Figure 2.4 generalized trapezoidal stress distribution between node $i$ and node $i+1$ acting normal to the I-Wall and/or sheet-pile wall segment $k$, designated as length $k$ in this figure. The equivalent nodal point forces at node $i$ and node $i+1$ are computed to be

$$
F_{i}^{-}=\text {length }_{k} *\left[\frac{\sigma_{i}^{-}}{3}+\frac{\sigma_{i+1}^{+}}{6}\right]
$$

and

$$
F_{i+1}^{+}=\text {length }_{k} *\left[\frac{\sigma_{i}^{-}}{6}+\frac{\sigma_{i+1}^{+}}{3}\right]
$$

length $k$ is the distance from node $i$ to node $i+1, \sigma_{i}$ is the stress normal to node $i$ on wall segment $k$, and $\sigma^{+} i+1$ is the stress normal to node $i+1$ on wall segment $k$. These two relationships for equivalent end forces $F_{i}^{-}$and $F_{i+1}^{+}$ will exactly reflect the trapezoidal pressure diagram along its nodal pair wall segment $k$. The sum of these two forces equals the area under the trapezodial pressure diagram. Moment equlibrium for the wall segment $k$ is statisfied for the applied trapezoidal pressure distribution and these two equivalent end normal forces. 
Figure 2.4. Distribution of stress acting normal to a discrete segment $k$ of the wall face.

(a) Trapezoidal pressure distribution. (b) Equivalent nodal point forces.

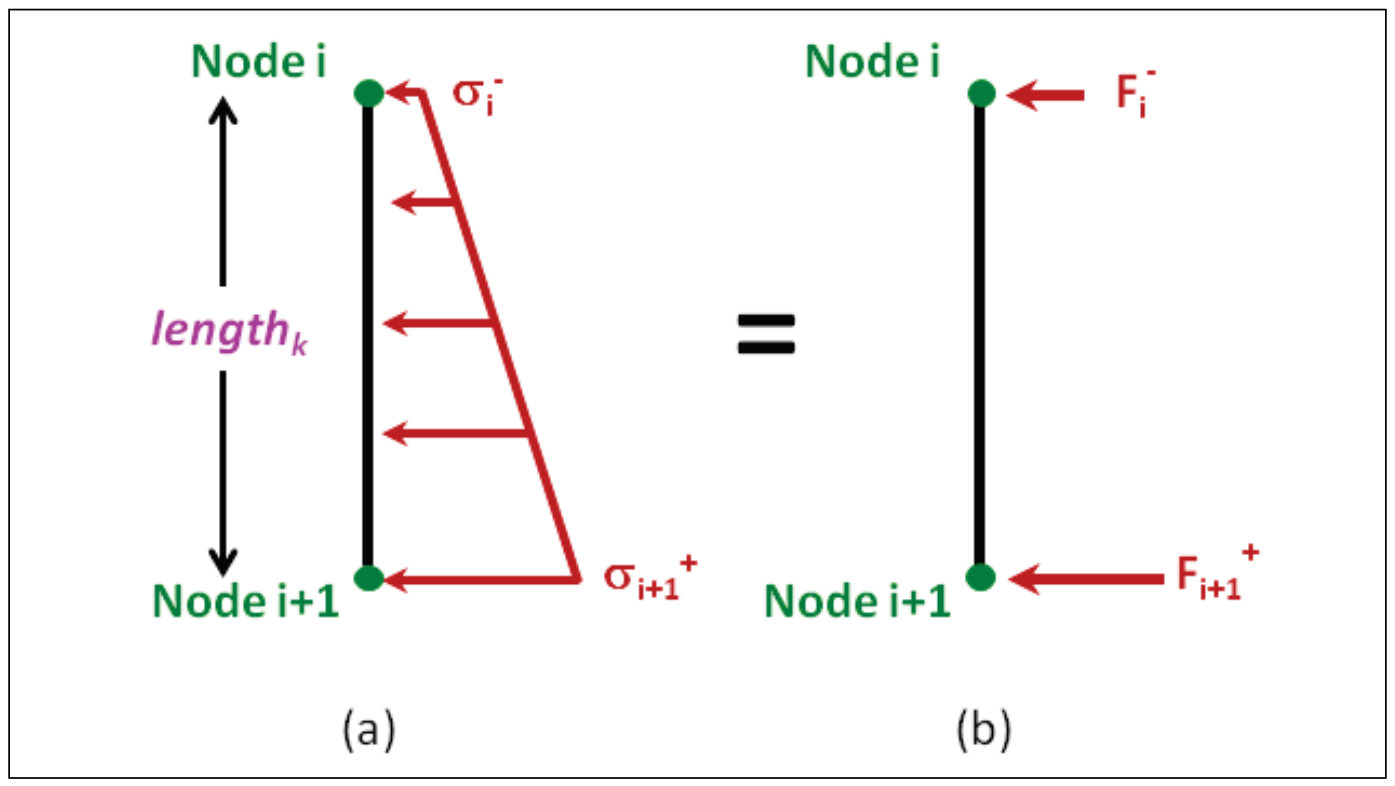

\subsection{Clockwise or counterclockwise I-Wall rotation}

Corps_I-Wall has the ability to accommodate either a clockwise or a counterclockwise I-Wall rotation. The direction of I-Wall rotation is dictated by the direction of net loading and its corresponding moment applied to the I-Wall. Logic was developed to ascertain the direction of I-Wall rotation based upon the flood and earth loading specified for the problem. This capability was specifically developed to accommodate the coastal problem for which the magnitude of user-specified wave or surge water pressure (i.e., height of design storm surge wave) can alter the direction of I-Wall rotation, relative to the calm water design case, especially when a soil berm exists on the landside of the I-Wall. The direction of rotation is determined internally by Corps_I-Wall and reported in the output information contain within the Analysis tab.

To determine the direction of I-Wall rotation, the moment for the net loading is computed about the lower of the RHS or LHS ground surfaces for level ground, for a retaining wall design with differential ground surfaces and for differential water surfaces. A resultant positive moment will produce a counterclockwise rotation, which should agree with mobilized active pressures on the RHS of the I-Wall. If this initial assumption is incorrect, mobilized active pressures on the LHS and mobilized passive pressures on the RHS of the I-Wall will be used to determine if a clockwise rotation will occur from a resultant negative moment. If this next calcula- 
tion does not agree, the sheet-pile I-Wall is deemed stable and no further rotational stability analysis is necessary.

\subsection{Net active pressure and net passive pressure}

In the design of a sheet-pile I-Wall, all applicable pressures are used to compute the net active and net passive pressures along the I-Wall for the final design. The Figure 2.5 schematic shows the I-Wall rotating in the counterclockwise direction with the net active and net passive pressure distributions. The net active pressure distribution with elevation is shown in red solid and dashed line segments while the net passive pressure distribution is shown by a green-dashed line segment. Above the RHS ground surface, the net passive and net active pressure distributions coincide. These two net pressures are determined by:

Net active pressure $=$ RHS soil active pressure - LHS soil passive pressure + net water pressure + surcharge pressure + distributed external horizontal pressure

Net passive pressure $=$ RHS soil passive pressure - LHS soil active pressure + net water pressure + surcharge pressure + external horizontal pressure

Figure 2.5b shows the net active pressures are calculated along the I-Wall from the surface water on the RHS until the elevation at the PR is reached (a solid red line segment). A linear interpolation is made between the net active pressure at elevation PR and the net passive pressure at the sheetpile tip elevation to calculate subsequent net pressures along the sheet-pile wall. This segment of net pressure is shown as a solid green line segment in Figure 2.5b.

\subsection{Factors of safety applied to each soil region}

Corps_I-Wall Version 1.0 allows the user to specify a value for the factors of safety used in both active and passive earth pressure calculations per soil region. The GUI allows the user to define default values for $F S_{\text {Active }}$ and $F S_{\text {Passive }}$ that will pre-populate the factors of safety per soil region, as will be discussed in the Chapter 3 Visual Modeler GUI. 
Figure 2.5. I-Wall rotation. (a) Counterclockwise rotation about a point of rotation PR due to flood loading applied to the right-hand side of the I-Wall. (b) The net water and earth pressures acting on the I-Wall during its rotation.

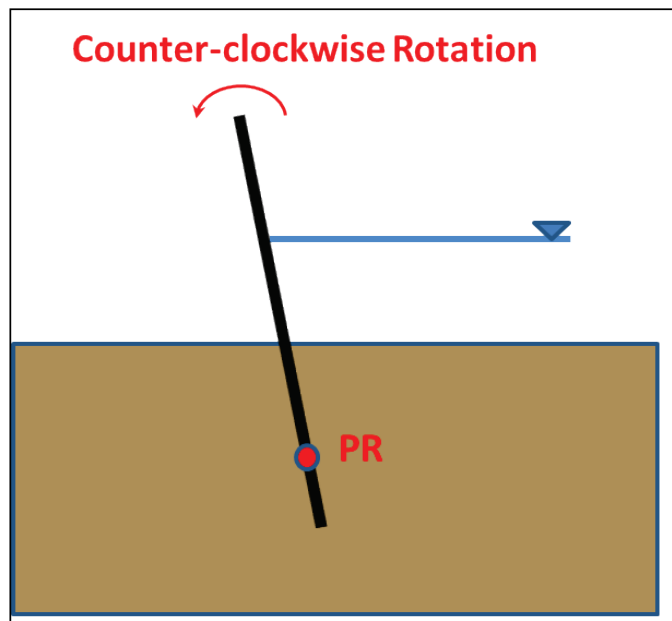

(a)

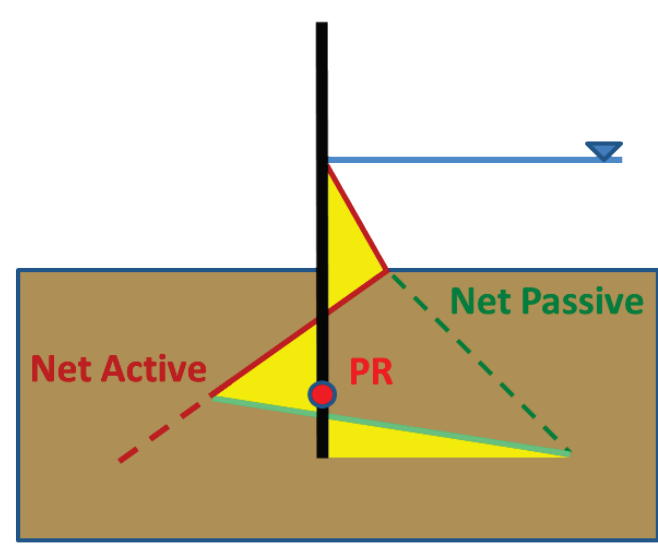

(b)

In a Design mode, the user specifies values for FSActive and FSPassive. The value assigned to $F S_{\text {Active }}$ is usualy 1.0. Guidance on the value to be assigned to $F S_{\text {Passive }}$ is given in EM 1110-2-2504 (HQUSACE 1994) and ETL 1110-2-575 (HQUSACE 2011b).

In an Analysis mode, the value assigned to $F S_{\text {Active }}$ is usually 1.0 and the software will compute the value for $F S_{\text {Passive }}$ assuming the same value is applied to all soil regions developing mobilized passive earth pressures. This engineering evaluation mode is typically used to evaluate the rotational stability of an existing I-Wall.

\subsection{Distributions of water pressures acting on the I-Wall and sheet- pile wall due to surface water and piezometric surfaces}

When surface water is specified by the user along the side of an I-Wall, hydrostatic water pressures are specified normal to the I-Wall from the water surface down to the ground surface. One surface water may be specified for the flood side and a second, independent, surface water may be specifed for the landside, as discussed in Chapter 3 .

When specifing water pressures to be used in computing pore water pressures internal to the soil regime, the user has two options: hydrostatic water pressures and water pressures computed assuming homogenous seepage or hetergeneous seepage. Figure 3.22 in Chapter 3 shows these 
three user options within the GUI. Water pressures internal to the soil are applied normal to the embedded sheet-pile wall when the soil region is specified as an effective stress material. No water pressures internal to the soil are applied normal to the sheet-pile wall when the soil region is specified as a total stress material. When a gap opens between the soil and the sheet-pile wall, hydrostatic water pressures (with the calculations starting at the elevation of the surface water) are applied normal to the sheet-pile wall irrespective of the soil region material specification (Appendix B).

Example 1: Figure 2.6 shows an example of the hydrostatic water pressure distributions acting normal to the sheet-pile wall for a user-selected hydrostatic water pressures option. Both soil regions (Ro1 and R02) represent a single, homogenous soil layer and are specified to be an effective stress material (E). The LHS soil region's piezometric surface is associated with the LHS surface water (designated as the LHS surface water number Po1) and the RHS soil region's piezometric surface is associated with the RHS surface water (designated as the RHS surface water number Po1). The water pressures above the LHS and RHS ground sufaces and the pore water pressures below these two ground surfaces increase in value at a rate of the unit weight of water (i.e., $\gamma_{\text {water }}=62.4 \mathrm{pcf}$ ) per unit of depth (i.e., $1 \mathrm{ft}$ in this case). Observe at the sheet-pile tip in Figure 2.6a that there is a discontinuity in the LHS and RHS hydrostatic pore water pressure values.

Figure 2.6. Distributions of water pressures acting normal to the I-Wall and sheet-pile wall hydrostatic water pressures. (a) Hydrostatic water and pore-water pressure distributions on the LHS and RHS. (b) The net water pressure distribution.

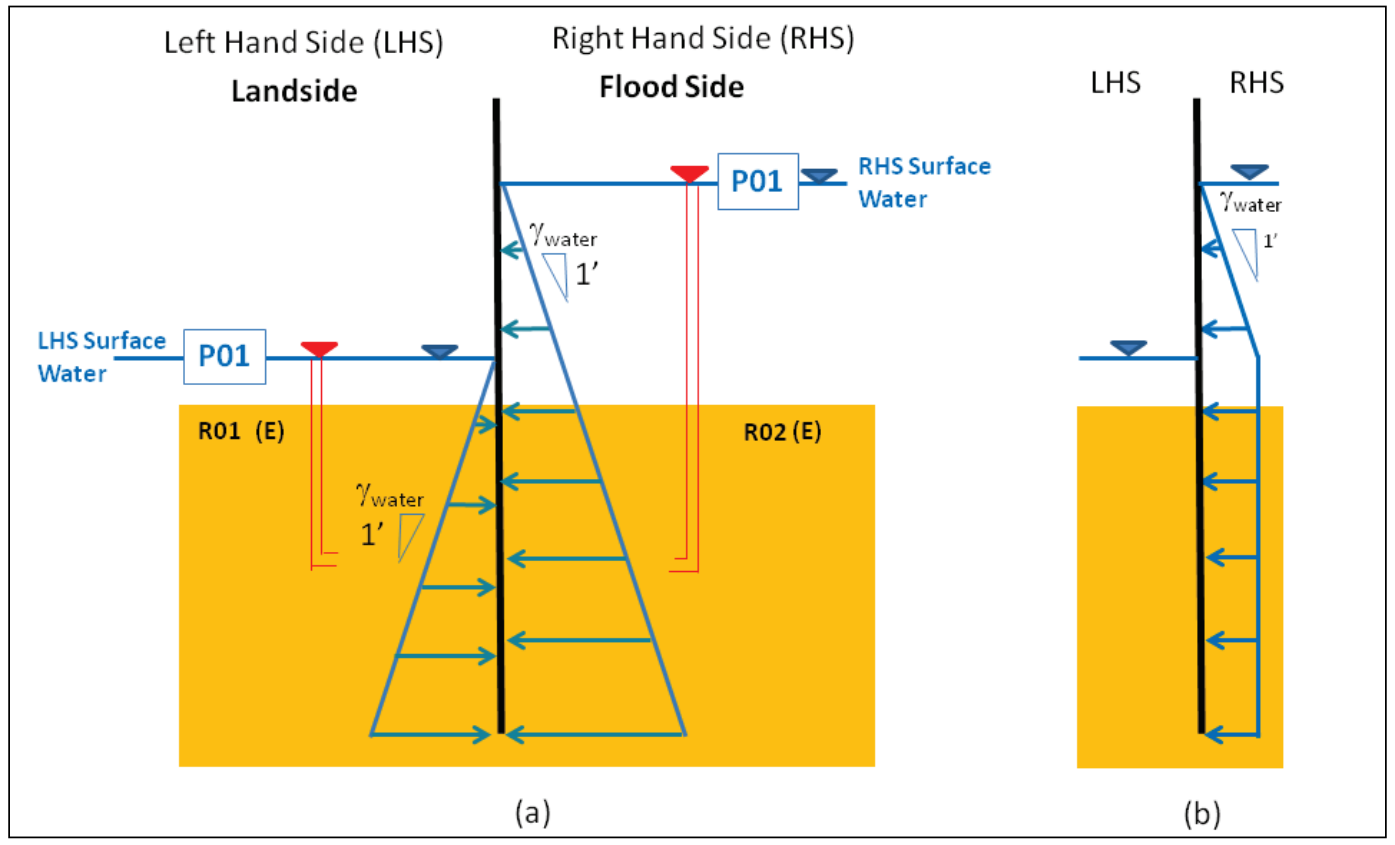


Figure 2.6b shows the distribution of net water pressures acting normal to the I-Wall and sheet-pile wall. The distribution of net water pressures is equal to the difference between the RHS and LHS Figure 2.6a water pressures. For the discontinuity shown in Figure 2.6a, pore-water pressures at the sheet-pile tip are reflected in the net water pressure diagram of Figure $2.6 \mathrm{~b}$ with a non-zero net water pressure.

Example 2: Under the Hydrostatic Water Pressures option (see Figure 3.22 in Chapter 3) and for sites with multiple soil regions, Corps_I-Wall Version 1.0 allows for the definition of more than one piezometric surface on each side. Consider the case shown in Figure 2.7 flood I-Wall. The foundation consists of three soil layers: upper medium sand, clay,and lower dense sand. Both sand layers are designated effective stress-based materials. The intermediate layer is clay and is designated as a total stress material. The software model requires a defintion of this site using six soil regions as shown in Figure 3.22. The LHS medium sand, Region 01, has a piezometric surface (designated P02) associated with it, and the dense sand, Region 03, has a piezometric surface (designated Po3) associated with it. The RHS medium sand, Region 04, has a piezometric surface (designated Po1) associated with it, and the dense sand, Region 06, has a piezometric surface (designated Po4) associated with it. Observe that the RHS Region Ro4 piezometric surface (Po1) corresponds to the RHS surface water of flood loading. The water pressures above the RHS ground surface and within Region 04 increase with decreasing elevation at a rate of $\gamma_{\text {water }}$ (i.e., 62.4 pcf). This is consistent with the Figure 3.22 Hydrostatic Water Pressures option selected by the user. A distribution of hydrostatic water pressure is also computed within the RHS dense sand of Region Ro6 but uses the piezometric surface of Po4 to compute the pore water pressures. The increase in water pressure with decreasing elevation in Region Ro6 is hydrostatic and at a rate of $\gamma_{\text {water. }}$ Observe that the elevation of Po4 is lower than Po1 so the pore water pressures in Region Ro6 are lower than what would occur if the piezometric surface would have been designated to be Po1 for this region. 
Figure 2.7. Distributions of hydrostatic water pressures acting normal to the I-Wall and sheetpile wall - hydrostatic water pressures. (a) Hydrostatic water and pore water pressure distributions on the LHS and RHS. (b) The net water pressure distribution.

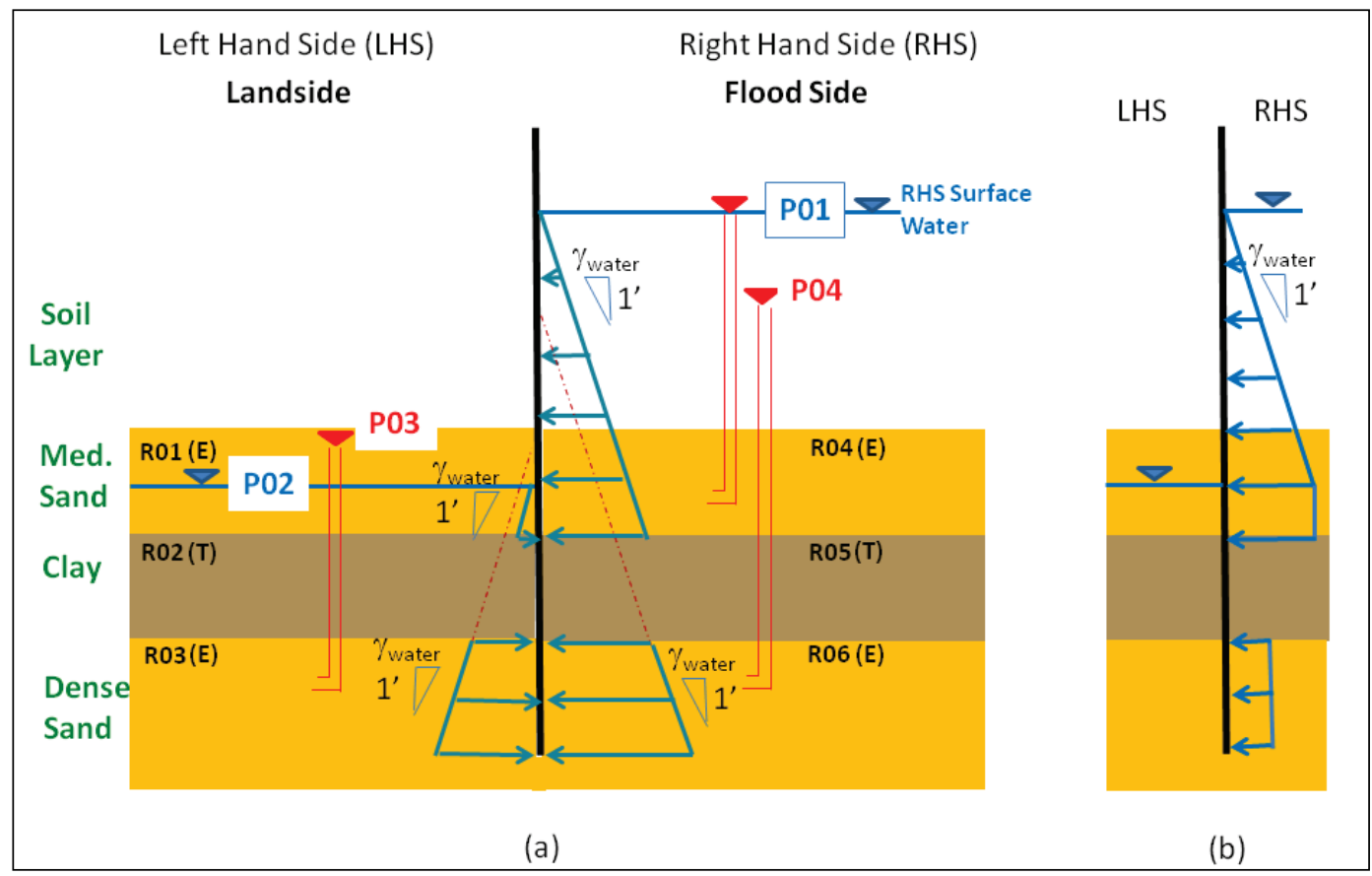

The LHS medium sand, Region 01, has a piezometric surface (designated Po2) associated with it, and the dense sand, Region 03, has a piezometric surface (designated Po3) associated with it. The water pressures start within Region 01 at its piezometric surface (Po2) elevation and increase with decreasing elevation at a rate of $\gamma_{\text {water. A distribution of hydrostatic }}$ water pressure is also computed within the LHS dense sand of Region Ro3 but using the piezometric surface of Po3 to compute the pore-water pressures. The increase in water pressure with decreasing elevation in Region Ro3 is hydrostatic and at a rate of $\gamma_{\text {water. }}$ Observe that the elevation of Po3 is higher than Po2 so the pore-water pressures in Region Ro3 are higher than what would occur if the piezometric surface would have been designated as Po2 for this region.

For stratified soils regions with different piezometric sufaces assigned to adjacent regions, there will be a disjoint in pore-water pressure values at the interface between the two regions under the (Figure 3.22) Hydrostatic Water Pressures option.

Figure 2.7b shows the distribution of net water pressures acting normal to the I-Wall and sheet-pile wall. The distribution of net water pressures is equal to the difference between the RHS and LHS Figure 2.7a water 
pressures. Observe that the discontinuity in the Figure 2.7a pore-water pressures at the sheet-pile tip is reflected in the net water pressure diagram of Figure $2.7 \mathrm{~b}$ with a non-zero net water pressure there.

Example 3: Figure 2.8a shows an example of the water pressure distributions acting normal to the sheet-pile wall for a user-selected Homogenous Seepage option. Both soil regions (Ro1 and Ro2) represent a single soil layer and are specified to be an effective stress material (E). The soil layer and both soil regions possess a single value for vertical hydraulic conductivity. The line of seepage (a.k.a., line of creep) is used to compute pore-water pressures for a steady-state seepage condition (Appendix C) within the soil starting at the ground surface on the high head RHS of the sheet-pile, down to the tip of the sheet pile, and then back up to the ground surface on the low head LHS of the sheet pile.

Figure 2.8. Distributions of water pressures acting normal to the I-Wall and sheet-pile wall - homogenous seepage. (a) Water and pore-water pressure distributions on the LHS and RHS. (b) The net water pressure distribution.

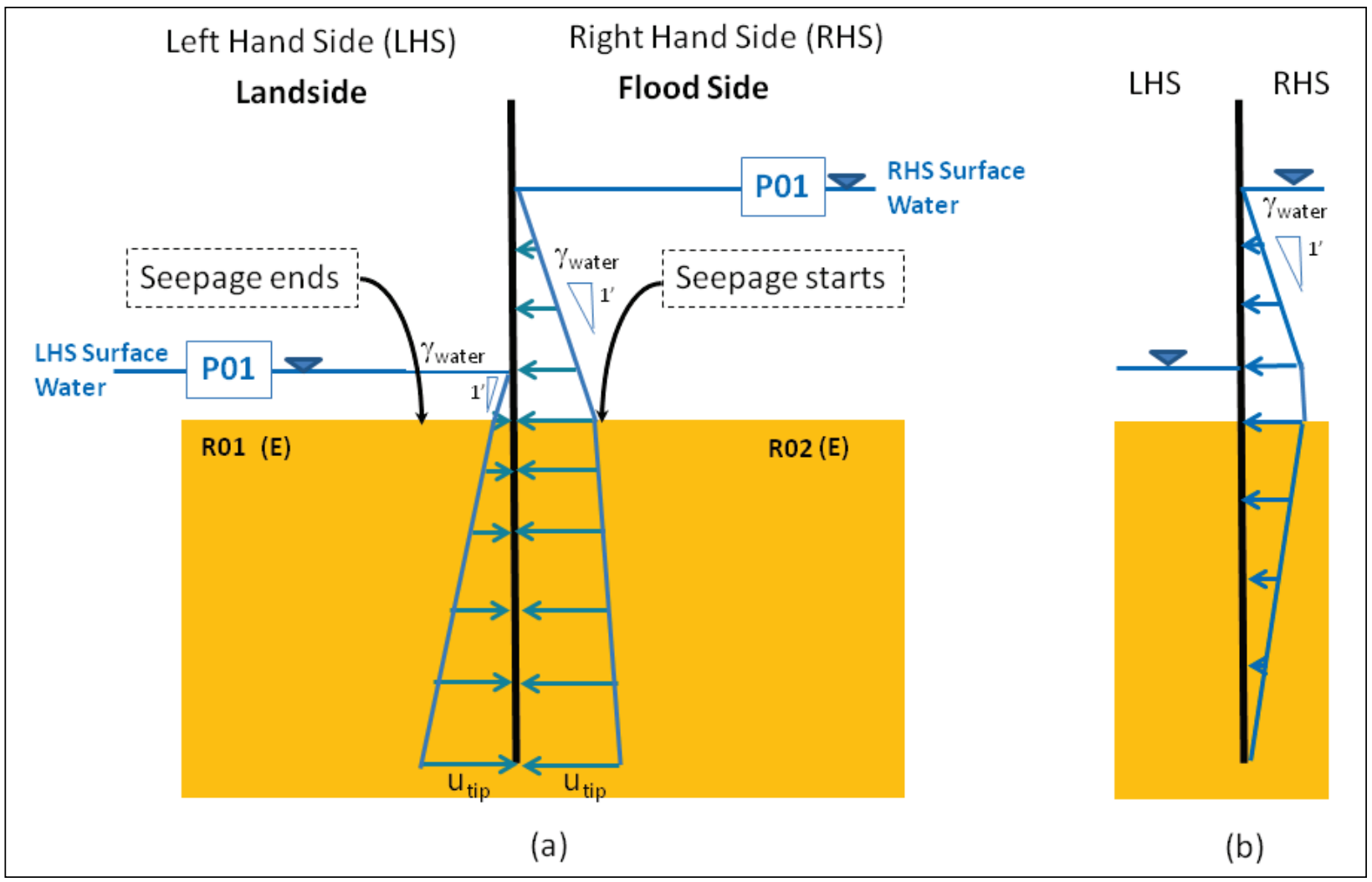

The Figure 2.8a LHS soil region's piezometric surface is associated with the LHS surface water (designated as the LHS surface water number Po1) and the RHS soil region's piezometric surface is associated with the RHS 
surface water (designated as the RHS surface water number P01). ${ }^{1}$ Hydrostatic water presures act against the I-Wall above both the LHS and RHS ground surfaces. These two (surface) water pressure distributions increase with decreasing elevation at a rate equal to the unit weight of water (i.e., $\gamma_{\text {water }}=62.4 \mathrm{pcf}$ ) per unit of depth (i.e., $1 \mathrm{ft}$ in this case). Because of head loss due to seepage, the pore-water pressures within the soil below the LHS and RHS ground increase in value with decreasing elevation at a rate equal to less than $\gamma_{\text {water }}$ per unit of depth. Observe at the sheet-pile tip in Figure 2.8a that there is continuity in the LHS and RHS hydrostatic pore-water pressure values.

Figure $2.8 \mathrm{~b}$ shows the distribution of net water pressures acting normal to the I-Wall and sheet-pile wall. The distribution of net water pressures is equal to the difference between the RHS and LHS Figure 2.8a water pressures. Observe the continuity in the Figure 2.8a pore-water pressures at the sheet-pile tip is reflected in the net water pressure diagram of Figure $2.8 \mathrm{~b}$ with a zero net water pressure there.

Example 4: Figure 2.9a shows an example of the water pressure distributions acting normal to the sheet-pile wall for a user-selected Heterogenous Seepage option. The site consists of two soil layers: a medium dense sand overlaying a dense sand. This software requires that the upper soil layer be modeled by two soil regions (Ro1 and Ro3), which are specified to be an effective stress material (E). This upper soil layer (and both soil regions) possess a single value for vertical hydraulic conductivity with a value of $k_{1}$ (in units of length/time; e.g., $\mathrm{ft} / \mathrm{sec}$ ). The lower soil layer is also modeled by two soil regions (Ro2 and Ro4), which are specified to be an effective stress material (E). This lower soil layer (and both soil regions) possess a single value for vertical hydraulic conductivity with a value of $k_{2}$. The value for $k_{2}$ is one-half the value of $k_{1}$.

\footnotetext{
${ }^{1}$ By the theory of steady-state seepage, the seepage of water through the soil surrounding the sheet-pile wall occurs down the high head side, around the tip of the sheet pile, and up the soil on the low head side. When a seepage analysis is specified, the software requires that only one piezometric surface (or surface water assigned as a piezometric surface) be assigned per side. This restriction is for either the Homogeneous or Heterogeneous Seepage option.
} 
Figure 2.9. Distributions of water pressures acting normal to the I-Wall and sheet-pile wall hetergenous seepage for two soil layers, (a) Water and pore-water pressure distributions on the LHS and RHS. (b) The net water pressure distribution.

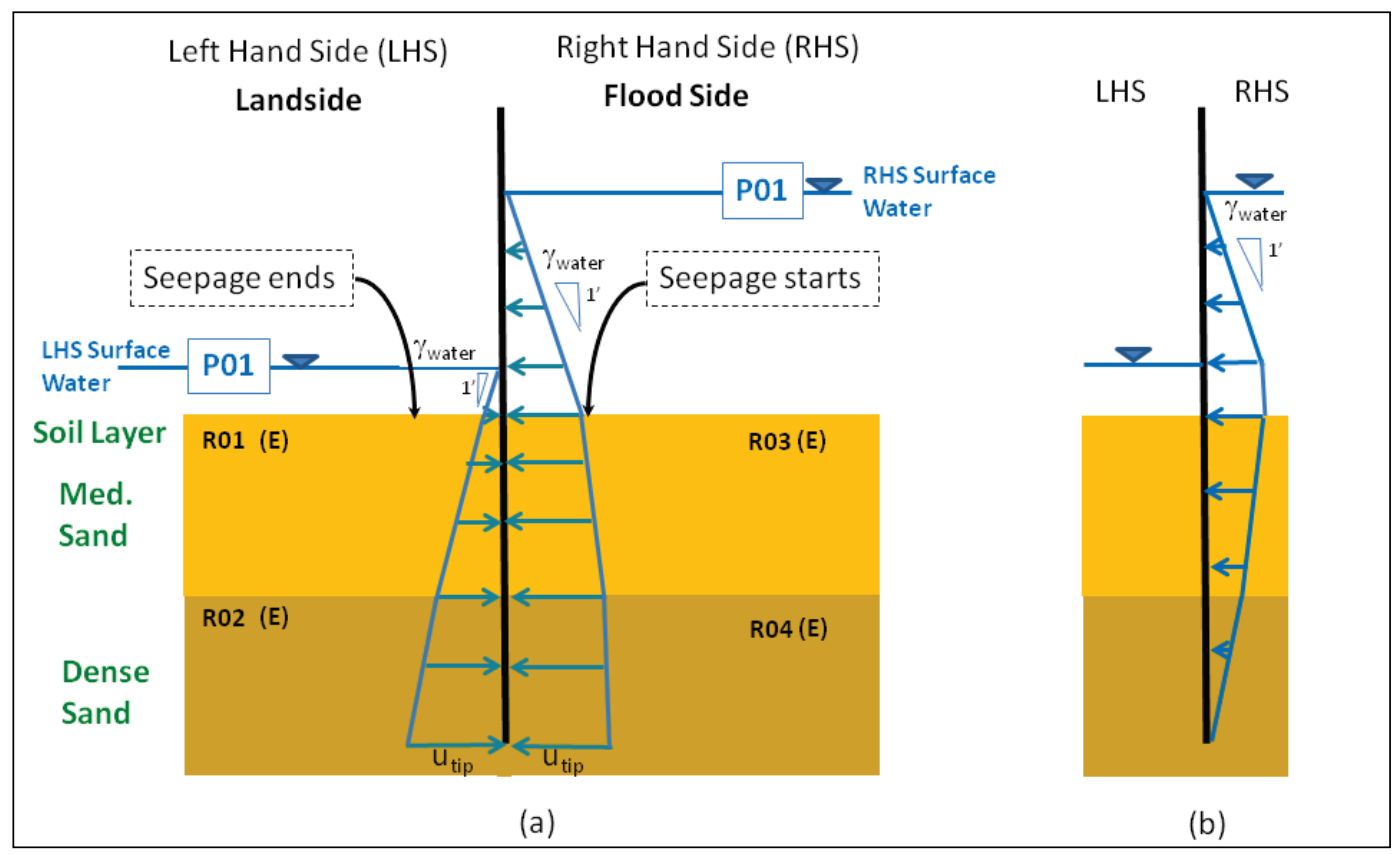

The Appendix C line of seepage (a.k.a., line of creep) is used to compute pore-water pressures for a steady-state seepage condition within the soil starting at the ground surface on the high head RHS of the sheet pile, down to the tip of the sheet pile, and then back up to the ground surface on the low head LHS of the sheet pile.

The two Figure 2.9a LHS soil region's (Ro1 and R02) are associated with a single piezometric surface that corresponds to the LHS surface water (designated as the LHS surface water number Po1). The two RHS soil region's (Ro3 and Ro4) are associated with a single piezometric surface that corresponds to the RHS surface water (designated as the RHS surface water number P01). Hydrostatic water pressures act against the I-Wall above both the LHS and RHS ground surfaces. These two (surface) water pressure distributions increase with decreasing elevation at a rate equal to the unit weight of water (i.e., $\gamma_{\text {water }}=62.4 \mathrm{pcf}$ ) per unit of depth (i.e., $1 \mathrm{ft}$ in this case). Because of head loss due to seepage, the pore-water pressures within the soil below the LHS and RHS ground increase in value with decreasing elevation at a rate equal to less than $\gamma_{\text {water }}$ per unit of depth. Additionally, at the interface between the upper soil region and the lower soil region there is a change in the rate of pore water pressure computed with depth by the Appendix $\mathrm{C}$ line of seepage procedure due to the contrast in hydraulic conductivity values for the two soil layers. Observe at the sheet-pile tip in 
Figure 2.9a that there is continuity in the LHS and RHS hydrostatic pore water pressure values.

Figure 2.9b shows the distribution of net water pressures acting normal to the I-Wall and sheet-pile wall. The distribution of net water pressures is equal to the difference between the RHS and LHS Figure 2.9a water pressures. Observe the continuity in the Figure 2.9a pore-water pressures at the sheet-pile tip is reflected in the net water pressure diagram of Figure $2.9 \mathrm{~b}$ with a zero net water pressure there.

\subsection{Surcharge pressures, horizontal pressures and line loads}

Externally applied pressures and line loads are used in Corps_I-Wall Version 1.o to compute equivalent horizontal pressures acting along the sheet-pile wall. These surcharges will increase the overall horizontal pressures. Horizontal distributed pressures and horizontal line loads always act normal to the sheet-pile wall and can be assigned by specifying line loads at particular elevations at the wall or by specifying one or a series of trapezoid pressures at elevations along the I-Wall. Vertical pressures and line loads always act normal to the ground surface ${ }^{1}$. There are six methods used to define externally applied pressures and line loads. These pressure distributions and line loads will compute a value of equivalent horizontal pressure at nodal points along the wall.

\subsubsection{Externally applied horizontal pressure distribution and line loading}

Horizontal pressures and line loads are usually introduced for an I-Wall rotational stability analysis when wave actions originating from storms with available wave loading information are being considered. The two types of user-defined horizontal pressure distribution and line loads are illustrated by Figure 2.10. Figure 2.10a represents a line load applied at elevation (el) with loading of HL force. Figure $2.10 \mathrm{~b}$ represents a pressure distribution of two horizontal pressures. The first is applied at elevation el ${ }_{1}$ with pressure of $\sigma_{\mathrm{H}_{1}}$ and the second at elevation $\mathrm{el}_{2}$ with pressure of $\sigma_{\mathrm{H}_{2}}$. Multiple line loads and combinations of distributed pressures can be assigned. A positive line load or pressure distribution acts toward the left ${ }^{2}$ and a negative line load or

\footnotetext{
${ }_{1}^{1}$ Analyses of I-Walls produced from Corps_I-Wall, Version 1.0., are on level ground; therefore, all pressures and forces are vertical and act normal to the ground surface.

2 Surcharge pressures or loads located (a) on the RHS of the I-Wall will act toward the I-Wall and (b) on the LHS of the I-Wall will act away from the I-Wall.
} 
pressure distribution will act toward the right ${ }^{1}$. Therefore, there can be positive or negative loads and pressure distributions on either side of the I-Wall.

Figure 2.10. External horizontal line load and pressures. (a) Line load. (b) Distributed pressures.

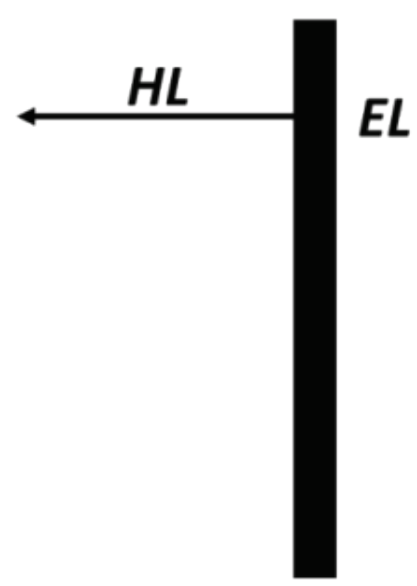

(a)

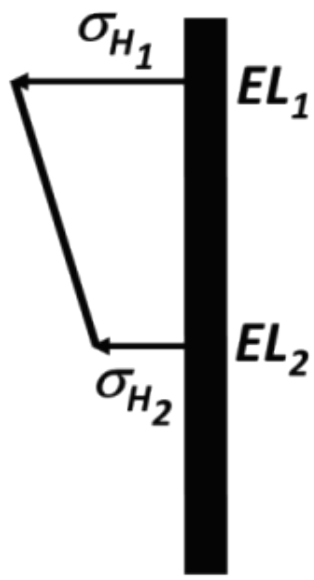

(b)

\subsubsection{Externally applied vertical pressure distribution and line loading}

Surface surcharge pressures and line loads are usually introduced for an I-Wall rotational stability analysis when external structures, such as buildings, construction equipment, vegetation, or stockpiles are considered. There is one definition for applying vertical line loads and four variations for describing possible applied vertical pressure distributions. Pressures due to line loads can be calculated using Figure 2.11. The two elastic half-space theory-based relationships given in this figure include a factor of 2 , as per Clough and Duncan's (1991) concept figure (Figure 6.14) for a wall retaining non-yielding soil. A uniform surface surcharge pressure (Q) is added directly as overburden pressure at the ground surface. The vertical stress will increase by the amount of the surcharge. Horizontal pressures from strip, ramp, triangular, and trapezoidal distributions can be calculated using Figure 2.12. These relationships are for a wall retaining yielding backfill. For walls retaining non-yielding backfills, a factor of 2 would be applied to these relationships. Horizontal pressures due to the various surcharge distributions can be calculated by applying the principle of superposition to the solutions.

\footnotetext{
${ }^{1}$ Surcharge pressures or loads located (a) on the RHS of the I-Wall will act away from the I-Wall and
} (b) on the LHS of the I-Wall will act toward the I-Wall. 
Figure 2.11. Vertical line load.

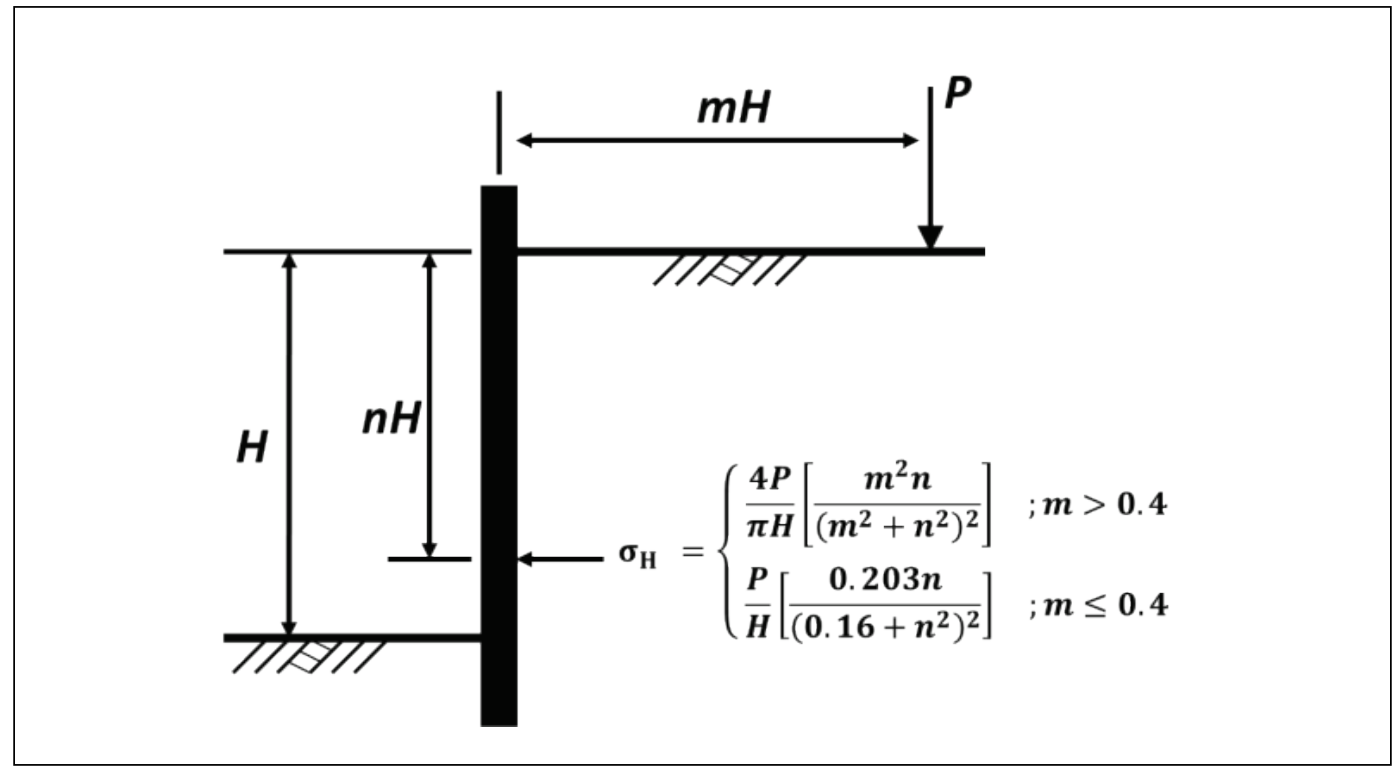

Figure 2.12. Vertical pressure distributions. (a) Strip pressure. (b) Ramp pressure. (c) Triangular pressure. (d) Trapezoidal pressure.
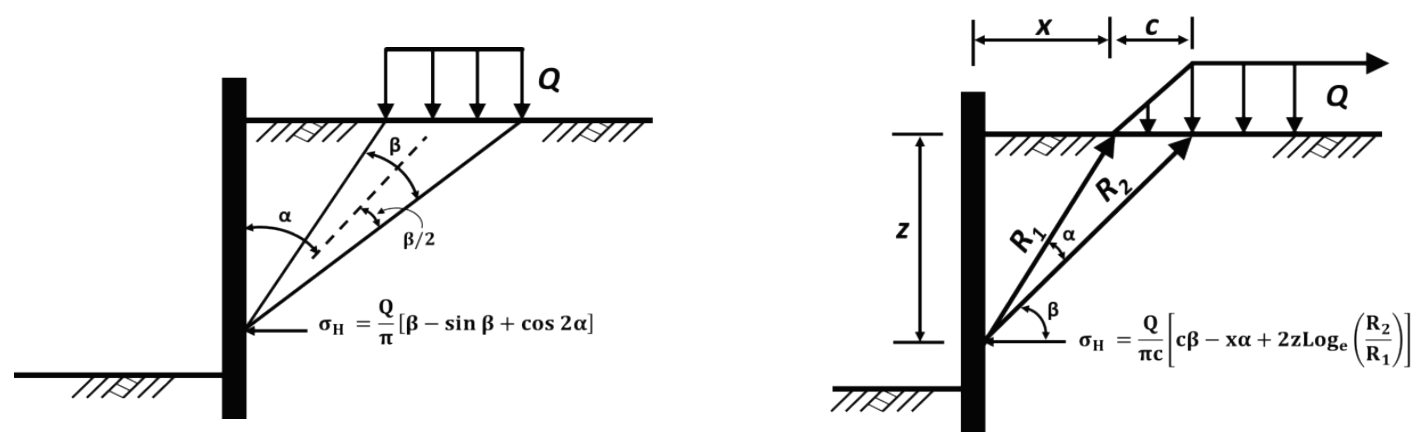

(a) (b)
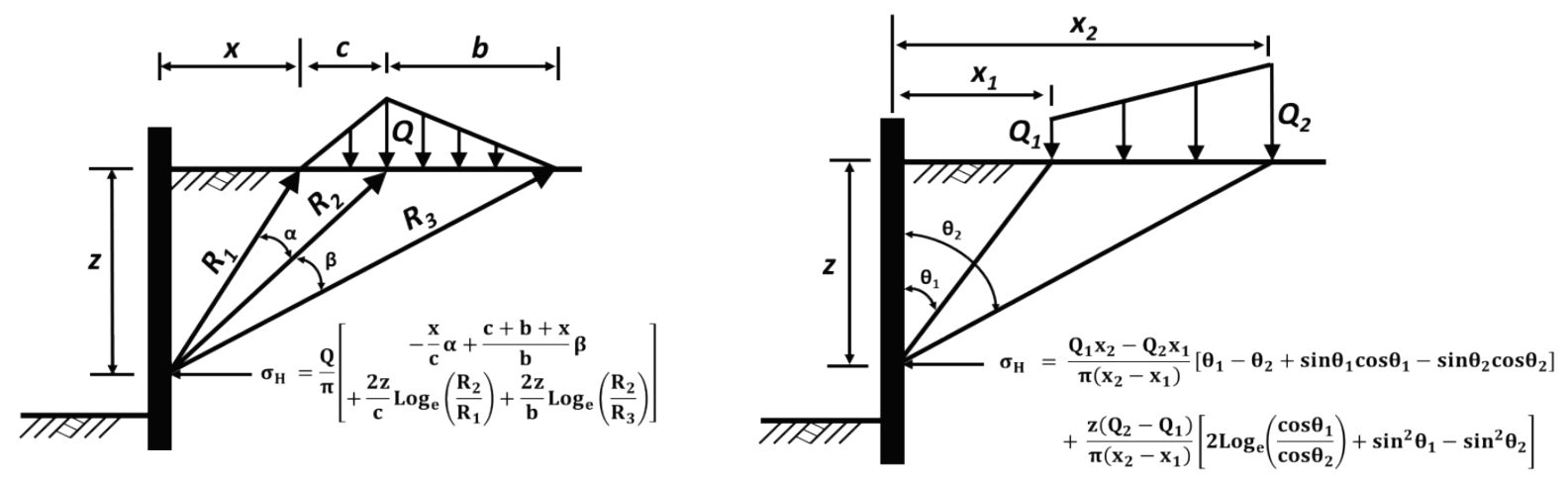

(c) (d) 


\subsection{Shear force and moment distribution internal to the I-Wall and sheet pile}

The shear force and moment internal to the I-Wall/sheet-pile wall are computed following the procedure outlined in Figures 2.13 and 2.14. From the top of the I-Wall down to the sheet-pile tip, wall segments are defined by each pair of adjacent nodes. Figure 2.13 depicts wall segment $k$ between node $i$ and node $i+1$. Similarly, Figure 2.14 depicts the Figure 2.13 wall segment $k$ and wall segment $k+1$ between node $i+1$ and node $i+2$. Because there can be a discontinuity in normal pressure at each interface between adjoining soil regions (e.g., at node $i+1$ in Figure 2.14), there is a need to maintain an upper and a lower earth pressure computation per node to accommodate this possibility (e.g., for node $i+1$, designating it as $\sigma_{i+1}^{+}$and $\sigma_{i+1}^{-}$in Figure 2.14).

For each wall segment the software converts the applied pressure distribution into equivalent nodal point forces $\left(F_{i}^{-}\right.$and $\left.F_{i+1}^{+}\right)$using the onethird/one-sixth force computational procedure described in Section 2.4. With the exception of the top node and the node at the sheet-pile tip, a plus and minus designated equivalent nodal point force is computed. At the top of I-Wall loaded wall segment, only a minus designated equivalent nodal point force is computed. Similarly, at the tip of the sheet-pile wall, only a plus designated equivalent nodal point force is computed.

Figure 2.13. Internal shear and moment at node $i+1$ for wall segment $k$. (a) Trapezoidal pressure distribution. (b) Equivalent nodal point forces.

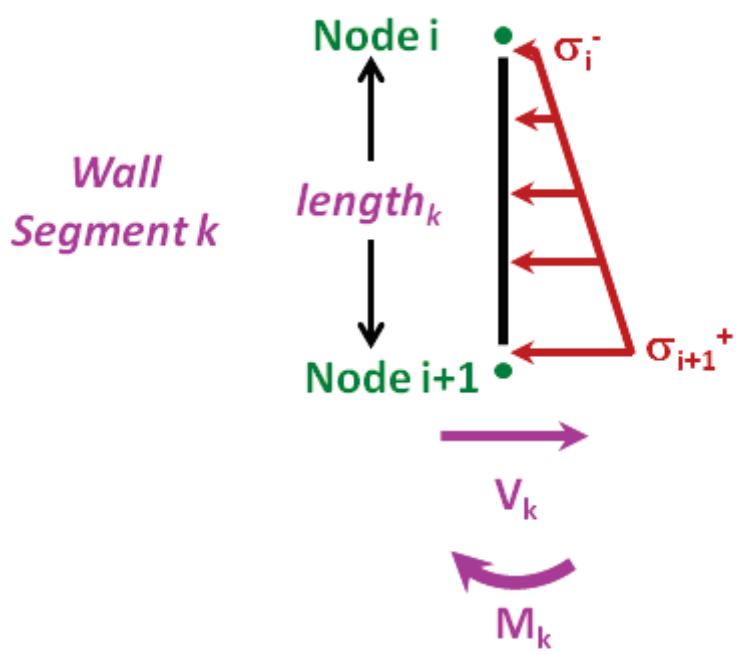

(a)

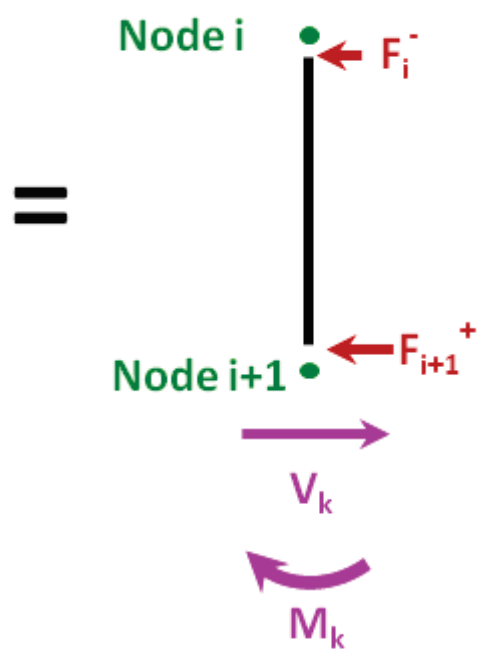

(b) 
Figure 2.14. Internal shear and moment at node $i+2$ for wall segment $k+1$. (a) Trapezoidal pressure distributions. (b) Equivalent nodal point forces.

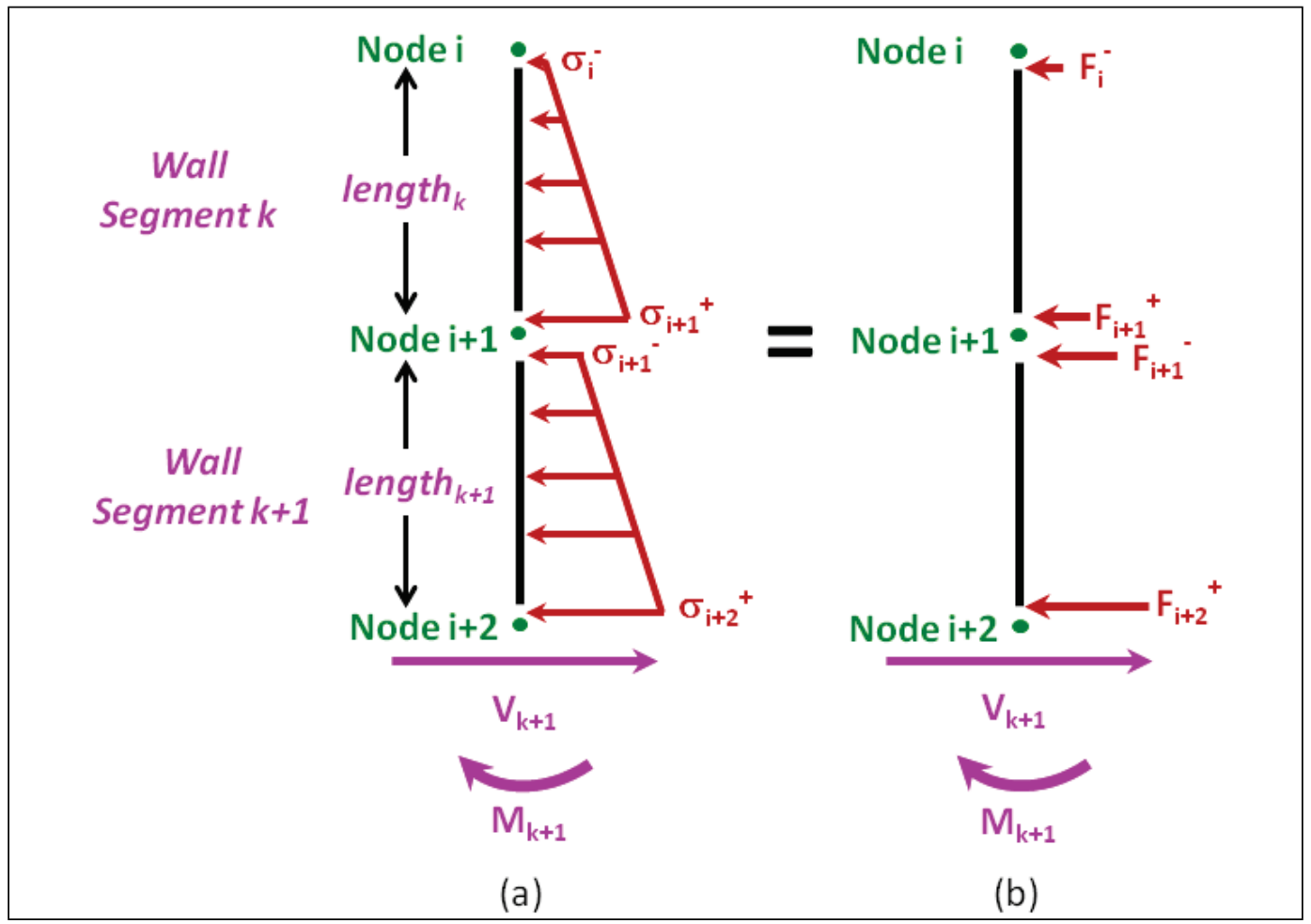

Internal shear force: The shear force $V_{k}$ at the bottom of the Figure 2.13 wall segment $k$ is equal to the sum of forces acting on wall segment $k$.

$$
V_{k}=F_{i}^{-}+F_{i+1}^{+}
$$

This shear force $V_{k}$ is assigned at the elevation of node $i+1$ within the software.

The shear force $V_{k+1}$ at the bottom of the Figure 2.14 wall segment $k+1$ is equal to the sum of forces acting on wall segments $k$ and $k+1$.

$$
V_{k+1}=F_{i}^{-}+F_{i+1}^{+}+F_{i+1}^{-}+F_{i+2}^{+}
$$

This shear force $V_{k+1}$ is assigned at the elevation of node $i+2$ within the software.

This calculation procedure is followed top down for all wall nodes to define the distribution of shear force internal to the wall. 
Internal moment:

The moment $M_{k}$ at the bottom of the Figure 2.13 wall segment $k$ is equal to the sum of forces acting on wall segment $k$.

$$
M_{k}=F_{i}^{-} \cdot \text { length }_{k}
$$

This moment $M_{k}$ is assigned at the elevation of node $i+1$ within the software.

The moment $M_{k+1}$ at the bottom of the Figure 2.14 wall segment $k+1$ is equal to the sum of forces acting on wall segments $k$ and $k+1$ times their respective lever arm.

$M_{k+1}=F_{i}^{-} \cdot\left(\right.$ length $_{k}+$ length $\left._{k+1}\right)+\left\{F_{i+1}^{+}+F_{i+1}^{-}\right\} *\left(\right.$ length $\left._{k+1}\right)$

This moment $M_{k+1}$ is assigned at the elevation of node $i+2$ within the software.

This calculation procedure is followed top down for all wall nodes to define the distribution of moment internal to the wall.

The resulting distributions of internal shear and moment are tabulated and presented in graphical form by the software, as shown in Figures 3.64 and 3.66 of Chapter 3 . 


\section{Visual Modeler Graphical User Interface User's Guide}

\subsection{Introduction}

This chapter discusses the Visual Modeler for the Corps_I-Wall software. The Visual Modeler allows the user to create the input that will be processed, to process the input to gather results, and to visualize the results in a meaningful fashion. An example problem is used to demonstrate the features for input and output visualization, so the user can see how the program works.

\subsection{Example problem}

The input and screen captures presented in this chapter are all built around an example problem. This example problem illustrates many of the more involved features in the GUI. This subsection defines the input parameters for the I-Wall section being analyzed.

Version 1.0 of the Corps_I-Wall software only works with level soil surfaces next to the I-Wall, so all of the soils will be defined as rectangular blocks close to the I-Wall. Future versions of the Corps_I-Wall software will allow for more involved analysis with more involved geometry (e.g., varying ground surface elevations e; varying soil strata interface elevations; and varying piezometric surface elevations), and the GUI has been defined with the more advanced features in mind.

The I-Wall section in the example problem (Figure 3.1) is $12 \mathrm{ft}$ high, has a layer of loose sand beginning at el o.o, and has another layer of dense sand beginning at el -10 ft. Because we are designing the sheet-pile section, the program will determine an appropriate depth of embedment for the I-Wall. Because Version 1.o of the Corps_I-Wall software deals only with the intersections of soils with the I-Wall, the modeled soils will only extend a short way from the I-Wall (for input with the GUI).

On the flood side of the I-Wall, which is arbitrarily chosen to be on the LHS of the I-Wall, there is surface water, which begins at el $10 \mathrm{ft}$. On the landside of the I-Wall (RHS), there is a water table, which is modeled as a piezometric surface at an el -1.0 ft. $\gamma_{\text {water }}$ is given as $62.4 \mathrm{pcf}$. 
Figure 3.1. Example problem for an I-Wall section used to illustrate features of the GUI.

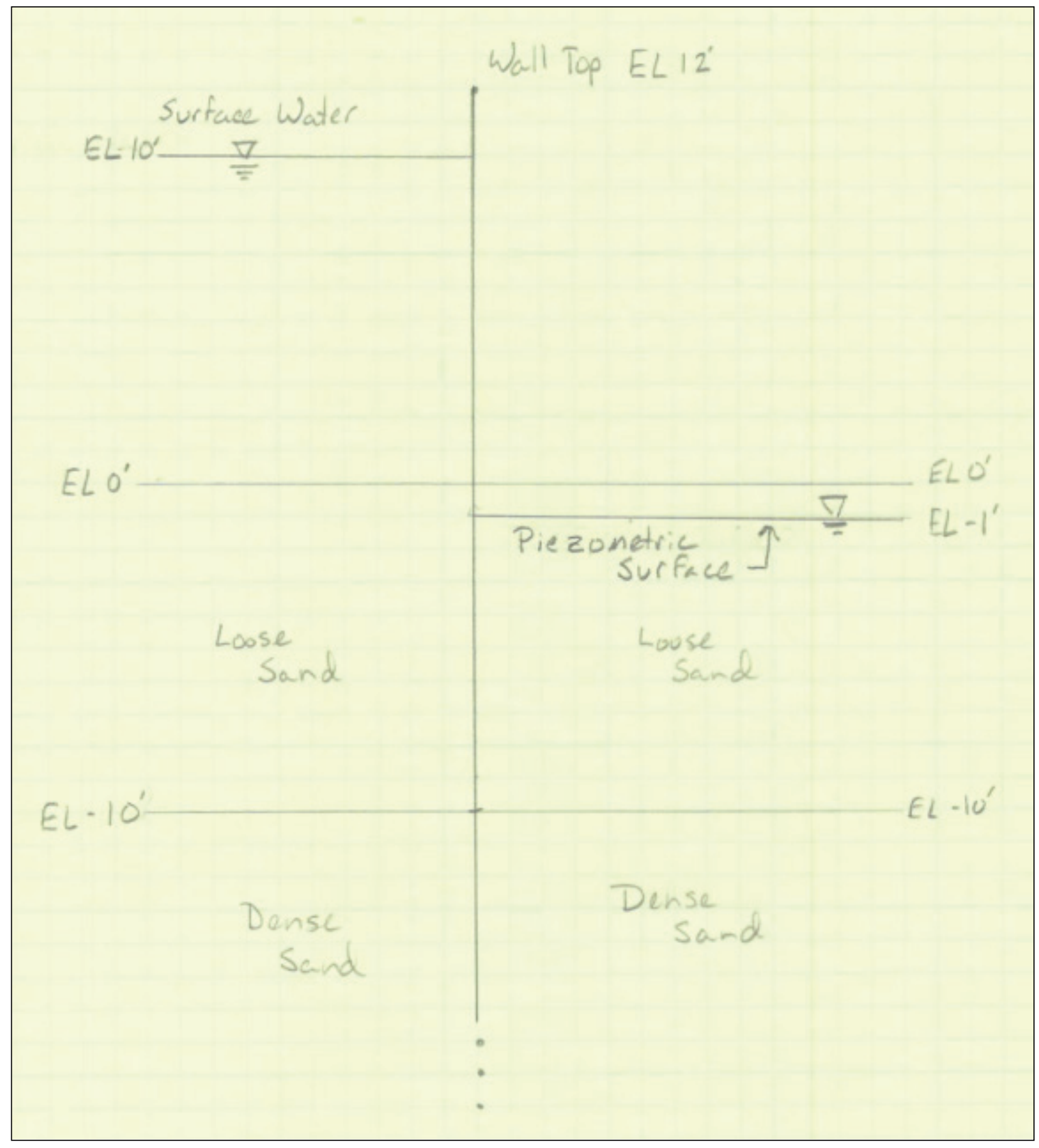

The soil properties for loose and dense sand use effective stress and are given in the following table: 
Table 3.1. Input soil properties for the example problem.

\begin{tabular}{|l|c|c|}
\hline Soil Layer & Loose Sand & Dense Sand \\
\hline Ymoist (pcf) & 110 & 115 \\
\hline Ysaturated (pcf) & 122.4 & 126 \\
\hline FSactive & 1.0 & 1.5 \\
\hline FS passive & 1.0 & 1.5 \\
\hline$C^{\prime}$ & 5.0 & 0.0 \\
\hline$\varphi^{\prime}$ & $30^{\circ}$ & $36^{\circ}$ \\
\hline$\delta^{\prime}$ & $15^{\circ}$ & $18^{\circ}$ \\
\hline $\mathrm{C}^{\prime}(\mathrm{psf})$ & 0.0 & 0.0 \\
\hline $\mathrm{K}(\mathrm{cm} / \mathrm{sec})$ & $3 \times 10^{-3}$ & $1 \times 10^{-3}$ \\
\hline $\mathrm{K}(\mathrm{ft} / \mathrm{sec})$ & 0.000098425 & 0.000032808 \\
\hline & & \\
\hline
\end{tabular}

Gap propagation is enabled, and heterogeneous seepage is enabled to show the effects of different soil properties.

\subsection{Introduction tab}

When the Corps_I-Wall program (Version 1.0) opens, the first display is the Introduction tab (Figure 3.2) and problem-specific input fields at the top of the page. The Introduction tab has an image that illustrates the kind of problems that Corps_I-Wall will be able to analyze.

The input data at the top of the window (which is available no matter which tab is selected) are a project title text entry box and radio button selectors for whether the current analysis will be deterministic or probabilistic. Each project should be given a unique title because multiple projects and analyses can be saved in the same directory. Because filenames can become cryptic, a good description of the problem in the project title can provide a way to differentiate files.

The selector for whether a project will be deterministic or probabilistic will be discussed in more detail in the section dealing with soil properties. 
Figure 3.2. Introduction tab.

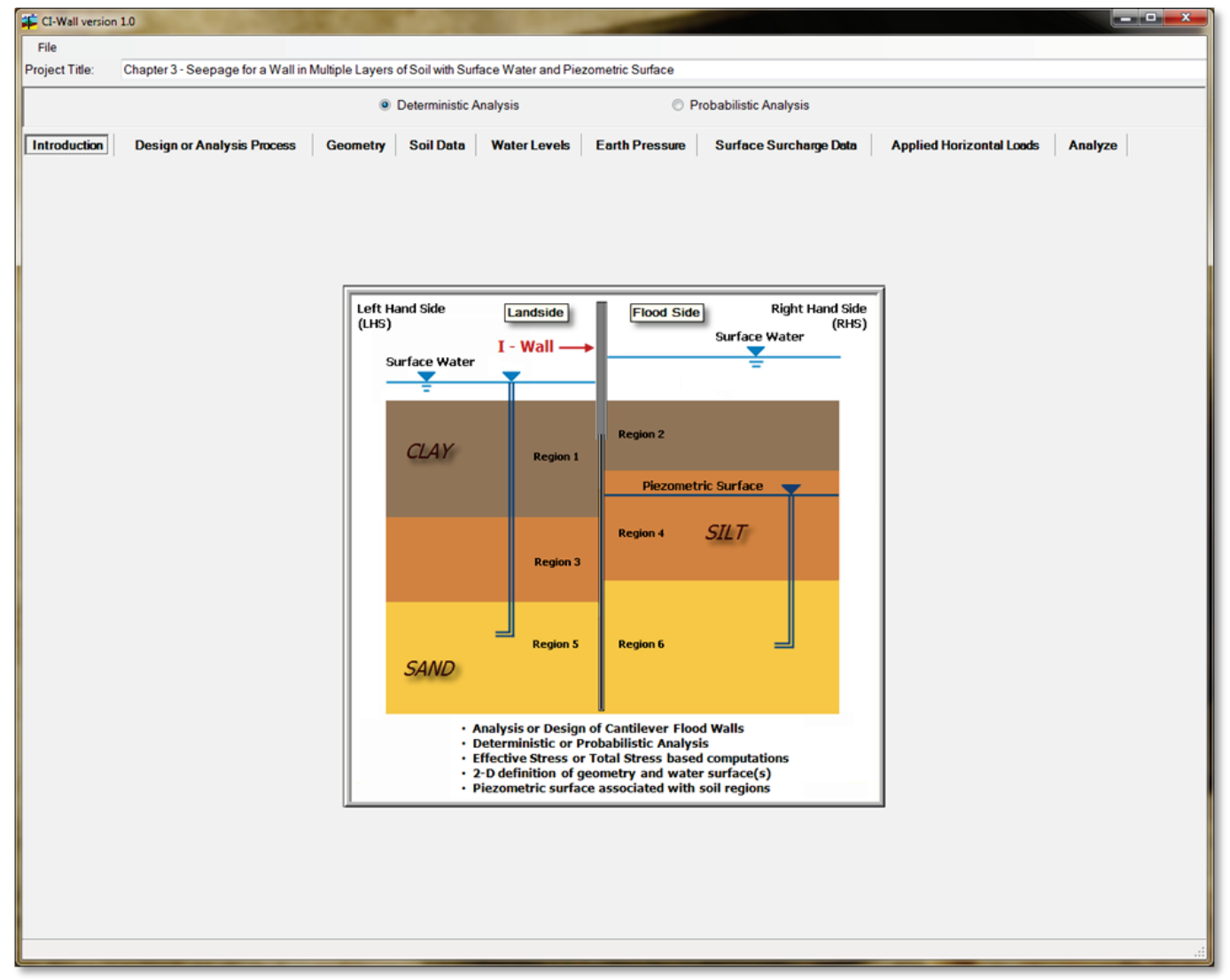

\subsection{Design or Analysis Process tab}

While named the Design or Analysis Process tab, this tab actually also has a geometric extent and unit information section. The geometric extents describe the area in which a problem will be solved. It is used for visualization purposes; the fully-defined model will be expected to reach the left and right extents and to be sitting on the bottom extent.

The area for unit selection and extent entry is to the left of the Design or Analysis Process tab (as shown in Figure 3.3). Radio buttons allow the user to choose the appropriate units of measure that will be used for the project. If the user selects metric units, all distance coordinates will be entered in with decimal meters. For English measurements, distance coordinate data entry will be in decimal feet.

The I-Wall extent entries are next. The first two entries for the I-Wall extents are the $\mathrm{Y}$ and $\mathrm{X}$ coordinates of the I-Wall top. These are given in absolute coordinates and establish the right-handed coordinate system for the I-Wall system, with the I-Wall extending along the y-axis. 
Figure 3.3. Design or Analysis Process - Design mode.

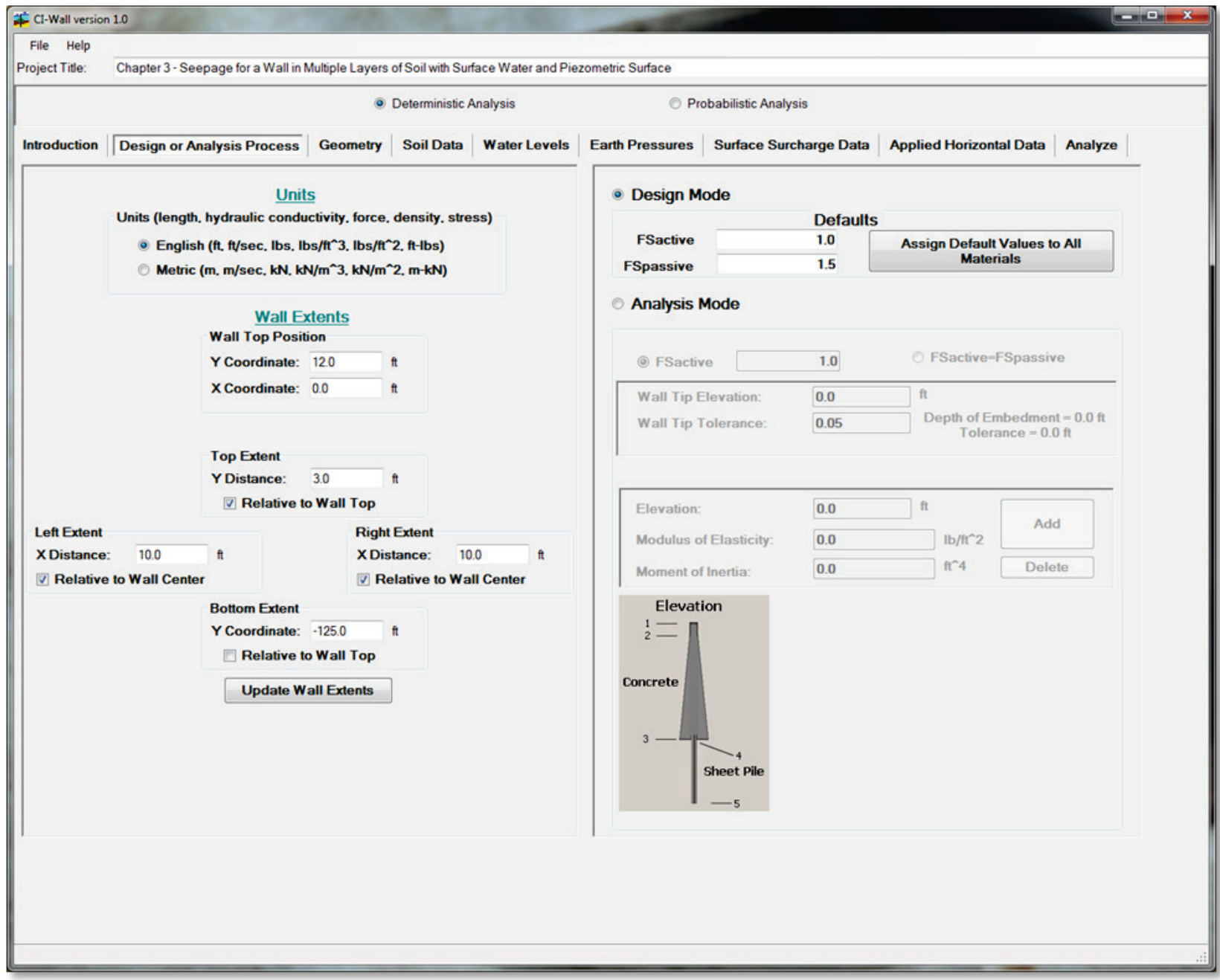

The next entry is the $\mathrm{Y}$ coordinate for the highest data point expected to be used for the model. Because some models may be built with water levels that exceed the height of the I-Wall, this elevation should be set to the higher of the two elevations. Notice that this entry does not create data points but only affects the highest point that may be created and shown in the Geometry tab discussed later. This coordinate can be entered with either relative coordinates (given by a distance above the I-Wall top) or as an absolute coordinate, depending on the checkbox below the input. Changing the checkbox also adjusts the current value to show the absolute or relative value of the extent. This same process is used for the left, right, and bottom extents.

The left and right extents give the maximum distance that soil-based regions can extend to in the X-coordinate. The Corps_I-Wall program uses a right-hand coordinate system, so the extents to the left are in the 
$-\mathrm{X}$ direction and the extents to the right are in the $+\mathrm{X}$ direction. These extents can be modified in the Geometry tab but, as a good rule-of-thumb, extents should be used to describe how much soil on either side of I-Wall will be needed to solve the problem. It is expected that regions will extend to the extents on either side of the I-Wall. Using relative distances, the extents should be specified with positive numbers. Therefore, if the I-Wall top has a 0.o X-coordinate, the relative left distance of 10.0 gives an absolute extent coordinate of -10.0.

The final entry for the extents of the model is the elevation at the bottom extent of the model. In the Design mode, where the program is attempting to determine the appropriate I-Wall tip depth of embedment, this extent should be set deeper than the deepest elevation expected for the I-Wall tip. In Analysis mode, this extent should be set to the I-Wall tip depth. In either circumstance, soil regions will be created extending from this elevation to the bottom of the lowest region drawn in the Geometry tab.

On the right side of the Design or Analysis Process tab is the entry for selecting either the Design or Analysis mode for the solver. Radio buttons allow the user to select the appropriate mode. Each mode has ancillary data that may be entered.

If the Design mode is selected, the user is able to set default values for the $F S_{\text {active }}$ and $F S_{\text {passive }}$ soil data values. When these values have changed, any new soil materials that are created will have these values set to the defaults, and previously existing materials that have the previous default value will be changed to the new default value. A button with the caption Assign Default Values to All Materials is next to the text entries for these defaults. If the button is pressed, all previously created soil materials will be assigned the current value of the default entries.

In future versions of Corps_I-Wall for the Analysis mode, the user can specify how the $F S_{\text {active }}$ is defined, either as a constant value, or equivalent to the each soil material's specified $F S$ passive value. At the moment, this option is grayed out. In Design mode, the Corps_I-Wall program is attempting to find a near-optimal I-Wall depth of embedment. In Analysis mode, the Corps_I-Wall program is attempting to analyze a fully specified I-Wall, therefore an I-Wall tip elevation should be input, with a tolerance. 
Finally, for future versions of Corps_I-Wall, in Analysis mode (shown in Figure 3.4), Corps_I-Wall will allow the user to analyze I-Walls with different properties at different depths (as shown in the input image at the bottom left of the Analysis mode section of the Design or Analysis Process tab). Future versions will allow the user to specify the I-Wall properties at different elevations. This option is grayed out for the current version. To specify the properties at any point of the I-Wall, the user enters an elevation, modulus of elasticity, and moment of inertia into the text boxes provided. Clicking the $A d d$ button puts those properties in the list describing the I-Wall, sorted by elevation. The properties at the top of the I-Wall are linearly interpolated to the next data point. If an elevation is entered more than once, then the interpolation ends at the first entered data point and proceeds from the last entered data point at that elevation.

Figure 3.4. Design or Analysis process- Analysis mode.

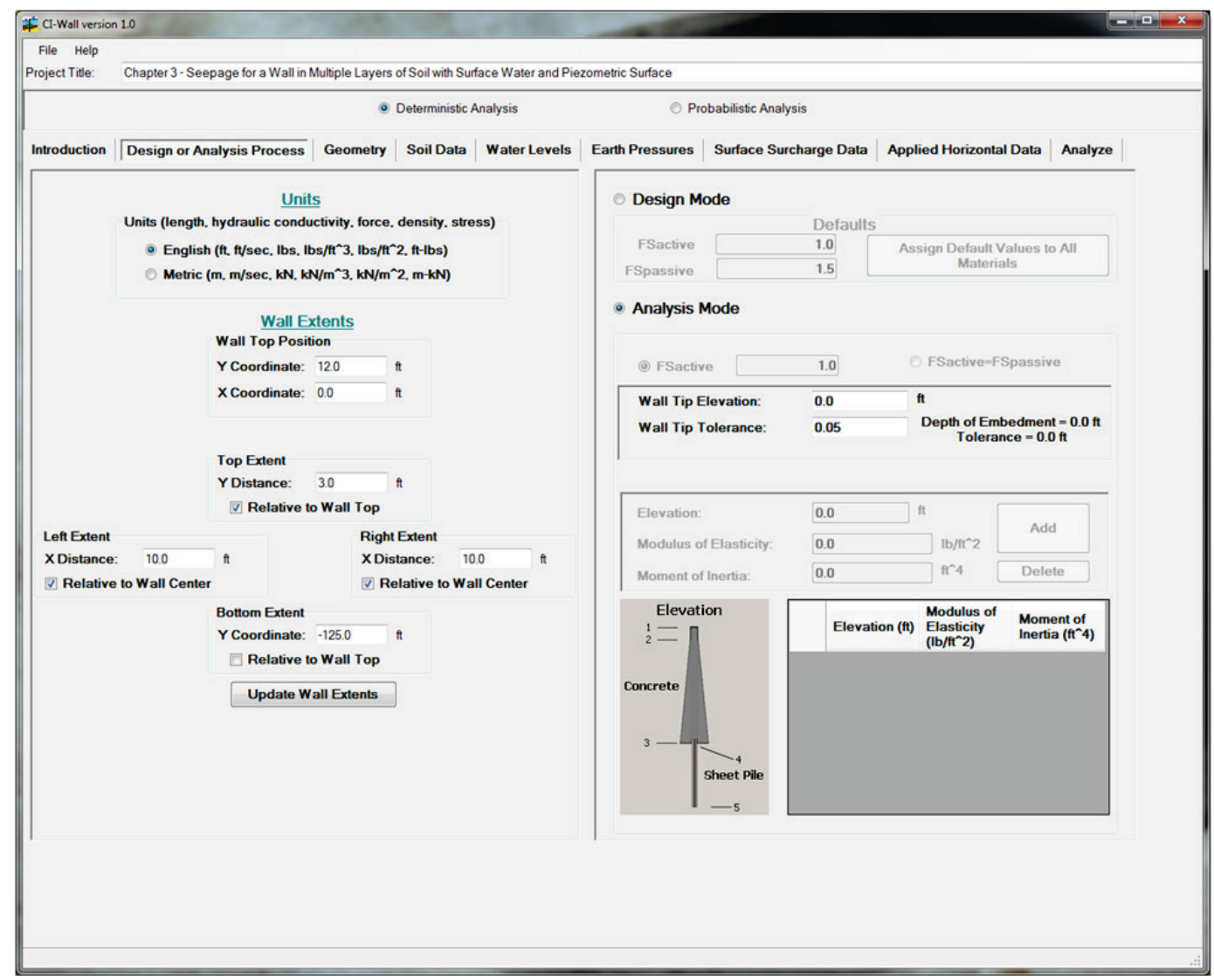

Clicking on the list allows the user to select a specific data point. The Delete button gives the user the option to remove points. This operation may be necessary if data points are specified in the wrong order, leading to errors in interpolation of I-Wall properties. 


\subsection{Geometry tab}

The Geometry tab (Figure 3.5) is where the shapes of the soil regions that lie on either side of the I-Wall are defined. These regions are described using nodes, edges, the I-Wall itself, and the extents entered on the Design or Analysis Process tab. Also on this tab is information that will help the user orient his model, designating the sides as either flood side or landside. While these orientation distinctions do not come to bear in the solver (which works from the materials and properties of the soil and water), they provide the means for the user to establish directionality in the model.

Figure 3.5. Creation of geometry is done with the Geometrytab.

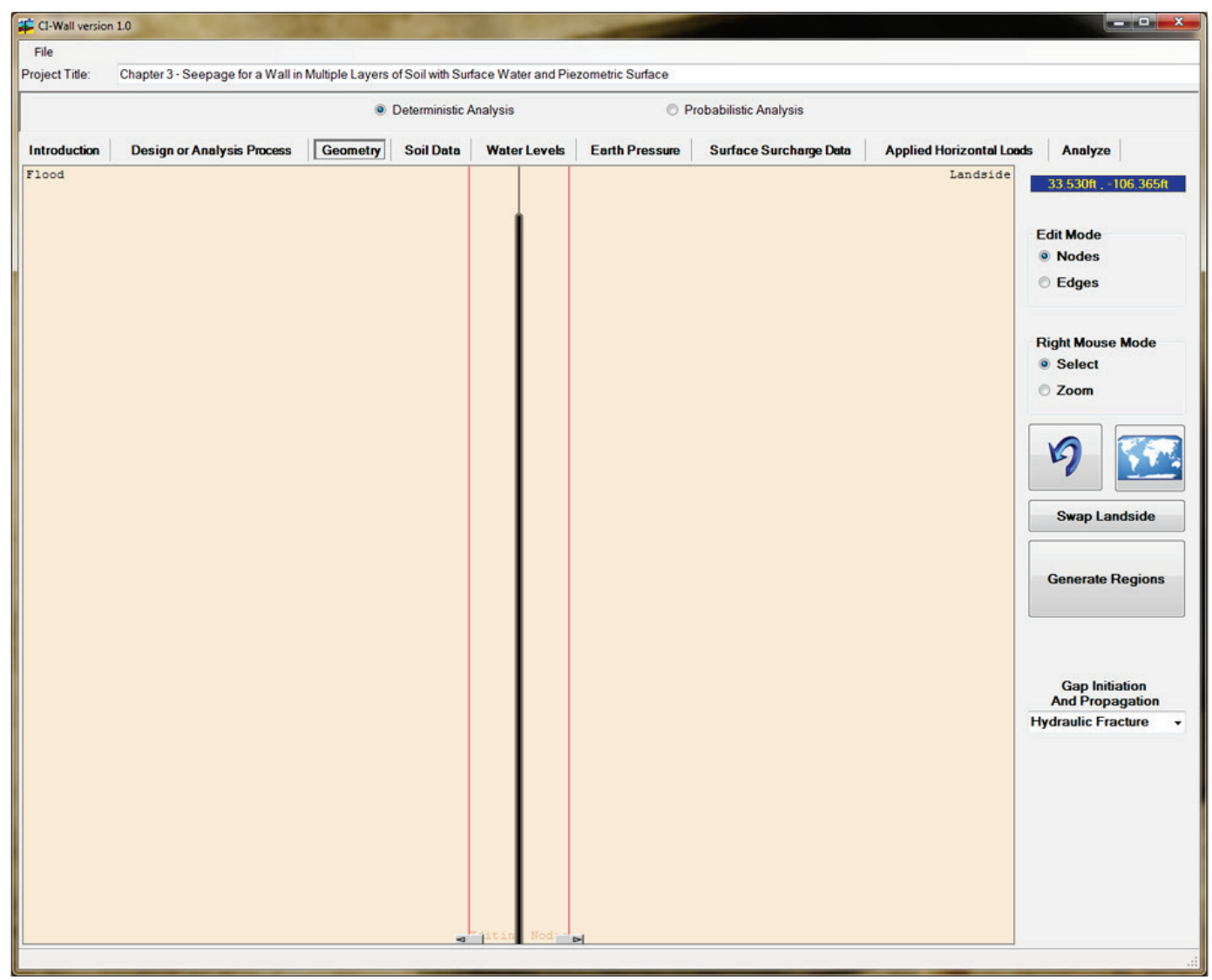

The most prominent feature of the tab is the area where the model is displayed. The I-Wall is displayed, as well as the left and right extents of the model, when a new model is created. When the mouse is moved across the model display area, its coordinates in model space are displayed in the upper right-hand corner of the Geometry tab in the units selected in the Design or Analysis Process tab. 
Below the coordinate display are boxes that set the input mode (either nodes or edges) and the right mouse button mode (either Zoom or Select), and buttons that Undo the previous actions, zoom to the current extents of the model, and create the actual regions that will be used from the edges, nodes, and extents of the model.

When the input mode is set to Nodes (Figure 3.6a), the user can left-click in the model display area or on the extents of the model. A dialog for node creation then appears with the current coordinates of the mouse, snapped to a certain tolerance (Figure 3.6b). These coordinates can be edited or left as they are in the dialog. Selecting Ok creates the node at the selected location (Figure 3.6c), and Cancel does not create the node.

If the left mouse button is pressed when the mouse is over an existing node, then that node will be moved with the mouse until the left mouse button is released. Again, a dialog appears with the new node position, which can be edited. Clicking Ok moves the node to the new position and clicking Cancel returns the node to its original position.

When the mouse is within a certain distance from the extents, the node positions will snap to the extent.

When the input mode is set to Edges (Figure 3.7a), the user can connect nodes via edges. To create an edge, the user must press the left mouse button when the mouse is on one node (Figure 3.7b), drag the mouse to another node (Figure 3.7c) and release the left mouse button over this other node (Figure $3.7 \mathrm{~d}$ ). Releasing the left mouse button when it is not over another node will not create an edge.

By default, the right mouse button is in the Select mode. By click-dragging the right mouse button, nodes or edges can be selected, depending on the input mode. Nodes are selected when the node is inside the box (or boxes) defined by the right mouse click-drag operation (Figure 3.8a and b). Edges are selected whenever any part of the edge is inside the box (or boxes) defined by the right mouse click-drag operation (Figure 3.9a and b). Multiple selection boxes can be created by holding the shift button while doing multiple right mouse button click-drag operations. To deselect all nodes or edges, the users can right-click anywhere away from any edges or nodes. When a node or edge is selected, it can be deleted by pressing the Delete key on the keyboard (Figure 3.8c). 
Figure 3.6 The procedure to input a node into the I-Wall geometry.

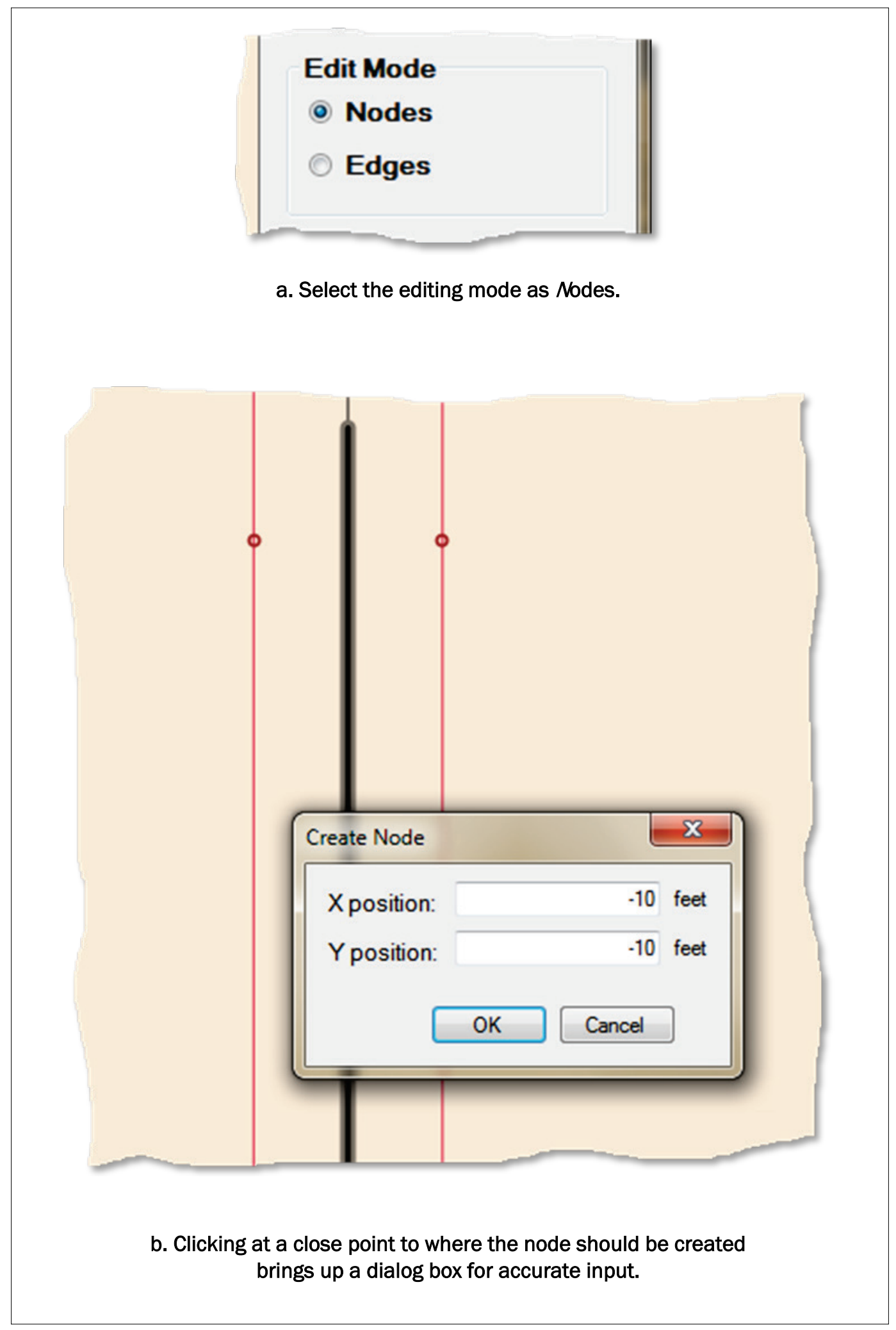




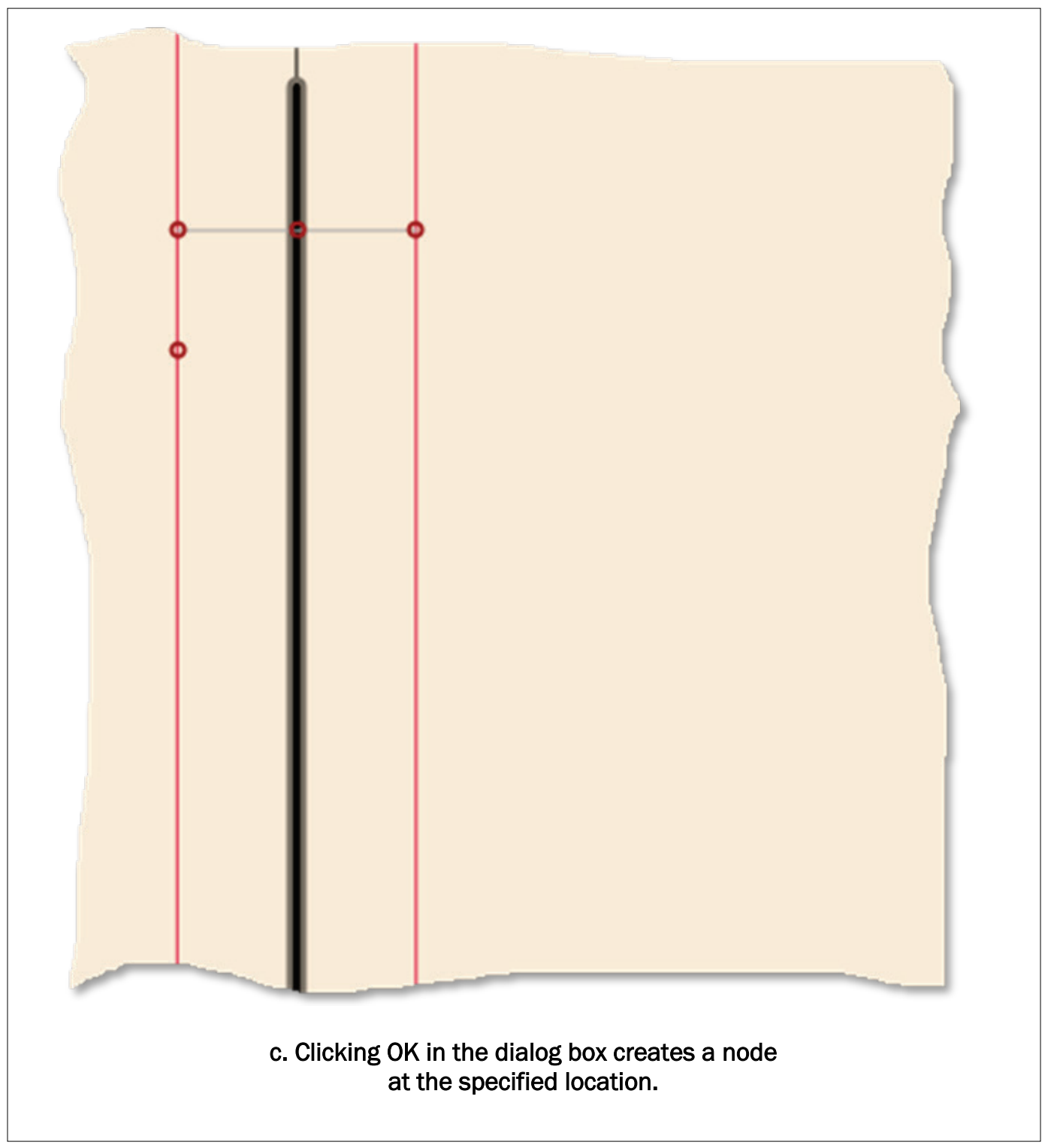


Figure 3.7a-d. The procedure for creating a new edge in the I-Wall geometry.

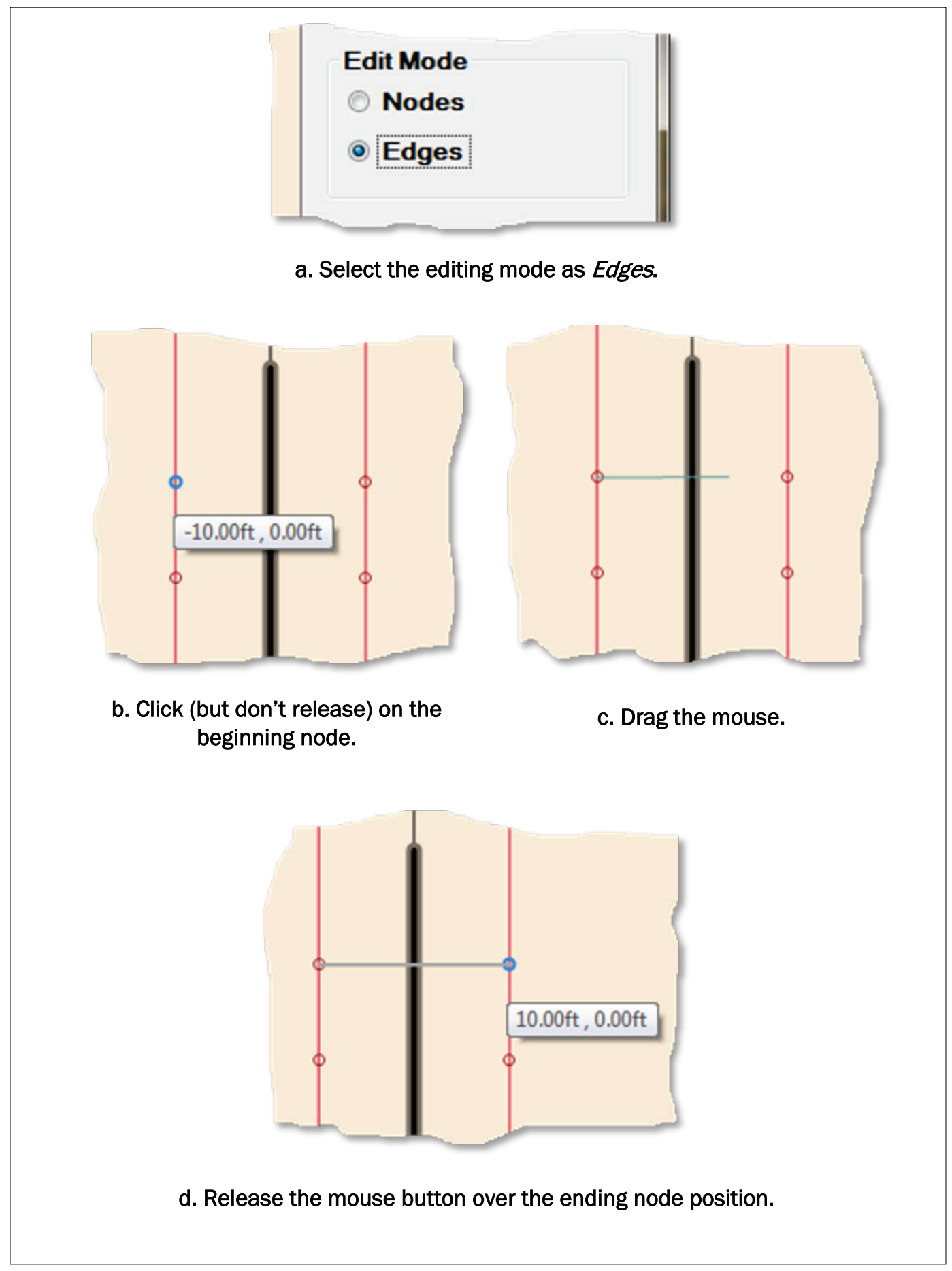


Figure 3.8. The procedures for selecting a node, and for deleting the node (and its connected edges).

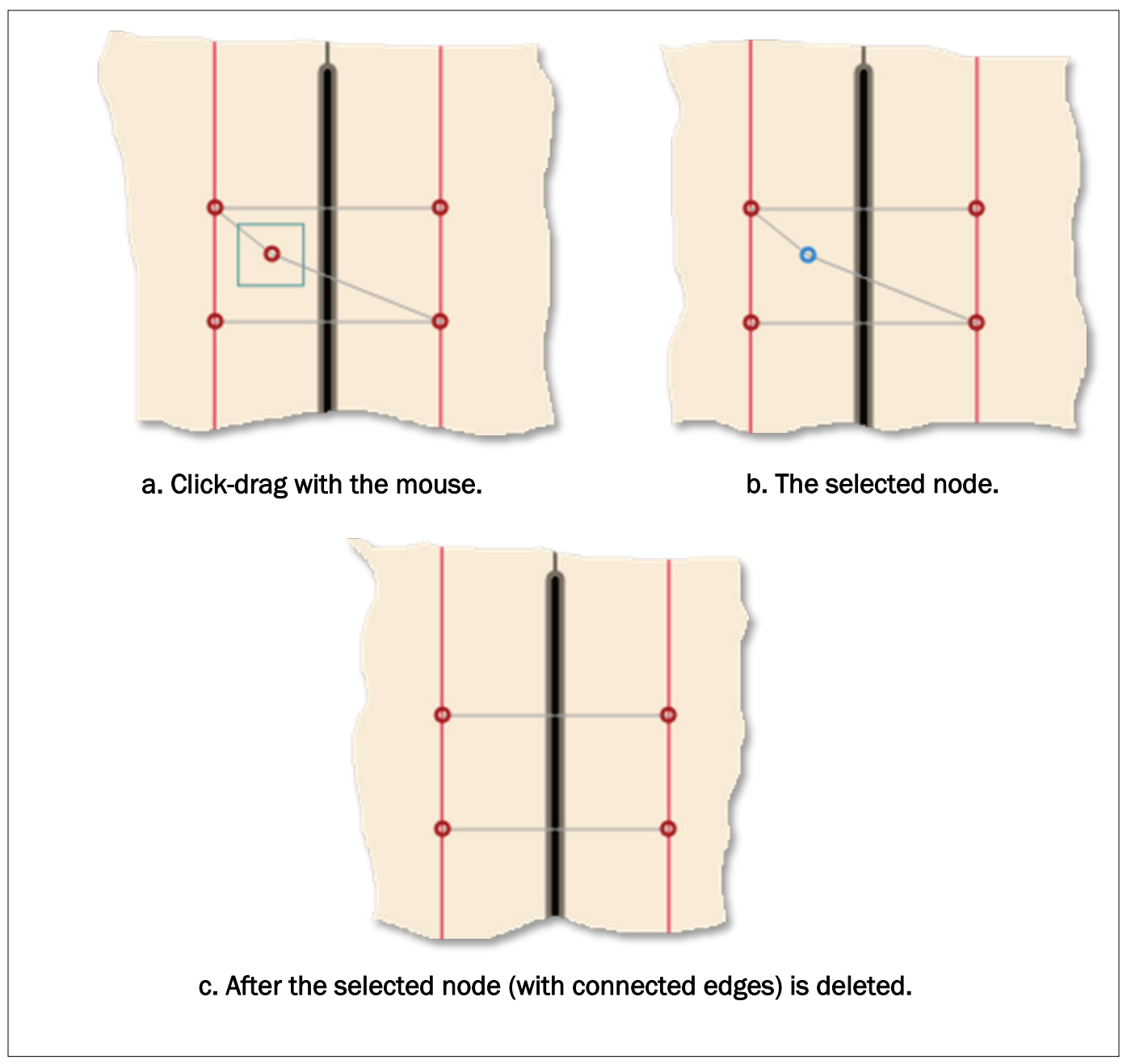

Figure 3.9. The procedure for selecting an edge.

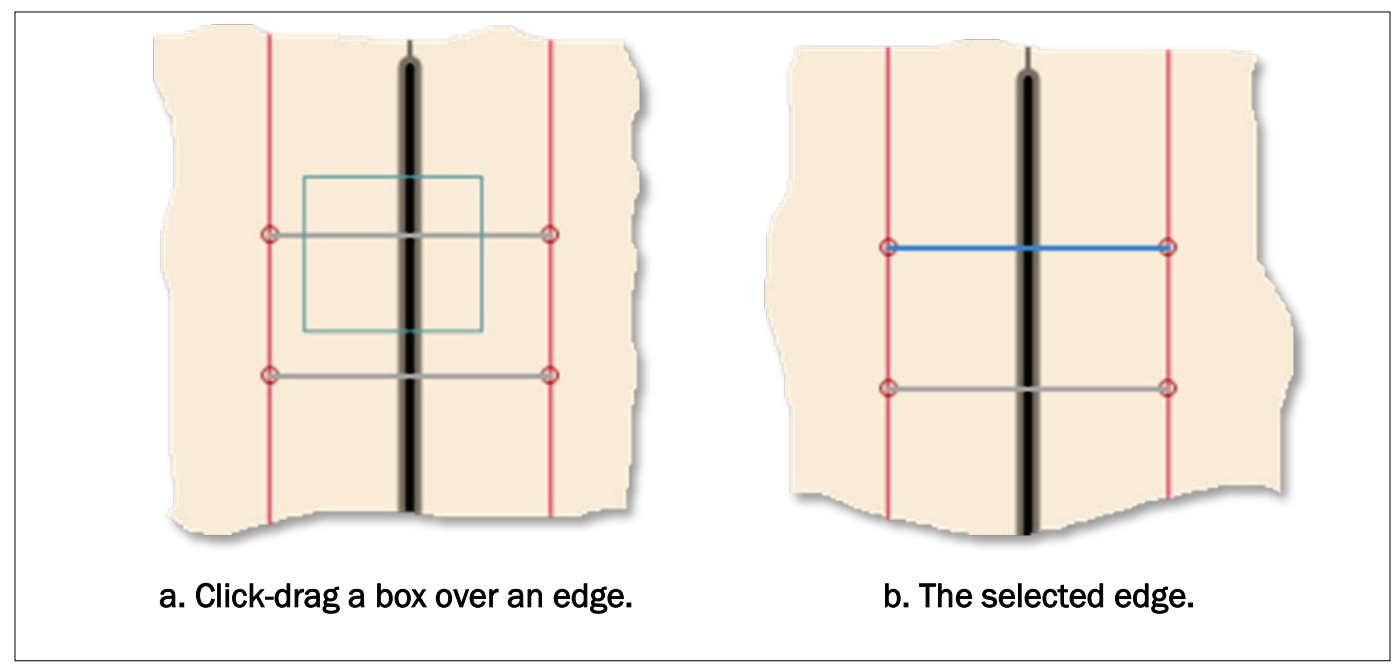


When the right mouse button mode is set to Zoom, a zoom region can be created by doing a click-drag operation in the model display area (Figure 3.10a and b). The area selected is zoomed and displayed in the model display area. To return to a full extent view, click on the Zoom Extents button (Figure 3.11a and b).

At the bottom of the each of the extent lines in the model display area is a slider that allows the extents to be widened (Figure 3.12). Sliding the extent causes any nodes that are on the original extent to share an edge at the original extent and will add a node at the same elevation at the new extent that is also connected with an edge to the node at the original extent. In effect, this creates all the information to describe a new rectangular region to the new extent. This feature, while available in Version 1.0 of the Corps_I-Wall program, does not serve a purpose in defining a Version 1.0 problem with level ground, and its use should be avoided.

An Undo button exists with multiple undo capabilities in a last-in, first-out queue (Figure 3.13a). In this way, the last node or edge created or deleted, or the last movement of an extent can be undone (Figure 3.13b). If a change that involves more than one element is performed (i.e., a node is deleted and therefore deletes two edges that are dependent on the node), then all of the elements are affected by the Undo (e.g., the node is replaced with the two edges as in Figure 3.13c).

There is a button that can change the orientation of the model for the user. This button swaps the landside and flood side of the I-Wall (Figure 3.14). By default, the landside of the I-Wall is to the LHS of the I-Wall (in the -X direction). Swapping the sides will not affect the solver operation, but will affect the location of flood and landside water surfaces defined in the Water Levels tab.

The last button on the Geometry tab is the Region Creation button (Figure 3.15). When this button is pressed, all of the edges and nodes and extents are processed to generate a set of regions (where a region is defined as a polygon that encloses an area). These regions will be assigned soil properties on the Soil Data tab.

In the Geometry tab is a drop-down menu to determine how gap initiation and propagation are handled by Corps_I-Wall (Figure 3.16). The options available in this version of Corps_I-Wall are No Propagation and Hydraulic Fracture. Gap development occurs when the I-Wall moves away from the containing soil regions and affects the pressure and seepage of the model. 
Figure 3.10a-b. The procedure for zooming with the right mouse.

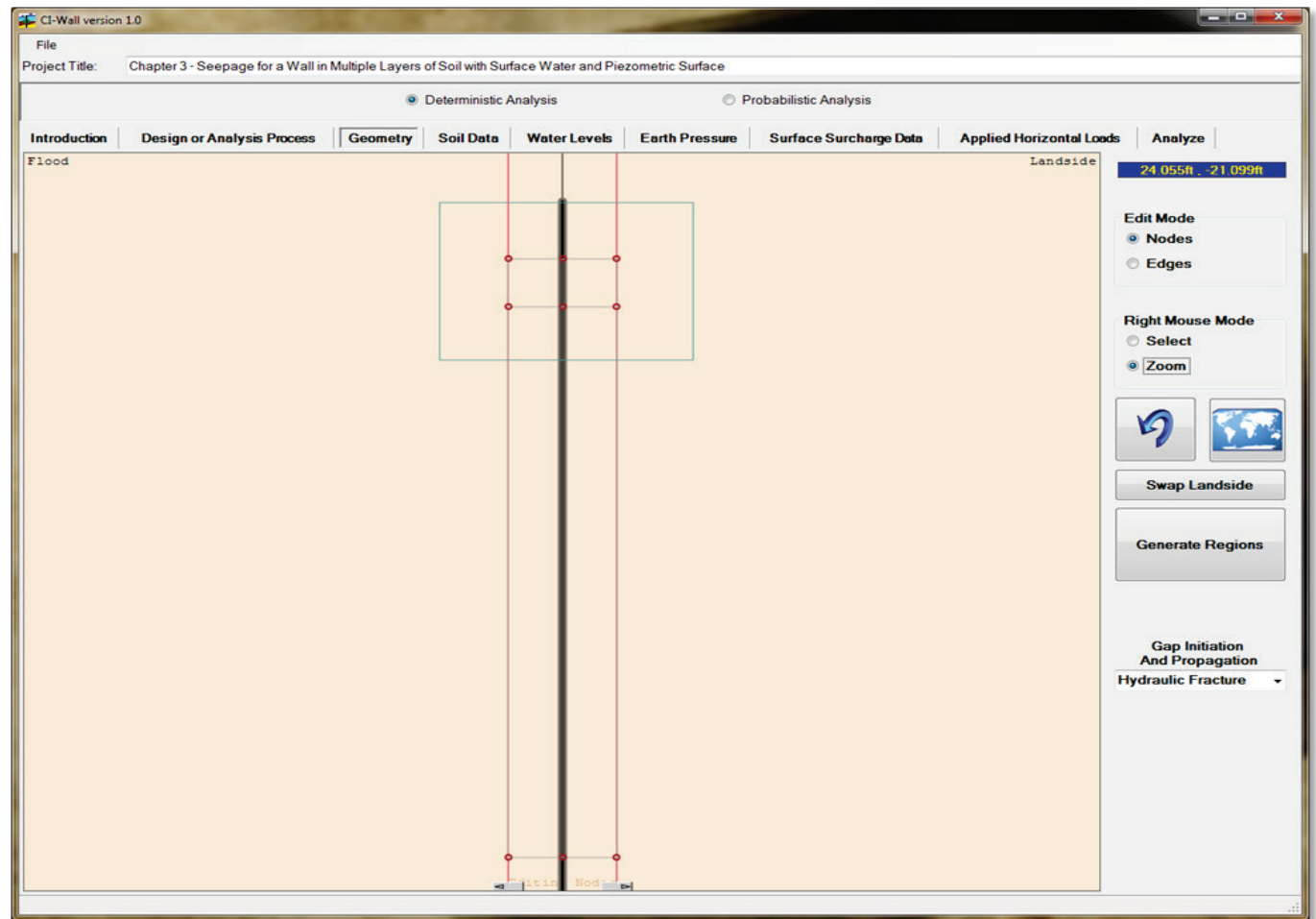

a. Click-drag with the right mouse button to define the zoomed area.

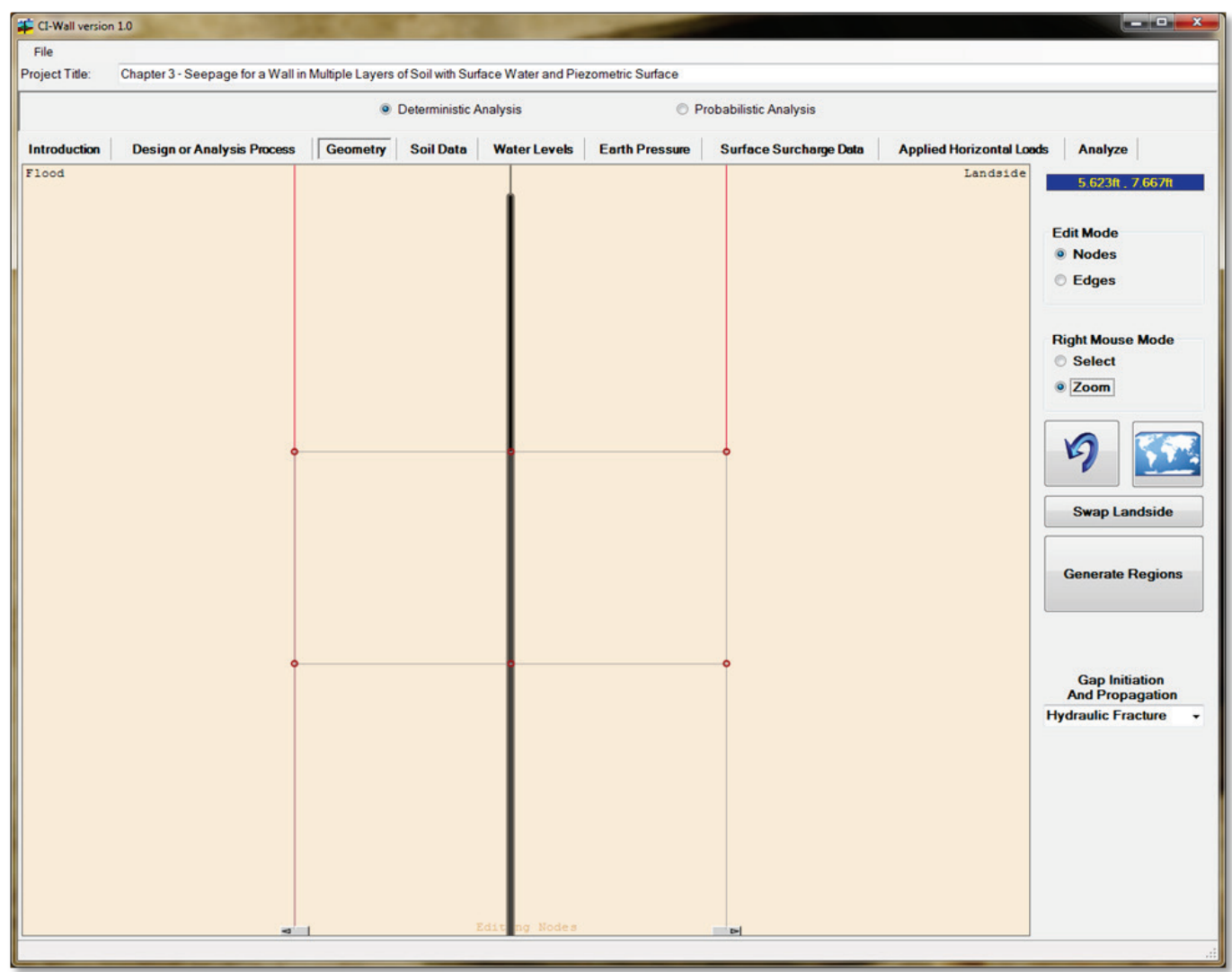

b. The drawing region is then zoomed to that area. 
Figure 3.11. Using the Zoom Extents button.

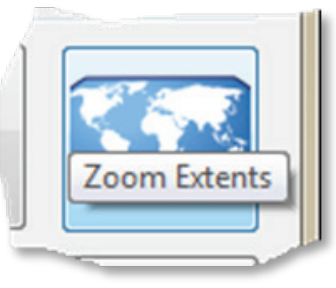

a. Clicking the Zoom Extents button will restore the full view of the I-Wall.

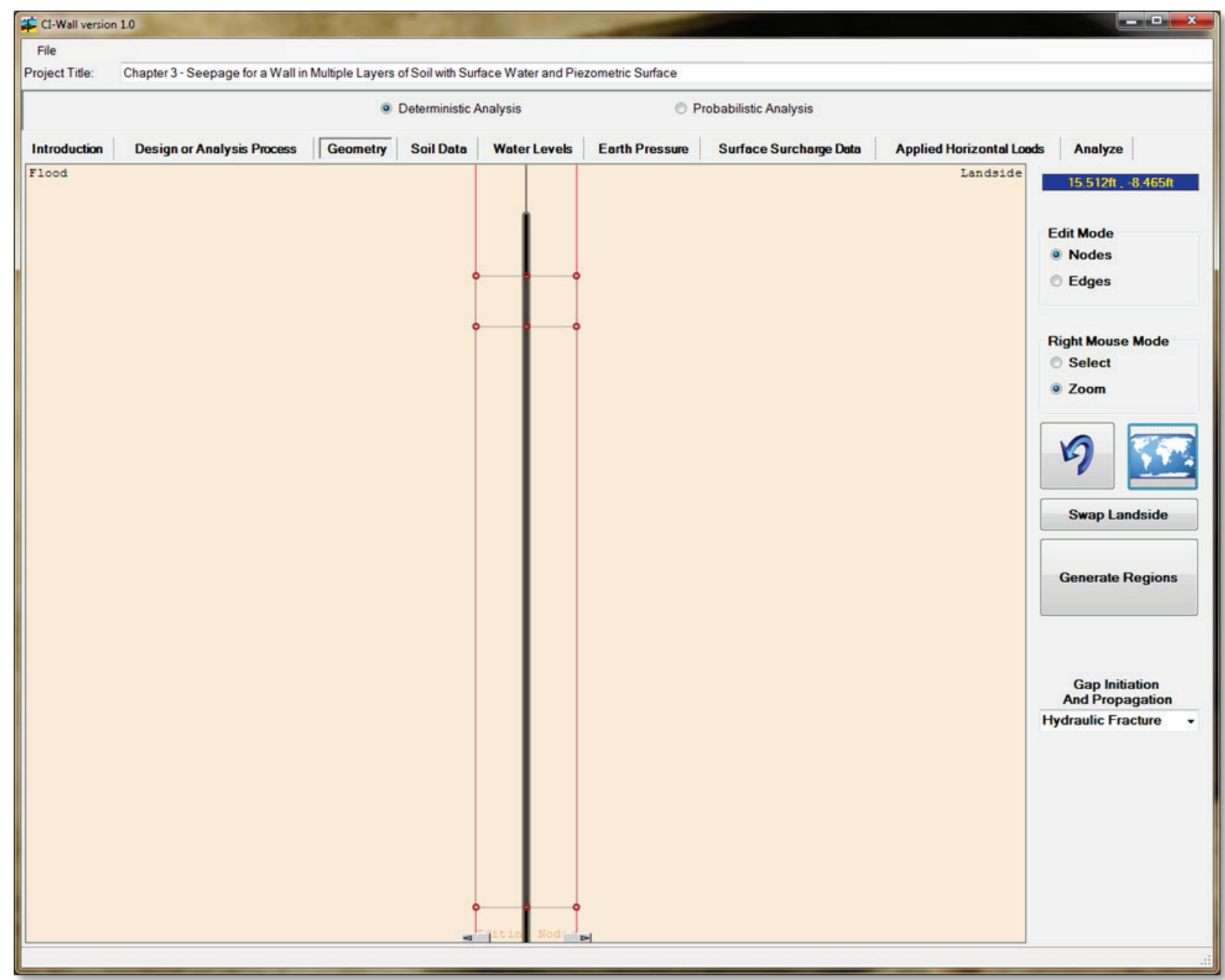

b. After the Zoom Extents button has been pressed. 
Figure 3.12. Modifying the extents of the soil regions to the sides of the I-Wall.

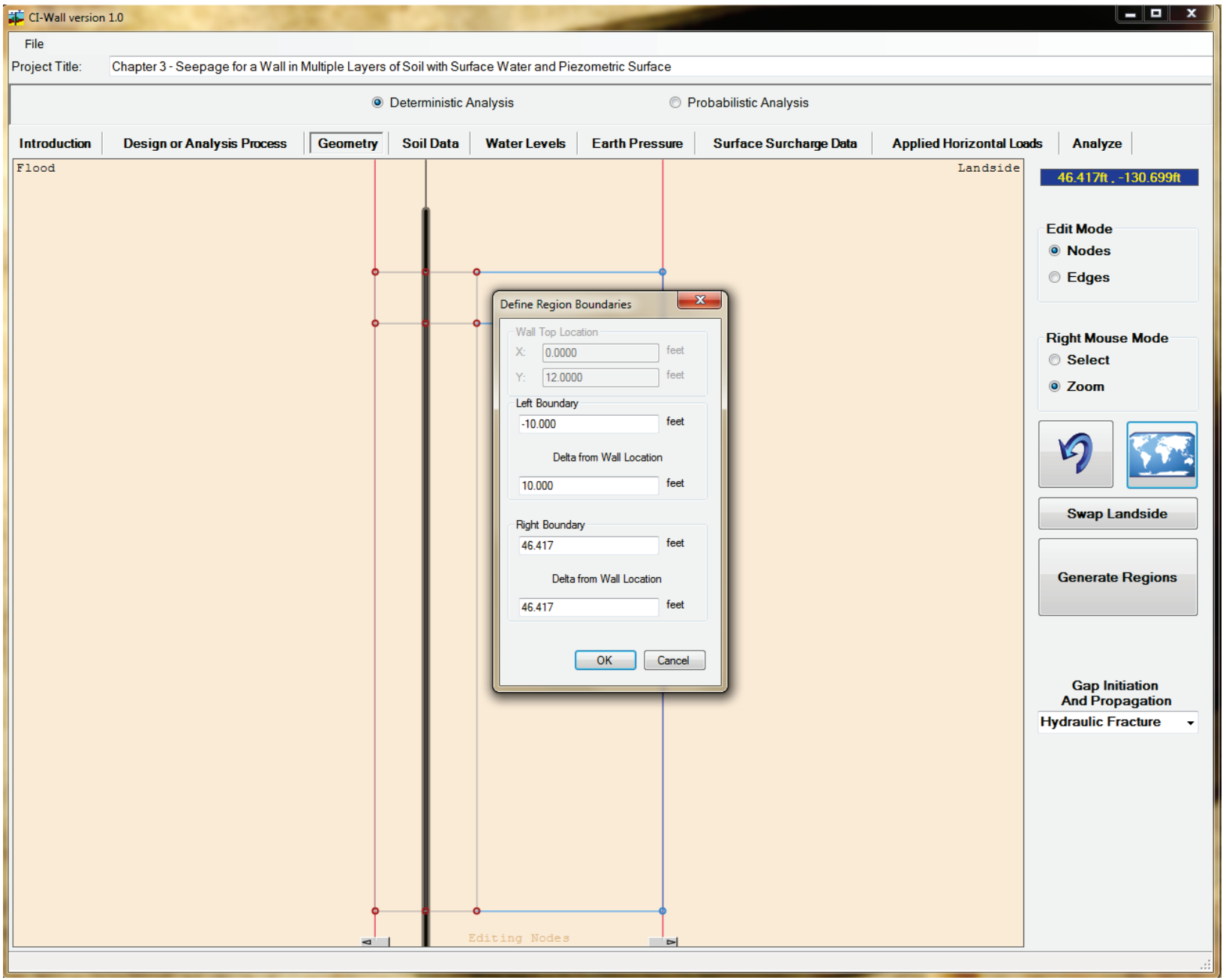


Figure 3.13a-c. Usage of the Undo button.

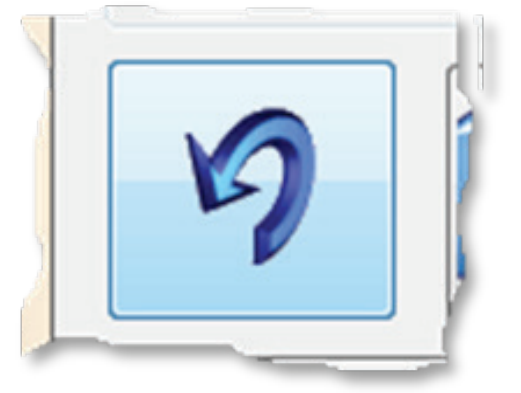

a. The Undo button.

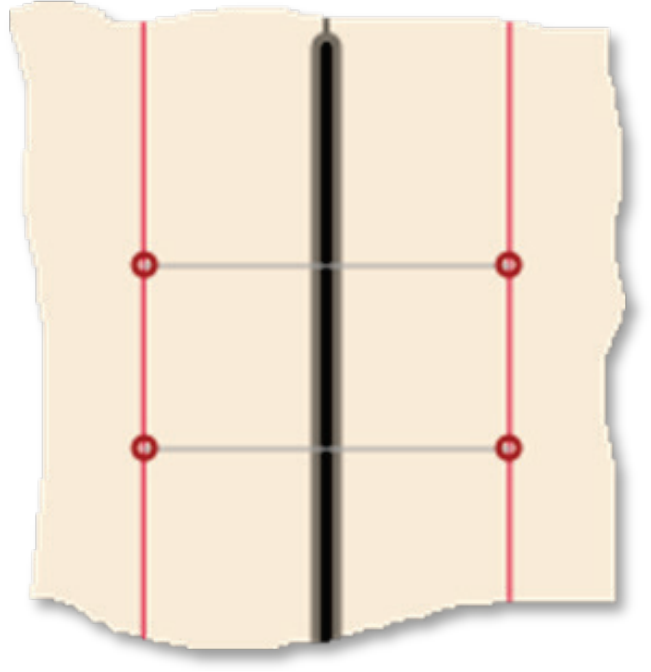

b. Before the Undo.

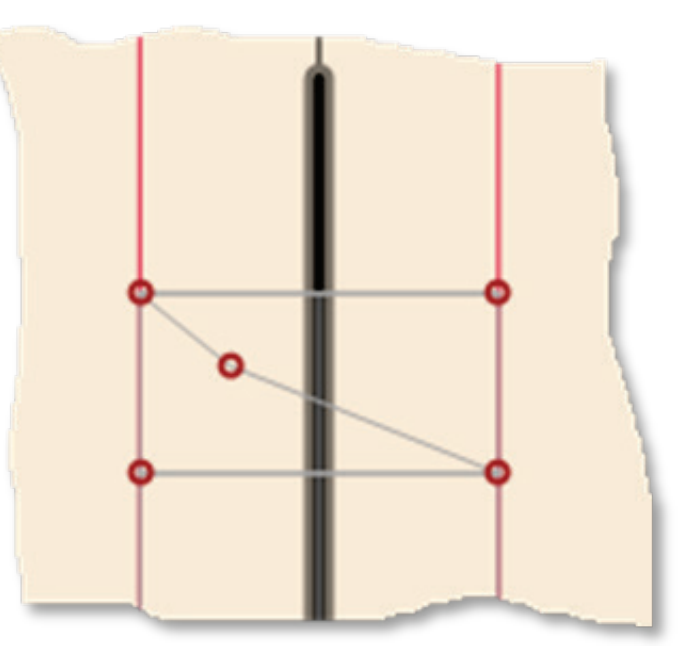

c. After the Undo. 
Figure 3.14. Using the Swap Landside button to reverse the Flood and Landsides of the structure.

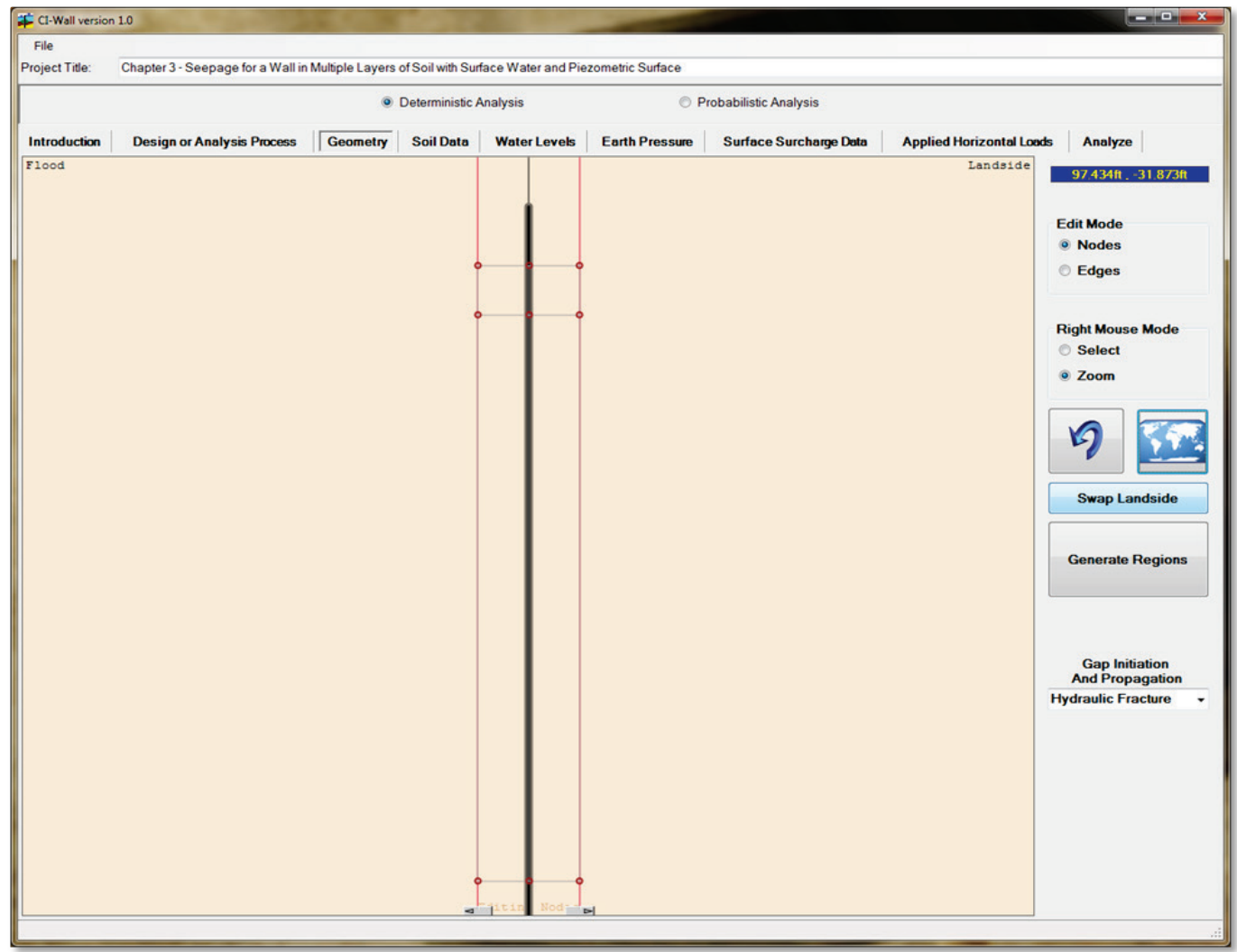

Figure 3.15 Generating regions adds new nodes and edges (to account for intersections with the I-Wall and other edges, and the full I-Wall area). Each area enclosed by edges is a region.

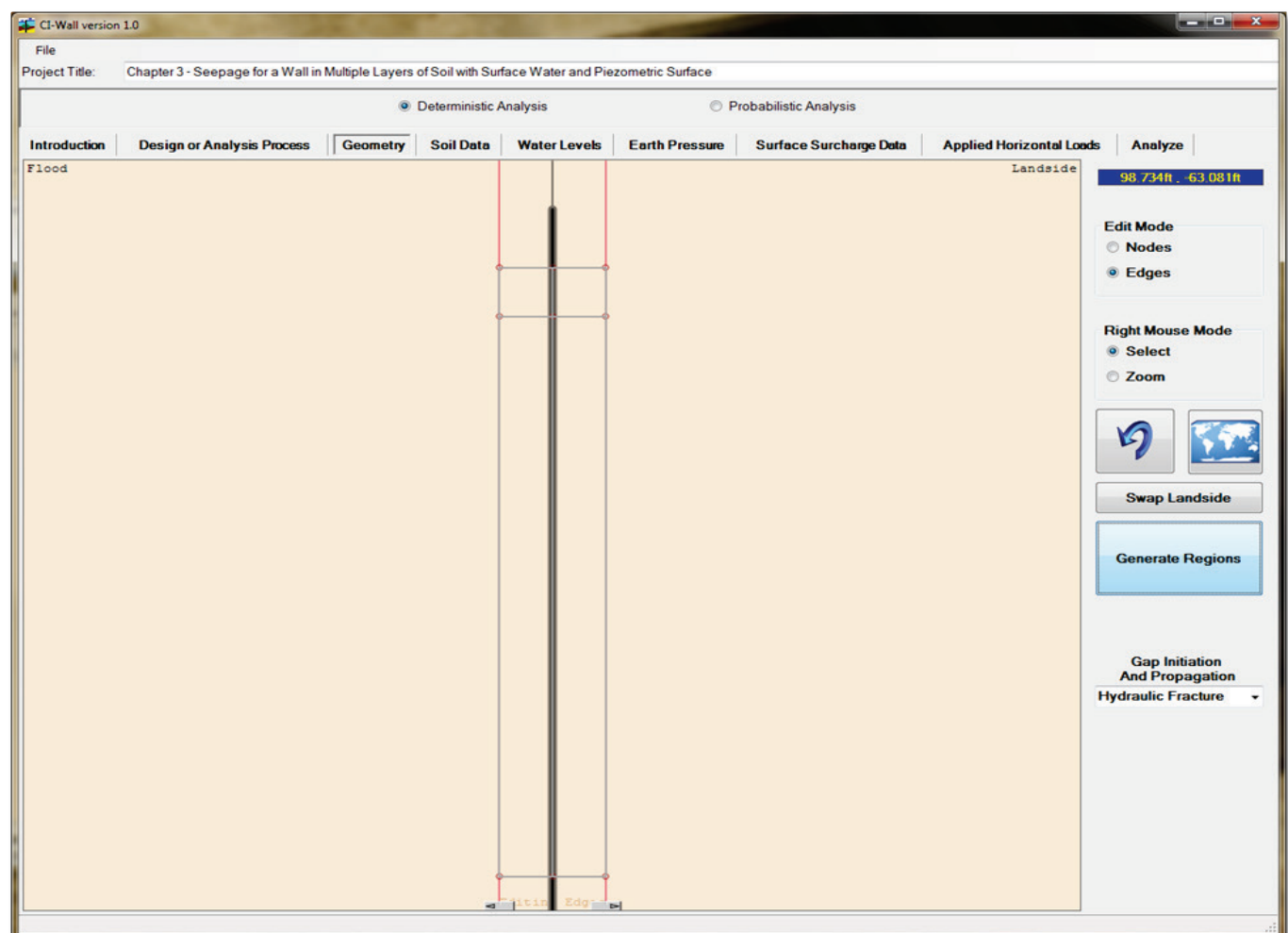


Figure 3.16 Choosing the method of gap creation.

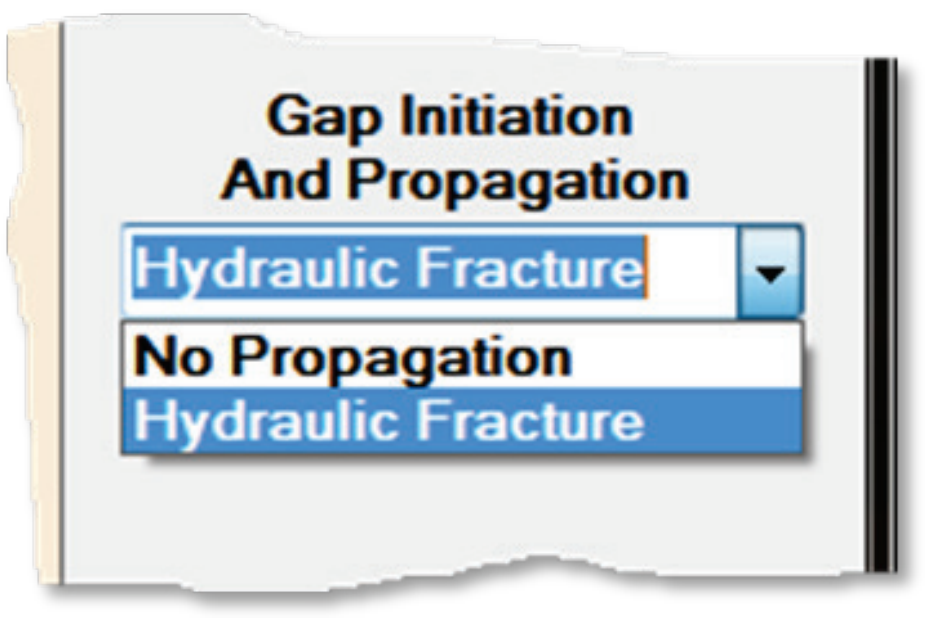

\subsection{Soil Data tab}

In the Soil Data tab, the user can create and edit soil materials deterministically and probabilistically and assign those materials to the soil regions created in the Geometry tab (Figure 3.17). For a new model, there are initially no new materials. Material IDs are the means by which soil properties will be assigned to each region that was generated according to the Section 3.5 instructions.

At the upper left corner of the Soil Data tab is a drop-down list, which allows the user to select the current material that is being defined. Next to that drop-down list is a button that allows the user to create a new material. When a new material is created, it is added to the bottom of the list and is set to the current material.

The button next to the Add New Material button shows a color. This color is the color with which that material will be displayed (once it is assigned to the region). Pressing that button brings up a color dialog box from which a color may be selected/defined. Default values for high-contrast materials are provided for selection. In order to avoid confusion, each material should be assigned a unique color.

Underneath these controls are the areas where the material properties for the currently selected material are defined. The Material Properties box has the input boxes and selectors for deterministic material properties. The unit weights and $F S_{\text {active }}$ and $F S_{\text {passive }}$ are absolute and are entered separately from the values that can be entered in due to relative stress. Recall that the $F S_{\text {active }}$ and $F S_{\text {passive }}$ defaults are defined in the Design or Analysis tab and that these values can change outside of the Soil Data tab. 
Figure 3.17. Entering region attributes with the Soil Data tab.

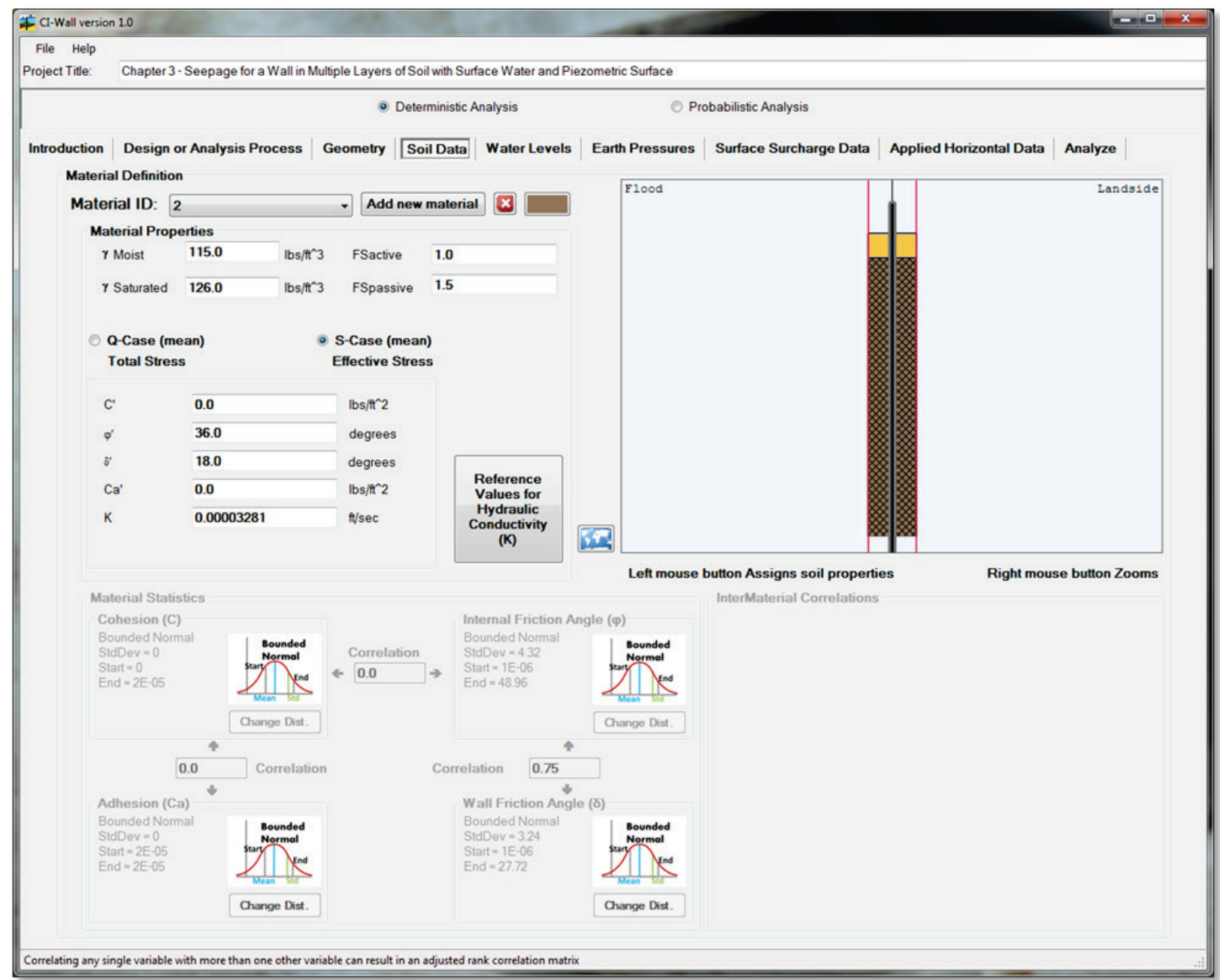

Clicking the button titled Reference Values for Hydraulic Conductivity $(K)$ brings up a window with tables containing typical hydraulic conductivity for standard materials (Figure 3.18). This table gives ranges of values in different units for generalized soil types, typical earthen materials, and compacted materials.

If the Probabilistic Analysis radio button is selected at the top of the screen, the Material Statistics data input section is enabled (Figure 3.19). This box allows the user to choose a statistical distribution as input for the currently selected material properties. For each property (cohesion $\mathrm{C}$, adhesion $\mathrm{Ca}$, internal friction angle $\varphi$, and soil-to-sheet-pile (wall) friction angle $\delta$ ), the user is able to specify a distribution method by clicking the Change Dist. button. When this button has been clicked, a new input dialog is opened (Figure 3.20). This dialog allows the user to select from seven distribution methods: constant, normal, bounded normal, lognormal, bounded 
lognormal, uniform, and triangular. The probability density function of the currently specified distribution is plotted at the upper left of the dialog box, and the cumulative density function is plotted in the upper right. Below these plots are the distribution selector and a symbolic representation of the currently selected distribution and its input values. If the currently selected distribution is either one of the bounded distributions, then a button, which fills the bounds with default values, is displayed. Below this area is where the input values are input for each distribution.

For the constant, normal, bounded normal, lognormal, and bounded log normal distributions, the mean value is fixed at the original value of the currently selected material properties. The normal and lognormal distributions also require a standard deviation to be entered. If the Figure 3.19 (input) mean value of the distributed variable is not o.o, then the coefficient of variation can be used to define a standard deviation value. For the bounded functions, the function will be defined by a certain region with a start and end point. Both the start and end points can be defined as actual values, or as multiples of the standard deviation. Both the start and end points must also be positive values, with the end value being greater than or equal to the start value. Clicking the Assign Default Start and End

Figure 3.18. Reference values for soil types.

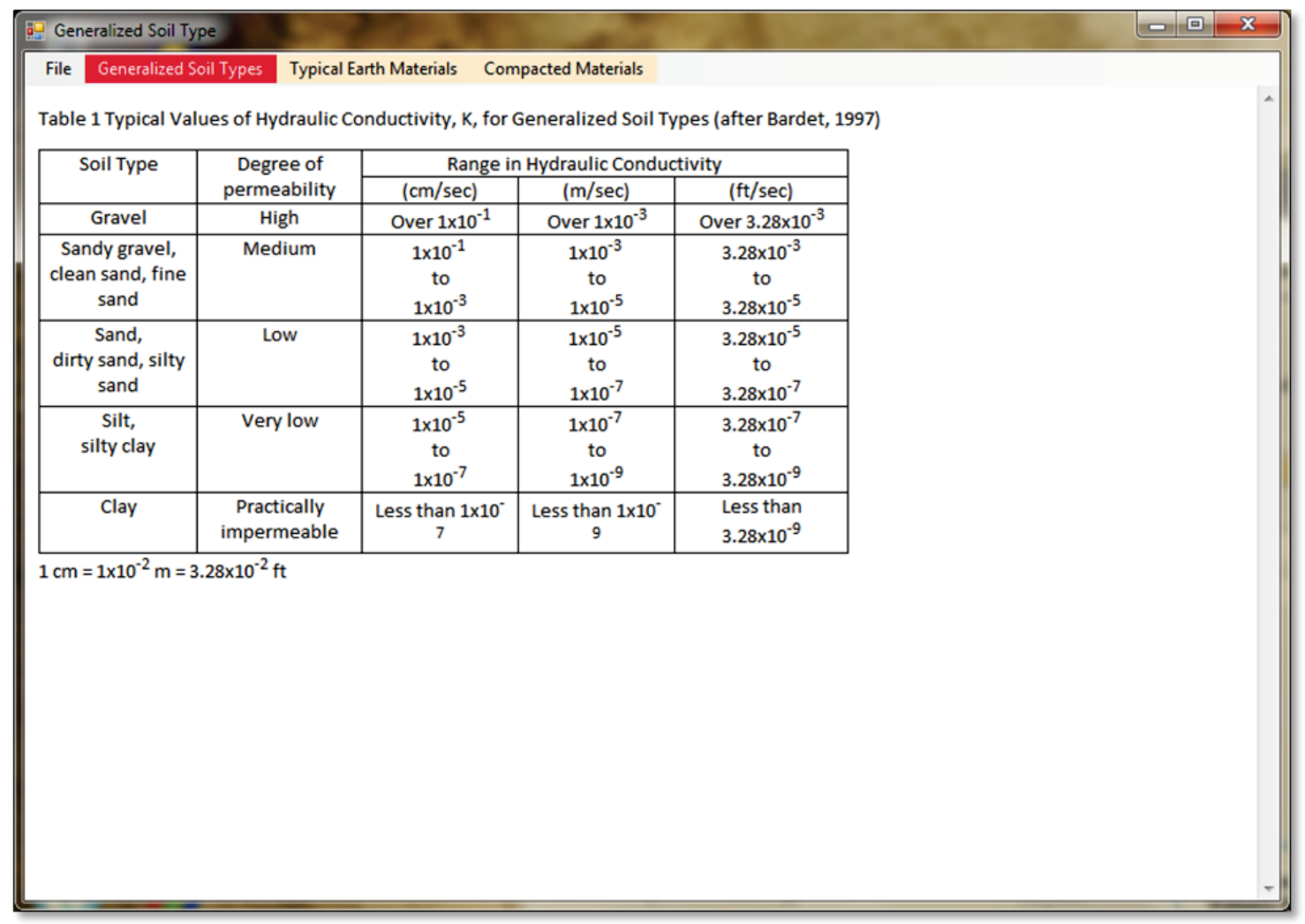


Figure 3.19. Soil material probabilistic input.

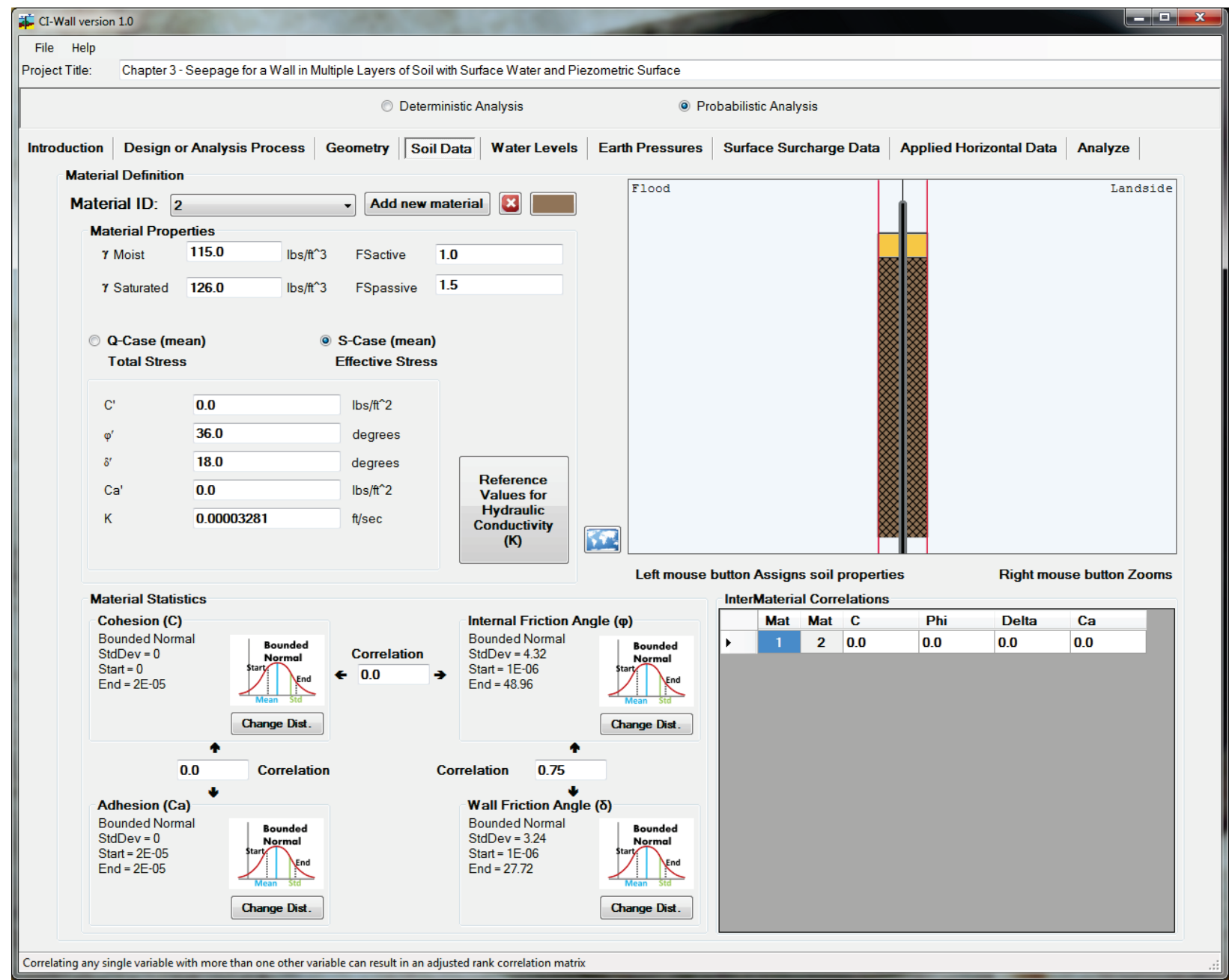

Values button populates the start value with 0.000001 and the end value with a value equal to 3.0 times the standard deviation plus the mean value, as shown in Figure 3.20, for one of the variables.

For a uniform distribution of a variable, only the start and end values of the distribution need to be specified. Both the start and end values must be greater or equal to o.o, and the end value must be greater than the start value.

For a triangular distribution of a variable, the start value, end value, and midpoint value of the distribution need to be entered. The start value must be greater than or equal to o.o. The end value must be greater than the start value. The midpoint value has to be in the range from start to end. 
Figure 3.20. Selecting/changing a distribution.

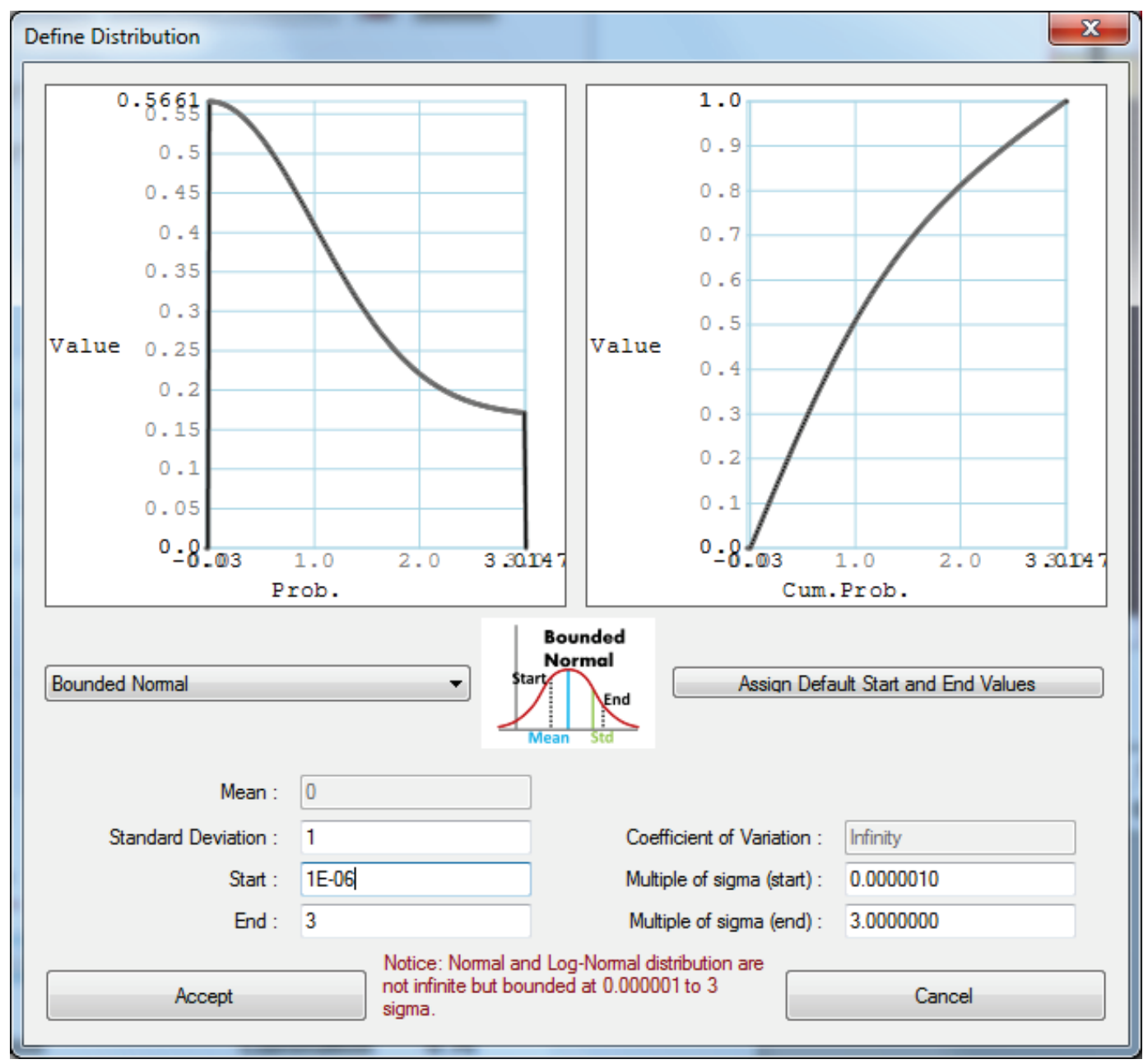

Internal-soil material correlations can also be assigned between adhesion and cohesion, cohesion and internal friction angle, and internal friction angle and soil-to-sheet-pile (wall) friction angle (Figure 3.19).

When the Probabilistic Analysis radio button is selected, the box titled InterMaterial Correlations is also enabled. Each permutation of pairs of soils is shown in a list. For example, if there are three soil materials, the pairs would be material $1 \& 2$, material $1 \& 3$, and material $2 \& 3$. The user is allowed to input the correlation coefficient value (from -1.0 to 1.0) for similar material properties between two regions. For example, the $\mathrm{C}^{\prime}$ material property of material 1 can be correlated with the $\mathrm{C}^{\prime}$ material property of material 3 but the $\mathrm{C}^{\prime}$ material property of material 1 cannot be correlated with the $\varphi^{\prime}$ property of material 3 . Entering an appropriate value in the table for the appropriate pair of materials and the appropriate material property sets the correlation factor between the materials for the property. 
For example, putting 0.65 into the $\mathrm{C}^{\prime}$ property of material $2 \& 3$ correlates the material $2 \& 3 \mathrm{C}^{\prime}$ values by 65 percent. A correlation coefficient value of o.o implies that there is no correlation between material properties. ${ }^{1}$

After a material is defined, it can be assigned to a soil region. In the upperright of the Soil Data tab is the soil assignment box. This box displays the I-Wall and the soil regions. By right-click-dragging a box, this image of the regions and the I-Walls can be zoomed to more effectively display small regions. The button at the bottom left of the soil assignment box (with a picture of the map of the world) is a Zoom Extents button to return a full view from the zoomed area.

To assign the currently selected material to any region, simply left-click the mouse button when the mouse button is inside the region. That enclosed region will then be displayed with the color of the currently selected region, affirming the selection. All of the regions with the currently selected material are shown with a pattern, so they can be easily recognized.

It is vital that each region be assigned a Material ID number, thus assigning soil properties to that region(s). The same Material ID number may be assigned to multiple regions.

\subsection{Water Levels tab}

The Water Levels tab is one of the most complicated tabs in the Corps_I-Wall software (Figure 3.21). Surface water levels can be input for lateral pressure and overburden pressures on the soil. Piezometric surfaces can be entered to reflect changes of water pressures as well as the determination whether soil is moist or saturated. Surface water may also serve to act as a piezometric surface (in an effective stress analysis) for port water pressure calculations. Finally, the effects of seepage can be defined on this tab.

At the left of the tab is the Soil Assignment Area. This area initially displays the regions with materials in muted colors. To the upper right of the tab is a Coordinate Indicator which shows the current position of the cursor in the Soil Assignment Area. Below the coordinate indicator is the right mouse

\footnotetext{
1 In statistics, a correlation coefficient of 0.0 does not mean that the variables are independent. But two independent variables will possess a correlation coefficient value of 0.0.
} 
mode selector box. The Assign to region option will be discussed later. When the right mouse mode is set to zoom, then right-click-dragging in the soil assignment area will select a region to zoom to. To zoom back to the extents of the model, simply click the world map button under the right mouse mode selector box. Zooming will allow the user to more closely see piezometric surfaces, as well as assign surfaces to small thin regions.

Figure 3.21. Water levels are defined either as level surface waters or piezometric surfaces described by piecewise linear elements.

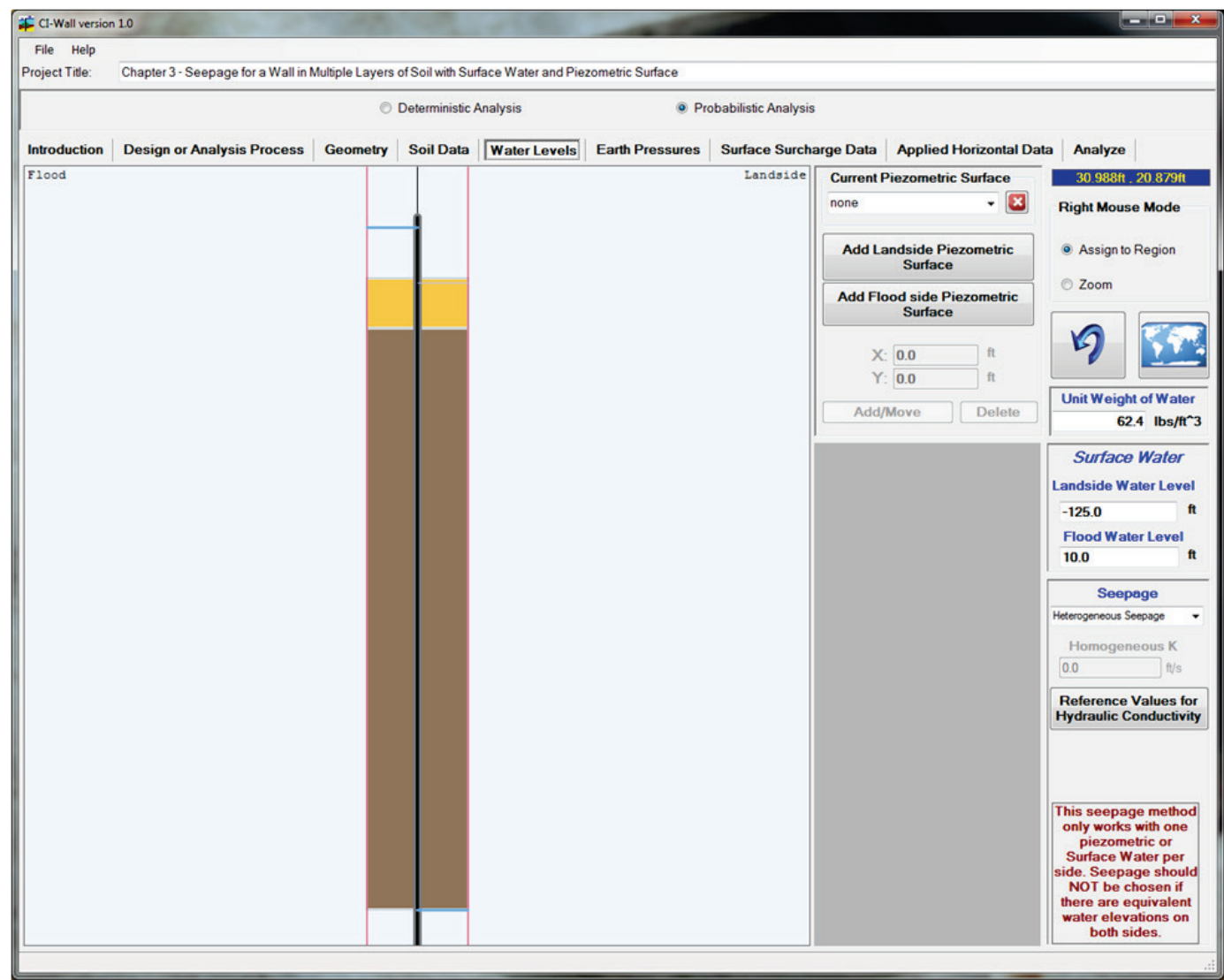

At any time in the creation, deletion, or assignment of water level and piezometric surfaces to regions, the previous operation can be undone using the Undo button immediately to the left of the Zoom Extents button.

Beneath the Undo and Zoom Extents button is the input box for the unit weight of water, and inputs for the elevation of the surface water levels on the flood and landside of the structure (as defined by the user). Changing the values of the surface water levels adjusts the plot of data in the Soil Assignment Area. Water surfaces determine hydrostatic water pressures against the I-Wall and overburden pressures on the uppermost regions in the model, but they are not applied to the wall in a soil region until they are 
assigned to the soil region because user-defined piezometric pressures may apply. The process of assigning surface water to regions will be discussed later.

At the bottom of the left-hand column of input on the Water Levels tab are the inputs to determine the seepage method to be used in computation (Figure 3.22). The selection box gives three methods for seepage: no seepage, homogeneous seepage, and heterogeneous seepage. If either homogeneous or heterogeneous seepage is selected, then there should only be two water surfaces assigned: one water surface or user-defined piezometric surface to all of the regions on each side (flood or landside) of the I-Wall. A message indicating this restriction is included in the GUI (Figure 3.23).

Figure 3.22. Choosing the appropriate seepage method.

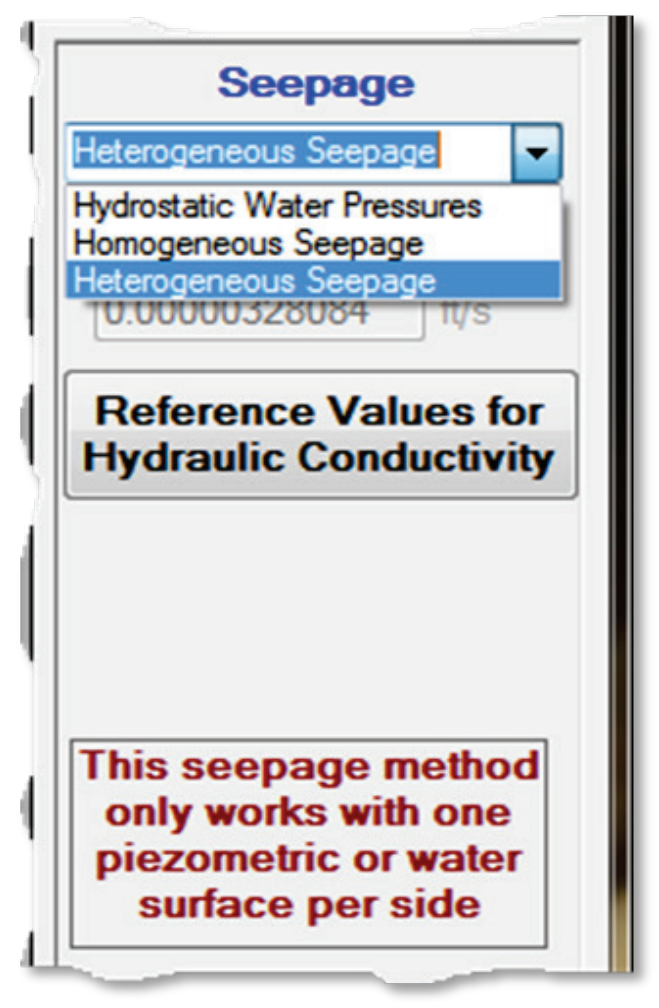

Figure 3.23. Entering a homogeneous seepage parameter (and the warnings associated with this method).

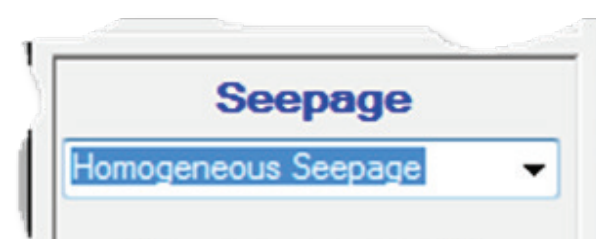

$$
\begin{aligned}
& \text { Homogeneous K } \\
& 0.00000328084 \mathrm{ft} / \mathrm{s}
\end{aligned}
$$

Reference Values for Hydraulic Conductivity

This Homogeneous $\mathrm{K}$ value will be assigned to all materials during processing

This seepage method only works with one piezometric or water surface per side

For heterogeneous seepage, each soil material's permeability is used to determine the head loss along the I-Wall as per the line of seepage (a.k.a., the line of creep) method of analysis discussed in Appendix C. 
Homogeneous seepage assumes that the permeability is consistent across all of the soil materials. When the homogeneous seepage method is chosen, the user is prompted to enter this common permeability value. When the in file is written for the solver, this permeability value is substituted as the permeability for each soil material. A message indicating this substitution is shown in the GUI in the Water Levels tab and a comment line is added to the in file noting this fact.

Near the middle of the Water Levels tab is the selectors and input for piezometric water levels (Figure 3.24), whether determined from water surfaces or user-defined. At the top of this input area is a selector for the current piezometric surface to assign to earth regions or edit for userdefined water levels for pore-water pressure calculations.

Assigning a water level to a soil region is performed by using the piezometric water surface selector to determine the current water surface to assign to a region (Figures 3.25 and 3.26). The currently assigned regions for that current water surface will be plotted with bold colors, and regions that are not assigned to that water surface will be shown with faded colors. For a new model, none of the soil regions has a piezometric water surface and so all of the regions are shown as bold with the selection of none in the piezometric water surface selector. To assign a region to the currently selected piezometric surface, the user only needs to left-click on the soil region to assign. Soil regions can only be assigned to the current piezometric surface if the current piezometric surface is on the same side of the I-Wall as the soil region. For instance, a soil region on the landside of the I-Wall cannot be assigned to a user-defined piezometric surface on the flood side of the structure.

The none and Water Surface piezometric levels that can be chosen with the selector apply to both sides of the structure and can thus be assigned to any of the soil regions. It should be noted that the Water Surface option applies the surfaces defined by the Water Level inputs in the rightmost input section of the tab, and that the appropriate level piezometric surface is assigned to the region based on the side of the region with respect to the structure. 
Figure 3.24 Creating a piezometric surface highlighting the selector and region creation buttons.

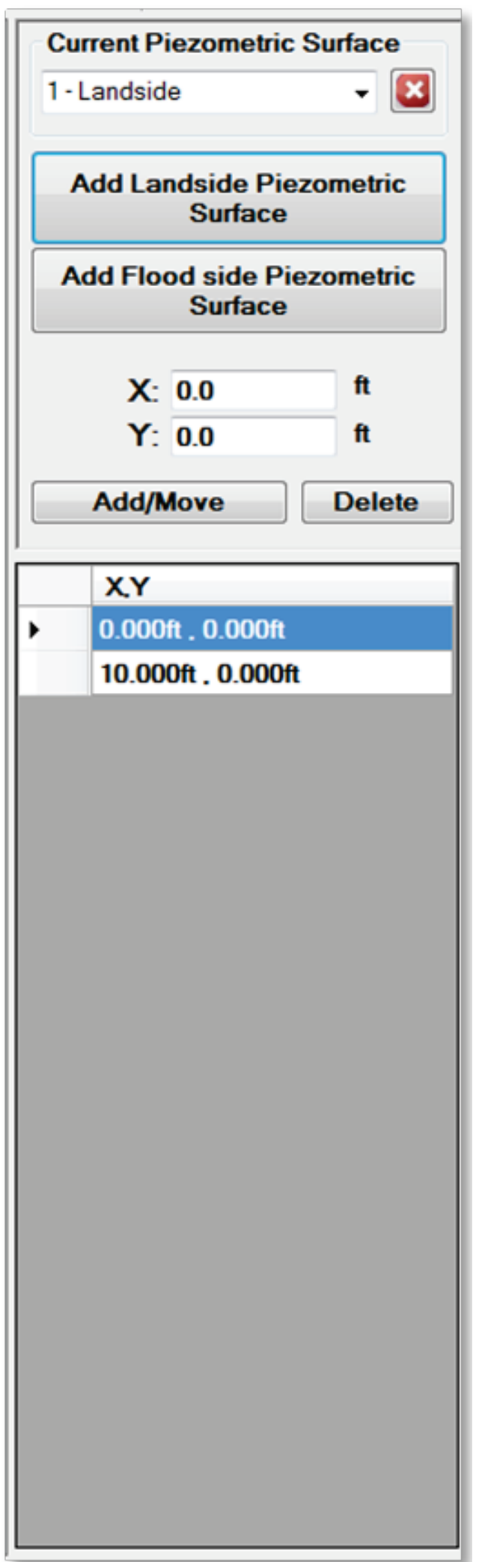


Figure 3.25. Assigning soil regions to a user-defined piezometric surface (whose point coordinates are in the piezometric area).

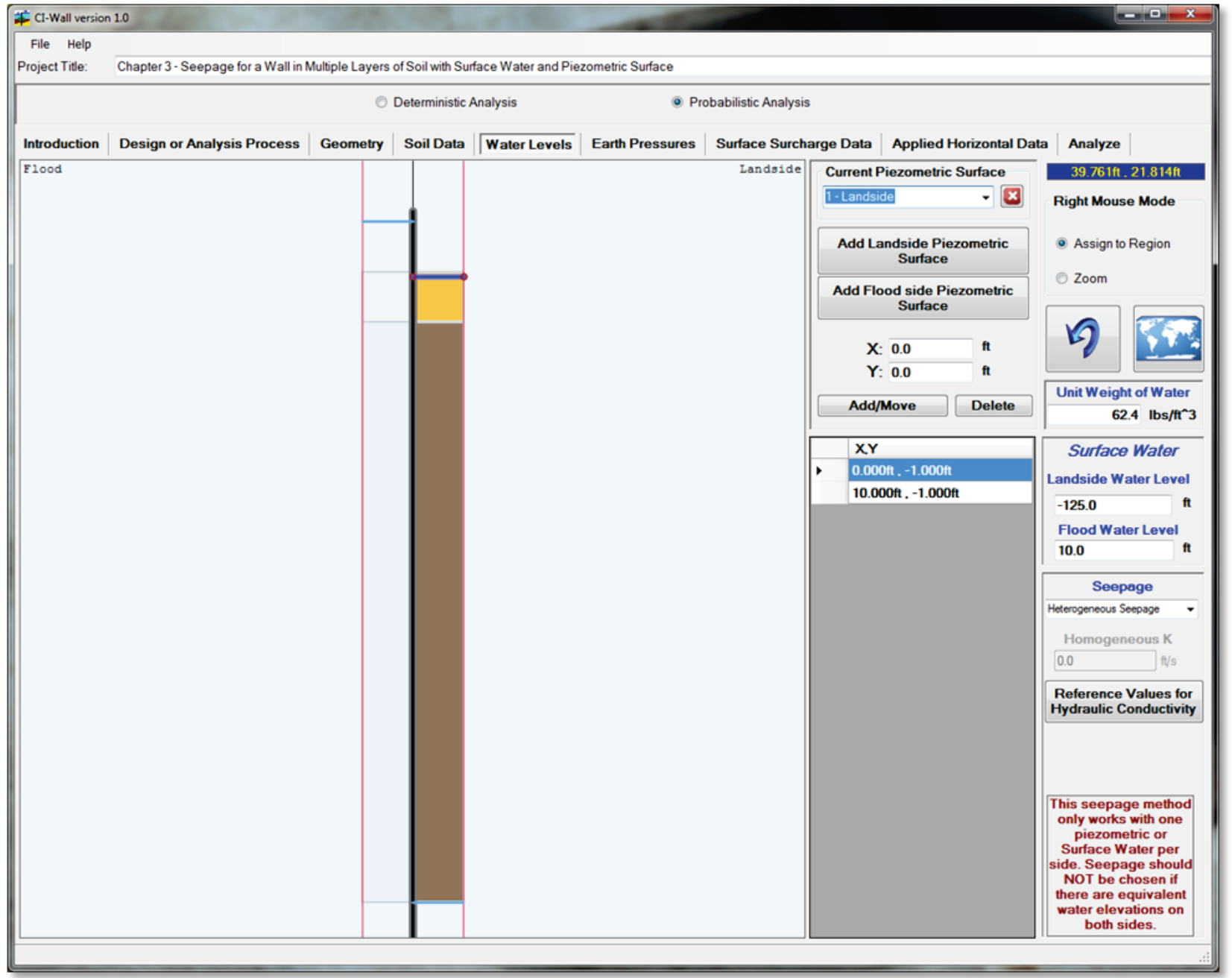

Notice: Applying Water Surface piezometric levels to a surface region means that the soil region can have a varying piezometric water table in a probabilistic analysis. This possibility will be discussed in the Analyze tab discussion.

The $X$ image button (with a red background) next to the current piezometric surface selector allows the user to delete the currently selected surface (if the currently selected surface is a user-defined surface). Clicking the button removes the currently selected surface (and its definition).

In order to create a new user-defined piezometric surface, the user can click either of the two buttons beneath the current piezometric surface selector. The buttons add a new user-defined piezometric surface to either the user-defined (in the Geometry tab) Flood or Landside of the structure, 
which are labeled in the Soil Assignment Area. The new surface is created with an appropriate water level of zero and proceeds from the I-Wall structure to the extent on the Flood or Landside (Figure 3.27). The coordinates of the two nodes (shown in red) used to define the landside piezometric surface are as listed in the data box (with the heading of $X, Y$ ).

Figure 3.26. Assigning soil regions to the flood side surface water.

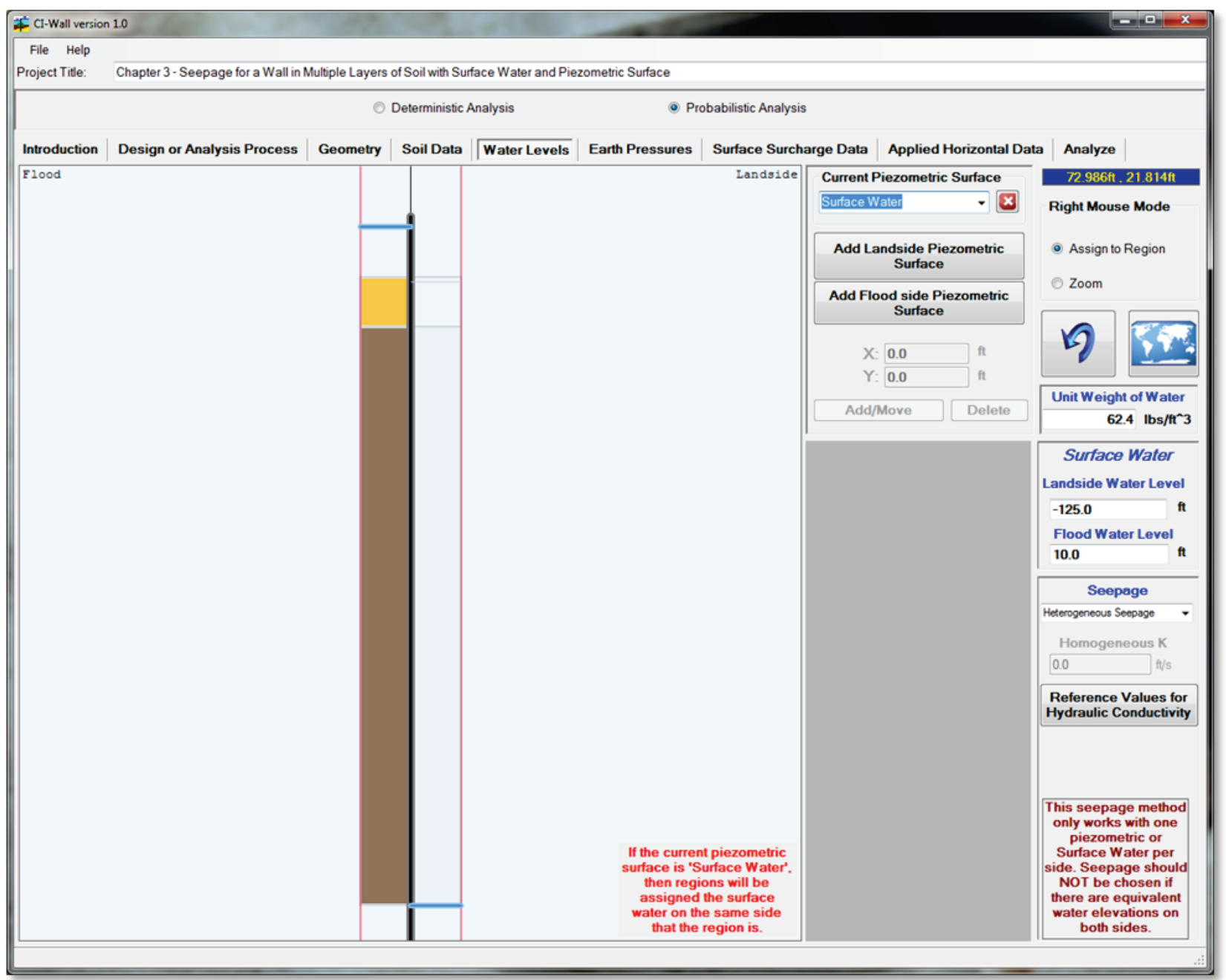

The piezometric water surface is assumed to have an elevation that is a function of distance from the I-Wall (i.e., $\mathrm{Y}=\mathrm{f}(\mathrm{X})$ ). In this vein, the piezometric surface is defined as a set of linear interpolations of $Y$ for points with continually increasing $\mathrm{X}$-values. These points are shown in the area below the Add/Move button, as shown in Figure 3.28. 
Figure 3.27. The piezometric surface immediately after the Add Landside Piezometric Surface button has been pushed.

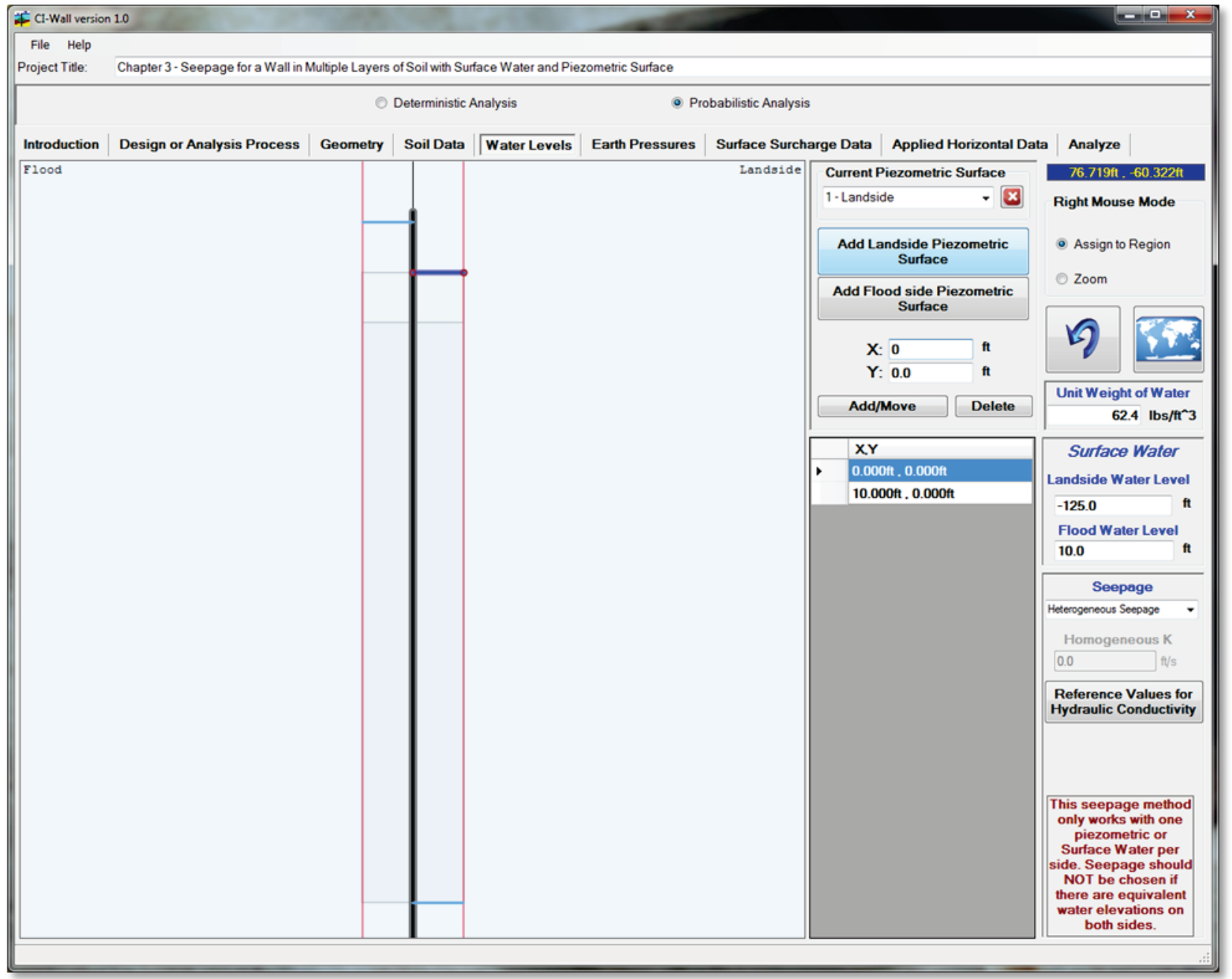

To edit the currently selected piezometric surface, a (X,Y) location should be entered into the text boxes below the buttons to create piezometric surfaces. Clicking the Add/Move button either adds the input point values to the currently selected surface sorted by $\mathrm{X}$, or replaces a point in the currently selected piezometric surface that shares the same $\mathrm{X}$ value (Figure 3.29 and 3.30). This input is shown by the changed surface shown in the Soil Assignment Area (the current piezometric surface is drawn with bold colors) and by the change of the data in the current piezometric surface list under the Add/Move button.

To remove a point from the currently selected piezometric surface, click on that point in the piezometric surface list (under the Add/Move button) and click the Delete button next to the Add/Move button. 
Figure 3.28. The points of the piezometric are in the grid below the point definition area.

\section{Current Piezometric Surface}

1 - Landside - $x$

Add Landside Piezometric Surface

Add Flood side Piezometric Surface

$X: 0.0 \quad f t$

$Y:-1.0 \quad f t$

Add/Move Delete

\begin{tabular}{|l|l|}
\hline & $X . Y$ \\
\hline & $0.000 f t .0 .000 f t$ \\
\hline & $10.000 f t .0 .000 f t$ \\
\hline
\end{tabular}


Figure 3.29. If an input point has an $X$ value that is already on the curve, then that point is moved to the new $Y$ location on confirmation.

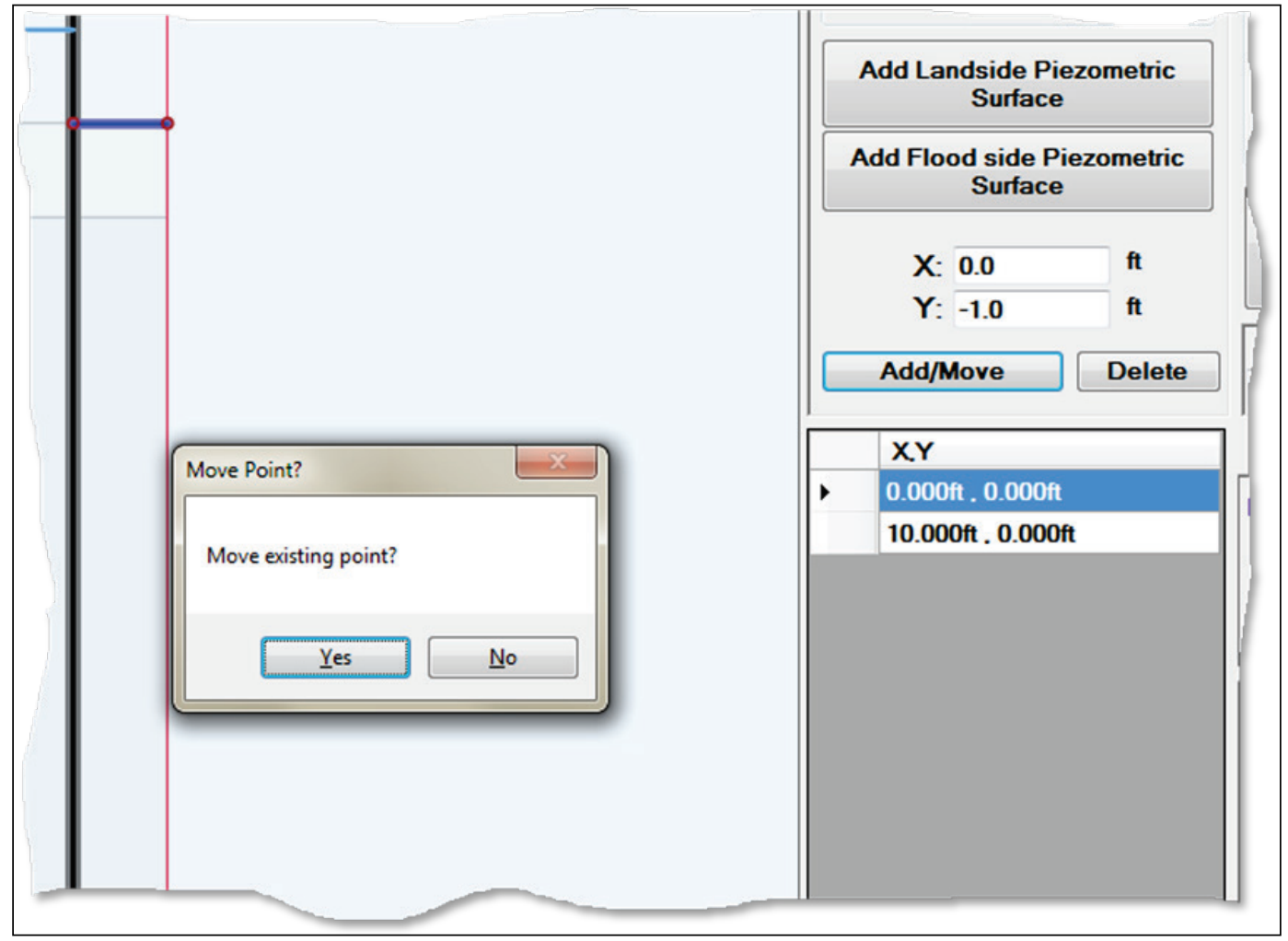

Figure 3.30. After both ends of the piezometric surface have been moved.

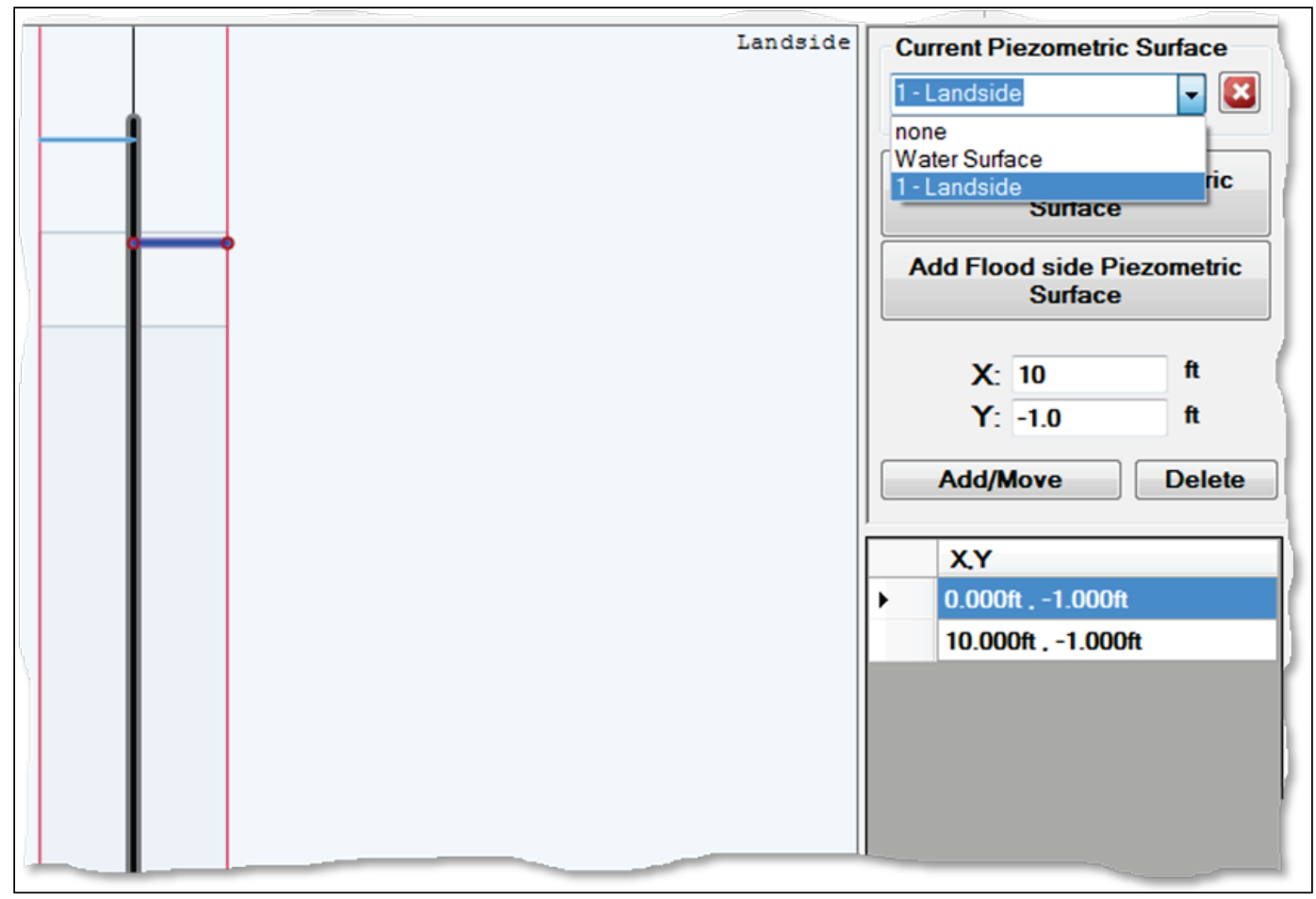




\subsection{Earth Pressures tab}

In the Earth Pressures tab, the user can decide whether the computation of earth pressures will be performed using the coefficient method or the wedge method by selecting the provided radio buttons. Selecting an option allows the user to specify information specific to that option.

In the coefficient method data input area, the only input required is the selection of a method to use from a drop-down menu (Figure 3.31). The methods and the requirements for those methods are presented in an image beneath the dropdown combo-box. For instance, if the logarithmic spiral coefficient method is selected, all soil materials would be required to have a value for $\delta \geq 0.0$. The default coefficient method is the Coulomb method. The coefficient method is used exclusively in Corps_I-Wall Version 1.0.

Figure 3.31. Choosing the coefficient method using the Earth Pressures tab.

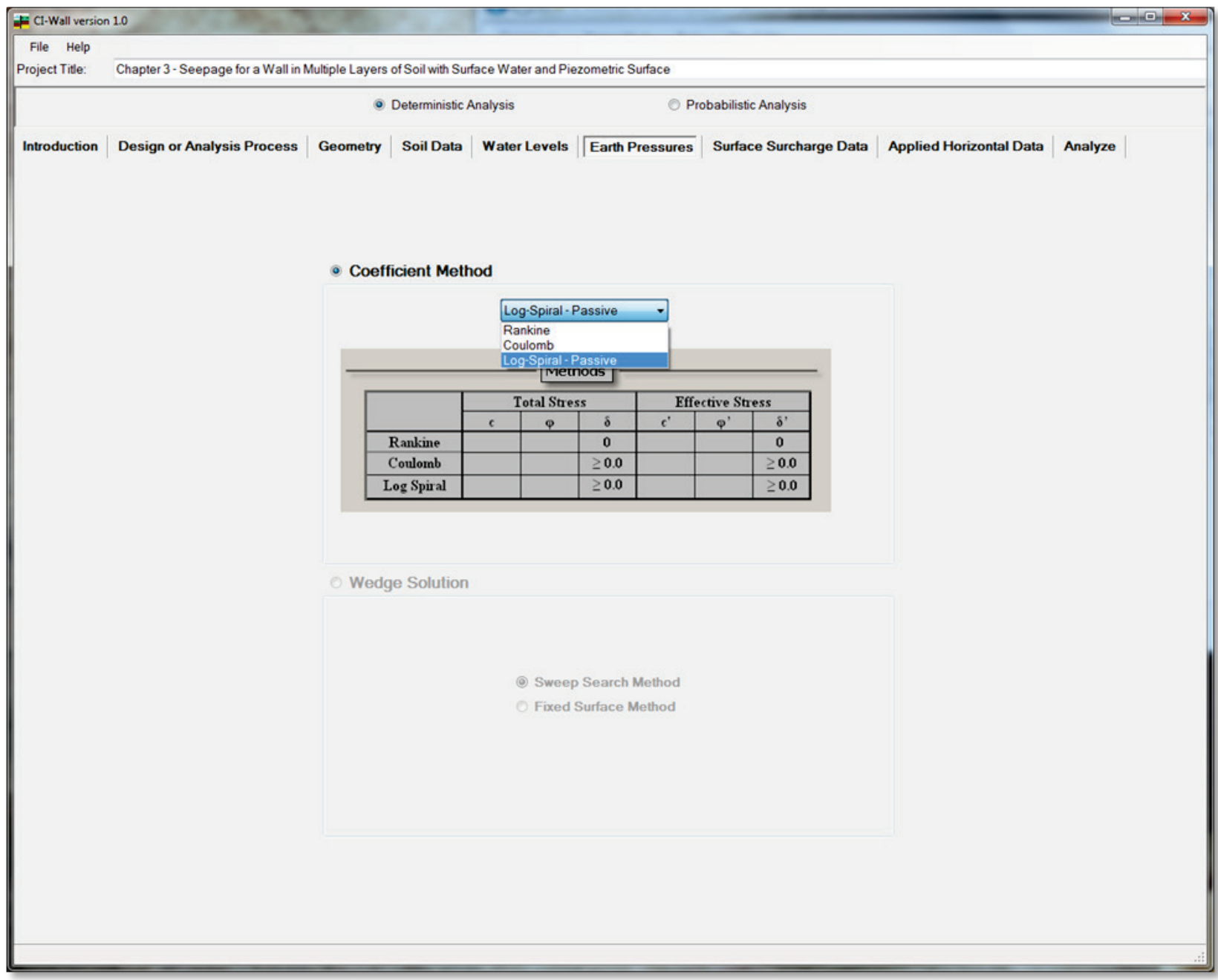


In the wedge method data input area, the user must select from either the sweep surface wedge method or the fixed surface wedge method. The sweep surface wedge method attempts to find the critical slip planes of the soil for active and passive earth pressure zones acting on the I-Wall, whereas the fixed surface wedge method proceeds based on a prescribed slip plane orientation. The wedge method is not implemented in Corps_I-Wall Version 1.o but will be in Corps_I-Wall Version 2.0.

\subsection{Surface Surcharge Data tab}

The Surface Surcharge Data tab allows the user to add line loads or distributed pressures that are applied vertically to the ground surface. Surface loads can be applied to the ground surface either to the left or right of the I-Wall, irrespective of which side is classified as the flood or landside (Figure 3.32).

Figure 3.32. Surface Surcharge Data tab.

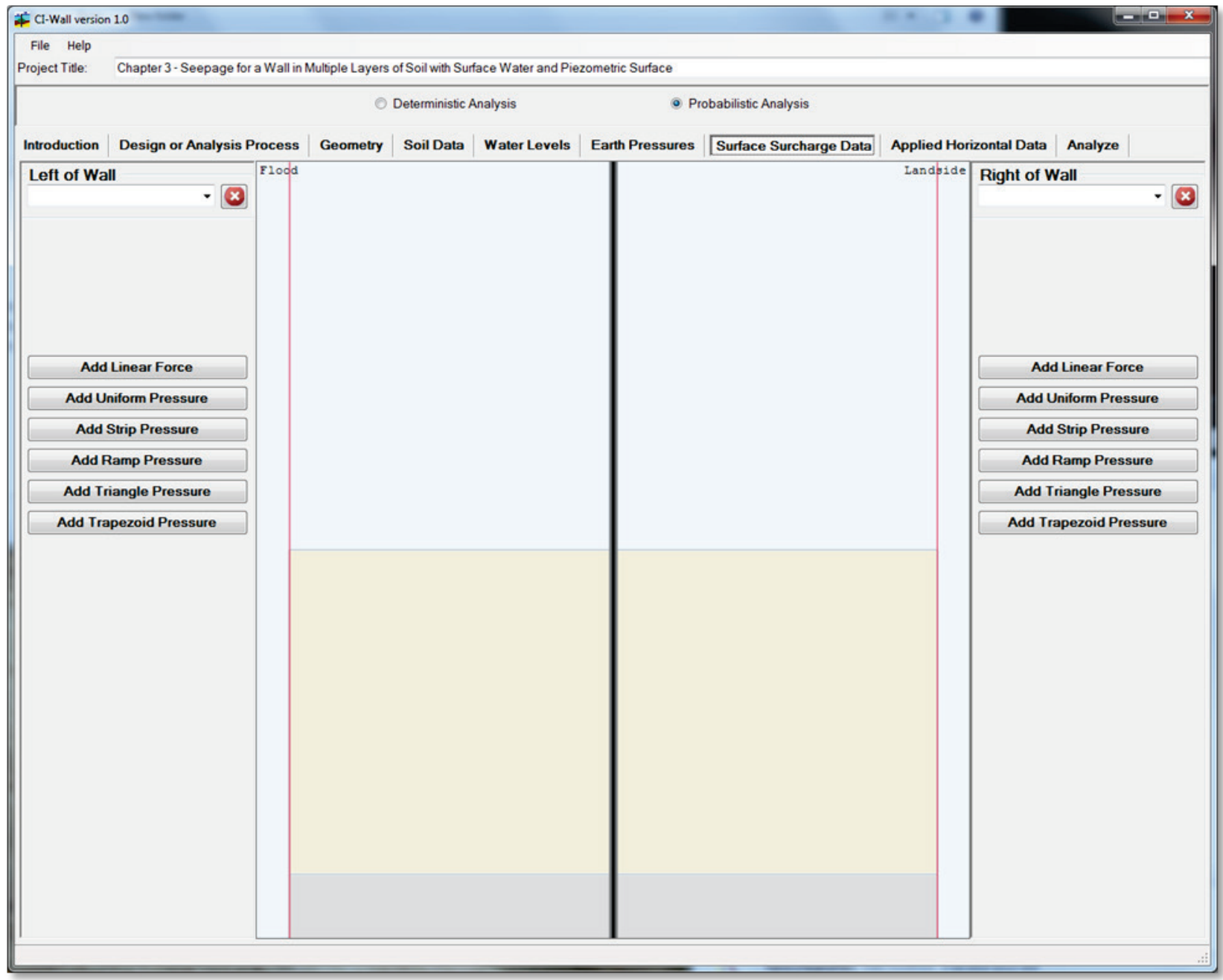


At the center of the tab is a display showing the model and zoomed to show the ground surface explicitly. The different loads are displayed on this plot. The loads are scaled to each other for the display. To the left and right of the display are the controls for selecting and adding loads to the existing model. Both sides behave in a similar manner, so the following discussion applies to both sides.

At the top of the vertical loads section is a drop-down combo-box, which has a list of the existing loads. When one of these loads is selected in the combo-box, that load becomes the current load. This load is displayed in an enhanced manner in the center screen for verification.

To the right of the combo-box is a button with an $X$ icon in a red box. Clicking that button deletes the currently selected vertical load from its respective list.

Below the current load selector and delete is the buttons that allow a new vertical load to be added to the model. Clicking either of these buttons brings up a dialog to create a new vertical load and add it to the bottom of the selector list.

These buttons allow for the creation of one of six types of vertical area loads: line load, uniform load, strip load, ramp load, triangle load, and trapezoid load. These loads follow the slope of the ground surface (for Version 2.0 and above).

The line load is a point vertical load acting on the ground surface. It is defined by giving a distance from the I-Wall and a load magnitude in relative mode and a specific position and magnitude otherwise (Figure 3.33).

The uniform load is a vertical load acting on the ground surface from the I-Wall position to an infinite distance to the I-Wall. It is defined by inputting a pressure magnitude (Figure 3.34).

The strip load is a pressure that is uniform in magnitude but acts in a certain range across the surface (Figure 3.35). The range is defined by a distance from the I-Wall for the start and end of the range in relative coordinates, and in actual start and ending X-distance from the I-Wall for actual coordinates. The consistent magnitude of the strip load pressure must also be input. 
Figure 3.33. The input window for line loads.

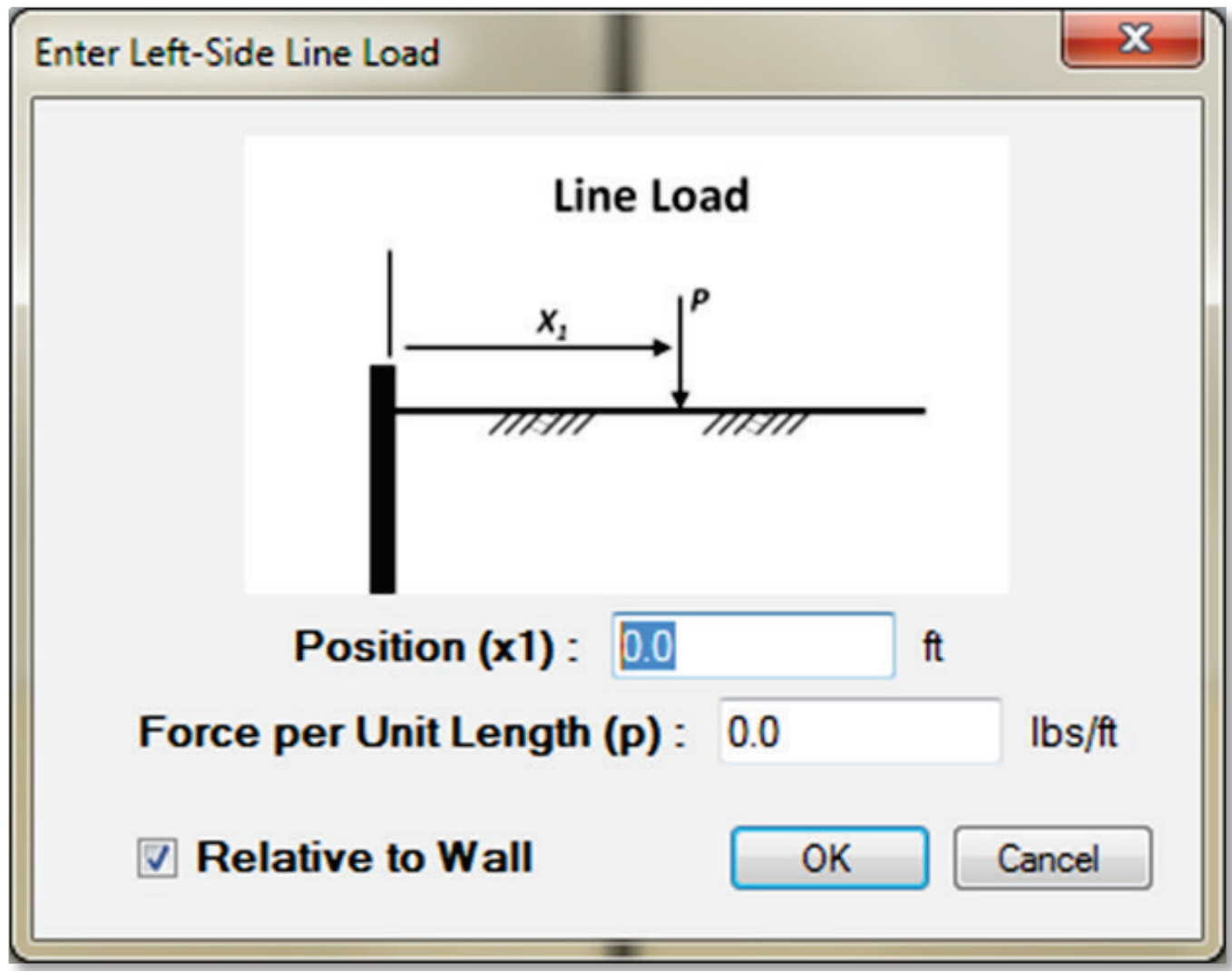

Figure 3.34. The input window for uniform loads.

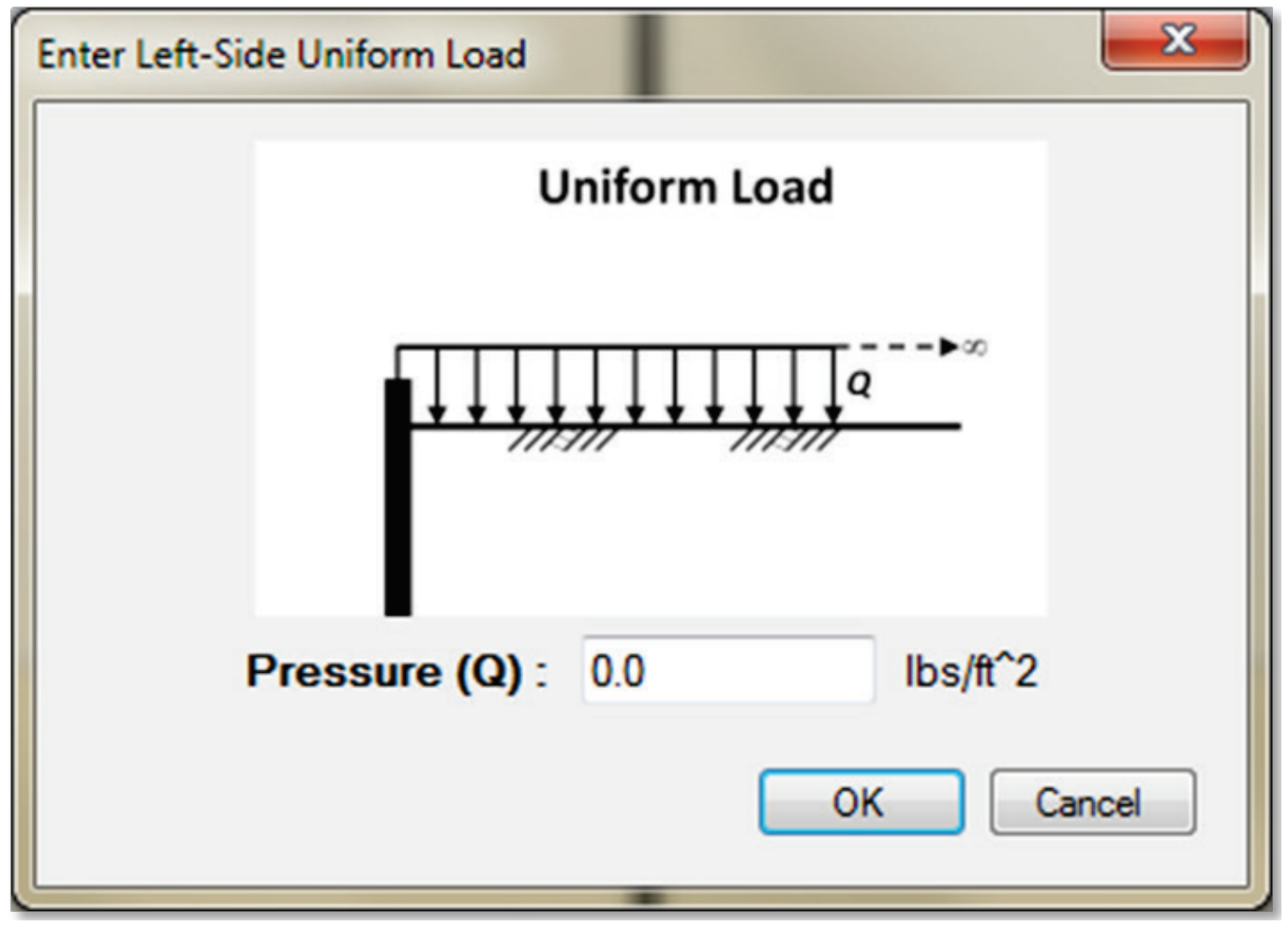


Figure 3.35. The input window for strip loads.

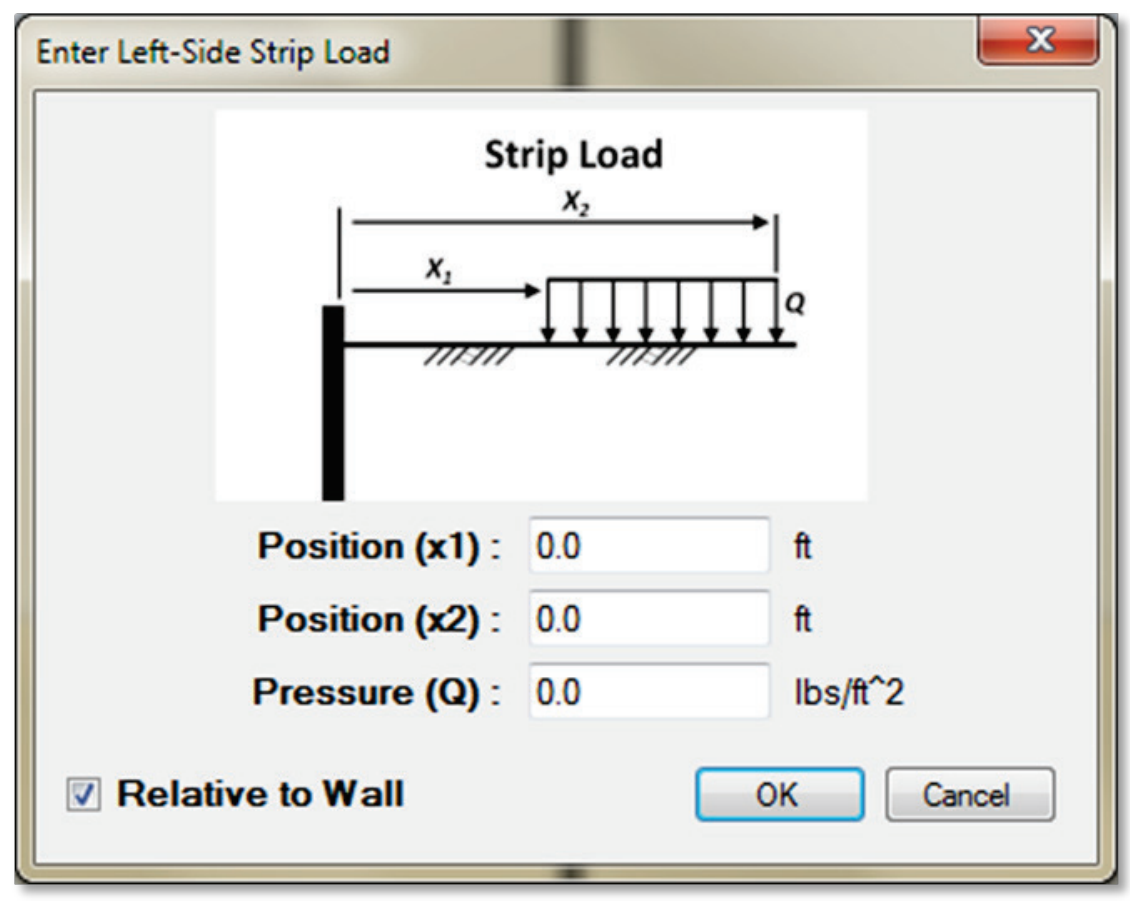

The ramp load is a vertical load. At the starting X coordinate of the load, the magnitude of the pressure is zero, but it increases to an input value by the ending $\mathrm{X}$ coordinate of the pressure. The magnitude of the pressure continues from that point to an infinite distance from the I-Wall, in the same manner as the uniform pressure. Both the start and ending points are entered in relative or absolute coordinates and the final pressure magnitude is also entered (Figure 3.36).

The triangle load has three $\mathrm{X}$-coordinates and a single pressure magnitude for entry (Figure 3.37). The first X-coordinate entered is the point at which the load begins. The magnitude of the pressure at its beginning point is zero. The second X-coordinate is the location at which the pressure has its peak magnitude, as input by the user. The last X-coordinate is the point at which the pressure ends (and has a magnitude of zero). The X-coordinates (as always) may be entered in relative or absolute coordinates. The peak magnitude must also be entered. The load is linearly interpolated from the start point to the peak point and from the peak point back down to the end point. In order to create a right triangular pressure distribution, the first or last X-coordinate can be the same as the peak X-coordinate. 
Figure 3.36. The input window for ramp loads.

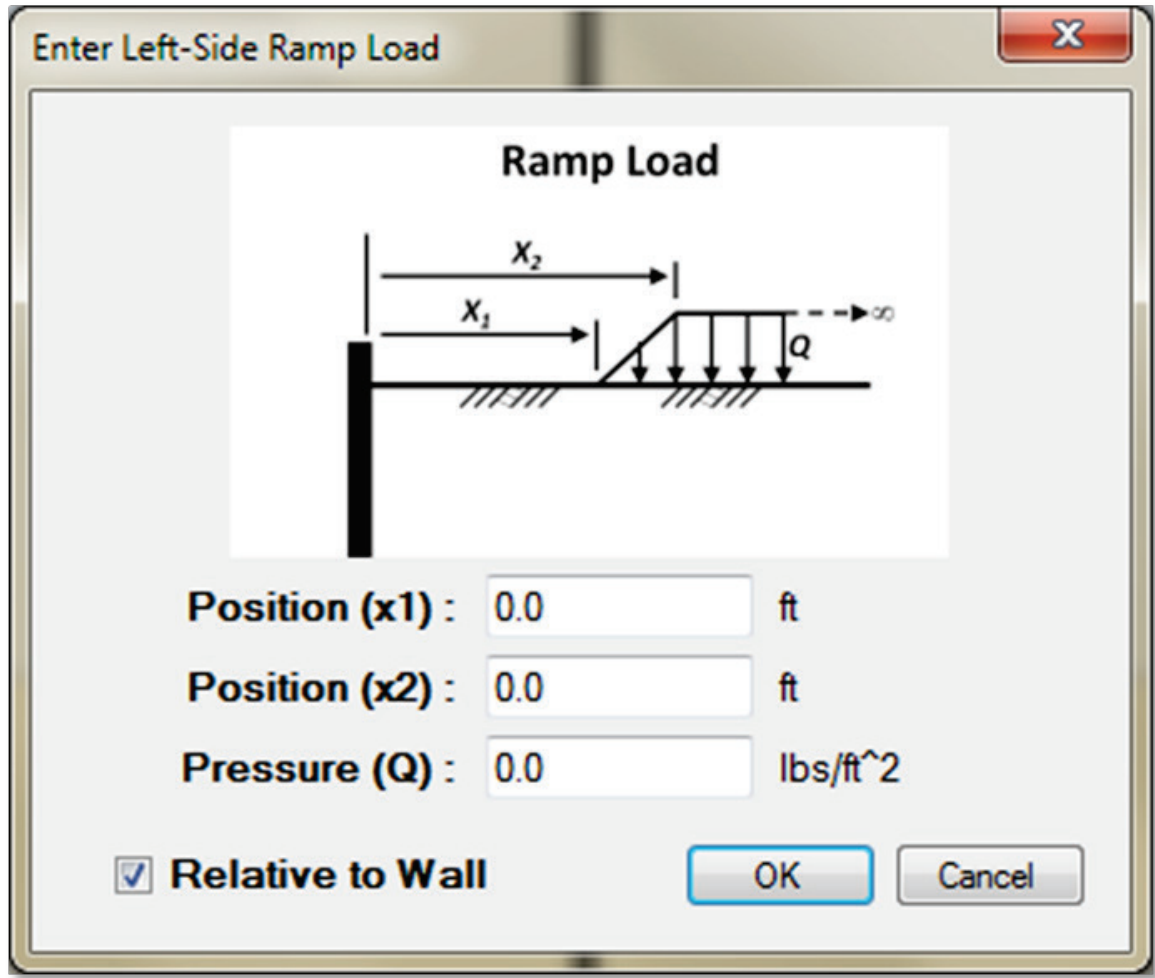

Figure 3.37. The input window for triangular loads.

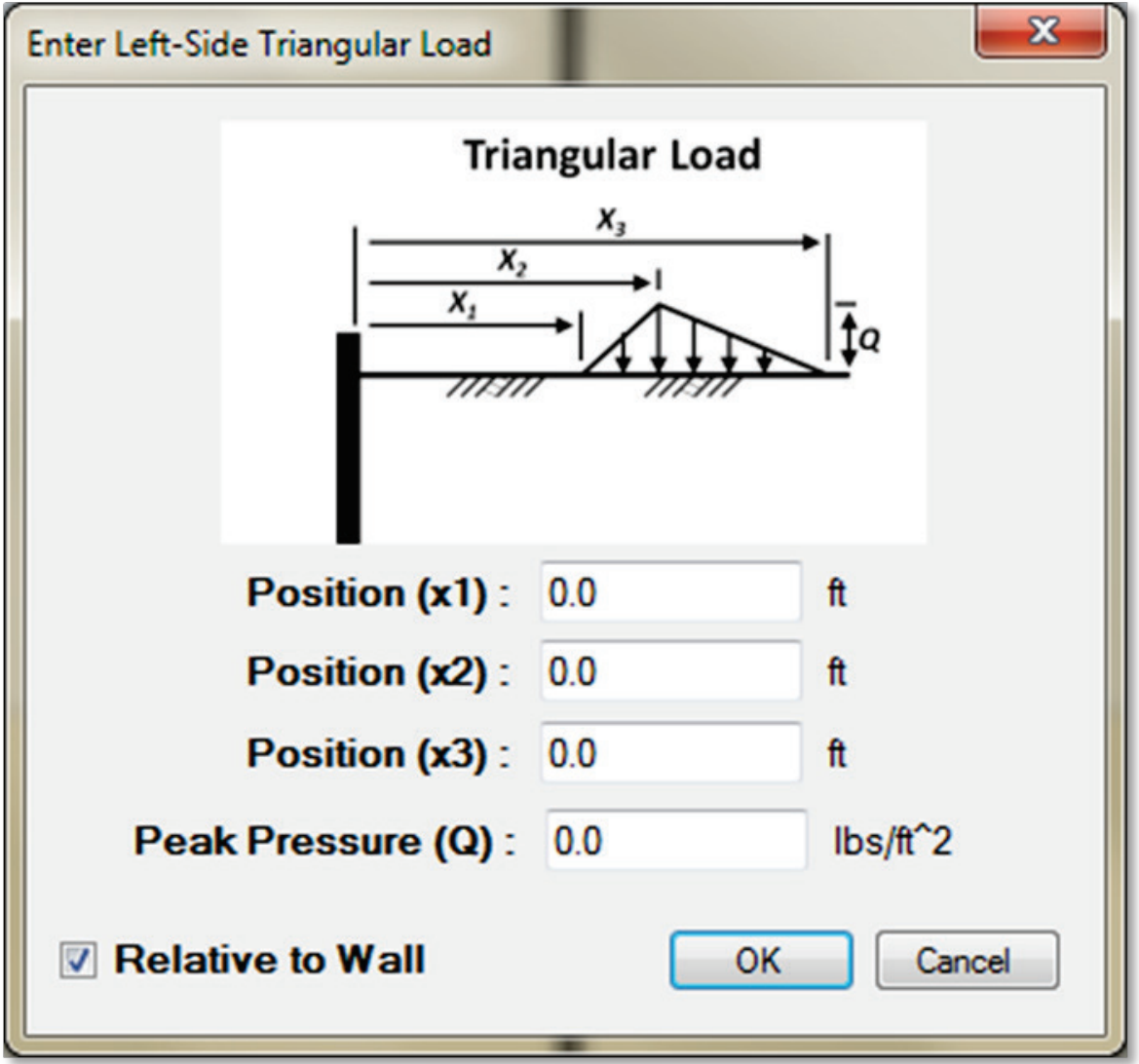


The last vertical load type is the trapezoid load (Figure 3.38). The trapezoid of pressure is defined by two points. Each point has an $\mathrm{X}$-coordinate (specified in either relative or absolute coordinates) and a magnitude at that X-coordinate. The pressure is linearly interpolated between the start point and the end point.

Figure 3.38. The input window for trapezoid/distributed loads.

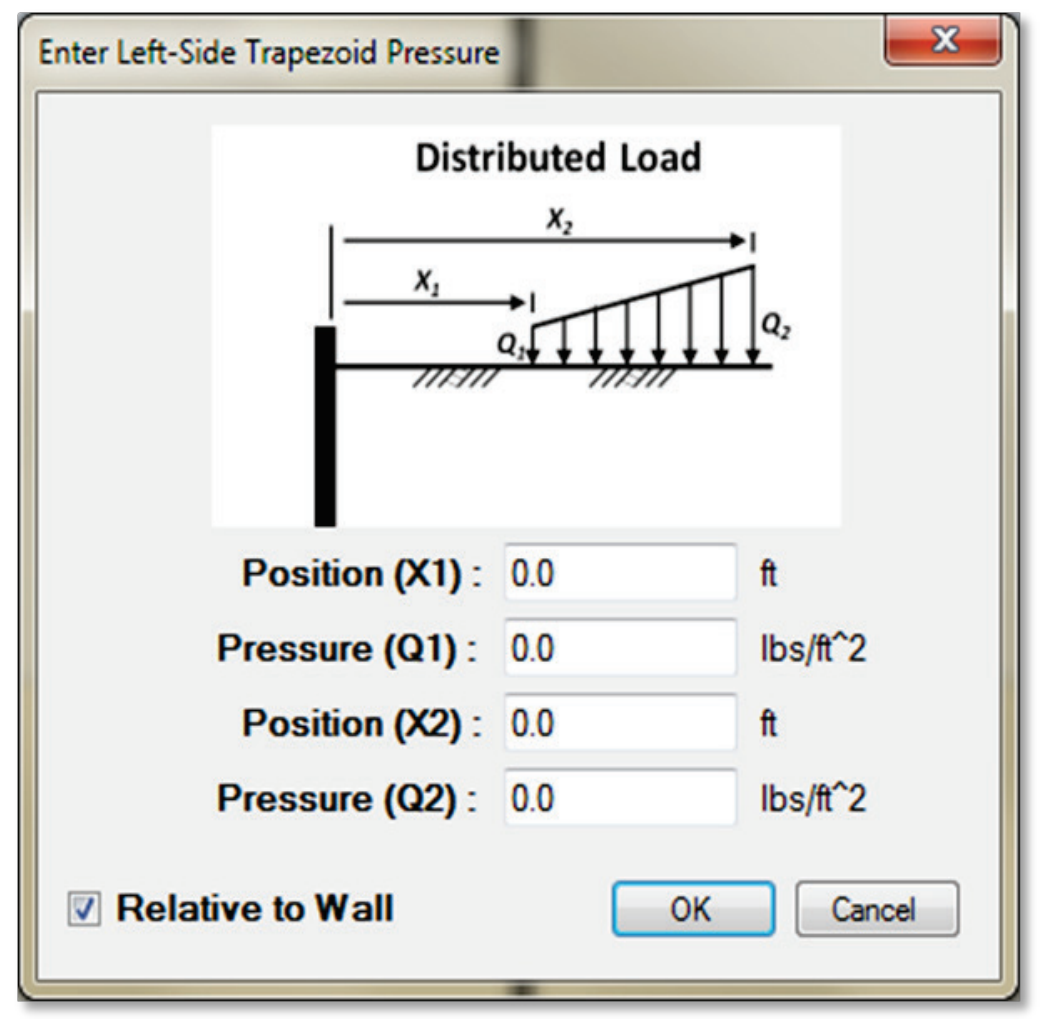

It is assumed that these loads will fall within the extents of the defined regions where materials are defined but for Version 1.0, loads can be applied up to $125 \mathrm{ft}$ away from the I-Wall or the extents of the regions whichever is greater.

Figure 3.39 shows the example problem with sample surcharge loads applied. The currently selected surcharge loads, on either side, are highlighted and the relevant information for that load is shown. 
Figure 3.39. Surface Surcharge Data tab with highlighted loads.

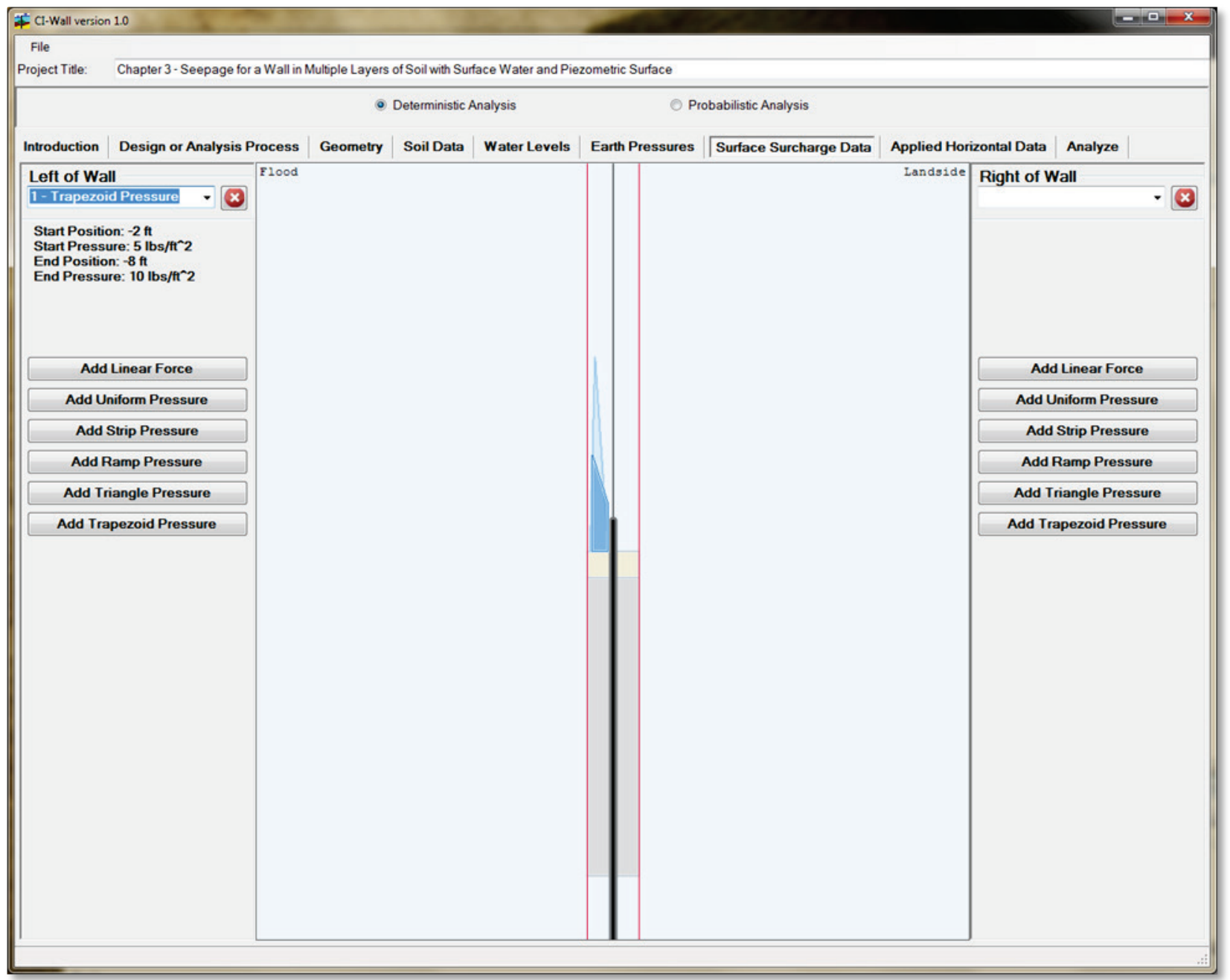

\subsection{Applied Horizontal Loads tab}

The Applied Horizontal Loads tab allows the user to specify loads that act horizontally directly against the I-Wall (Figure 3.40). Line loads and distributed loads (which are interpolated between elevation and magnitude points) can be entered. Distributed loads are input to the left of the display window and line loads are input to the right of the display window. The display window shows all of the loads acting upon the I-Wall. The entire I-Wall is displayed. The visualized loads are scaled with respect to each other. The currently selected distributed load is highlighted in the display.

Notice that pressures and loads that act toward the right are negative and that positive pressures and loads act toward the leftward direction (opposite of the global coordinate system $\mathrm{x}$-axis). Because a right-handed coordinate system is being used, positive forces acting against a surface act 
to the right of the surface definition tangent. Because the I-Wall is defined from the I-Wall top down to the I-Wall tip, the positive direction is to the right of the I-Wall looking down the global y-axis and therefore in the negative global $\mathrm{x}$-axis direction. The notes in red at the bottom of the tab serve as reminders of this unintuitive situation.

Figure 3.40. The Applied Horizontal Loads tab allows input of point loads (on the right) and linearly distributed loads (on the left).

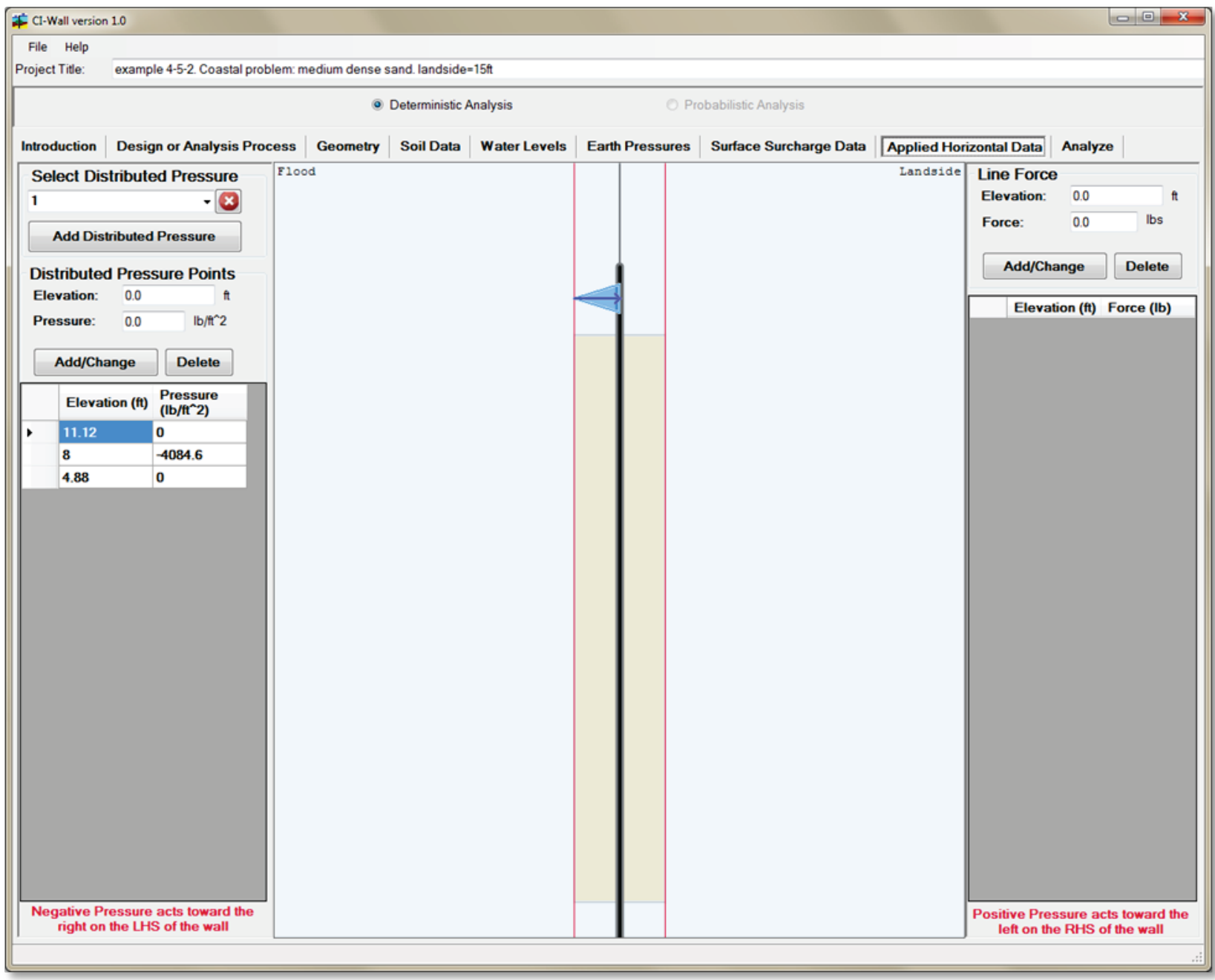

A list of distributed loads can be created in much the same way that surface surcharge loads can be created to either side of the structure. A selector exists that allows the user to select the currently active distributed load. This currently active load case is shown enhanced in the display window. This currently active load can be deleted by clicking the $X$ button (in the red box) next to the selection drop-down list for the currently active distributed load. The list of loads is renumbered sequentially when the currently active load is deleted from the list. 
Distributed horizontal loads are created as a set of piecewise linear curves. In order to start the generation of a piecewise linear distributed horizontal load, click the Add Distributed Load button. This creates a new distributed load at the end of the currently active load selector and selects it. At the beginning, the distributed load is empty.

To change the currently active distributed load, an elevation must be entered with the pressure at that elevation, under the Distributed Load Point label. When these numbers have been entered, clicking the Add/ Change button will place the horizontal pressure load at that elevation. This pressure will be displayed in the display window and in the list below the entry. As additional entries are added to the list, the list is sorted by elevation and the resulting surface shown. If an elevation is entered that is the same as an existing elevation, the existing elevation and its pressure data are overwritten with the new values.

If an elevation/pressure pair is in error and needs to be removed, the user can left mouse-click the pair anywhere on the list beneath the Add/Change button and click the Delete button, which is to the right of the Add/Change button.

Line loads are entered to the right of the display screen. These line loads are horizontal loads that act on the I-Wall with a prescribed force and at a certain elevation. All of the existing line loads are shown in the list underneath the line load creation entry text boxes and buttons. Individual line loads can be created by entering an elevation and horizontal pressure acting at that elevation. Clicking the Add/Change button adds that line load to the line load list or overwrites existing loads at that elevation. To remove a line load, click anywhere on that load in the list and click the Delete button in the line load region. Line loads are displayed in a purple color in the display window.

\subsection{Analyze tab}

The Analyze tab has the final input information for simulation runs necessary to perform a probabilistic run of Corps_I-Wall, as well as controls for running the FORTRAN processing code and viewing the results of a run. When the program is performing a deterministic calculation, the tab looks like Figure 3.41. The probabilistic input is shown in Figure 3.42. 
Figure 3.41. The Analyze tab at the beginning of a deterministic analysis.

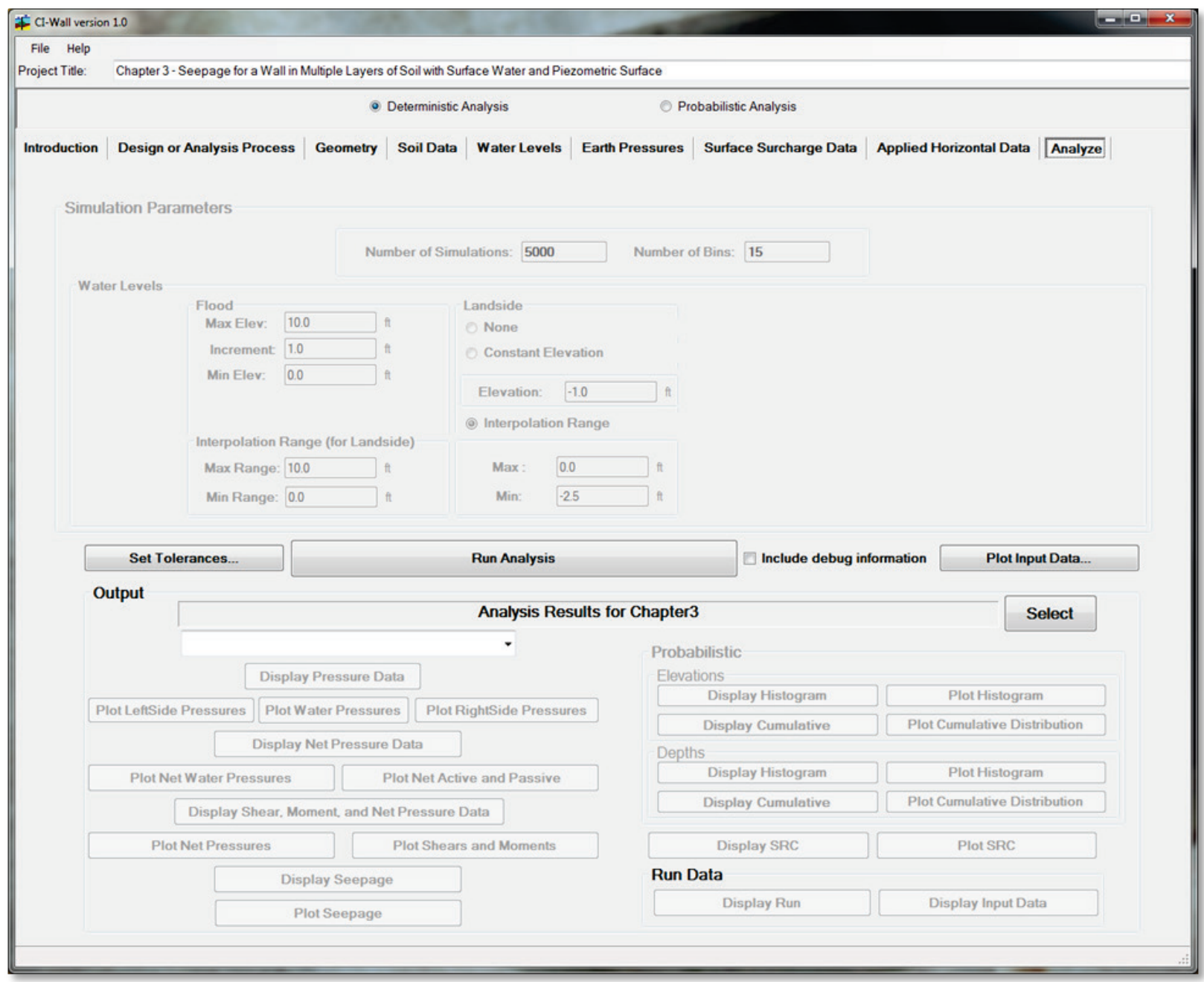

The simulation parameters to be input for probabilistic analysis are at the top of the Analyze tab. The number of simulations that will run and the number of bins used in a Latin hypercube simulation are the first input boxes. Increasing the number of bins increases the resolution and therefore, precision of the resulting simulations. Increasing the number of simulations used for each flood elevation increases the accuracy of the computations but also lengthens the time for execution.

The water levels can also be varied during the simulation runs, resulting in a system response curve, also referred to as a fragility curve. This presents the probability of performance rotational limit state as a function of load conditions (i.e., flood elevation). 
Figure 3.42. The Analyze tab for a probabilistic analysis without landside water levels.

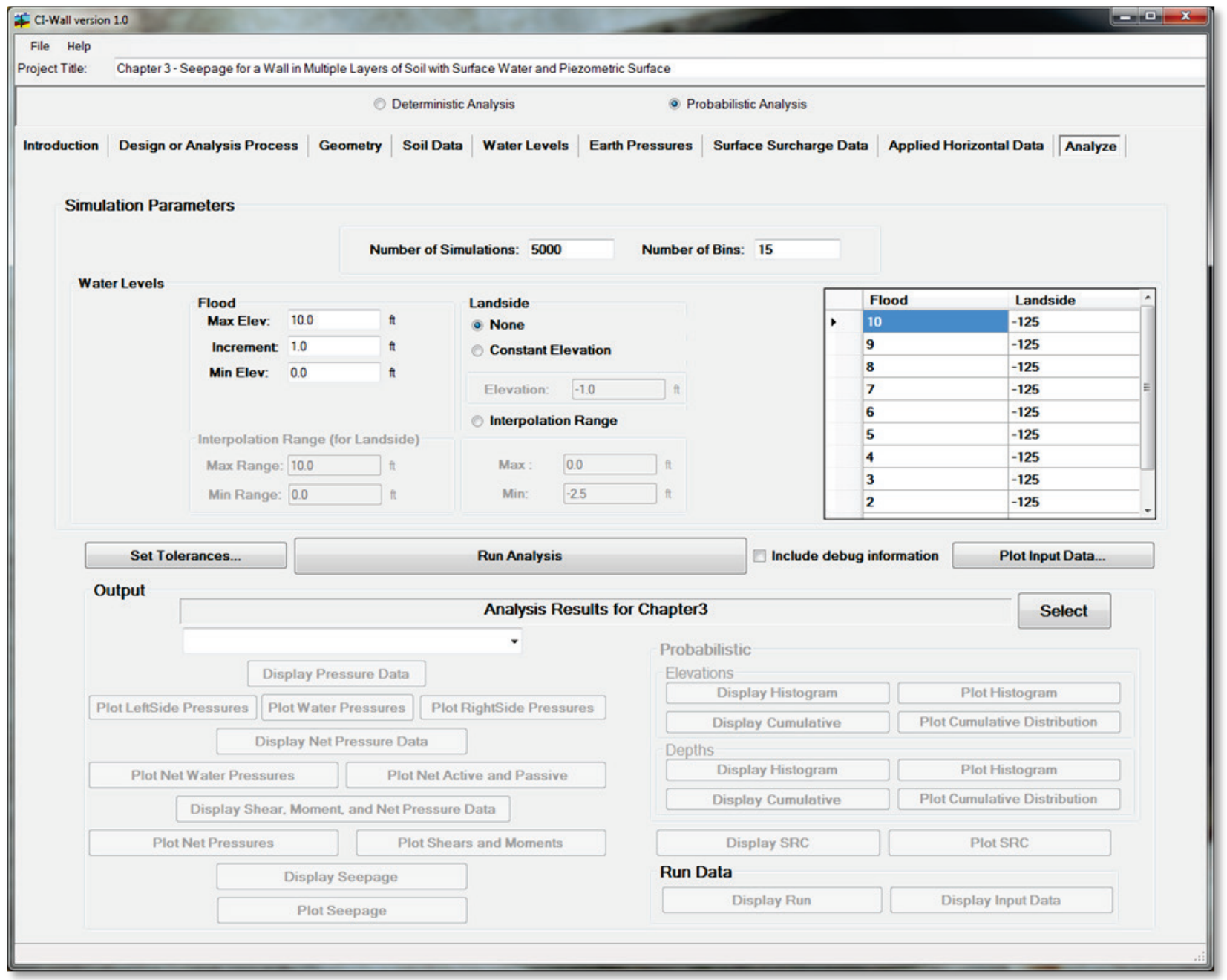

The levels are set differently for both the flood and landside of the I-Wall. The flood levels are set by giving a range of elevations, defined by the minimum and maximum elevations, and an increment of elevations from the minimum to the maximum. The set of elevations created is shown in a table to the right side of the tab.

Landside water elevations can be defined by either no water level, constant water level, or a range of interpolated levels that vary with the flood elevations. One of these methods is chosen using radio buttons.

Selecting the radio button labeled None sets the landside water elevation to the base of the bottom soil region on the landside for every flood elevation (Figure 3.42). This means that the water levels on that side of the structure have no impact on the final solution. 
Selecting the radio button labeled Constant Elevation allows the user to enter a constant elevation that will be used for the landside water levels (Figure 3.43). This value is used for every flood level simulation.

Figure 3.43. The Analyze tab for a probabilistic analysis with constant landside water level.

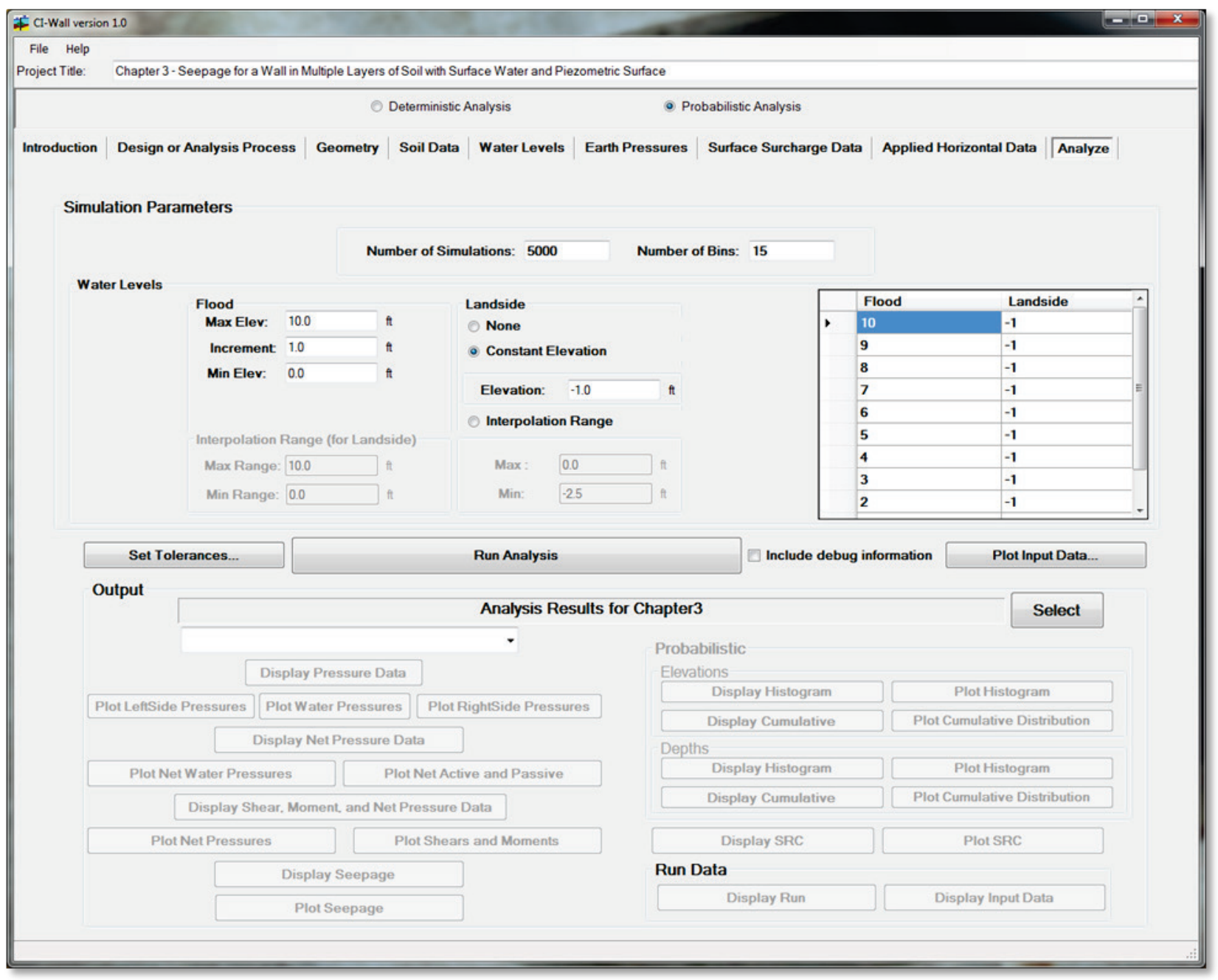

Choosing the Interpolation Range radio button for assigning the landside causes a set of input windows to be opened. Data for the flood and landside are requested. This is because the values of the landside water levels can vary over a range of possible floodwater level elevations. The minimum and maximum water elevations for the landside are entered under the Interpolation Range radio button. The landside increment is determined by specifying the lower and upper limits of the flood elevations (Figure 3.44a). 
Figure 3.44. (a) The Analyze tab. (b) Its procedure for a probabilistic analysis with interpolated landside water levels.

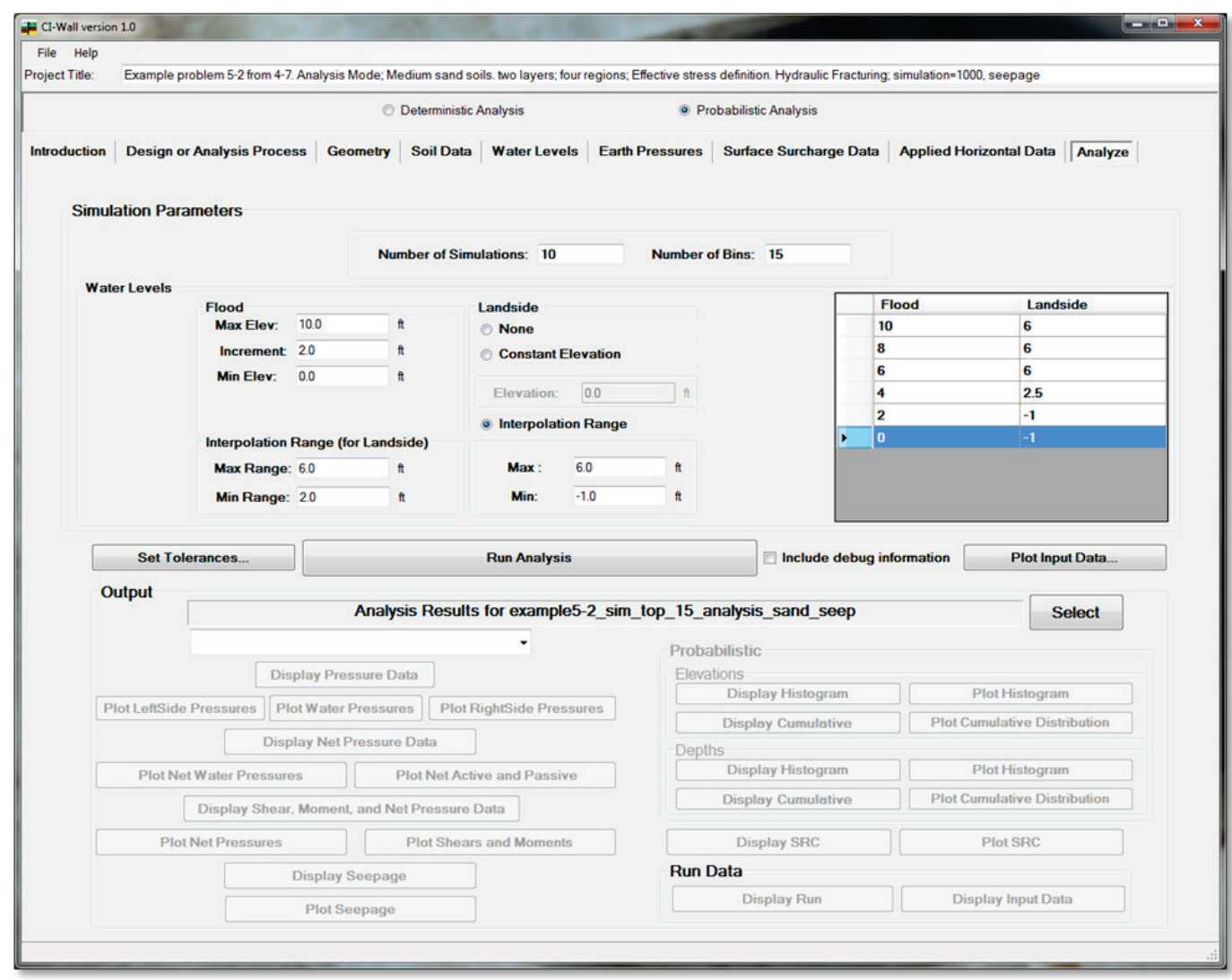

a.

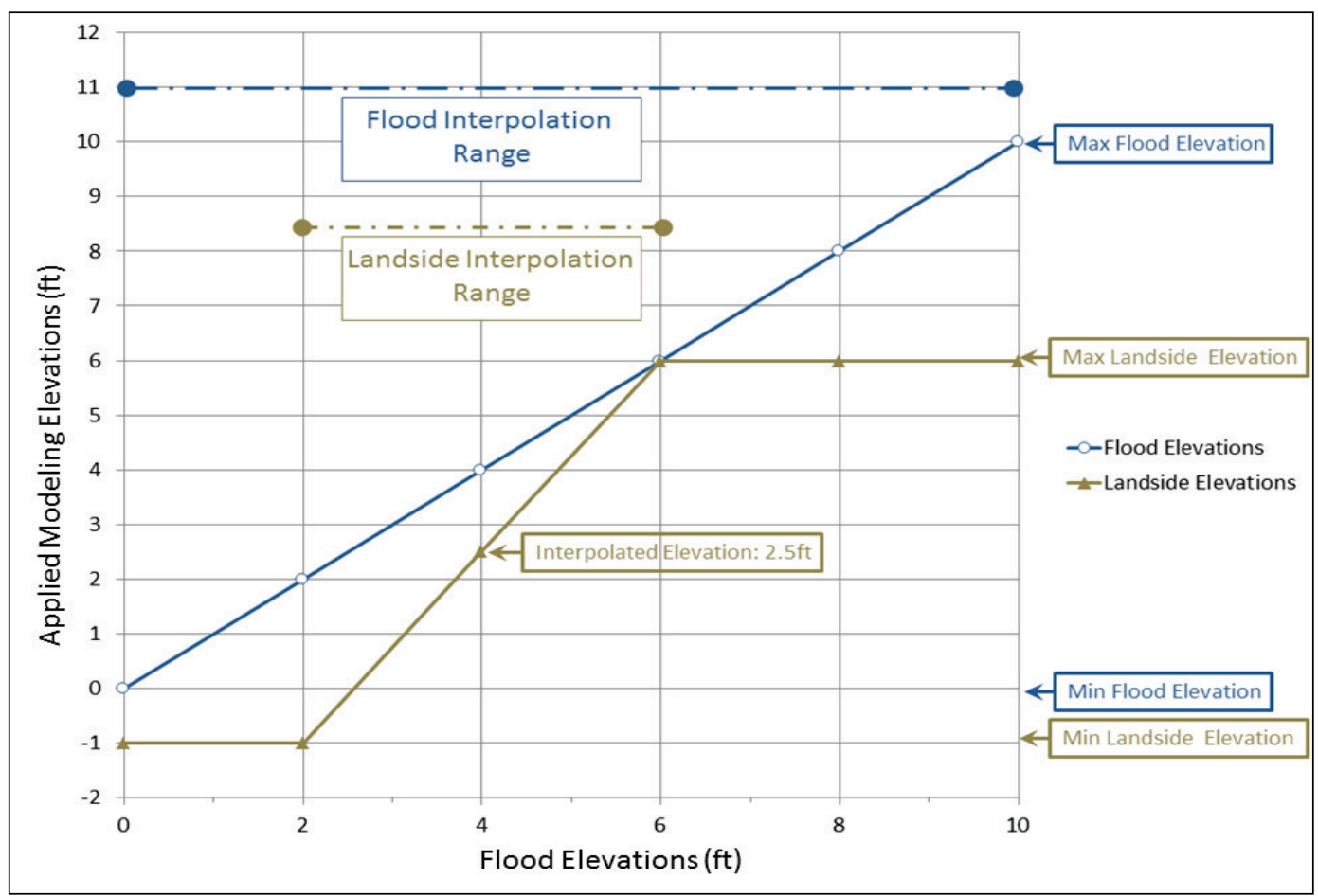

b. 
Figure 3.44a and $\mathrm{b}$ shows a probabilistic run that is generated using the Interpolation Range option. The flood levels are defined by a minimum elevation of $0.0 \mathrm{ft}$ with increments of $2.0 \mathrm{ft}$ to the maximum elevation of $10 \mathrm{ft}$ (input in the Flood section). This will result in six x-values of flood els: $0,2,4,6,8,10 \mathrm{ft}$. Therefore, six $\mathrm{x}$-values of landside water elevations are needed as well. The landside elevation range of values for water levels go from a minimum of $-1 \mathrm{ft}$ to a maximum of $6 \mathrm{ft}$ (input in the Interpolation Range section at the lower right of the Simulation Parameters input section), but these values are interpolated for only a subrange of the flood elevations. The lower left input of Flood Side Interpolation Range of a Min Range value of $2 \mathrm{ft}$ and a Max Range value of $6 \mathrm{ft}$ establishes the subrange of flood elevations over which the landside water elevations will be linearly interpolated.

Figure 3.44b shows the water elevations for the flood and landside of the I-Wall as a function of the flood side water elevations. Because the flood side has a 1-to-1 correspondence to itself in this figure, the flood elevations plot at a 45-deg angle. The landside water elevation is a three part, piecewise linear function of flood side water elevations. The landside water elevations are linearly interpolated when the flood side elevations are in the range between 2 and $6 \mathrm{ft}$. For these two flood elevations the corresponding landside water elevations are -1 and $6 \mathrm{ft}$, respectively. Outside of the flood side range of 2 to $6 \mathrm{ft}$, the values for the landside water elevations are horizontal with the landside values of -1 and $6 \mathrm{ft}$, respectively. Between these two flood elevations of 2 and $6 \mathrm{ft}$, a linear interpolation is required. In this case, the value for the landside water elevation has to be calculated for the corresponding flood side elevation of $4 \mathrm{ft}$. Between flood elevations of 2 and $6 \mathrm{ft}$, the landside water elevation relationship follows the linear equation

$$
y=m * x+b
$$

After substitutions, the relationship becomes

$$
y=\{6--1\}\{6-2\} * 4-2+-1=1.75 * 2-1=2.5
$$

So for a flood elevation of $4 \mathrm{ft}$, the corresponding landside water elevation is $2.5 \mathrm{ft}$. This linear interpolation computation is made by the CI-Wall GUI software. If the range had included more flood elevations between the extremes, multiple landside water elevations would have been computed 
using the linear interpolation based on Equation 3.1. The table to the right in Figure 3.44a summarizes the results of this interpolation for the six pairs of flood side and landside water elevations.

It is possible to enter values for the interpolation range to extend outside of the range of flood values, but this will usually lead to unexpected results and is discouraged.

The buttons under the simulation parameter box allow the user to run the Corps_I-Wall FORTRAN processor. The Plot Input button gives the user a final chance to see the input geometry of the input data as a verification step. Figure 3.45 shows a resulting plot for a deterministic analysis. At the top of the plot is a list of the material descriptions. The first column has a material ID (e.g., Mo2) that is displayed at the top of each surface region in the plot below. Used materials are shown in black and unused materials, which may be input but not assigned to a region, are in gray.

Figure 3.45. Plotting the input model for a deterministic analysis.

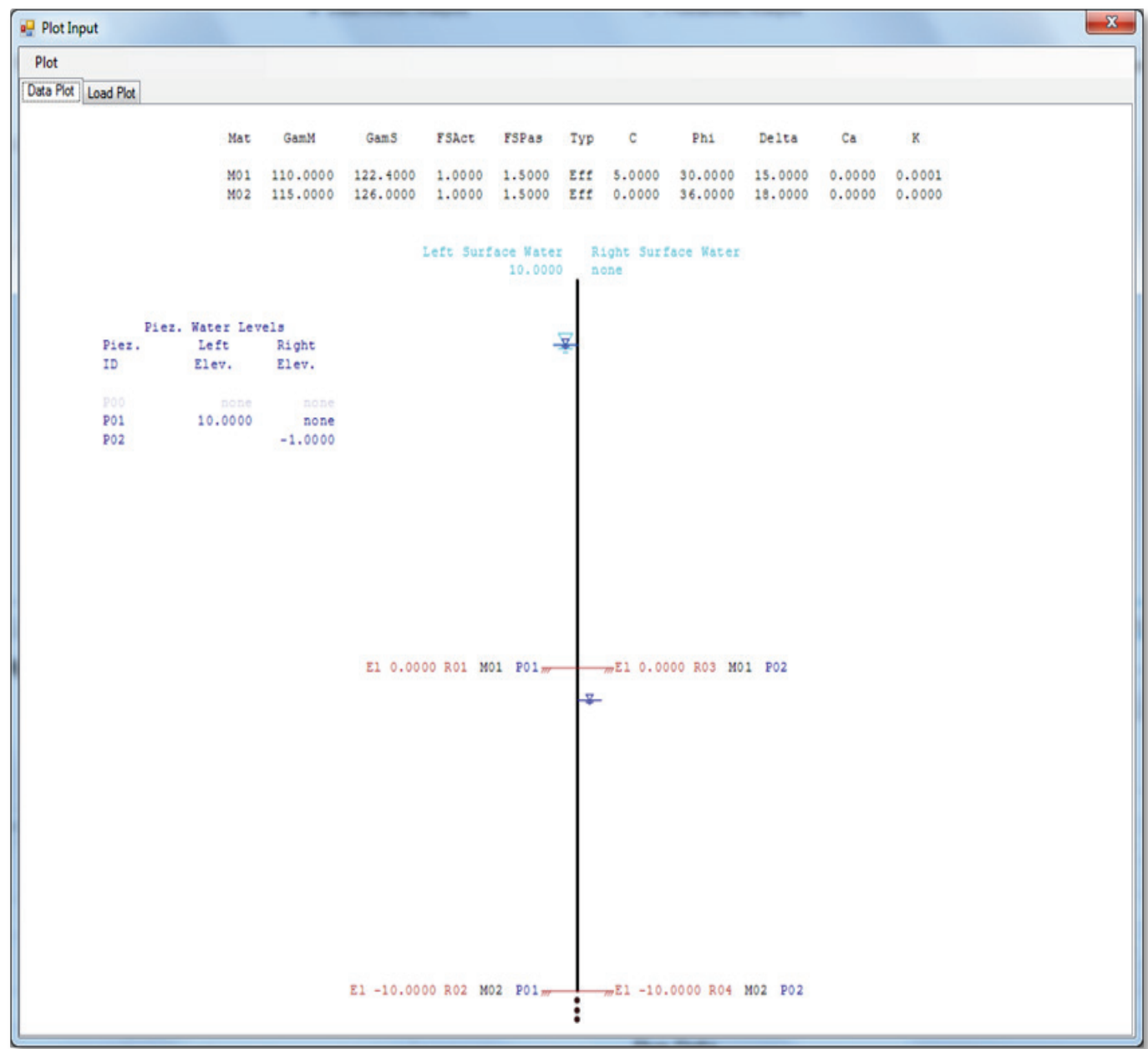


Immediately below the material definitions are the water surface elevations for the LHS and RHS of the I-Wall in cyan. A table of defined piezometric surfaces is given to the left of the plot in blue. Assigned piezometric surfaces are shown in dark blue, and unassigned piezometric surfaces are shown in light blue. The first two piezometric surfaces are special. The first piezometric surface is only applied if there is no water pressure acting on a material region. The second piezoemetric surface acts as if the surface water were acting on the side of the I-Wall as the material region, so both surface elevations are shown. The rest of the piezometric surfaces are user-defined surfaces and only apply to one side of the I-Wall, so they only have a value on that side of the I-Wall. The piezometric surface ID (e.g., Po1) is given in the leftmost column.

The plot shows the I-Wall, at least to the top of the lowest soil material layer or the lowest water elevation. The surface water elevations are displayed with a typical water symbol in cyan. Assigned piezometric surfaces are displayed in a like manner with a dark blue color. For each surface material region, a brown line extends from the I-Wall with an earth symbol. The elevation is marked, and the region ID (e.g., Ro1) is given. Also shown are the material properties assigned to the region (acting below the surface to the top of the next surface) and the piezometric surface exerting pore water pressure on this region.

Figure 3.46 shows the results of plotting the input for a probabilistic run of the Corps_I-Wall program. The only additions to this plot are the table to the left of the various water surface elevations that will be tested for the simulation runs and these elevations on the plot itself. These additions are in a green color. These water levels not only provide different overburdens but also affect the material regions that are assigned the piezometric surface water (Po1). This is represented in the region plots by displaying the piezometric surface water Ro1 in green.

If surface surcharge or applied horizontal loads are specified, they can be viewed in the same fashion as they are displayed in the GUI by selecting the Load Plot tab (Figure 3.47). 
Figure 3.46. Plotting the input model for a probabilistic range of both flood and landside water levels.

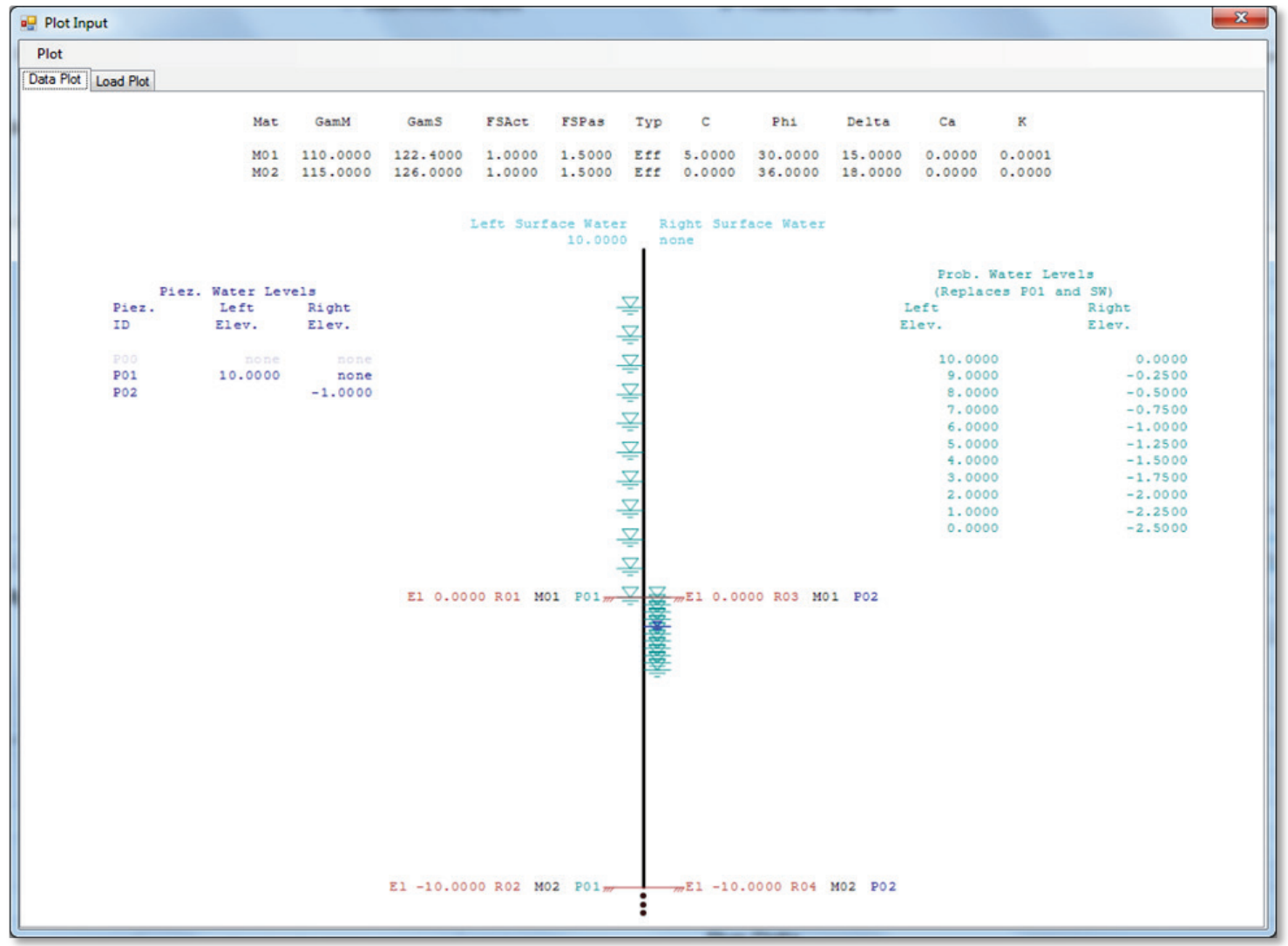

Figure 3.47. View of the pile without horizontal and vertical loads.

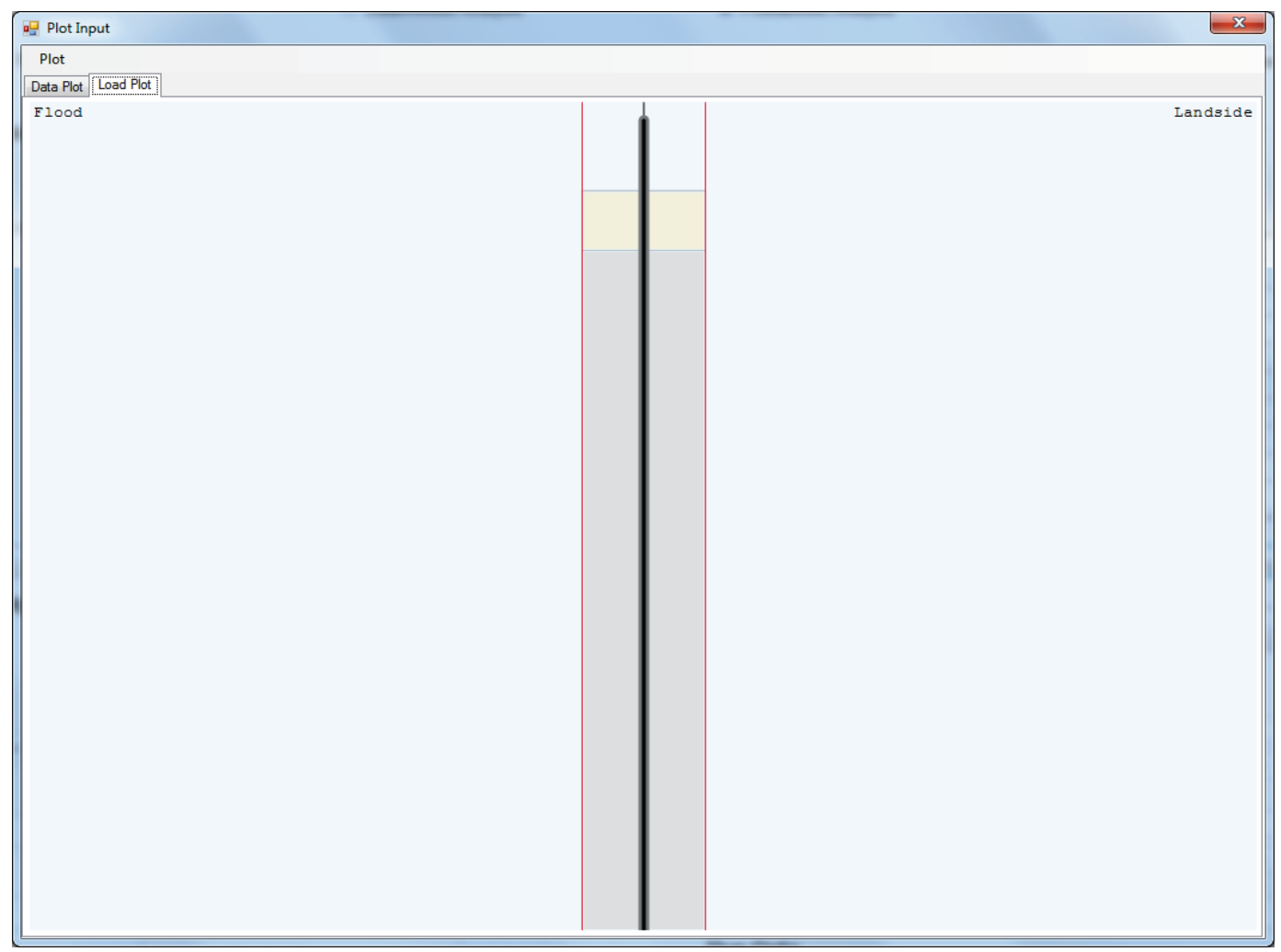


The Set Tolerances button opens the Set Tolerances dialog box (Figure 3.48), allowing for the input of the initial node spacing along the I-Wall, the node spacing increment (so that Corps_I-Wall can meet convergence criteria), and the minimum space allowed between nodes. There is also input of moment calculation tolerance, which will allow Corps_I-Wall to converge to a solution faster. The number of iterations allowed to calculate the I-Wall tip depth is also given. It is anticipated that these default values will be adequate for the majority of I-Wall analyses.

Figure 3.48. Tolerance input dialog.

\begin{tabular}{|c|c|c|}
\hline Set Tolerances & & $x$ \\
\hline \multicolumn{3}{|l|}{ Nodal Limits/Tolerances } \\
\hline Initial Node Spacing: & 1.0 & \multirow[t]{5}{*}{$\mathrm{ft}$} \\
\hline Node Spacing Increment Factor: & 0.01 & \\
\hline \multicolumn{2}{|c|}{ Node Spacing Increment $=0.01 \mathrm{ft}$} & \\
\hline Minimum Node Spacing Factor: & 0.7 & \\
\hline \multicolumn{2}{|c|}{ Minimum Node Spacing $=0.7 \mathrm{ft}$} & \\
\hline \multicolumn{2}{|l|}{ Moment Calculation Tolerance } & \multirow[b]{2}{*}{$\mathrm{ft}$-lbs } \\
\hline Moment Calculation Tolerance: & 10.0 & \\
\hline \multicolumn{2}{|l|}{ Wall Tip Max Iterations } & \\
\hline \multirow[t]{2}{*}{ Wall Tip Max Iterations: } & 100 & \\
\hline & OK & Cancel \\
\hline
\end{tabular}

The Run Analysis button performs the analysis as requested for Analysis or Design mode, and for deterministic or probabilistic mode. The Include debug information checkbox allows the user to specify the output to include debug information when the FORTRAN processor runs (Figure 3.49). When the processor has finished, a database of output files is created. It is not anticipated that a user will request execution in debug mode. This option was put in place for use by program developers.

There is a caveat to this procedure. In probabilistic mode, it is possible to specify correlations that are impossible to fulfill when cross-correlation occurs. Cross-correlation occurs when a variable (e.g., material property $\mathrm{Ca}$ ) is correlated with more than one other material property variable or 
correlated across materials. When a cross-correlation is recognized by the GUI, a message dialog will pop up warning that this condition may occur (Figure 3.50).

Figure 3.49. The Corps_I-Wall processor in action.

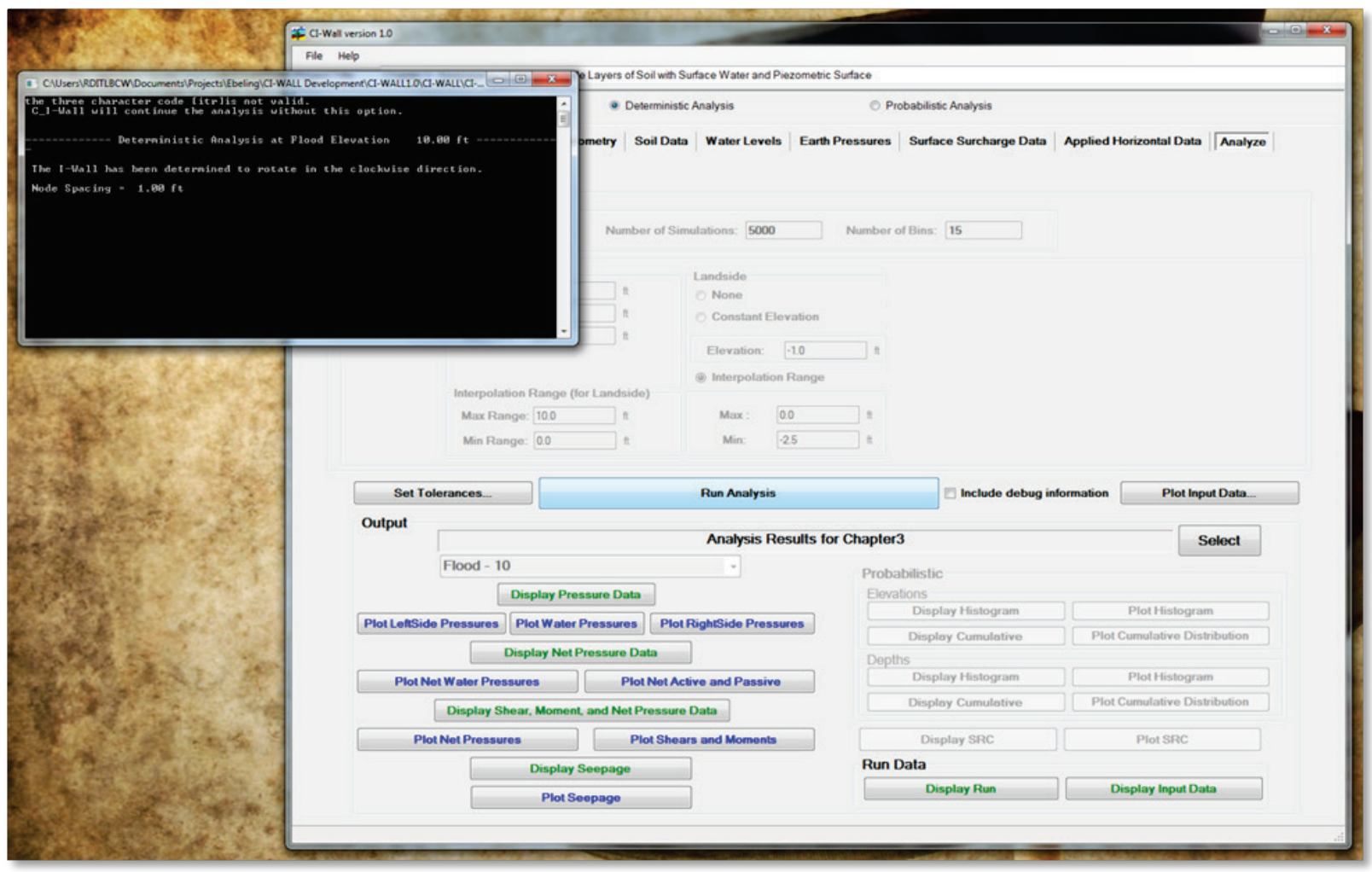

Figure 3.50. Adjusting rank correlation dialog.

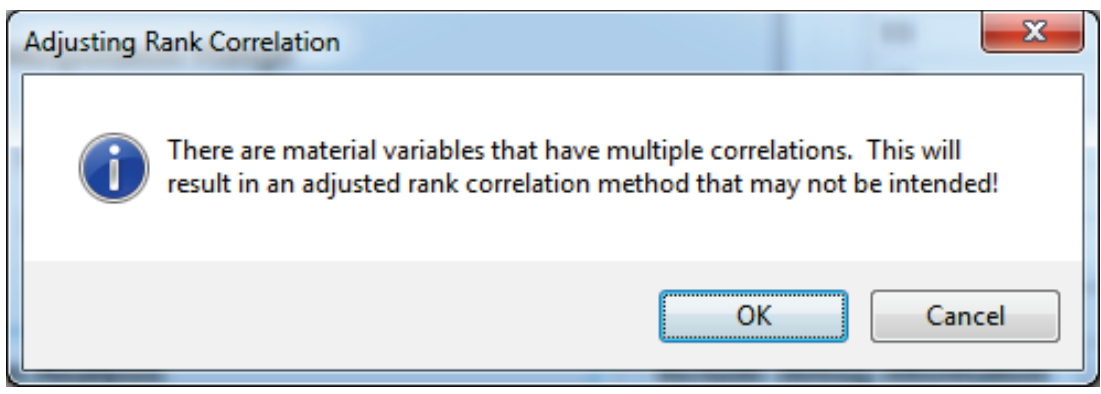

At this point, the user can choose to abort the attempted run to check his correlations or continue to run. If the run is continued, the software will attempt to adjust the offending correlations so that there are no conflicts and to remain as close to the specified correlations as possible. If this is successful, the run will complete with the adjusted correlations. If the values cannot be adjusted to achieve a meaningful set of correlations, a message dialog will appear informing the user that the correlations must be adjusted in the GUI (Figure 3.51), and the run will not be completed. 
Figure 3.51. If problems occur during adjustment of rank correlation, this dialog appears.

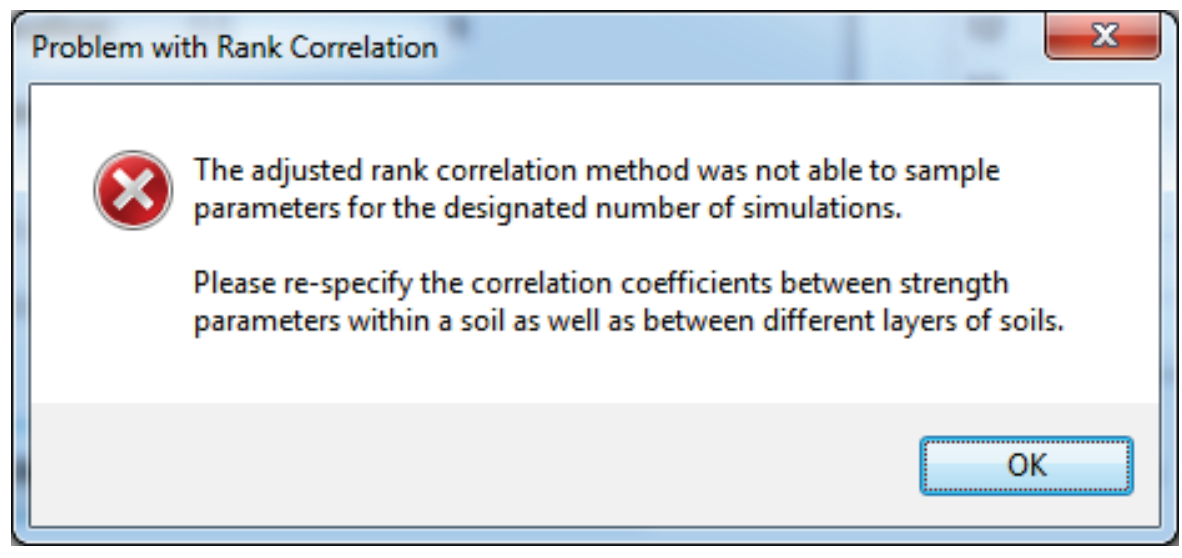

The Output section of the Analyze tab allows the user to view outputs from any runs that have been performed (Figure 3.52). At the top of this form is a label showing the currently selected output data set. The Select button to the right of this label allows the user to search for existing output data sets that may exist on their computer. The file that designates the data set has an .ohd (Output Header Designation) extension. When an .ohd file has been selected, that data set is populated in the Output section, and the output options are enabled for each option that has a valid output file. For instance, if a deterministic analysis was performed the probabilistic section options will remain disabled, but if a probabilistic analysis was performed those options would be enabled.

NOTICE: If an error occurred during an analysis, then only the Run Data subsection options will be enabled.

When the Run Analysis button has been performed, the .ohd for that run is automatically selected and the Output section is automatically populated with the values of that data set. This enables the user to proceed directly to analyzing the results from the existing input immediately.

Each output option begins with either the word Display or Plot. The Display options show the relevant data as a text file. The display window shows the data and allows the user to print the data or save it to another file.

The Plot options display the data in the files in some graphical manner. At this time, the drawn data cannot be sent to a printer or the clipboard, but these options will be added in later versions. 
Figure 3.52. After the processor has finished, output options are made available.

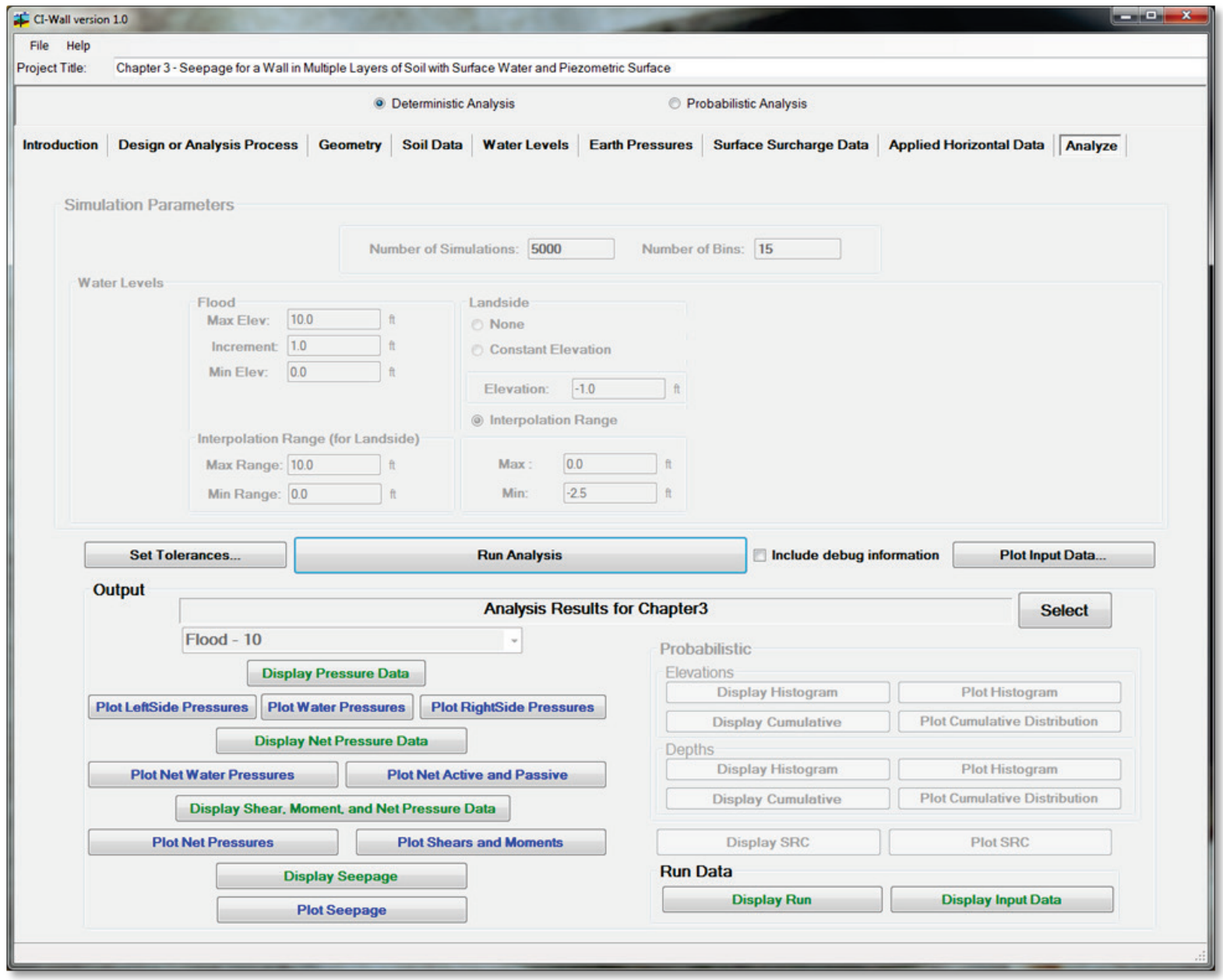

At the bottom left of the Output section of the Analyze tab is the Run Data subsection (Figure 3.53). The Display Run button in this subsection brings up another window that has a permanent copy of the running output of the FORTRAN processor, as well as any debug information if it was requested (Figure 3.54). Pertinent information, such as the elevation of rotation, pile tip elevation, and gap depth are presented.

Figure 3.53. The Run Data subsection allows the user to see processor input, results, and debugging information.

\section{Run Data}

Display Run

Display Input Data 
Figure 3.54. The display run window shows the output and debugging information from an analysis.

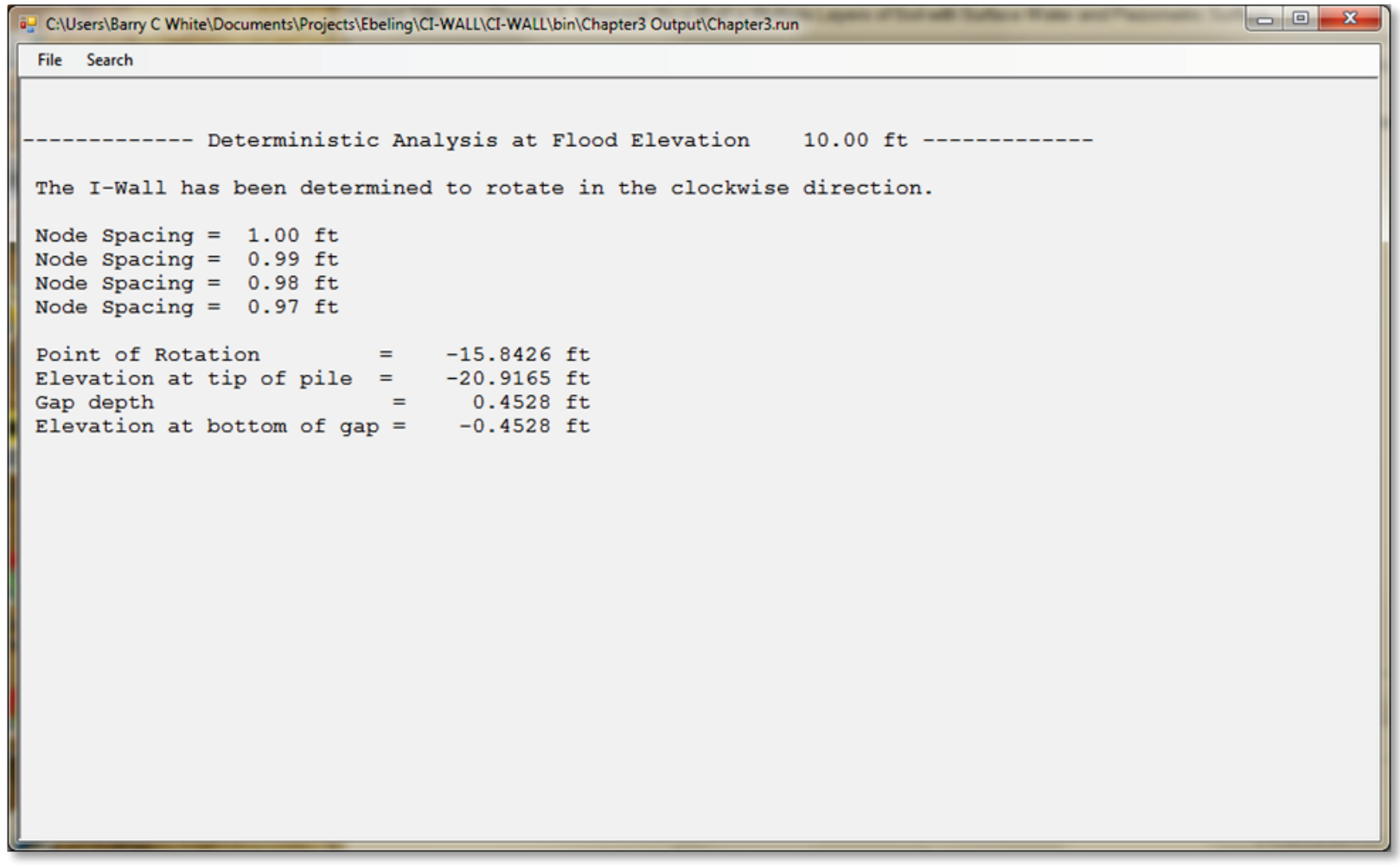

The Display Input Data option of the Run Data subsection allows the user to review the input file for the FORTRAN processor that has been generated by the visual pre-processor (Figure 3.55). The file format for this input file is discussed in Appendix D.

To the left of the Run Data subsection are the buttons that display the output file data (Figure 3.56). Above these buttons is a selector that allows the user to select and view data for a flood level elevation. For a deterministic run, this selector will only have one value and the selector will be disabled. For a probabilistic run, multiple values will be available. The currently selected flood elevation output data will be the data displayed and plotted by the buttons shown.

The example problem is deterministic, and the following plots are for the fixed flood elevation of $10 \mathrm{ft}$.

Clicking the Display Pressure Data opens the output file related to water pressures on the LHS and RHS of the I-Wall (Figure 3.57). If gap propagation has been enabled, information about the gap is presented. 
Figure 3.55. The display input data button allows the user to see the processor input file.

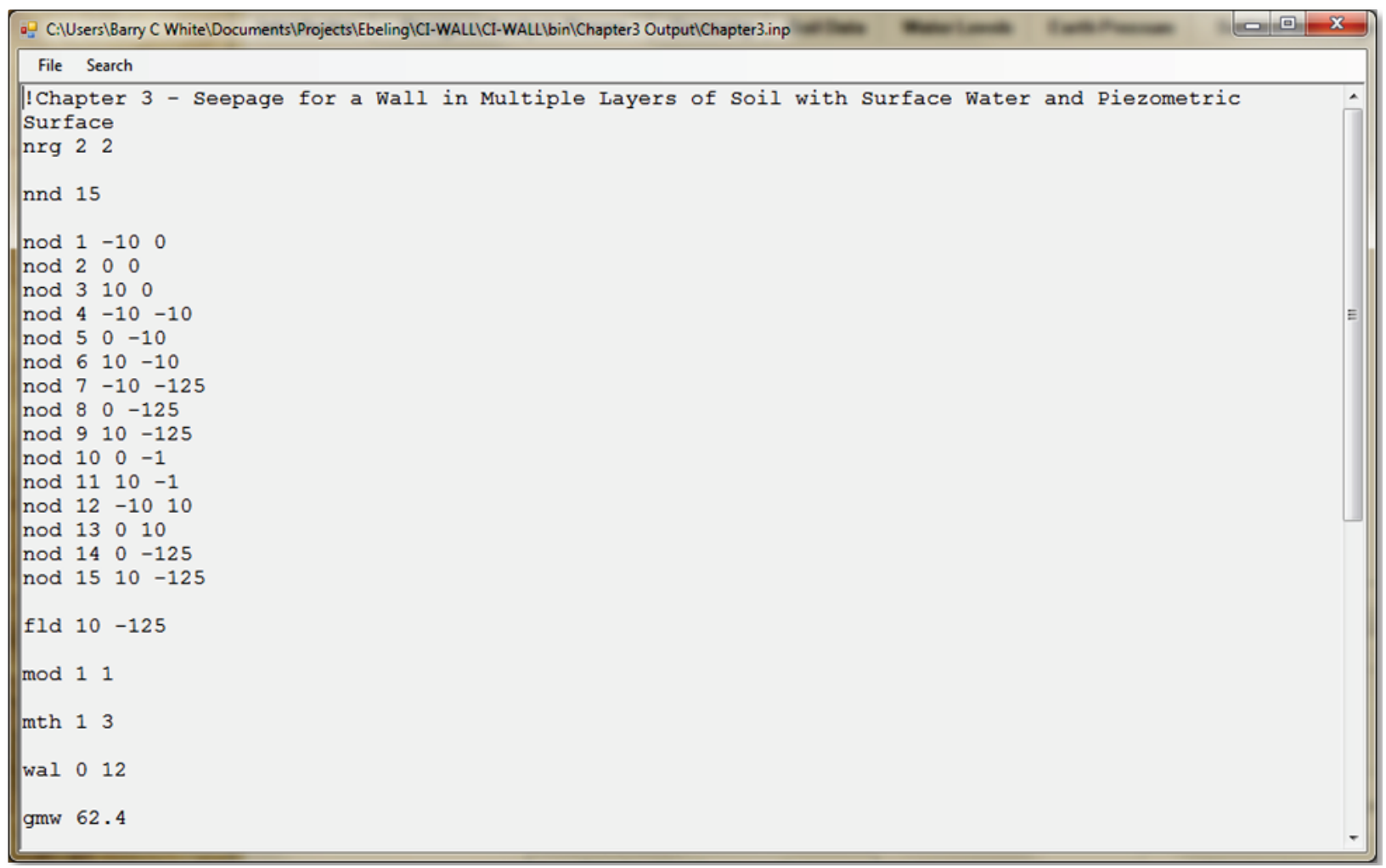

Figure 3.56. The output plot subsection allows the user to see data based on a flood elevation.

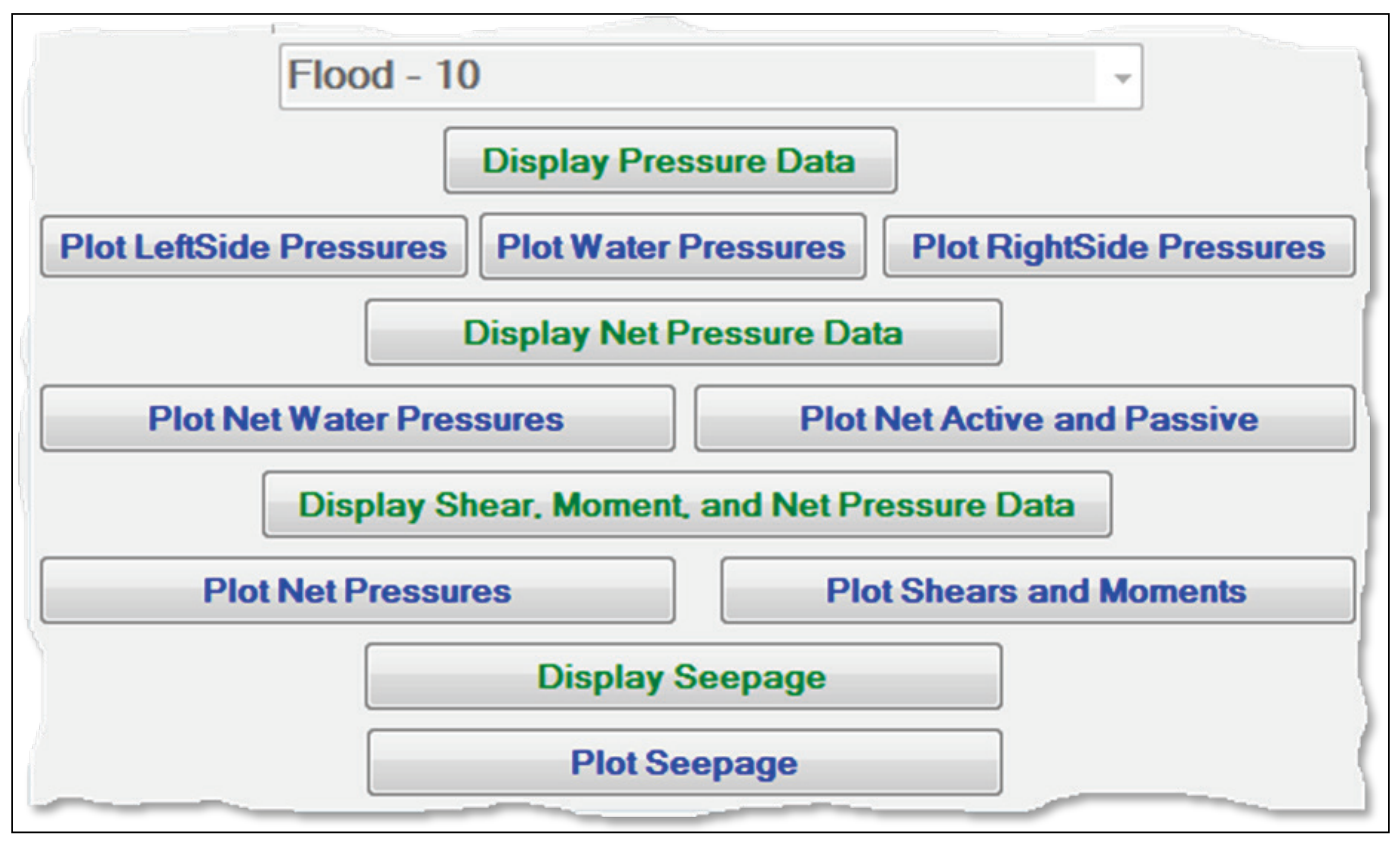


Figure 3.57. Pressure data for both the left and right sides of the I-Wall.

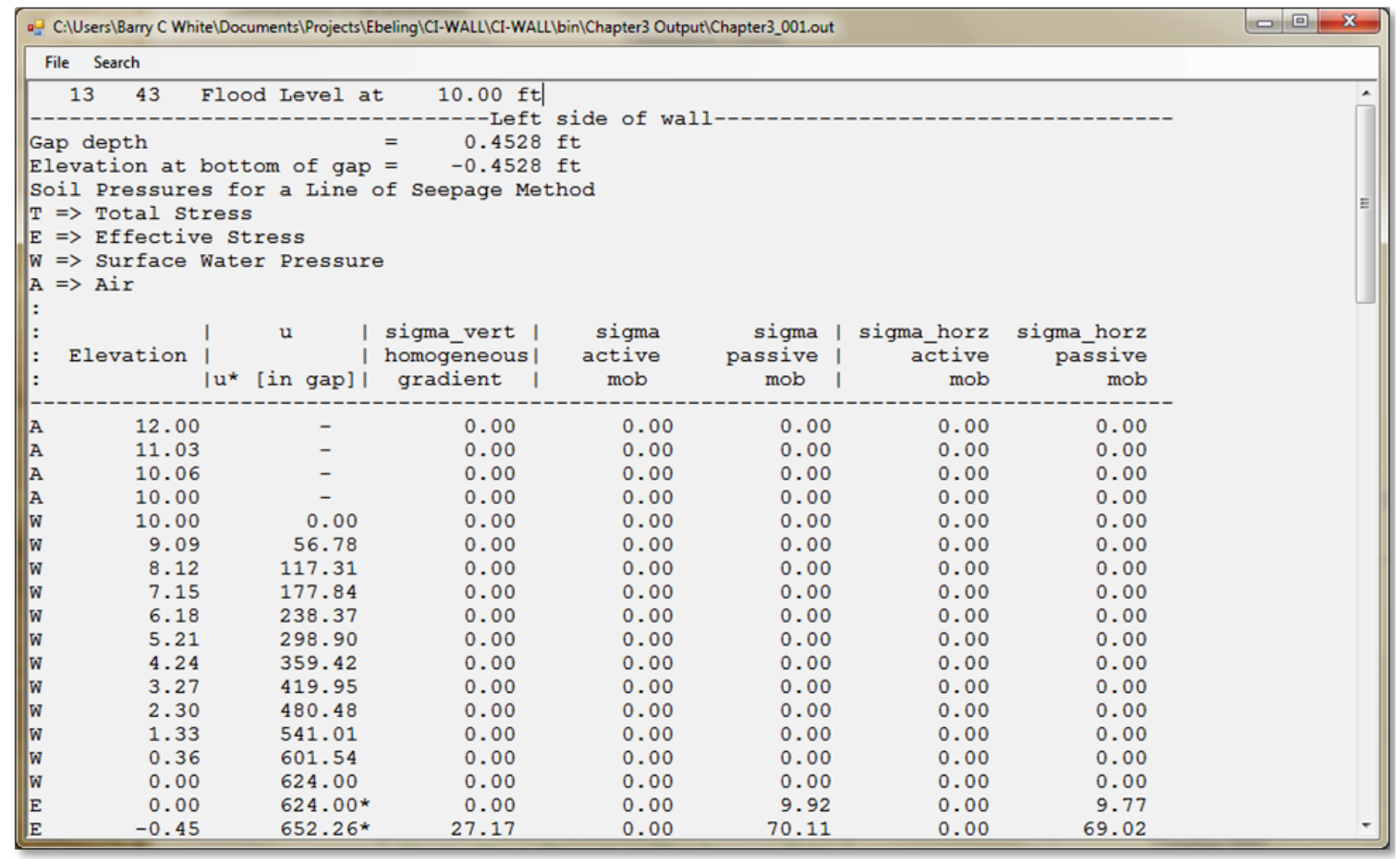

Each side of the I-Wall has a table presenting a series of elevations from the top of the I-Wall to the calculated tip of the I-Wall. A one letter tag (at the left of the table) tells how the I-Wall is loaded at each elevation. After the elevation data, data are presented for the pressure (u), the vertical homogeneous gradient, the active and passive mobilizing pressure, and the mobilized active and passive pressure acting in the horizontal direction (parallel to the ground surface).

Figures 3.58 through 3.60 show the plots of data from selecting the Plot Leftside Data, Plot Rightside Data, and Plot Water Pressures, respectively. The LHS (Figure 3.58) and RHS (Figure 3.59) data displayed are the horizontal active and passive pressures in red and green, respectively. The heights of the regions for each side are presented with brick-red lines and a region number with the $R \#$ format. These plots go from the top of the I-Wall to the tip. When the user moves the cursor over the plot, the y-coordinate of the point is determined, and the relevant pressure is interpolated from the piecewise linear curve representing the pressure. These data are presented in a tooltip at the cursor location.

Figure 3.60 shows the water pressures based on the head and region materials for each side of the I-Wall. The data for Figures 3.58 through 3.60 are presented with the Display Pressure Data button presented above the Plot buttons. 
Figure 3.58. Left-side horizontal mobilized passive and active pressures.

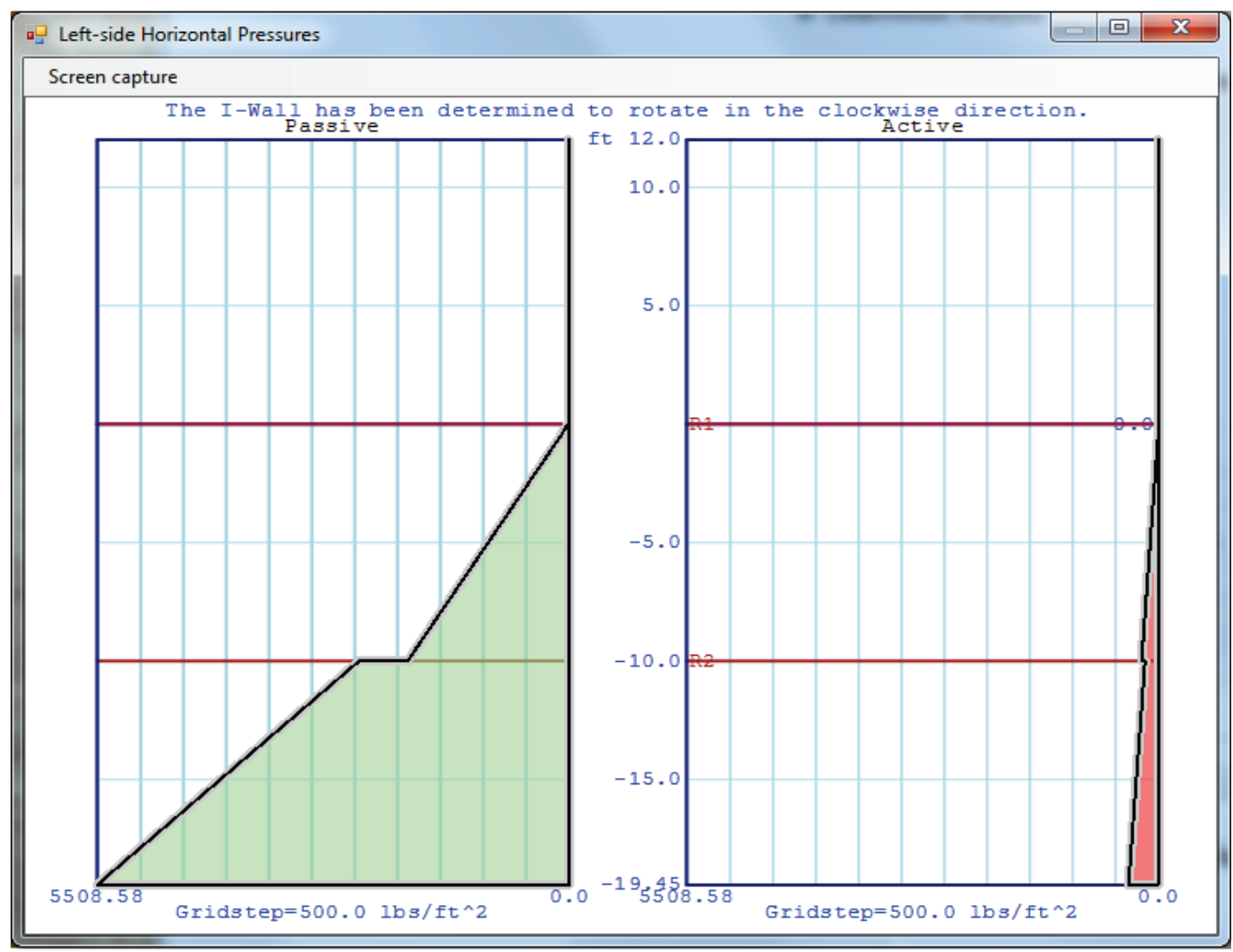

Figure 3.59. Right-side horizontal mobilized active and passive pressures.

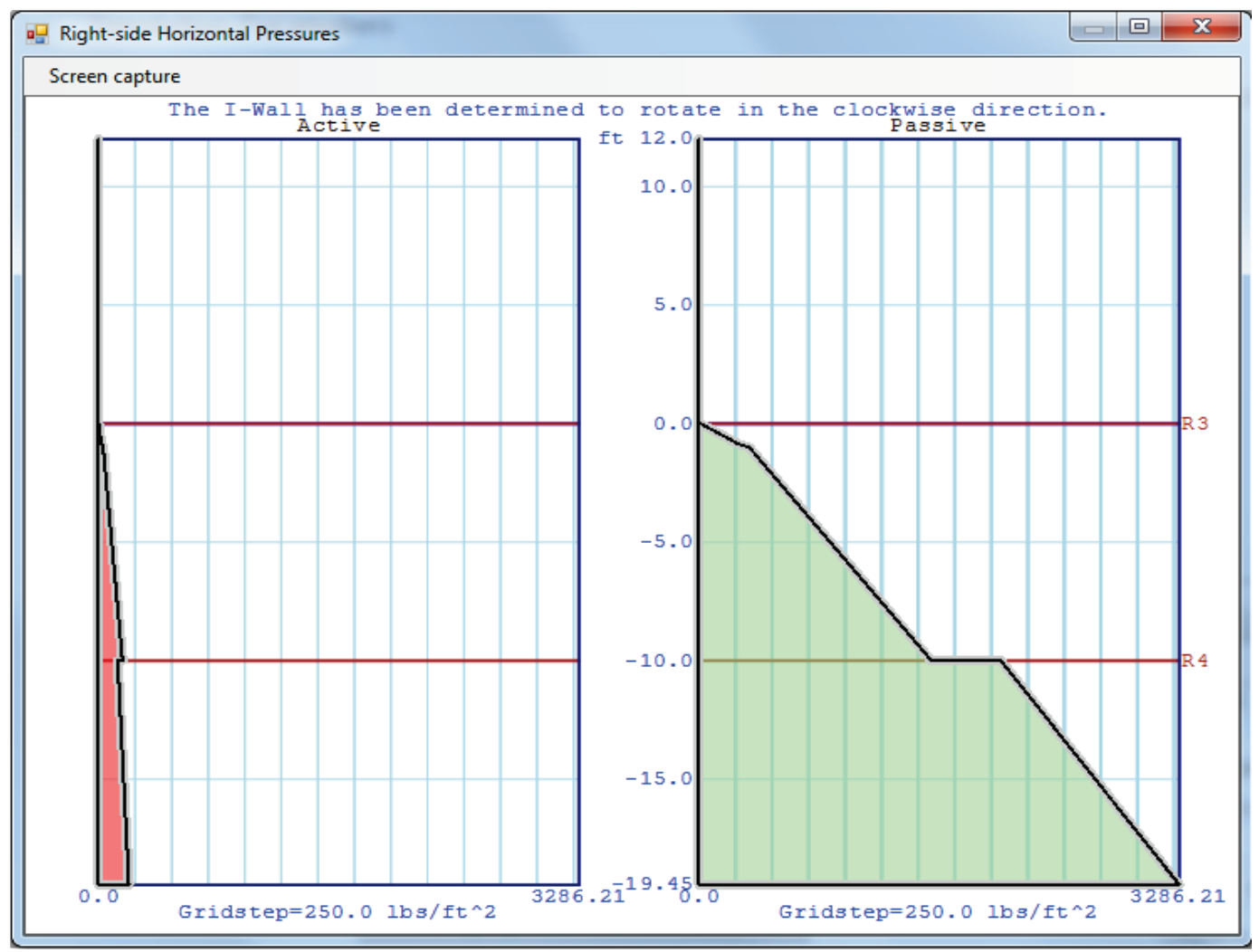


Figure 3.60. Left- and right-side water pressures.

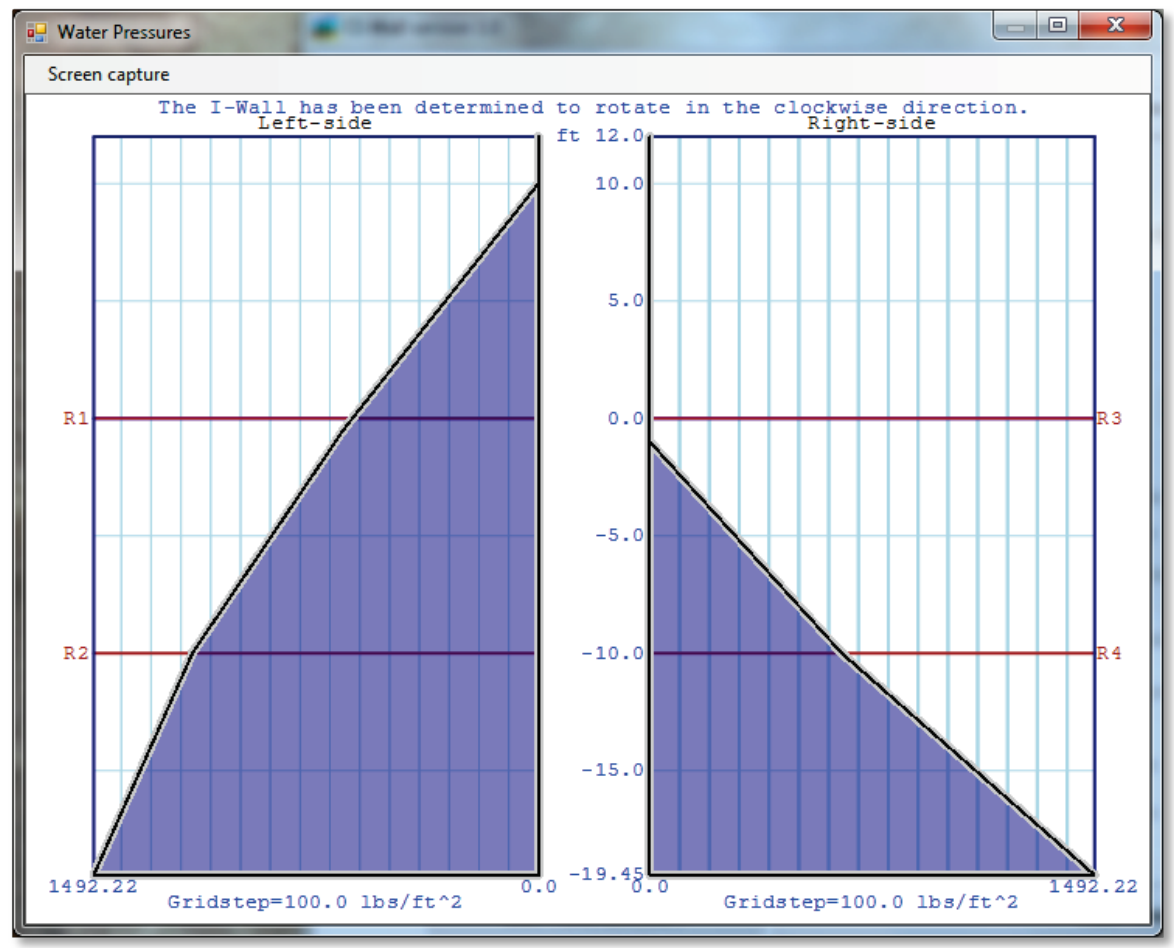

The Net Pressure Data combine the information from the water pressures plot with the display data. The data contain the I-Wall elevations and the summed water pressures and mobilized active and passive horizontal pressures. These net data are presented in summed form with the Display Net Pressures button (Figure 3.61), the Display Side Net Water Pressures (Figure 3.62), and plotted with the Plot Net Water Pressures button (Figure 3.63). Again, a tooltip tracks the location of the map and interpolates the piecewise linear net_u information.

Both the net active and passive pressures are listed in Figure 3.64 along with shear and moment values internal to the I-Wall.

Figures 3.65 and 3.66 show how the combined net pressure against the I-Wall from soil and water, shear, and moment along the I-Wall are presented to the user. The plots have the active tooltip interpolation for the piecewise linear curves. 
Figure 3.61. Displaying the net pressure data.

\begin{tabular}{|c|c|c|c|}
\hline \multicolumn{4}{|c|}{ 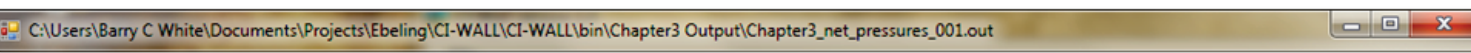 } \\
\hline File Search & & & \\
\hline 143 & Flood Level at & $10.00 \mathrm{ft}$ & A \\
\hline wall_ele & net_u net_a & net_p & \\
\hline $1 \overline{2} .00$ & $0 . \overline{0} 0$ & $0 . \overline{0} 0$ & \\
\hline 11.03 & 0.00 & 0.00 & \\
\hline 10.06 & 0.00 & 0.00 & \\
\hline 10.00 & 0.00 & 0.00 & \\
\hline 10.00 & 0.00 & 0.00 & \\
\hline 9.09 & 56.78 & 56.78 & \\
\hline 8.12 & 117.31 & 117.31 & \\
\hline 7.15 & 177.84 & 177.84 & \\
\hline 6.18 & 238.37 & 238.37 & \\
\hline 5.21 & 298.90 & 298.90 & $\equiv$ \\
\hline 4.24 & 359.42 & 359.42 & \\
\hline 3.27 & 419.95 & 419.95 & \\
\hline 2.30 & 480.48 & 480.48 & \\
\hline 1.33 & 541.01 & 541.01 & \\
\hline 0.36 & 601.54 & 601.54 & \\
\hline 0.00 & 624.00 & 624.00 & \\
\hline 0.00 & 624.00 & 633.77 & \\
\hline-0.45 & 652.26 & 712.07 & \\
\hline-0.61 & 660.78 & 738.92 & \\
\hline-1.00 & 681.93 & 801.95 & \\
\hline-1.58 & 672.46 & 869.93 & \\
\hline-2.55 & 656.61 & 983.64 & \\
\hline-3.52 & 640.77 & 1097.34 & \\
\hline-4.49 & 624.93 & 1211.04 & \\
\hline-5.46 & 609.08 & 1324.74 & \\
\hline-6.43 & -175.94 & 1438.45 & \\
\hline-7.40 & -282.16 & 1552.15 & \\
\hline-8.37 & -388.39 & 1665.85 & \\
\hline-9.34 & -494.62 & 1779.56 & \\
\hline-10.00 & -566.90 & 1856.92 & - \\
\hline
\end{tabular}

Figure 3.62. Active and passive net pressures.

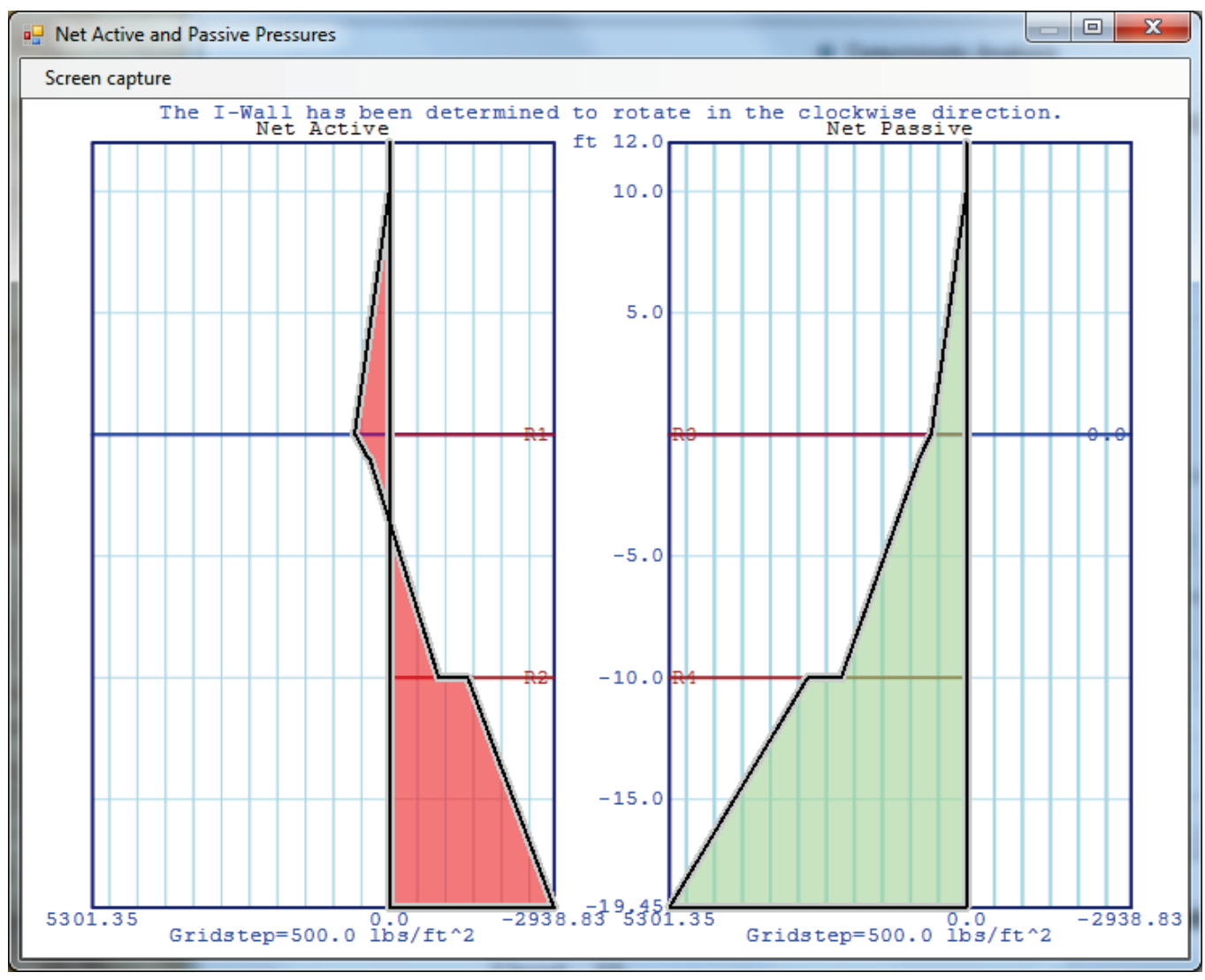


Figure 3.63. Displaying the net water pressures.

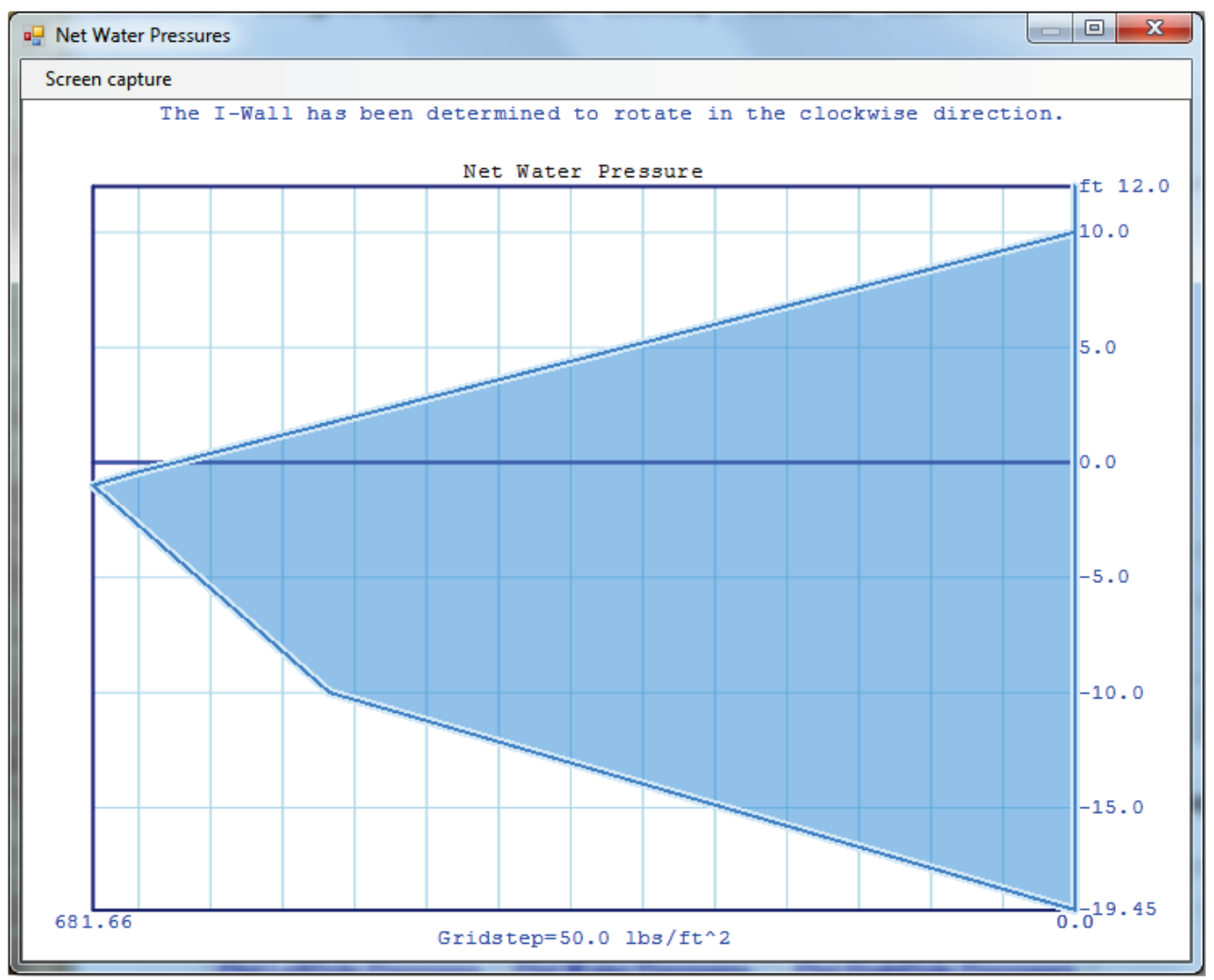

Figure 3.64. Shear, moment, and combined net pressures.

\begin{tabular}{|c|c|c|c|c|}
\hline \multicolumn{4}{|c|}{ 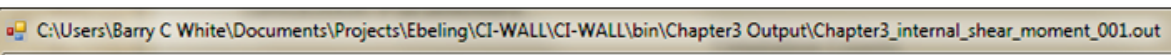 } & \multirow[t]{2}{*}{\begin{tabular}{|l|l|l|}
0 & 0 & $x$ \\
\end{tabular}} \\
\hline Search & & & & \\
\hline 144 & Flood Level at & $10.00 \mathrm{ft}$ & & 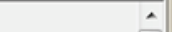 \\
\hline Elevation & Shear & Moment & Net Pressure & \\
\hline 12.00 & 0.000000 & 0.000000 & 0.000000 & \\
\hline 11.03 & 0.000000 & 0.000000 & 0.000000 & \\
\hline 10.06 & 0.000000 & 0.000000 & 0.000000 & \\
\hline 10.00 & 0.000000 & 0.000000 & 0.000000 & \\
\hline 9.09 & 25.836720 & 7.837138 & 56.784000 & \\
\hline 8.12 & 110.273280 & 69.104589 & 117.312000 & \\
\hline 7.15 & 253.422000 & 240.750900 & 177.840000 & \\
\hline 6.18 & 455.282880 & 579.726867 & 238.368000 & \\
\hline 5.21 & 715.855920 & 1142.983286 & 298.896000 & $E$ \\
\hline 4.24 & 1035.141120 & 1987.470950 & 359.424000 & $\equiv$ \\
\hline 3.27 & 1413.138480 & 3170.140657 & 419.952000 & \\
\hline 2.30 & 1849.848000 & 4747.943200 & 480.480000 & \\
\hline 1.33 & 2345.269680 & 6777.829375 & 541.008000 & \\
\hline 0.36 & 2899.403520 & 9316.749978 & 601.536000 & \\
\hline 0.00 & 3120.000000 & 10400.000000 & 624.000000 & \\
\hline-0.45 & 3382.164806 & 11873.760905 & 533.869055 & \\
\hline-0.61 & 3464.225089 & 12411.797925 & 510.421708 & \\
\hline-1.00 & 3645.408887 & 13799.338794 & 418.725973 & \\
\hline-1.58 & 3869.849662 & 15980.544401 & 355.207735 & \\
\hline-2.55 & 4162.880209 & 19884.747610 & 248.978959 & \\
\hline-3.52 & 4352.868843 & 24023.215121 & 142.750183 & \\
\hline-4.49 & 4439.815564 & 28295.996280 & 36.521407 & \\
\hline-5.46 & 4423.720373 & 32603.140431 & -69.707369 & \\
\hline-5.46 & 4423.720373 & 32603.140431 & -69.707369 & \\
\hline-6.43 & 4304.583269 & 36844.696919 & -175.936145 & \\
\hline-7.40 & 4082.404252 & 40920.715087 & -282.164921 & \\
\hline-8.37 & 3757.183322 & 44731.244282 & -388.393697 & \\
\hline-9.34 & 3328.920480 & 48176.333848 & -494.622473 & \\
\hline-10.00 & 2978.617454 & 50260.445107 & -566.901846 & \\
\hline-10.00 & 2978.617454 & 50260.445107 & -1410.162110 & - \\
\hline
\end{tabular}


Figure 3.65. Combined net pressures with soil and water pressures.

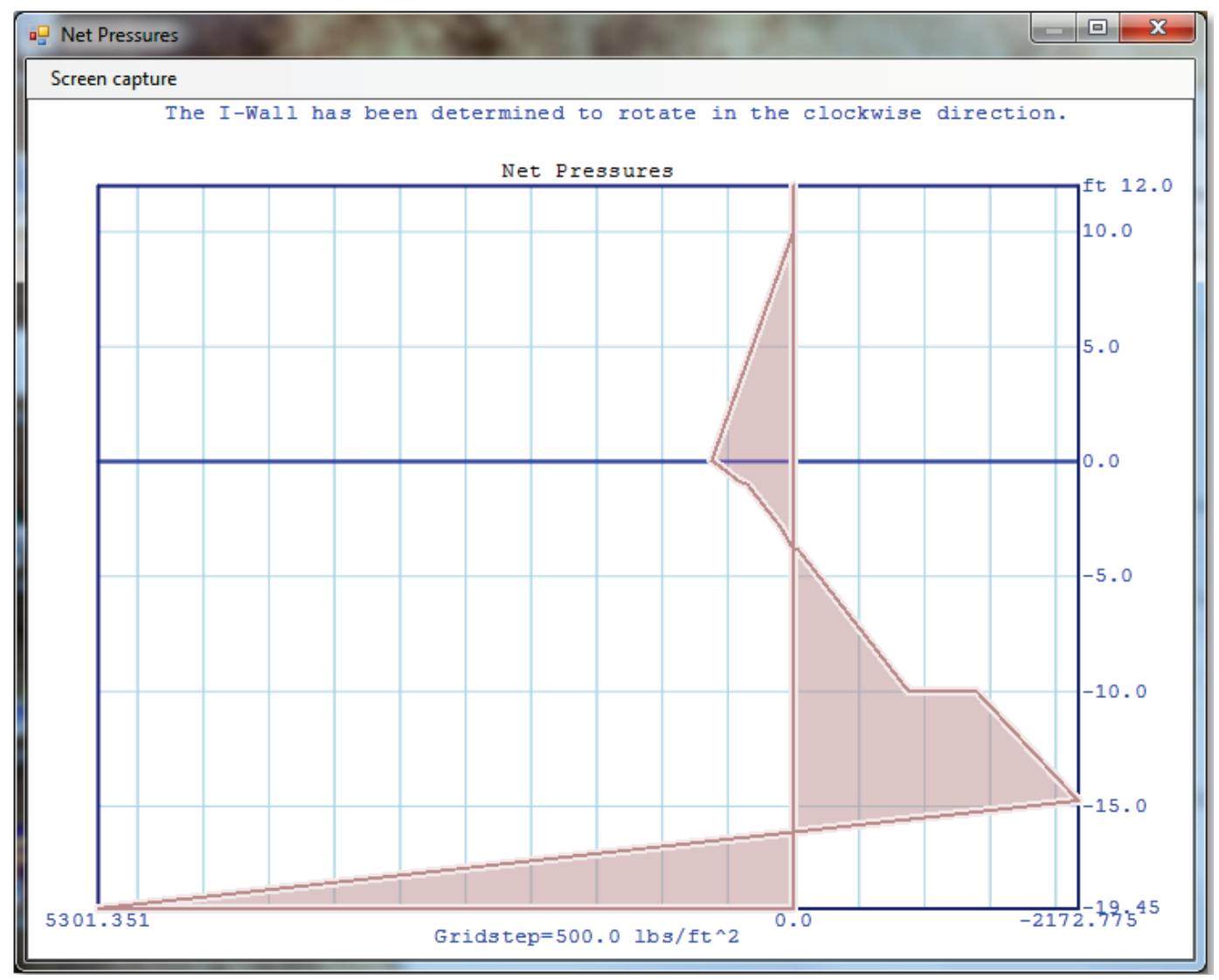

Figure 3.66. Shear and moment diagrams for the I-Wall.

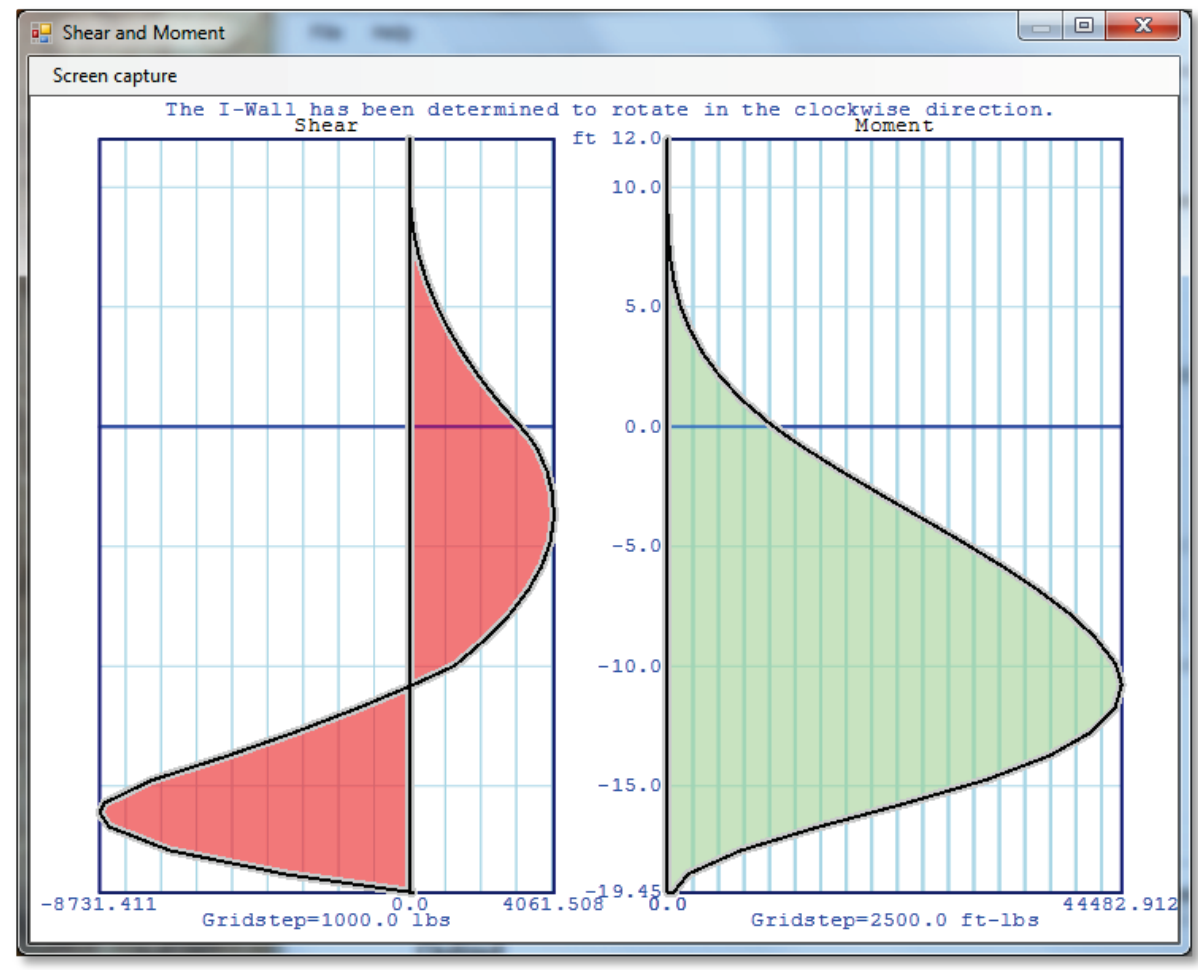


The last two buttons in the Output section are the Display Seepage and Plot Seepage buttons. The Display Seepage button opens a window as shown in Figure 3.67. In the header of the file, after numbers that aid the reading of the data, is the information for the seepage analysis. This information includes: the units for elevation, the ground surface elevations for the LHS and RHS of the structure, the water surface elevations for the LHS and RHS of the structure, the side and elevation for the start of seepage, the side and elevation for the point where seepage stops, the constant seepage gradient (for transformed coordinates), the pile tip elevation, and the gap depth (on the side where seepage starts) and elevation.

Figure 3.67. Display of seepage data for both the left and right sides of the I-Wall.

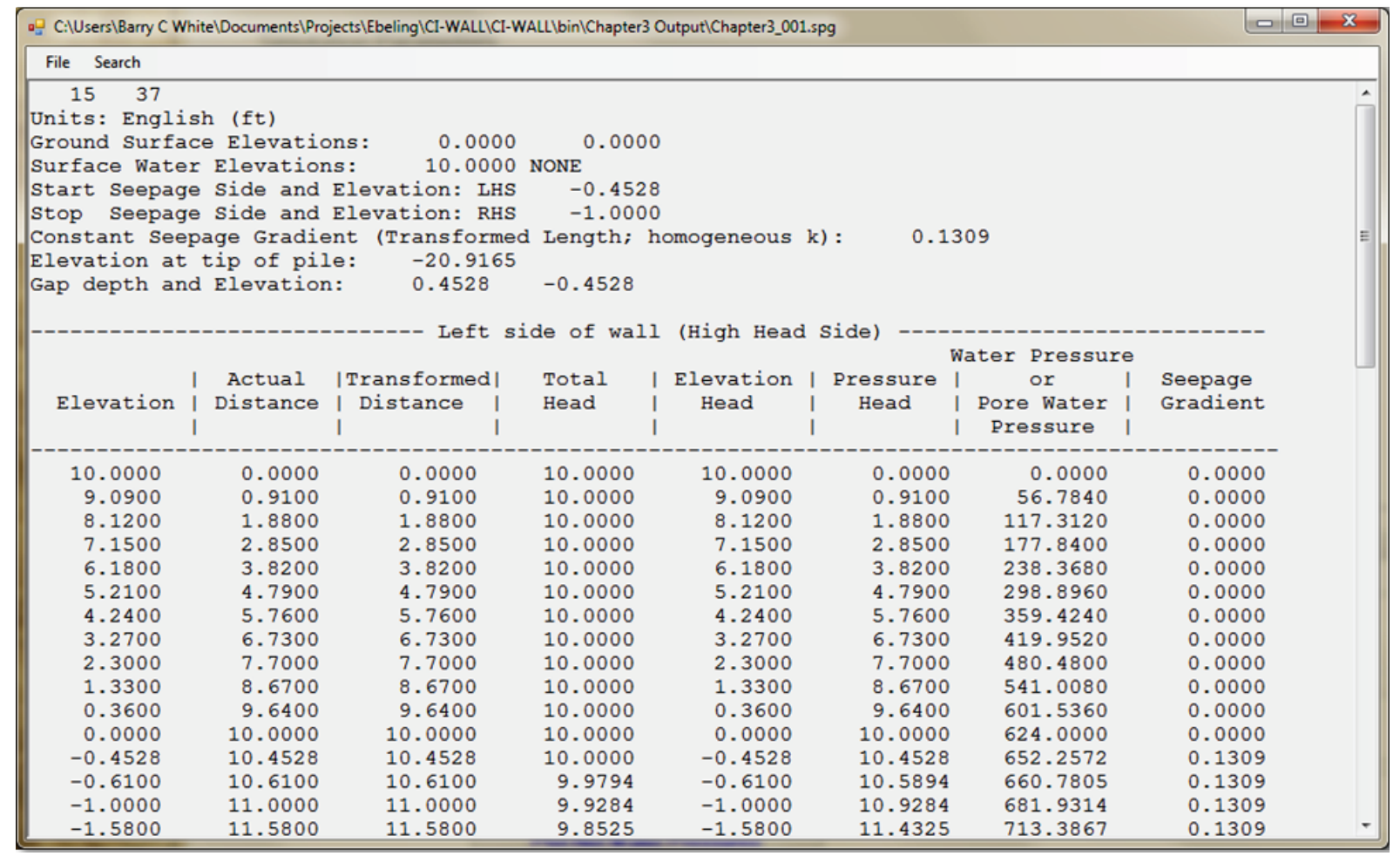

The constant seepage gradient is explained in Appendix C, but a brief description is as follows. For homogeneous soils, the head loss due to seepage is linear along the line of seepage (from the high head top of soil or gap depth to the pile tip and then back up to the low head soil level or piezometric surface level). This line has a constant gradient. A set of heterogeneous soils will have different permeabilities, and therefore the gradient will vary although the head values at the start and end of seepage will remain the same. In order to model this behavior linearly, a transformed space with distances based on the permeabilities of the soil regions is created. Thus, a single line will represent the change in head 
from the start-to-end seepage region, reducing the problem to a single constant gradient in the transformed space.

After the header information, tables are presented for each side of the I-Wall. This table has data points at elevations from the top of the water surface on that side of the I-Wall to the pile tip. The columns have information about distance for the line of seepage (both actual distance and transformed distance according to soil region properties), the heads (total, elevation, and pressure) that are acting on the system, the pore water pressures acting on the structure, and the actual seepage gradient based on soil region properties.

Figure 3.68 is the initial plot shown by Plot Seepage button. This plot has five main areas: the line of seepage plot, the header information, the plot type indicator, the plot, and the axes for the plot. The line of seepage plot is in the white area to the left of the window. This plot provides a scaled overview of the I-Wall and how seepage occurs. On this plot are surface waters (if they exist), piezometric water surfaces, soil region information, the bottom of the gap, the pile tip, and the line of seepage with direction arrows.

The header information is a repeat of the header information from the file.

The plot type indicator reveals what information is being displayed (as discussed later) and whether the plot is in transformed space or not.

The plot is a simple linear function plot, showing either head information (Total, Elevation, or Pressure), pore water pressures, or the seepage gradient.

The axes at the bottom of the plot show (1) either the actual or transformed distances, and (2) the positions of points of interest along the structure (surface water, piezometric surface, region tops, gap location, and pile tip location).

Figures 3.69 and 3.70 show the menu options for selecting the data to display on the vertical axis and horizontal axis, respectively.

Figure 3.71 shows the result of plotting the total head in transformed coordinates for heterogeneous soil regions. As expected, the seepage occurs along a single line with a constant gradient. 
Figure 3.68. Seepage plot of total head loss in actual (untransformed) space.

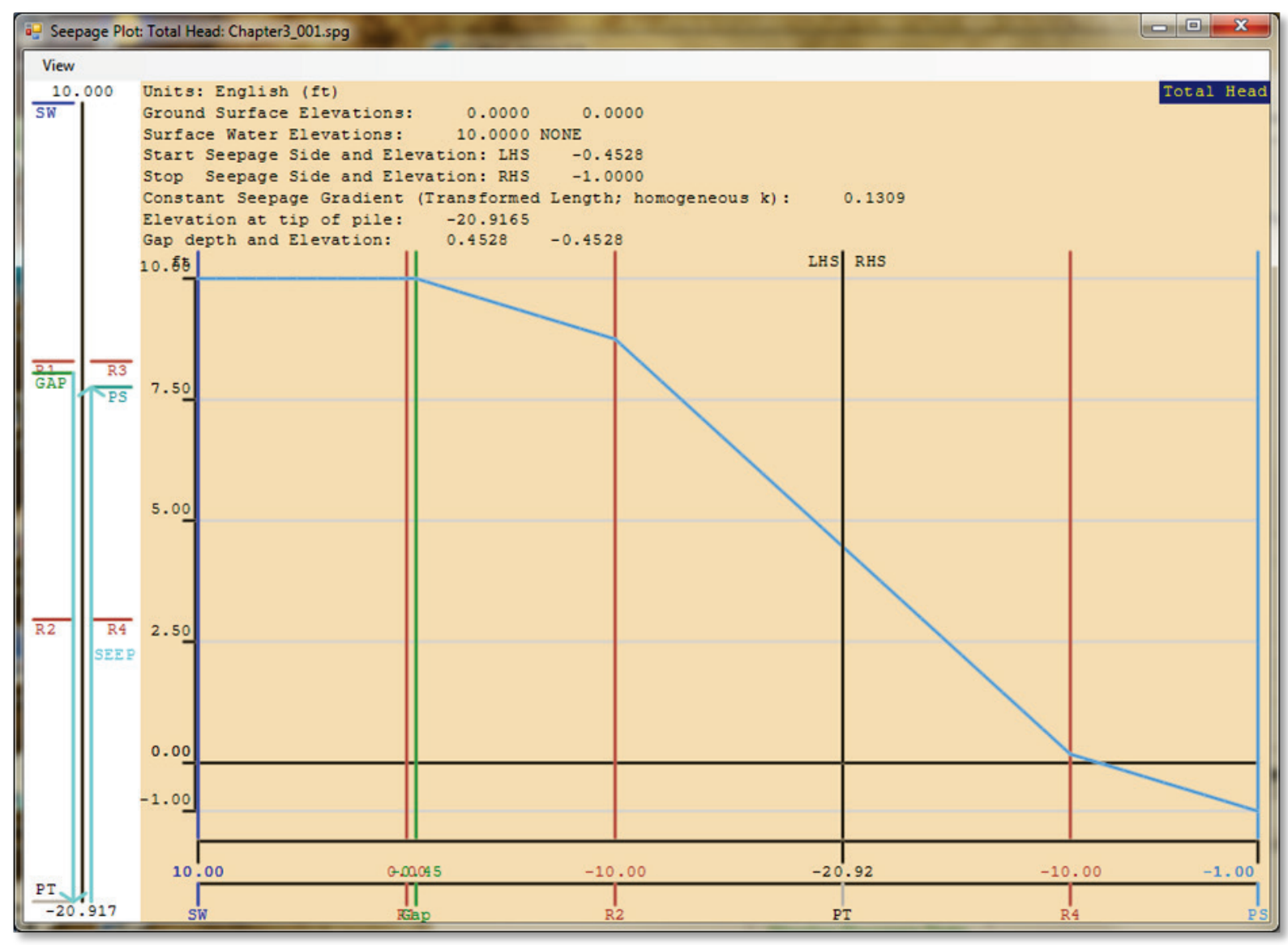

Figure 3.69. Seepage plot items to display.

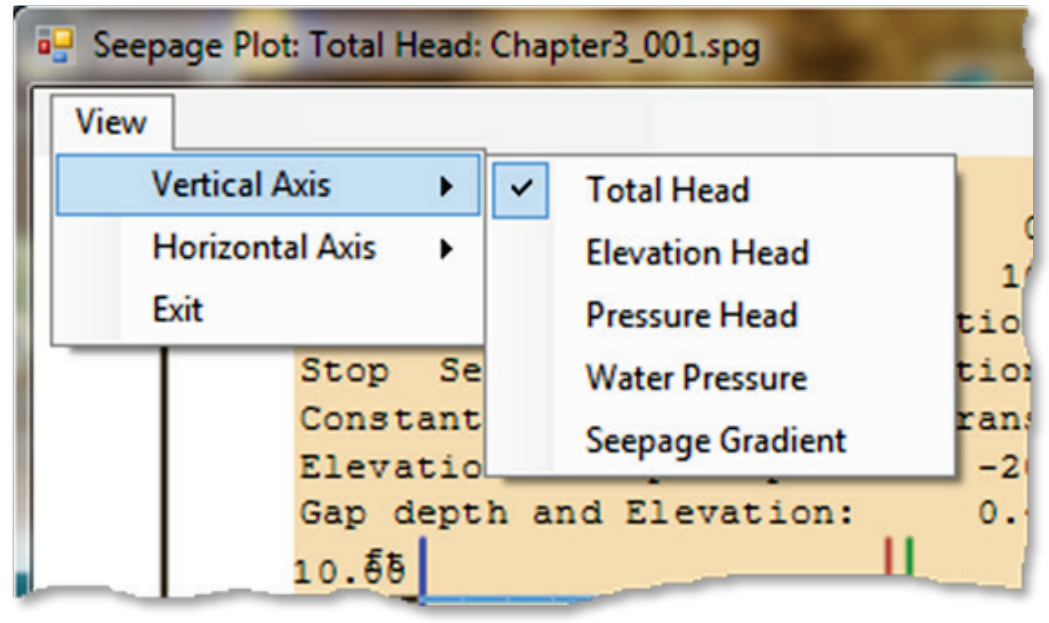


Figure 3.70. Seepage plots selector for untransformed or transformed space.

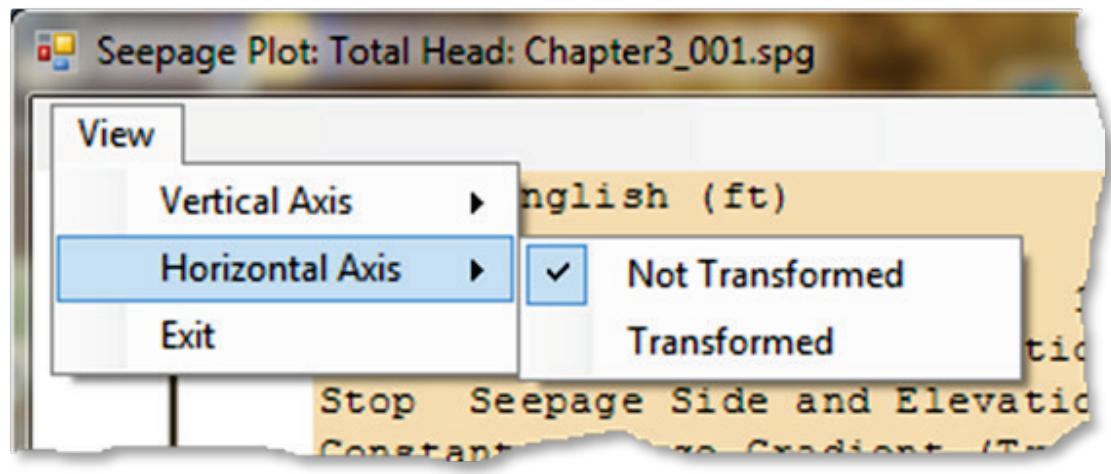

Figure 3.71. Seepage plot of total head loss in transformed space.

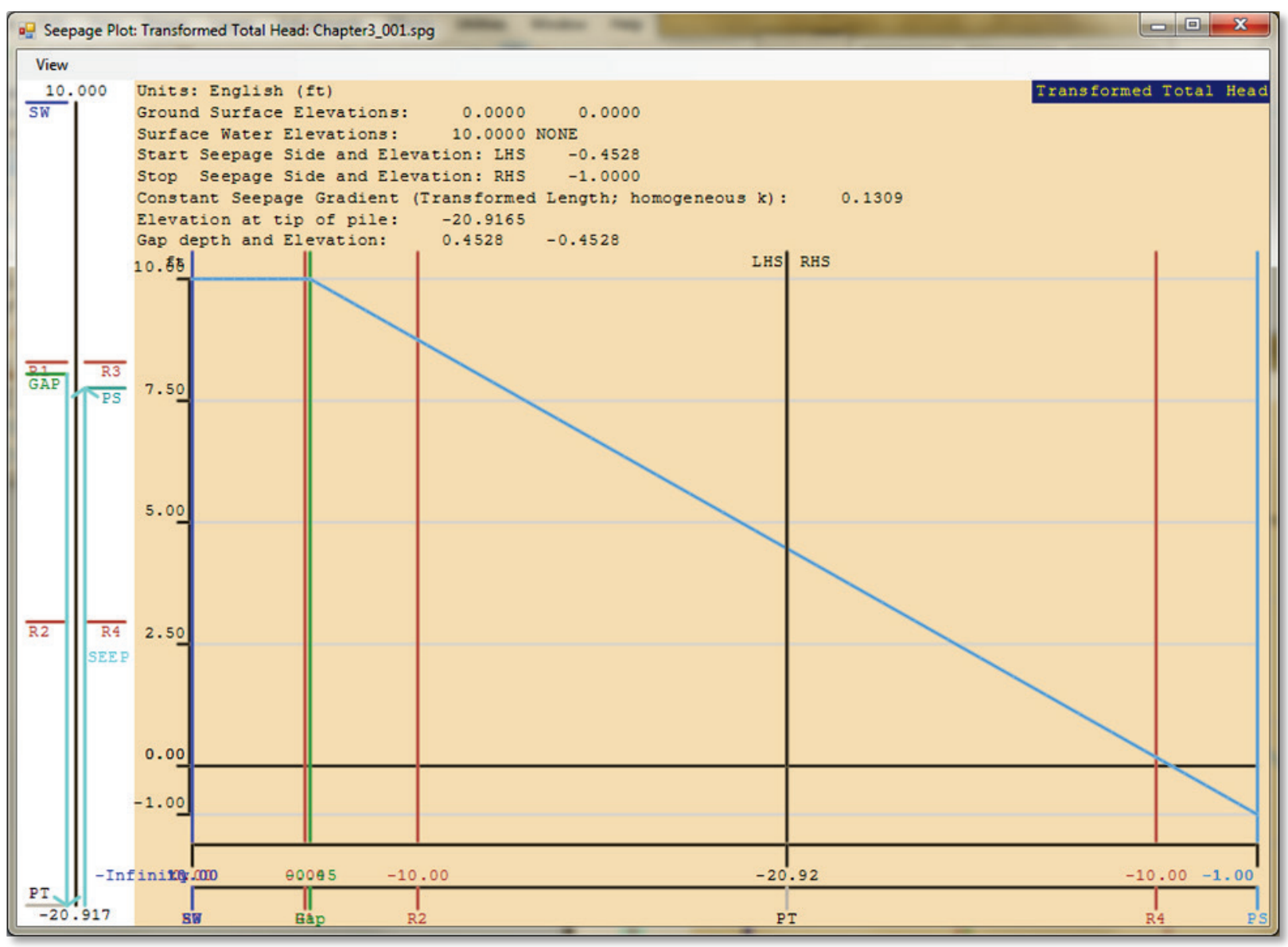

Figure 3.72 shows the result of displaying the seepage gradient data across the structure. The gradients are constant across a soil region. These are the actual gradients formed by taking the constant gradient from the transformed space back into the untransformed space. 
Figure 3.72. Seepage plot of seepage gradient due to soil region properties (untransformed).

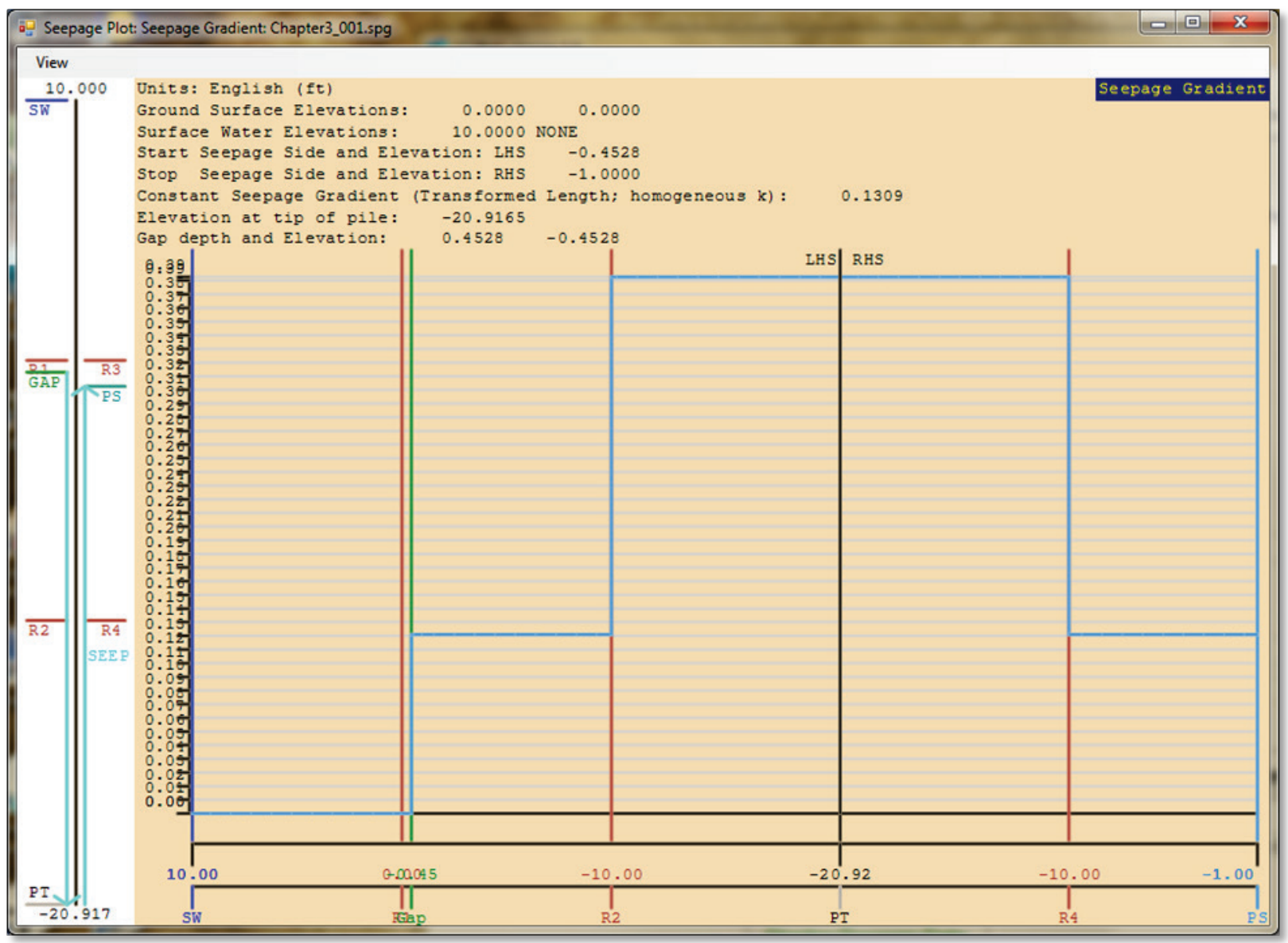

Of interest for this chapter are the correlations between variables. In this case, the internal friction angle and the soil-to-sheet-pile (wall) friction angles have a correlation coefficient of 0.7. This correlation is the same for both materials, although only one material is shown in Figure 3.73.

The intra-material correlations are also shown at the bottom right of Figure 3.73. The Internal Friction Angle between material 1 and material 2 has a correlation coefficient of 0.6 , as does the soil-to-sheet-pile Friction Angle between material 1 and material 2.

These inputs can lead to contradictory solutions when probabilistic inputs are created because when a variable is correlated to more than one other variable, it may not be possible to maintain the correlation factors with both other variables. For this example, the material 1 Internal Friction Angle correlated with the material 1 soil-to-sheet-pile (wall) Friction Angle using a correlation coefficient of 0.7. The material 1 Internal Friction Angle is also correlated with the material 2 Internal Friction Angle using a correlation 
coefficient of 0.6. These constraints may be contradictory and lead to a reformulation of the correlation matrix to get the correlation factors as close as possible. The user is warned when this instance may occur.

Figure 3.74 shows the probabilistic input for variations of water levels for the Analysis option run. The varying water levels for Example 5.2 from Chapter 5 allow the analysis to determine a safe penetration depth for the I-Wall to withstand the changing I-Wall pressures due to changes of water elevation. In this example, the water levels on the flood side range from an elevation of $15 \mathrm{ft}$ to an elevation of $4 \mathrm{ft}$, while the water elevation on the landside is fixed at an elevation is $\mathrm{oft}$.

Figure 3.73. Defining internal and external material correlations for probabilistic analysis for Example 5.2 input.

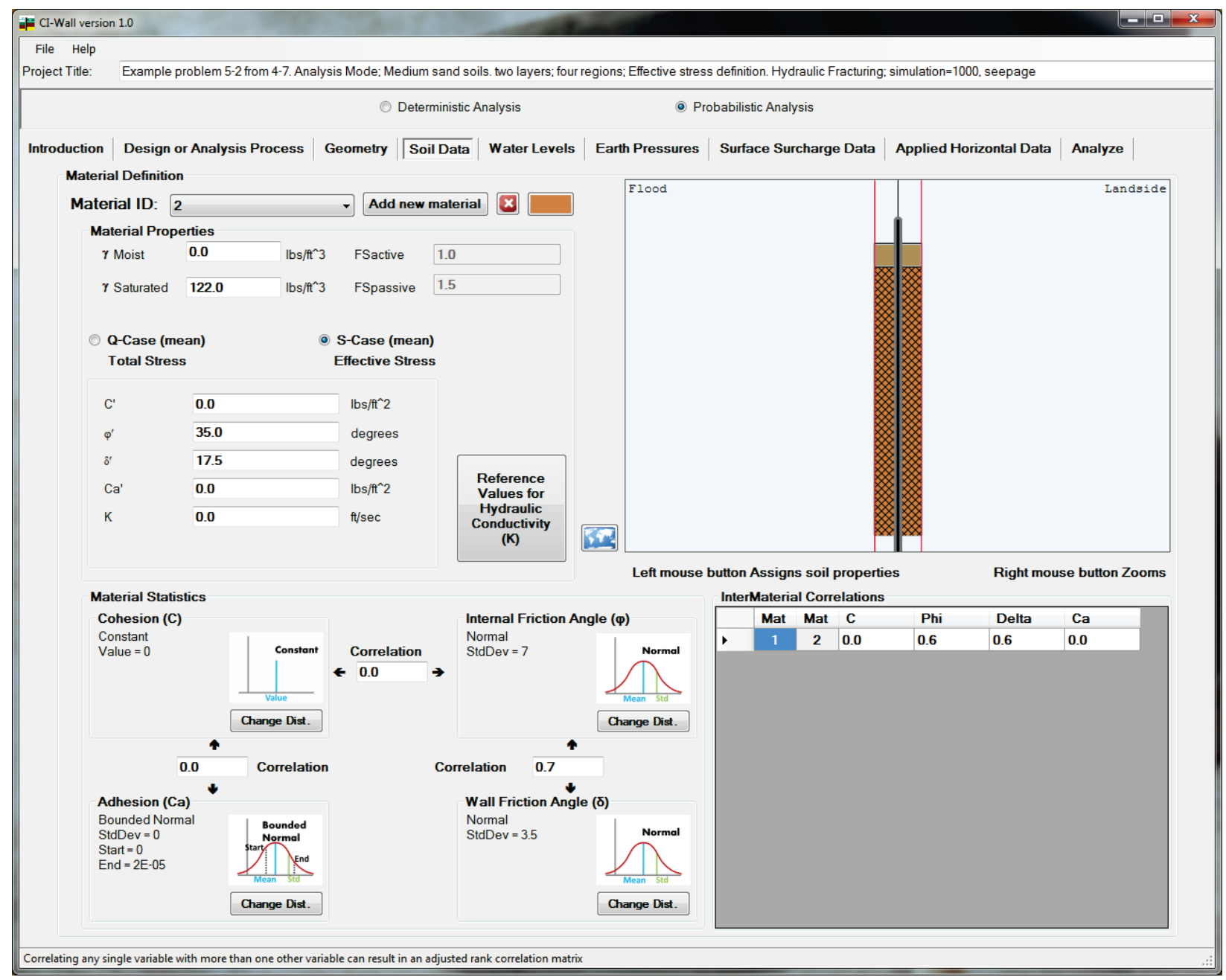


Figure 3.74. Probabilistic analysis options for the Analysis option run for Example 5.2 from Chapter 5.

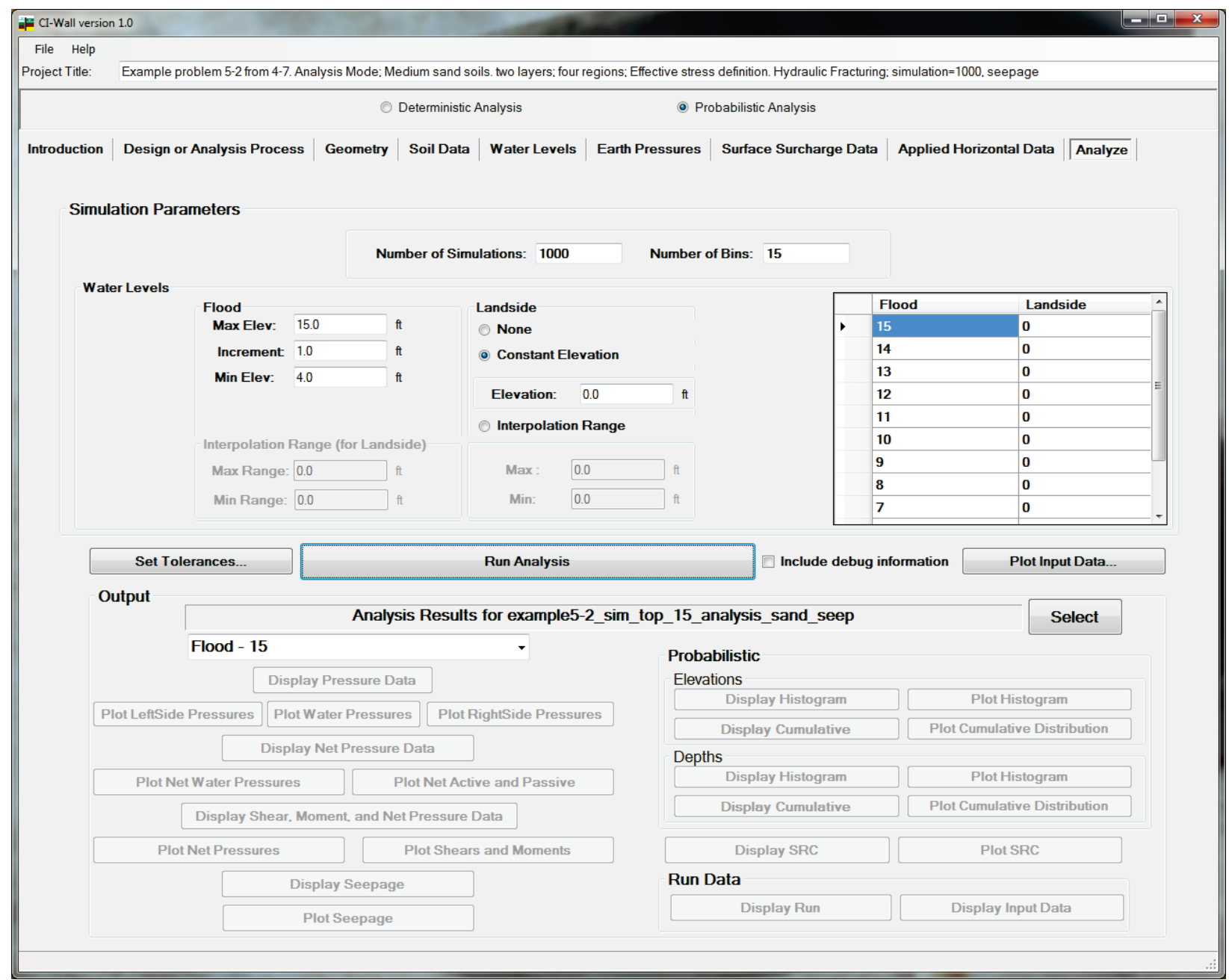

The probabilistic analysis will consist of 1,000 simulations at each set of water elevations, and the output will be divided into 15 bins. The greater the number of simulations, the more accurate the results will be, but the analysis will take longer to perform. Performance times are presented in Chapter 5 and range from minutes to hours. Chapter 5 also provides a strategy for performing simulations to reduce the amount of run-time involved. The greater the number of bins, the more precision can be gathered at each level of the output. If the number of bins is increased, the number of simulations should also be increased because each bin is susceptible to noise in the generated input.

If multiple correlations exist between variables (as is the case with the Example 5.2 problem of Chapter 5), clicking the Run Analysis button will cause the dialog box of Figure 3.75 to appear. This dialog is a warning that the correlations specified by the user may not be maintained as the program 
is run. If the user is content to let the software alter the correlation matrix between the variables, clicking the $O K$ button will allow the probabilistic analysis to continue. By clicking the Cancel button, the user can go back to the input and vary the correlations to remove the likelihood of contradictions between correlations.

Figure 3.75. Warning that the correlation matrix between variables may change.

\begin{tabular}{|l|l|}
\hline Adjusting Rank Correlation & \\
\hline \\
There are material variables that have multiple correlations. This will \\
result in an adjusted rank correlation method that may not be intended!
\end{tabular}

If the user continues to run the analysis after receiving the warning of Figure 3.75, then it is possible that the error dialog of Figure 3.76 will appear. When this error appears, then the Corps_I-Wall software was not able to adjust contradictory correlations in the correlation matrices to maintain a close set of correlations to what the user specified. In this case, the analysis is not performed, and the user will have to change one or more correlations in the soil material input window to reduce the contradictions between correlations.

Figure 3.76. Error dialog that occurs when the software altered correlations cannot match the user input correlations.

Problem with Rank Correlation

The adjusted rank correlation method was not able to sample parameters for the designated number of simulations.

Please re-specify the correlation coefficients between strength parameters within a soil as well as between different layers of soils. 
The probabilistic analysis output is dependent on whether a Design or Analysis mode problem is run. Figure 3.77 show the probabilistic output area of the Analyze tab after a Design mode probabilistic run has been performed. For a Design mode probabilistic run, probabilistic data are returned for the range of tip depths that are required at each flood elevation. The flood elevations are selected using the Flood selector box directly to the left of the word Probabilistic on the frame in Figure 3.77.

Figure 3.77. Active probabilistic output options in the Analyze tab after execution of a Design mode.

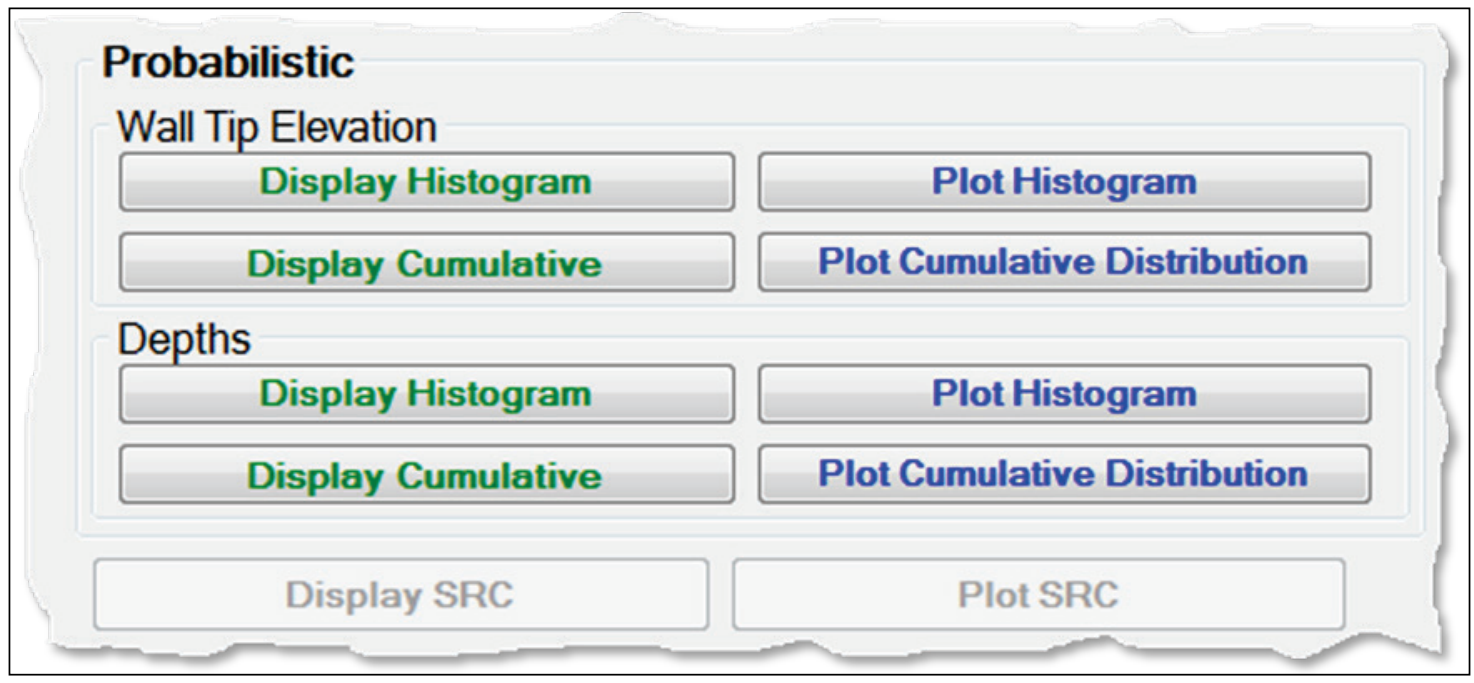

The output probabilistic data after the Design mode are reported using two datums: elevations of the I-Wall tip (based on user input) and depths below the lower ground surface of the I-Wall tip (on either the LHS or RHS of the I-Wall). Elevation data use the coordinate system established by the user, which may be based on a datum, such as mean sea level. These data are reported (histograms and cumulative distribution passive Factor of Safety data) for the number of bins specified over the range of simulations for the selected flood elevation.

The Display buttons for each of these options opens a dialog that displays the contents of the file containing the data to be viewed. This dialog has menu options to allow the user to save a copy of the file to a different location, to print the file, and to exit the dialog. The Plot buttons behave in a different fashion depending on whether a histogram is plotted or cumulative distributions are plotted, as discussed below.

Figures 3.78 and 3.79 provide the histogram data and a plot of the histogram data for I-Wall tip elevations, respectively. The histogram data have three columns: bin number, midpoint elevation of that bin, and 
probability that that I-Wall tip elevation will occur. Because the full range of possible I-Wall tip elevations is divided into the number of calculated sheetpile design depths contained within each of the bins, the summation of these probabilities will always be unity (1.0).

The histogram data are presented with the flood level elevation, the number of simulations, the minimum, maximum, and mean elevations generated by the Design mode analysis, as well as the standard deviation and coefficient of variation of elevations.

The bin with the greatest probability is bin number 12, which is centered about the I-Wall tip elevation of $-29.27 \mathrm{ft}$. The computed mean gives an elevation of $-31.51 \mathrm{ft}$, and the standard deviation is $5.9 \mathrm{ft}$.

Histogram data can either be shown as filled bins centered about a data point (elevation, in this case) or as a linear interpolation of the data through the midpoints of the bins. The Histogram plot dialog gives the user the means to choose either or both. Figure 3.79 shows the data as filled bins, Figure 3.80 reveals the data as a linear interpolation, and Figure 3.81 shows the data with both options. These options, and the option to exit the dialog, are available through the menu.

Figure 3.78. Displayed sheet-pile tip elevation histogram data after execution of a Design mode.

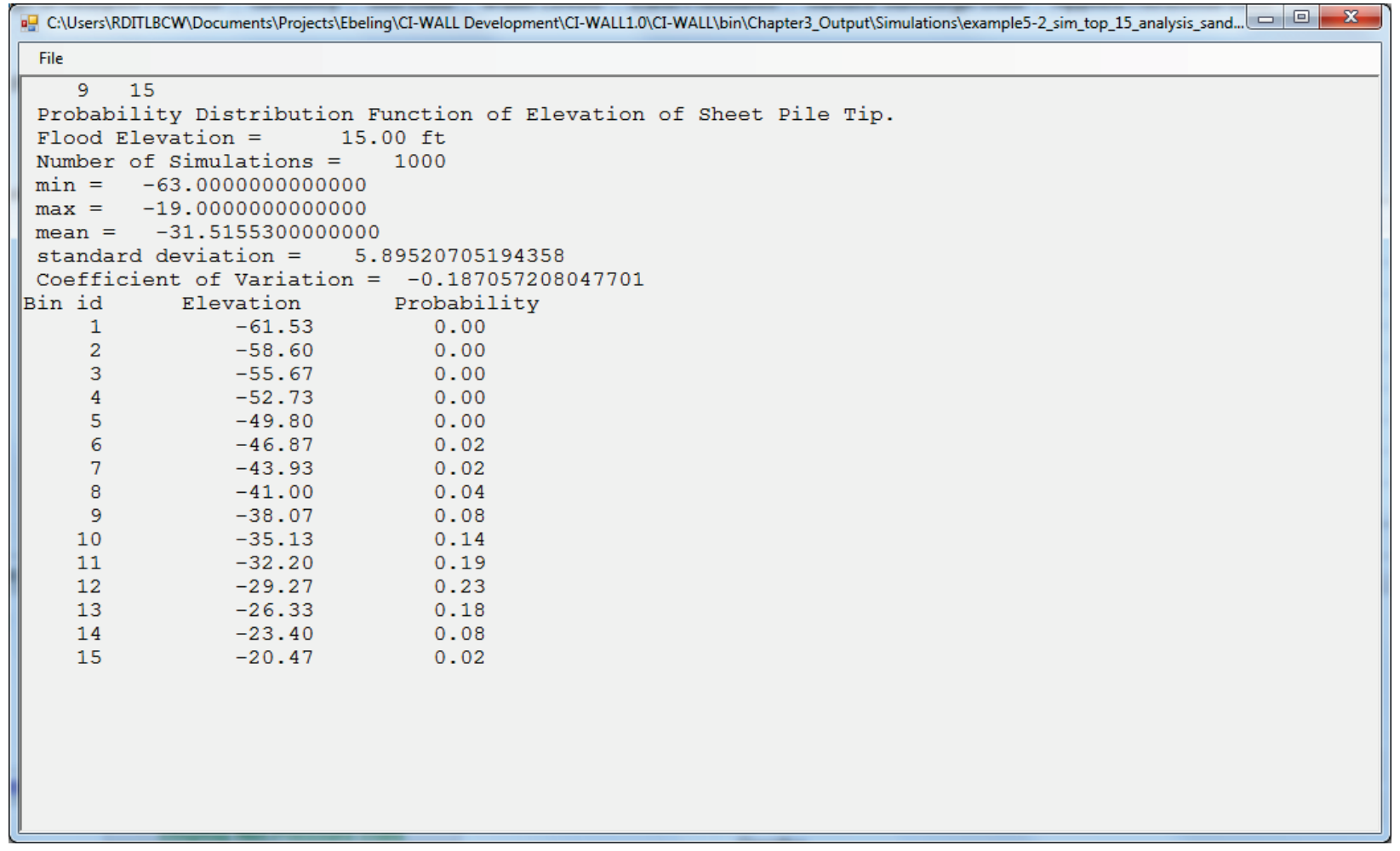


Figure 3.79. Plotted sheet-pile tip elevation histogram data after execution of a Design mode.

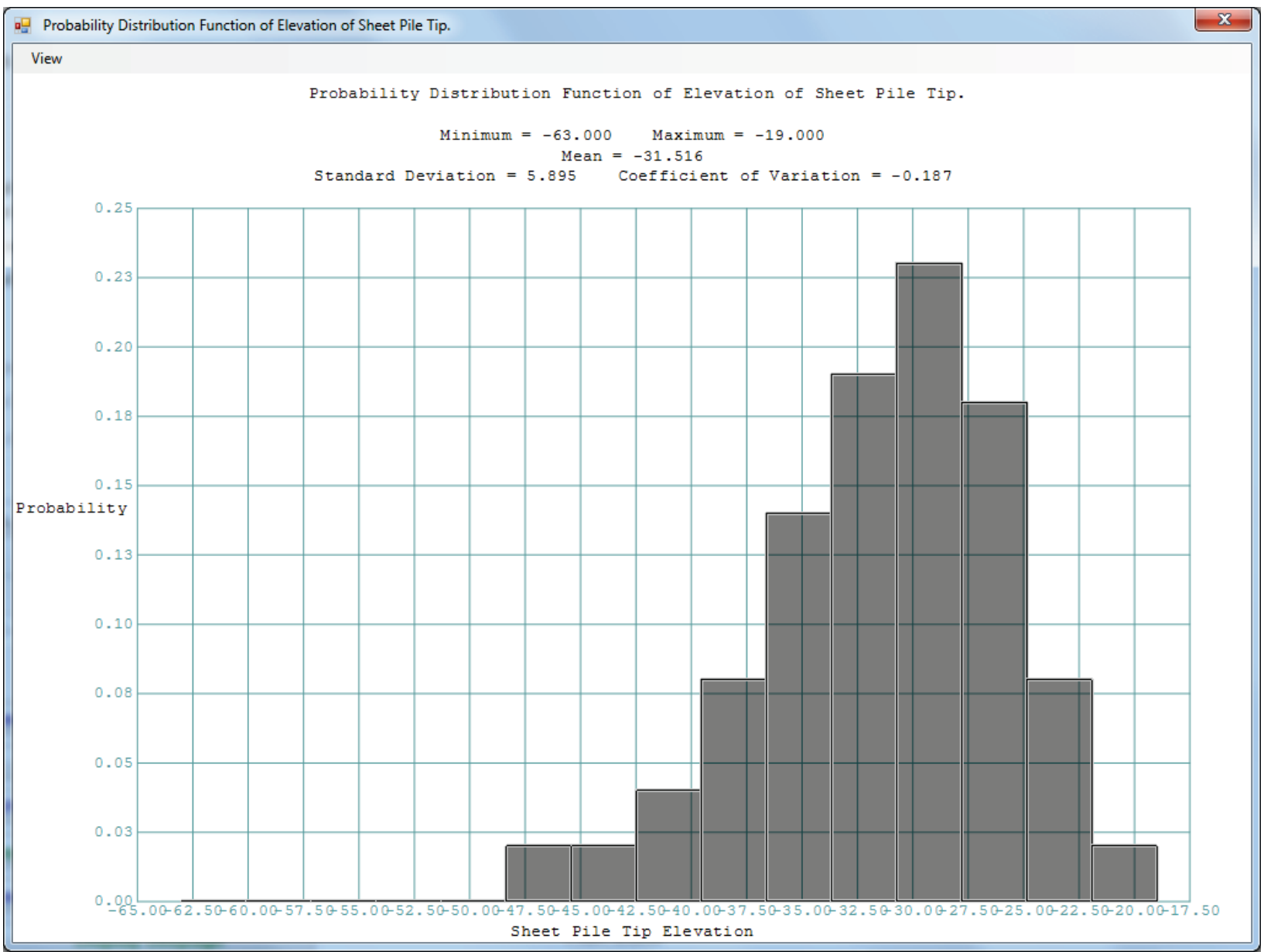

Figures 3.82 and 3.83 provide the cumulative distribution of elevation of sheet-pile tip data and a plot of the cumulative distribution data, respectively. In these figures, 1,000 simulation results for calculated tip elevation are gathered in 15 bins for a flood elevation of $15 \mathrm{ft}$, which is $15 \mathrm{ft}$ above the ground surface for this example.

The computed mean and standard deviation stay the same, as these data are just a cumulative summation of the histogram probabilities.

Because the cumulative distribution data are based on a summation of the bin probabilities, the data are best plotted as a linear interpolation of the data. Therefore, the dialog box has no options for other methods of plotting the data and must be closed by using the Windows close dialog button at the upper right of the dialog. 
Figure 3.80. Plotting the sheet-pile tip elevation histogram data as a set of linear segments passing through the center of each bin after execution of a Design mode.

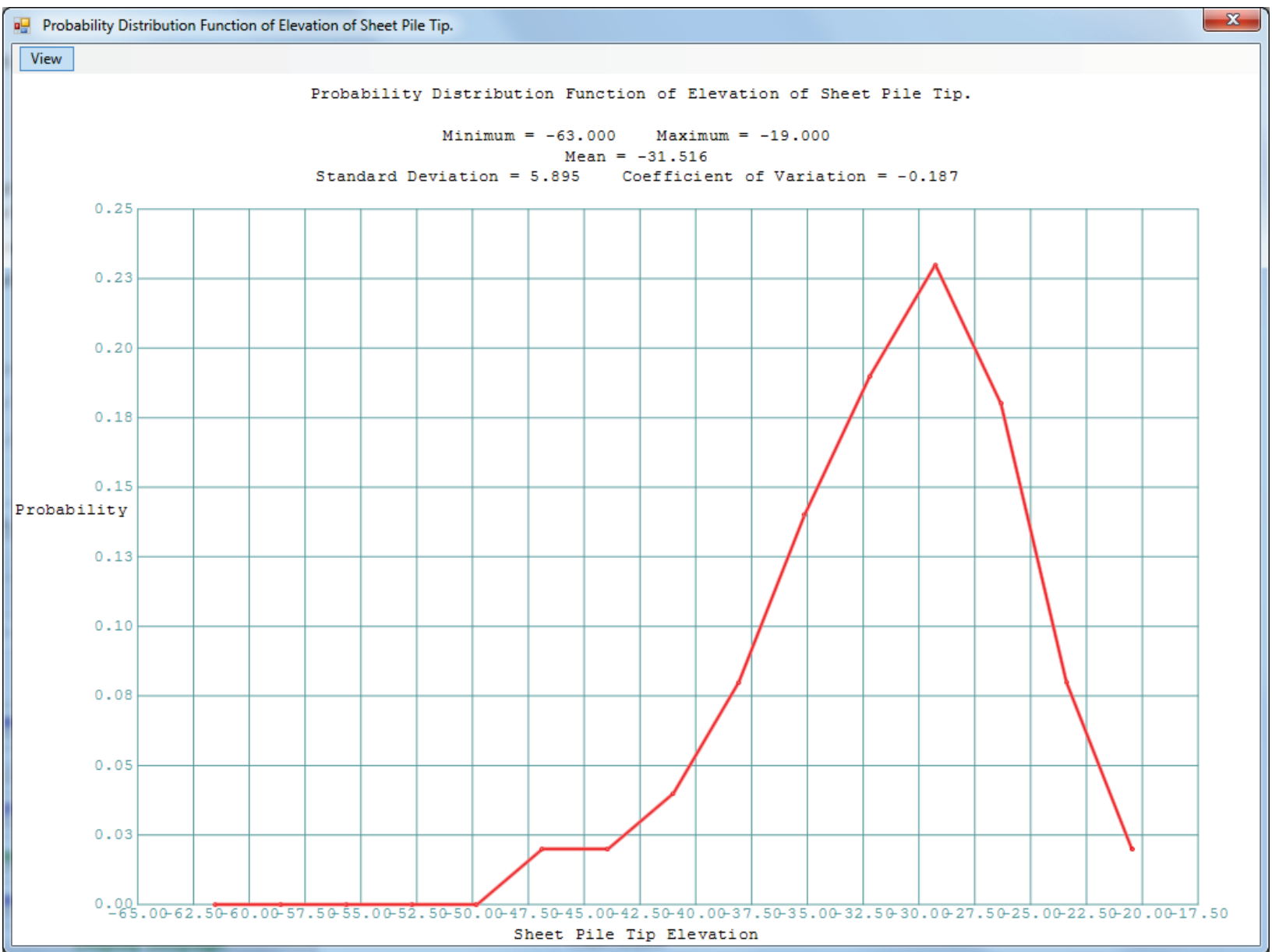

Figures 3.84 and 3.85 provide the histogram data and a plot of the histogram data for I-Wall tip depths, respectively. The histogram data have three columns: bin number, midpoint depth of that bin, and probability that the I-Wall tip depth will occur. Because the full summation of possible I-Wall tip depths is divided into the number of calculated sheet-pile design depths contained within each of the bins, the summation of these probabilities will always be unity (1.0). Notice that these data are essentially the same as the elevation data, but the elevation information has been transformed to depth, resulting in a different base and an inverse scale factor. Therefore, the values in the bins are in the reverse order.

The histogram data are presented with the flood level elevation, the number of simulations that do not exceed the rotational limit state of the I-Wall, the minimum, maximum, and mean passive Factor of Safety, as well as the standard deviation and coefficient of variation of the passive Factor of Safety. 
Figure 3.81. The combined plot of bins and linear interpolation of sheet-pile tip elevation histogram data after execution of a Design mode.

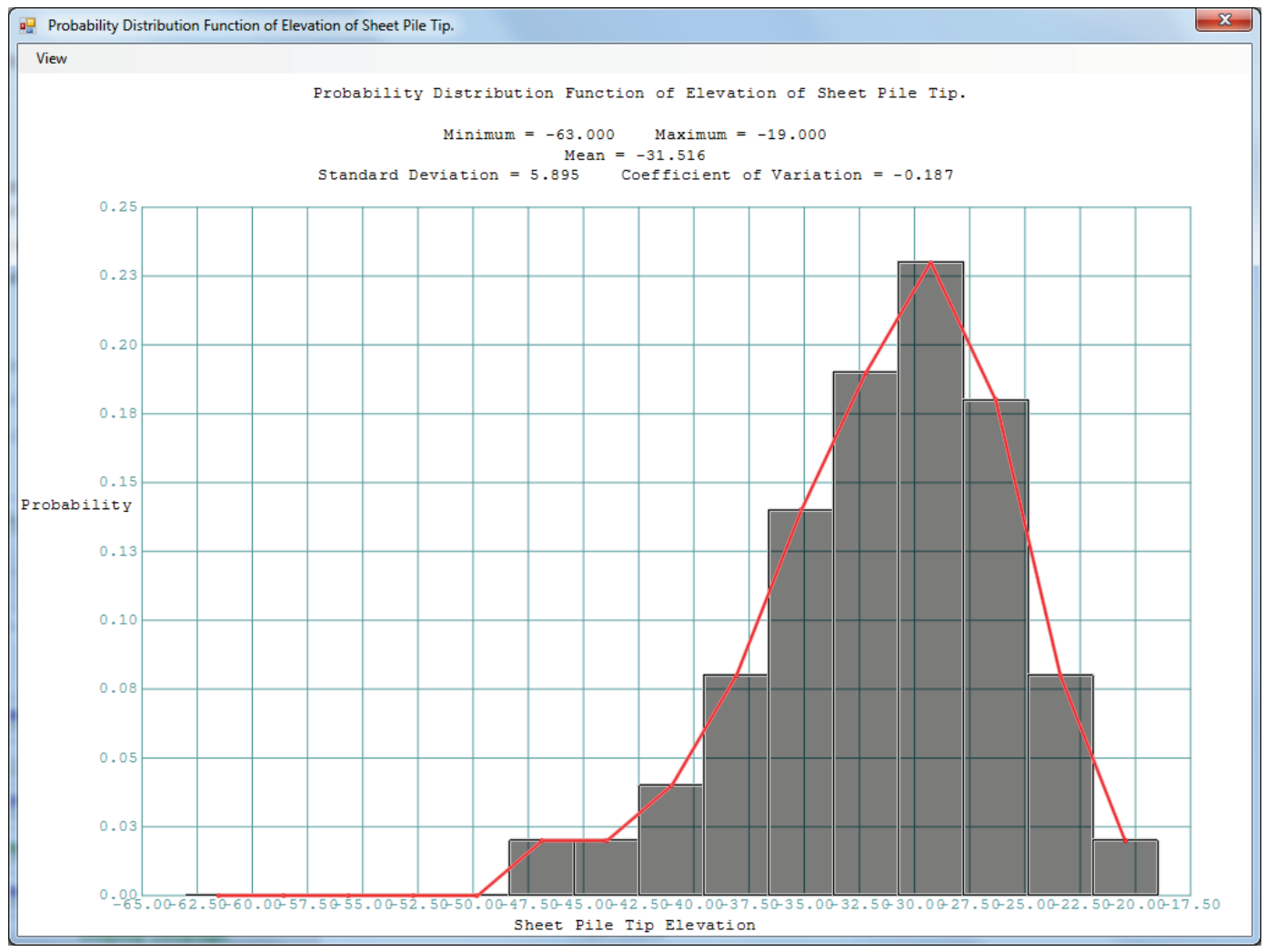

Figure 3.82. Displayed cumulative sheet-pile tip elevation distribution data after execution of a Design mode.

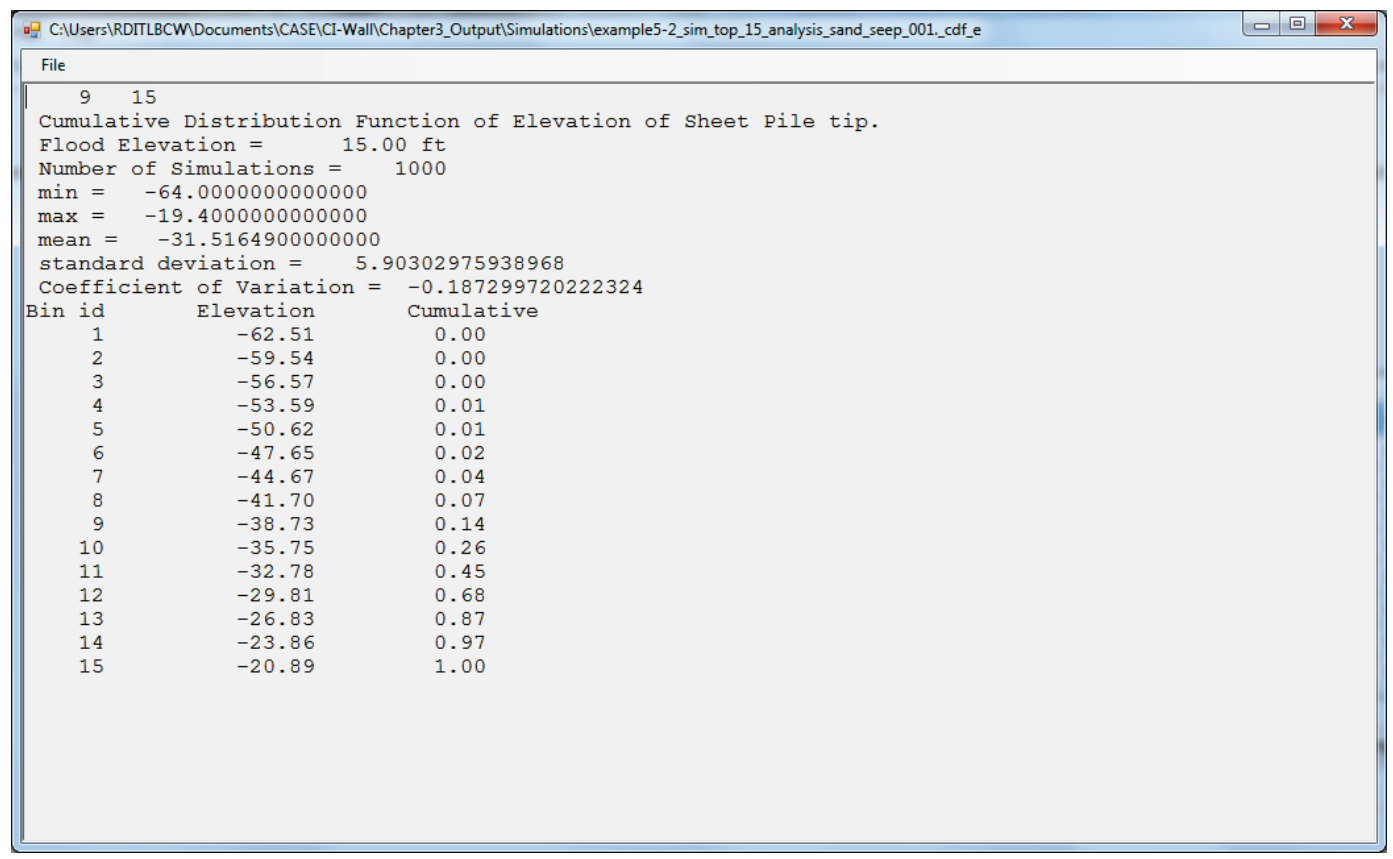


Figure 3.83. Plotted cumulative sheet-pile tip elevation distribution data after execution of a Design mode.

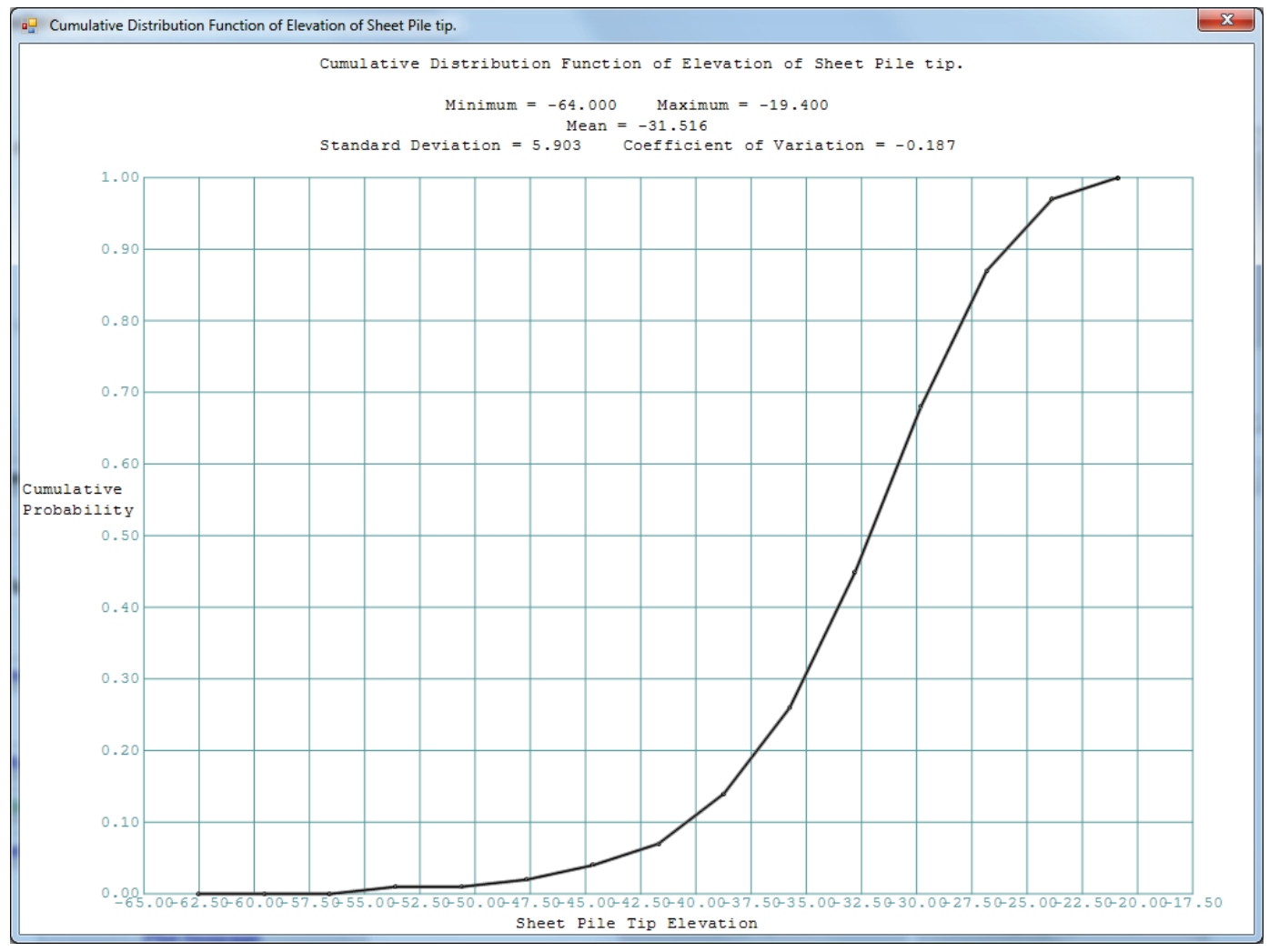

Figure 3.84. Displayed sheet-pile tip depth histogram data after execution of a Design mode.

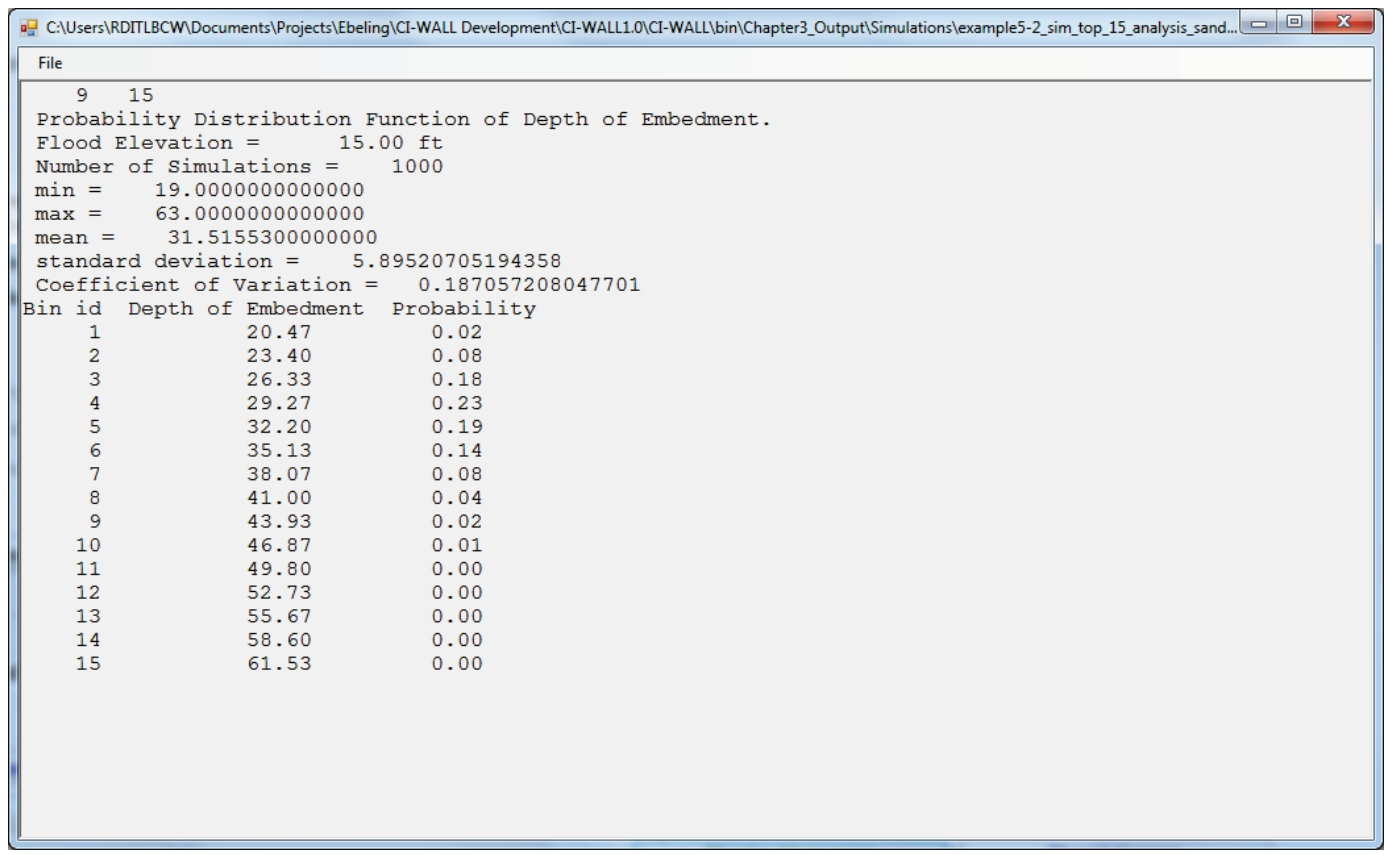


Figure 3.85. Plotted sheet-pile tip depth histogram data after execution of a Design mode.

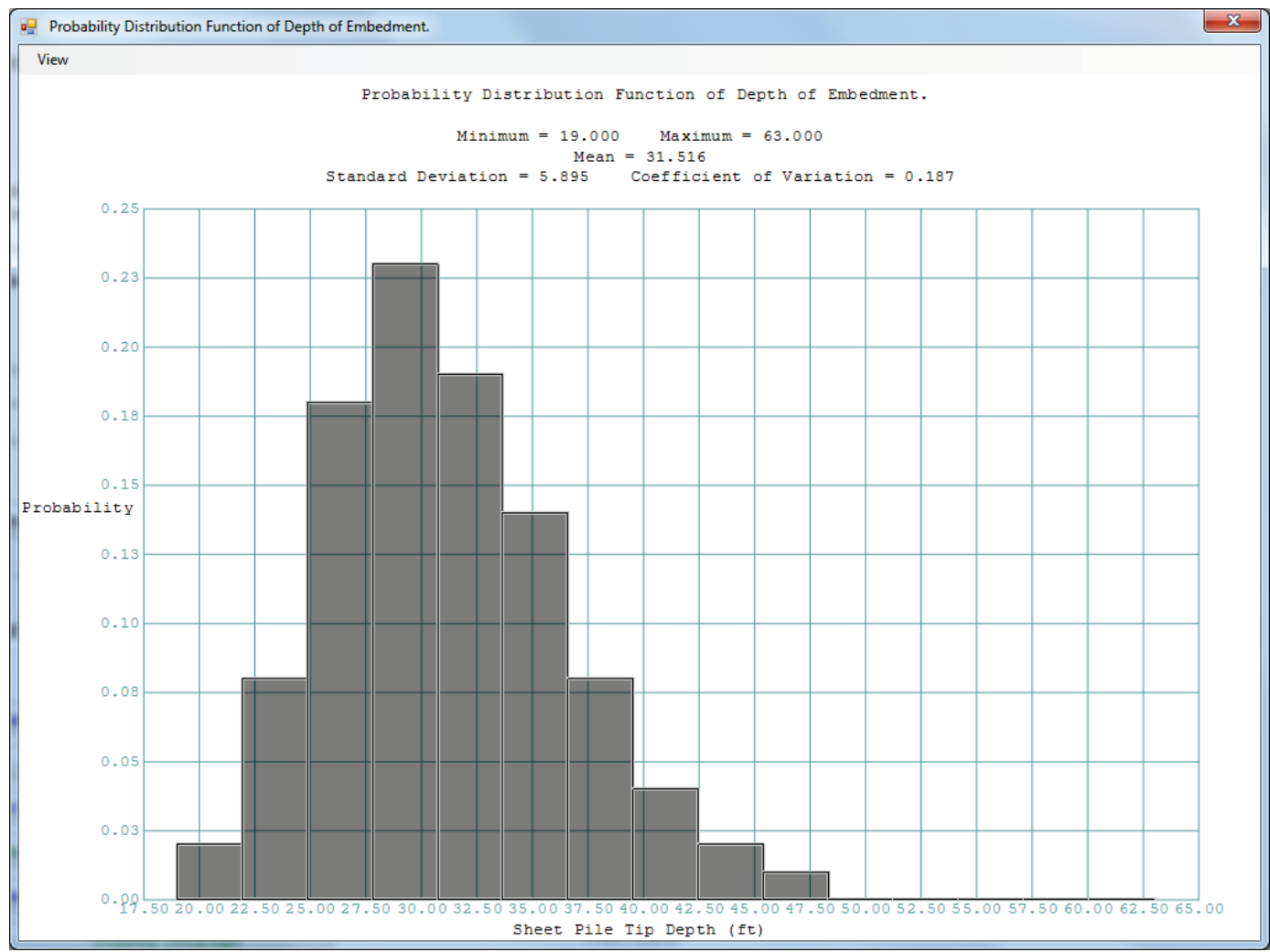

The histogram data are presented with the flood level elevation, the number of simulations that do not exceed the rotational limit state of the I-Wall, the minimum, maximum, and mean passive Factor of Safety, as well as the standard deviation and coefficient of variation of the passive Factor of Safety.

Figures 3.86 and 3.87 provide the cumulative distribution data and a plot of the cumulative distribution data for the depth based coordinates, respectively. In these figures, results from 994 simulations are gathered in 15 bins for a flood elevation of $15 \mathrm{ft}$, which is $15 \mathrm{ft}$ above the ground surface for this example.

The computed mean and standard deviation stay the same, as these data are just a cumulative summation of the histogram probabilities.

For an Analysis mode solution, the probabilistic output area of the Analyze tab looks like Figure 3.88. The Passive Factor of Safety section has the output that is specific to the Flood Level selector on the left side of the Analyze tab (immediately left of the word Probabilistic. Once an elevation is chosen, the user can select one of the Passive Factor of Safety options for information about the passive Factor of Safety for that flood elevation. 
Figure 3.86. Displayed cumulative sheet-pile tip depth data after execution of a Design mode.

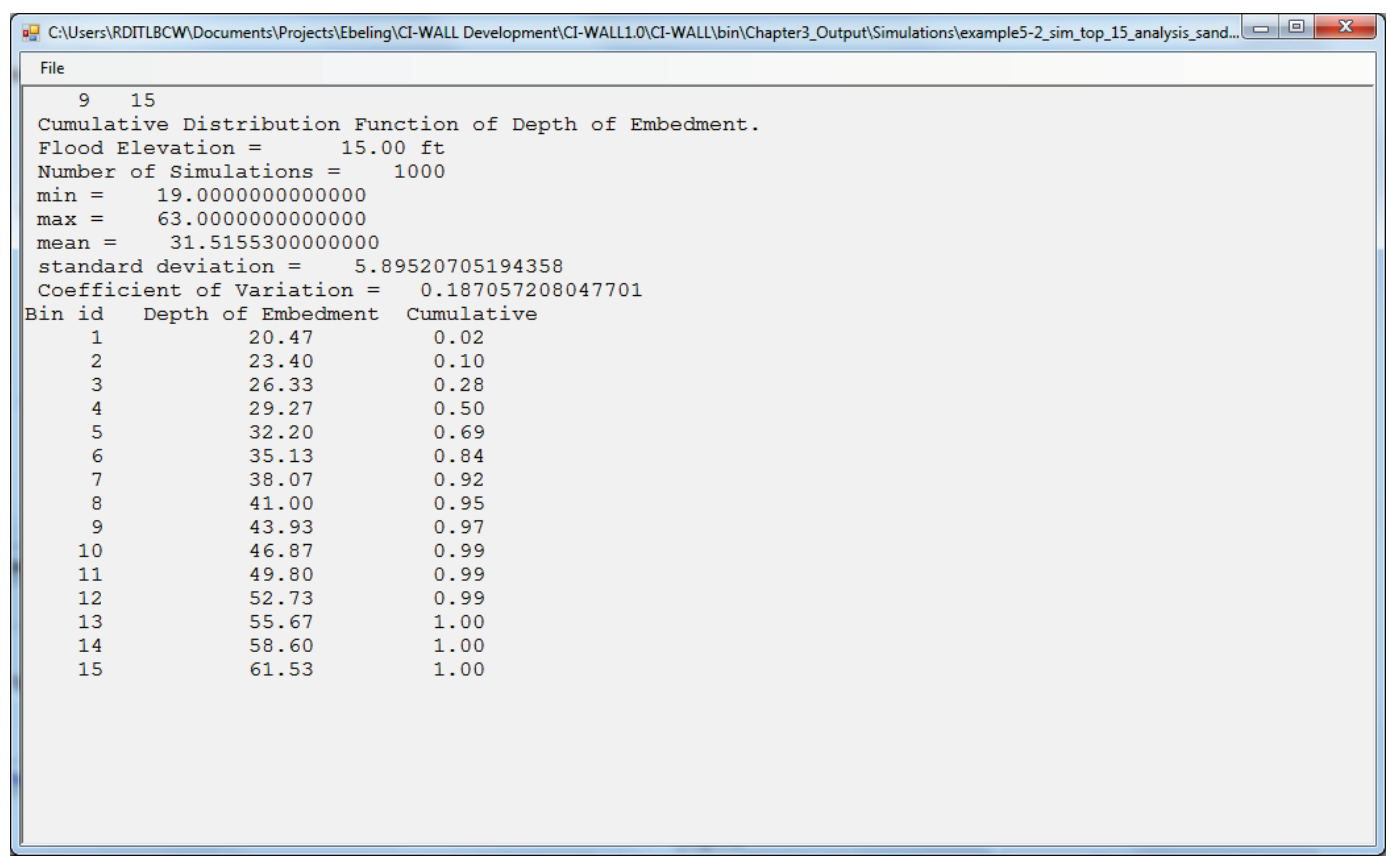

Figure 3.87. Plotted cumulative sheet-pile tip depth data after execution of a Design mode.

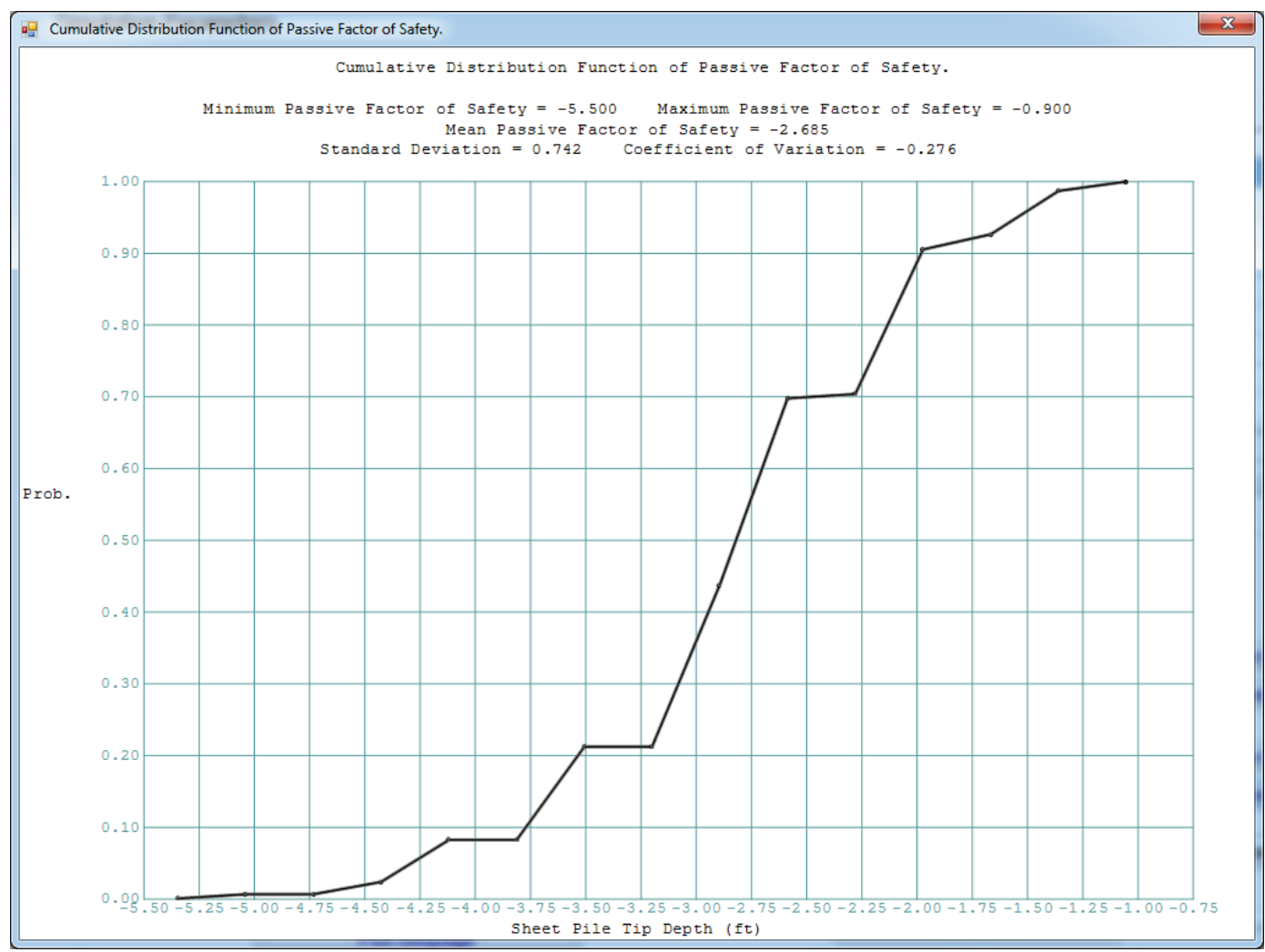


Figure 3.88. The selection of probabilistic output for an Analysis mode problem.

\section{Probabilistic}

Passive Factor of Safety

\section{Display Histogram}

Display Cumulative
Plot Histogram

Plot Cumulative Distribution

\section{Display SRC}

\section{Plot SRC}

Figures 3.89 and 3.90 show a display and plot of the histogram data showing the discrete probabilities (divided into bins) covering a range of passive factors of safety for the Analysis mode example problem with a flood elevation of $4 \mathrm{ft}$. This is the typical means for viewing histogram data for all of the elevations where data were returned. If an elevation has no successful runs, then the buttons for viewing that data are grayed out. The passive Factor of Safety with the greatest probability of occurrence is 2.52 (bin 6) with a probability of 0.25. The mean passive Factor of Safety is at 2.69 and the standard deviation is 0.76 .

Figure 3.89. Histogram data of passive Factor of Safety probabilities for flood elevation $4 \mathrm{ft}$ after execution of an Analysis mode.

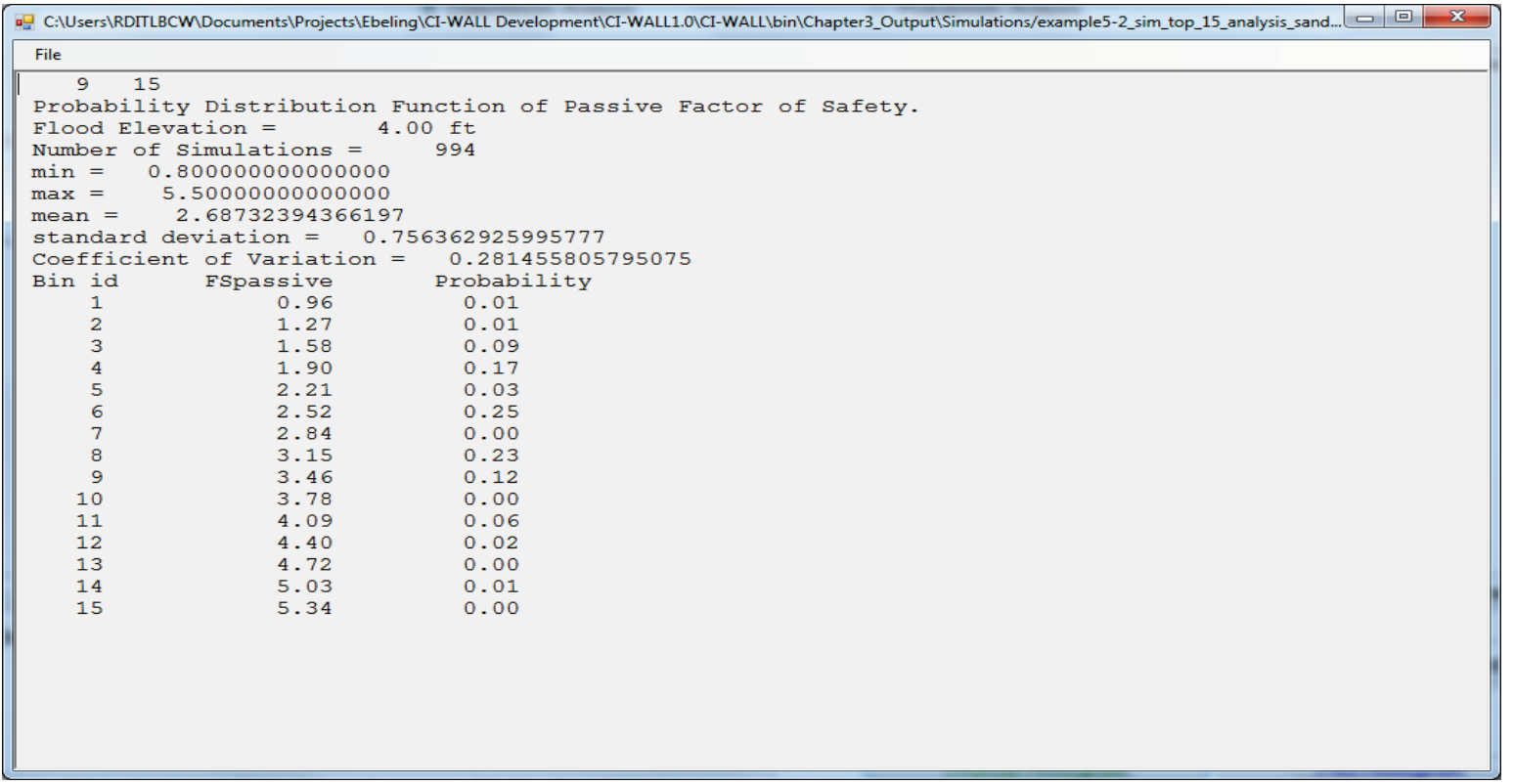


Figure 3.90. Histogram plot of passive Factor of Safety probabilities for flood elevation $4 \mathrm{ft}$ after execution of an Analysis mode.

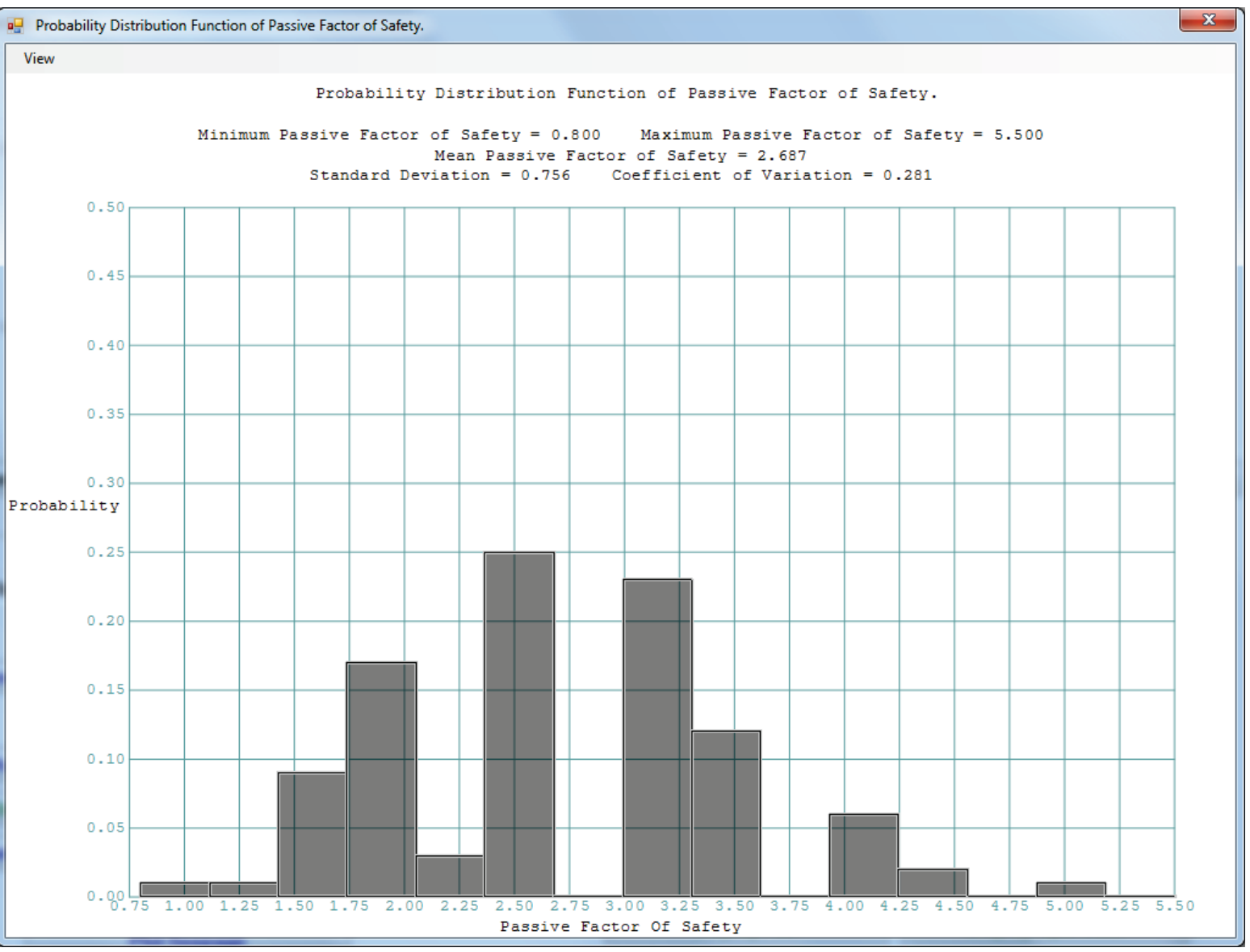

Figures 3.91 and 3.92 show a display and plot of the cumulative distribution data of the discrete probabilities (divided into bins) covering a range of passive factors of safety for the elevation at $4 \mathrm{ft}$. This is the typical means for viewing histogram data for all of the elevations where data were returned. If an elevation has no successful runs, then the buttons for viewing that data are grayed out. Again, the mean passive Factor of Safety is at 2.69 and the standard deviation is 0.76 .

The system response curve (SRC) data and plot shows the probability of exceeding the rotational limit state for an I-Wall over a series of flood elevations (entered by the user). A system response curve is only created in a probabilistic Analysis mode, where the sheet-pile tip depth of the I-Wall is known. For the example problem, 12 flood elevations were entered, ranging from 15 to $4 \mathrm{ft}$. Figure 3.93 shows the computed system response curve values, with the first column being the case number, the second column being the flood elevation, and the last column being the probability that the limits state will be exceeded. These data can be saved, printed, or the dialog 
exited from the menu. Figure 3.94 is a plot of the system response curve, showing the trend as the flood elevation increases. The Plot window for the SRC curve is similar to the Plot window for the cumulative distribution data, in that the user can close the dialog only with the controls at the upper right of the window.

Figure 3.91. Cumulative distribution data of probabilities given a passive Factor of Safety for flood elevation $4 \mathrm{ft}$ after execution of an Analysis mode.

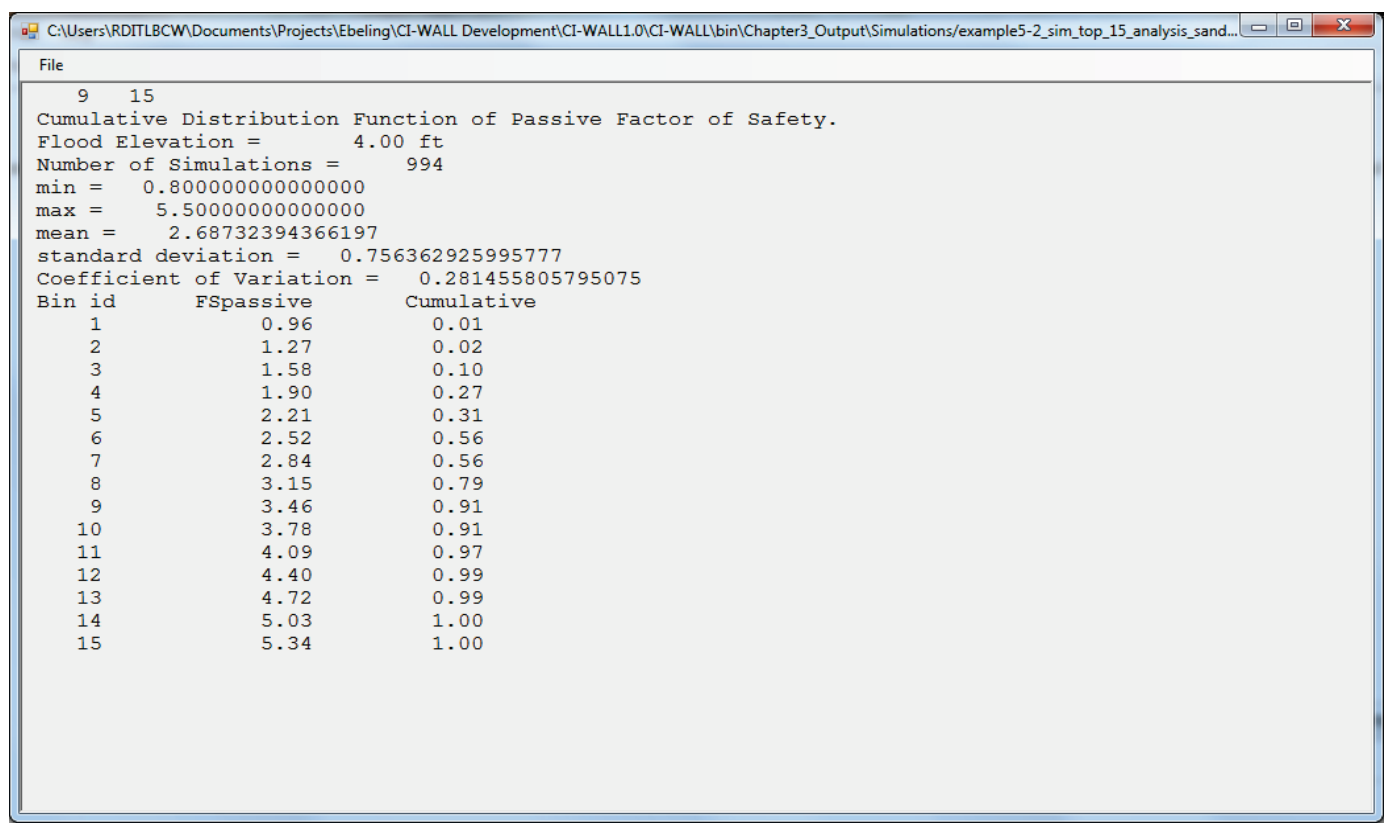

Figure 3.92. Cumulative distribution plot of probabilities given a passive Factor of Safety for flood elevation $4 \mathrm{ft}$ after execution of an Analysis mode.

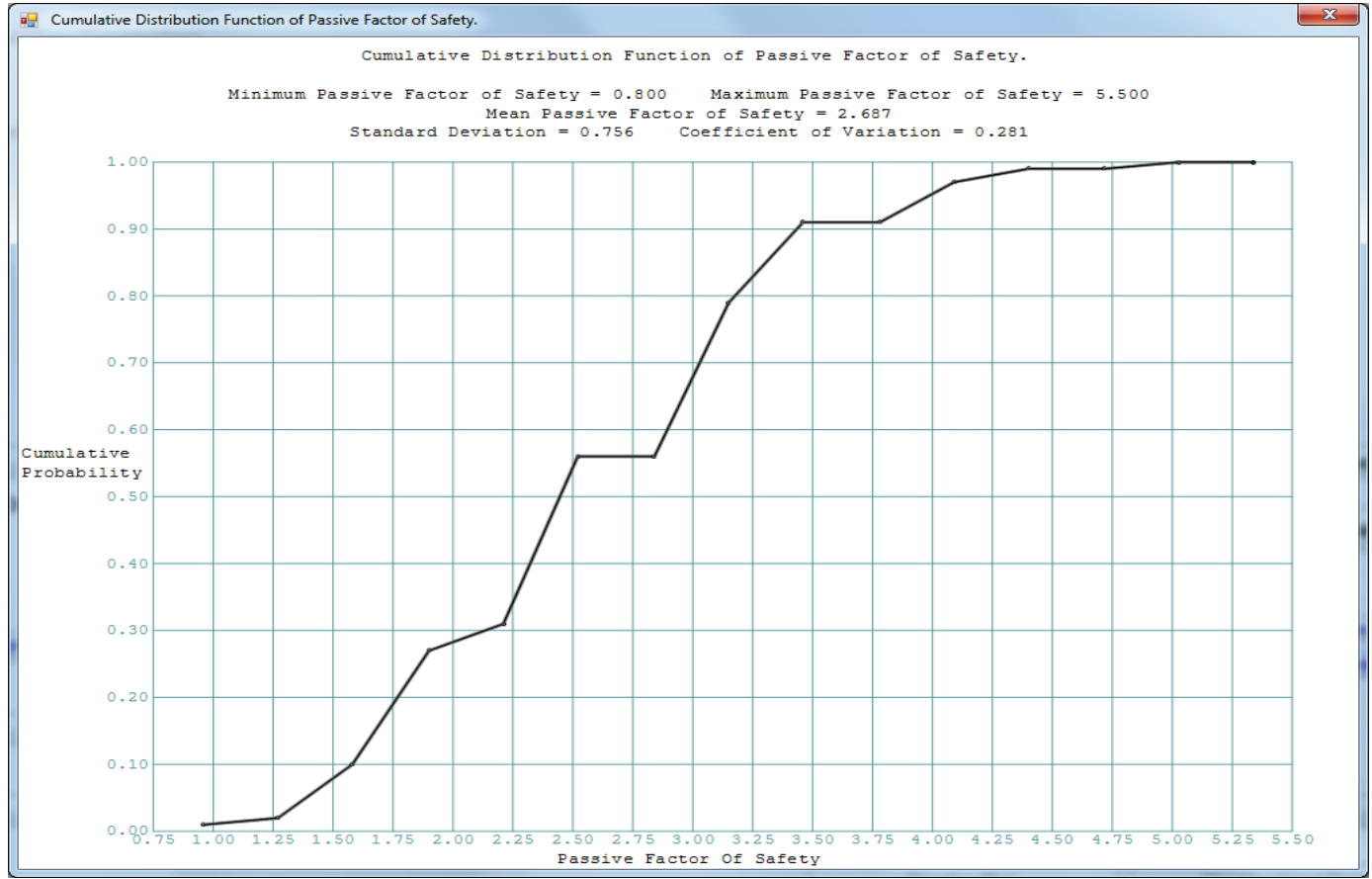


Figure 3.93 Displayed system response curve for 1,000 simulations per flood elevation with 15 bins.

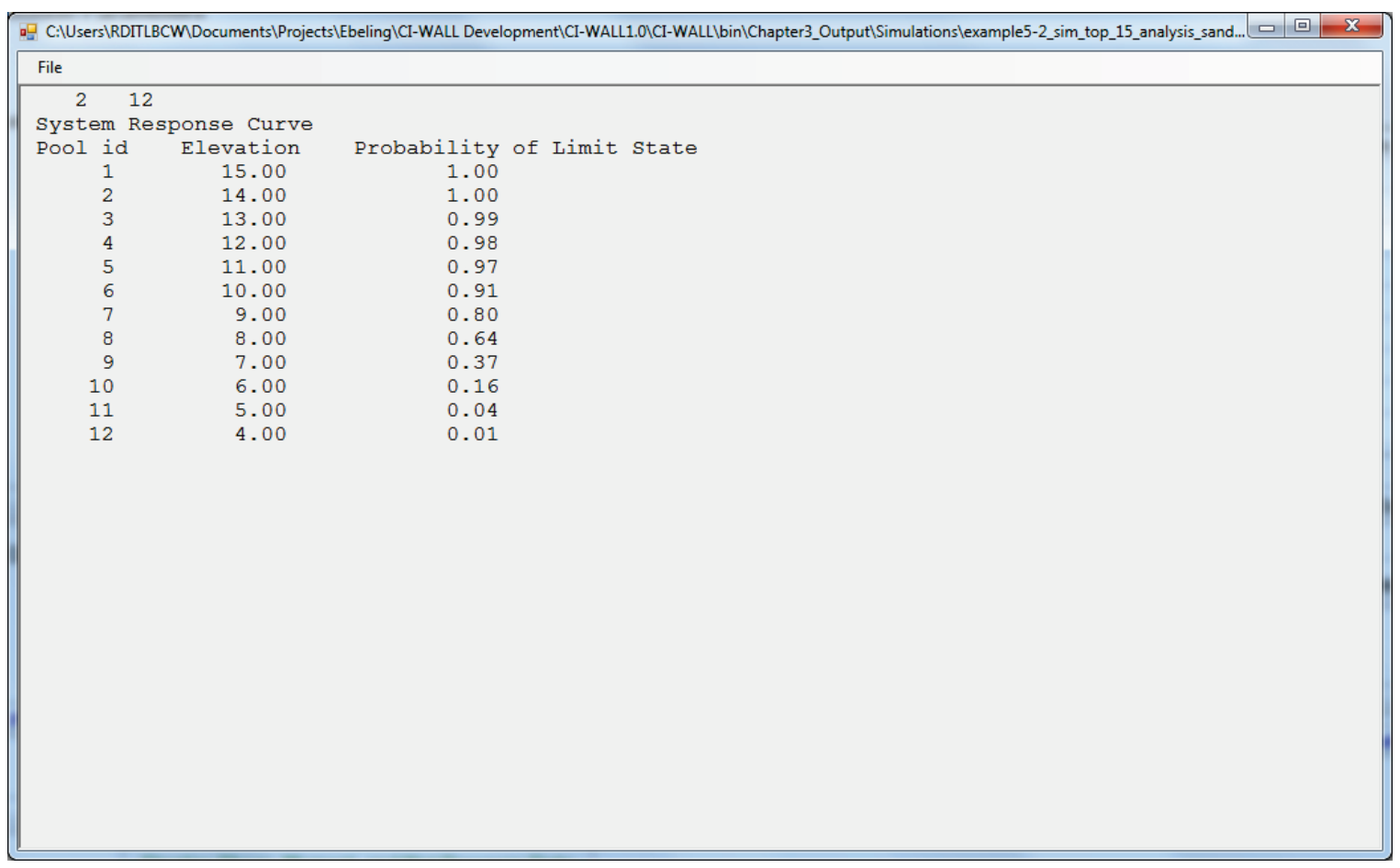

Figure 3.94. Plotted system response curve for 1,000 simulations per flood elevation with 15 bins.

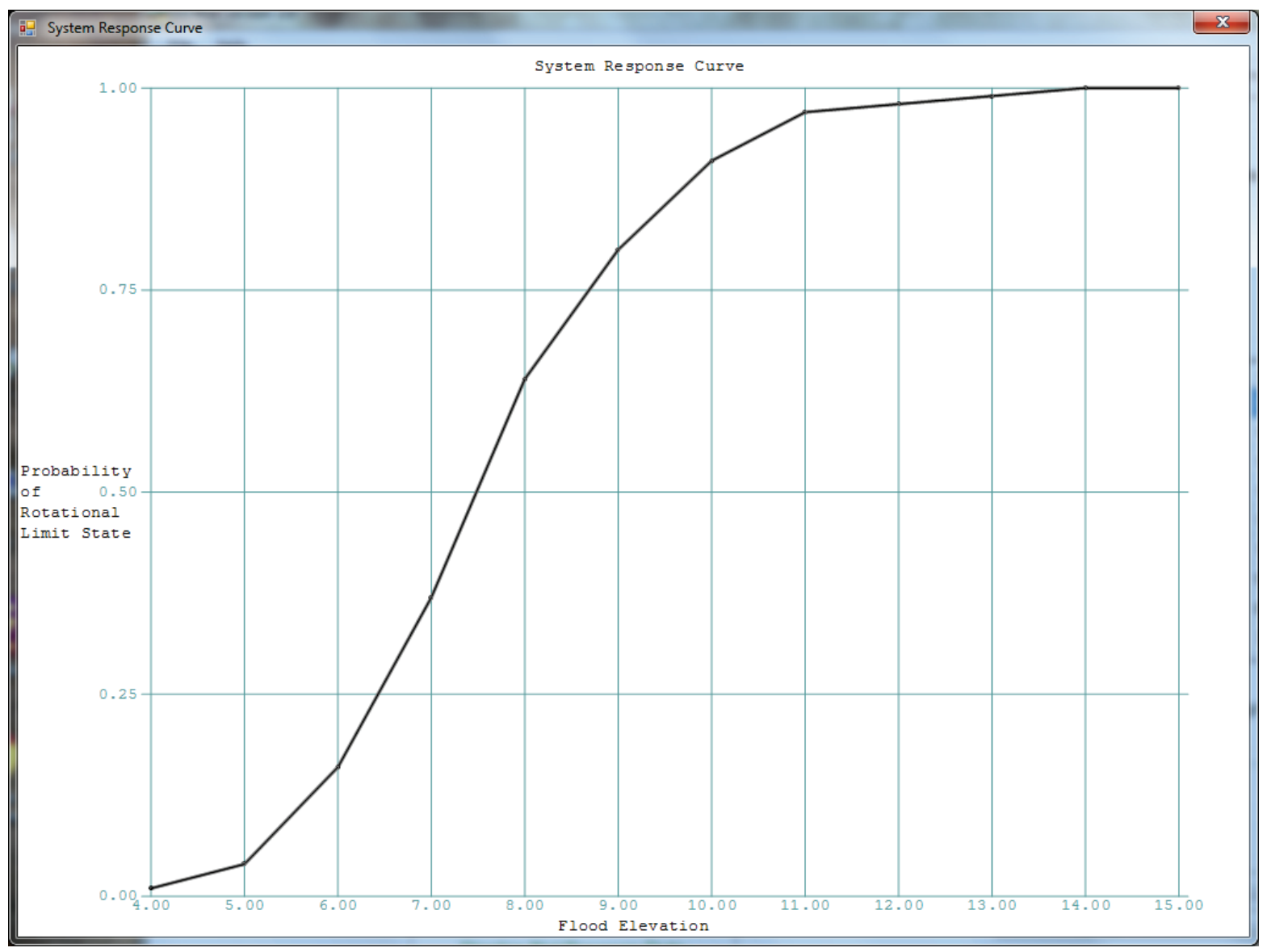




\section{Deterministic Example Problems}

\subsection{Example 4.1 sand site, effective stress strength definition with $\varphi^{\prime}=30, c^{\prime}=0$, with either hydrostatic pore water pressures or homogeneous seepage}

An effective stress definition with $\varphi^{\prime}=30 \mathrm{deg}$ and $c^{\prime}=0$ is illustrated by a series of five design problems in order to determine the depth of embedment (d) of the sheet-pile wall embedded into a sand site. These example problems apply a Factor of Safety of 1.0 for computing active earth pressures and a Factor of Safety of 1.5 for computing passive earth pressures beneath the soil layers. In this example, I-Wall has an exposed height of $10 \mathrm{ft}$. The water surfaces at both sides of the I-Wall are at elevation, el $40 \mathrm{ft}$ on the flood side and el $34 \mathrm{ft}$ on the landside. The homogeneous sand layer of soil has a level ground surface on both sides of the I-Wall at $30 \mathrm{ft}$ with saturated unit weight of $122.4 \mathrm{pcf}$ and an effective angle of internal friction $\left(\varphi^{\prime}\right)$ of $30 \mathrm{deg}$. Figure 4.1 outlines the basic geometry and structural parameters for the sheet-pile wall, and Figure 4.2 illustrates the schematic produced by Corps_I-Wall for the same problem. The top row of text listed in Figure 4.2 documents the input values of all parameters associated with each material type. For this problem, there is only one material assigned for the sand site and recorded as "Mo1," the "M" signifies the material type and is followed by the ID, which starts at "01." All information concerning material types are written in black text. The representation of a layer of soil on each side of the I-Wall is characterized as a region. Regions are labeled with an "R" and followed by an ID starting from "O1." For this problem two soil regions are required to represent each soil layer; Region 1 (as Ro1), is located on the LHS of the sheet-pile wall and Region 2 (as Ro2) is located on the RHS of the sheet-pile wall. Each region and its surface elevation are written in red text. Piezometric surfaces are labeled as "P" and followed by an ID starting from 01. Piezometric water surfaces are listed in the upper left-hand corner and drawn with the water symbol at its defined elevation and shown in blue. Observe that piezometric surface ID, Po1, is assigned to both regions. The LHS piezometric surface elevation is assigned as the LHS surface water elevation. Similarly, the RHS piezometric surface elevation is assigned as the RHS surface water elevation. This allows for the assignment of submerged unit weights to the soil regions. This geometry will be used for the following example problems and are described in the following subsections. 
Figure 4.1. Sheet-pile wall in sand site.

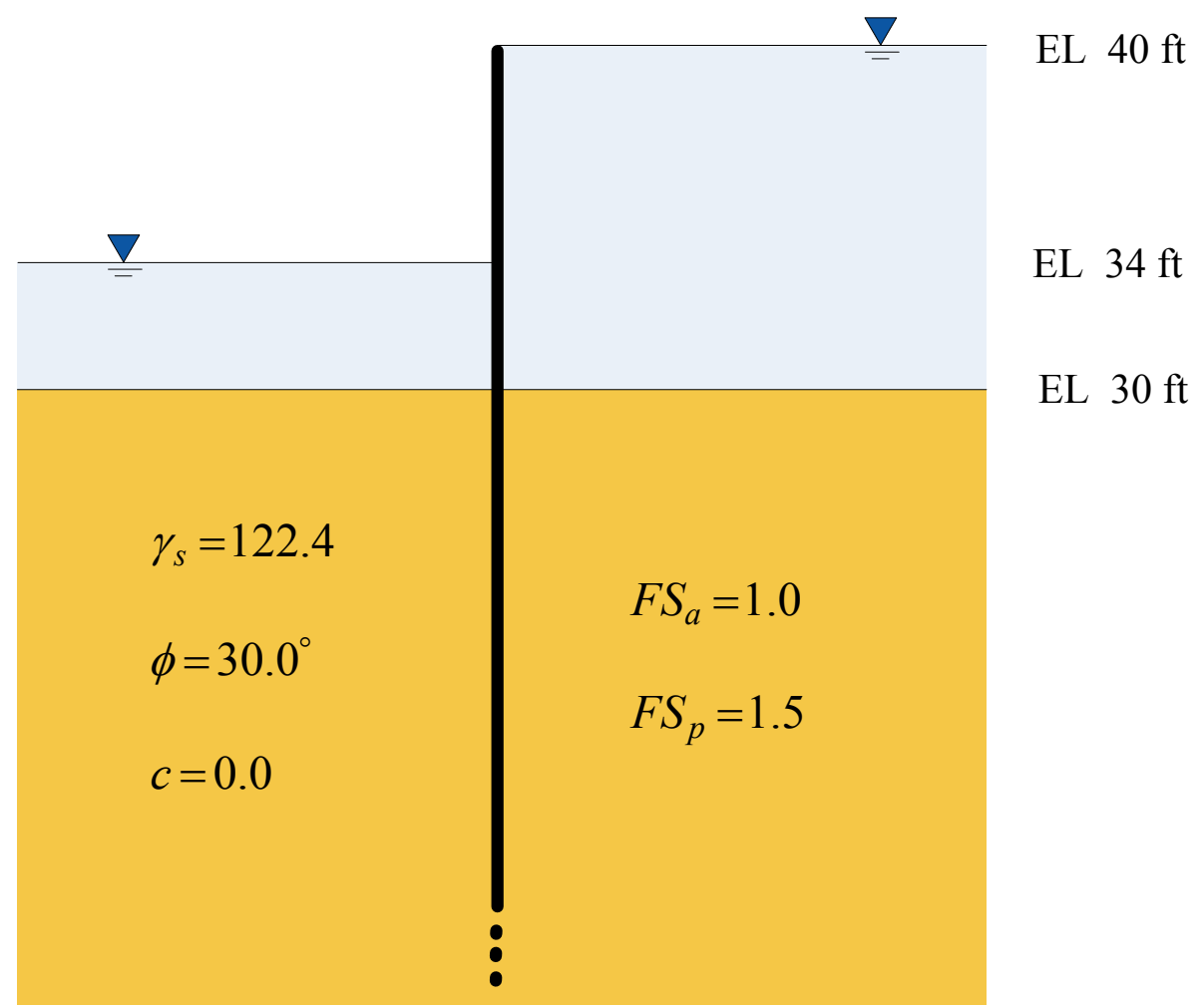

Figure 4.2. Corps_I-Wall schematic of sheet-pile wall in sand site.

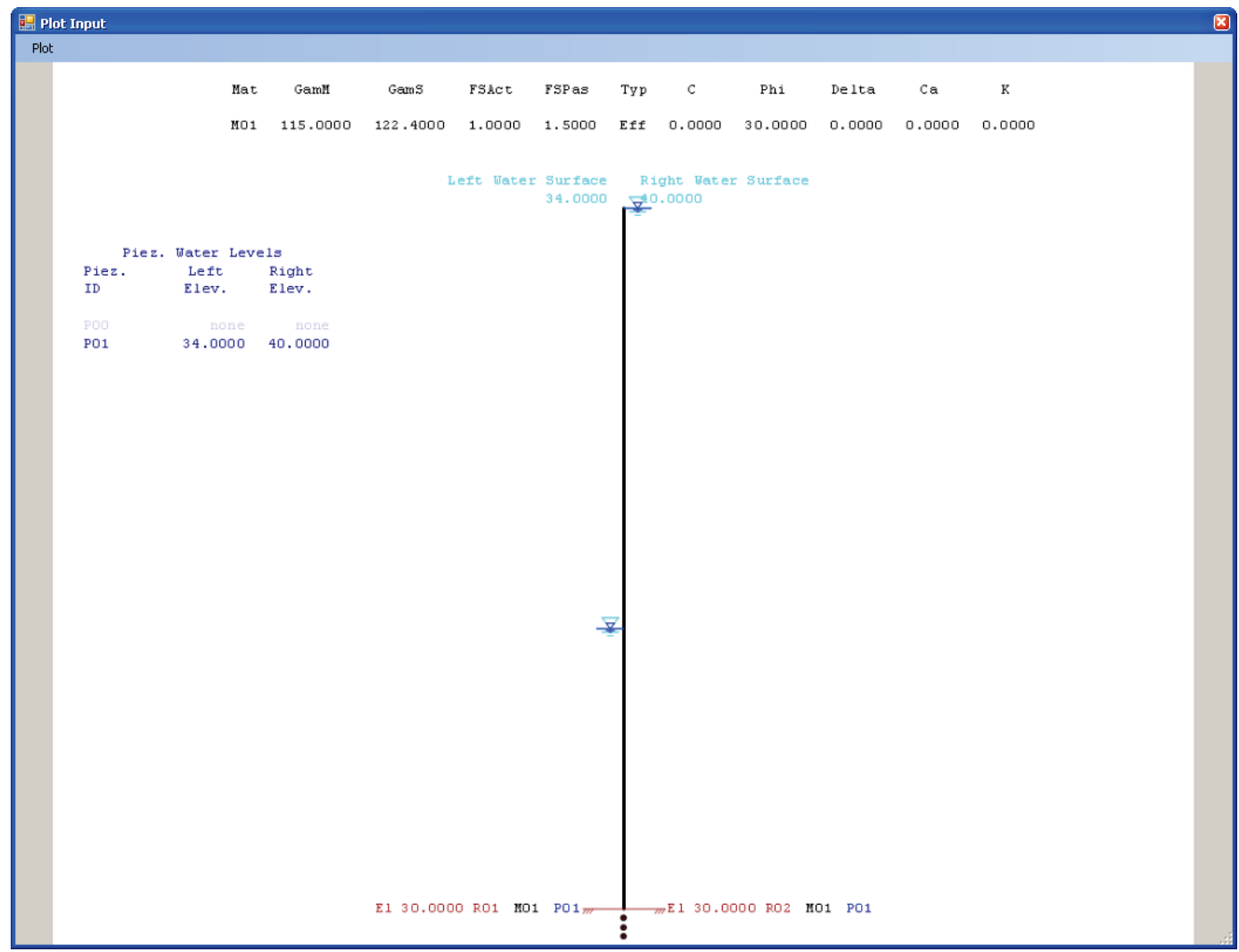




\subsubsection{Example 4.1.1}

An effective stress definition with $\varphi^{\prime}=30 \mathrm{deg}$ and $c^{\prime}=0$ as illustrated in Figure 4.2 is considered for the evaluation of the depth of embedment. Homogeneous soil, hydrostatic pore water pressures, effective angle of wall friction $\left(\delta^{\prime}\right)$ of o deg as well as the Coulomb earth pressure coefficient method of analysis (for $K_{a}$ and $K_{p}$ ) form the basis for this example ${ }^{1}$. Hydraulic fracturing is not included as an option for this analysis.

This analysis resulted in a computed active earth pressure coefficient $\left(K_{a}\right)$ of 0.3333 and a "mobilized" passive pressure coefficient $\left(K_{p}\right)$ of $2.1212 .{ }^{2}$ The distribution of active and passive earth pressures with elevation for both sides of the sheet-pile wall are illustrated in Figures 4.3a and 4.3b. 3 The hydrostatic water pressures acting on both sides of the sheet-pile wall are shown in Figure $4.4 \mathrm{a}^{4}$ with the resultant net water pressure calculated by taking the difference between the water pressures acting on both sides of the wall (Figure 4.4a) and shown in Figure 4.4b.5 Observe the differential water pressure at the tip of the sheet pile ( $=6 \mathrm{ft}$ times $62.4 \mathrm{pcf})$; a common outcome when the piezometric surface elevations (defined as equal to the surface water elevations in this problem) for the two sides of the I-Wall differ (e.g., $34 \mathrm{ft}$ on the LHS and $40 \mathrm{ft}$ on the RHS) and hydrostatic water pressures are specified by the user.

From these pressures (Figures 4.3 and 4.4), the net active and net passive pressures are constructed and presented in Figure 4.5a. ${ }^{6}$ For the applied flood loading, the I-Wall will rotate counterclockwise. The net active pressure is determined from the difference between the RHS-mobilized active earth pressure and the LHS-mobilized passive earth pressure (i.e., acting on the other side of the wall) with the addition of any net water pressures and any external horizontal net pressures and overburden pressures (if any). The net passive pressure is determined by the difference between the RHS- mobilized passive earth pressure and the LHSmobilized active earth pressure acting on the other side of the sheet-pile wall with the addition of any net water pressures and any external

\footnotetext{
1 For no soil-to-I-Wall interface friction (i.e., $\delta=0 \mathrm{deg}$ ), the Coulomb solution for active and passive earth pressure coefficients corresponds to the Rankine earth pressure coefficients.

2 A FSpassive value equal to 1.5 is used in this example, consistent with EM 1110-2-2504 (HQUSACE 1994).

3 The earth pressures in all four Figure 4.3 diagrams act toward the wall.

4 The water pressures in the two Figure 4.4a diagrams act toward the wall.

5 The net water pressure in the Figure $4.4 \mathrm{~b}$ diagram acts toward the wall.

6 The net earth pressures in the two Figure 4.5a diagrams act towards the wall.
} 
horizontal net pressures and overburden pressures (if any). The net pressure diagram of Figure $4.5 \mathrm{~b}$ is derived from the replication of all values of the net active pressures, from the top of the wall until the elevation at the point of rotation (el $14.4079 \mathrm{ft}$ ), at which instant the remaining values of the net pressure diagram is assumed to be linear with elevation between (1) the net active pressure value at the elevation of the point of rotation and (2) the net mobilized passive pressure at the elevation of the approximated sheet-pile tip (i.e., el 8.0195). ${ }^{1}$

Figure 4.3. Active and passive earth pressures. (a) LHS of wall. (b) RHS of wall.

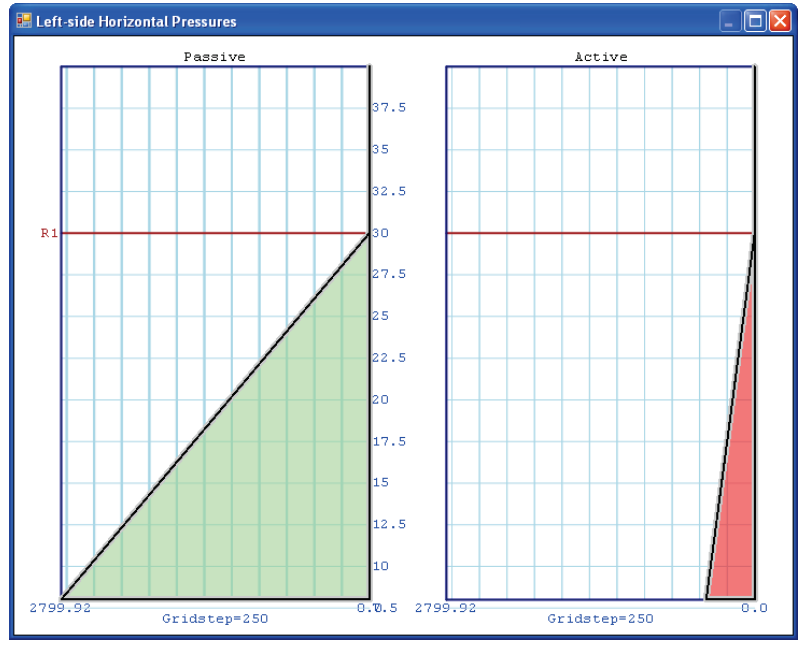

(a)

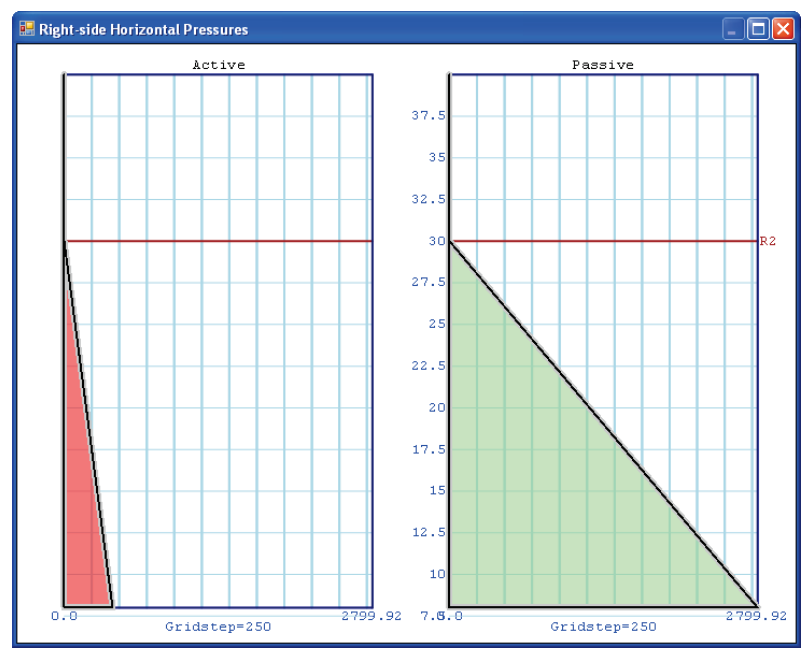

(b)

Figure 4.4. (a) Pore water pressures for the left- and right-hand side of the wall. (b) Net water pressures.

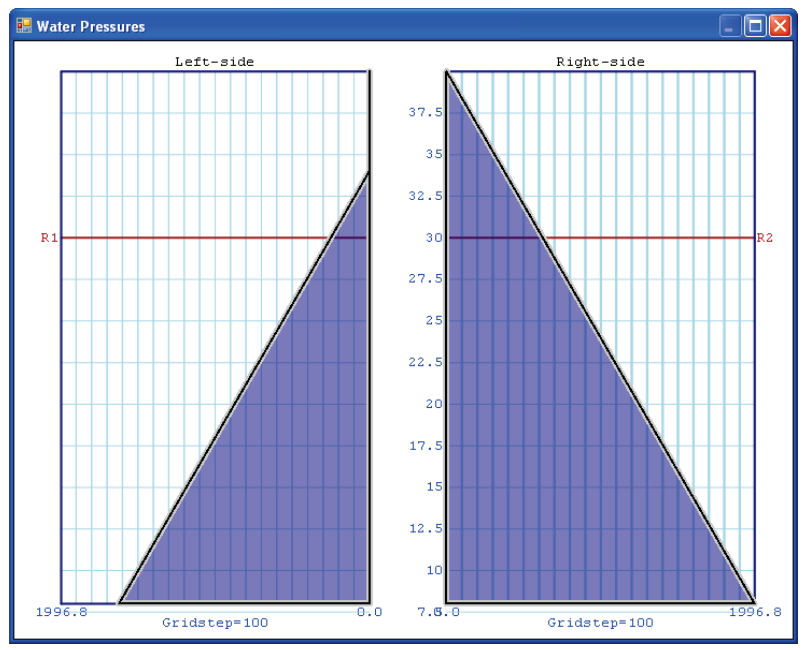

(a)

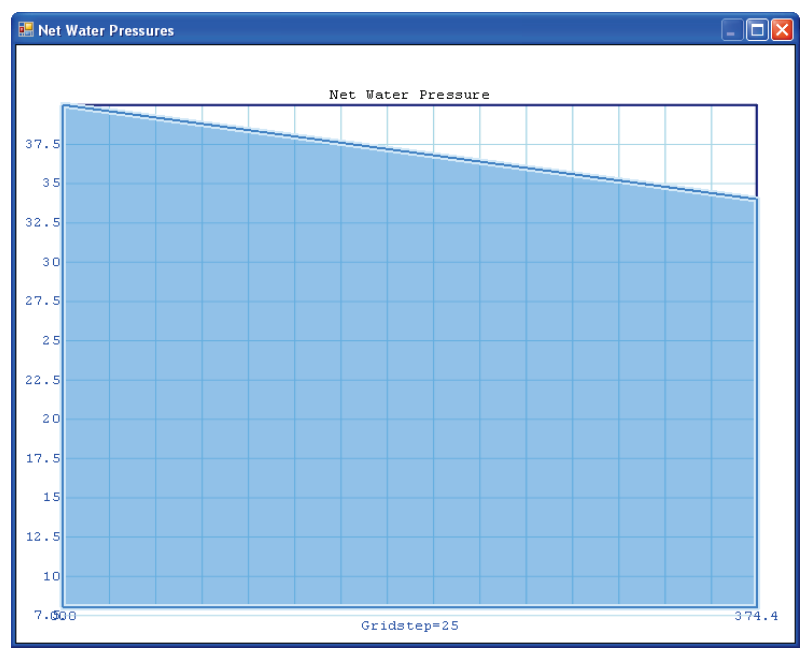

(b)

\footnotetext{
1 Thus, only one mobilized net passive pressure value in Figure 4.5a is used to construct the Figure 4.5b net pressure diagram; corresponding to the sheet-pile tip elevation at el 8.0195. The majority of the net pressure diagram is derived from the Figure 4.5a net active pressure diagram.
} 
Figure 4.5. Net pressures. (a) Net active and net passive. (b) Net pressure diagram.

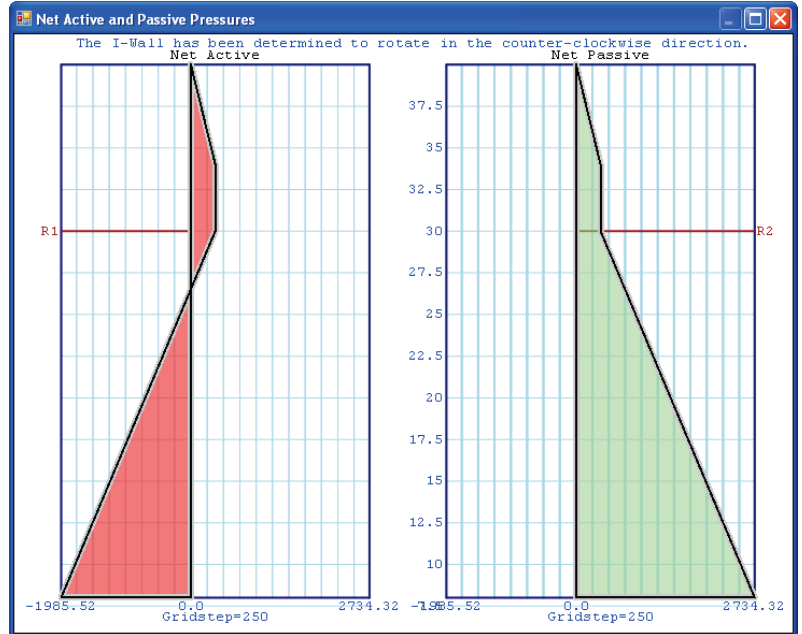

(a)

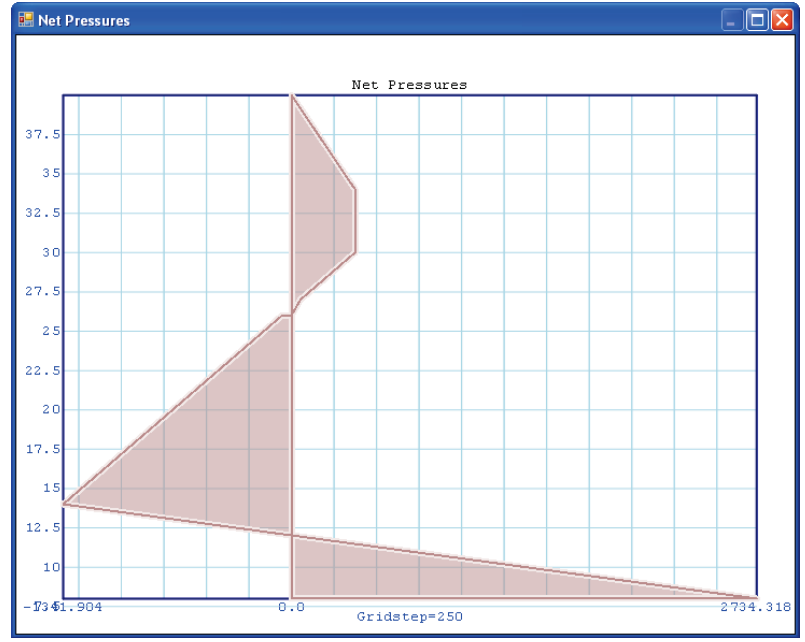

(b)

Figure $4.5 \mathrm{~b}$ is an illustration of how the net pressure diagram was formed. ${ }^{1}$ The final results are illustrated in Figure 4.6 by the presentation of the net pressure diagram Figure 4.6a (replica of Figure 4.5b) and 4.6b, the shear and moment diagrams. Figure $4.6 \mathrm{~b}$ shows that the maximum bending moment occurs at the zero crossing of the shear diagram.

Figure 4.6. (a) Net pressure diagram. (b) Shear and moment.

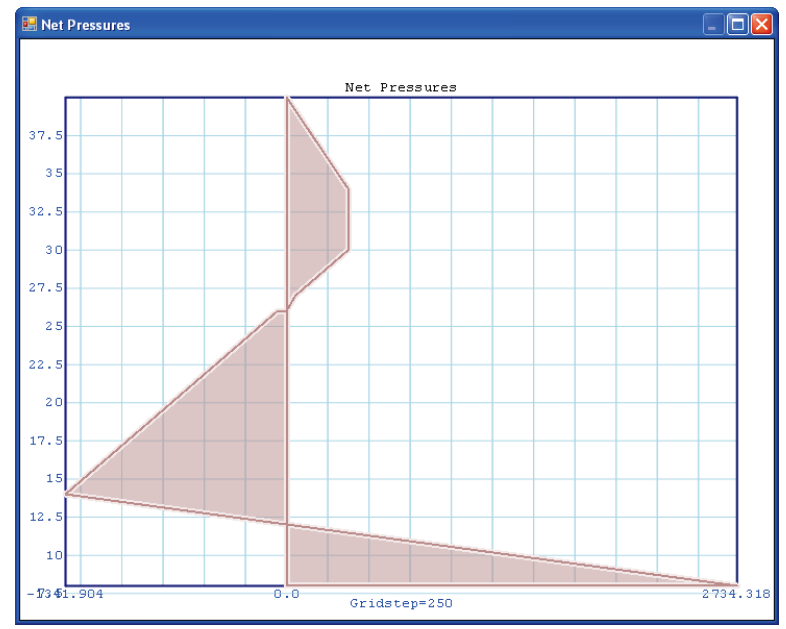

(a)

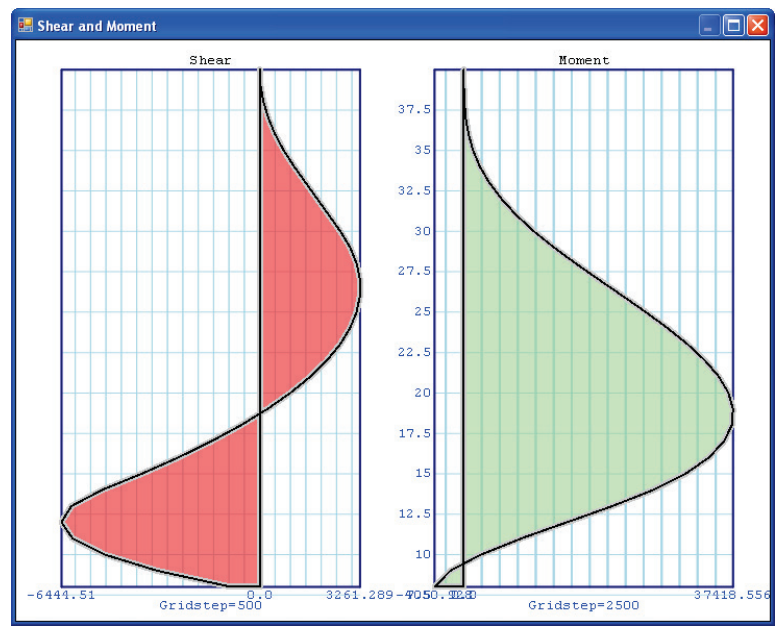

(b)

The various input conditions and final results for Example 4.1.1 are tabulated as check problem 1 in Table 4.1. The sheet-pile tip elevation is computed at $8.02 \mathrm{ft}$ with a calculated depth of embedment of $21.98 \mathrm{ft}$.

\footnotetext{
1 The net pressures in the Figure $4.5 \mathrm{~b}$ diagram act towards the wall.
} 
Table 4.1. Examples of effective stress strength definitions (sands with $c^{\prime}=0$ ).

\begin{tabular}{|l|l|l|l|l|l|l|l|l|l|l|}
\hline $\begin{array}{l}\text { Check } \\
\text { Problems }\end{array}$ & $\begin{array}{l}\text { Coulomb (C) or } \\
\text { Logarithmic } \\
\text { spiral (L) }\end{array}$ & $\begin{array}{l}\text { Hydraulic } \\
\text { Fracture } \\
\text { Option }\end{array}$ & $\begin{array}{l}\text { Seepage } \\
\text { Gradient }\end{array}$ & $\delta^{\prime}(\mathrm{deg})$ & $K_{a}$ mob & $K_{p}$ mob & $\frac{2 c^{\prime}}{\sqrt{K_{a}} \gamma_{b u o y}}$ & $\begin{array}{l}\text { Hydraulic } \\
\text { Fracture } \\
\text { Depth }\end{array}$ & $\begin{array}{l}\text { Elevation at } \\
\text { Point of } \\
\text { Rotation }\end{array}$ & $\begin{array}{l}\text { Elevation at } \\
\text { Tip of Pile }\end{array}$ \\
\hline 1 & C & Off & HY & 0 & 0.3333 & 2.1212 & 0.0000 & NG & 14.4079 & 8.0195 \\
\hline 2 & C & Off & HY & 15 & 0.3014 & 2.7681 & 0.0000 & NG & 17.3497 & 11.7993 \\
\hline 3 & L & Off & HY & 15 & 0.3014 & 2.7645 & 0.0000 & NG & 17.3374 & 11.7835 \\
\hline 4 & L & On & HY & 15 & 0.3014 & 2.7645 & 0.0000 & 0.0000 & 17.3374 & 11.7835 \\
\hline 5 & L & On & 0.1603 & 15 & 0.3014 & 2.7645 & 0.0000 & 0.0000 & 16.2400 & 11.2900 \\
\hline
\end{tabular}

Note: All check problems have a single sand layer of homogeneous soil with an effective angle of internal friction $\left(\varphi^{\prime}\right)$ of 30 deg. Factor of Safety is 1.0 for active earth pressures and Factor of Safety is 1.5 for passive earth pressures. All elevations and depths are reported in units of feet.

$N G$ - Hydraulic fracturing (gap) not considered.

HY - Hydrostatic pore water pressure. 


\subsubsection{Example 4.1.2}

An effective stress definition with $\varphi^{\prime}=30 \mathrm{deg}$ and $c^{\prime}=0$ as illustrated in Figure 4.2 is considered for the evaluation of the depth of embedment. Homogeneous soil, hydrostatic pore water pressures, effective angle of soil-to-I-Wall friction $\left(\delta^{\prime}\right)$ of 15 deg as well as the Coulomb coefficient method of analysis (for $K_{a}$ and $K_{p}$ ) form the basis for this example. Hydraulic fracturing is not included as an option for this analysis. This Example 4.1.2 differs from Example 4.1.1 by a $\delta^{\prime}$ of $15 \mathrm{deg}$.

The analysis resulted in a computed mobilized active earth pressure coefficient $\left(K_{a}\right)$ of 0.3014 and a mobilized passive pressure coefficient $\left(K_{p}\right)$ of 2.7681. The active and passive earth pressures for both sides of the sheetpile wall will be slightly higher with the same triangular shape as Example 4.1.1 Figures 4.3a and 4.3b. The hydrostatic water pressures acting on both sides of the sheet-pile wall are similar to those shown in Figure 4.4a with the resultant net water pressure calculated by taking the difference between the water pressures acting on both sides of the wall (Figure 4.4a) and similar to that shown in Figure 4.4b for the Example 4.1.1 problem.

From the calculated pressures, the net active and net passive pressures are estimated. The net pressure diagram (not shown) is similar to Figure 4.5 b and is derived from the replication of all values of the net active pressures, from the top of the wall until the elevation at the point of rotation (el $17.3497 \mathrm{ft}$ ), at which instant the remaining values of the net pressure diagram is assumed to be linear with elevation between (1) the net active pressure value at the elevation of the point of rotation and (2) the net mobilized passive pressure at the elevation of the approximated sheet-pile tip (i.e., el 11.7993). For the applied flood loading, the I-Wall will rotate counterclockwise.

The various input conditions and final results for Example 4.1.2 are tabulated as check problem 2 in Table 4.1. The sheet-pile tip elevation is computed at $11.80 \mathrm{ft}$ with a calculated depth of embedment of $18.20 \mathrm{ft}$. With the addition of soil-to-I-Wall friction of $15 \mathrm{deg}$ as compared with Example 4.1.1, the approximated sheet-pile tip elevation is higher than the 8.02 ft of Example 4.1.1, which is acceptable as the mobilized passive earth pressures have increased along the sheet-pile wall and thereby resulted in a shallower depth of embedment by nearly $3.8 \mathrm{ft}$. 


\subsubsection{Example 4.1.3}

An effective stress definition with $\varphi^{\prime}=30 \mathrm{deg}$ and $\mathrm{c}^{\prime}=0$ as illustrated in Figure 4.2 is considered for the evaluation of the depth of embedment. Homogeneous soil, hydrostatic pore water pressures, effective angle of soil-to-I-Wall friction $\left(\delta^{\prime}\right)$ of 15 deg as well as the passive earth pressure coefficient obtained from the logarithmic spiral procedure form the basis for this example. Hydraulic fracturing is not included as an option for this analysis. This Example 4.1.3 differs from Example 4.1.2 by the logarithmic spiral procedure for the calculation of mobilized passive earth pressures with $\delta^{\prime}=\varphi^{\prime} / 2$ or $15 \mathrm{deg}$.

The analysis resulted in a computed mobilized active earth pressure coefficient $\left(K_{a}\right)$ of 0.3014 and a mobilized passive pressure coefficient $\left(K_{p}\right)$ of 2.7645. There is a slight change in $K_{p}$ values as compared to example 4.1.2; this is anticipated because there is less than a 10 percent difference between the Coulomb and logarithmic spiral $K_{p}$ solutions when $\delta^{\prime}$ is less than or equal to $\varphi^{\prime} / 2$. The active and passive earth pressures for both sides of the sheet-pile wall and all other net pressures are calculated to be similar in shape to those for Example 4.1.2. ${ }^{1}$ The various input conditions and final results for Example 4.1.3 are tabulated as check problem 3 in Table 4.1. The sheet-pile tip elevation is computed at $11.78 \mathrm{ft}$ with a calculated depth of embedment of $18.22 \mathrm{ft}$. With the change from Coulomb coefficient method to the logarithmic spiral method for calculating the passive earth pressures, the calculated sheet-pile tip elevation is approximately the same as that of Example 4.1.2.

For an appreciation of the logarithmic spiral $K_{p}$ solution, $\varphi^{\prime}$ will be assigned a larger value (i.e., $\delta^{\prime}>\varphi^{\prime} / 2$ ). Table 4.2 has three check problems that tabulate the results for three wall friction angles, (i.e., $\delta^{\prime}=\varphi^{\prime} / 2, \delta^{\prime}=3 / 4 \varphi^{\prime}$, and $\delta^{\prime}=\varphi^{\prime}$ ). The first check problem in Table 4.2 is the same as check problem 3 of Table 4.1. As the wall friction angle increased from 15 to $22.5 \mathrm{deg}$, the calculated passive earth pressure coefficient increased. With an increase in earth pressure within the Figure 1.1 passive zones, the system is more stable and the sheet-pile tip elevation is reduced, moved higher, from elevation 11.78 to $12.92 \mathrm{ft}$. This trend is further established by the assignment of the effective angle of soil-to-I-Wall friction of $30 \mathrm{deg}$, the same value as the effective angle of internal friction. Here the sheet-pile tip elevation is reduced to $13.25 \mathrm{ft}$. Increasing the effective angle of wall friction $\delta^{\prime}$ from 15 to 30 deg reduced the required depth of embedment by nearly $1.5 \mathrm{ft}$.

1 For the applied flood loading, the I-Wall will rotate counterclockwise. 
Table 4.2. Results from wall friction angles with $K_{p}$ coefficients from logarithmic spiral procedure.

\begin{tabular}{|l|l|l|l|l|l|l|l|}
\hline $\begin{array}{l}\text { Check } \\
\text { Problems }\end{array}$ & $\delta^{\prime}$ (deg) & $K_{a}$ mob & $K_{p}$ mob & $\frac{2 \boldsymbol{c}^{\prime}}{\sqrt{\boldsymbol{K}_{\boldsymbol{a}}} \boldsymbol{\gamma}_{\text {buoy }}}$ & $\begin{array}{l}\text { Hydraulic } \\
\text { Fracture } \\
\text { Depth }\end{array}$ & $\begin{array}{l}\text { Elevation at } \\
\text { Point of } \\
\text { Rotation }\end{array}$ & $\begin{array}{l}\text { Elevation at } \\
\text { Tip of Pile }\end{array}$ \\
\hline 1 & 15 & 0.3014 & 2.7645 & 0.0000 & NG & 17.3374 & 11.7835 \\
\hline 2 & 22.5 & 0.2963 & 3.0952 & 0.0000 & NG & 18.2122 & 12.9167 \\
\hline 3 & 30 & 0.2972 & 3.2792 & 0.0000 & NG & 18.4685 & 13.2500 \\
\hline
\end{tabular}

Note: All check problems have a single homogeneous sand layer of soil with an effective angle of internal friction $\left(\varphi^{\prime}\right)$ of 30 deg. Factor of Safety is 1.0 for active earth pressures and Factor of Safety is 1.5 for passive earth pressures. Hydrostatic pore water pressures. Passive earth pressure coefficients obtained from logarithmic spiral procedure. All elevations and depths are reported in units of feet.

NG - Hydraulic fracturing (gap) not considered.

\subsubsection{Example 4.1.4}

An effective stress definition with $\varphi^{\prime}=30 \mathrm{deg}$ and $\mathrm{c}^{\prime}=0$ as illustrated in Figure 4.2 is considered for the evaluation of the depth of embedment. Homogeneous soil, hydrostatic pore water pressures, effective angle of soil-to-I-Wall friction $\left(\delta^{\prime}\right)$ of $15 \mathrm{deg}$ as well as the mobilized passive earth pressure coefficient obtained from the logarithmic spiral procedure form the basis for this example. Hydraulic fracturing is considered an option for this analysis. This Example 4.1.4 differs from Example 4.1.3 with the estimation of hydraulic fracturing using the procedure discussed in Appendix B.

The analysis resulted in a computed mobilized active earth pressure coefficient $\left(K_{a}\right)$ of 0.3014 and a mobilized passive earth pressure coefficient $\left(K_{p}\right)$ of 2.7645. The active and passive earth pressures for both sides of the sheet-pile wall and all other net pressures are calculated to be those from Example 4.1.3. ${ }^{1}$ The various input conditions and final results for Example 4.1.4 are tabulated as check problem 4 in Table 4.1. The sheetpile tip elevation is computed at $11.78 \mathrm{ft}$ with a calculated depth of embedment of $18.22 \mathrm{ft}$.

For this analysis with hydrostatic pore water pressures and a cohesion value of zero, the estimated gap depth is $0.0 \mathrm{ft}$ with the elevation at bottom of gap at $30 \mathrm{ft}$ (i.e., at ground surface on the flood side of the I-Wall). The hydraulic fracture procedure (outlined in Appendix B) implemented in Corps_I-Wall provides the same gap depth as the standard equation (which is valid for hydrostatic water pressures only) of Table 4.1 and is given by

\footnotetext{
1 For the applied flood loading, the I-Wall will rotate counterclockwise.
} 


$$
\text { Gap }=\frac{2 c^{\prime}}{\sqrt{K_{a}} \gamma_{\text {buoy }}}
$$

Observe in Equation 4.1 that in order for the standard equation to indicate a non-zero gap depth, the soil must be assigned a value for effective cohesion, $c^{\prime}$, greater than zero.

\subsubsection{Example 4.1.5}

An effective stress definition with $\varphi^{\prime}=30 \mathrm{deg}$ and $\mathrm{c}^{\prime}=0$, as illustrated in Figure 4.2 is considered for the evaluation of the depth of embedment. Homogeneous soil, homogeneous seepage by the line of seepage method (a.k.a., line of creep), effective angle of soil-to-I-Wall friction $\left(\delta^{\prime}\right)$ of $15 \mathrm{deg}$ as well as the coefficients obtained from the logarithmic spiral procedure and hydraulic fracturing form the basis for this example. Example 4.1.5 differs from Example 4.1.4 because of the addition of homogenous seepage.

The analysis resulted in a computed mobilized active earth pressure coefficient $\left(K_{a}\right)$ of 0.3014 and a mobilized passive pressure coefficient $\left(K_{p}\right)$ of 2.7645. The hydrostatic water pressures acting on both sides of the sheetpile wall are shown in Figure 4.7a with the resultant net water pressure calculated by taking the difference between the water pressures acting on both sides of the wall (Figure 4.7a) and shown in Figure 4.7b. Homogeneous seepage in this problem is clearly shown by the net water pressure diagram of Figure $4.7 \mathrm{~b}$, which differs from the net water pressure diagram of Figure $4.4 \mathrm{~b}$ for hydrostatic pore water pressures. The net water pressure diagram of Figure $4.7 \mathrm{~b}$ is first calculated from hydrostatic water pressures from el $40 \mathrm{ft}$ to el $34 \mathrm{ft}$, resulting in the uppermost triangular distribution between these two elevations. Between el $34 \mathrm{ft}$ and el $30 \mathrm{ft}$, the increase in water pressure on both sides of the wall is the same, resulting in a uniform net pressure diagram between these two elevations. At the ground surface elevation of $30 \mathrm{ft}$, which coincides with a gap depth elevation of $30 \mathrm{ft}$, homogeneous seepage calculations are initiated from RHS ground surface down the RHS to the sheet-pile tip and then up the LHS of the sheet pile, concluding at the LHS ground surface. Corps_I-Wall line of seepage (a.k.a., line of creep) computations result in a net water pressure of $0.0 \mathrm{psf}$ at the sheet-pile tip elevation of $11.29 \mathrm{ft}^{1}$

\footnotetext{
1 This Figure 4.7b zero net water pressure computed at the sheet-pile tip from steady-state seepage contrasts with the 374.4 psf net water pressure value at sheet-pile tip for the hydrostatic water pressure condition shown in Figure 4.4b. Additionally, the line of seepage results in a linear variation in net water pressure from the ground surface to the sheet-pile tip in Figure $4.7 \mathrm{~b}$ while the hydrostatic water pressure condition results in a uniform net water pressure distribution from the ground surface to the sheet-pile tip (Figure 4.4b). Higher net water pressures act on the embedded portion of sheet-pile wall when hydrostatic water pressure is specified.
} 
Figure 4.7. Pore water pressures. (a) Left- and right-hand side of wall. (b) Net water pressures.

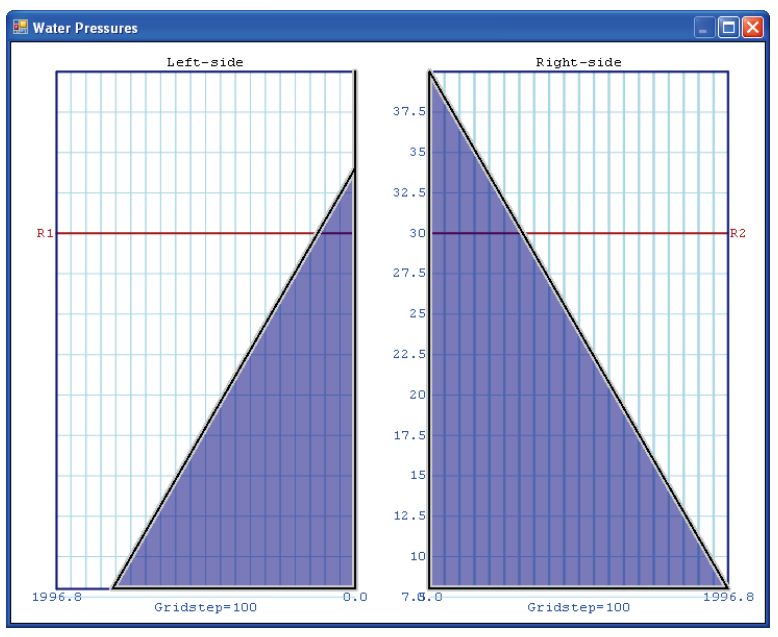

(a)

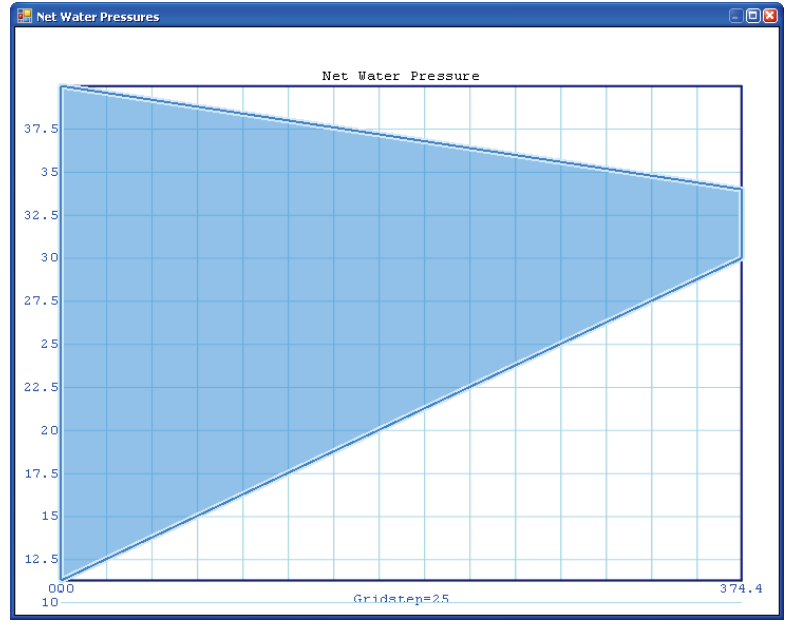

(b)

The active and passive earth pressures for both sides of the sheet-pile wall and all other net pressures are shown to have the same shape as those from Example 4.1.3. For the applied flood loading, the I-Wall will rotate counterclockwise. The various input conditions and final results for Example 4.1.5 are tabulated as check problem 5 in Table 4.1. The sheet-pile tip elevation is computed at $11.29 \mathrm{ft}$ with a depth of embedment of $18.71 \mathrm{ft}$. With the addition of homogeneous seepage, the approximated sheet-pile tip elevation is deeper by $0.5 \mathrm{ft}$. than the $11.78 \mathrm{ft}$ of Example 4.1.4.

This problem required the analysis with homogeneous seepage. An input hydraulic conductivity for sand (i.e., $3.28 \mathrm{e}-5 \mathrm{ft} / \mathrm{sec}$ ) was entered and the constant seepage gradient was calculated to be 0.1603 . Figure 4.8 provides an interpretation of the line of seepage (a.k.a, line of creep) method by a graph of the total head along the seepage path. The first graph (Figure 4.8a), shows that seepage starts at the ground surface of R2 at the RHS of the wall (i.e., flood side), continues down to the sheet-pile tip at $11.29 \mathrm{ft}$, then continues upward towards the ground surface of R1 at the LHS (i.e., landside) of the sheet-pile wall. The second graph (Figure $4.8 \mathrm{~b}$ ) displays total head values of flow along the distance travelled. Total heads are constant from el $40.0 \mathrm{ft}$ to the ground surface at $30.0 \mathrm{ft}$. (vertical red line). The slope of 0.1603 starts at el $30 \mathrm{ft}$ continues past the sheet-pile tip and stops at el $30 \mathrm{ft}$ (vertical green line). Total head values are constant from el $30.0 \mathrm{ft}$ to the water surface at el $34 \mathrm{ft}$ on the LHS of the wall. Further details on the line of seepage method are given in Appendix C. 
Figure 4.8. Line of seepage. (a) Direction of flow. (b) Total head along distance of flow.

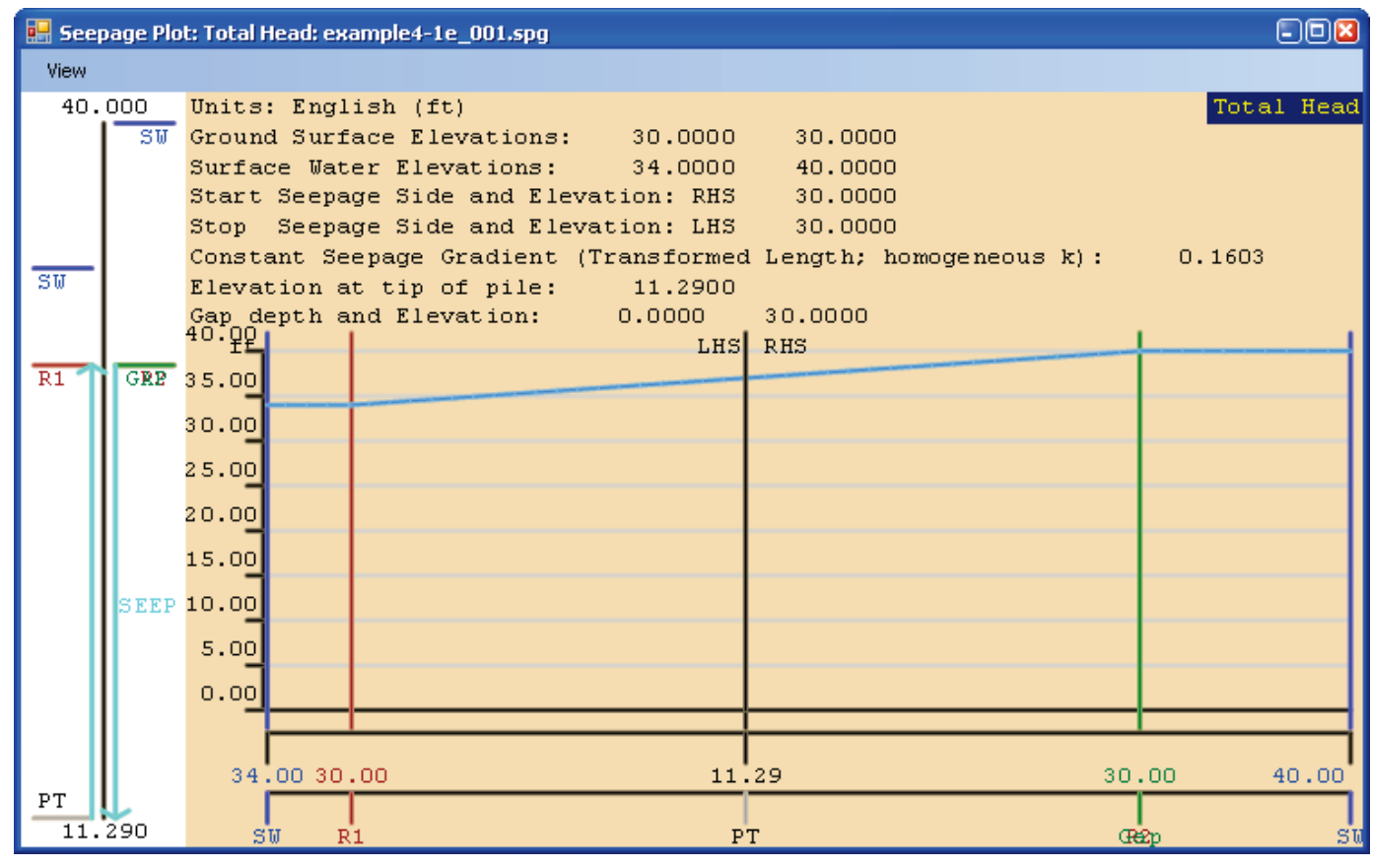

(a)

(b)

\subsection{Example 4.1 sand site, effective stress strength definition with $\varphi^{\prime}=30 \mathrm{deg}$ and $c^{\prime}=125$ psf, with either hydrostatic pore water pressures or homogeneous seepage}

An effective stress strength definition with $\varphi^{\prime}=30$ deg and $c^{\prime}=125 \mathrm{psf}$ (lightly cohesive) is illustrated by a series of three design problems in order to determine the depth of embedment (d) of the sheet-pile wall embedded into a sand site. These example problems apply a Factor of Safety of 1.0 for computing active earth pressures and a Factor of Safety of 1.5 for computing the mobilized passive earth pressures beneath the soil layers.

Figure 4.9 provides a schematic of the basic geometry and structural parameters for the sheet-pile wall. This I-Wall system has an exposed height of $10 \mathrm{ft}$. The water surfaces at both sides of the I-Wall are at $40 \mathrm{ft}$ on the flood side and $34 \mathrm{ft}$ on the landside. The homogeneous sand layer of soil has a level ground surface on both sides of the I-Wall at $30 \mathrm{ft}$ with saturated unit weight of $122.4 \mathrm{pcf}$ and an effective angle of internal friction of $30 \mathrm{deg}$. The top row of listed text documents the input values of all parameters associated with each material type. For this problem, there is only one material assigned for the sand site and recorded as "Mo1"; the "M" signifies the material type and is followed by the ID, which starts from "01." All information concerning material types is written in black text. The 
representation of a soil structure is characterized by a region. Regions are labeled with an "R" and followed by an ID starting from "01." For this problem, two regions are assigned; Region 1 (as Ro1), is located on the LHS of the sheet-pile wall, and Region 2 (as Ro2) is located on the RHS of the sheet-pile wall. Each region and its surface elevation are written in red text. Piezometric surfaces are labeled as "P" and followed by an ID starting from 01. Piezometric water surfaces are listed in the upper left-hand corner and drawn with the water symbol at its defined elevation and shown in blue. Observe that piezometric surface ID, Po1, is assigned to both regions. The LHS piezometric surface elevation is assigned as the LHS surface water elevation. Similarly, the RHS piezometric surface elevation is assigned as the RHS surface water elevation. This allows for the assignment of submerged unit weights to the soil regions. This geometry will be used in Example 4.2.1 and Example 4.2.3.

Figure 4.9. Corps_I-Wall schematic of sheet-pile wall in sand site.

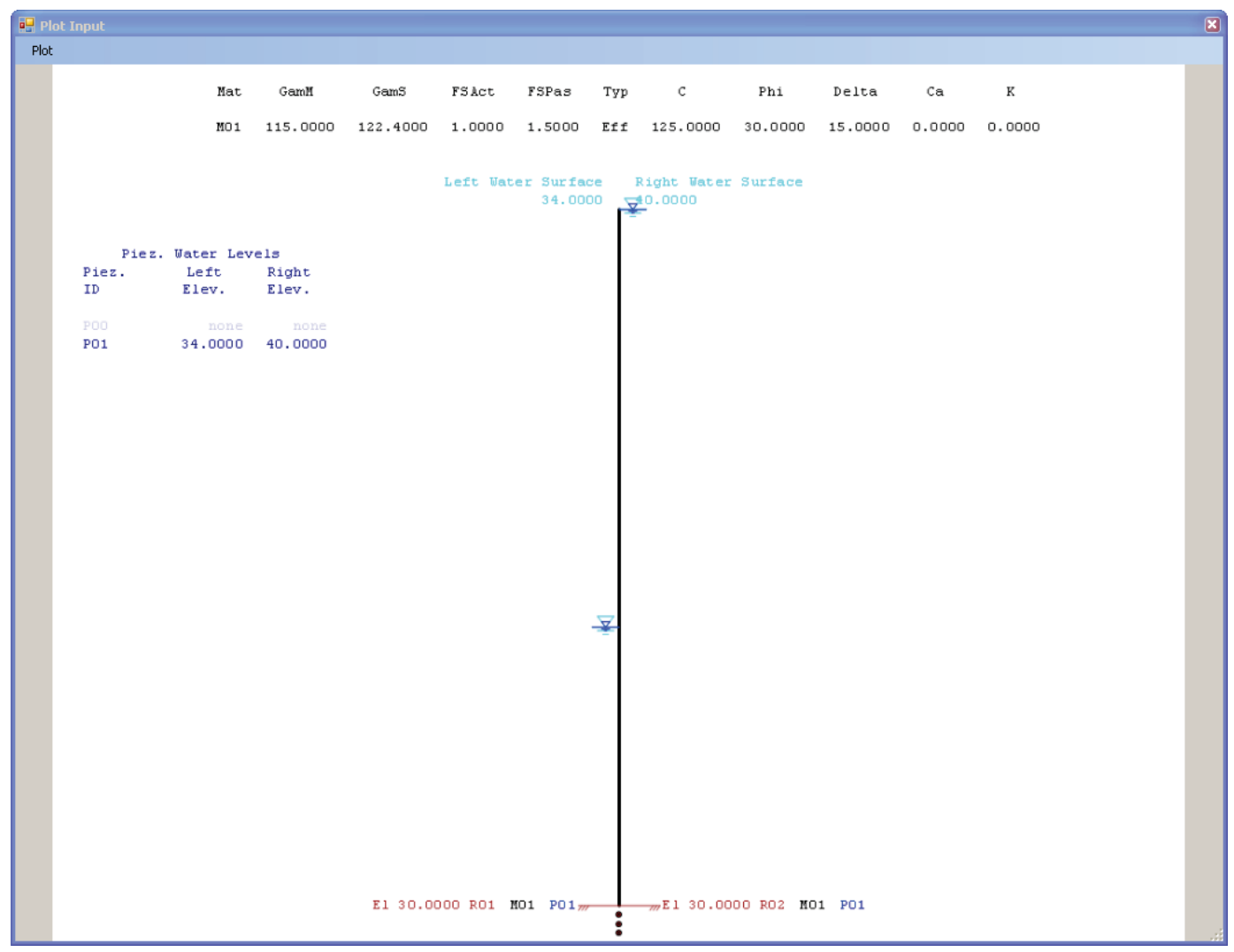

The series of subsection 4.2 problems differ from the series of subsection 4.1 problems in that an effective cohesion value of $125 \mathrm{psf}$ is assigned to the homogenous sand site in subsection 4.2 problems. 


\subsubsection{Example 4.2.1}

An effective stress definition with $c^{\prime}=125$ (lightly cohesive) as illustrated in Figure 4.9 is considered for the evaluation of the depth of embedment. Homogeneous soil, hydrostatic pore water pressures, effective angle of internal friction $\left(\varphi^{\prime}\right)$ of $30 \mathrm{deg}$, effective angle of soil-to-I-Wall friction $\left(\delta^{\prime}\right)$ of $15 \mathrm{deg}$, hydraulic fracturing as well as the logarithmic spiral procedure for calculating $K_{p}$ form the basis for this example.

The analysis resulted in a mobilized active earth pressure coefficient $\left(K_{a}\right)$ of 0.3014 and a mobilized passive pressure coefficient $\left(K_{p}\right)$ of $2.7645 .{ }^{1}$ The active and passive earth pressures for both sides of the sheet-pile wall are illustrated in Figures 4.10a and 4.10b. The hydrostatic water pressures acting on both sides of the sheet-pile wall are shown in Figure 4.11a with the resultant net water pressure calculated by taking the difference between the water pressures acting on both sides of the wall (Figure 4.11a) and shown in Figure 4.11b.

From these pressures (Figures 4.10 and 4.11), the net active and net passive pressures are constructed and presented in Figure 4.12a. For the applied flood loading, the I-Wall will rotate counterclockwise. The net active pressure is determined from the difference between the RHS-mobilized active earth pressure and the LHS-mobilized passive earth pressure (i.e., acting on the other side of the wall) with the addition of any net water pressures and any external horizontal net pressures and overburden pressures (if any). The net passive pressure is determined by the difference between the RHS-mobilized passive earth pressure and the LHS- mobilized active earth pressure acting on the other side of the sheet-pile wall with the addition of any net water pressures and any external horizontal net pressures and overburden pressures (if any). The net pressure diagram of Figure 4.12b is derived from the replication of all values of the net active pressures, from the top of the wall until the elevation at the point of rotation (el $20.6843 \mathrm{ft}$ ), at which instant the remaining values of the net pressure diagram is assumed to be linear with elevation between (1) the net active pressure value at the elevation of the point of rotation and (2) the netmobilized passive pressure at the elevation of the approximated sheet-pile tip (i.e., el 15.8704). ${ }^{2}$

\footnotetext{
${ }^{1}$ Recall that an $F S_{\text {passive }}$ value equal to 1.5 was specified in this example.

2 Thus, only one mobilized net passive pressure value in Figure 4.12a is used to construct the Figure 4.12b net pressure diagram; corresponding to the sheet-pile tip elevation at el 15.8704. The majority of the net pressure diagram is derived from the Figure 4.12a net active pressure diagram.
} 
Figure 4.10. Active and passive earth pressures. (a) Left-hand side of wall. (b) Right-hand side of wall.

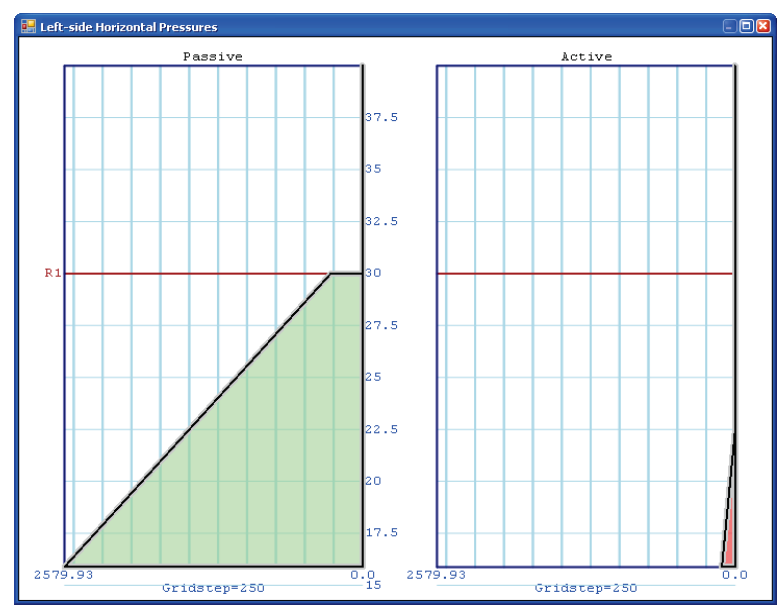

(a)

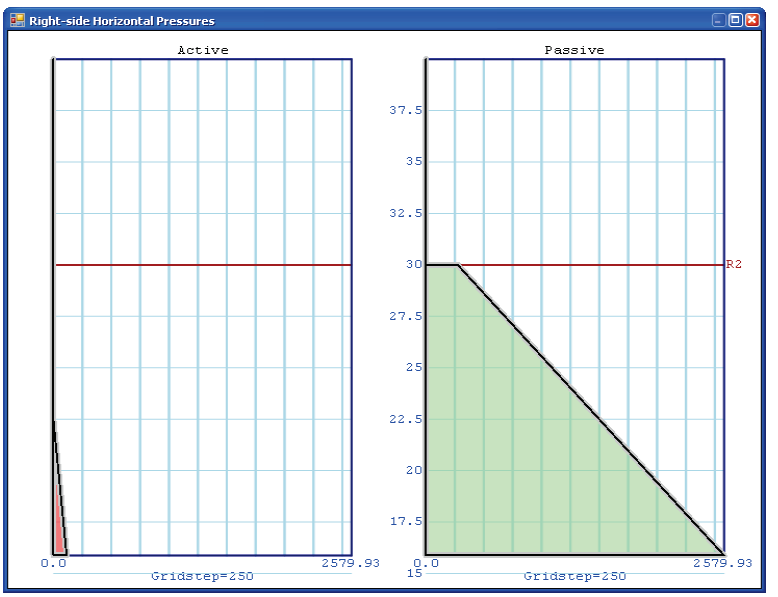

(b)

Figure 4.11. Pore water pressures. (a) Left- and right-hand side of wall. (b) Net water pressures.

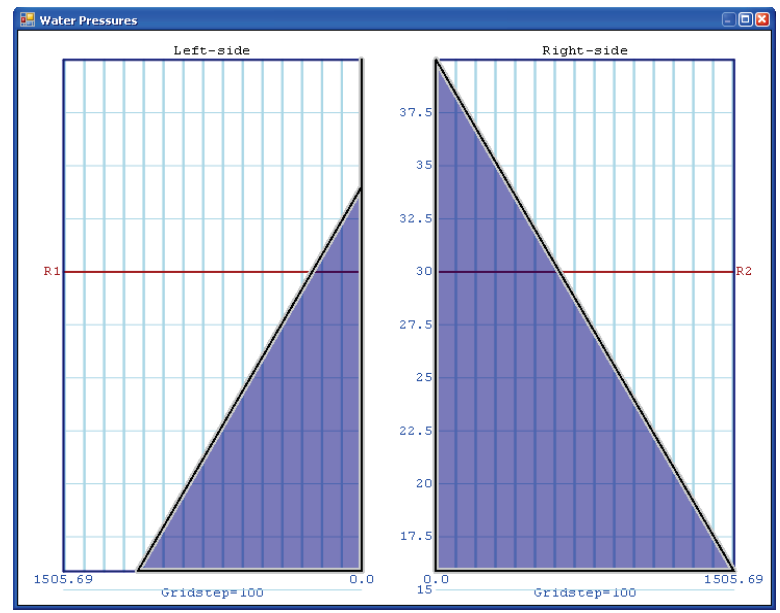

(a)

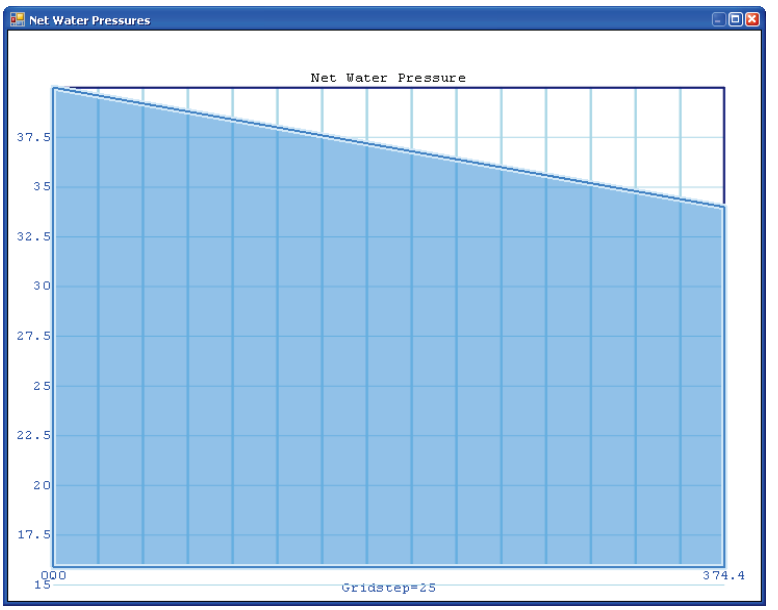

(b)

Figure 4.12. Net pressures. (a) Net active and net passive. (b) Net pressure diagram.

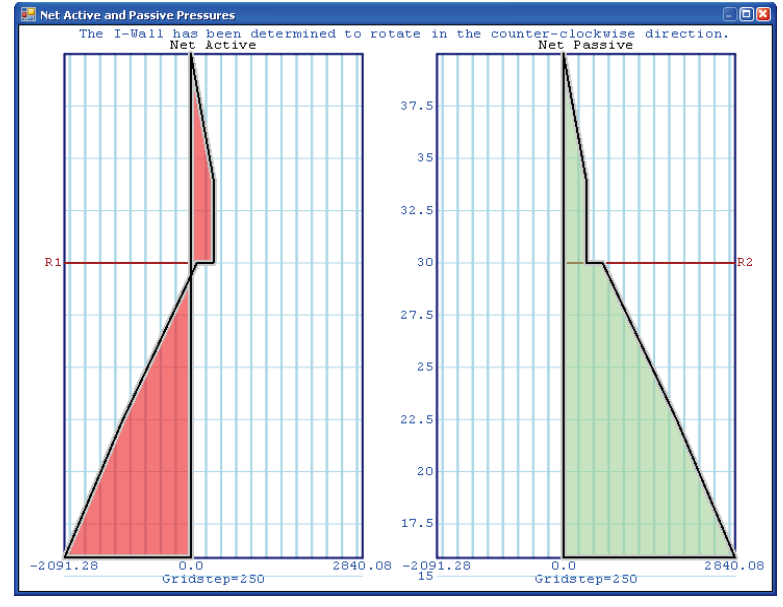

(a)

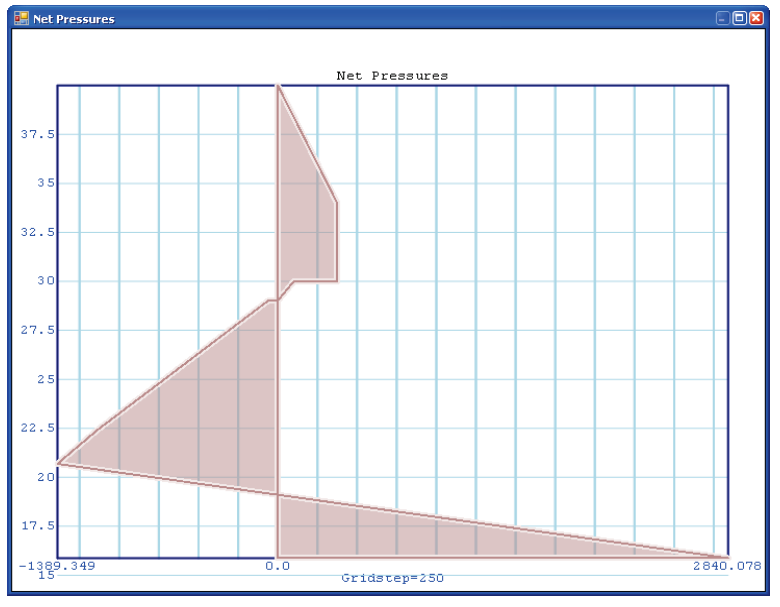

(b) 
The final results are illustrated in Figure 4.13 by the presentation of the net pressure diagram Figures 4.13a (replica of Figure 4.12b) and 4.13b, the shear and bending moment diagrams. Figure $4.13 \mathrm{~b}$ shows that the maximum bending moment occurs at the zero crossing of the shear diagram.

Figure 4.13. (a) Net pressure diagram. (b) Shear and moment.

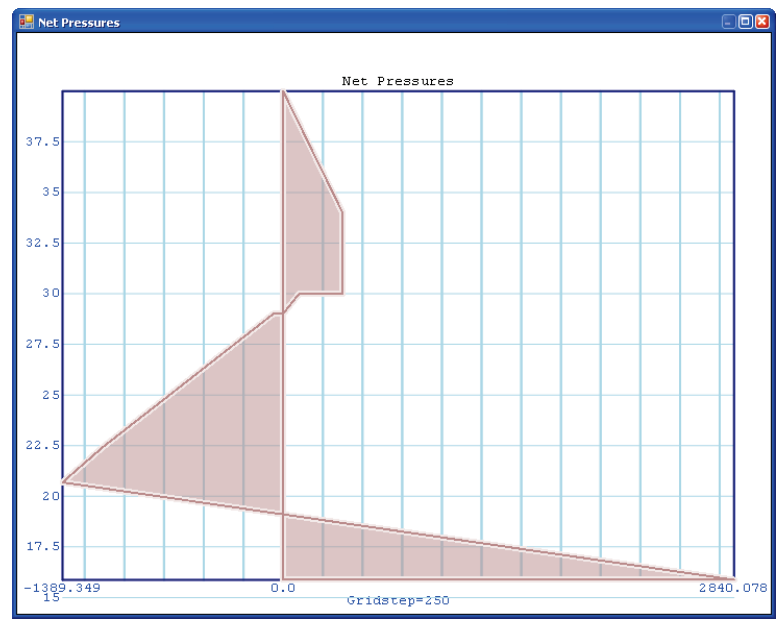

(a)

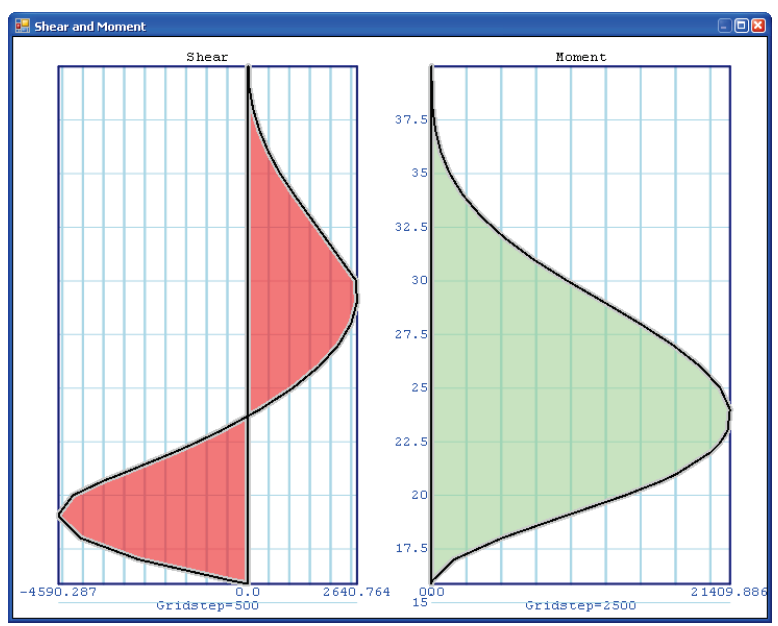

(b)

The various input conditions and final results for Example 4.2.1 are tabulated as check problem 1 in Table 4.3. For a gap depth of approximately $7.59 \mathrm{ft}$, the sheet-pile tip elevation is computed at $15.87 \mathrm{ft}$ with a calculated depth of embedment of $14.13 \mathrm{ft}$.

Table 4.3. Examples of effective stress strength definitions (sands with $\left.c^{\prime}=125\right)$.

\begin{tabular}{|c|c|c|c|c|c|c|c|c|c|c|}
\hline $\begin{array}{l}\text { Check } \\
\text { Problems }\end{array}$ & $\begin{array}{l}\text { Seepage } \\
\text { Gradient }\end{array}$ & $\begin{array}{l}\text { No. } \\
\text { Layers }\end{array}$ & $\begin{array}{l}c 1^{\prime} \\
\text { (psf) }\end{array}$ & $\begin{array}{l}c 2^{\prime} \\
\text { (psf) }\end{array}$ & $K_{a} \mathrm{mob}$ & $K_{p}$ mob & $\frac{2 c^{\prime}}{\sqrt{K_{a}} \gamma_{b u o y}}$ & $\begin{array}{l}\text { Hydraulic } \\
\text { Fracture } \\
\text { Depth }\end{array}$ & $\begin{array}{l}\text { Elevation } \\
\text { at Point of } \\
\text { Rotation }\end{array}$ & $\begin{array}{l}\text { Elevation } \\
\text { at Tip of } \\
\text { Pile }\end{array}$ \\
\hline 1 & $\mathrm{HY}$ & 1 & 125 & - & 0.3014 & 2.7645 & 7.5894 & 7.5894 & 20.6843 & 15.8704 \\
\hline 2 & $\mathrm{HY}$ & 2 & 125 & 0 & 0.3014 & 2.7645 & 7.5894 & 5.0000 & 19.1238 & 14.2894 \\
\hline 3 & 0.2760 & 1 & 125 & - & 0.3014 & 2.7645 & 7.5894 & 8.2576 & 19.0000 & 15.0000 \\
\hline
\end{tabular}

Note: All check problems have sand layers of soil with an effective angle of internal friction $\left(\varphi^{\prime}\right)$ of 30 deg and effective angle of wall friction $\left(\delta^{\prime}\right)$ of 15 deg. Factor of Safety is 1.0 for active earth pressures and Factor of Safety is 1.5 for passive earth pressures. Passive earth pressure coefficients obtained from logarithmic spiral procedure. HY refers to Hydraulic Fracturing. All elevations and depths are reported in units of feet.

HY - Hydrostatic pore water pressure.

\subsubsection{Example 4.2.2}

A sand site using an effective stress definition with $\varphi^{\prime}=30 \mathrm{deg}$ as illustrated in Figure 4.14 is considered for the evaluation of the depth of embedment. A two-layer sand soil with $\mathrm{c}^{\prime}=125 \mathrm{psf}$ (lightly cohesive) for 
the upper sand layer at el $30 \mathrm{ft}$ and $\mathrm{c}^{\prime}=\mathrm{o}$ for the lower layer sand at el $25 \mathrm{ft}$. Hydrostatic pore water pressures, effective angle of soil-to-I-Wall friction $\left(\delta^{\prime}\right)$ of $15 \mathrm{deg}$, hydraulic fracturing as well as the logarithmic spiral procedure form the basis for this example. Example 4.2.2 differs from Example 4.2.1 by the introduction of a second layer of sandy soil with $\mathrm{c}^{\prime}=\mathrm{O}$ and all other soil parameters retaining the same values.

Figure 4.14. Corps_I-Wall schematic of sheet-pile wall in sand site with two layers.

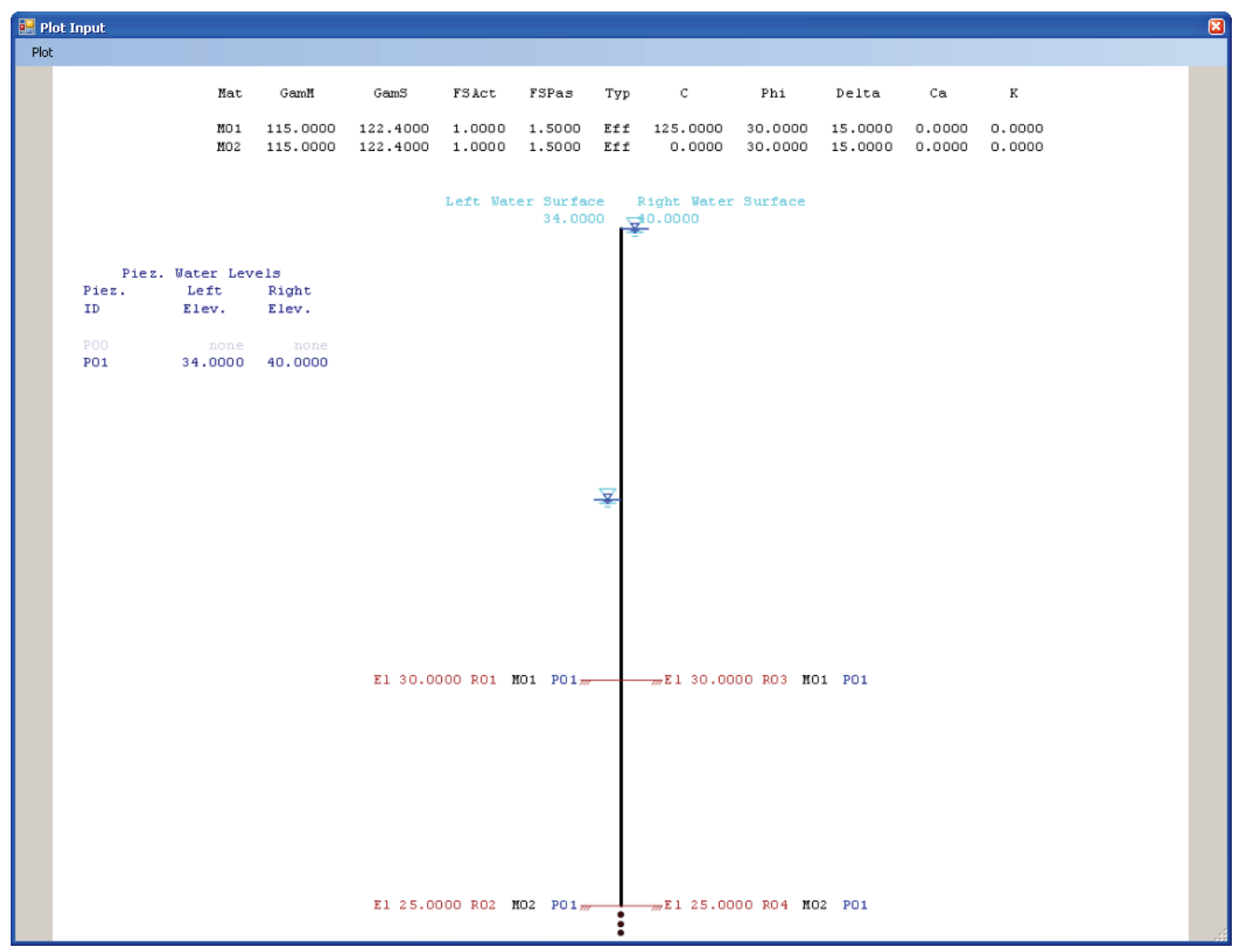

Figure 4.14 provides a schematic of the basic geometry and structural parameters for the sheet-pile wall. This I-Wall has an exposed height of $10 \mathrm{ft}$. The water surfaces at both sides of the I-Wall are at $40 \mathrm{ft}$ on the flood side and $34 \mathrm{ft}$ on the landside. There are two material types and four regions. Each sand layer has a level ground surface on both sides of the I-Wall at $30 \mathrm{ft}$ and at $25 \mathrm{ft}$ with saturated unit weight of $122.4 \mathrm{pcf}$ and an effective angle of internal friction of $30 \mathrm{deg}$.

The top row of text listed documents the input values of all parameters associated with each material type. For this problem, there is only one material assigned for the sand site and recorded as "Mo1," the "M" signifies the material type and is followed by the ID which starts from "01." All 
information concerning material types are written in black text. The representation of a soil structure is characterized by a region. Regions are labeled with an "R" and followed by an ID starting from "01." For this problem two regions are assigned, Region 1 (as Ro1), is located on the LHS of the sheet-pile wall and Region 2 (as Ro2) is located on the RHS of the sheet-pile wall. Each region and its surface elevation are written in red text. Piezometric surfaces are labeled as "P" and followed by an ID starting from 01. Piezometric water surfaces are listed in the upper left-hand corner, drawn with the water symbol at its defined elevation, and shown in blue. Observe that piezometric surface ID, Po1, is assigned to all four regions. The LHS piezometric surface elevation is assigned as the LHS surface water elevation. Similarly, the RHS piezometric surface elevation is assigned as the RHS surface water elevation. This allows for the assignment of submerged unit weights to the four soil regions.

The analysis resulted in a computed mobilized active earth pressure coefficient $\left(K_{a}\right)$ of 0.3014 and a mobilized passive pressure coefficient $\left(K_{p}\right)$ of 2.7681. The active and passive earth pressures for both sides of the sheetpile wall are illustrated in Figures $4.15 \mathrm{a}$ and $4.15 \mathrm{~b}$ for this two soil layer problem. Note the change in earth pressures at el $25 \mathrm{ft}$, which is the interface of Region 1 and Region 2 (labeled R2). The hydrostatic water pressures acting on both sides of the sheet-pile wall are the same as the water pressures of Example 4.2.1 and shown in Figure 4.11a with the resultant net water pressure calculated by taking the difference between the water pressures acting on both sides of the wall (Figure 4.11a) and shown in Figure 4.11b.

Figure 4.15. Active and passive earth pressures. (a) Left-hand side of wall. (b) Right-hand side of wall.

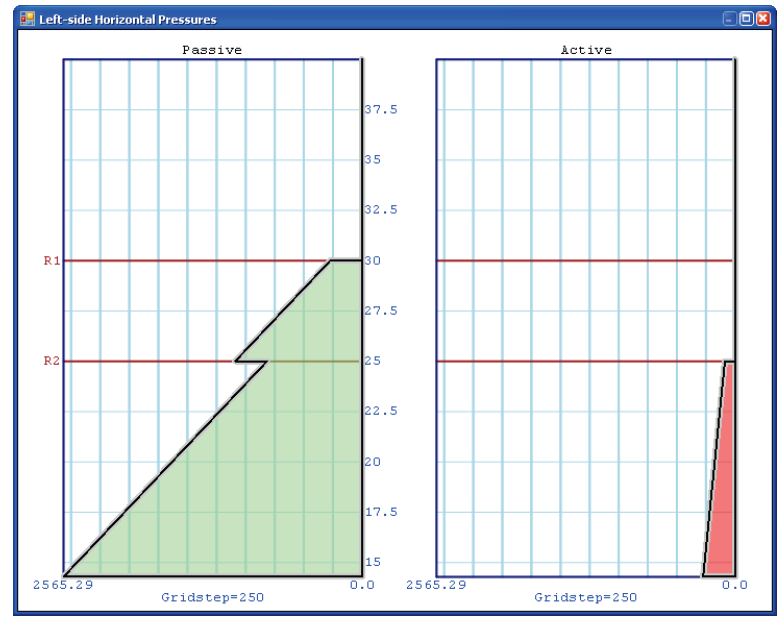

(a)

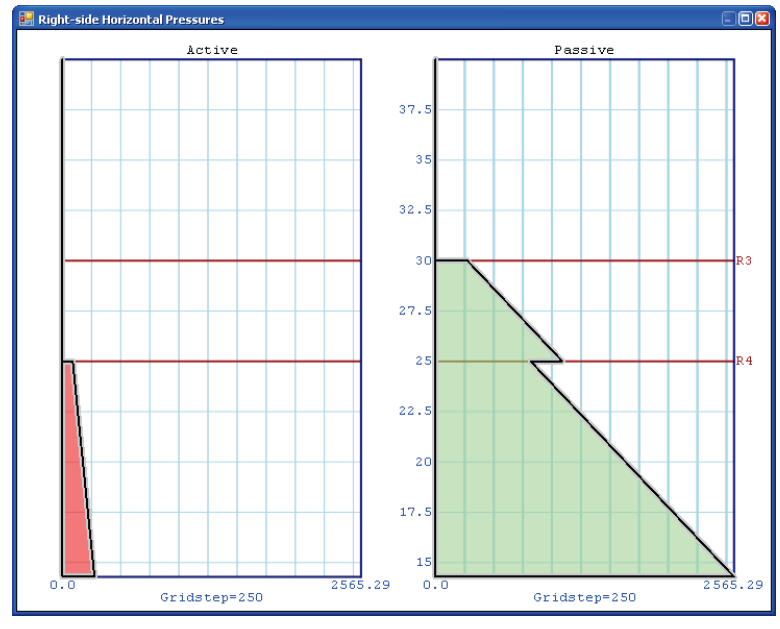

(b) 
From the calculated pressures, the net active and net passive pressures are estimated. For the applied flood loading, the I-Wall will rotate counterclockwise. The net pressure diagram of Figure 4.16b is derived from the replication of all values of the net active pressures, from the top of the wall until the values of the net pressure diagram is assumed to be linear with elevation between (1) the net active pressure value at the elevation of the point of rotation and (2) the net mobilized passive pressure at the elevation of the approximated sheet-pile tip (i.e., el 14.2894). ${ }^{1}$ The final results are illustrated in Figure 4.17 by the presentation of the net pressure diagram Figures 4.17a (replica of Figure 4.16b) and 4.17b, the shear and bending moment diagrams. Figure $4.17 \mathrm{~b}$ shows that the maximum bending moment occurs at the zero crossing of the shear diagram.

The various input conditions and final results for Example 4.2.2 are tabulated as check problem 2 in Table 4.3. The sheet-pile tip elevation is computed at $14.29 \mathrm{ft}$ with a calculated depth of embedment of $15.71 \mathrm{ft}$. With the addition of second sand layer with $\mathrm{c}^{\prime}=0$ at el $25 \mathrm{ft}$ as compared with Example 4.2.1, the approximated sheet-pile tip elevation is calculated higher than the $15.87 \mathrm{ft}$ of Example 4.2.1. This is reasonable as the change in effective cohesion of $\mathrm{c}^{\prime}=\mathrm{O}$ at el $25 \mathrm{ft}$ "arrests" (i.e., stops) the gap, at which the (positive) active RHS earth pressures will start and increase with decreasing elevation along the sheet-pile wall until the point of rotation is reached (at el $19.12 \mathrm{ft}$ ). Because (active) earth pressures acting on the wall occur at a higher (RHS) elevation, the sheet-pile tip will be calculated at a higher elevation and the I-Wall will have a shallower depth of embedment.

Figure 4.16. Net pressures. (a) Net active and net passive. (b) Net pressure diagram.

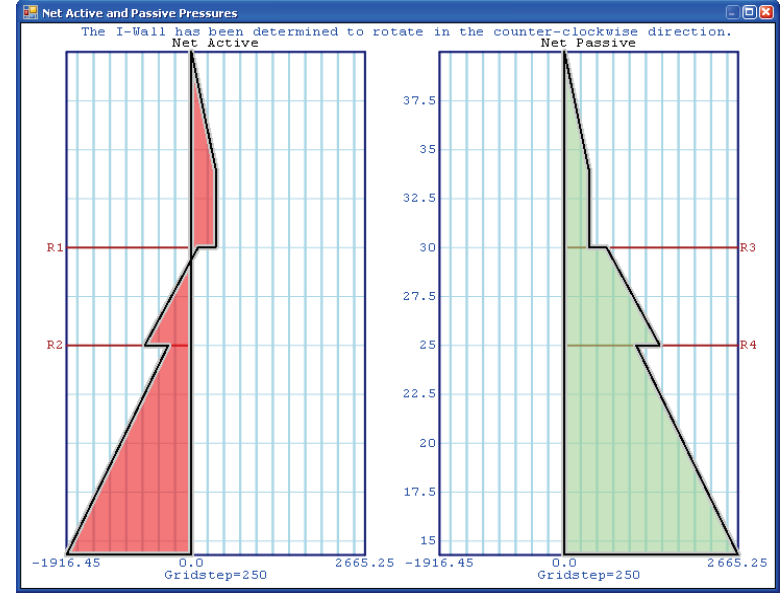

(a)

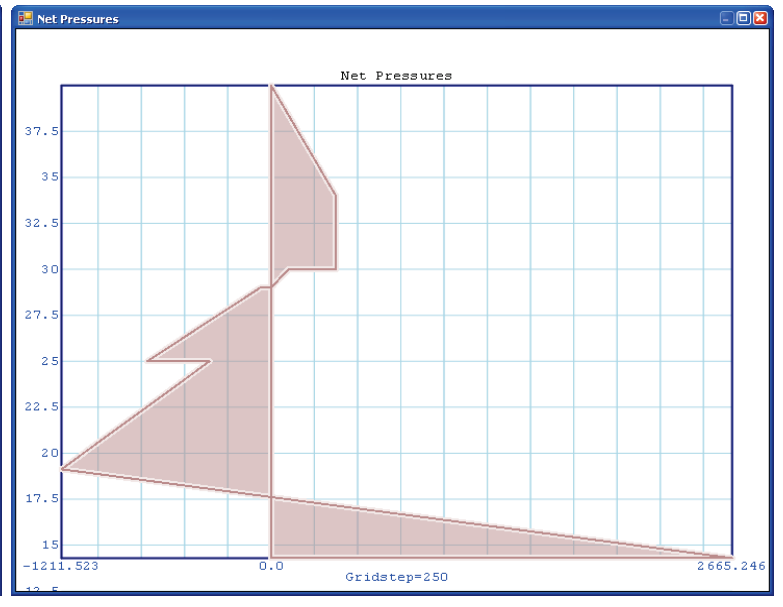

(b)

1 Thus, only one mobilized net passive pressure value in Figure 4.16a is used to construct the Figure 4.16b net pressure diagram, corresponding to the sheet-pile tip elevation at el 14.2894. The majority of the net pressure diagram is derived from the Figure 4.16a net active pressure diagram. 
Figure 4.17. (a) Net pressure diagram. (b) Shear and moment.

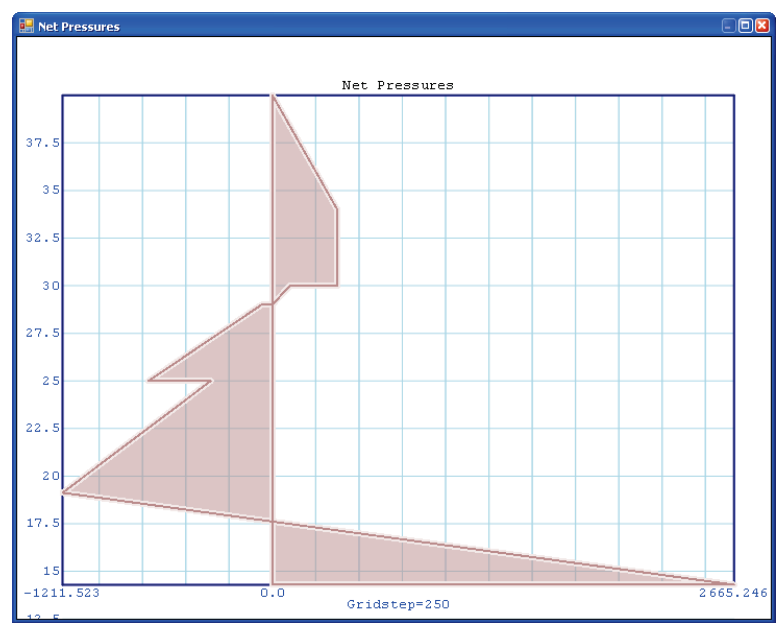

(a)

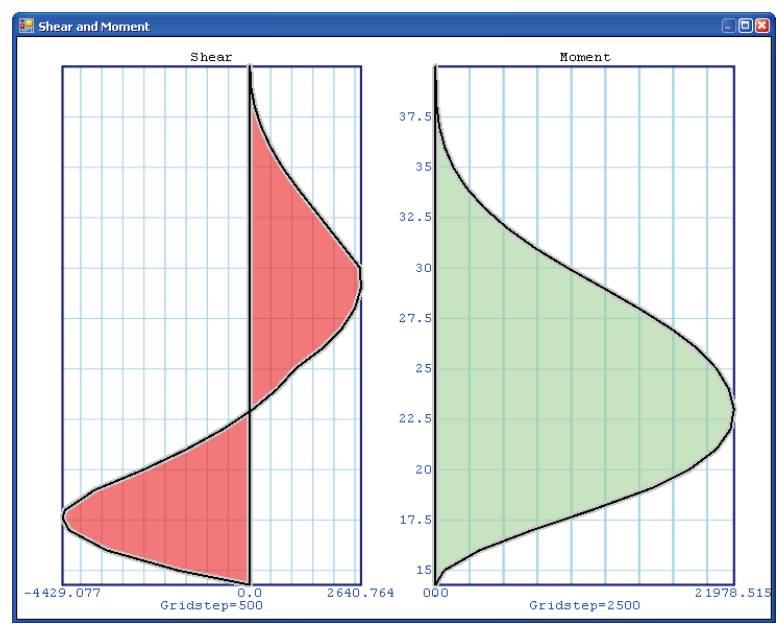

(b)

\subsubsection{Example 4.2.3}

A sand site using an effective stress definition with $\varphi^{\prime}=30 \mathrm{deg}$ and $\mathrm{c}^{\prime}=$ $125 \mathrm{psf}$ (lightly cohesive), as illustrated in Figure 4.9 is considered for the evaluation of the depth of embedment. Homogeneous soil, homogeneous seepage by the line of seepage method, effective angle of soil-to-I-Wall friction $\left(\delta^{\prime}\right)$ of $15 \mathrm{deg}$, as well as the passive earth pressure coefficient obtained from the logarithmic spiral procedure and hydraulic fracturing, form the basis for this example. Example 4.2.3 differs from Example 4.2.1 with the addition of homogenous seepage (versus hydrostatic pore water pressures).

The analysis resulted in a computed mobilized active earth pressure coefficient $\left(K_{a}\right)$ of 0.3014 and a mobilized passive pressure coefficient $\left(K_{p}\right)$ of 2.7645. The active and passive earth pressures for both sides of the sheet-pile wall are illustrated in Figure 4.18. Note the earth pressures at the gap elevation of $21.74 \mathrm{ft}$ (i.e., a gap depth of $8.26 \mathrm{ft}$ ).

The hydrostatic water pressures acting on both sides of the sheet-pile wall are shown in Figure 4.19a with the resultant net water pressure calculated by taking the difference between the water pressures acting on both sides of the wall (Figure 4.19a) and shown in Figure 4.19b. Homogeneous seepage in this problem is clearly shown by the net water pressure diagram of Figure $4.19 \mathrm{~b}$ which differs from the net water pressure diagram of Figure 4.11b for hydrostatic pore water pressures. The net water pressure diagram of Figure 4.19b is first calculated by evaluating hydrostatic water pressures from el $40 \mathrm{ft}$ to el $30 \mathrm{ft}$. A gap is initiated at the ground surface 
elevation (i.e., $30 \mathrm{ft}$ ). Corps_I-Wall computed a gap depth equal to $8.26 \mathrm{ft}$; corresponding to a gap tip el of $21.74 \mathrm{ft}$. Figure 4.19a shows the RHS water pressures to be hydrostatic from el $40 \mathrm{ft}$ down to the gap tip el of $21.74 \mathrm{ft}$. The net water pressures will decrease linearly under this hydrostatic condition until the gap is arrested at el $21.74 \mathrm{ft}$. Homogeneous seepage calculations are then initiated on the RHS; these steady-state seepage calculations will continue down the I-Wall where the net water pressure will decrease to zero at the sheet-pile tip elevation of $11.29 \mathrm{ft}$; then upwards on the LHS face of the sheet-pile wall until the LHS ground surface is met at el $30 \mathrm{ft}$.

Figure 4.18. Active and passive earth pressures. (a) Left-hand side of wall, (b) Right-hand side of wall.

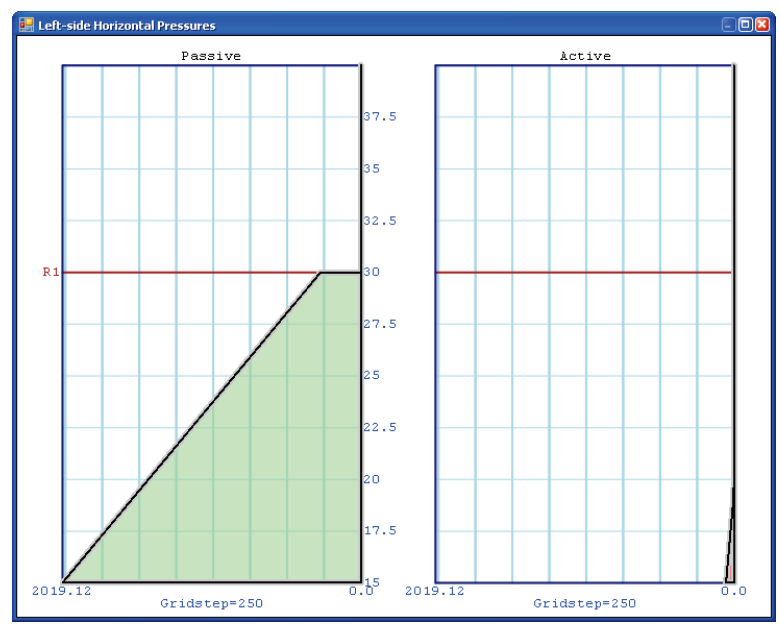

(a)

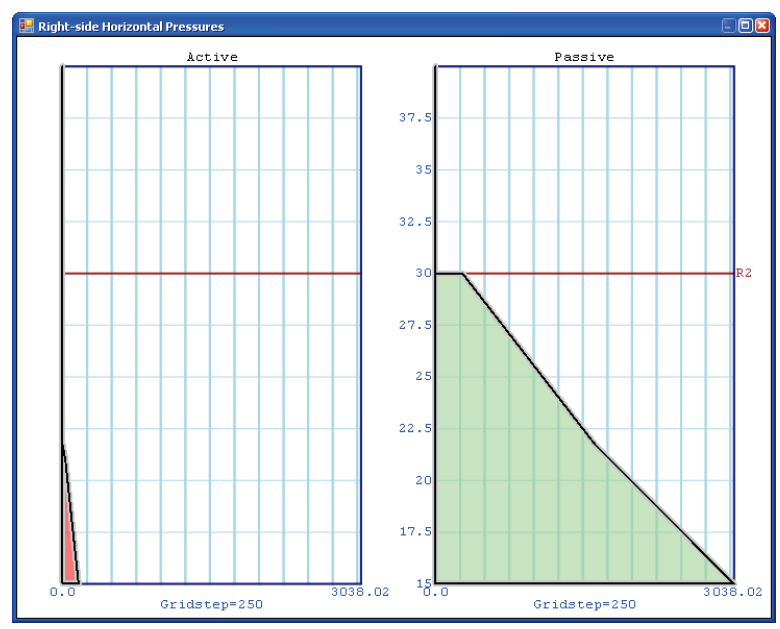

(b)

Figure 4.19. Pore water pressures. (a) Left- and right-hand side of wall. (b) Net water pressures.

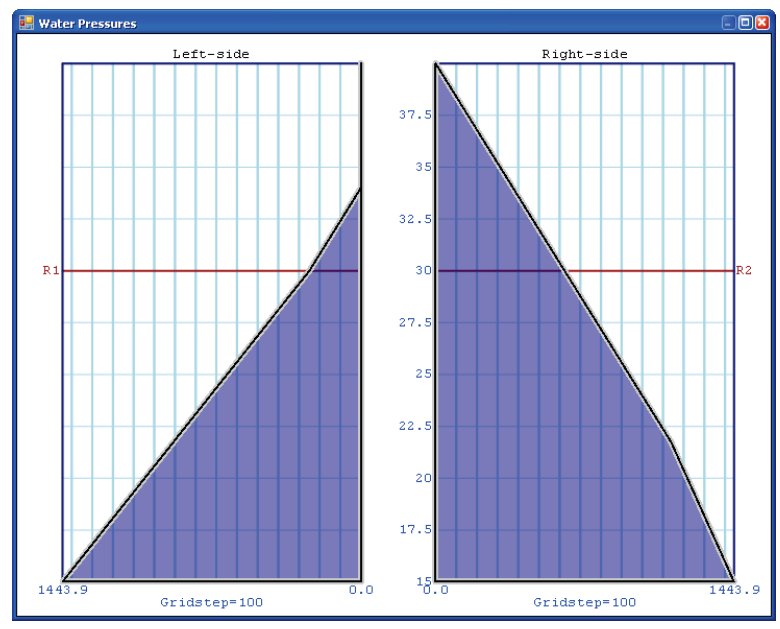

(a)

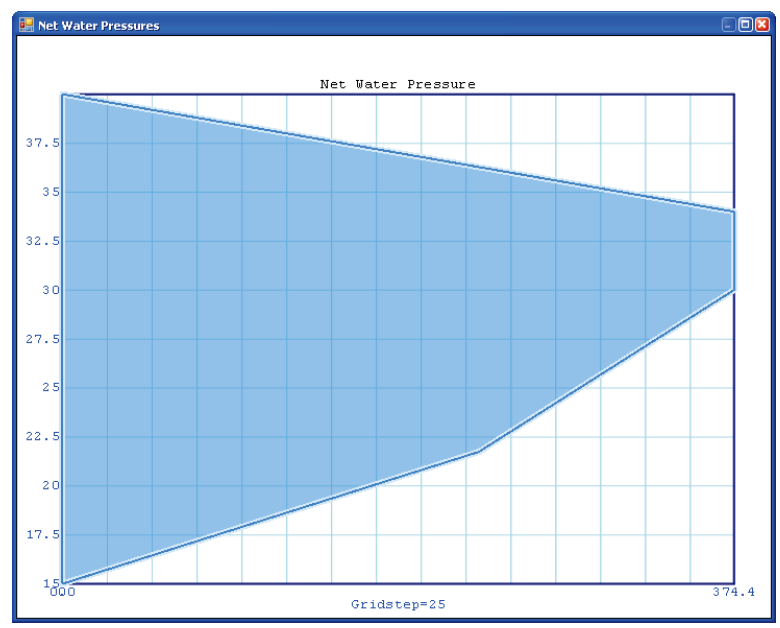

(b) 
The active and passive earth pressures for both sides of the sheet-pile wall and all other net pressures are shown to have the same shape as those from Example 4.1.3. ${ }^{1}$ The resulting net active and net passive earth pressures as well as the net pressure diagram is shown in Figure 4.20. Figure 4.21 shows the resulting distribution shear and moment internal to the sheet-pile wall. The various input conditions and final results for Example 4.1.5 are tabulated as check problem 5 in Table 4.1. The sheet-pile tip elevation is computed at $15.00 \mathrm{ft}$ with a depth of embedment of $15.00 \mathrm{ft}$. With the addition of homogeneous seepage, the approximated sheet-pile tip elevation is shallower by $0.87 \mathrm{ft}$ than the $15.87 \mathrm{ft}$ of Example 4.2.1.

Figure 4.20. Net pressures. (a) Net active and net passive. (b) Net pressure diagram.

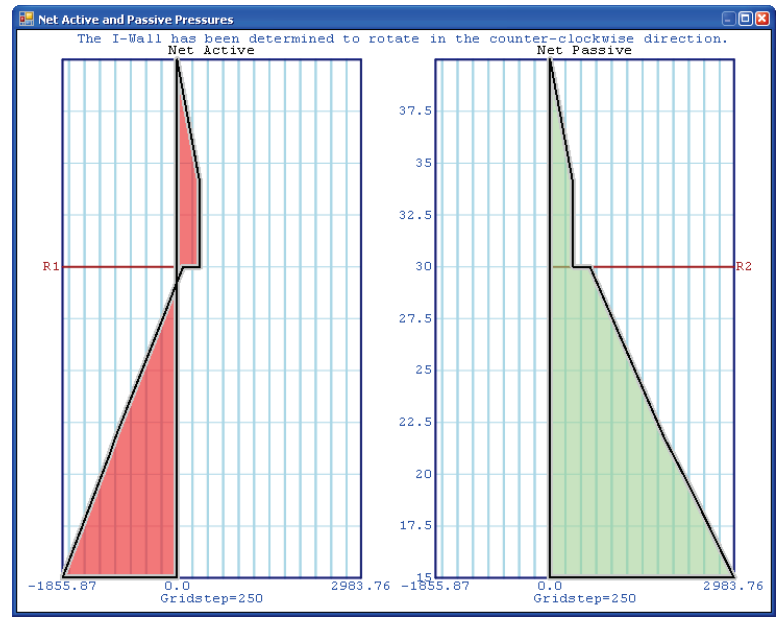

(a)

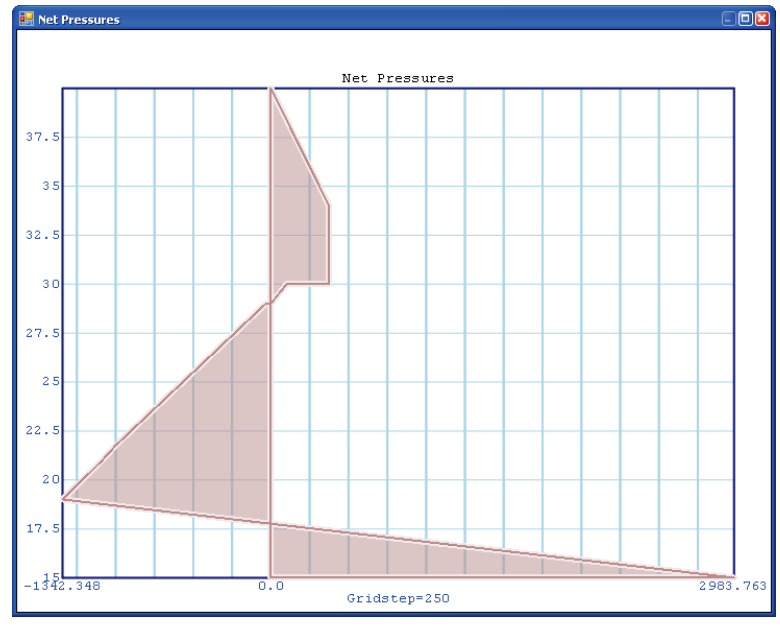

(b)

Figure 4.21. (a) Net pressure diagram. (b) Shear and moment.

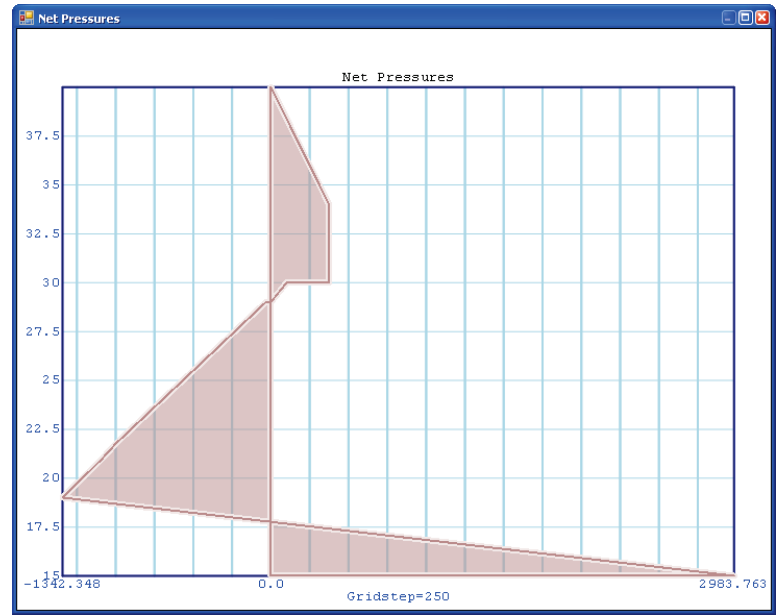

(a)

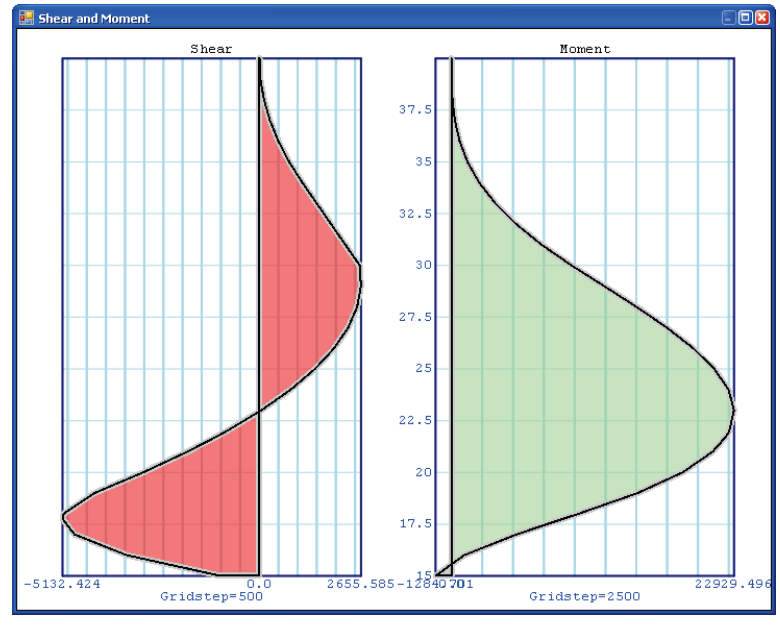

(b)

\footnotetext{
${ }^{1}$ For the applied flood loading, the I-Wall will rotate counterclockwise.
} 
Due to seepage and the presence of lightly cohesive soil regions, this problem required the analysis with homogeneous seepage and with hydraulic fracturing. An input hydraulic conductivity for sand (i.e., 3.28e$5 \mathrm{ft} / \mathrm{sec}$ ) was entered and the constant seepage gradient was calculated to be 0.2760 . Figure 4.22 provides an interpretation of the line of seepage method by a graph of the total head along the seepage path. The first graph or Figure 4.22a, shows that seepage starts the gap elevation of $21.74 \mathrm{ft}$ of R2 at the RHS of the wall (i.e., flood side), continues down to the sheetpile tip at $15.00 \mathrm{ft}$, then continues upward towards the ground surface of R1 at the LHS (i.e., landside) of the sheet-pile wall. The second graph or Figure 4.22b displays total head values of flow along the distance travelled.

Figure 4.22. Line of seepage. (a) Direction of flow. (b) Total head along distance of flow.

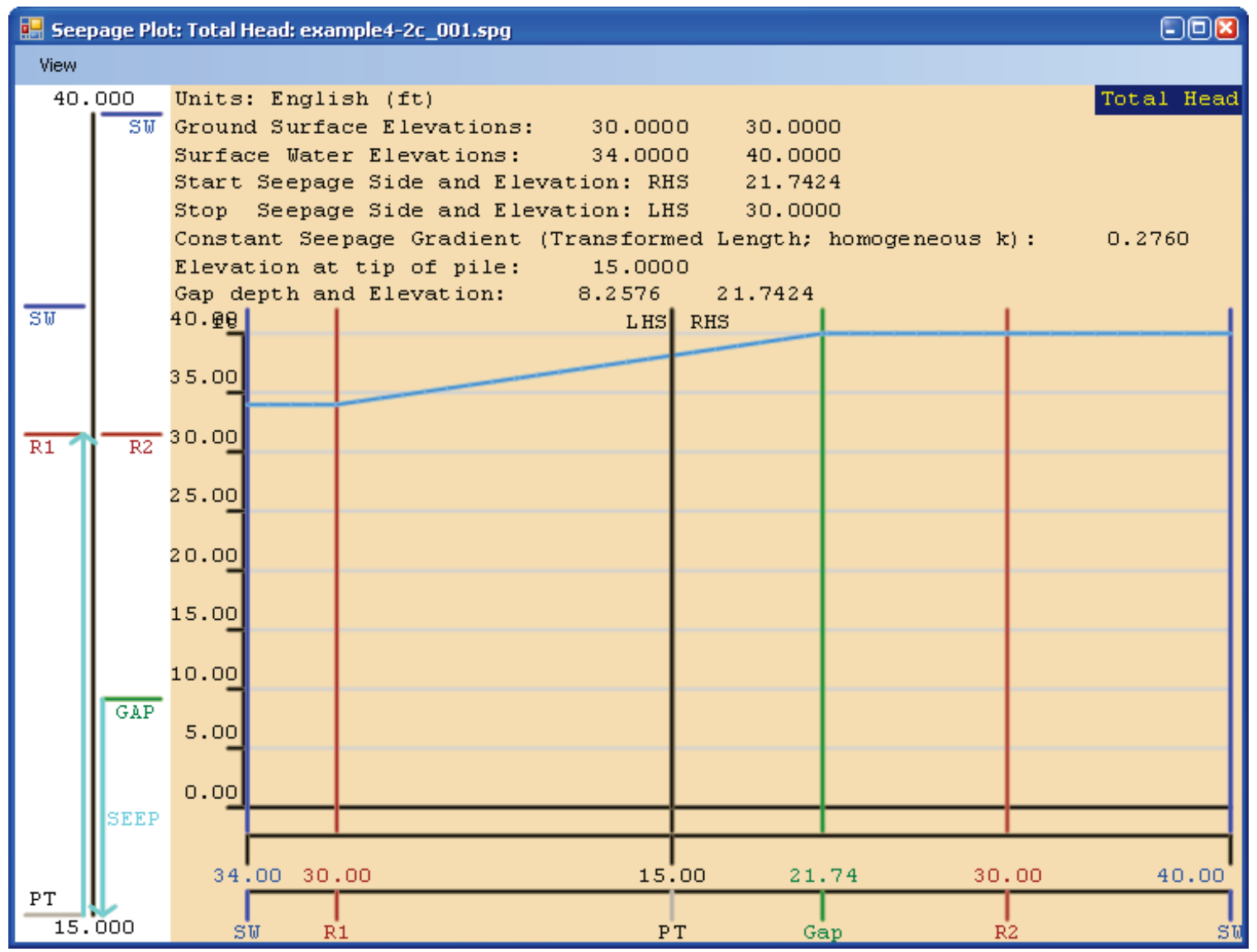

(a)

(b)

Total heads are constant from el $40.0 \mathrm{ft}$ to the gap elevation at $21.74 \mathrm{ft}$ (vertical red line at R2). The slope of 0.2760 starts at the gap elevation of $21.74 \mathrm{ft}$ on the LHS, continues past the sheet-pile tip and stops at el $30 \mathrm{ft}$ (vertical green line) on the RHS. Total head values are constant from el $30.0 \mathrm{ft}(\mathrm{R} 1)$ to the water surface at el $34 \mathrm{ft}$ on the LHS of the wall. 


\subsection{Example 4.3 clay site, total stress strength definition with $\phi=0$}

A total stress definition with the angle of internal friction of ( $\varphi$ ) of o deg and two material layers, where the upper clay layer differs with the undrained shear strength (c) of $300 \mathrm{psf}$ and the lower clay layer with $\mathrm{c}=400 \mathrm{psf}$ is illustrated by this design problem to determine the depth of embedment. In this example problem, a Factor of Safety of 1.0 is assigned for computing active earth pressures and a Factor of Safety of 1.5 is assigned for computing passive earth pressures within the two clay soil layers. This I-Wall system has an exposed height of $10 \mathrm{ft}$. The water surfaces on both sides of the I-Wall are at $10 \mathrm{ft}$ on the flood side and o ft on the landside. In this example the flood side corresponds to the LHS of the I-Wall. In this scenario, the I-Wall will rotate clockwise during flood loading. The clay layers have a level ground surface on both sides of the I-Wall at el o ft with saturated unit weight of $122 \mathrm{pcf}$ and at el $10 \mathrm{ft}$ with saturated unit weight of 124 pcf.

Figure 4.23 outlines the basic geometry and structural parameters for the sheet-pile wall. The top row of text documents the input values of all parameters associated with each material type. For this problem, two materials are assigned and listed as Mo1 and Mo2; the "M" signifies the material type and is followed by the ID, which starts from "01." All information concerning material types are written in black text. The representation of a portion of a layer of soil on each side of the I-Wall is characterized as a region. Two soil regions are required to represent each soil layer.

Regions are labeled with an "R" and followed by an ID starting from "01." For this problem four regions are assigned; Region 1 and Region 2 (as Ro1 and Ro2), are located on the LHS of the sheet-pile wall and Region 3 and Region 4 (as Ro3 and Ro4) are located on the RHS of the sheet-pile wall. Each region and its surface elevation are written in red text. Surface water on either side of the sheet-pile wall are drawn with the water symbol at its defined elevation and labeled as "Left Water Surface" and "Right Water Surface" at the top of the wall in light blue text. Piezometric surfaces are labeled as "P" and followed by an ID starting from 01. Piezometric water surfaces are listed in the upper left-hand corner and drawn with the water symbol at its defined elevation and shown in blue. Observe that piezometric surface ID, Po1, is assigned to all four regions. The LHS piezometric surface elevation is assigned as the left-hand side surface water elevation. Similarly, the RHS piezometric surface elevation is assigned as the RHS surface water elevation. This allows for the assignment of submerged unit weights to the four soil regions. 
Figure 4.23. Corps_I-Wall schematic of sheet-pile wall in clay site.

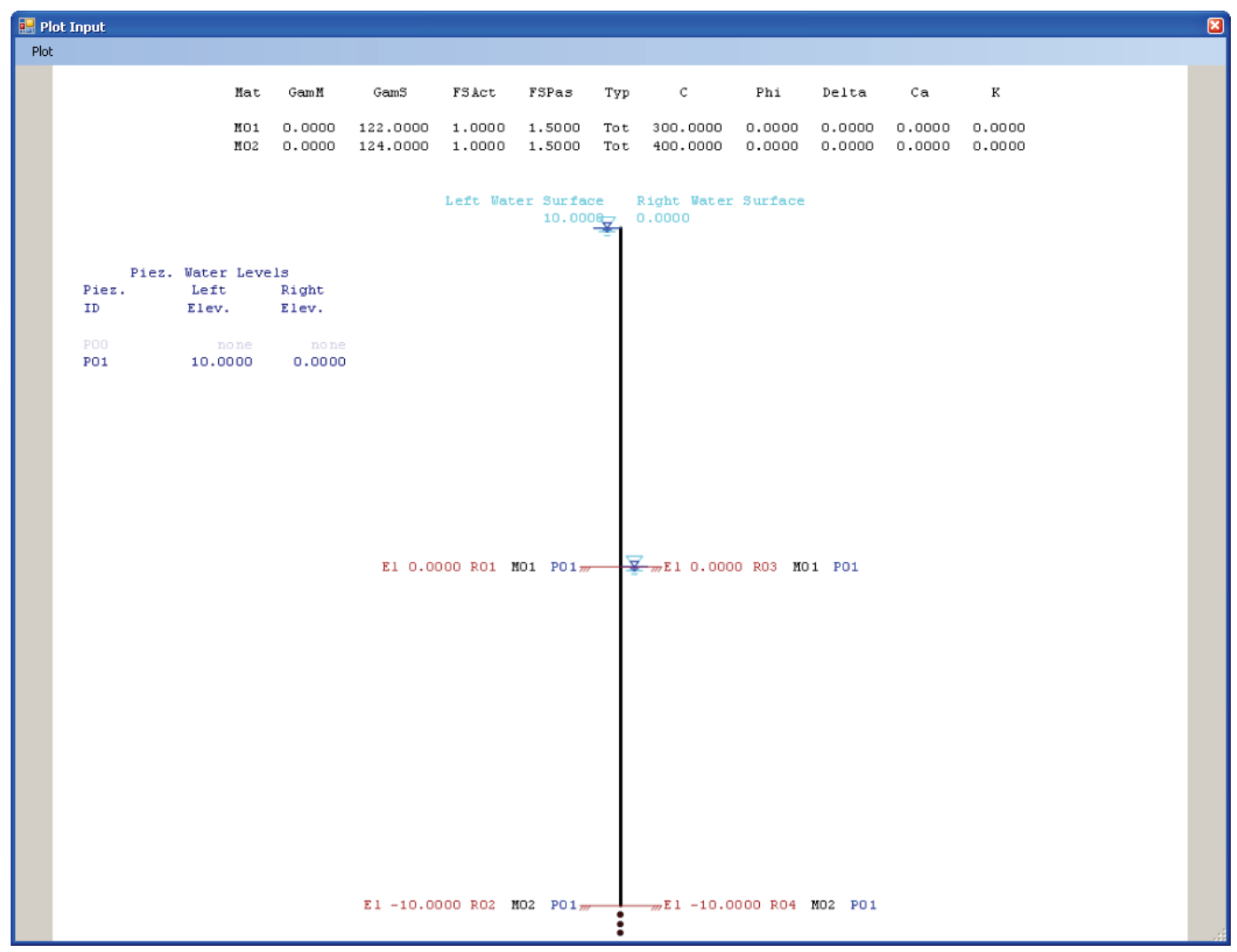

A clay site using a total stress definition with $\varphi=0$ as illustrated in Figure 4.23 is considered for the "design" computation of the depth of embedment. Hydrostatic water pressures as well as the Coulomb coefficient method of analysis and hydraulic fracturing form the basis for this example ${ }^{1}$.

This $\varphi=0$ analysis resulted in a computed mobilized active earth pressure coefficient $\left(K_{a}\right)$ of 1.0 and a mobilized passive pressure coefficient $\left(K_{p}\right)$ of 1.0. ${ }^{2}$ The active and passive earth pressures for both sides of the sheet-pile wall are illustrated in Figure 4.24. The hydraulic fracturing procedure discussed in Appendix B estimates a gap depth of $13.3117 \mathrm{ft}$. Active earth pressures only develop at the gap depth or where the fracturing terminates, this is shown by the (LHS) active earth pressure diagram of Figure 4.24a. Note the change in earth pressures at el -10 ft; at the interface of Region 1 and Region 2 of the passive earth pressure of Figure 4.24a and also at the

\footnotetext{
1 For no soil-to-I-Wall interface friction (i.e., $\delta=0 \mathrm{deg}$ ), the Coulomb solution for active and passive earth pressure coefficients corresponds to the Rankine earth pressure coefficients.

2 For a total stress definition, $\varphi=0$ deg, the active and passive earth pressure coefficients will equal to unity $\left(K_{a}=K_{p}=1.0\right)$.
} 
interface of Region 3 and Region 4 of Figure 4.24b. Please refer to Chapter 2 for a detailed description of multilayered soils. The hydrostatic water pressures acting on both sides of the sheet-pile wall are shown in Figure 4.25a with the resultant net water pressure calculated by taking the difference between the water pressures acting on both sides of the wall (Figure 4.25a) and shown in Figure 4.25b. In a total stress analysis, only overburden water pressure is included in the analysis (i.e., there is no pore water pressures calculated within the soil below the gap on the LHS and below the ground surface on the RHS). Also note that boundary water pressures acting on the flood wall for this total stress clay site increase with the propagation of the fracture down to the gap depth.

Figure 4.24. Active and passive earth pressures for undrained shear strength. (a) Left-hand side of wall. (b) Right-hand side of wall.

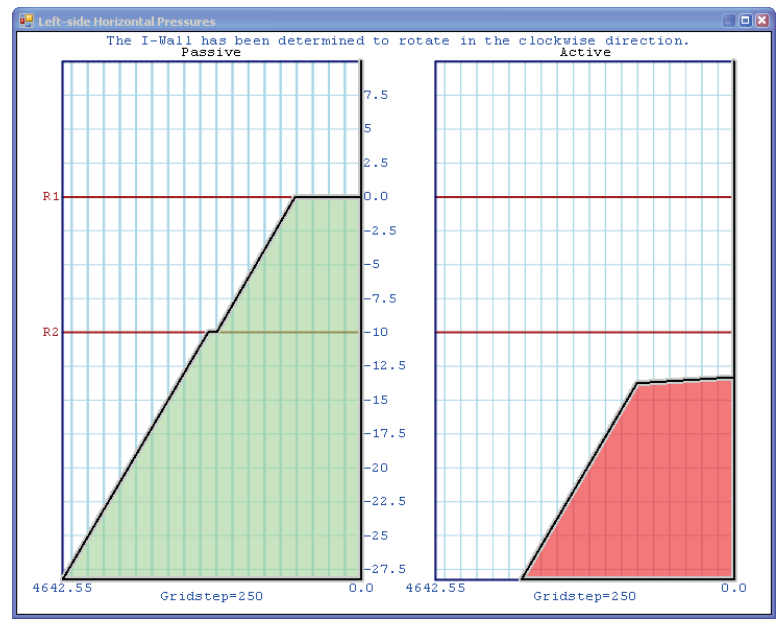

(a)

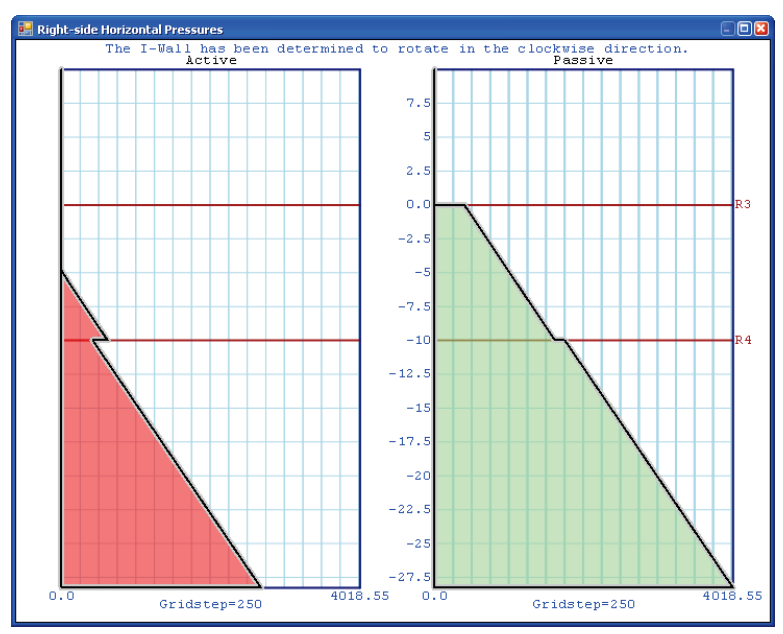

(b)

Figure 4.25. Pore water pressures. (a) Left- and right-hand side of wall. (b) Net water pressures.

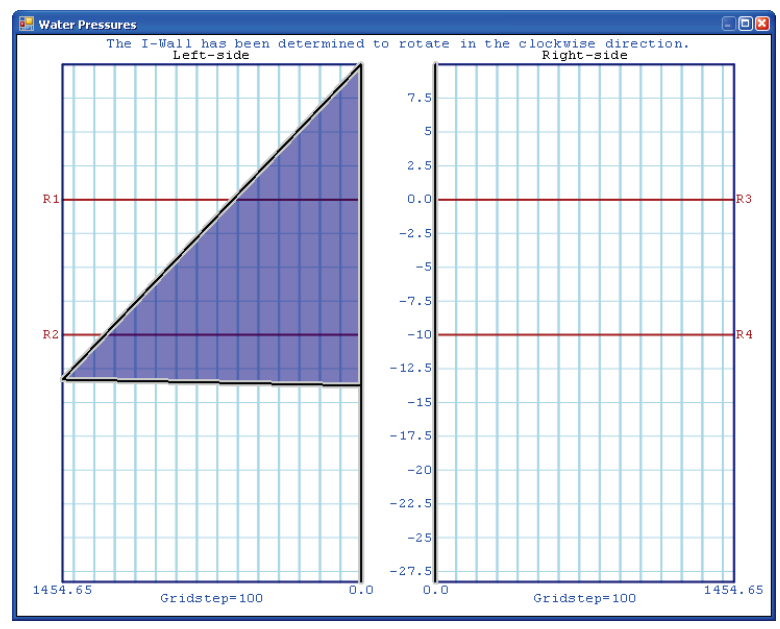

(a)

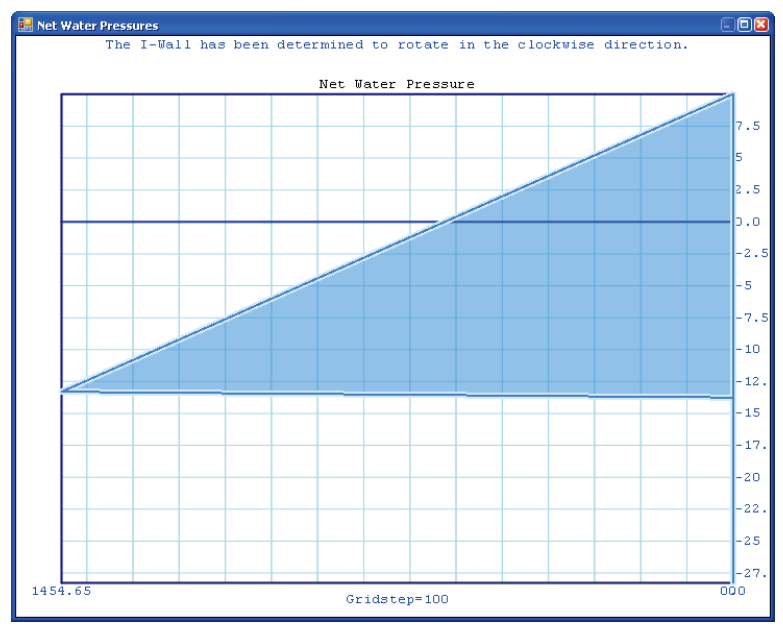

(b) 
From the calculated pressures of Figures 4.24 and 4.25, the net active and net passive pressures are constructed and presented in Figure 4.26a. for the prescribed LHS flood loading, the I-Wall will rotate in a clockwise direction; the net active pressure is determined from the difference between the LHS-mobilized active earth pressure and the RHS-mobilized passive earth pressure (i.e., acting on the other side of the wall) with the addition of the net water pressures and any external horizontal net pressures and overburden pressures (if any). The net passive pressure is determined by the difference between the LHS-mobilized passive earth pressure and the RHS-mobilized active earth pressure acting on the other side of the sheet-pile wall with the addition of any net water pressures and any external horizontal net pressures and overburden pressures (if any). The calculated net active and net passive pressures above the ground surface el o (and above Ro1 and Ro3) of Figure 4.26 are solely derived from the net water pressures of Figure 4.25b.

Figure 4.26. Net pressures. (a) Net active and net passive. (b) Net pressure diagram.

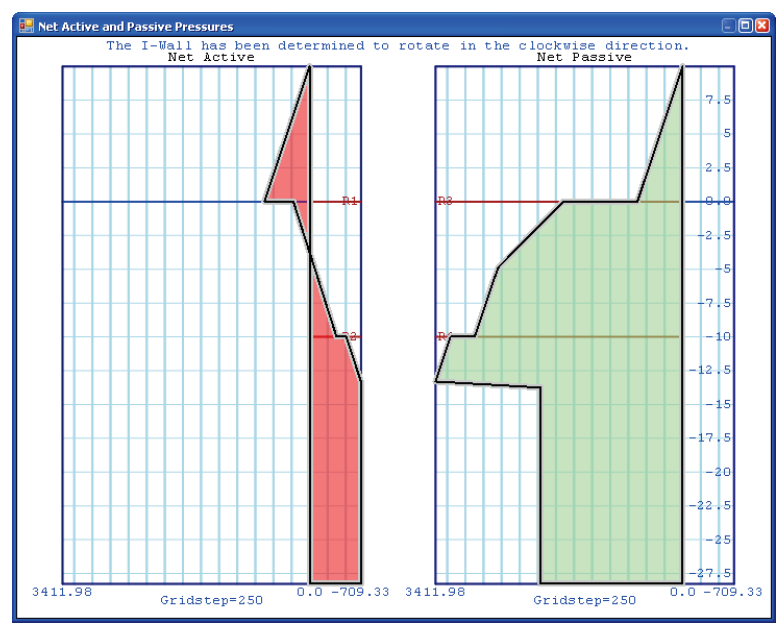

(a)

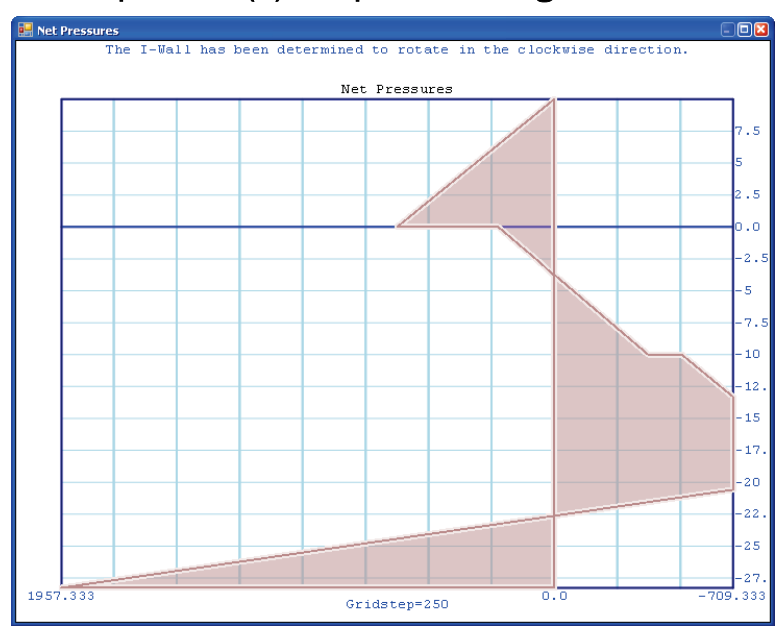

(b)

The net pressure diagram of Figure 4.26b is derived from the replication of all values of the net active pressures, from the top of the wall until the elevation at the point of rotation (at el $-20.5877 \mathrm{ft}$ ), at which instant the remaining values of the net pressure diagram is assumed to be linear with elevation between (1) the net active pressure value at the elevation of the point of rotation and (2) the net mobilized passive pressure at the elevation of the approximated sheet-pile tip (i.e., el -28.2679). ${ }^{1}$ Figure $4.26 \mathrm{~b}$ is an

1 Thus, only one mobilized net passive pressure value in Figure 4.26a is used to construct the Figure 4.26b net pressure diagram, corresponding to the sheet-pile tip elevation at el $-28.2679 \mathrm{ft}$. The majority of the net pressure diagram is derived from the Figure 4.26a net active pressure diagram. 
illustration of how the net pressure diagram was formed. Because the I-Wall has been determined to rotate in a clockwise direction, the net pressure diagram of Figure 4.26b shows that the upper zone of net active pressures are plotting on the LHS and the lower zone are plotting on the RHS. The net passive pressures are plotted on the LHS of the sheet-pile wall. ${ }^{1}$ The final results are illustrated in Figure 4.27 by the presentation of the net pressure diagram 4.27a (replica of Figure 4.26b) and 4.27b, the shear and moment diagrams. Figure $4.27 \mathrm{~b}$ shows that the maximum bending moment occurs at the zero crossing of the shear diagram. The sheet-pile tip elevation is computed at $-28.2679 \mathrm{ft}$ with a calculated depth of embedment of approximately $28.27 \mathrm{ft}$.

Figure 4.27. (a) Net pressure diagram. (b) Shear and moment.

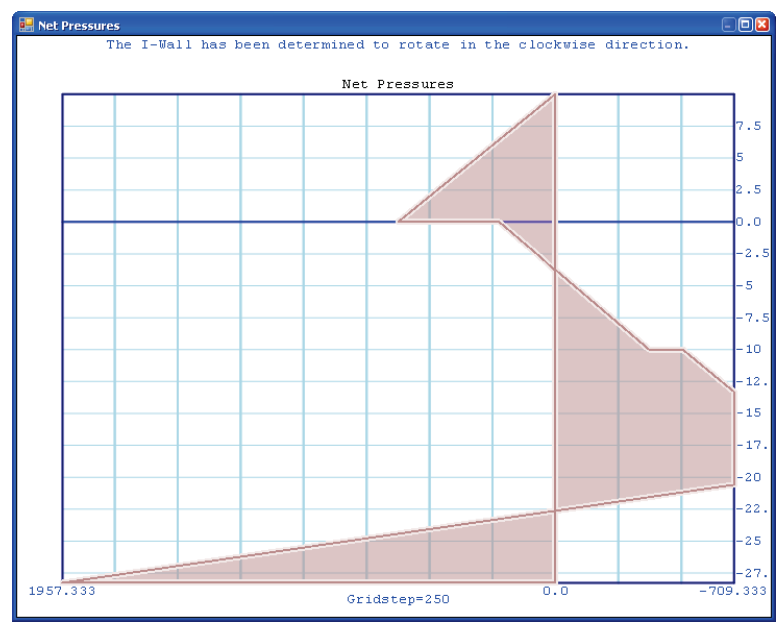

(a)

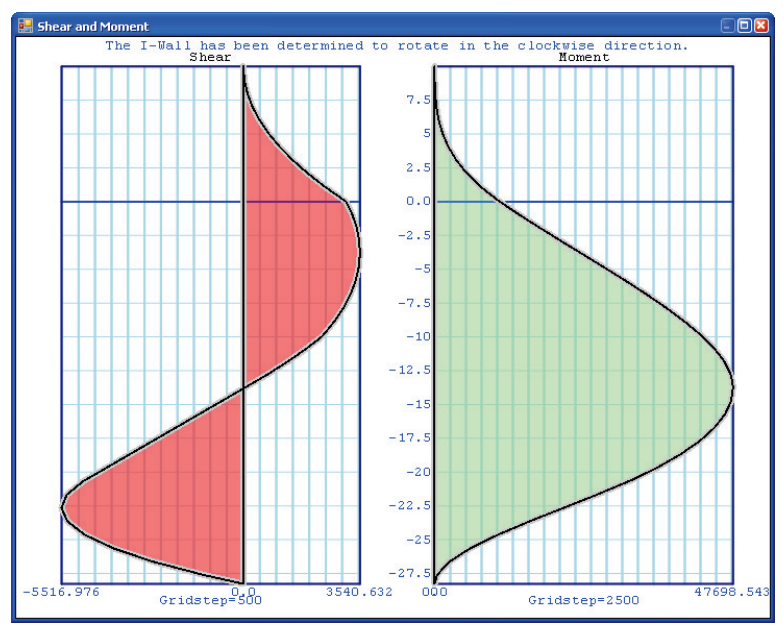

(b)

\subsection{Example 4.4 mixed total and effective stress based shear strength definitions in layered soils site}

Mixed layered soils of total stress and effective stress definitions where clay layers differ with the undrained shear strength (c) between 400 and $475 \mathrm{psf}$ and cohesionless soil comprise sand layers with varying effective angles of internal friction $\left(\varphi^{\prime}\right)$ of 30 and $32 \mathrm{deg}$. Ten soil layers are presented for this design problem to determine the depth of embedment. A Factor of Safety of 1.0 is assigned for computing active earth pressures and a Factor of Safety of 1.5 is assigned for computing passive earth pressures within the soil layers. The ground surface of the I-Wall is at o ft on the flood side and at $15 \mathrm{ft}$ on the landside. Surface water on the flood

\footnotetext{
1 The net earth pressures in the two Figure 4.26a diagrams and the Figure $4.26 \mathrm{~b}$ net pressures act towards the wall.
} 
side is at $10 \mathrm{ft}$. Piezometric surfaces are at el $7 \mathrm{ft}$ and el $10 \mathrm{ft}$ on the landside and at el $6 \mathrm{ft}$ on the flood side of the I-Wall. In this problem, the flood side corresponds to the LHS of the I-Wall and the I-Wall will rotate counterclockwise. Saturated unit weights range from $115 \mathrm{pcf}$ of $122 \mathrm{pcf}$.

Figure 4.28 outlines the basic geometry and structural parameters for the sheet-pile I-Wall. The top row of text documents the input values of all parameters associated with each material type. For this problem, 10 materials are assigned and listed as Mo1 through M1O; the "M" signifies the material type and is followed by the ID, which starts from "o1." All information concerning material types are written in black text. The representation of a portion of a layer of soil on each side of the I-Wall is characterized as a region. A soil region represents a soil layer. Regions are labeled with an "R" and followed by an ID starting from "01." The flood side consists of four regions: Region 1 through Region 4 (as Ro1 to R04), and the landside is assigned six regions; Region 5 through Region 10 (as Ro5 to R10). Each region and its top surface elevation are written in red text. Piezometric surface information is listed at the left side of Figure 4.28 (shown in blue) and are labeled as "P" and followed by an ID. Their respective locations are represented by water symbols at user-defined elevations along the wall. Po1 is associated with surface water on both sides of the sheet-pile wall. It is drawn with the water symbol located at the defined elevation. Surface water elevations are also listed at the top of the I-Wall in light blue text and labeled as "Left Water Surface" and "Right Water Surface." Piezometric surfaces, Po2 and higher, refer to piezometric surfaces other than surface water. Observe that piezometric surface ID, Po1, is associated with regions Ro1 thru Ro3; these soil regions have water levels that relate to the surface water at el $10 \mathrm{ft}$ on the LHS. Also, piezometric surface ID Po2 at el $10 \mathrm{ft}$, is associated with regions Ro5 thru Ro8 on the RHS.

Clay sites using a total stress definition with $\varphi=0$ and sand sites using an effective stress definition with $c^{\prime}=0$ are illustrated in Figure 4.28 and are considered for the "design" computation of the depth of embedment. Hydrostatic water pressures, as well as the Coulomb coefficient method of analysis, form the basis for this example ${ }^{1}$. Please refer to Chapter 2 for a detailed description of multilayered soils.

\footnotetext{
1 For no soil-to-I-Wall interface friction (i.e., $\delta=0 \mathrm{deg}$ ), the Coulomb solution for active and passive earth
} pressure coefficients corresponds to the Rankine earth pressure coefficients. 
Figure 4.28. Corps_I-Wall schematic of sheet-pile wall in clay site.

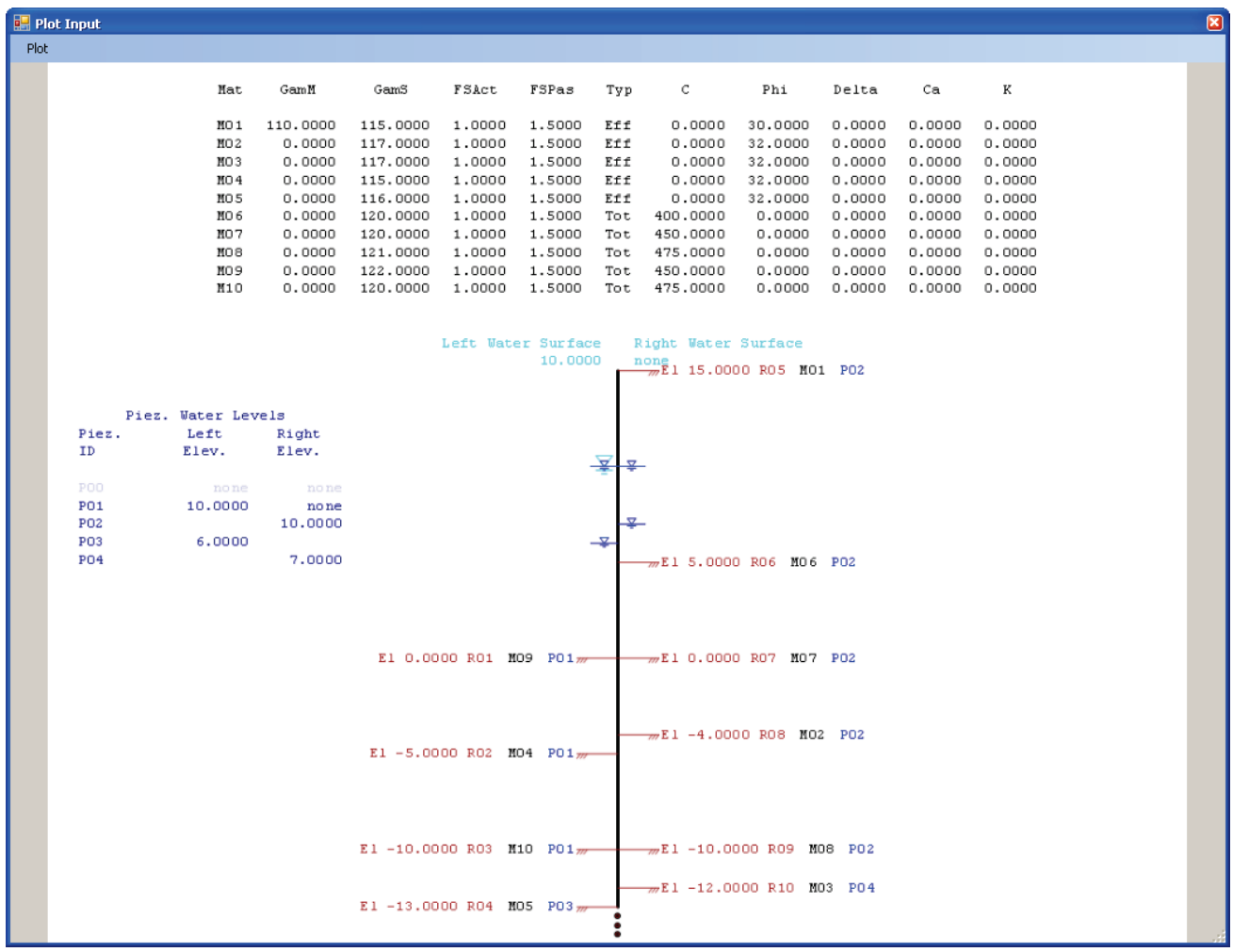

The active and passive earth pressures for both sides of the sheet-pile wall are illustrated in Figure 4.29. Note the change in earth pressures at the region interfaces at el -5, el -10 and el -13 ft of Figure 4.29a, illustrating active and passive earth pressures on the LHS, as well as the change in earth pressures at the region interfaces at el 5, el o, el -4, el -10 and el -12 ft as shown in Figure 4.29b.

Figure 4.29. Active and passive earth pressures. (a) Left-hand side of wall. (b) Right-hand side of wall.

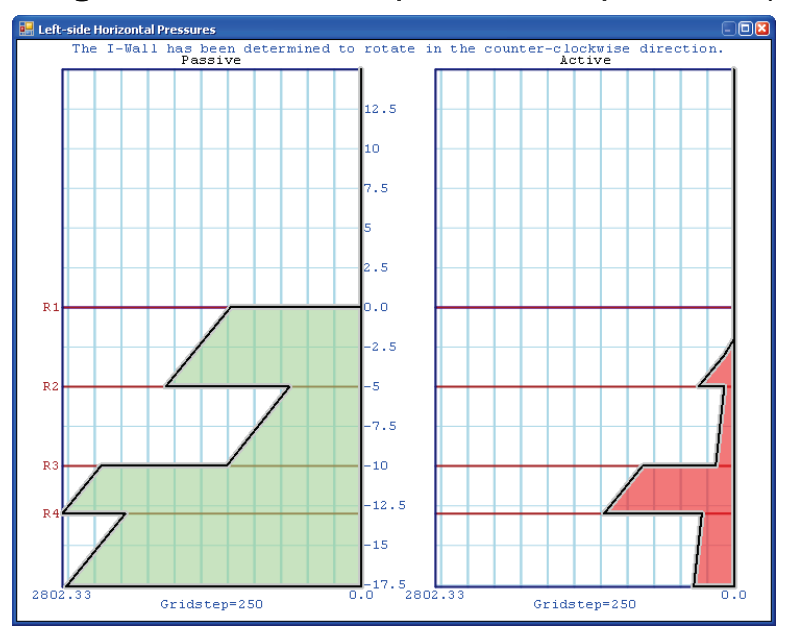

(a)

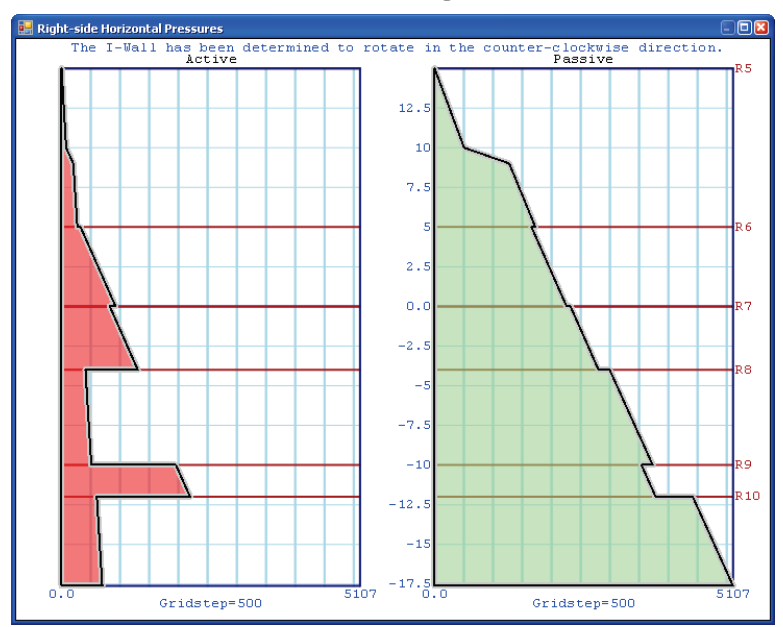

(b) 
The hydrostatic water pressures acting on both sides of the sheet-pile wall are shown in Figure 4.30a with the resultant net water pressure calculated by taking the difference between the water pressures acting on both sides of the wall (Figure 4.30a) and shown in Figure 4.30b.

Figure 4.30. Pore water pressures. (a) Left- and right-hand side of wall. (b) Net water pressures.

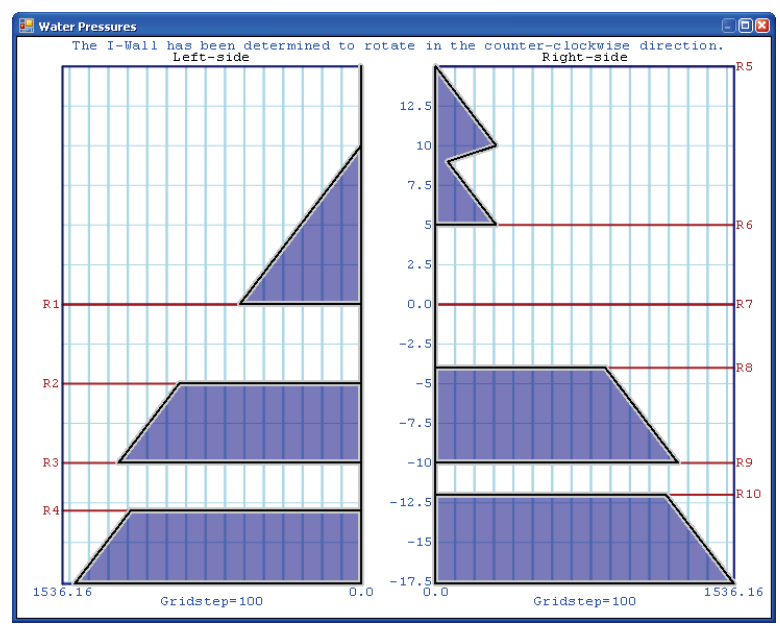

(a)

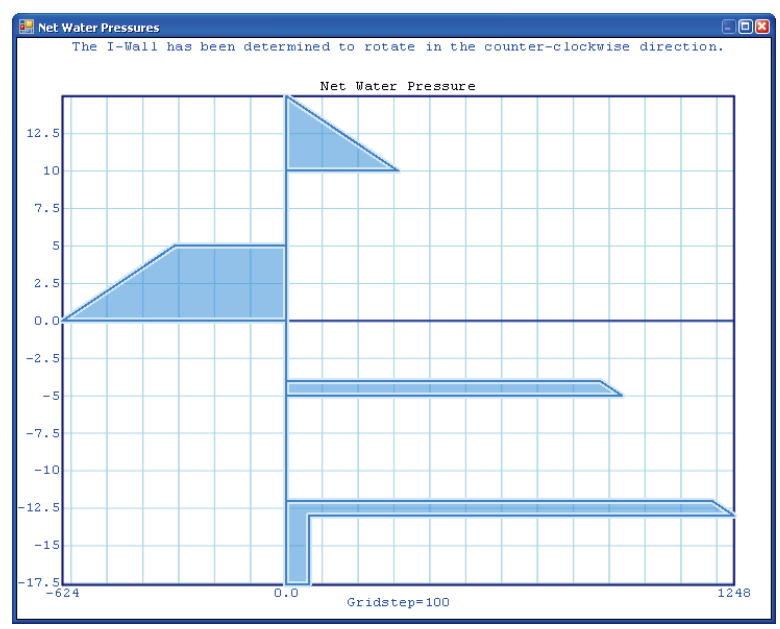

(b)

From the calculated pressures of Figures 4.29 and 4.30, the net active and net passive pressures are constructed and presented in Figure 4.31a. For the prescribed LHS flood loading, the I-Wall will rotate in a counterclockwise direction. The net active pressure is determined from the difference between the LHS-mobilized active earth pressure and the RHS-mobilized passive earth pressure (i.e., acting on the other side of the wall) with the addition of the net water pressures and any external horizontal net pressures and overburden net pressures (if any). The net passive pressure is determined by the difference between the LHS-mobilized passive earth pressure and the RHS-mobilized active earth pressure acting on the other side of the sheet-pile wall with the addition of any net water pressures and any external horizontal net pressures and overburden net pressures (if any). The net pressure diagram of Figure $4.31 \mathrm{~b}$ is derived from the replication of all values of the net active pressures, from the top of the wall until the elevation at the point of rotation (at el $-13.71 \mathrm{ft}$ ), at which instant the remaining values of the net pressure diagram are assumed to be linear with elevation between (1) the net active pressure value at the elevation of the point of rotation and (2) the net mobilized passive pressure at the elevation of the approximated sheet-pile tip (i.e., el -15.69). ${ }^{1}$ Figure $4.31 \mathrm{~b}$ is an

\footnotetext{
1 Only one mobilized net passive pressure value in Figure 4.31a is used to construct the Figure 4.31b net pressure diagram corresponding to the sheet-pile tip elevation at el $-15.69 \mathrm{ft}$. The majority of the net pressure diagram is derived from the Figure 4.31a net active pressure diagram.
} 
illustration of how the net pressure diagram was formed. Because the I-Wall has been determined to rotate in a counterclockwise direction, the net pressure diagram of Figure 4.31b shows that the upper zone of net active pressures is plotted on the RHS and the lower zone is plotted on the LHS. The net passive pressures are plotted on the RHS of the sheet-pile wall. The final results are illustrated in Figure 4.32 by the presentation of the net pressure diagram Figures 4.32a (replica of Figure 4.31b) and 4.32b, the shear and moment diagrams. Figure $4.32 \mathrm{~b}$ shows that the maximum bending moment occurs at the zero crossing of the shear diagram.

Figure 4.31. Net pressures. (a) Net active and net passive. (b) Net pressure diagram.

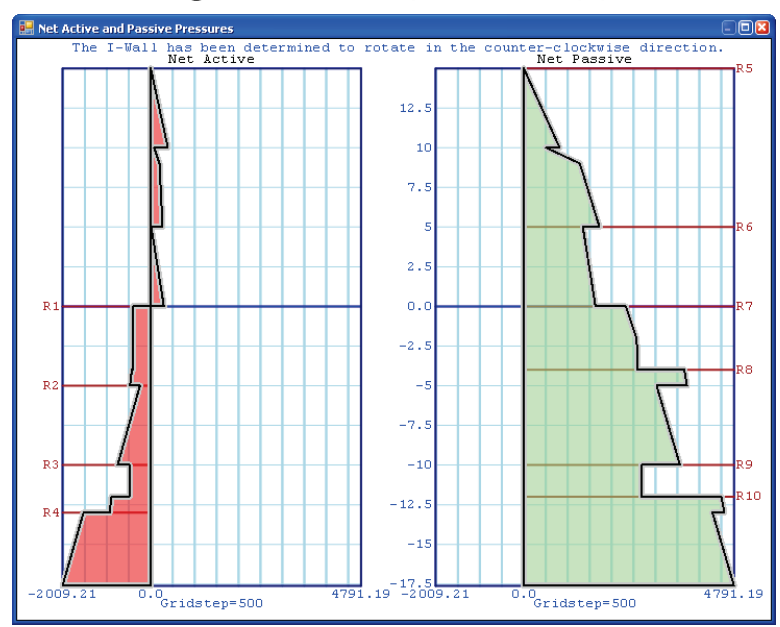

(a)

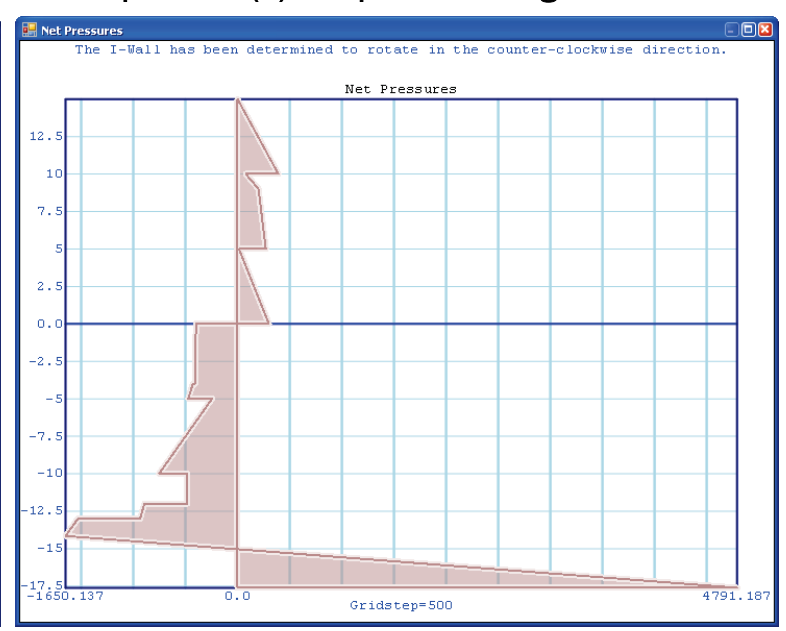

(b)

Figure 4.32. (a) Net pressure diagram. (b) Shear and moment.

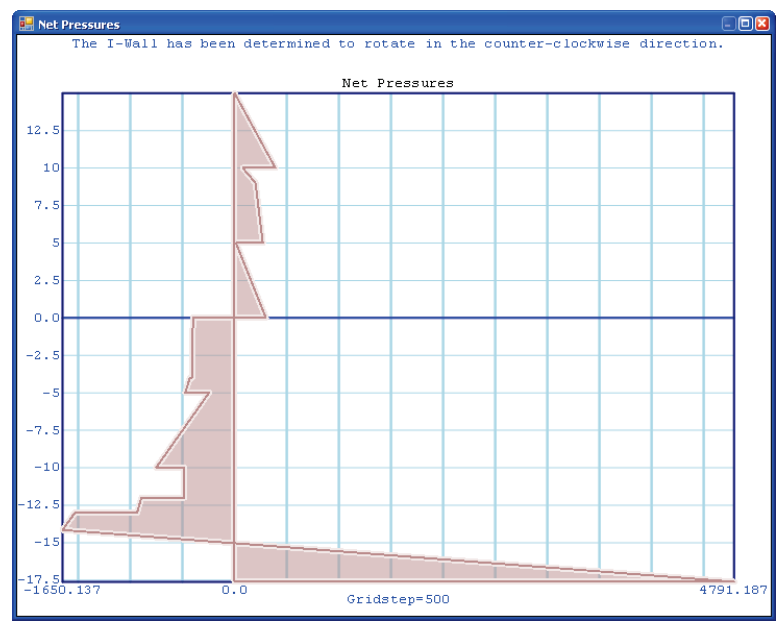

(a)

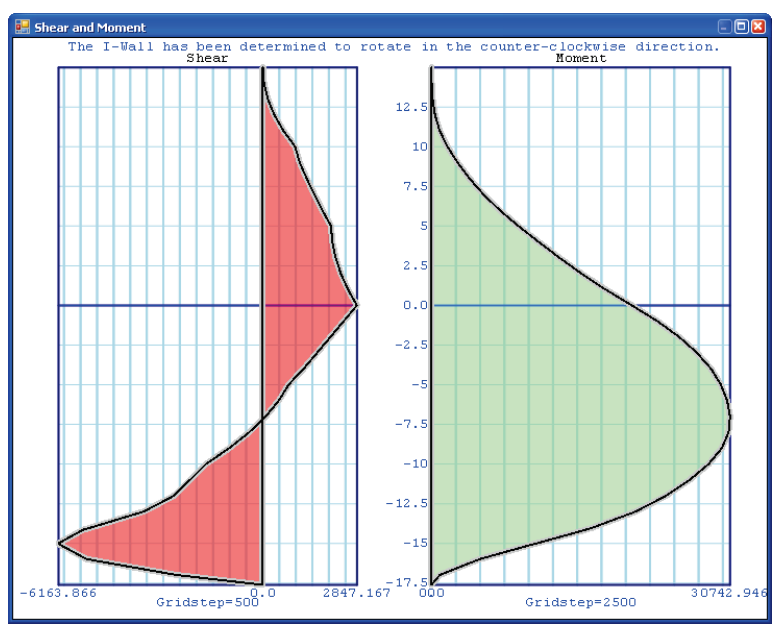

(b)

The various input conditions for the 10 layers of soil and final results for Example 4.4 are tabulated in Table 4.4. 
Table 4.4. Multilayered soils with effective stress strength definitions (sands with $c^{\prime}=0$ ) and total stress strength definitions $($ clays with $\varphi=0$ ).

\begin{tabular}{|c|c|c|c|c|c|c|c|c|c|c|c|}
\hline $\begin{array}{l}\text { Region } \\
\text { ID }\end{array}$ & $\begin{array}{l}\text { Material } \\
\text { Layer ID }\end{array}$ & Soil Type & $\begin{array}{l}\text { Piezometric } \\
\text { Surface ID }\end{array}$ & $\begin{array}{l}\varphi^{\prime} \\
\text { (deg) }\end{array}$ & $\begin{array}{l}c \\
\text { (psf) }\end{array}$ & $\underset{(p c f)}{\text { Y_moist }}$ & $\begin{array}{c}\text { Y_sat } \\
\text { (pcf) }\end{array}$ & $\mathrm{K}_{\mathrm{a}} m o b$ & $\mathrm{~K}_{\mathrm{p}} m o b$ & $\begin{array}{l}\text { Elevation at } \\
\text { Point of } \\
\text { Rotation }\end{array}$ & $\begin{array}{l}\text { Elevation at Tip } \\
\text { of Pile }\end{array}$ \\
\hline 1 & 9 & Clay & 1 & 0 & 450 & 0 & 122 & 1.0000 & 1.0000 & \multirow{10}{*}{-13.71} & \multirow{10}{*}{-15.69} \\
\hline 2 & 4 & Sand & 1 & 32 & 0 & 0 & 115 & 0.3073 & 2.2496 & & \\
\hline 3 & 10 & Clay & 1 & 0 & 475 & 0 & 120 & 1.0000 & 1.0000 & & \\
\hline 4 & 5 & Sand & 3 & 32 & 0 & 0 & 116 & 0.3073 & 2.2496 & & \\
\hline 5 & 1 & Sand & 2 & 30 & 0 & 110 & 115 & 0.3333 & 2.1212 & & \\
\hline 6 & 6 & Clay & 2 & 0 & 400 & 0 & 120 & 1.0000 & 1.0000 & & \\
\hline 7 & 7 & Clay & 2 & 0 & 450 & 0 & 120 & 1.0000 & 1.0000 & & \\
\hline 8 & 2 & Sand & 2 & 32 & 0 & 0 & 117 & 0.3073 & 2.2496 & & \\
\hline 9 & 8 & Clay & 2 & 0 & 475 & 0 & 121 & 1.0000 & 1.0000 & & \\
\hline 10 & 3 & Sand & 4 & 32 & 0 & 0 & 117 & 0.3073 & 2.2496 & & \\
\hline
\end{tabular}

The angle of wall friction $(\delta)$ and the adhesion (Ca) for all soil layers are zero. The Coulomb earth pressure coefficient method is being used to compute the earth pressures acting on the faces of the sheet-pile wall and hydrostatic pore water pressures are being used in the effective stress

calculations. The design Factor of Safety is 1.0 for active earth pressures, and for passive earth pressures the design Factor of Safety is 1.5. Elevations

and depths are reported in units of feet. 
The sheet-pile tip elevation is computed at $-15.69 \mathrm{ft}$ with a calculated depth of embedment of approximately $15.69 \mathrm{ft}$.

\subsection{Coastal site evaluation, designing for wave loads}

For coastal sites, I-Wall designs must take into account the effect of storm surges and the action of waves against the I-Walls. The design of the I-Wall should proceed in steps to assure that the I-Wall can remain stable with normal loads, as well as with storm surge loads. If unsatisfactory performance results due to the loading of the I-Wall, it may be necessary to remediate the I-Wall design with the addition of a stabilizing berm on the landside of the I-Wall to counter the detrimental effects of storm surge action. If the I-Wall design is enhanced by the addition of a berm, analysis of the new system under normal and surge loads will be performed.

For the four design examples discussed in this subsection, the I-Wall is assumed to be constructed with $\mathrm{PZ}_{3} 8$ sections of steel having a yield stress of:

$$
\sigma_{\text {Yield }}=39 \frac{\mathrm{kips}}{\mathrm{in.}^{2}}
$$

By EM-1110-2-2504 (HQUSACE 1994), the allowable bending stress is:

$$
\sigma_{\text {Allowable }}=0.5 \times \sigma_{\text {Yield }}=0.5 \times 39 \frac{\mathrm{kips}}{\mathrm{in.}^{2}}=19.5 \frac{\mathrm{kips}}{\mathrm{in.} .^{2}}
$$

The allowable bending moment $\left(M_{\text {Allowable }}\right)$ is computed as:

$$
M_{\text {Allowable }}=S \times \sigma_{\text {Allowable }} \text { per foot run of wall }
$$

Where $S$ = Section modulus per foot run of wall. Given a PZ38 section with $S=46.8$ in. ${ }^{3}$ per foot run of wall:

$$
M_{\text {Allowable }}=46.8 \mathrm{in.}^{3} \times 19.5 \frac{\mathrm{kips}}{\mathrm{in.} .^{2}}=912.6 \mathrm{kip}-\mathrm{in} . \div 12 \frac{\mathrm{in} .}{\mathrm{ft}}=76.1 \mathrm{kip}-\mathrm{ft}
$$

Therefore, a demand bending moment is required to be less than 76.1 kip-ft. 


\subsubsection{Example 4.5.1: Coastal site, effective stress strength definition with $\varphi^{\prime}=35^{\circ}$}

This first design problem for a coastal I-Wall is for the determination of the depth of embedment for an I-Wall with an exposed height of $15 \mathrm{ft}$ above the ground surface on the flood side in dense sand. The ground surface is defined to have an elevation of $0.0 \mathrm{ft}$. The dense sand has an effective stress definition with no cohesion, an effective angle of internal friction of $\left(\varphi^{\prime}\right)$ of $35 \mathrm{deg}$, a soil-to-sheet-pile effective interface friction angle $\left(\delta^{\prime}\right)$ of $17.5 \mathrm{deg}\left(=\varphi^{\prime} / 2\right)$, and a hydraulic conductivity $\left(K^{\prime}\right)$ of $0.0000328 \mathrm{ft} / \mathrm{sec}$ in a single material layer definition. In this design example problem, a Factor of Safety of 1.0 is assigned for computing active earth pressures and a Factor of Safety of 1.5 is assigned for computing passive earth pressures within the soil layer for designing the I-Wall. The water surfaces on both sides of the I-Wall are at $8.0 \mathrm{ft}$ on the flood side and $0.0 \mathrm{ft}$ on the landside. For this example, the flood side corresponds to the LHS of the I-Wall. In this analysis, it is assumed that the I-Wall will rotate clockwise (i.e., away from the floodwater) during design loading. The sand layers have a level ground surface on both sides of the I-Wall at elevation $0.0 \mathrm{ft}$ and assigned unsaturated (not used) and saturated unit weights of $125 \mathrm{pcf}$. Because the effective cohesion ( $\mathrm{c}^{\prime}$ ) is 0.0 psf, there will be no gap formation, but the hydraulic fracture option is enabled because it will be used with future runs. Homogeneous seepage is specified in the design calculations.

Figure 4.33 outlines the basic geometry and structural parameters for the sheet-pile wall. A design computation run is performed to determine the embedment depth that will allow this wall to be stable for the required Factor of Safety applied to the passive earth pressures of the sand foundation (i.e., $F S_{\text {Passive }}=1.50$ ).

The resulting depth of embedment is shown in Figure 4.34. The I-Wall becomes stable when the pile tip is embedded to a depth of $15.79 \mathrm{ft}$ because the soil surface is defined at an elevation of o ft. The point about which the wall will rotate is at a depth of $12.06 \mathrm{ft}$ below the ground surface. 
Figure 4.33. Corps_I-Wall schematic of sheet-pile wall in sand site.

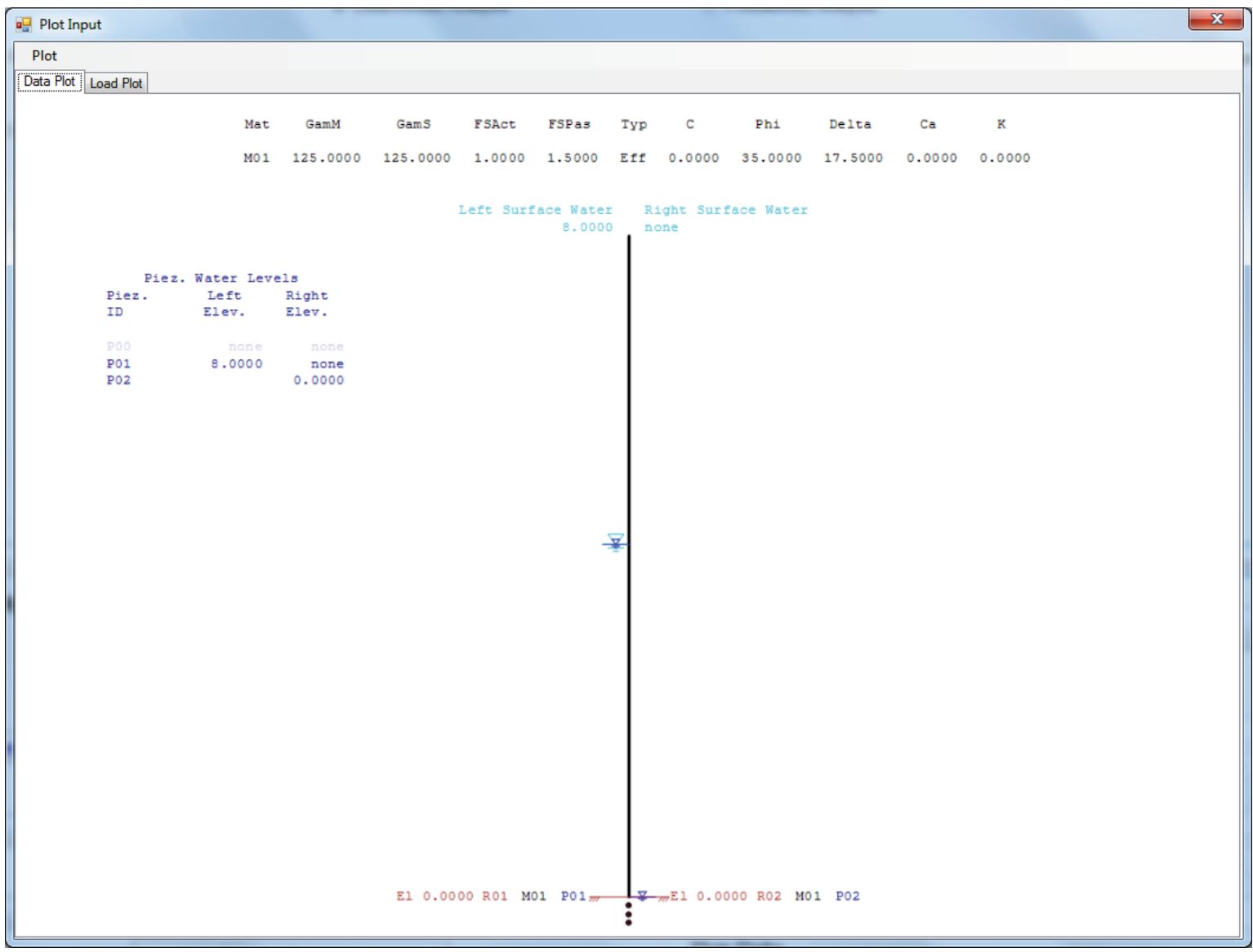

Figure 4.34. Corps_I-Wall run file for the specified input.

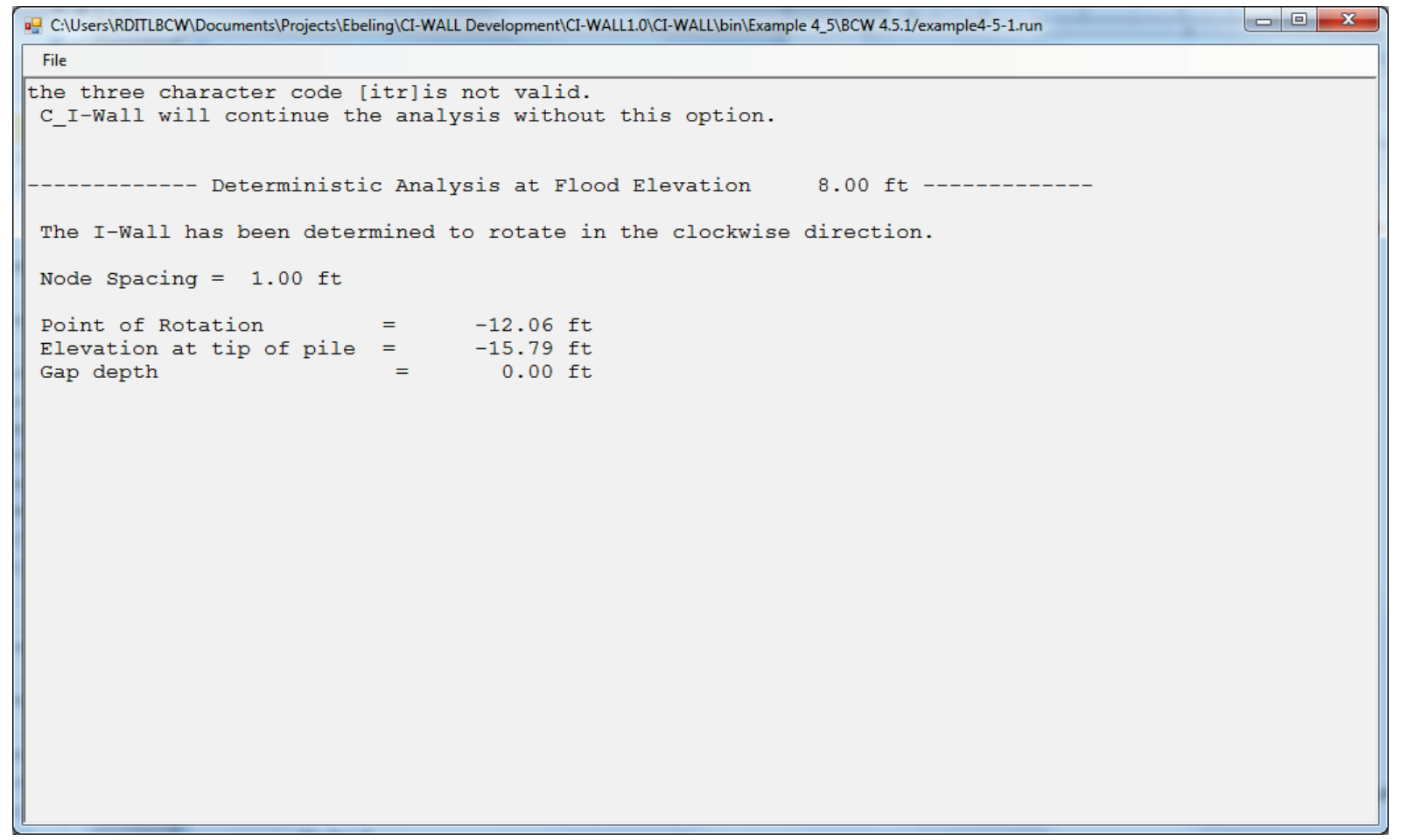


From the calculated pressures of Figures 4.35 and 4.36, the net active and net passive pressures are constructed and presented in Figure 4.37a. For the prescribed LHS flood loading, the I-Wall will rotate in a clockwise direction. The net active pressure is determined from the difference between the LHS-mobilized active earth pressure and the RHS-mobilized passive earth pressure (i.e., acting on the other side of the wall) with the addition of the net water pressures and any external horizontal net pressures and overburden pressures (if any). The net passive pressure is determined by the difference between the LHS-mobilized passive earth pressure and the RHS-mobilized active earth pressure acting on the other side of the sheet-pile wall with the addition of any net water pressures and any external horizontal net pressures and overburden pressures (if any). Both the calculated net active and net passive pressures above the ground surface el 0.o (and above Ro1 and Ro2) of Figure 4.37 are solely derived from the net water pressures of Figure 4.36b.

Figure 4.35. Corps_I-Wall run file for the specified input.
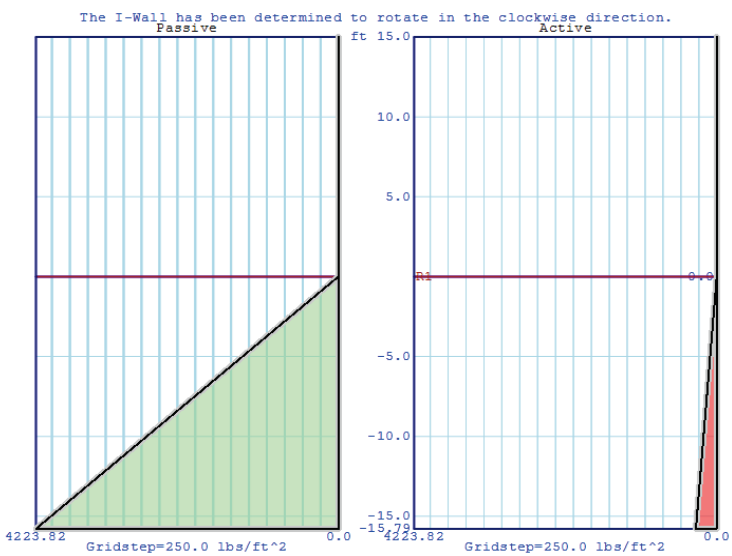

(a)

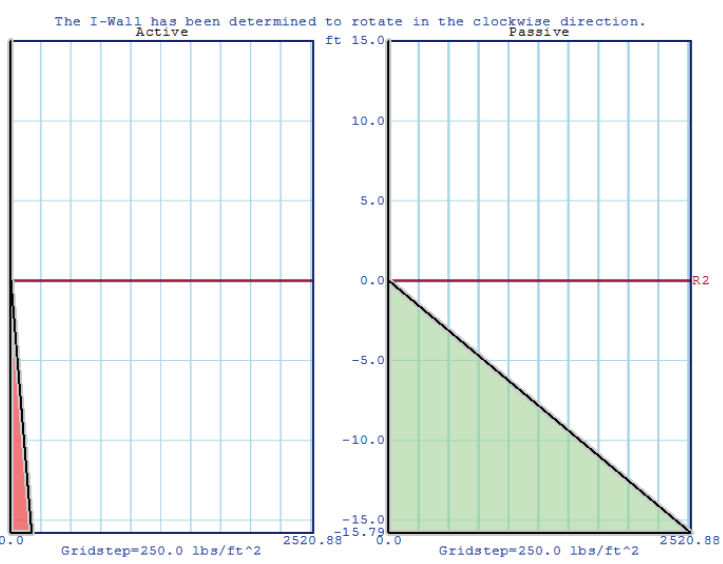

(b)

Figure 4.36. Pore water pressures. (a) Left- and right- hand side of wall. (b) Net water pressures.
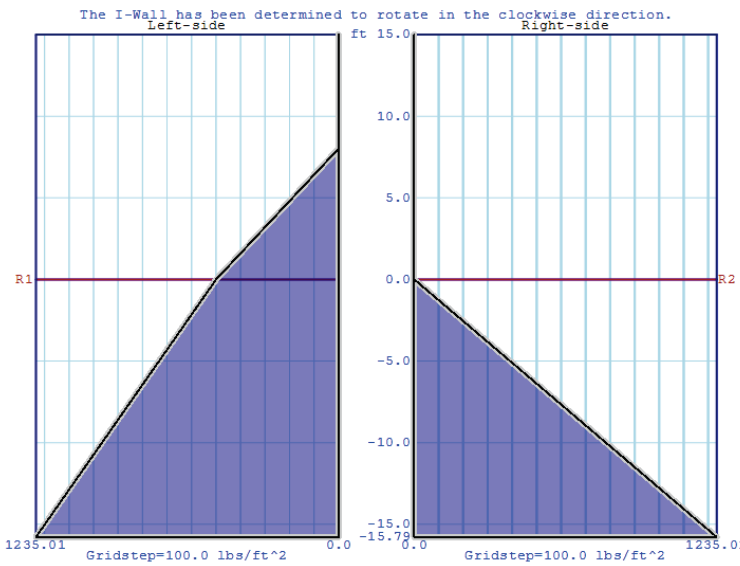

(a)

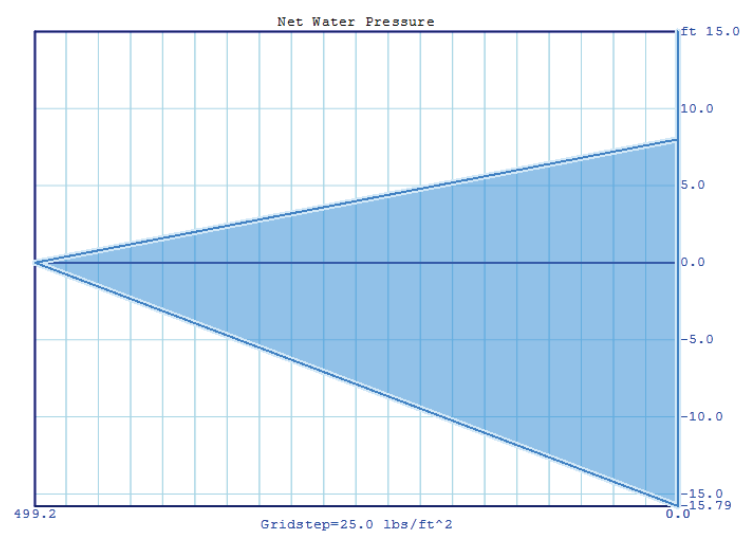

(b) 
Figure 4.37. Net pressures. (a) Net active and net passive. (b) Net pressure diagram.

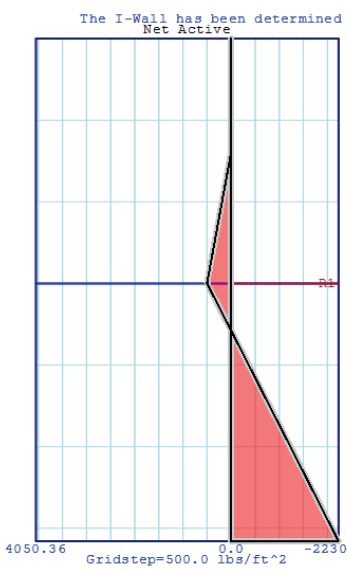

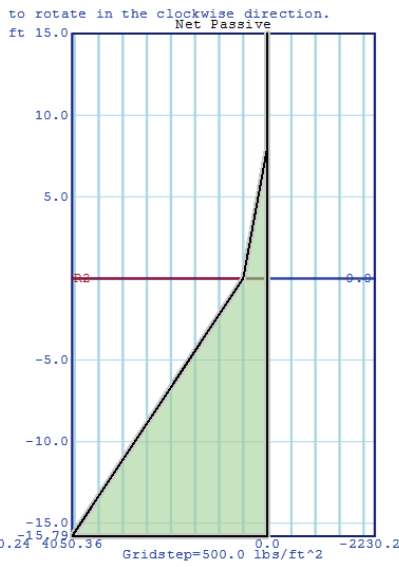

(a)

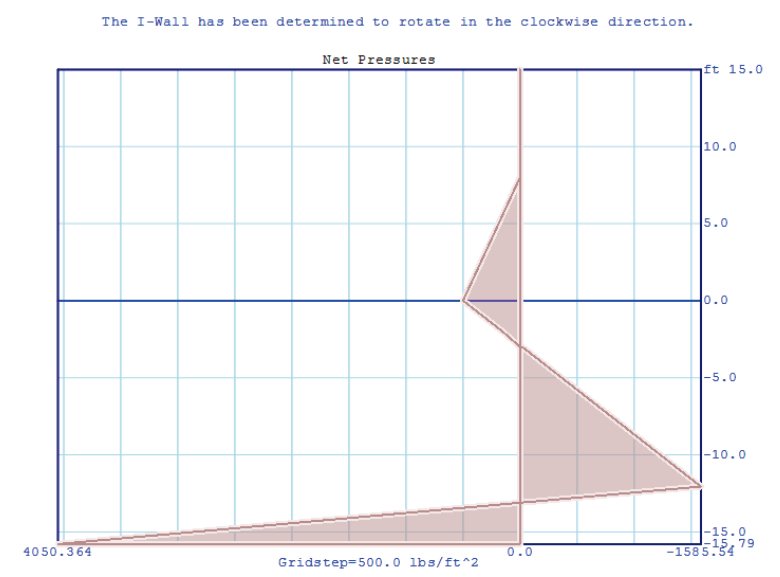

(b)

The net pressure diagram of Figure $4.37 \mathrm{~b}$ is derived from the replication of all values of the net active pressures, from the top of the wall until the elevation at the point of rotation (at el -12.06 ft), at which instant the remaining values of the net pressure diagram is assumed to be linear with elevation between (1) the net active pressure value at the elevation of the point of rotation and (2) the net-mobilized passive pressure at the elevation of the approximated sheet-pile tip (i.e., el -15.79). ${ }^{1}$ Figure $4.37 \mathrm{~b}$ is an illustration of how the net pressure diagram was formed. Because the I-Wall has been determined to rotate in a clockwise direction, the net pressure diagram of Figure 4.37b shows that the net active pressures plot on the LHS in the upper zone and on the RHS in the lower zone. The net passive pressures plot on the LHS of the sheet-pile wall. The final results are illustrated in Figure 4.38 by the presentation of the net pressure diagram Figure 4.38a (replica of Figure 4.37b) and Figure 4.38b, the shear and moment diagrams. Figure $4.38 \mathrm{~b}$ shows that the maximum bending moment occurs at the zero crossing of the shear diagram. This maximum bending moment is $22.5 \mathrm{kip}-\mathrm{ft}$. This moment calculation is below the allowable bending moment of $76.1 \mathrm{kip}-\mathrm{ft}$, so the wall will withstand the pressures that it is under. The sheet-pile tip elevation is computed at $-15.79 \mathrm{ft}$ with a calculated depth of embedment of approximately $15.79 \mathrm{ft}$.

\footnotetext{
1 Thus, only one mobilized net passive pressure value in Figure 4.37a is used to construct Figure 4.37b net pressure diagram; corresponding to the sheet-pile tip elevation at el $-15.79 \mathrm{ft}$. The majority of the net pressure diagram is derived from Figure 4.37a net active pressure diagram.
} 
Figure 4.38. (a) Net pressure diagram, (b) Shear and moment.

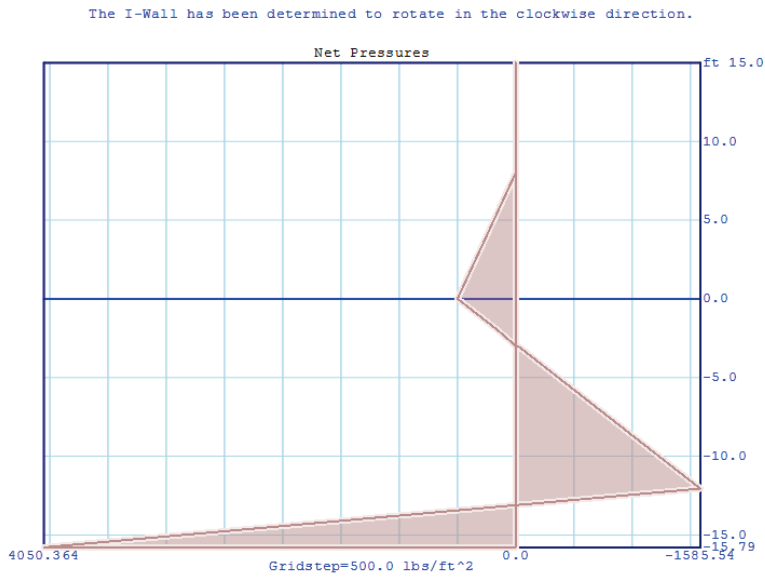

(a)

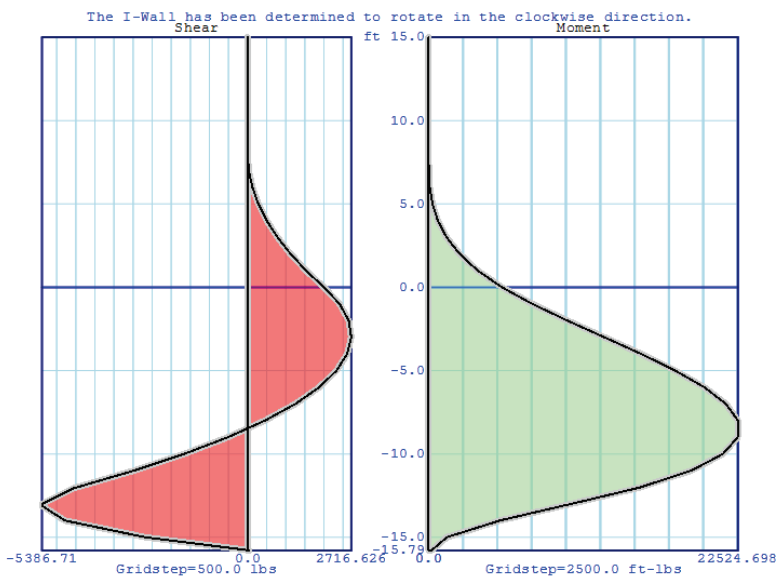

(b)

A diagram of the drop in total head is given in Figure 4.39. To the left is a diagram of the wall showing the surface water, soil region surfaces, gap definition, wall depth, and the direction of seepage. The drop in total heads due to seepage is given in the right side plot. This plot follows the line of seepage along the wall from one side to the other on the horizontal axis, and displays the head values on the vertical axis. The head loss occurs as water seeps from el o.o, down the LHS foundation sands to the sheet-pile tip and then up the RHS foundation sands to the ground surface (at el o.o) and is represented by the blue line. Because this site possesses a homogeneous value of hydraulic conductivity (i.e., a $\mathrm{K}^{\prime}$ value of $0.0000328 \mathrm{ft} / \mathrm{sec}$ specified for the single material definition applied to the LHS and RHS dense sand regions), the head loss with distance shown in Figure 4.39 is linear along the line of seepage, as anticipated.

\subsubsection{Example 4.5.2 Coastal site with surge loading}

The second design problem for a coastal I-Wall is determining the depth of embedment for an I-Wall with a 15 -ft exposed height embedded in dense sand (at elevation $0.0 \mathrm{ft}$ ) and subjected to a surge loading. The dense sand has an effective stress definition with no cohesion, an effective angle of internal friction of $\left(\varphi^{\prime}\right)$ of $35 \mathrm{deg}$, a soil-to-sheet-pile effective interface friction angle $\left(\delta^{\prime}\right)$ of $17.5 \mathrm{deg}\left(=\varphi^{\prime} / 2\right)$, and a hydraulic conductivity $\left(\mathrm{K}^{\prime}\right)$ of $0.0000328 \mathrm{ft} / \mathrm{sec}$ in a single material layer definition. In this design example problem, a Factor of Safety of 1.0 is assigned for computing active earth pressures and a Factor of Safety of 1.5 is assigned for computing passive earth pressures within the soil layer. This I-Wall system has an exposed height of $15 \mathrm{ft}$. The sand layers have a level ground surface on 
both sides of the I-Wall at el $0.0 \mathrm{ft}$ and assigned unsaturated (not used) and saturated unit weights of 125 pcf. Because the effective cohesion $\left(\mathrm{c}^{\prime}\right)$ is o.o psf, there will be no gap formation, but the hydraulic fracture option is enabled because it will be used with future runs. Homogeneous seepage is specified in the design calculations.

Figure 4.39. Drop in total heads due to seepage diagram.

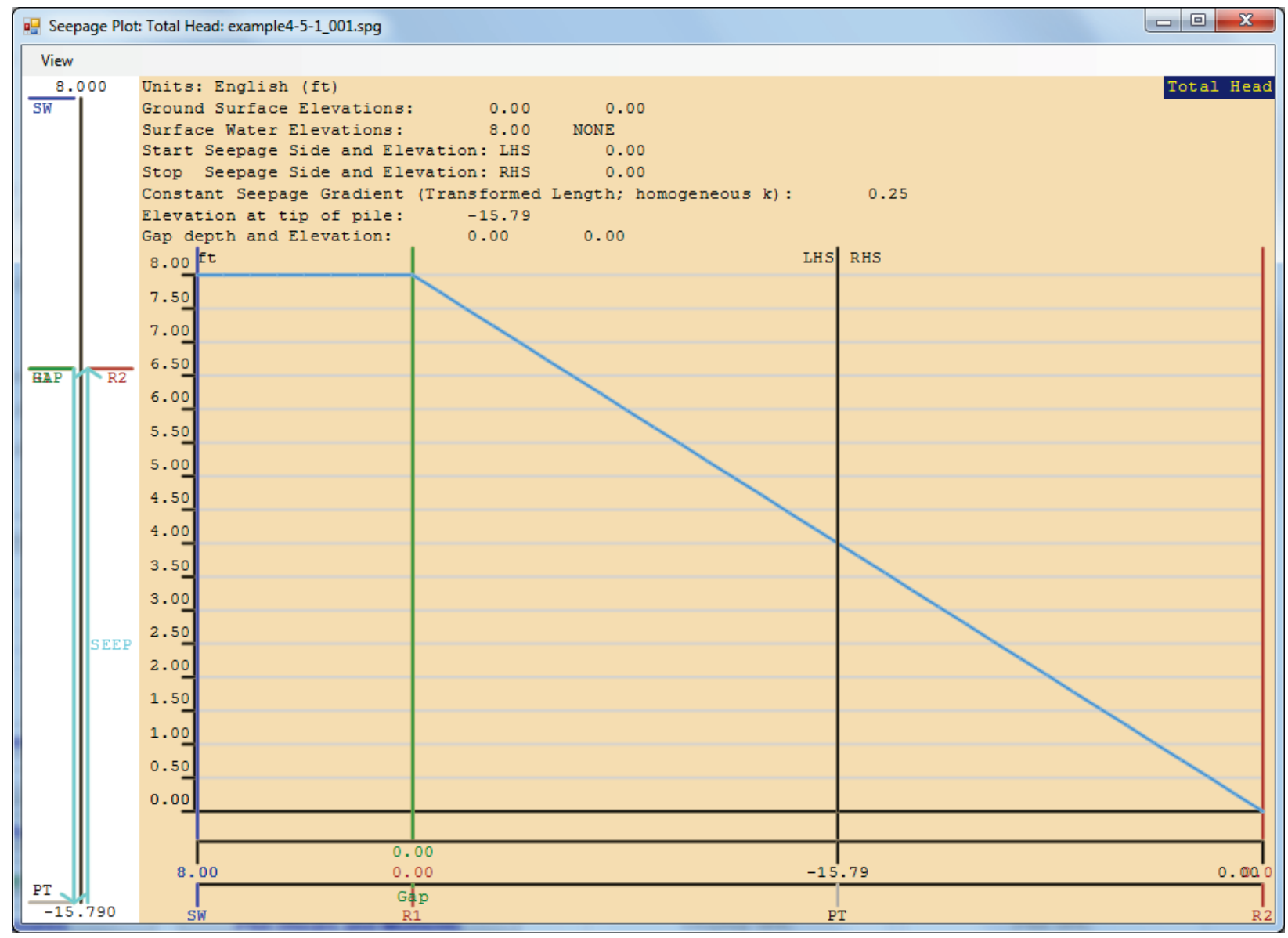

The water surface on the LHS of the I-Wall is determined by the possible surge wave size calculation in Appendix F, using the Minikin breaking wave method for determining forces. The Minikin method is diagrammed in Figure F.2. In essence, a pseudostatic pressure distribution is applied to the wall composed of the dynamic component of the breaking wave and the hydrostatic pressure component acting on the wall. The hydrostatic pressures acting on the LHS of the wall are equivalent to the pressure as if the swell height was the actual water level acting on the wall. The dynamic pressures are caused by the effects of the forward motion of the water in the wave as it collides with the wall. 
The Minikin method is used to compute the peak dynamic pressure and wave height based on the average still-water level and the period of the wave. The dynamic pressure component is modeled as a triangular distributed pressure that begins with a pressure of 0.0 at half the height of the wave above the still-water level, reaches the maximum pressure at the still- water level, and returns to a pressure of 0.0 at the elevation of the still-water level minus one-half the height of the wave.

For Corps_I-Wall Version 1.o, the hydrostatic component is modeled by setting the water surface as half the height of the wave above the stillwater level as it breaks against the wall. This elevation is then used to calculate pressures.

These pressures are shown in Figure 4.40. Figure 4.40a shows the dynamic wave as a horizontal pressure distribution entered into Corps_I-Wall Version 1.o. Figure 4.40b adds a representation of hydrostatic pressures when the water surface elevation is increased to the still-water level plus half the height of the breaking wave (in brown).

Because the swell height is the elevation of the still-water level added to half of the breaking wave height ( $\sim 6.24$ from Equation F.2 and Table F.3), the water level on the flood side is set equal to the swell height of $8 \mathrm{ft}$ $+3.12 \mathrm{ft}=11.12 \mathrm{ft}$ and at $\mathrm{oft}$ on the landside. The additional horizontal pressure due to the motion of the wave is accounted for by having a triangular horizontal load that goes from $0.0 \mathrm{lb} / \mathrm{ft}^{2}$ at $11.12 \mathrm{ft}$ to a maximum pressure of $4,084.6 \mathrm{lb} / \mathrm{ft}^{2}$ (computed using equation F.4) at $8 \mathrm{ft}$ and reducing to $0.0 \mathrm{lb} / \mathrm{ft}^{2}$ at $4.88 \mathrm{ft}(8 \mathrm{ft}-3.12 \mathrm{ft}$ ). For this example, the flood side corresponds to the LHS of the I-Wall. In this analysis, it is assumed that the I-Wall will rotate clockwise during flood loading.

Figure 4.41 outlines the basic geometry and structural parameters for the sheet-pile wall. A design computation analysis is performed to determine an adequate depth of embedment for stability of the sheet-pile wall.

The resulting depth of embedment is shown in Figure 4.42. Because of the higher water levels and the additional wave loading (applied as a horizontal surcharge load), the I-Wall becomes stable when the pile tip is embedded to a depth of $-36.37 \mathrm{ft}$, with the soil surface defined at an elevation of $0.0 \mathrm{ft}$. The point about which the wall will rotate is at a depth of $-25.41 \mathrm{ft}$ below the ground surface. 
Figure 4.40. The components of Minikin wave pressure diagram.

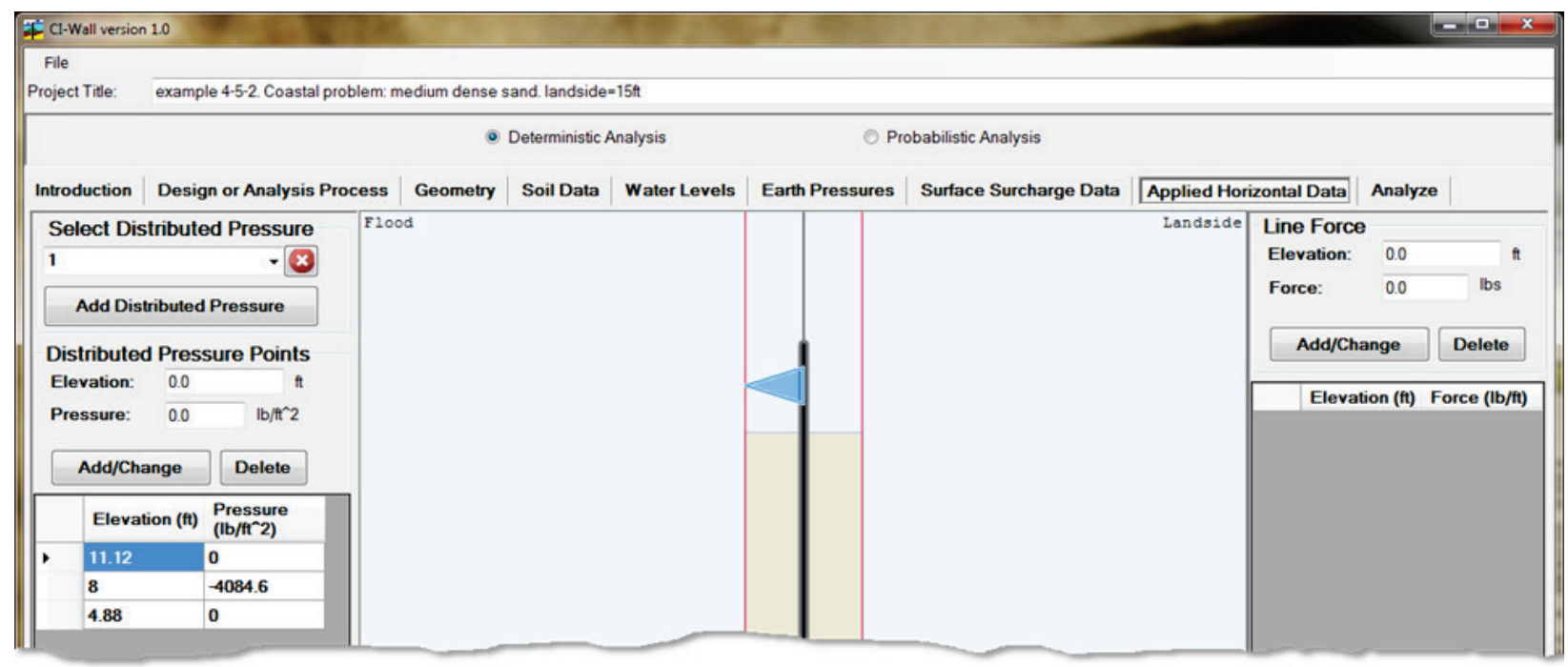

(a) Corps_I-Wall input for the Minikin dynamic pressure component.

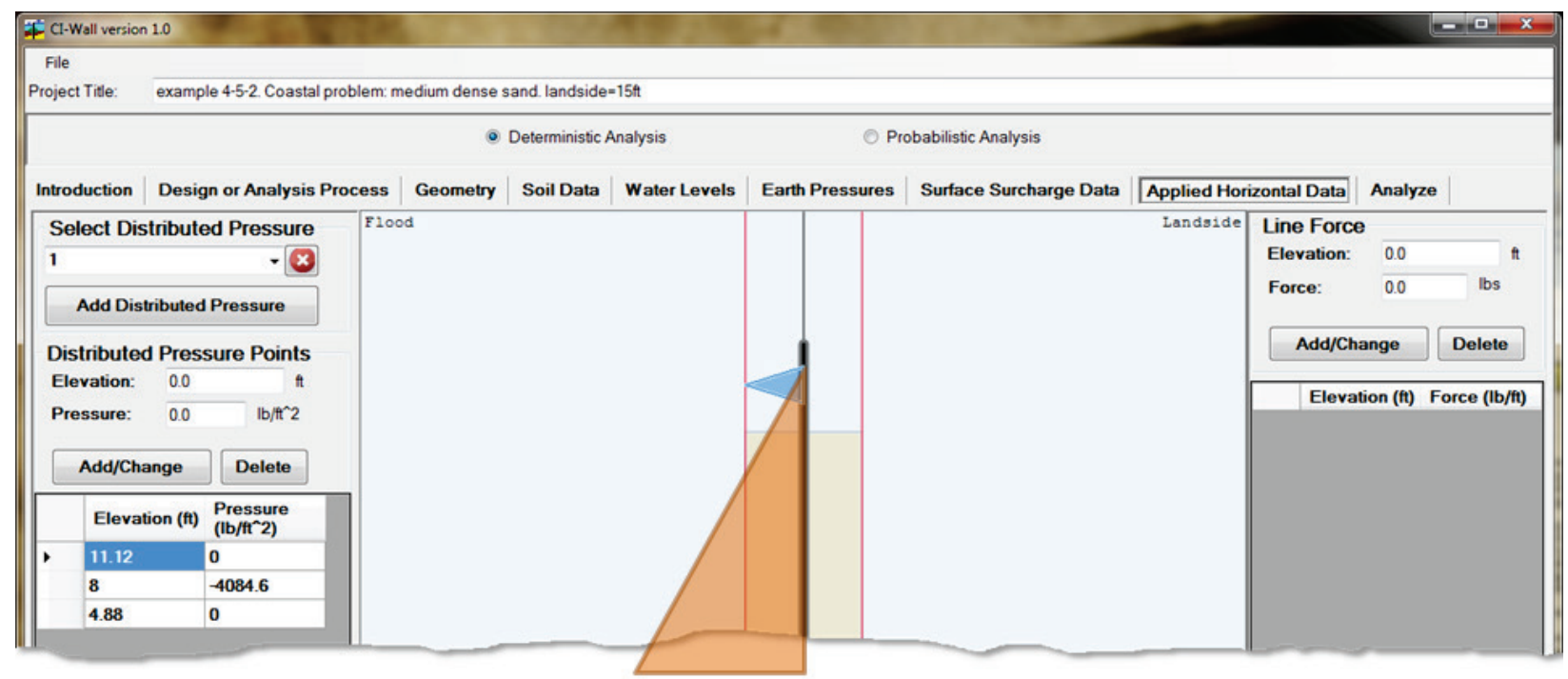

(b) The dynamic pressure component input with a representation of the hydrostatic pressure component (altered based on the still-water level and wave height).

This calculation shows that the sheet-pile wall tip is embedded more than twice as deep as its exposed height. This situation also has the point of rotation at a depth lower than the exposed height. 
Figure 4.41. Corps_I-Wall. (a) Schematic of sheet-pile wall in sand site. (b) Wave load applied to flood side (LHS) of sheet-pile wall.

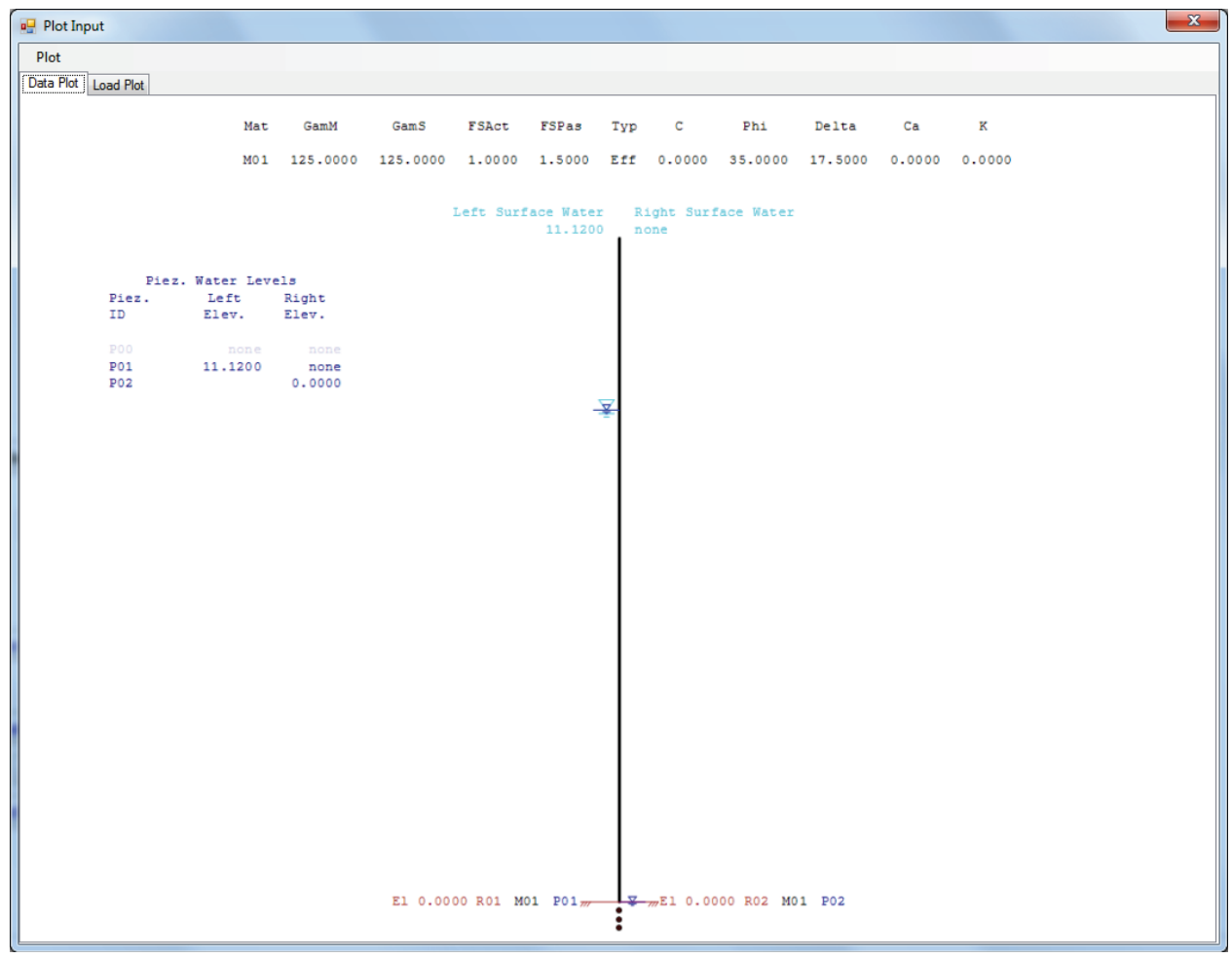

(a)

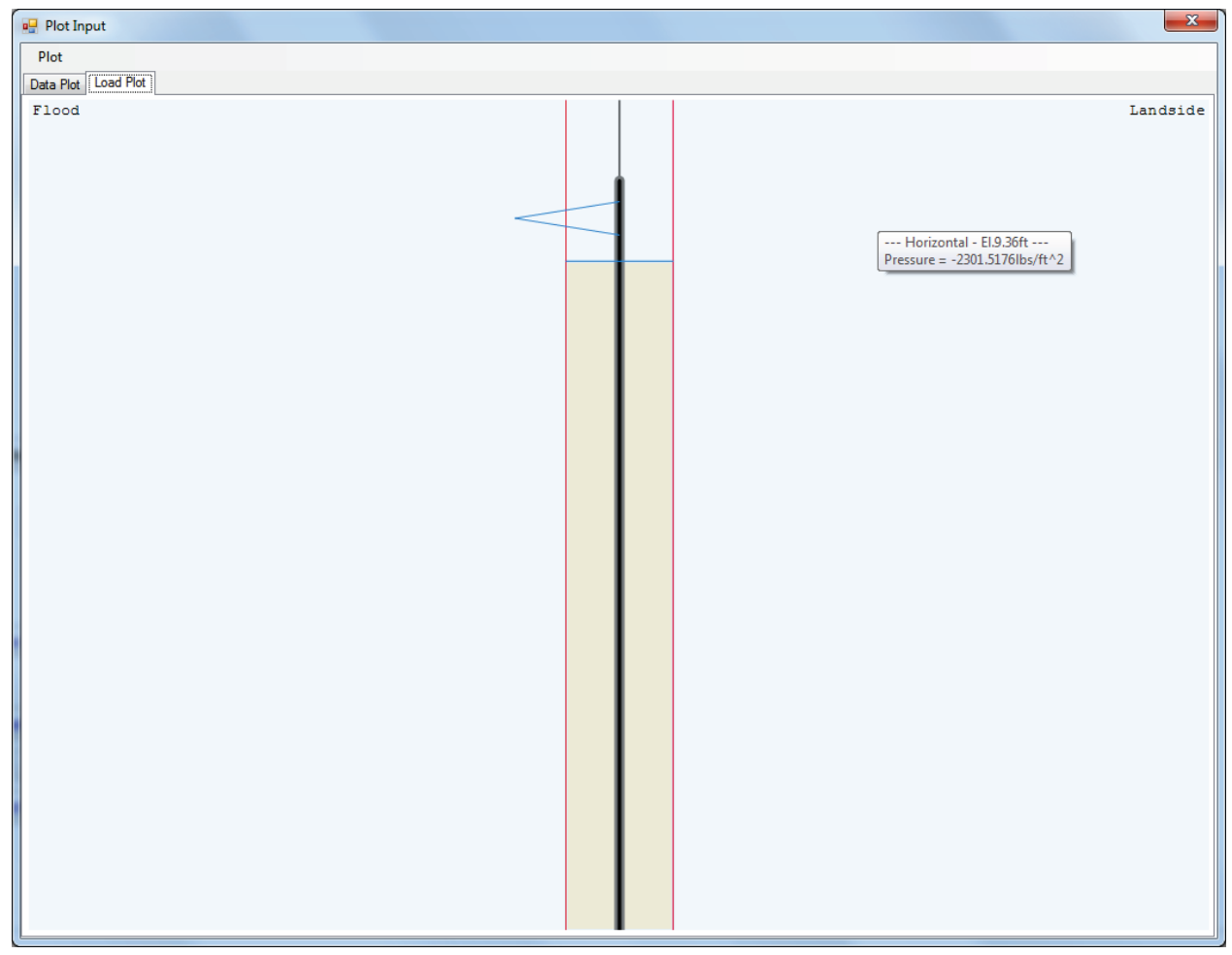

(b) 
Figure 4.42. Corps_I-Wall run file for the specified input.

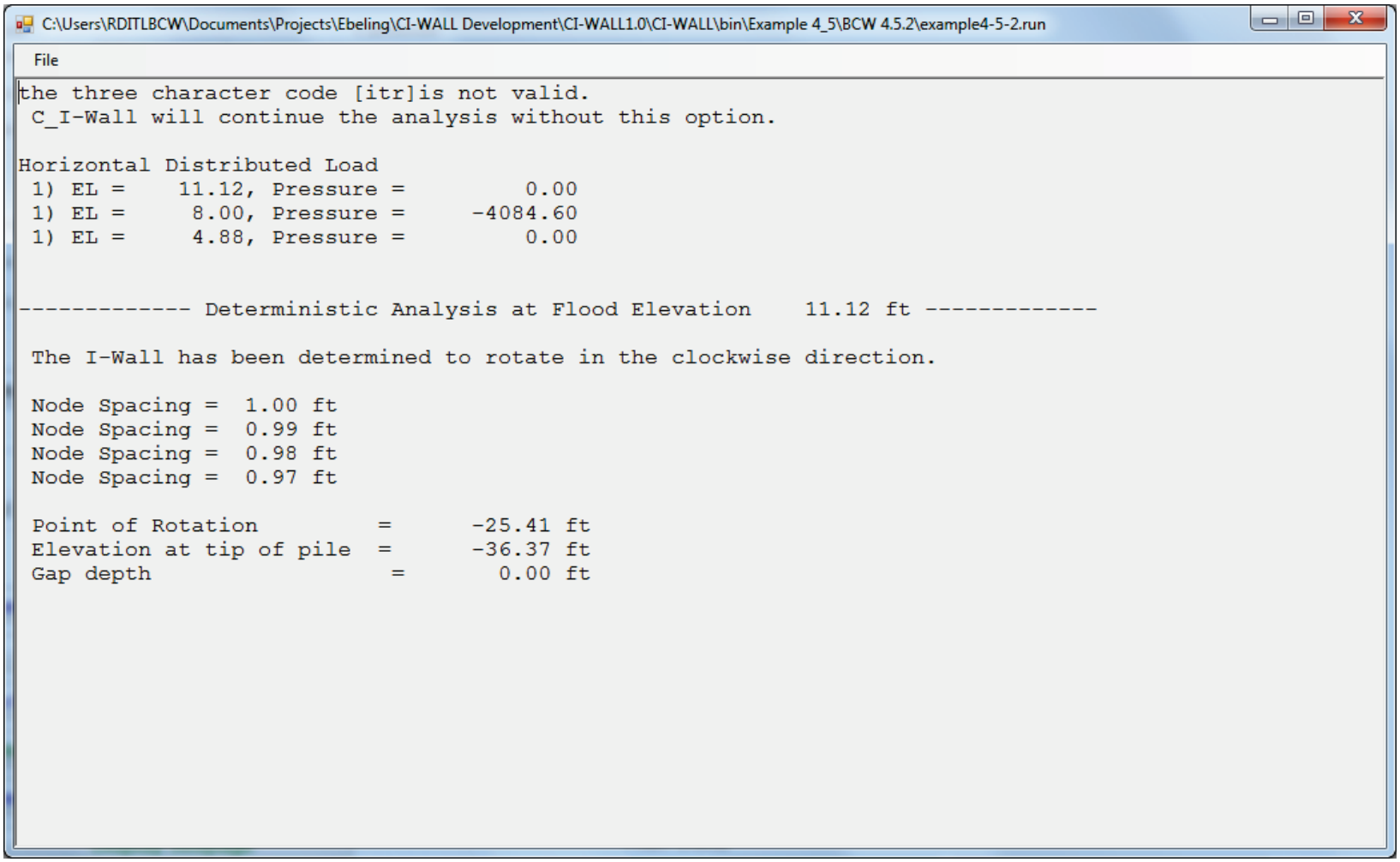

From the calculated pressures of Figures 4.43 and 4.44, both the net active and net passive pressures are constructed and presented in Figure 4.45a. For the prescribed LHS flood loading, the I-Wall will rotate in a clockwise direction. The net active pressure is determined from the difference between the LHS-mobilized active earth pressure and the RHS-mobilized passive earth pressure (i.e., acting on the other side of the wall) with the addition of the net water pressures and any external horizontal net pressures and overburden pressures (if any). The net passive pressure is determined by the difference between the LHS-mobilized passive earth pressure and the RHS-mobilized active earth pressure acting on the other side of the sheet-pile wall with the addition of any net water pressures and any external horizontal net pressures and overburden pressures (if any). Both the calculated net active and net passive pressures above the ground surface el 0.0 (and above Ro1 and R02) of Figure 4.45 are solely derived from the net water pressures of Figure 4.44b. 
Figure 4.43. Active and passive earth pressures for drained shear strength. (a) Left-hand side of wall. (b) Right-hand side of wall.

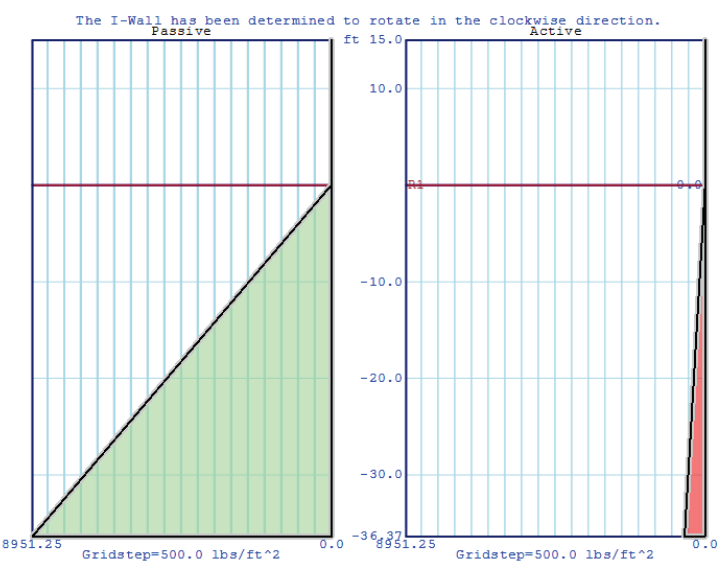

(a)

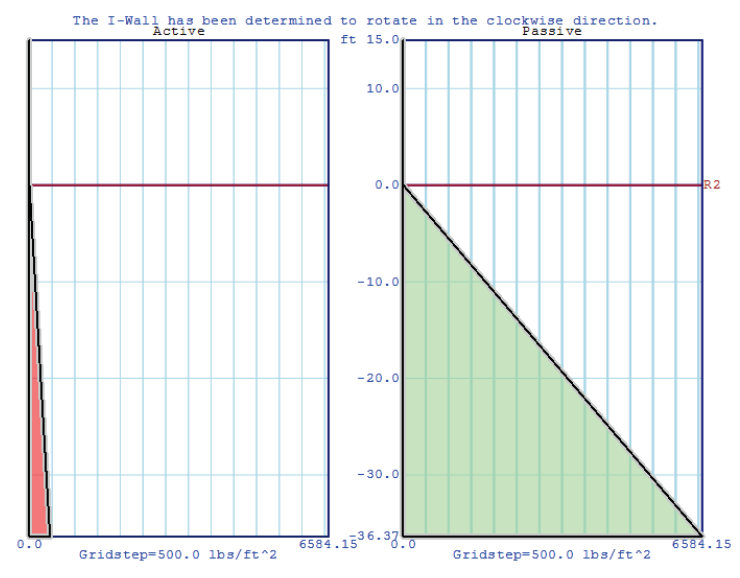

(b)

Figure 4.44. Pore water pressures. (a) Left- and right-hand side of wall. (b) Net water pressures.
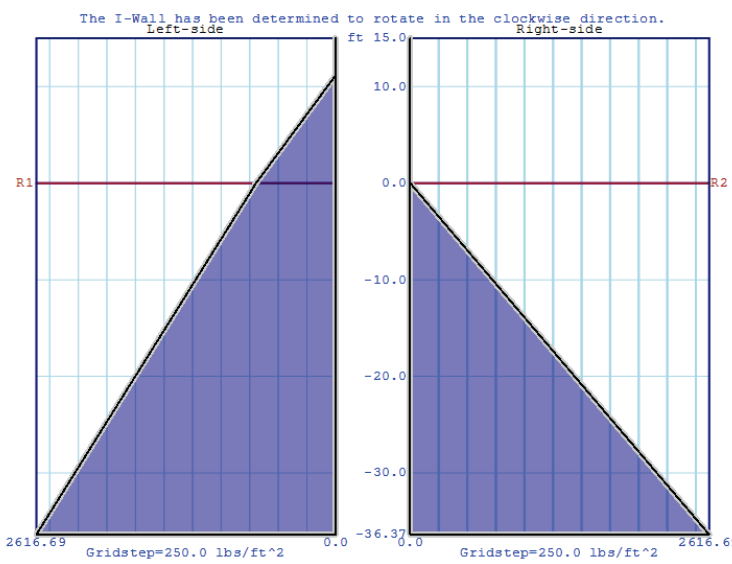

(a)

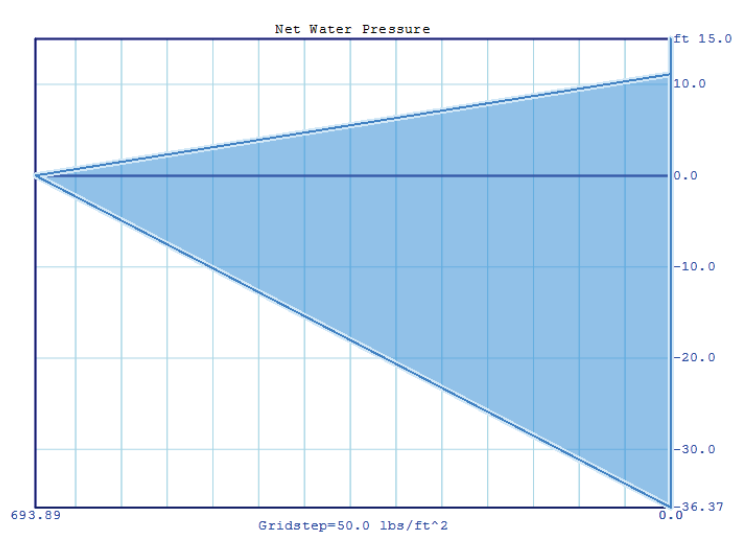

(b)

Figure 4.45. Net pressures. (a) Net active and net passive. (b) Net pressure diagram.

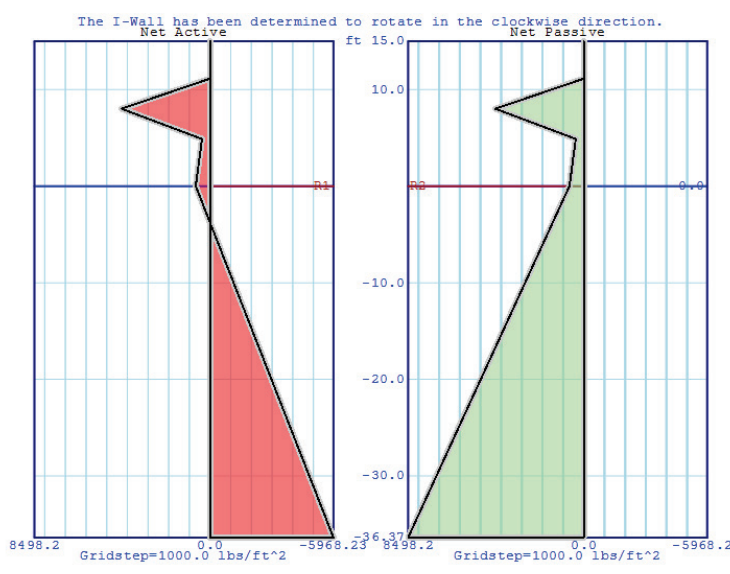

(a)

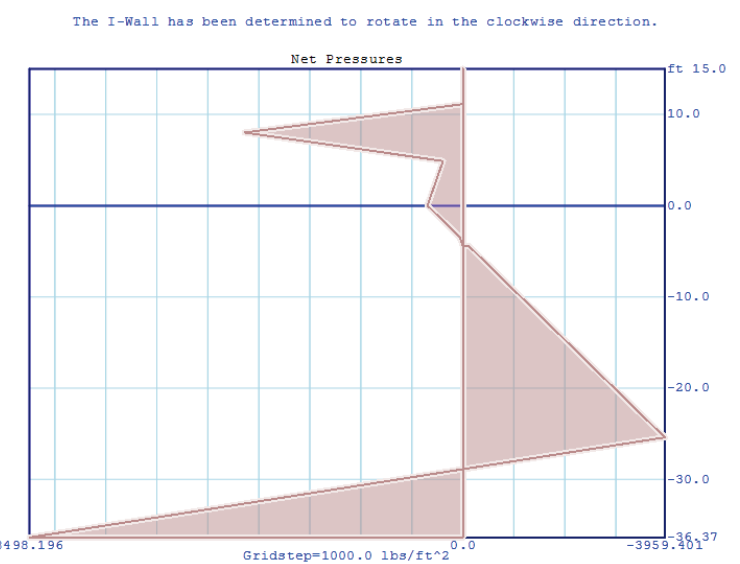

(b) 
The net pressure diagram of Figure $4.45 \mathrm{~b}$ is derived from the replication of all values of the net active pressures, from the top of the wall until the elevation at the point of rotation (at el -25.41 ft), at which instant the remaining values of the net pressure diagram are assumed to be linear with elevation between (1) the net active pressure value at the elevation of the point of rotation and (2) the net mobilized passive pressure at the elevation of the approximated sheet-pile tip (i.e., el -36.37). ${ }^{1}$ Figure $4.45 \mathrm{~b}$ is an illustration of how the net pressure diagram was formed. Because the I-Wall has been determined to rotate in a clockwise direction, the net pressure diagram of Figure $4.45 \mathrm{~b}$ shows that the net active pressures plotting on the LHS in the upper zone and on the RHS in the lower zone. The net passive pressures plot on the LHS of the sheet-pile wall. The final results are illustrated in Figure 4.46 by the presentation of the net pressure diagram Figures 4.46a (replica of Figure 4.45b) and 4.46b, the shear and moment diagrams.

Figure 4.46. (a) Net pressure diagram. (b) Shear and moment.

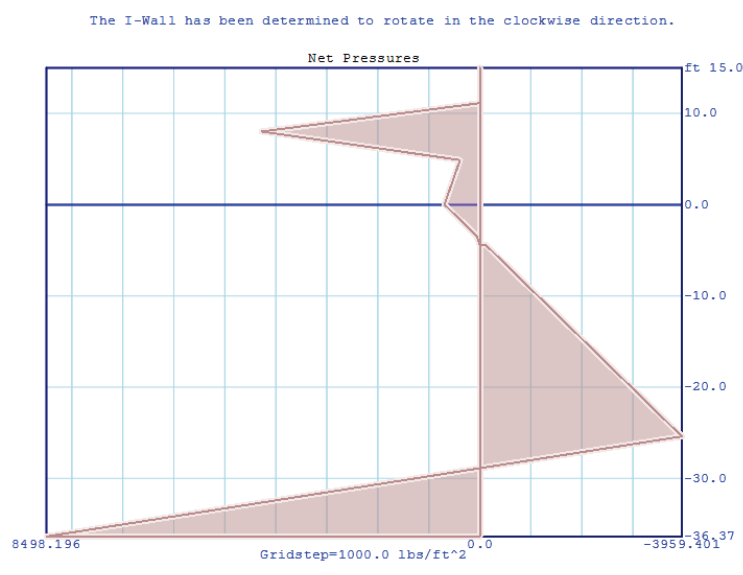

(a)

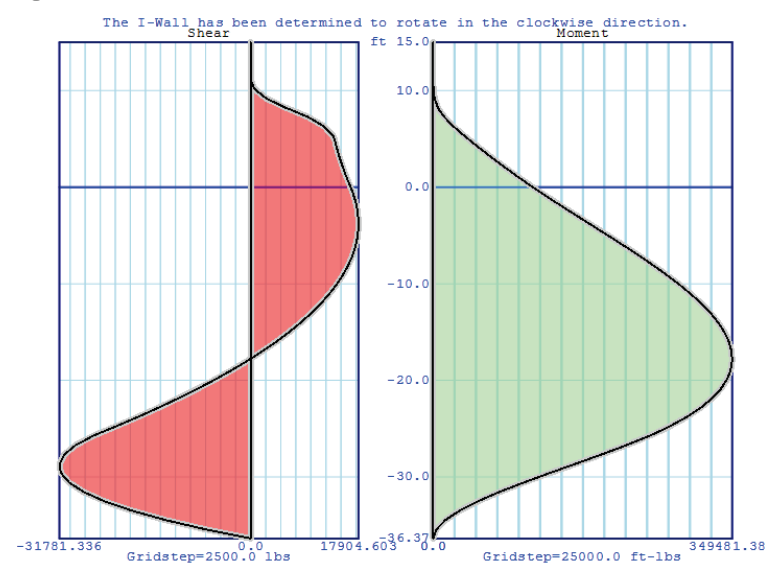

(b)

Figure $4.46 \mathrm{~b}$ shows that the maximum bending moment occurs at the zero crossing of the shear diagram. The maximum moment here is much higher than the previous example, with a value of $349.5 \mathrm{kip}-\mathrm{ft}$. This value is much higher than the allowable bending moment of $76.1 \mathrm{kip}-\mathrm{ft}$, therefore the wall will yield under the specified wave and pressure loads. The sheet-pile tip elevation is computed at $-36.37 \mathrm{ft}$ with a calculated depth of embedment of approximately $36.37 \mathrm{ft}$, which is more than twice the exposed height of $15 \mathrm{ft}$.

\footnotetext{
1 Thus, only one mobilized net passive pressure value in Figure 4.45a is used to construct the Figure $4.45 \mathrm{~b}$ net pressure diagram, corresponding to the sheet-pile tip elevation at el $-36.37 \mathrm{ft}$. The majority of the net pressure diagram is derived from the Figure $4.45 a$ net active pressure diagram.
} 
A diagram of drop in total head is given in Figure 4.47. To the left is a diagram of the wall showing the surface water, soil region surfaces, gap definition, wall depth, and the direction of seepage. The drop in total heads due to steady-state seepage is given in the right side plot. This plot follows the line of seepage along the wall from one side to the other on the horizontal axis and displays the head values on the vertical axis. The head loss occurs as water seeps from el o.o, down the LHS foundation sands to the sheet-pile tip and then up the RHS foundation sands to the ground surface (at el o.o), which is shown in the blue line. Because this site possesses a homogeneous value of hydraulic conductivity, the Figure 4.47 head loss with distance is linear along the line of seepage.

Figure 4.47. Drop in total heads due to seepage diagram.

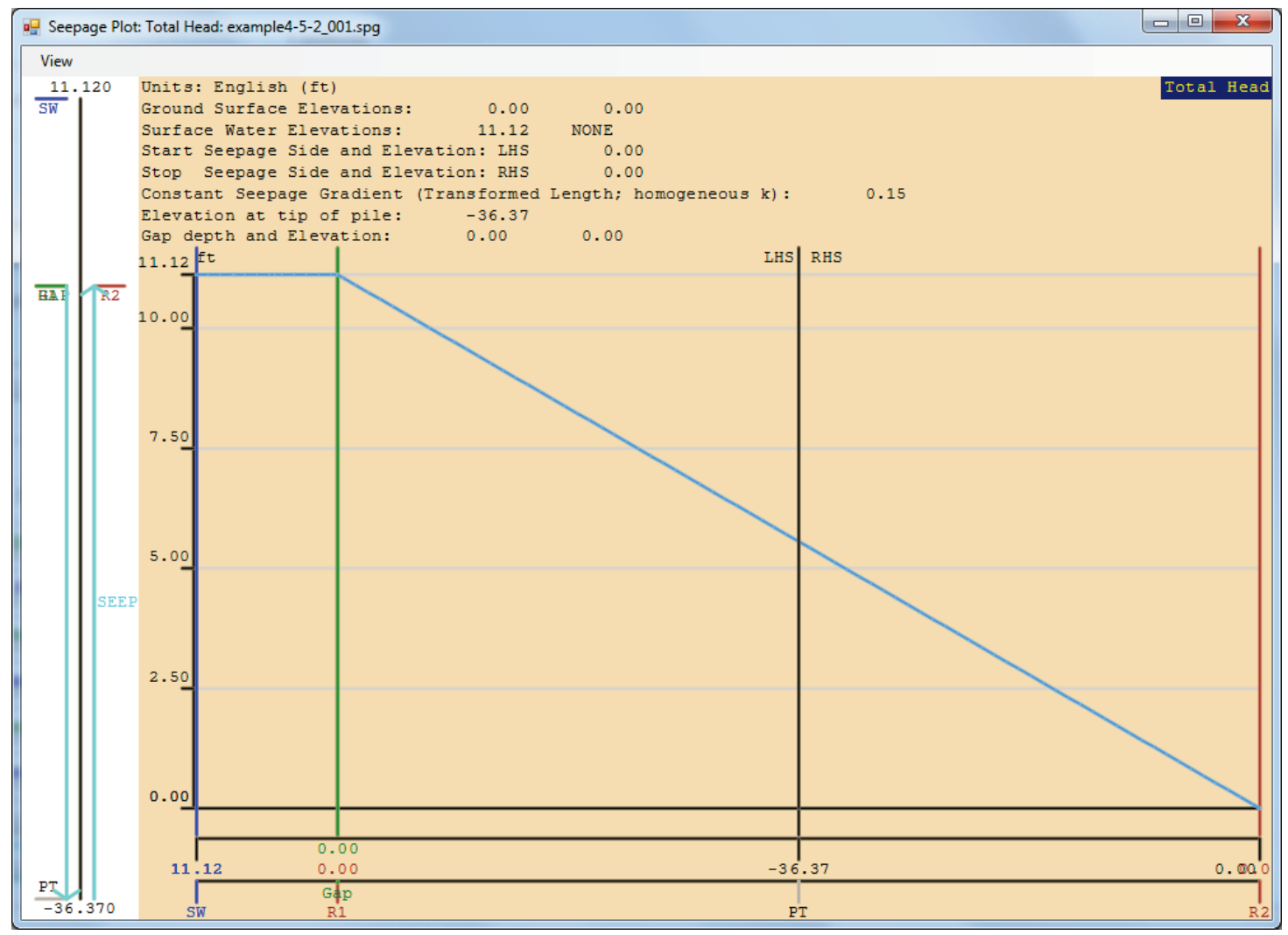

\subsubsection{Example 4.5.3 Coastal site including backfill}

The third design problem for a coastal I-Wall is determining the depth of embedment for a 15-ft-high I-Wall in dense sand (at elevation $0.0 \mathrm{ft}$ ) with a berm constructed above the $\mathrm{o}-\mathrm{ft}$ ground surface on the RHS of the I-Wall. The placement of a berm is being investigated to counter the effect of the wave loading that was first introduced in the previous example. The calculations made in this example do not yet include the wave loading. The 
performances of the I-Wall and berm are being investigated first. The dense sand has an effective stress definition with no cohesion, an effective angle of internal friction of $\left(\varphi^{\prime}\right)$ of $35 \mathrm{deg}$, a soil-to-sheet-pile effective interface friction angle $\left(\delta^{\prime}\right)$ of $17.5 \mathrm{deg}\left(=\varphi^{\prime} / 2\right)$, and a hydraulic conductivity $\left(\mathrm{K}^{\prime}\right)$ of 0.0000328 in all sand layers. The berm material consists of compacted dense sand with $\varphi^{\prime}$ of $35 \mathrm{deg}$ and an effective cohesion $\left(\mathrm{c}^{\prime}\right)$ value of 100.0 lb/ $\mathrm{ft}^{2}$.

In this example problem, a Factor of Safety of 1.0 is assigned for computing the mobilized active earth pressures and a Factor of Safety of 1.5 is assigned for computing the mobilized passive earth pressures within the soil layers. This I-Wall system has an exposed height of $15 \mathrm{ft}$ on the flood side. The water surfaces on both sides of the I-Wall are at $8 \mathrm{ft}$ (flood side and landside). For this example, the flood side corresponds to the LHS of the I-Wall. In this analysis, it is assumed that the I-Wall will rotate counterclockwise due to the berm loading. The sand layers have an unsaturated and saturated unit weight of 125 pcf. With a berm effective cohesion $\left(c^{\prime}\right)$ of 100.o psf, a gap can form, so hydraulic fracturing is enabled. Homogeneous seepage is allowed although seepage would not occur with coincident water levels.

Figure 4.48 outlines the basic geometry and structural parameters for the sheet-pile wall. As can be seen in Figure $4.48 \mathrm{~b}$, there are no external pressures (i.e., wave load) exerting on the RHS of the sheet-pile wall. A design computation run is performed to determine the embedment depth that will allow this wall to be stable for the required Factor of Safety applied to the passive earth pressures of the sand foundation (i.e., $F S_{\text {Passive }}=1.50$ ).

The resulting depth of embedment is shown in Figure 4.49. The I-Wall becomes stable when the sheet-pile tip is embedded to a depth of $-11.55 \mathrm{ft}$, because the soil surface is defined at an elevation of $0.0 \mathrm{ft}$. This is a much lower required depth of embedment when compared with the results from Example 4.5.1 of $-16 \mathrm{ft}$, because the wall is supported on the landside by the berm, and by the surface water on the flood side. The point about which the wall will rotate is at a depth of $-9.77 \mathrm{ft}$ below the ground surface. 
Figure 4.48. Sheet-pile wall with berm on landside (RHS). (a) Schematic of sheet-pile wall in sand site. (b) Plot of external loads.

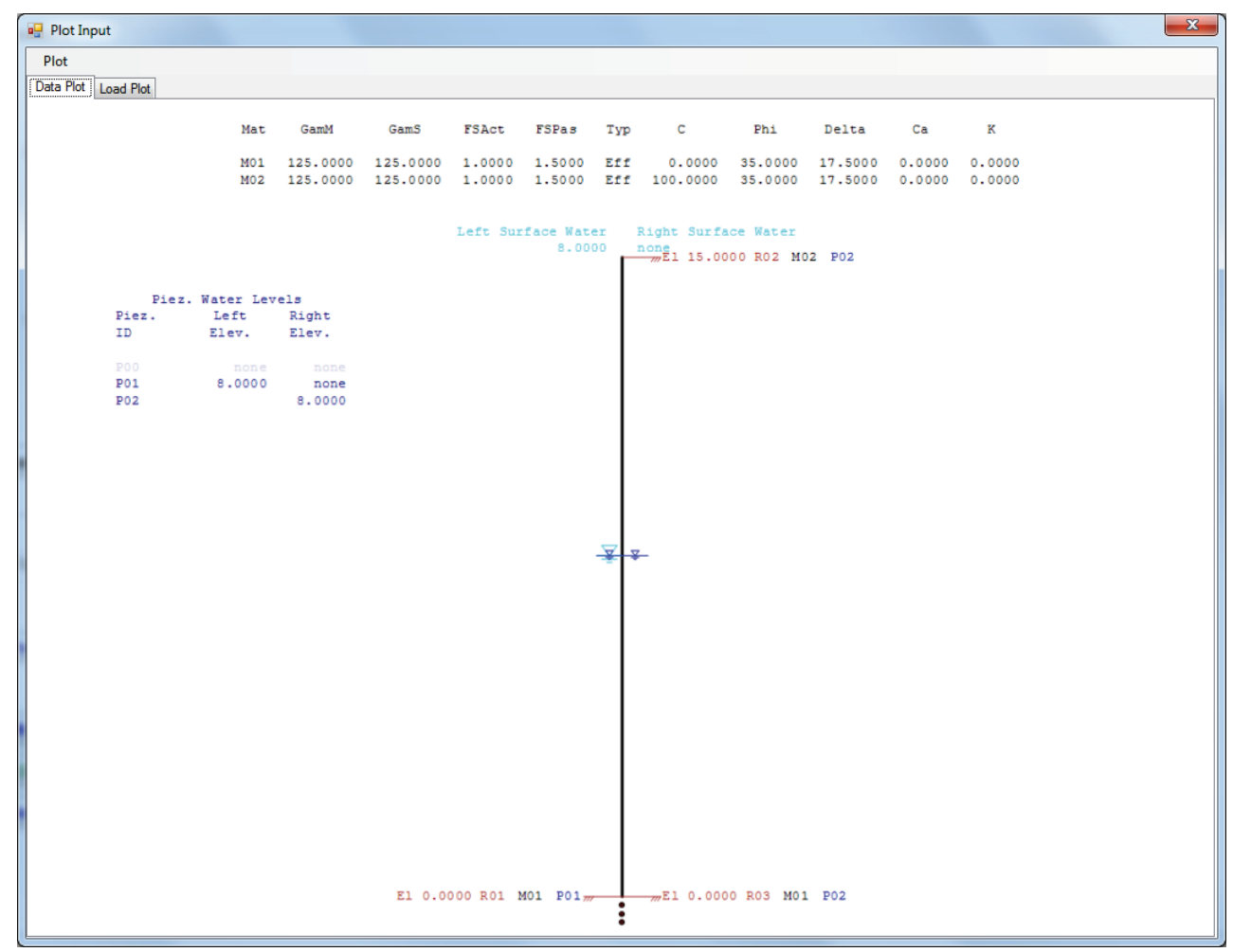

(a)

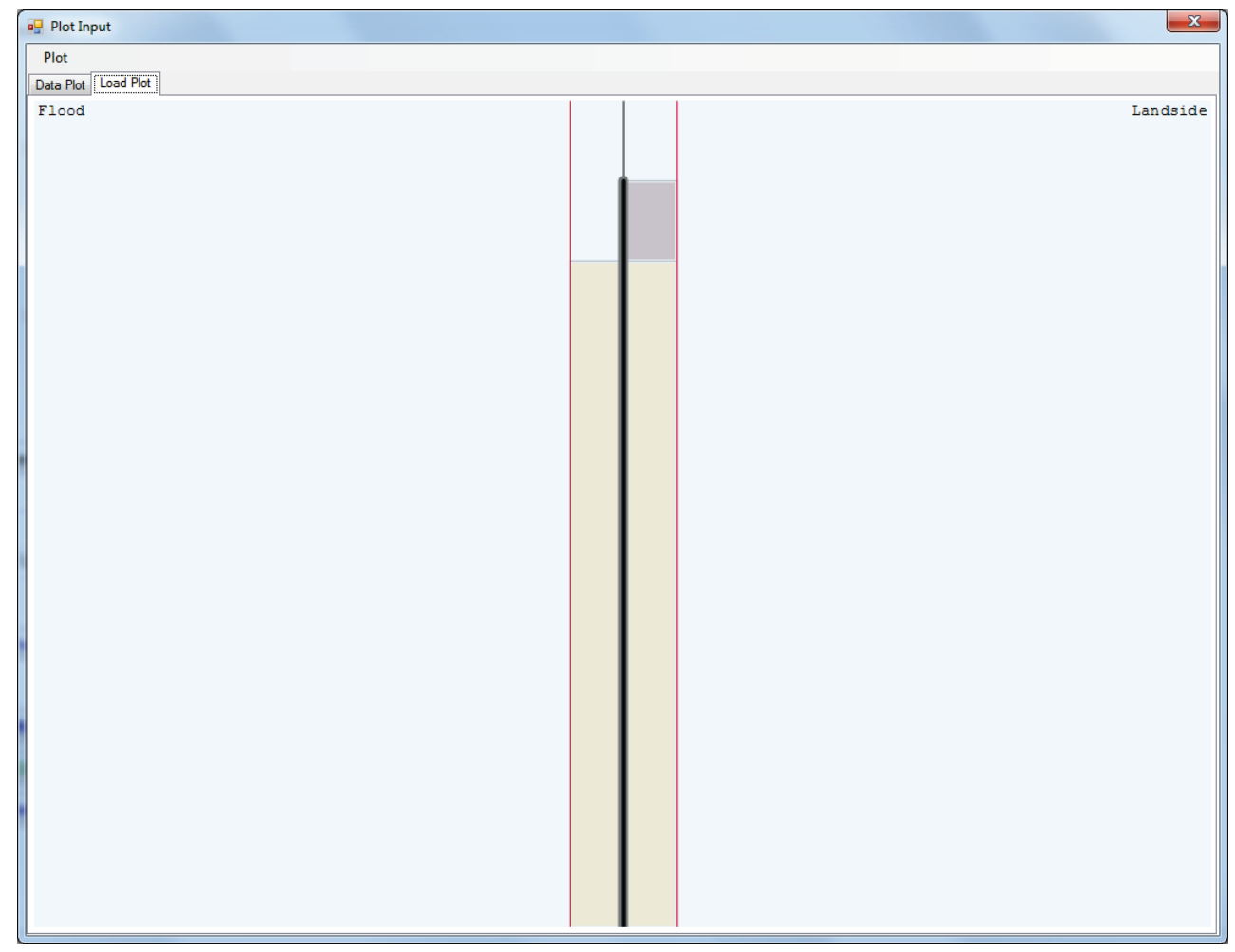

(b) 
Figure 4.49. Corps_I-Wall run file for the specified input.

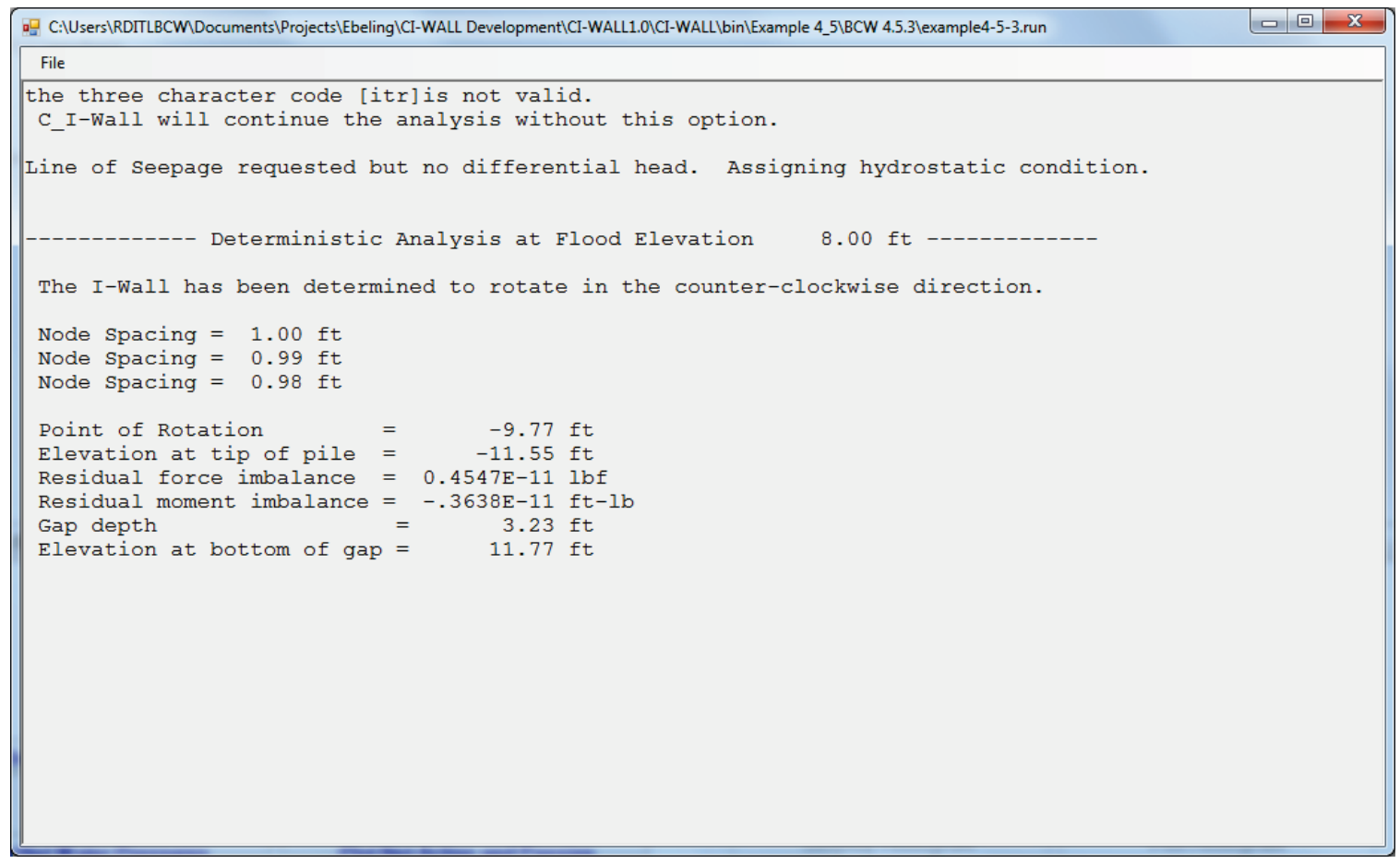

From the calculated pressures of Figures 4.50 and 4.51, both the net active and net passive pressures are constructed and presented in Figure 4.52a for the prescribed earth (berm) loading, and the I-Wall will rotate in a counterclockwise direction. The net active pressure is determined from the difference between the RHS-mobilized active earth pressure and the LHSmobilized passive earth pressure (i.e., acting on the other side of the wall) with the addition of the net water pressures (zero in this case) and any external horizontal net pressures and overburden pressures (if any). The net passive pressure is determined by the difference between the RHSmobilized passive earth pressure and the LHS-mobilized active earth pressure acting on the other side of the sheet-pile wall with the addition of any net water pressures and any external horizontal net pressures and overburden pressures (if any). Both the calculated net active and net passive pressures above the ground surface el 0.o (and above Ro1 and Ro2) of Figure 4.52 are solely derived from the net active soil pressures of Figure 4.52a.

The net pressure diagram of Figure $4.52 \mathrm{~b}$ is derived from the replication of all values of the net active pressures, from the top of the wall until the elevation at the point of rotation (at el $-9.77 \mathrm{ft}$ ), at which instant the remaining values of the net pressure diagram are assumed to be linear with elevation between (1) the net active pressure value at the elevation of the 
point of rotation and (2) the net mobilized passive pressure at the elevation of the approximated sheet-pile tip (i.e., el -11.55). ${ }^{1}$ Figure $4.52 \mathrm{~b}$ is an illustration of how the net pressure diagram was formed. Because the I-Wall has been determined to rotate in a clockwise direction, the net pressure diagram of Figure 4.52b shows that in the upper zone the net active pressures plot on the RHS and on the LHS in the lower zone. The net passive pressures plot on the RHS of the sheet-pile wall. The final results are illustrated in Figure 4.53 by the presentation of the net pressure diagram Figures 4.53a (replica of Figure 4.52b) and 4.53b, the shear and moment diagrams.

Figure 4.50. Active and passive earth pressures for drained shear strength. (a) Left-hand side of wall. (b) Right-hand side of wall.

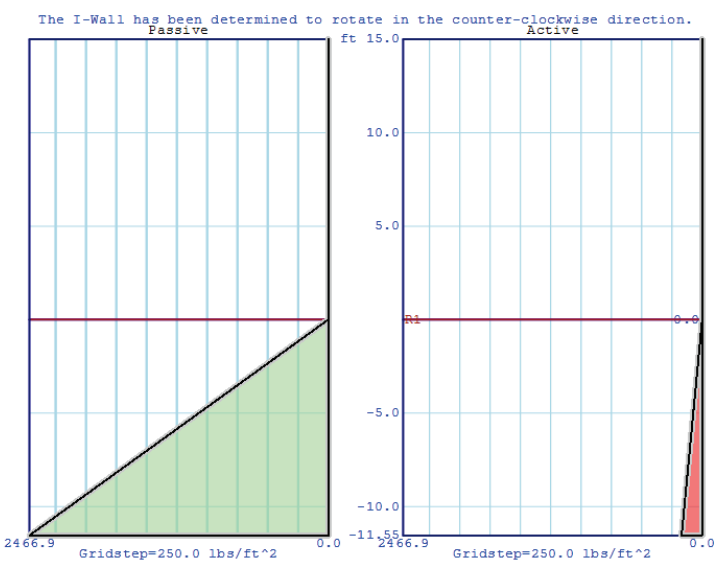

(a)

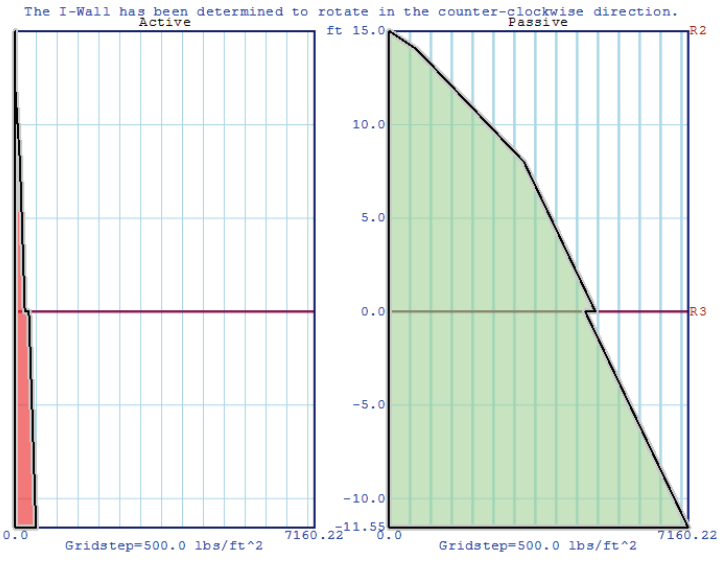

(b)

Figure 4.51. Pore water pressures. (a) Left- and right-hand side of wall. (b) Net water pressures.

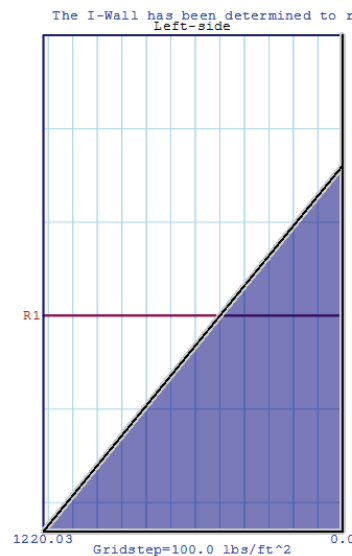

Gridstep $=100.0$ 1bs/ft^2

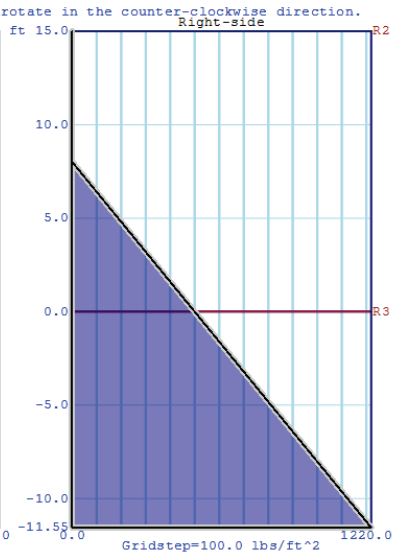

(a)

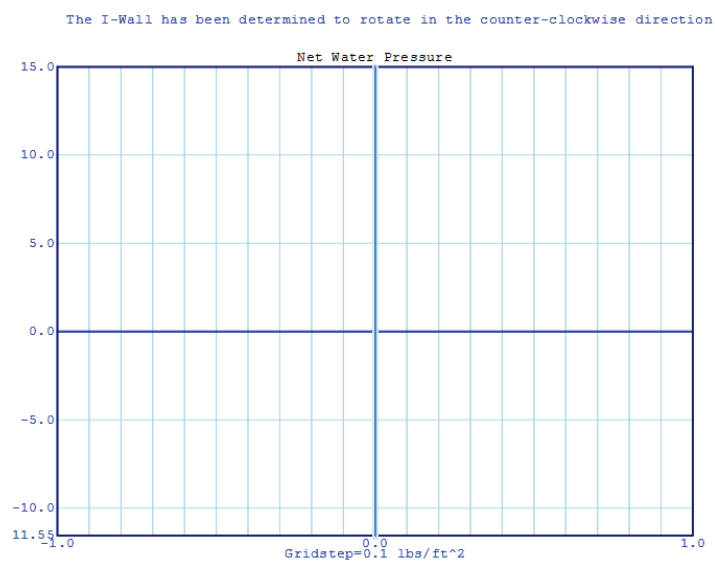

(b)

\footnotetext{
1 One mobilized net passive pressure value in Figure 4.52a is used to construct the Figure 4.53a net pressure diagram, corresponding to the sheet-pile tip elevation at el $-11.55 \mathrm{ft}$. The majority of the net pressure diagram is derived from the Figure 4.52 a net active pressure diagram.
} 
Figure 4.52. Net pressures. (a) Net active and net passive. (b) Net pressure diagram.

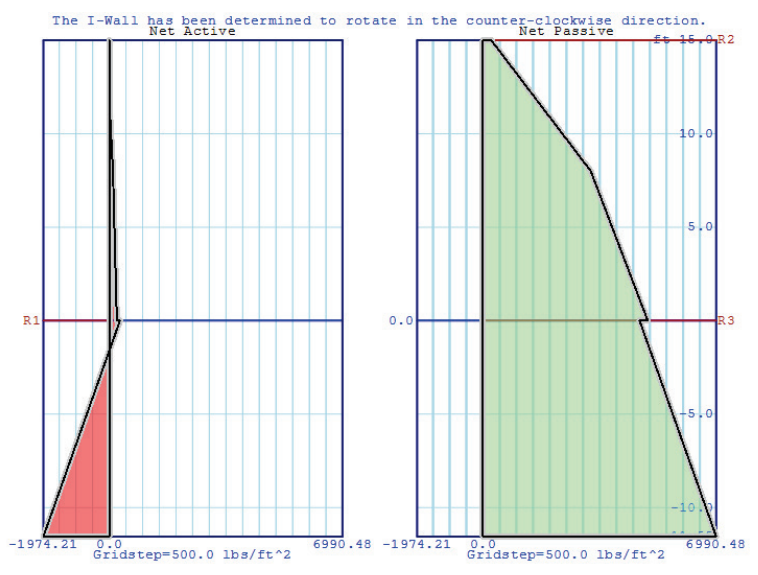

(a)

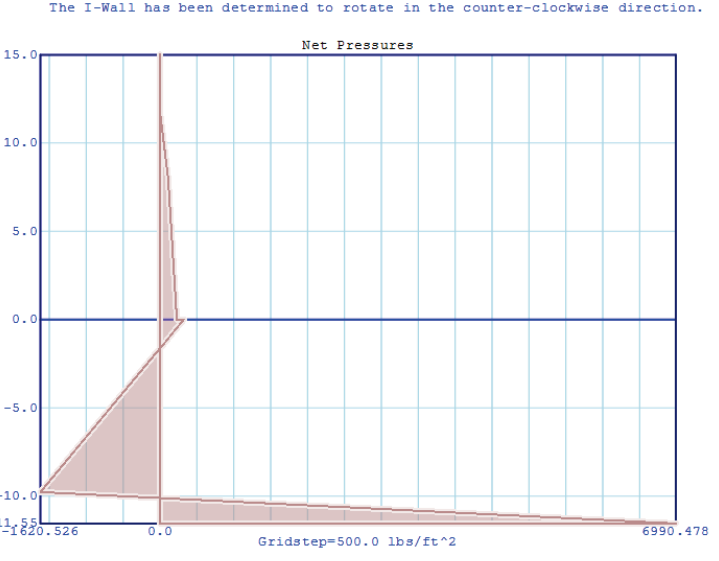

(b)

Figure 4.53. (a) Net pressure diagram. (b) Shear and moment.

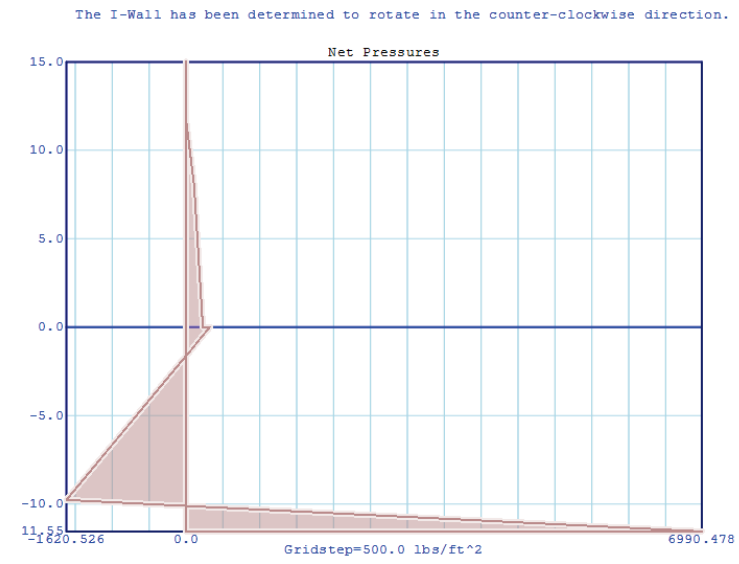

(a)

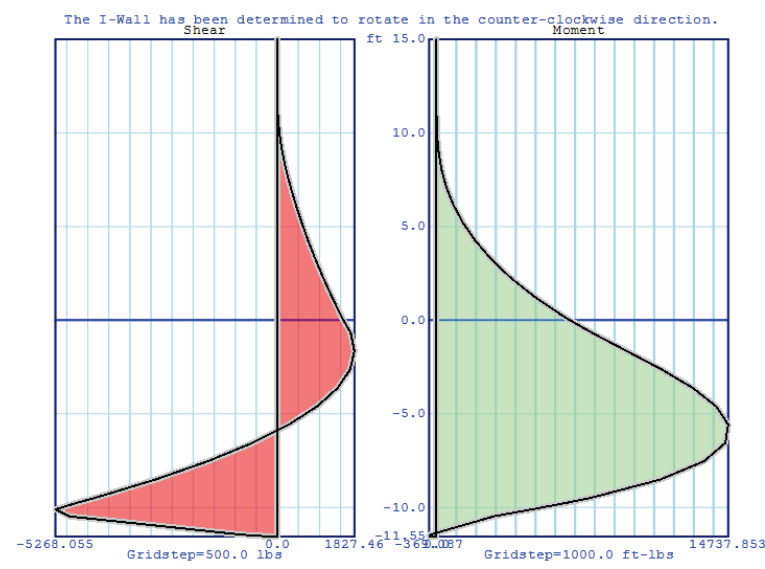

(b)

Figure $4.53 \mathrm{~b}$ shows that the maximum bending moment occurs at the zero crossing of the shear diagram. The maximum moment has a value of $14.8 \mathrm{kip}-\mathrm{ft}$, which is much less than the allowable bending moment of $76.1 \mathrm{kip}-\mathrm{ft}$. The sheet-pile tip elevation is computed at $-11.55 \mathrm{ft}$ with a calculated depth of embedment of approximately $11.55 \mathrm{ft}$.

Because the water elevations are the same for the flood side and landside of the wall, the net water pressures and therefore seepage are o.o. Seepage calculations are automatically stopped in Corps_I-Wall when this condition occurs and the problem is run with hydrostatic conditions. 


\subsubsection{Example 4.5.4 Coastal site including backfill with surge loading}

The fourth design problem for a coastal I-Wall is determining the depth of embedment for an I-Wall with 15 - $\mathrm{ft}$ height exposed to surge loading. The I-Wall is founded in dense sand (at elevation $0.0 \mathrm{ft}$ - Figure 4.54) and retaining a berm on the RHS of the I-Wall. The intent of the berm is to counter the detrimental effects of wave loading. The dense sand at o.o elevation and below has an effective stress definition with no effective cohesion, an effective angle of internal friction of $\left(\varphi^{\prime}\right)$ of $35 \mathrm{deg}$, a soil-tosheet-pile effective interface friction angle $\left(\delta^{\prime}\right)$ of $17.5 \mathrm{deg}\left(=\varphi^{\prime} / 2\right)$, and a hydraulic conductivity $\left(\mathrm{K}^{\prime}\right)$ of $0.0000328 \mathrm{ft} / \mathrm{sec}$ in all sand layers. In this example problem, a Factor of Safety of 1.0 is assigned for computing active earth pressures and a Factor of Safety of 1.5 is assigned for computing passive earth pressures within the soil layer. The sand layers are unsaturated (not used in the analysis) and saturated unit weights of $125 \mathrm{pcf}$. The compacted dense sand that forms the berm on the landside of the I-Wall has an effective cohesion ( $\left.\mathrm{c}^{\prime}\right)$ of $100.0 \mathrm{psf}$, so hydraulic fracture option is enabled. Homogeneous seepage is also allowed.

The flood side corresponds to the LHS of the I-Wall. The water surface on the LHS of the I-Wall is determined by the possible surge wave size calculation in Appendix F and detailed in Example 4.5.2 using the Minikin formulation for breaking waves. Because the swell height is the elevation of the average still-water level added to half of the breaking wave height ( $~ 6.24 \mathrm{ft}$ from Equation F.2 and Table F.3), the water level on the flood side is set equal to the swell height of $8 \mathrm{ft}+3.12 \mathrm{ft}=11.12 \mathrm{ft}$ on the flood side and o-ft landside. The additional horizontal pressure due to the motion of the wave is accounted for by having a triangular horizontal pressure distribution that ranges from $0.0 \mathrm{lb} / \mathrm{ft}^{2}$ at $11.12 \mathrm{ft}$ to a maximum pressure of $4,084.6 \mathrm{lb} / \mathrm{ft}^{2}$ (computed using equation $\mathrm{F} .4$ ) at $8 \mathrm{ft}$ to $0.0 \mathrm{lb} / \mathrm{ft}^{2}$ at $4.88 \mathrm{ft}(8 \mathrm{ft}-3.12 \mathrm{ft})$. In this analysis, it is assumed that the I-Wall will rotate clockwise during flood and surge loading. However, this is not guaranteed and, as will be discussed, the wall was found to show no rotation whatsoever. 
Figure 4.54. Sheet-pile wall with berm on landside (RHS). (a) Schematic of sheet-pile wall in sand site. (b) Wave load applied to flood side (LHS) of sheet-pile wall.

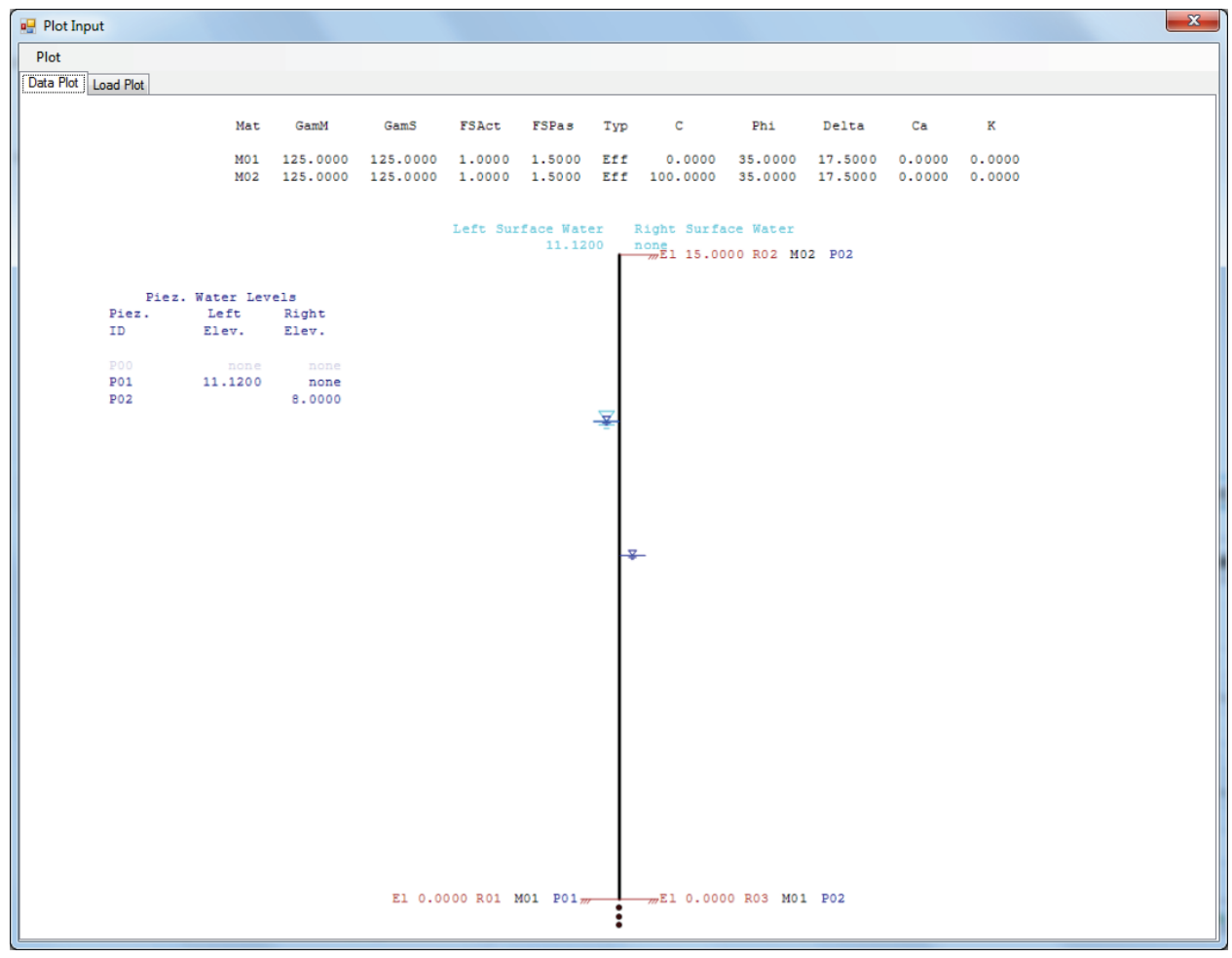

(a)

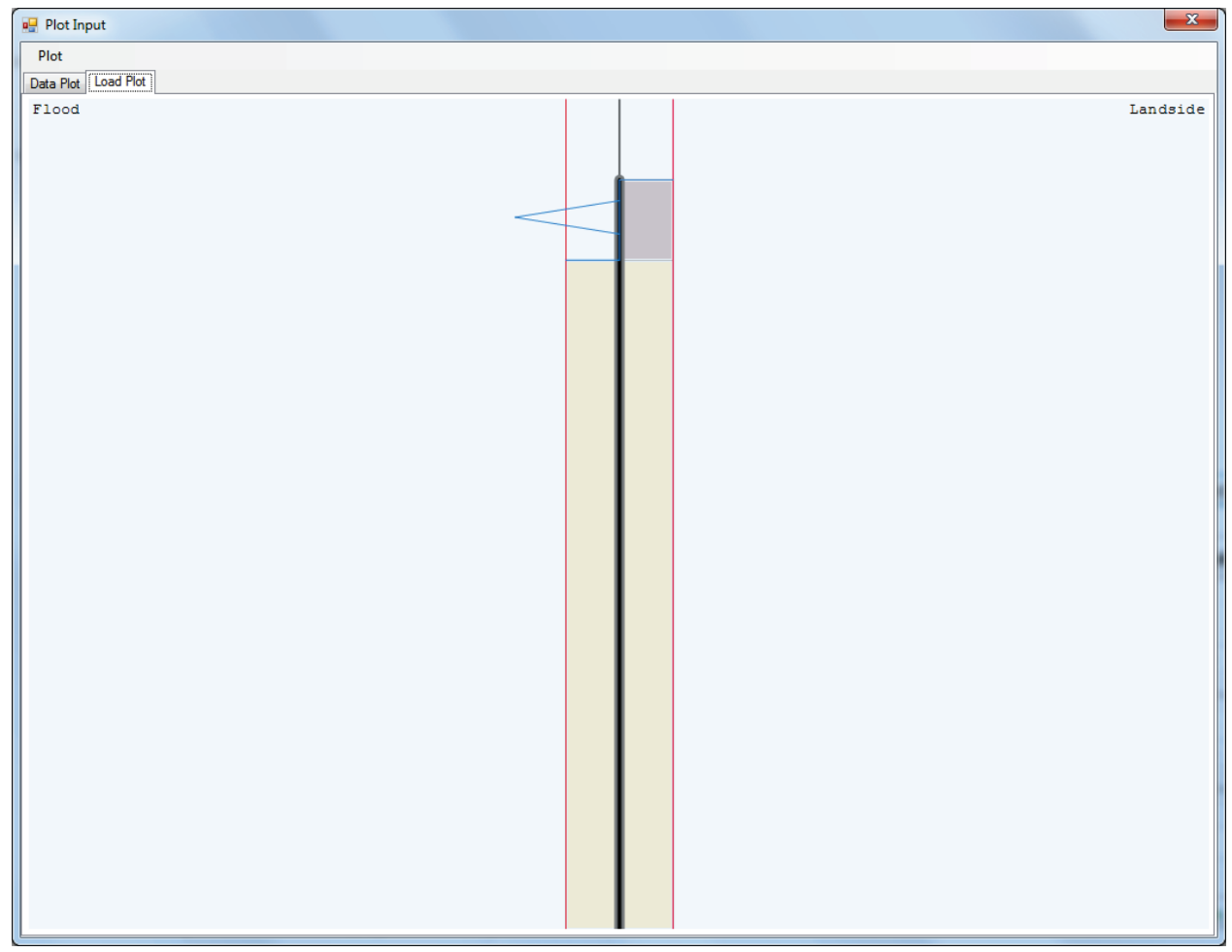

(b) 
Figures 4.55 and 4.56 show the results from the run specified, given seepage and for an assumed clockwise I-Wall rotation. For this example, the RHS-mobilized passive earth pressure forces balance the LHS net water and dynamic forces, meaning that the I-Wall does not have to resist any of the applied forces through a transfer of loading into the foundation via bending of the sheet pile. The I-Wall is simply a separator between the RHS soil berm and the LHS water loading. In this situation, the computed Factor of Safety for the structure becomes higher than the required value of 1.5 for the passive earth pressure region, and there is no means to compute a sheet-pile tip elevation because the I-Wall is held in place by the water pressure on one side and the earth loading provided by the berm on the other side. In practical terms, it is sufficient to build the wall to the depth for normal water levels (Example 4.5.3) because the wall is superfluous when wave loading is applied. The sheet-pile wall does serve the purpose to prevent soil erosion due to wave action applied to the earthen berm.

Figure 4.55. Corps_I-Wall run file for the specified input (with seepage enabled).

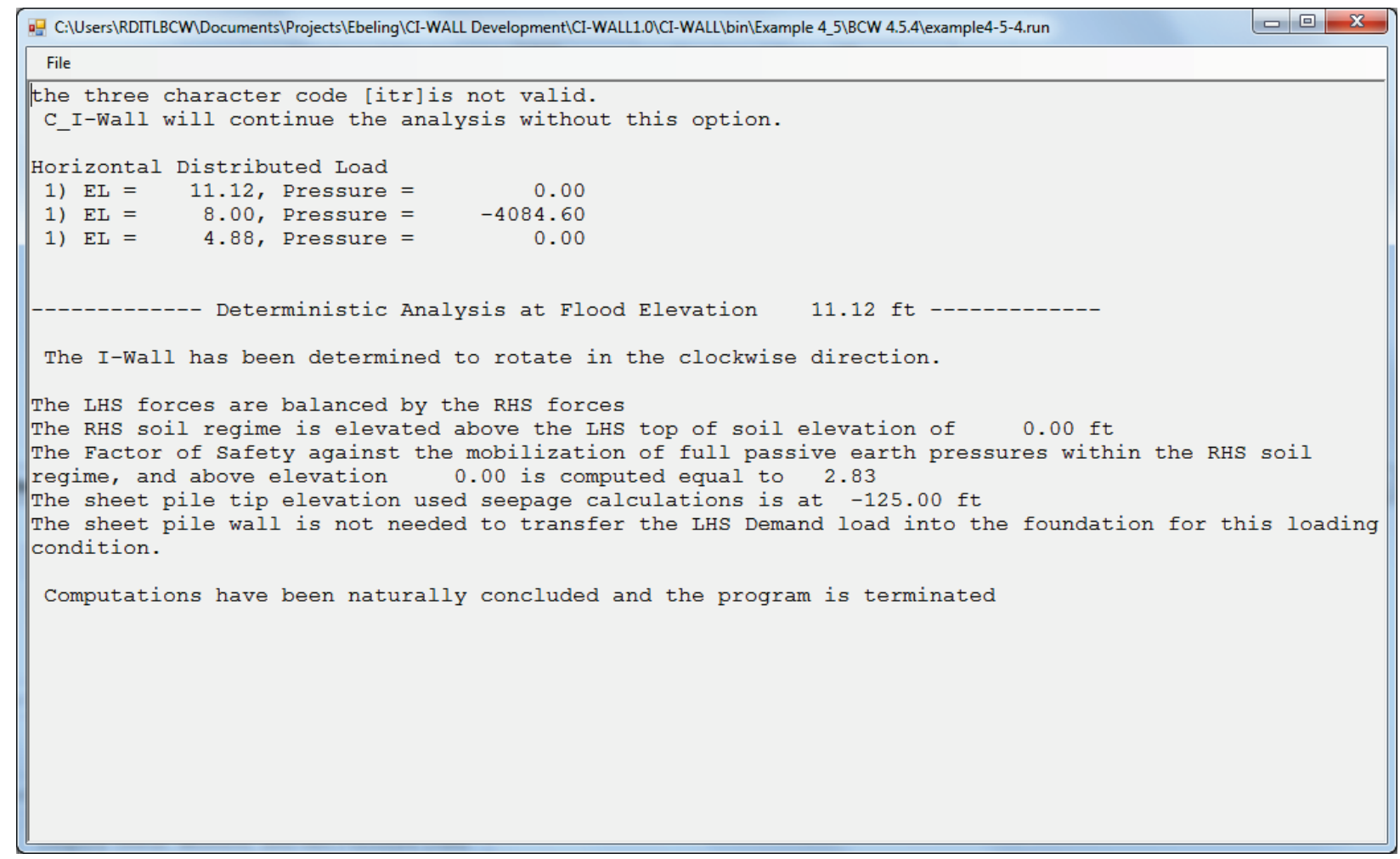


Figure 4.56. Corps_I-Wall run file for the specified input (without seepage).

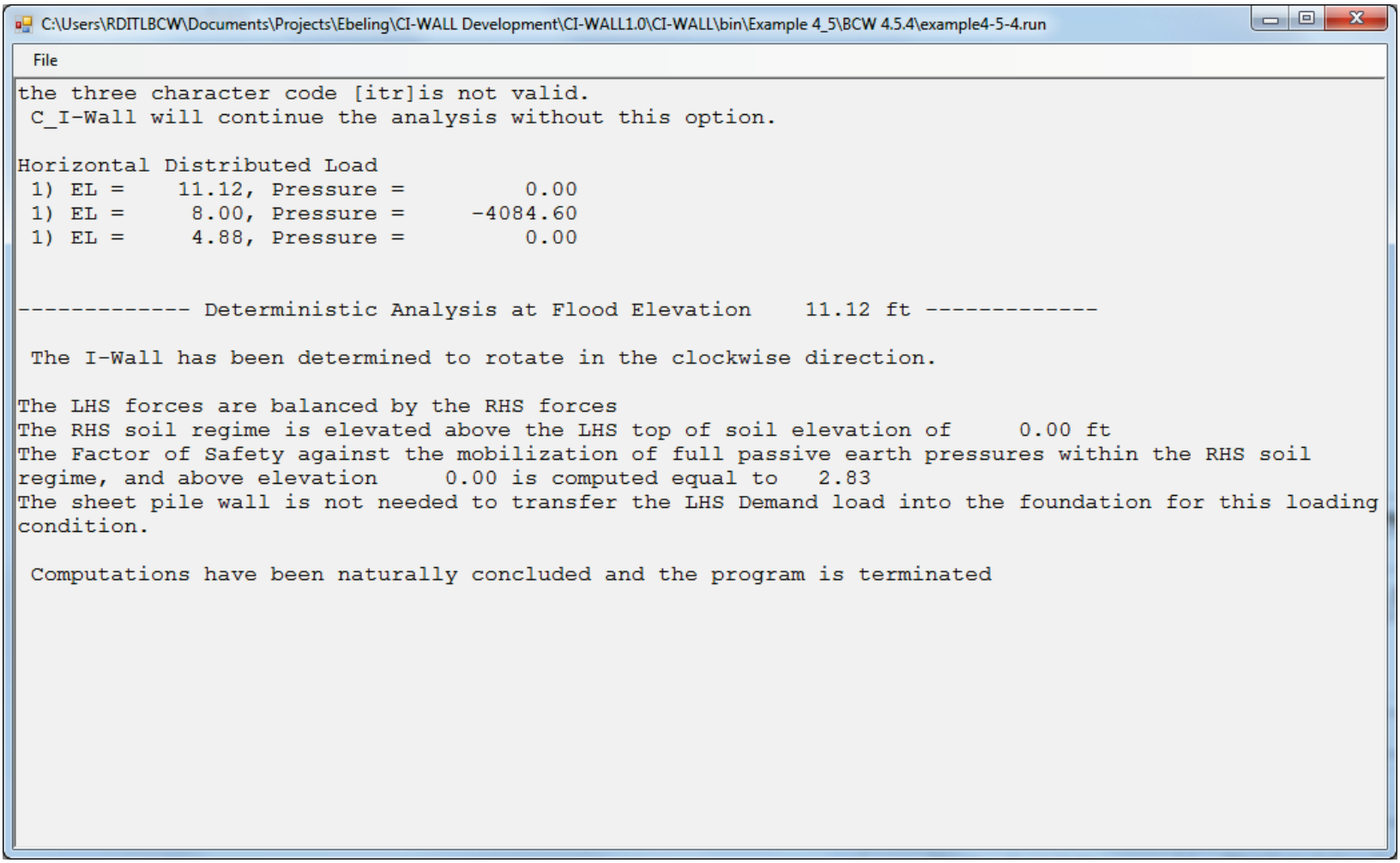

\subsubsection{Comparison and interpretation of backfill with surge loading}

Figure 4.57 shows the loads that are acting on the I-Wall of Example 4.5.4, which is expected to rotate clockwise about a point at or below the datum elevation. This model is for pseudostatic wave pressures acting on the I-Wall. Hand calculations can be performed to confirm the calculations made by Corps_I-Wall Version 1.o that determine the Factor of Safety for the I-Wall. In order for the I-Wall to be unengaged, the net moment acting on the wall must be $0.0 \mathrm{ft}-\mathrm{lb}$ with a Factor of Safety greater than 1.5 .

Two hydrostatic water pressures act on the wall, the pressure due to the landside piezometric water level at elevation $8 \mathrm{ft}$ above the datum, and the flood side water level determined by applying the Minikin method as an elevation of $11.12 \mathrm{ft}$. The pressures from both sides are given in Table 4.5. These hydrostatic pressures are added to form the net hydrostatic water pressures, detailed in Table 4.6.

The dynamic water pressures resulting from the moving water in the wave colliding with the I-Wall is calculated by the Minikin method. These values are shown in Table 4.7. 
Figure 4.57. Representation (not to scale) of both the water and earth pressures acting on the I-Wall in Example 4.5.4.

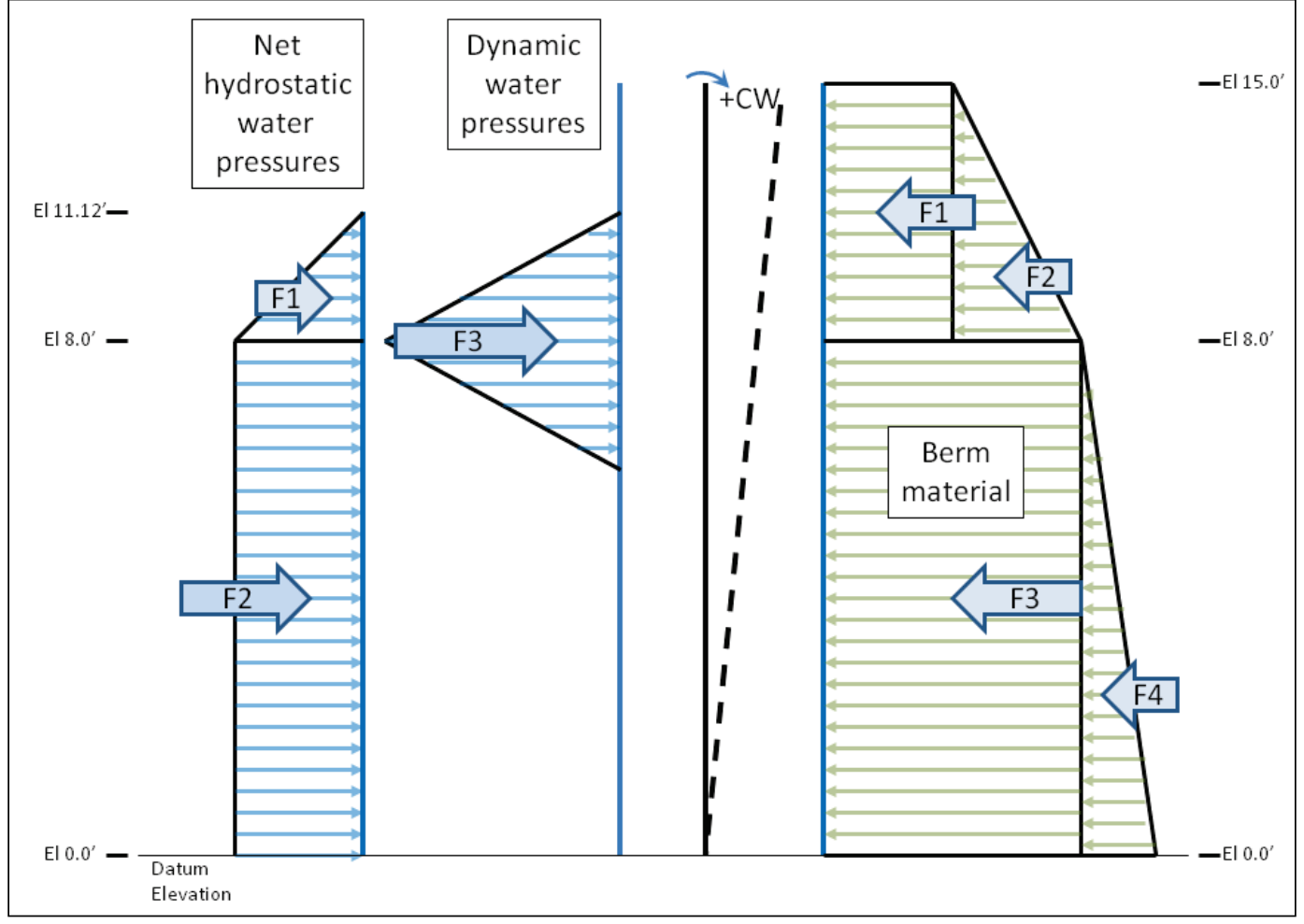

Table 4.5. Hydrostatic pressures with elevations from the site.

\begin{tabular}{|l|l|l|l|}
\hline \multicolumn{4}{|c|}{ Hydrostatic Water Pressures } \\
\hline \multicolumn{3}{|c|}{ Left-Hand Side } & \multicolumn{2}{c|}{ Right-Hand Side } \\
\hline Elevation (ft) & Pressure (psf) & Elevation (ft) & Pressure (psf) \\
\hline 11.12 & 0.00 & & \\
\hline 8.0 & 194.69 & 8.0 & 0 \\
\hline 0.0 & 693.89 & 0.0 & 499.2 \\
\hline
\end{tabular}

Table 4.6. Combining the left-hand side and right-hand side hydrostatic pressures to get the net pressure.

\begin{tabular}{|l|l|l|}
\hline \multicolumn{3}{|c|}{ Net Hydrostatic Water Pressure } \\
(Applied as water pressure on the left-hand side) \\
\hline Elevation (ft) & Depth (ft) & Pressure (psf) \\
\hline 11.12 & 0.0 & 0.00 \\
\hline 8.0 & 3.12 & 194.69 \\
\hline 0.0 & 11.12 & 194.69 \\
\hline
\end{tabular}


Table 4.7. Dynamic water pressures with elevations calculated with the Minikin method.

\begin{tabular}{|l|l|c|}
\hline \multicolumn{3}{|c|}{ Dynamic Water Pressures } \\
(Applied as water pressure on the left-hand side) \\
\hline Elevation (ft) & Depth (ft) & Pressure (psf) \\
\hline 11.12 & 0.0 & 0.00 \\
\hline 8.0 & 3.12 & $4,084.60$ \\
\hline 4.88 & 6.24 & 0.00 \\
\hline
\end{tabular}

Figure 4.57 gives a representation (not to scale) of these pressures acting on the LHS of an I-Wall. These pressures can be represented as a set of resultant forces (F1, F2, and $\left.\mathrm{F}_{3}\right)$ acting at locations shown along the wall for determining the moment at the datum of the wall with elevation 0.0 for this example. The resulting forces and resultant moments are given in Table 4.8.

Table 4.8. Forces and moments calculated from water pressure distributions at the datum elevation.

\begin{tabular}{|l|r|l|r|}
\hline & Force (lb) & Moment Arm (ft) & Moment (ft-lb) \\
\hline F1 & 303.71 & 9.04 & $2,745.57$ \\
\hline F2 & $1,557.50$ & 4.0 & $6,230.02$ \\
\hline F3 & $12,743.95$ & 8.0 & $101,951.60$ \\
\hline Sum_f & $14,605.16$ & Sum_m & $110,927.19$ \\
\hline
\end{tabular}

Assuming a Clockwise Rotation (CW), the RHS berm material properties must be taken into account to determine the mobilized passive earth pressures, the resulting forces, and moments acting as "passive" resistance to the water pressures on the LHS of the wall. In this example problem, a Factor of Safety of 1.0 is assigned for computing active earth pressures, and a Factor of Safety of 1.5 is assigned for computing passive earth pressures within the soil layer. This I-Wall system has an exposed height of $15 \mathrm{ft}$ on the flood side. In this analysis, it is assumed that the I-Wall will rotate clockwise due to the wave loads. The sand layers have an effective angle of internal friction of $\left(\varphi^{\prime}\right)$ of 35 deg, a soil-to-sheet- pile effective interface friction angle $\left(\delta^{\prime}\right)$ of $17.5 \mathrm{deg}\left(=\varphi^{\prime} / 2\right)$, a hydraulic conductivity $\left(K^{\prime}\right)$ of $0.0000328 \mathrm{ft} / \mathrm{sec}$, ground surface slope $(\beta)$ of $0.0 \mathrm{deg}$, and unsaturated and saturated unit weights of $125 \mathrm{pcf}$. With a berm effective cohesion $\left(\mathrm{c}^{\prime}\right)$ of 100.0 psf, a gap can be formed, so hydraulic fracturing is enabled. 
The resisting moment provided by the earth retaining structural berm can meet or exceed the driving moment caused by flood water pressures on the wall. If this occurs the wall serves only to provide erosion protection for the berm, and does not need to provide moment or force resistance.

In order to determine the resisting moment provided by the earth retaining structural berm, the Factor of Safety (passive) is applied to the berm's material properties. The LHS driving moment of the flood water pressures and the RHS resisting moment provided by the berm are computed.

If the resisting moment, which is acting in the opposite direction as the driving moment is less than the driving moment, then the I-Wall must also add its resistance (through a transfer of loading into the foundation soils) to counteract the driving moment of the water loading. If the resisting moment is greater than the driving moment, then the Factor of Safety (passive) can be raised iteratively resulting in different computed values for the mobilized berm material properties until the resisting moment is virtually equivalent to the driving moment.

The Factor of Safety is applied to the material properties in the following ways to determine the actual mobilized passive earth pressure properties:

$$
\begin{gathered}
c_{\text {mobilized }}^{\prime}=\frac{c^{\prime}}{F S_{\text {passive }}} \\
\tan \varphi_{\text {mobilized }}^{\prime}=\frac{\tan \varphi^{\prime}}{F S_{\text {passive }}} \\
\tan \delta_{\text {mobilized }}^{\prime}=\frac{\tan \delta^{\prime}}{F S_{\text {passive }}}
\end{gathered}
$$

From Coulomb's equation, the passive earth pressure coefficient is

$$
K_{p}=\frac{\cos ^{2}\left(\varphi_{\text {mobilized }}^{\prime}\right)}{\cos \left(\delta_{\text {mobilized }}^{\prime}\right)\left[1-\sqrt{\frac{\sin \left(\varphi_{\text {mobilized }}^{\prime}+\delta_{\text {mobilized }}^{\prime} \sin \left(\varphi_{\text {mobilized }}^{\prime}\right)\right.}{\cos \left(\delta_{\text {mobilized }}^{\prime}\right)}}\right]}
$$

For a Factor of Safety (passive) of 1.5, the mobilized material values are:

$$
\begin{aligned}
& c_{\text {mobilized }}^{\prime}=66.67 \mathrm{psf} \\
& \varphi_{\text {mobilized }}^{\prime}=25.02 \mathrm{deg} \\
& \delta_{\text {mobilized }}^{\prime}=11.87 \mathrm{deg}
\end{aligned}
$$


The RHS earth pressures can be computed based on Coulomb's equation, as shown in Table 4.9.

Table 4.9. Determining earth pressures on the right-hand side of the wall with Factor of Safety 1.5.

\begin{tabular}{|c|l|l|l|l|l|}
\hline \multicolumn{4}{|c|}{ RHS Earth Pressures } & \multicolumn{1}{|c|}{$\boldsymbol{K}_{p} * \sigma_{v^{\prime}}+2 \boldsymbol{c}_{\text {mobilized }}^{\prime} \sqrt{\boldsymbol{K}_{p}}$} & $\sigma_{p_{\text {mob }}} * \cos \boldsymbol{\delta}_{\text {mobilized }}^{\prime}$ \\
\hline $\begin{array}{l}\text { Elevation } \\
\text { (ft) }\end{array}$ & $\begin{array}{l}\text { Depth } \\
\text { (ft) }\end{array}$ & $\begin{array}{l}\boldsymbol{\sigma}_{v^{\prime}} \\
\text { (psf) }\end{array}$ & $\begin{array}{l}\boldsymbol{K}_{p} * \boldsymbol{\sigma}_{\boldsymbol{v}^{\prime}} \\
\text { (psf) }\end{array}$ & $\begin{array}{l}\boldsymbol{\sigma}_{\boldsymbol{p}_{\text {mob }}} \\
\text { (psf) }\end{array}$ & $\begin{array}{l}\text { horz } \\
\left(\mathbf{p s f} \boldsymbol{\sigma}_{p_{\text {mob }}}\right.\end{array}$ \\
\hline 15 & 0 & 0.0 & 0.00 & 248.94 & 243.62 \\
\hline 8 & 7 & 875.0 & $3,050.16$ & $3,299.10$ & $3,228.54$ \\
\hline 0 & 15 & $1,375.8$ & $4,795.89$ & $5,044.83$ & $4,936.94$ \\
\hline
\end{tabular}

These pressures are trapezoidal, as shown in the RHS pressures in Figure 4.57. Each pressure trapezoid can be divided into rectangular and right triangular distribution, resulting in easier calculations for the equivalent acting force and its moment arm to the datum. For the Factor of Safety (passive) of 1.5, the resulting forces and moment are shown in Table 4.10.

Table 4.10. Forces and moments acting on the right-hand side of the wall with Factor of Safety 1.5 .

\begin{tabular}{|l|c|l|c|}
\hline & Force $(\mathrm{lb})$ & Lever arm (ft) & Moment (ft-lb) \\
\hline F1 & $1,705.32$ & 11.50 & $19,611.15$ \\
\hline F2 & $10,447.24$ & 10.33 & $107,954.83$ \\
\hline F3 & $25,828.34$ & 4.00 & $103,313.37$ \\
\hline F4 & $6,833.60$ & 2.67 & $18,222.95$ \\
\hline Sum_f & $44,814.51$ & Sum_m & $249,102.30$ \\
\hline
\end{tabular}

For the Factor of Safety (passive) of 1.5, the total moment at the datum (i.e., el o.o) is much higher due to cumulative effects of the earth pressure distribution on the RHS of the retaining structure $(249,102.30 \mathrm{ft}-\mathrm{lb})$ than the total driving moment due to water pressures $(110,927.19 \mathrm{ft}-\mathrm{lb})$. In this case, the Factor of Safety (passive) can be iterated to find the case at which the moments are virtually equivalent.

When the Factor of Safety (passive) is set equal to 3.7581 , the moments on both sides of the wall are virtually equivalent at 110,900 $\mathrm{ft}-\mathrm{lb}$. The results are shown in Tables 4.11 and 4.12 and are consistent with the Corps_I-Wall computations. 
Table 4.11. Determining earth pressures on the right-hand side of the wall with Factor of Safety 3.7581.

\begin{tabular}{|c|c|c|c|c|c|}
\hline \multicolumn{4}{|c|}{ RHS Earth Pressures } & \multirow{2}{*}{$\begin{array}{l}K_{p} * \sigma_{v^{\prime}}+2 c_{m o b i l i z e d}^{\prime} \sqrt{K_{p}} \\
\begin{array}{l}\sigma_{p_{m o b}} \\
(\mathrm{psf})\end{array}\end{array}$} & \multirow{2}{*}{$\begin{array}{l}\sigma_{p_{m o b}} * \cos \delta_{\text {mobilized }}^{\prime} \\
\text { horz } \\
\text { (psf) }\end{array}$} \\
\hline $\begin{array}{l}\text { Elevation } \\
\text { (ft) }\end{array}$ & $\begin{array}{l}\text { Depth } \\
\text { (ft) }\end{array}$ & $\begin{array}{l}\sigma_{v^{\prime}} \\
\text { (psf) }\end{array}$ & $\begin{array}{l}\boldsymbol{K}_{\boldsymbol{p}} * \boldsymbol{\sigma}_{\boldsymbol{v}^{\prime}} \\
(\mathrm{psf})\end{array}$ & & \\
\hline 15 & 0 & 0.0 & 0.00 & 67.24 & 243.62 \\
\hline 8 & 7 & 875.0 & $1,396.92$ & $1,464.17$ & $3,228.54$ \\
\hline 0 & 15 & $1,375.8$ & $2,196.44$ & $5,044.83$ & $2,263.69$ \\
\hline
\end{tabular}

Table 4.12. Forces and moments acting on the right-hand side of the wall with Factor of Safety 3.7581 .

\begin{tabular}{|l|r|l|r|}
\hline & Force $(\mathrm{lb})$ & Lever arm $(\mathrm{ft})$ & Moment $(\mathrm{ft}-\mathrm{lb})$ \\
\hline F1 & 469.05 & 11.50 & $5,394.07$ \\
\hline F2 & $4,872.12$ & 10.33 & $50,345.23$ \\
\hline F3 & $11,672.33$ & 4.00 & $46,689.31$ \\
\hline F4 & $3,186.88$ & 2.67 & $8,498.35$ \\
\hline Sum_f & $20,200.38$ & Sum_m & $110,926.97$ \\
\hline
\end{tabular}

For a Factor of Safety (passive) of 3.7581, the mobilized material values are:

$$
\begin{aligned}
c_{\text {mobilized }}^{\prime} & =26.67 \mathrm{psf} \\
\varphi_{\text {mobilized }}^{\prime} & =10.58 \mathrm{deg} \\
\delta_{\text {mobilized }}^{\prime} & =4.81 \mathrm{deg}
\end{aligned}
$$

In summary, the 15 -ft-high soil berm resists the applied wave loading force. The sheet-pile wall is not needed to transfer the applied loading into the foundation through bending action. The sole purpose of the presence of the I-Wall in this case would be to protect the berm soil from erosion due to wave action.

The earth pressure relationships implemented in Corps_I-Wall, Version 1.o, are for level ground conditions. The lateral extent needed at the top of the RHS berm is approximated using the planar Rankine passive earth pressure's slide plane relationship of

$$
\alpha_{\text {mobilized }}=45^{\circ}-\frac{\varphi_{\text {mobilized }}^{\prime}}{2}
$$


For a Factor of Safety (passive) of 3.7581, this angle would be computed as

$$
\alpha_{\text {mobilized }}=45^{\circ}-\frac{10.58^{\circ}}{2}=39.71
$$

The lateral extent for the top of a berm with an elevation of $15 \mathrm{ft}$ would be

$$
\text { extent }=\frac{15 \mathrm{ft}}{\tan 39.71^{\circ}}=18.06 \mathrm{ft}
$$

And, with a rise over run for the advancing slope of 1 to 2, the berm would extend another $30 \mathrm{ft}$, as shown in Figure 4.58.

The next stage of the analysis would be an investigation to reduce the required height of the berm because a $F S_{\text {Passive }}$ equal to 3.76 is larger than what is normally required. Reducing the height of berm may require the I-Wall to be engaged and some of the applied loading may be transferred into the foundation through bending action of the sheet piling.

Figure 4.58. Results of lateral berm extent calculations for a 15-ft-high berm and a Factor of Safety (passive) within the berm of 3.7581 .

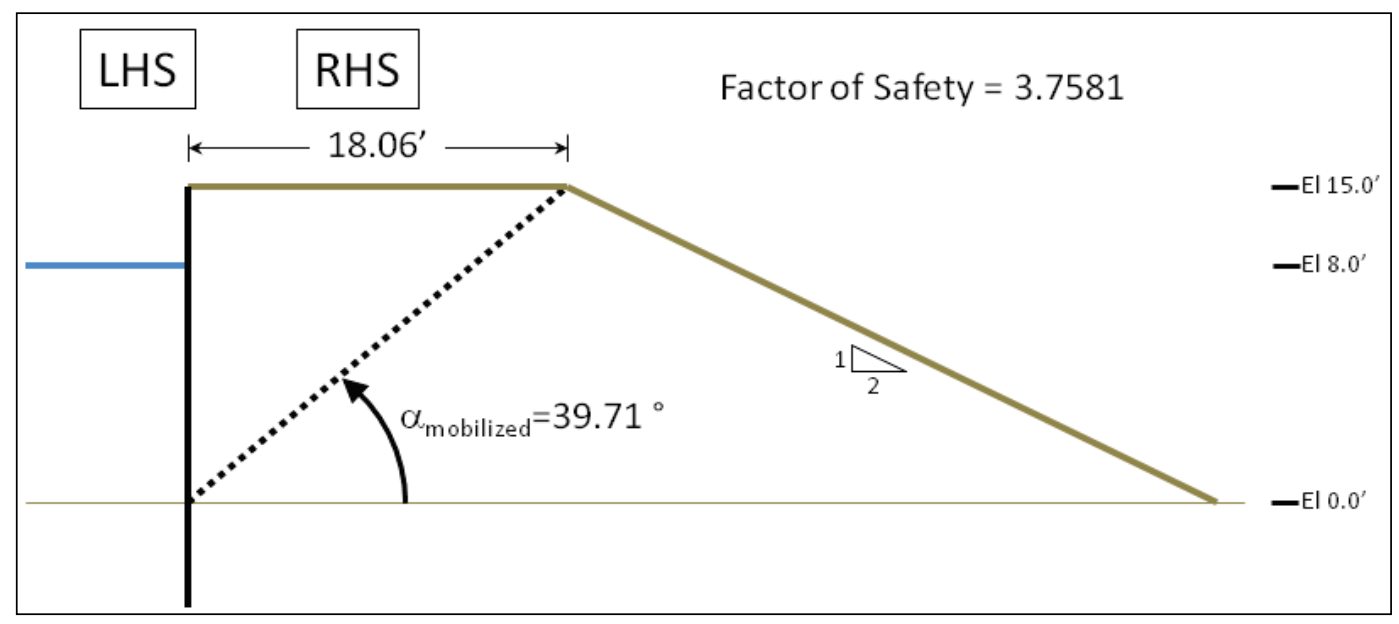

\subsection{Example 4.6 comparison to Corps_I-Wall results contained within other technical publications (Das; Bowles; Pace)}

This section discusses Corps_I-Wall results when compared with design problems from Das (2007), Bowles (1968), and Pace et al. (2012) for the determination of the depth of embedment. An effective stress definition of granular soils; a total stress definition with undrained shear strength, $S_{u}$, for clays; and a mixture of sand above saturated clays constitute the various design problems analyzed. The Das example problems of Sections 4.6.1 and 4.6.2 include granular soils as well as sandy soils over 
saturated clay. Bowles has similar examples in Sections 4.6.3 and 4.6.4. Section 4.6.5 examines a total stress definition of clays and the evaluation of gap depth from hydraulic fracturing.

\subsubsection{Example 4.6.1 sheet-pile wall penetrating sand (Das)}

To determine the depth of embedment of sheet-pile walls in granular soil, Figure 4.59 is examined. This example problem (Das 2007) represents the case of homogeneous sandy soil below the dredge line on the flood side (LHS) and retained above the dredge line on the landside (RHS) of the sheet-pile wall. The dense sand has an effective stress definition with no cohesion, an effective angle of internal friction $\left(\varphi^{\prime}\right)$ of $40 \mathrm{deg}$. The sand layers are assigned a moist unit weight of $120 \mathrm{pcf}$ and a saturated unit weight of $129.4 \mathrm{pcf}$. Hydrostatic water pressures are at el $20 \mathrm{ft}$ on both sides of the sheet-pile wall. For the Das calculation steps in a CI-wall Design mode, the specified Factors of Safety on both the active and passive pressures are assigned 1.0. ${ }^{1}$ An effective angle of wall to soil interface friction $\left(\delta^{\prime}\right)$ of o deg as well as the Coulomb coefficient method of analysis (for $K_{a}$ and $K_{p}$ ) form the basis for this example ${ }^{2}$. Hydraulic fracturing is not included as an option for this analysis. The Corps_I-Wall representation of this problem is presented by the schematic of Figure 4.60. This figure outlines and describes the geometry and structural parameters for the sheet-pile wall.

This analysis resulted in a computed mobilized active earth pressure coefficient $\left(K_{a}\right)$ of 0.2174 and a mobilized passive pressure coefficient $\left(K_{p}\right)$ of 4.5989. Figure 4.61 displays the results obtained from this analysis with the sheet-pile tip elevation computed at $-21.71 \mathrm{ft}$ for a calculated depth of embedment of $21.71 \mathrm{ft}$. The final results for this Example 4.6.1 has been recorded as check problem 1 in Table 4.13.

The distribution of active and passive earth pressures for both sides of the sheet-pile wall are illustrated in Figures 4.62a and 4.62b. The hydrostatic water pressures acting on both sides of the sheet-pile wall are shown in Figure 4.63a with the resultant net water pressure calculated by taking the difference between the water pressures acting on both sides of the wall. In the case when the piezometric surface elevation is equal to the surface water elevation, no resultant net water pressure will be calculated as shown in Figure 4.63b.

1 Assigning a value for FSpassive of 1.0 is not consistent with Corps design criteria.

2 For no soil-to-I-Wall interface friction (i.e., $\delta=0 \mathrm{deg}$ ), the Coulomb solution for both active and passive earth pressure coefficients corresponds to the Rankine earth pressure coefficients. 
Figure 4.59. Sheet-pile wall penetrating sand.

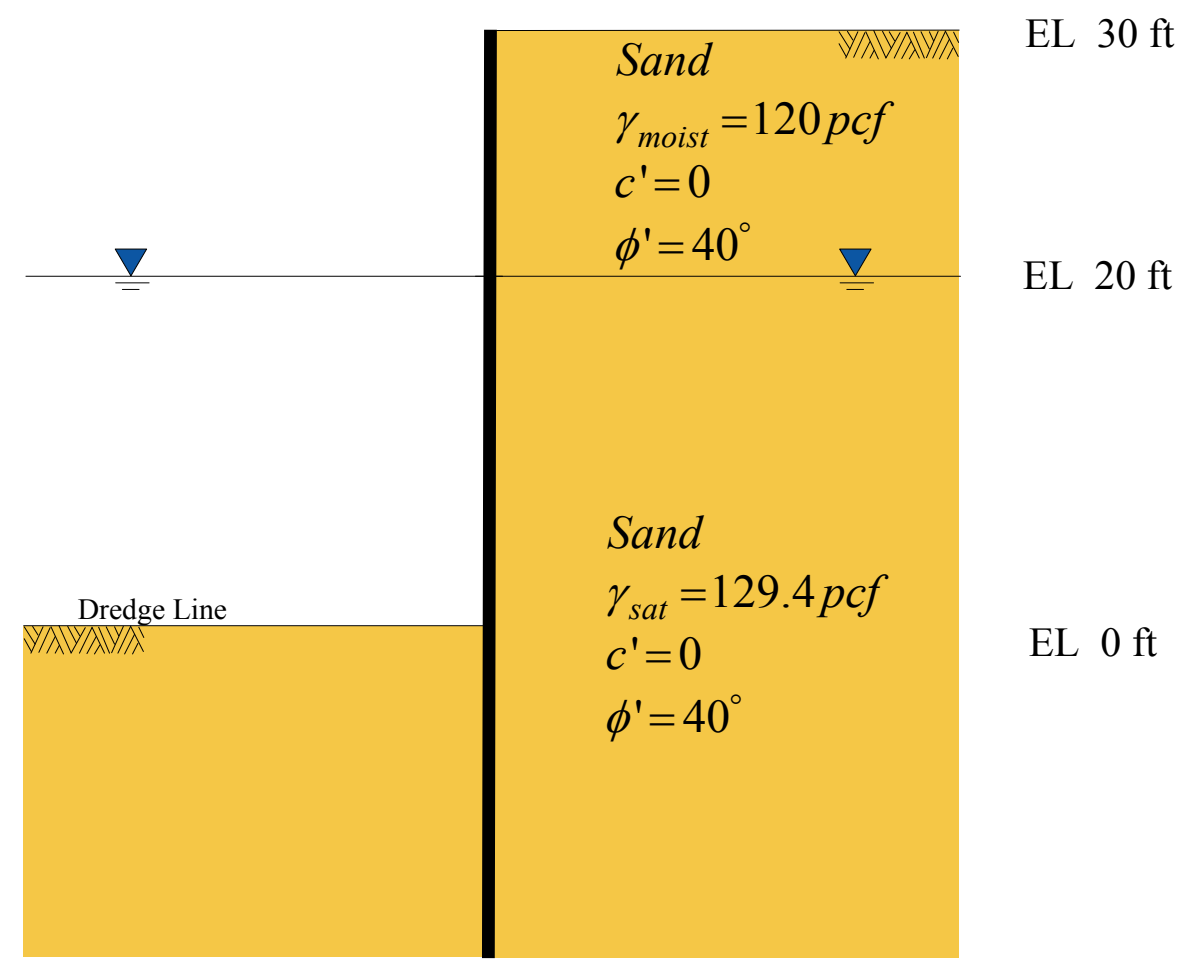

Figure 4.60. Corps_I-Wall schematic of sheet-pile wall in sand site.

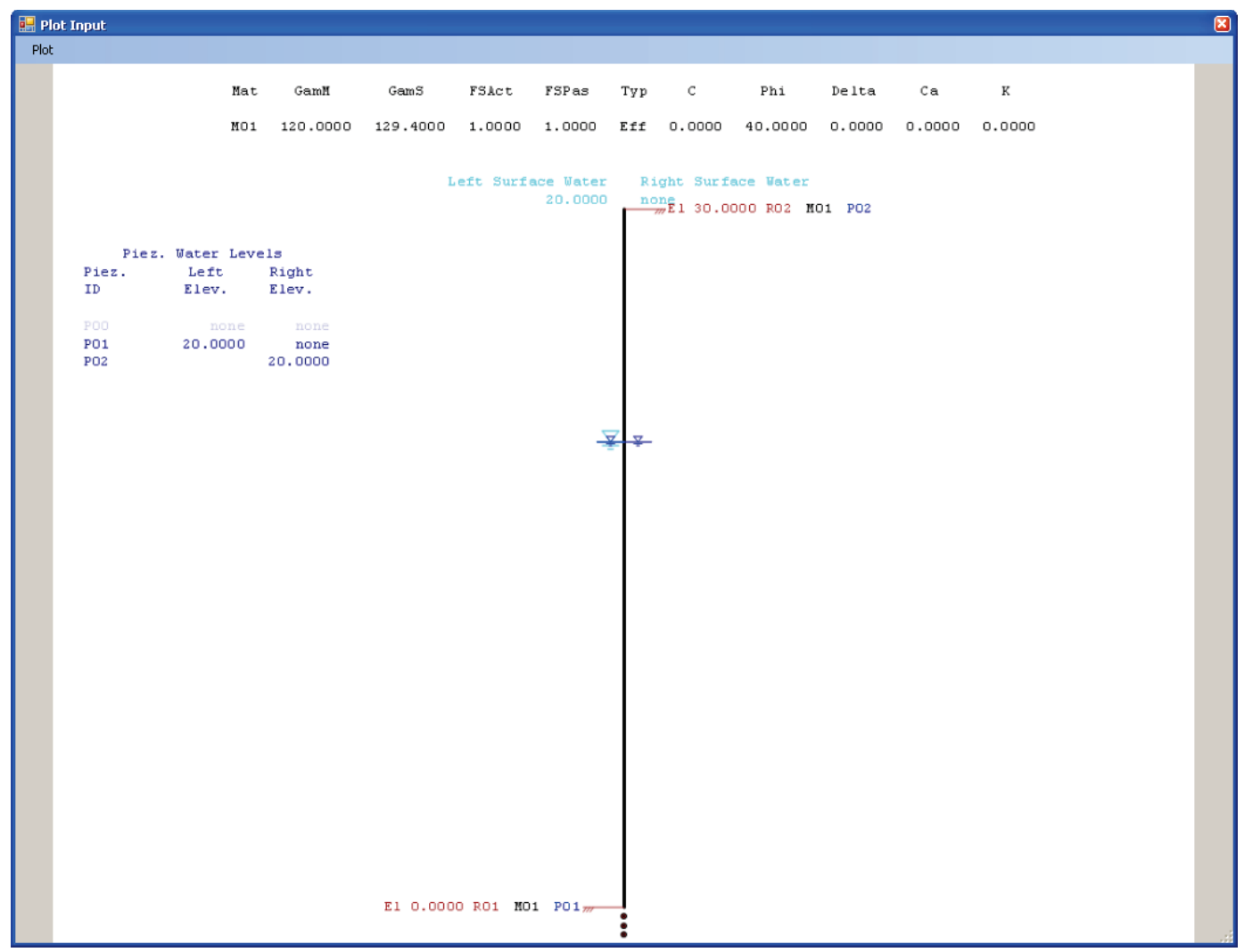


Figure 4.61. Corps_I-Wall output information.

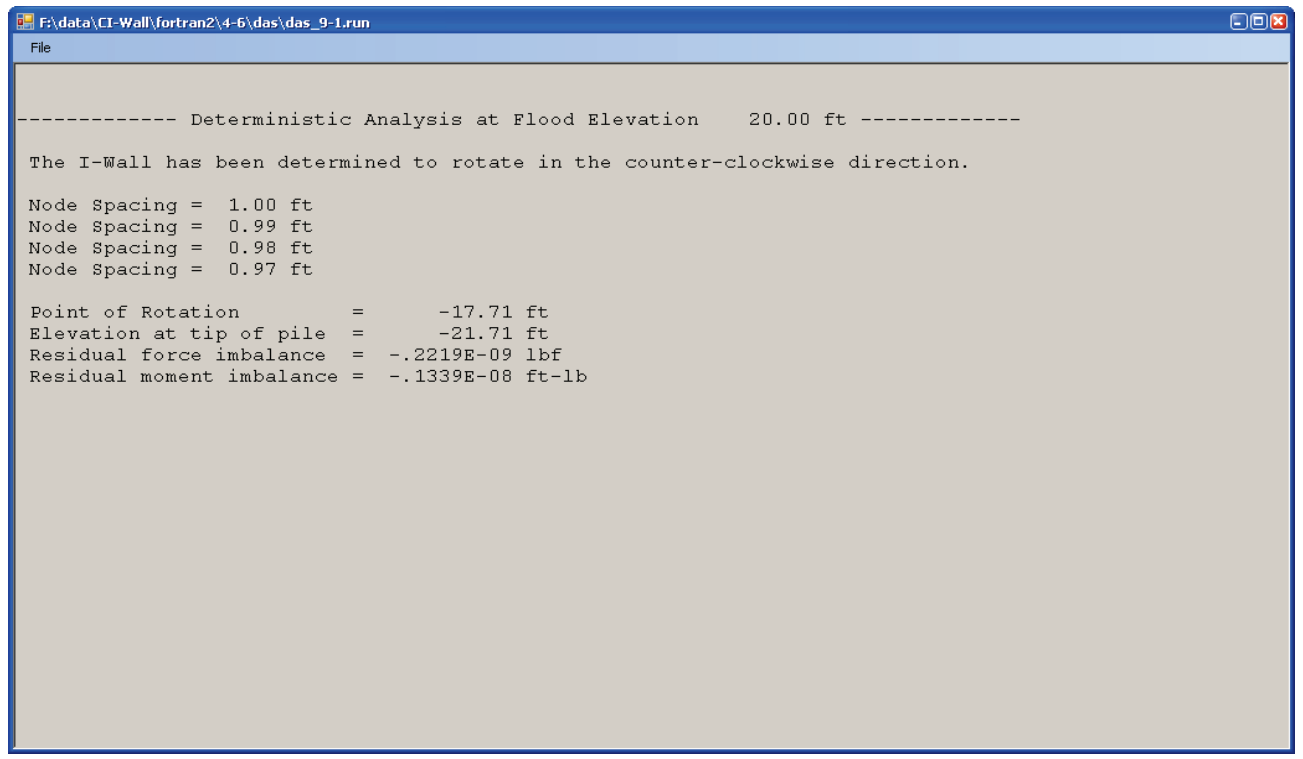

Figure 4.62. Active and passive earth pressures for drained shear strength. (a) Left-hand side. (b) Right-hand side.

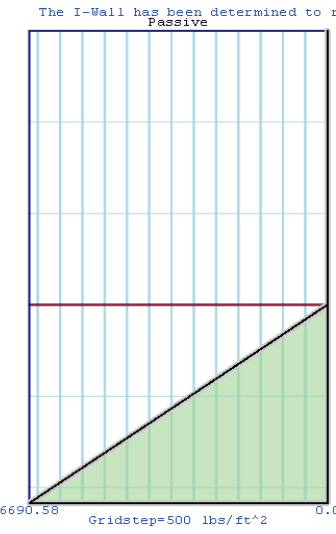

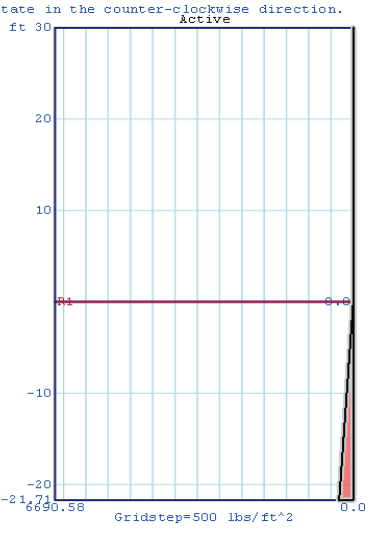

(a)

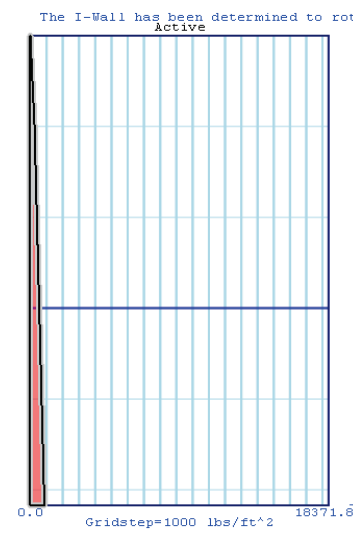

(b)

Figure 4.63. Hydrostatic water pressures. (a) Left- and right-hand side. (b) Net water pressures.

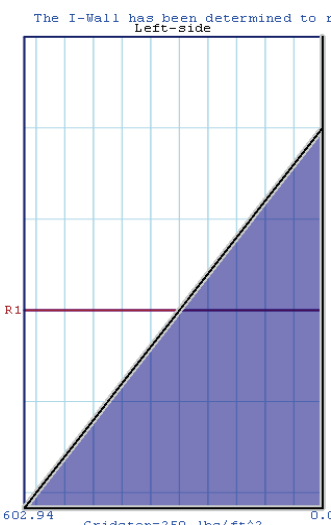

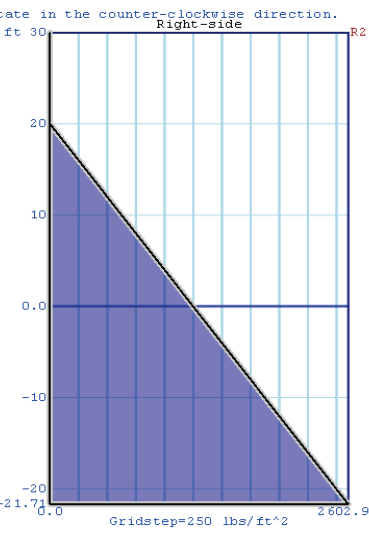

(a)

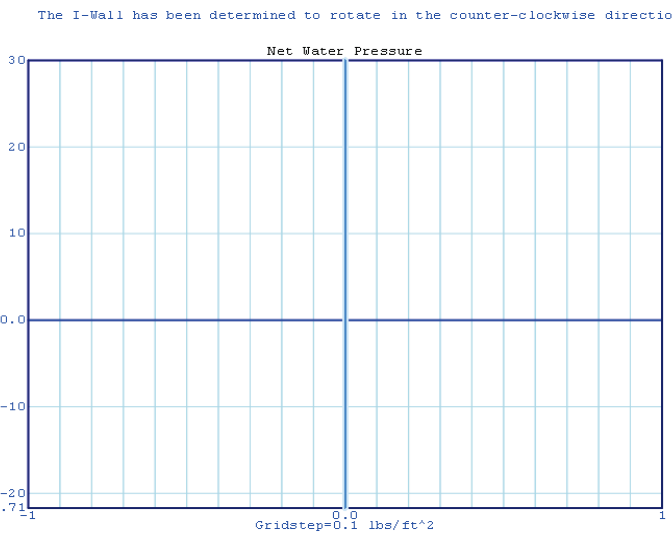

(b) 
Table 4.13. Comparisons of I-Wall results contained within Das (2007).

\begin{tabular}{|c|c|c|c|c|c|c|c|c|c|c|c|c|c|c|}
\hline \multirow{3}{*}{$\begin{array}{l}\text { Check } \\
\text { Problem }\end{array}$} & \multirow{3}{*}{$\begin{array}{l}\text { Das (2007) } \\
\text { Principles of } \\
\text { Foundation }\end{array}$} & \multirow{3}{*}{$\begin{array}{l}\text { Layer } \\
\text { No. }\end{array}$} & \multirow{3}{*}{$\begin{array}{l}\text { Layer } \\
\text { Soil } \\
\text { Type }\end{array}$} & & & \multirow{3}{*}{$\begin{array}{l}\varphi^{\prime} \\
\text { (deg) }\end{array}$} & \multirow{3}{*}{$\begin{array}{l}\mathrm{l}^{\prime} \\
(\mathrm{psf}) \text { or } \\
\left(\mathrm{kN} / \mathrm{m}^{2}\right)\end{array}$} & \multicolumn{4}{|c|}{ Point of Rotation } & \multirow{2}{*}{\multicolumn{2}{|c|}{$\frac{\text { Sheet-pile tip }}{\text { EL }}$}} & \multirow[b]{3}{*}{ Unit } \\
\hline & & & & Moist & Sat & & & \multicolumn{2}{|c|}{ EL } & \multicolumn{2}{|r|}{ z } & & & \\
\hline & & & & \multicolumn{2}{|c|}{$(p c f)$ or $\left(k N / m^{3}\right)$} & & & Reference & Corps_I-Wall & Reference & Corps_I-Wall & Reference & Corps_I-Wall & \\
\hline 1 & 9-1 & 1 & Sand & 120 & 129.4 & 40 & 0 & -17.81 & -17.71 & 4.07 & 4.00 & -21.88 & -21.71 & $\mathrm{ft}$ \\
\hline \multirow{2}{*}{2} & \multirow{2}{*}{$9-2$} & 1 & Sand & 15.9 & 19.33 & 32 & 0 & \multirow{2}{*}{-0.96} & \multirow{2}{*}{-0.91} & \multirow{2}{*}{1.17} & \multirow{2}{*}{1.28} & \multirow{2}{*}{-2.13} & \multirow{2}{*}{-2.19} & \multirow{2}{*}{$\mathrm{m}$} \\
\hline & & 2 & Clay & 15.9 & 19.33 & 0 & 47 & & & & & & & \\
\hline
\end{tabular}

Reference is Das (2007) principles of foundation for check problems 1 and 2. Corps_--Wall has an active Factor of Safety of 1.0 and a passive Factor of Safety of 1.0. Check problem 1 is in English units and check problem 2 is in metric. 
From these pressures (Figures 4.62 and 4.63), both the net active and net passive pressures are constructed and presented in Figure 4.64a. For the applied flood loading, the I-Wall will rotate counterclockwise. The net active pressure is determined from the difference between the RHSmobilized active earth pressure and the LHS-mobilized passive earth pressure (i.e., acting on the other side of the wall) with the addition of any net water pressures and any external horizontal net pressures and overburden pressures (if any). The net passive pressure is determined by the difference between the RHS-mobilized passive earth pressure and the LHS- mobilized active earth pressure acting on the other side of the sheetpile wall with the addition of any net water pressures and any external horizontal net pressures and overburden pressures (if any).

The net pressure diagram of Figure $4.64 \mathrm{~b}$ is derived from the replication of all values of the net active pressures, from the top of the wall until the elevation at the point of rotation (el $-17.71 \mathrm{ft}$ ), at which instant the remaining values of the net pressure diagram are assumed to be linear with elevation between (1) the net active pressure value at the elevation of the point of rotation and (2) the net mobilized passive pressure at the elevation of the approximated sheet-pile tip (i.e., el -21.71). ${ }^{1}$ Figure $4.64 \mathrm{~b}$ is an illustration of how the net pressure diagram was formed. The final results are illustrated in Figure 4.65 by the presentation of the net pressure diagram Figures 4.65a (replica of Figure 4.64b) and 4.65b, the shear and moment diagrams. Figure $4.65 \mathrm{~b}$ shows that the maximum bending moment occurs at the zero crossing of the shear diagram.

Figure 4.64. (a) Net pressure diagram. (b) Shear and moment diagram.

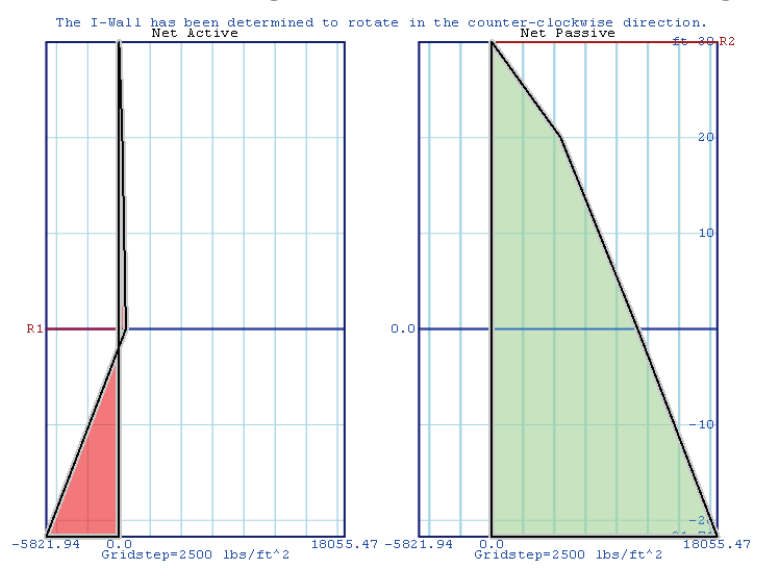

(a)

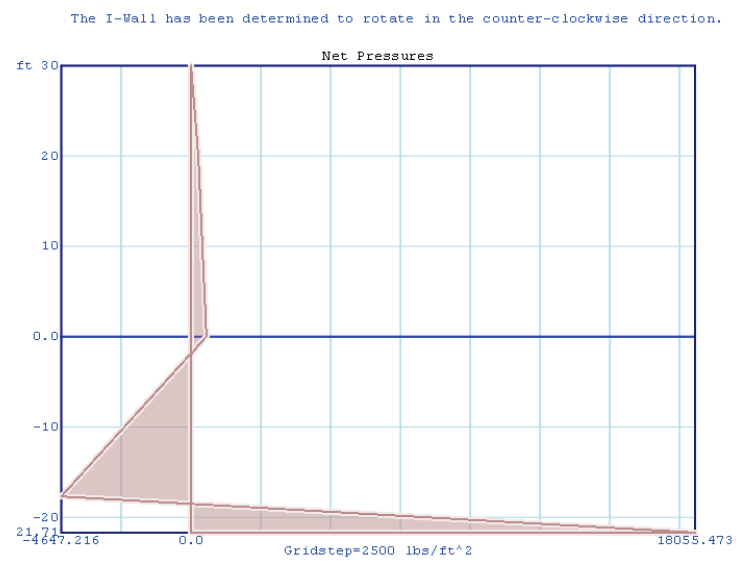

(b)

1 Thus, only one mobilized net passive pressure value in Figure 4.64a is used to construct the Figure $4.64 \mathrm{~b}$ net pressure diagram, corresponding to the sheet-pile tip elevation at el -21.71 . The majority of the net pressure diagram is derived from the Figure 4.64a net active pressure diagram. 
Figure 4.65. (a) Net pressure diagram. (b) Shear and moment diagram.

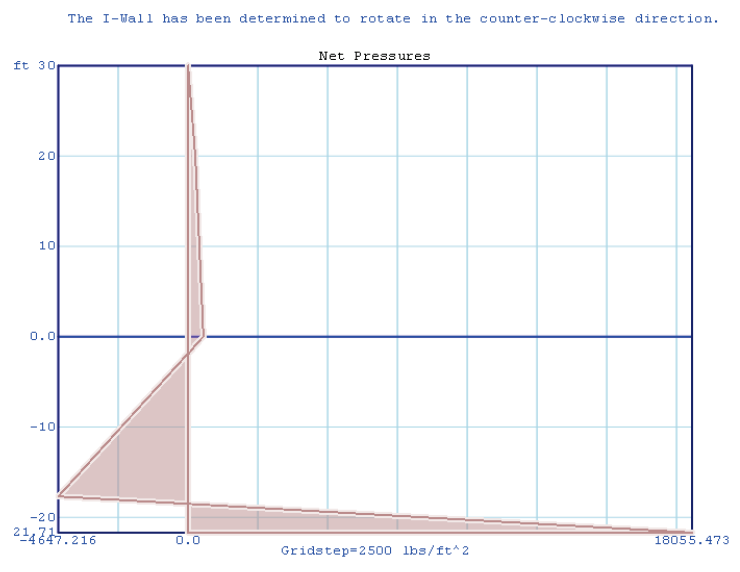

(a)

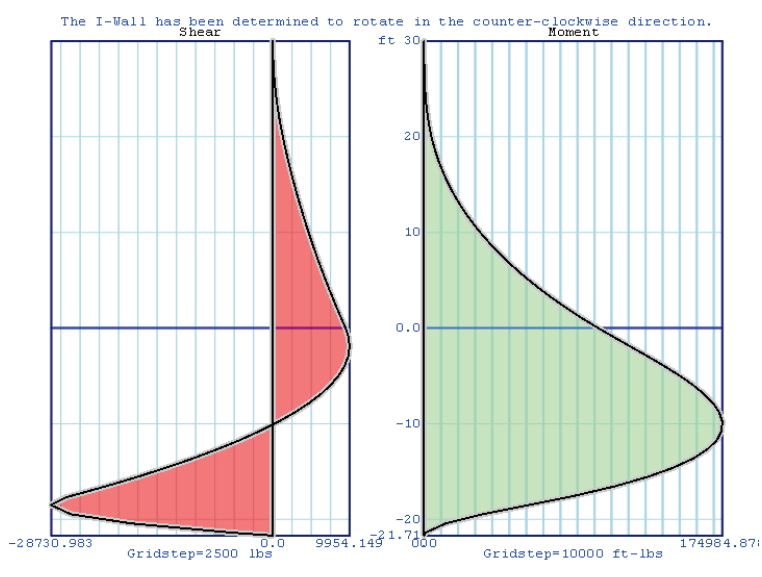

(b)

\subsubsection{Example 4.6.2 sheet-pile wall penetrating saturated clay (Das)}

To determine the depth of embedment of a sheet-pile wall in two-layered soil, Figure 4.66 is examined. This example problem from Das (2007) represents the case of saturated clay soil below the dredge line on both sides of the sheet-pile wall with retained sandy soil above the dredge line on the landside (RHS) of the sheet-pile wall. The sand has an effective stress definition with no cohesion and an effective angle of internal friction $\left(\varphi^{\prime}\right)$ of $32 \mathrm{deg}$. The sand is assigned a moist unit weight of $15.9 \mathrm{kN} / \mathrm{m}^{3}$ and a saturated unit weight of $19.33 \mathrm{kN} / \mathrm{m}^{3}$. The clay soil layer has a total stress definition with $\varphi=0$ and cohesion (c) of $47 \mathrm{kN} / \mathrm{m}^{2}$. Hydrostatic water pressures are at el $3 \mathrm{~m}$ on both sides of the sheet-pile wall. For this design, the required factors of safety on both the active and passive pressures are assigned 1.0. ${ }^{1}$ An effective angle of wall to soil interface friction $\left(\delta^{\prime}\right)$ of o deg as well as the Coulomb coefficient method of analysis (for $K_{a}$ and $K_{p}$ ) form the basis for this example ${ }^{2}$. Hydraulic fracturing is not included as an option for this analysis. The Corps_I-Wall representation of this problem is presented by the schematic of Figure 4.67. This figure outlines and describes the geometry and structural parameters for the sheet-pile wall.

\footnotetext{
${ }^{1}$ Assigning a value for FS Passive of 1.0 is not consistent with Corps' design criteria.

2 For no soil-to-I-Wall interface friction (i.e., $\delta=0 \mathrm{deg}$ ), the Coulomb solution for active and passive earth pressure coefficients corresponds to the Rankine earth pressure coefficients.
} 
Figure 4.66. Sheet-pile wall penetrating saturated clay.

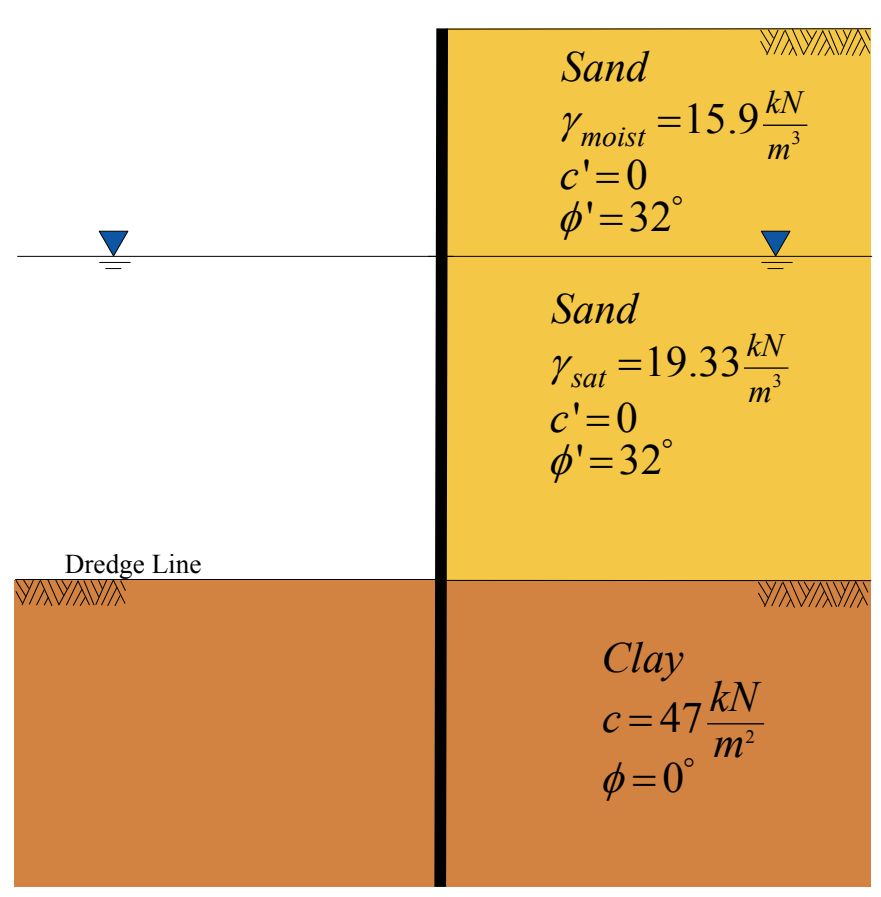

EL $5 \mathrm{~m}$

EL $3 \mathrm{~m}$

EL $0 \mathrm{~m}$

Figure 4.67. Corps_I-Wall schematic of sheet-pile wall in sand and saturated clay site.

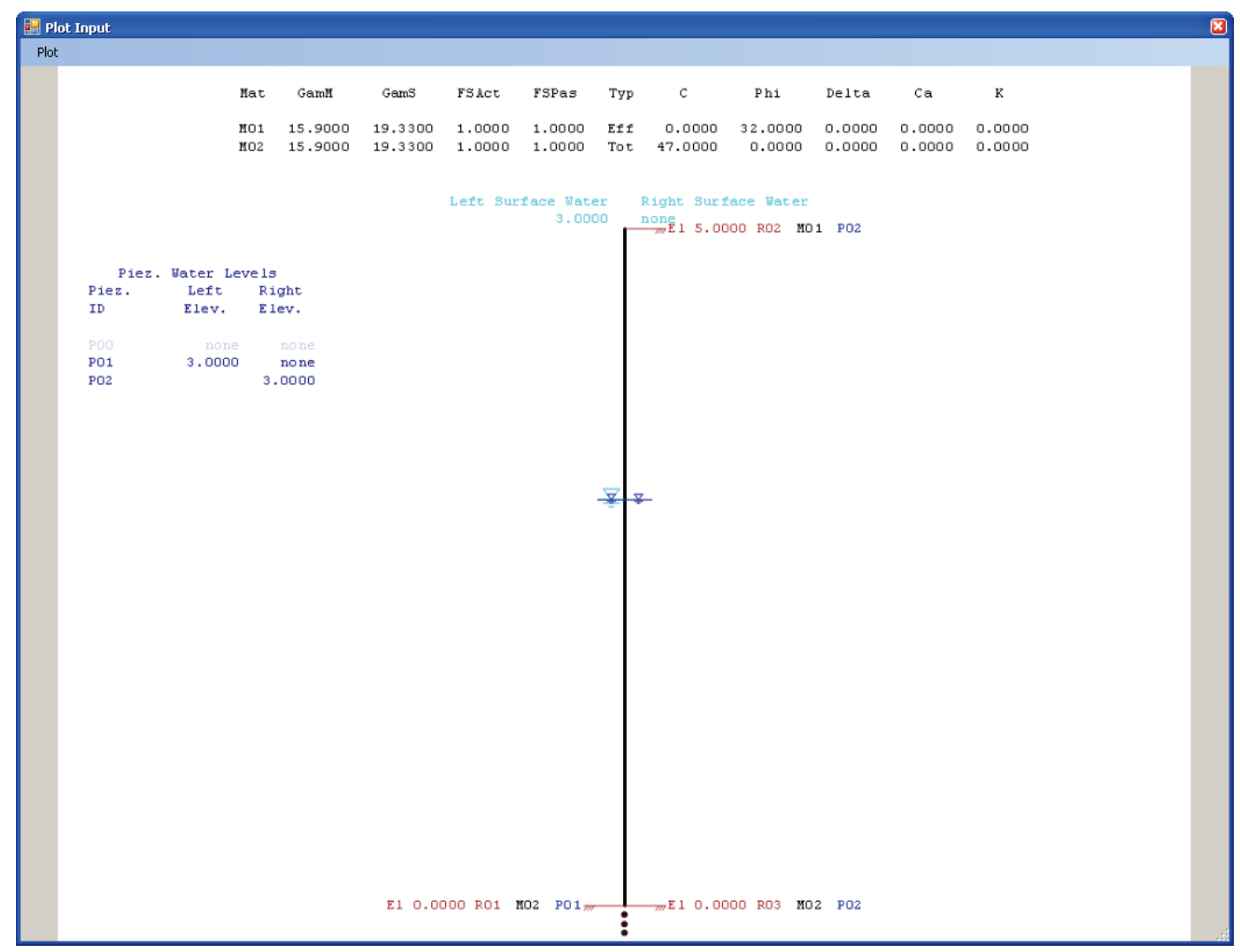


This analysis resulted in a computed mobilized active earth pressure coefficient $\left(K_{a}\right)$ of 0.30726 and a mobilized passive pressure coefficient $\left(K_{p}\right)$ of 3.25459 for the sandy soil on the retained side above the dredge line. For the clay layers (with $\varphi=0$ ) below the dredge line, $K_{a}$ and $K_{p}$ obtained values of 1.o. Figure 4.68 displays the results obtained from this analysis with the sheet-pile tip elevation computed at $-2.19 \mathrm{~m}$ for a calculated depth of embedment of $2.19 \mathrm{~m}$. The final results for this Example 4.6.2 has been recorded as check problem 2 in Table 4.13.

Figure 4.68. Corps_I-Wall output information.

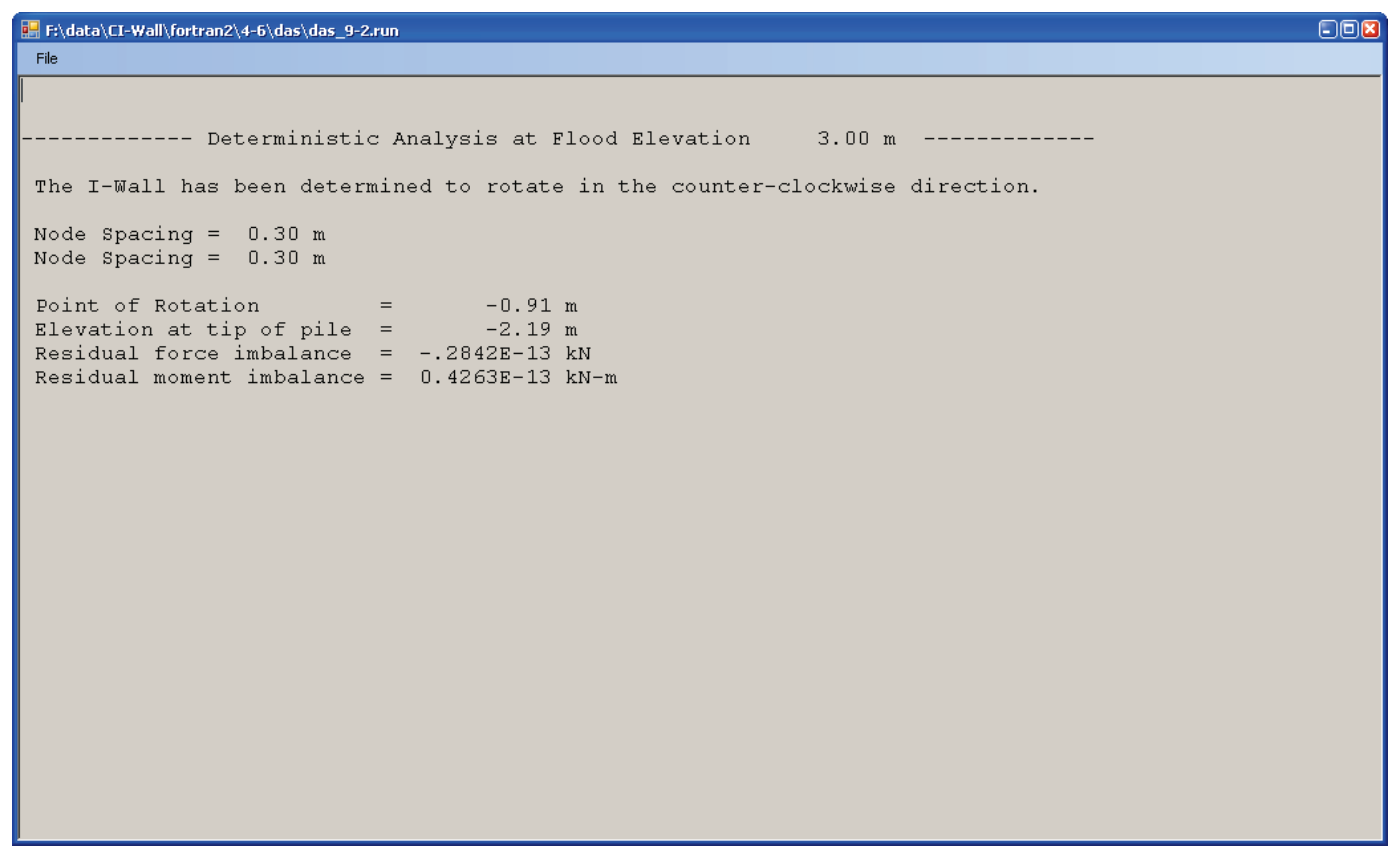

The distribution of both active and passive earth pressures for both sides of the sheet-pile wall are illustrated in Figures 4.69a and 4.69b. The hydrostatic water pressures acting on both sides of the sheet-pile wall are shown in Figure 4.70a with the resultant net water pressure calculated by taking the difference between the water pressures acting on both sides of the wall. In the case when the piezometric surface elevation is equal to the surface water elevation, no resultant net water pressure will be calculated as shown in Figure 4.70b.

From these pressures (Figures 4.69 and 4.70), the net active and net passive pressures are constructed and presented in Figure 4.71a. For the applied flood loading, the I-Wall will rotate counterclockwise. The net active pressure is determined from the difference between the RHSmobilized active earth pressure and the LHS-mobilized passive earth 
pressure (i.e., acting on the other side of the wall) with the addition of any net water pressures and any external horizontal net pressures and overburden pressures (if any). The net passive pressure is determined by the difference between the RHS-mobilized passive earth pressure and the LHS- mobilized active earth pressure acting on the other side of the sheetpile wall with the addition of any net water pressures and any external horizontal net pressures and overburden pressures (if any).

Figure 4.69. Active and passive earth pressures. (a) Left-hand side. (b) Right-hand side.

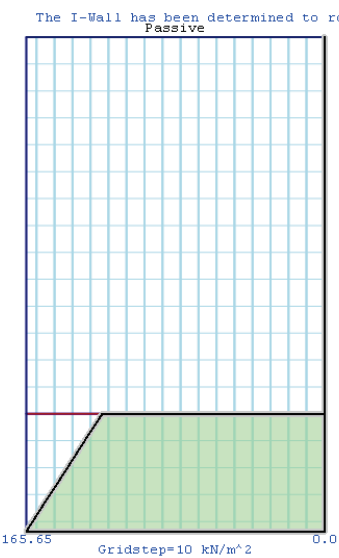

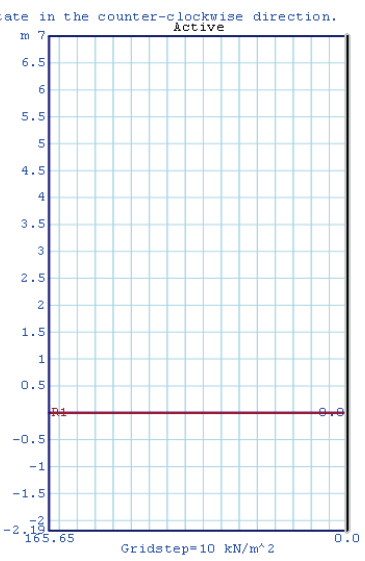

(a)
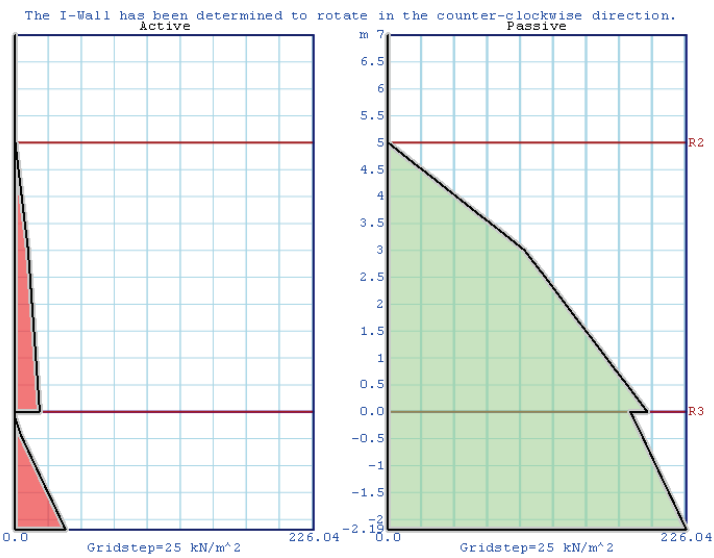

(b)

Figure 4.70. Hydrostatic water pressures. (a) Left- and right-hand side. (b) Net water pressures.

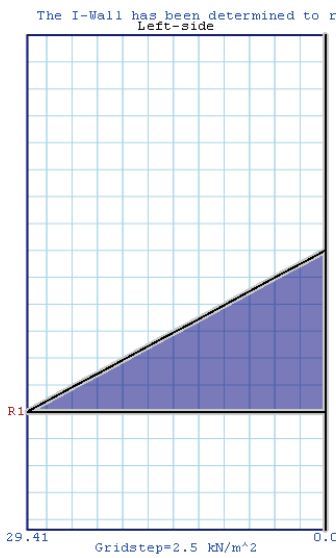

Gridstep $=2.5 \mathrm{kN} / \mathrm{m}^{\wedge} 2$

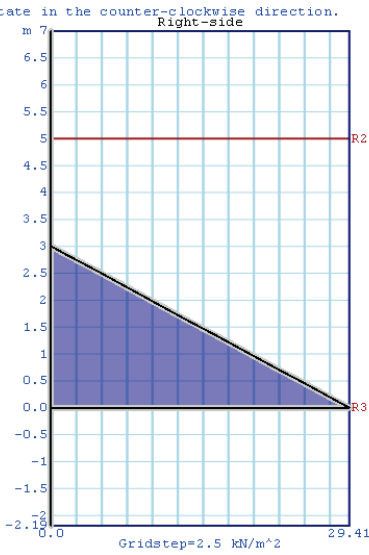

(a)

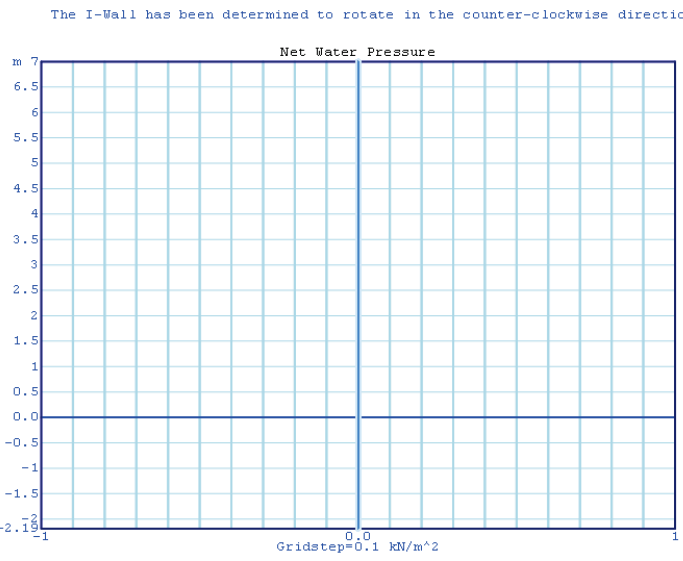

(b) 
Figure 4.71. Net pressures. (a) Net active and net passive pressures. (b) Net pressure diagram.
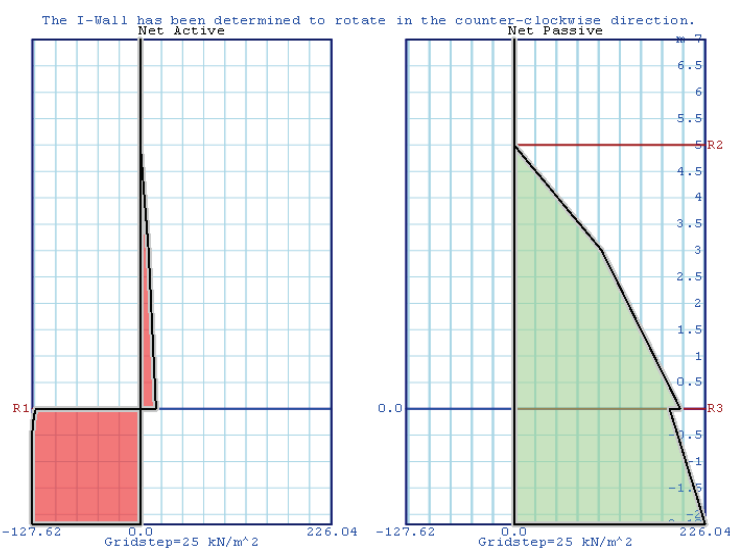

(a)

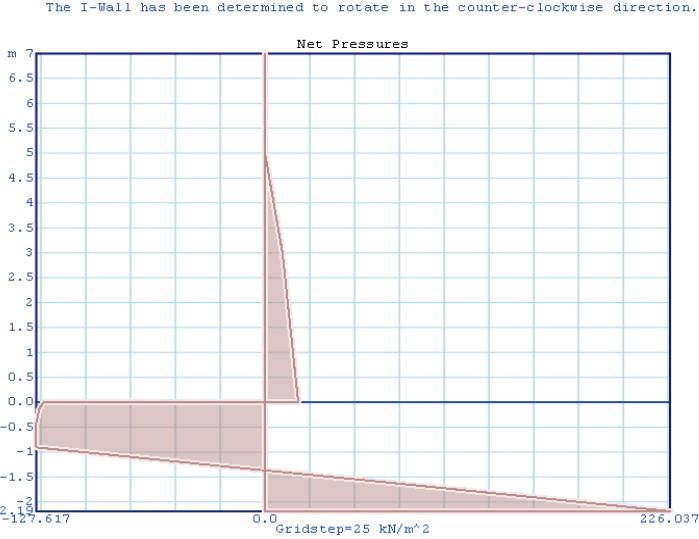

(b)

The net pressure diagram of Figure $4.71 \mathrm{~b}$ is derived from the replication of all values of the net active pressures, from the top of the wall until the elevation at the point of rotation (el -0.91 m), at which instant the remaining values of the net pressure diagram is assumed to be linear with elevation between (1) the net active pressure value at the elevation of the point of rotation and (2) the net mobilized passive pressure at the elevation of the approximated sheet-pile tip (i.e., el -2.19 m). ${ }^{1}$ Figure $4.71 \mathrm{~b}$ is an illustration of how the net pressure diagram was formed. The final results are illustrated in Figure 4.72 by the presentation of the net pressure diagram Figures 4.72a (replica of Figure 4.71b) and 4.72b, the shear and moment diagrams. Figure $4.72 \mathrm{~b}$ shows that the maximum bending moment occurs at the zero crossing of the shear diagram.

Figure 4.72. (a) Net pressure diagram. (b) Shear and moment diagram.

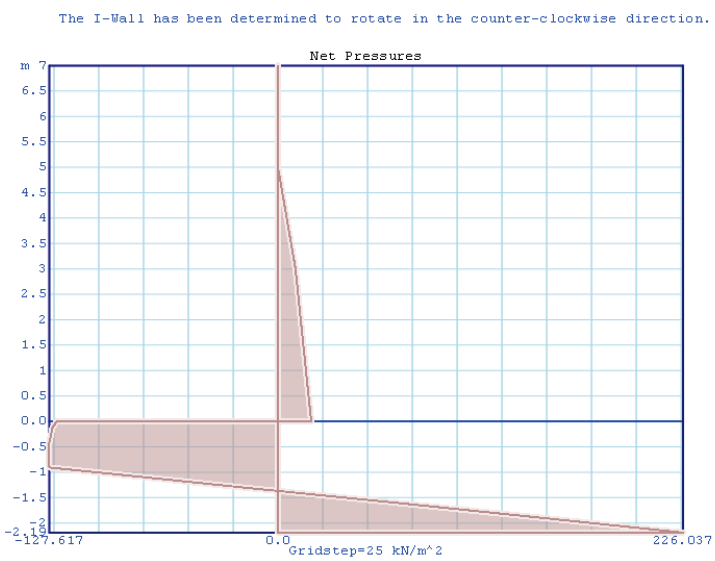

(a)
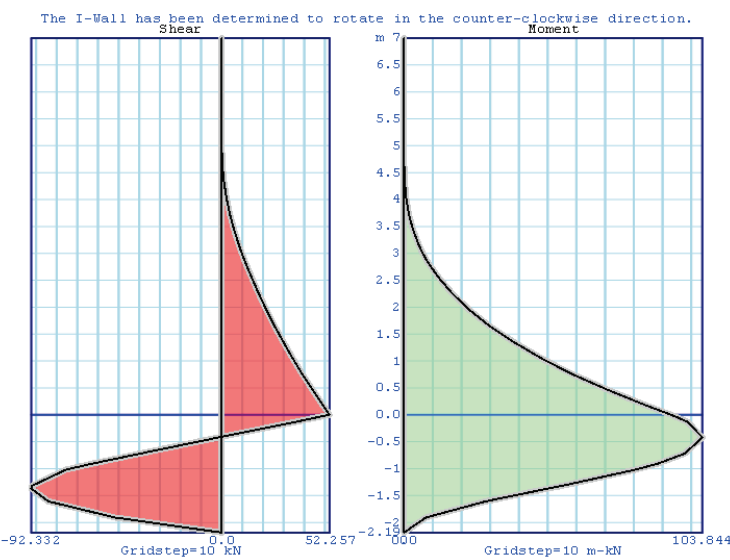

(b)

\footnotetext{
1 Thus, only one mobilized net passive pressure value in Figure 4.71a is used to construct the Figure $4.71 \mathrm{~b}$ net pressure diagram; corresponding to the sheet-pile tip elevation at el $-2.19 \mathrm{~m}$. The majority of the net pressure diagram is derived from the Figure 4.71a net active pressure diagram.
} 
The Corps_I-Wall computed results for point of rotation and sheet-pile tip agree with those from Das (2007) for the two sheet-pile walls analyzed.

\subsubsection{Sheet-pile wall penetrating sand (Bowles)}

To determine the depth of embedment of sheet-pile walls in granular soil, two problems, Examples 4.6.3 and 4.6.4, are examined. This pair of Bowles (1968) example calculations represents the case of homogeneous sandy soil that has an effective stress definition with no cohesion, an effective angle of internal friction $\left(\varphi^{\prime}\right)$ of $30 \mathrm{deg}$. These two problems differ with the assignment of the Factor of Safety for passive earth pressures of 1.0 (Section 4.6.3.1) ${ }^{1}$ and of 1.633 (Section 4.6.3.2). Table 4.14 shows the material properties for the problems.

\subsubsection{Example 4.6.3 sheet-pile wall penetrating sand (Bowles)}

To determine the depth of embedment of sheet-pile walls in granular soil, Figure 4.73 summaries an example problem of a cantilever sheet-pile wall retaining moist and saturated sand. The Corps-Wall idealization of this problem is shown in Figure 4.74. This example problem (Bowles 1968) represents the case of homogeneous sandy soil below the dredge line on the flood side (LHS) and retained above the dredge line on the landside (RHS) of the sheet-pile wall. The sand has an effective stress definition with no cohesion, an effective angle of internal friction $\left(\varphi^{\prime}\right)$ of $30 \mathrm{deg}$. The sand layers are assigned a moist unit weight of 110 pcf and a saturated unit weight of $122.4 \mathrm{pcf}$. Hydrostatic water pressures are at el $10 \mathrm{ft}$ on both sides of the sheet-pile wall. For this design, the required Factors of Safety on both the active and passive pressures are assigned 1.o. An effective angle of wall to soil interface friction $\left(\delta^{\prime}\right)$ of o deg as well as the Coulomb coefficient method of analysis (for $K_{a}$ and $K_{p}$ ) form the basis for this example 2 . Hydraulic fracturing is not included as an option for this analysis. The Corps_I-Wall representation of this problem is presented by the schematic of Figure 4.61. This figure outlines and describes the geometry and structural parameters for the sheet-pile wall.

\footnotetext{
1 Assigning a value for FS Passive of 1.0 is not consistent with Corps' design criteria.

2 For no soil-to-I-Wall interface friction (i.e., $\delta=0 \mathrm{deg}$ ), the Coulomb solution for both active and passive earth pressure coefficients corresponds to the Rankine earth pressure coefficients.
} 
Table 4.14. Comparisons of I-Wall results contained within Bowles (1968).

\begin{tabular}{|c|c|c|c|c|c|c|c|c|c|c|c|c|c|}
\hline \multirow{3}{*}{$\begin{array}{l}\text { Check } \\
\text { Problem }\end{array}$} & \multirow{3}{*}{$\begin{array}{l}\text { Bowles (1968) } \\
\text { Foundation } \\
\text { Analysis and } \\
\text { Design }\end{array}$} & \multirow{3}{*}{$\begin{array}{l}\text { Layer } \\
\text { No. }\end{array}$} & \multirow{3}{*}{$\begin{array}{l}\text { Layer } \\
\text { Soil } \\
\text { Type }\end{array}$} & \multirow{2}{*}{\multicolumn{2}{|c|}{$\begin{array}{c}\text { Corps_I-Wall Factors } \\
\text { of Safety }\end{array}$}} & \multirow{3}{*}{$\begin{array}{l}\varphi^{\prime} \\
\text { (deg) }\end{array}$} & \multirow{3}{*}{$\begin{array}{l}c^{\prime} \\
\text { (psf) }\end{array}$} & \multicolumn{4}{|c|}{ Point of Rotation } & \multirow{2}{*}{\multicolumn{2}{|c|}{$\begin{array}{c}\text { Sheet Pile Tip } \\
\text { EL }\end{array}$}} \\
\hline & & & & & & & & \multicolumn{2}{|c|}{ EL } & \multicolumn{2}{|r|}{$\mathbf{z}$} & & \\
\hline & & & & Active & Passive & & & Reference & Corps_I-Wall & Reference & Corps_I-Wall & Reference & Corps_I-Wall \\
\hline 1 & $8-1 a$ & 1 & Sand & 1 & 1 & 30 & 0 & -20.64 & -20.73 & 4.96 & 4.99 & -25.60 & -25.72 \\
\hline 2 & $8-1 b$ & 1 & Sand & 1 & 1.633 & 30 & 0 & -27.65 & -27.62 & 6.85 & 6.87 & -34.50 & -34.50 \\
\hline \multirow{2}{*}{3} & \multirow{2}{*}{$8-2 a$} & 1 & Sand & 1 & 1 & 30 & 0 & \multirow{2}{*}{-4.83} & \multirow{2}{*}{-4.61} & \multirow{2}{*}{4.98} & \multirow{2}{*}{5.43} & \multirow{2}{*}{-9.81} & \multirow{2}{*}{-10.04} \\
\hline & & 2 & Clay & 1 & 1 & 0 & 1200 & & & & & & \\
\hline \multirow{2}{*}{4} & \multirow{2}{*}{$8-2 b$} & 1 & Sand & 1 & 1 & 30 & 0 & \multirow{2}{*}{-10.05} & \multirow{2}{*}{-10.07} & \multirow{2}{*}{5.05} & \multirow{2}{*}{5.06} & \multirow{2}{*}{-15.10} & \multirow{2}{*}{-15.14} \\
\hline & & 2 & Clay & 1.5 & 1.5 & 0 & 1200 & & & & & & \\
\hline
\end{tabular}

Reference is Bowles (1968). Foundation Analysis and Design for check problems $1-4$. The moist unit weight of soil $\left(\gamma_{\text {moist }}\right)$ is 100 pcf and the saturated unit weight of soil $\left(\gamma_{\text {sat }}\right)$ is 122.4 pcf. All check problems are in English units. 
Figure 4.73. Sheet-pile wall penetrating sand.

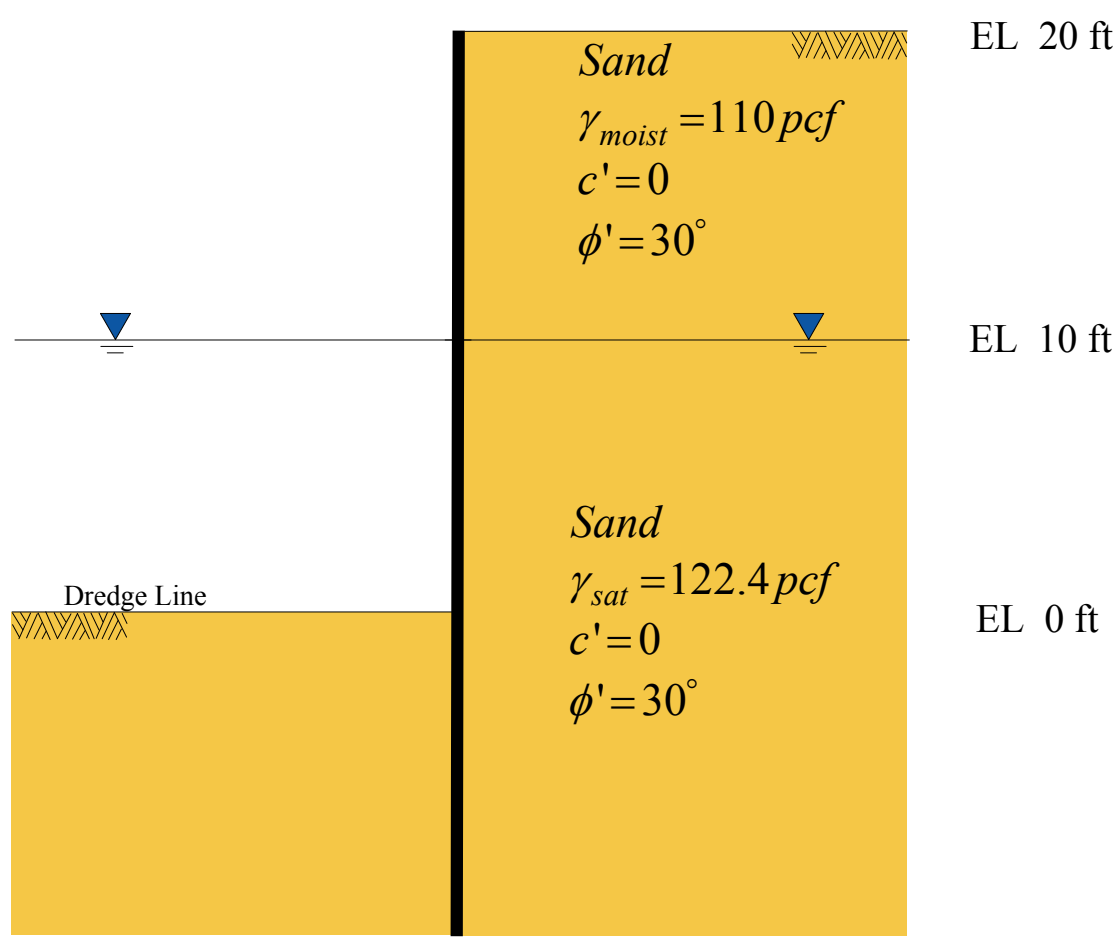

Figure 4.74. Corps_I-Wall schematic of sheet-pile wall in sand site.

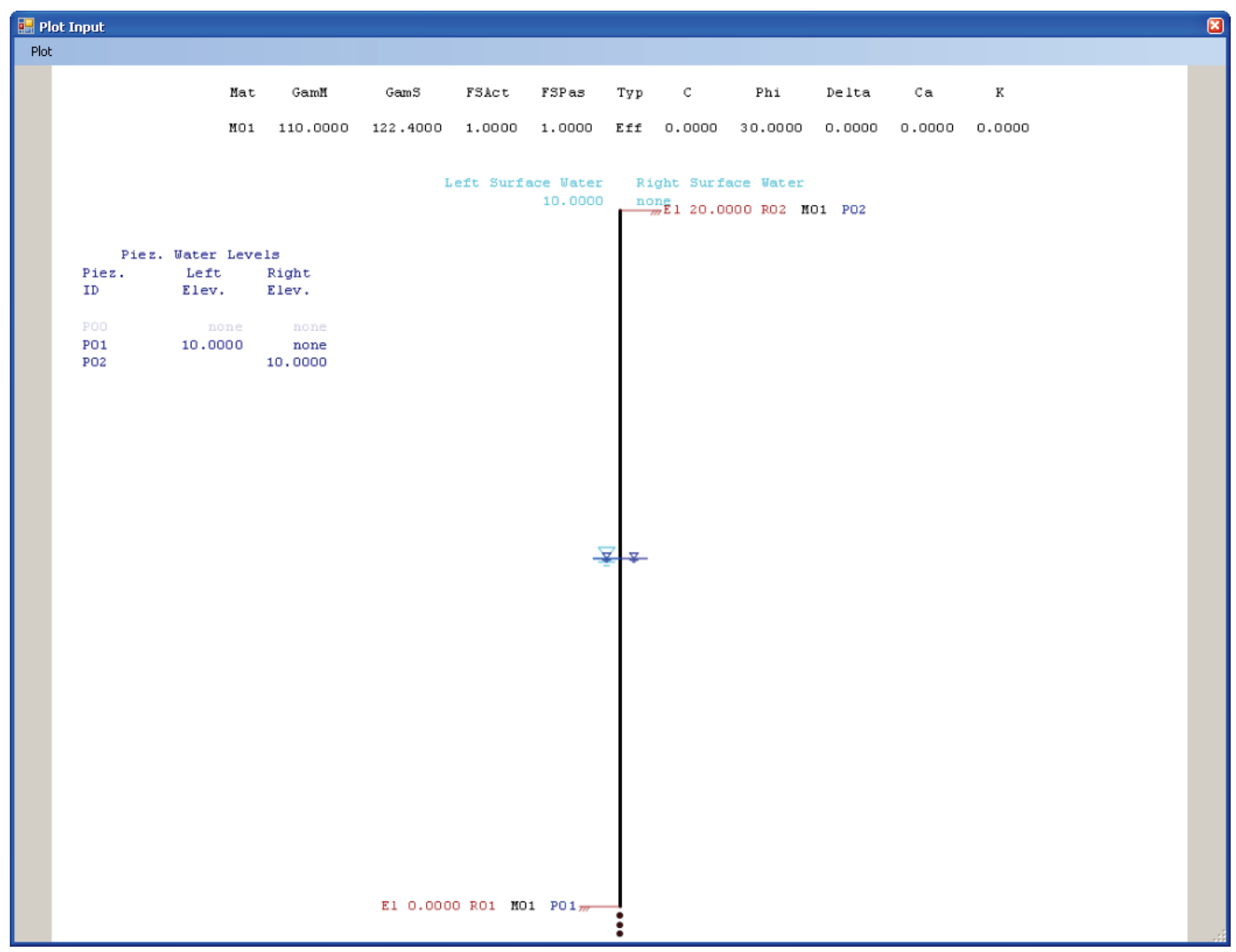


This analysis resulted in a computed mobilized active earth pressure coefficient $\left(K_{a}\right)$ of 0.3333 and a mobilized passive pressure coefficient $\left(K_{p}\right)$ of 3.0. Figure 4.75 displays the results obtained from this analysis with the

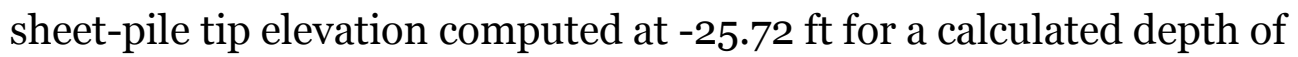
embedment of $25.72 \mathrm{ft}$. The final results for this Example 4.6.3 has been recorded as check problem 1 in Table 4.14.

Figure 4.75. Corps_I-Wall output information.

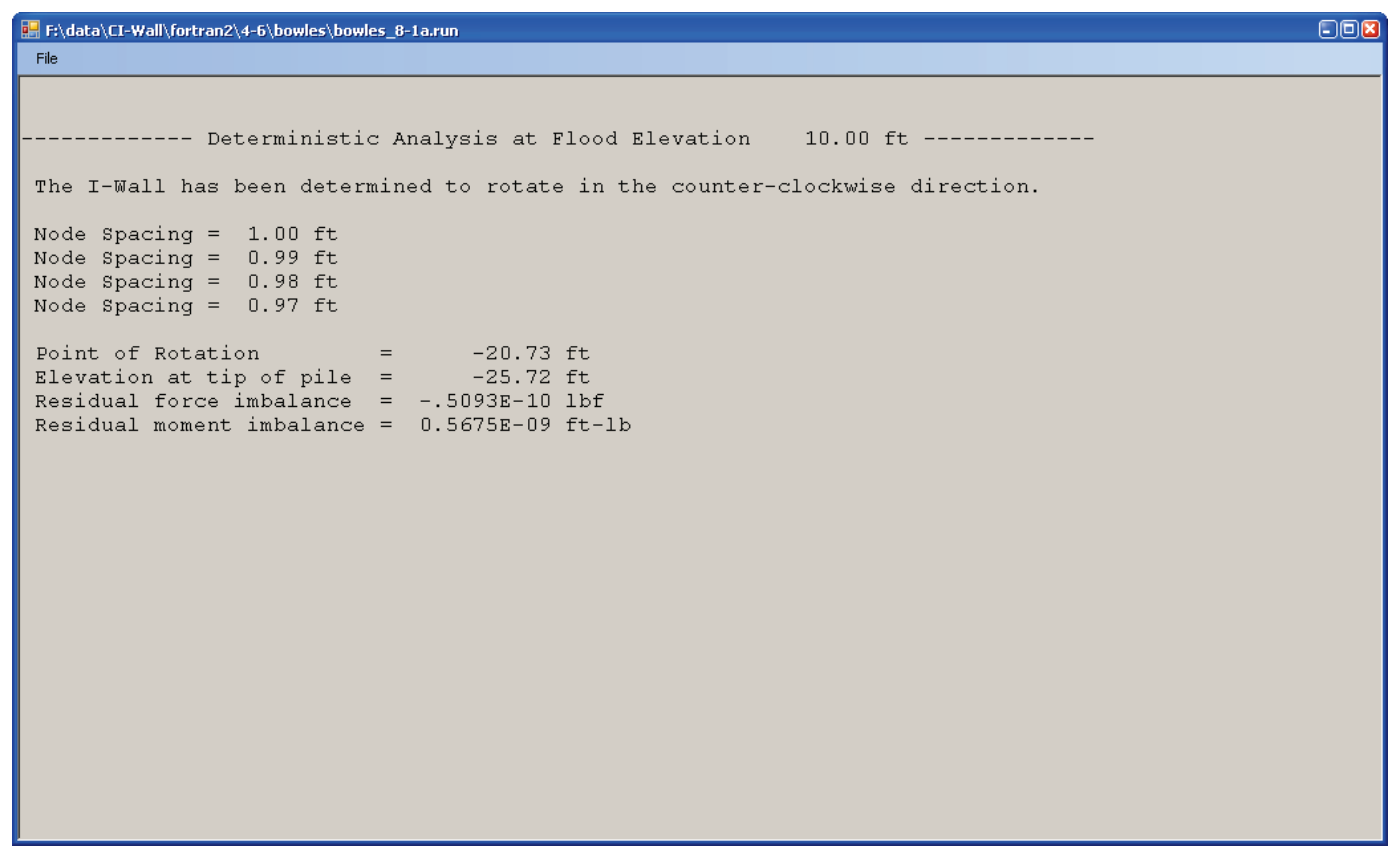

The distribution of both active and passive earth pressures for both sides of the sheet-pile wall are illustrated in Figures 4.76a and 4.76b. The hydrostatic water pressures acting on both sides of the sheet-pile wall are shown in Figure 4.77a with the resultant net water pressure calculated by taking the difference between the water pressures acting on both sides of the wall. In the case when the piezometric surface elevation is equal to the surface water elevation, no resultant net water pressure will be calculated as shown in Figure 4.77b.

From these pressures (Figures 4.76 and 4.77), both the net active and net passive pressures are constructed and presented in Figure 4.78a. For the applied flood loading, the I-Wall will rotate counterclockwise. The net active pressure is determined from the difference between the RHSmobilized active earth pressure and the LHS-mobilized passive earth pressure (i.e., acting on the other side of the wall) with the addition of any net water pressures and any external horizontal net pressures and 
overburden pressures (if any). The net passive pressure is determined by the difference between the RHS-mobilized passive earth pressure and the LHS- mobilized active earth pressure acting on the other side of the sheetpile wall with the addition of any net water pressures and any external horizontal net pressures and overburden pressures (if any).

Figure 4.76. Active and passive earth pressures for drained shear strength. (a) Left-hand side. (b) Right-hand side.

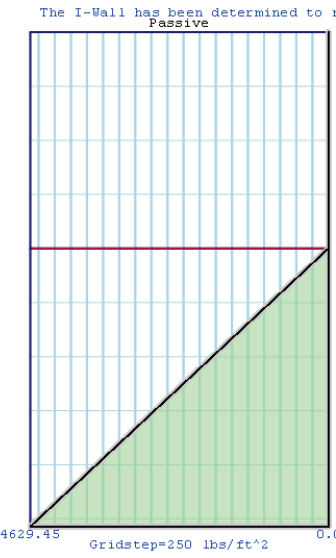

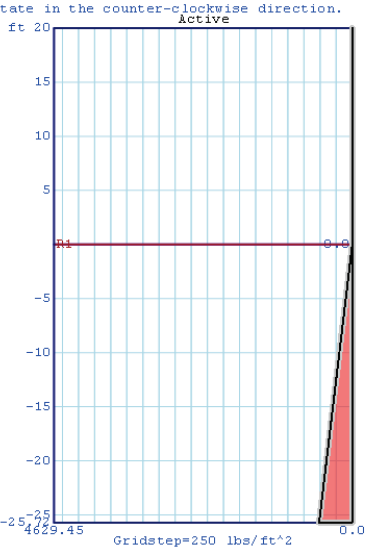

(a)

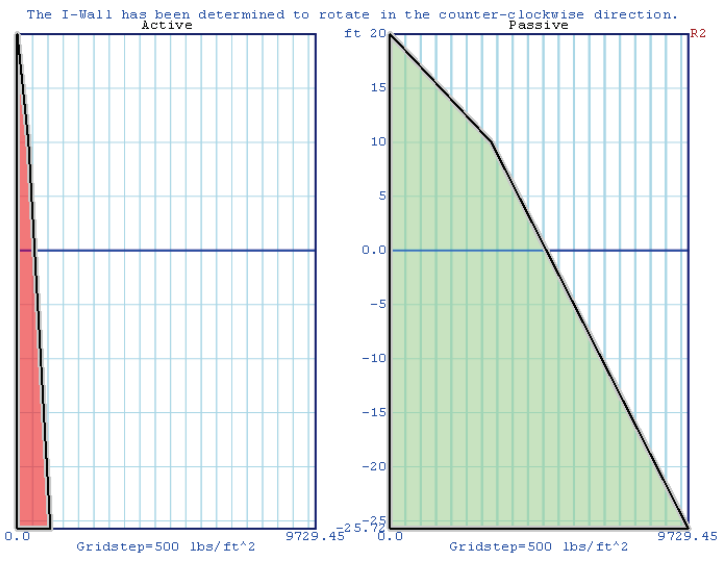

(b)

Figure 4.77. Hydrostatic water pressures. (a) Left- and right-hand side. (b) Net water pressures.

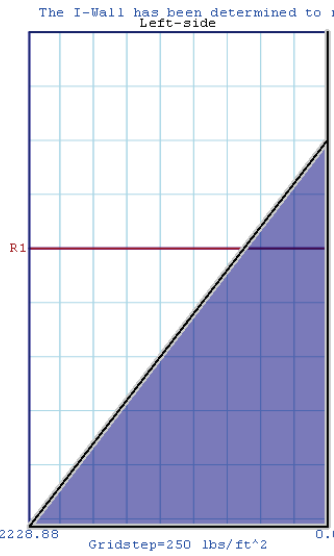

Gridstep $=250 \quad 1 b s / \mathrm{ft}^{\wedge} \mathrm{2}$

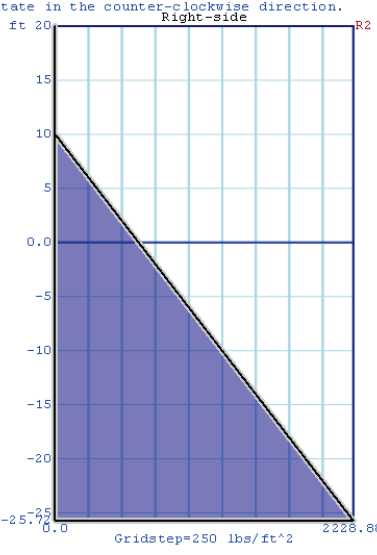

(a)

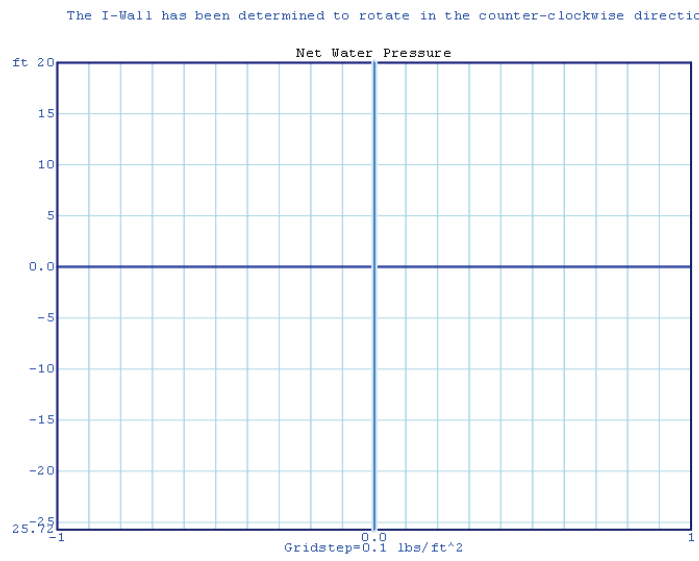

(b)

The net pressure diagram of Figure $4.78 \mathrm{~b}$ is derived from the replication of all values of the net active pressures, from the top of the wall until the elevation at the point of rotation (el-20.73 ft), at which instant the remaining values of the net pressure diagram is assumed to be linear with elevation between (1) the net active pressure value at the elevation of the point of rotation and (2) the net mobilized passive pressure at the 
elevation of the approximated sheet-pile tip (i.e., el -25.73). ${ }^{1}$ Figure $4.78 \mathrm{~b}$ is an illustration of how the net pressure diagram was formed. The final results are illustrated in Figure 4.79 by the presentation of the net pressure diagram Figure 4.79a (replica of Figure 4.78b) and 4.79b, the shear and moment diagrams. Figure 4.79b shows that the maximum bending moment occurs at the zero crossing of the shear diagram.

Figure 4.78. Net pressures. (a) Net active and net passive pressures. (b) Net pressure diagram.

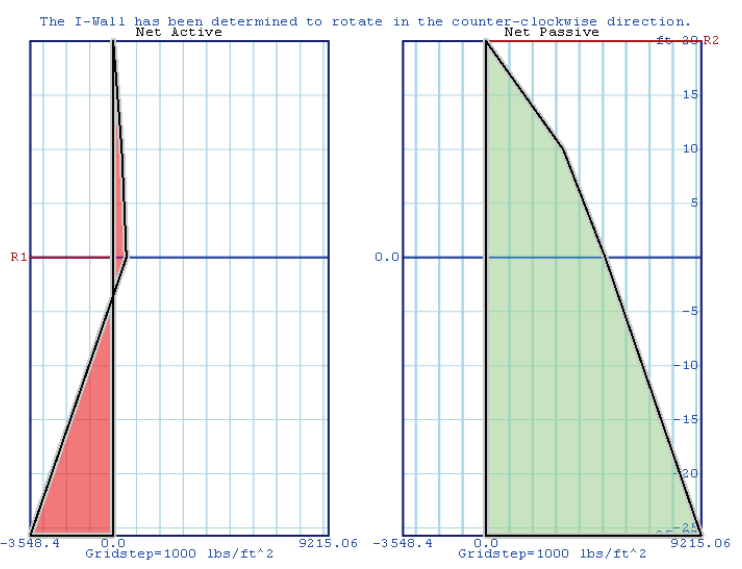

(a)

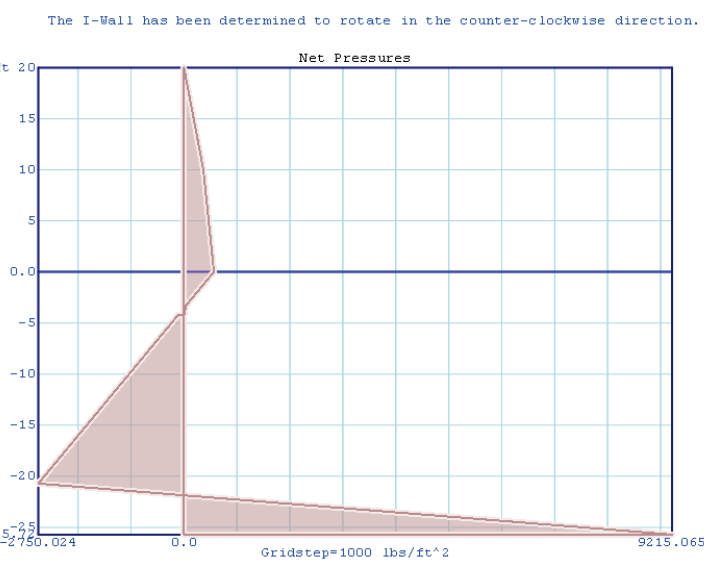

(b)

Figure 4.79. (a) Net pressure diagram. (b) Shear and moment diagram.

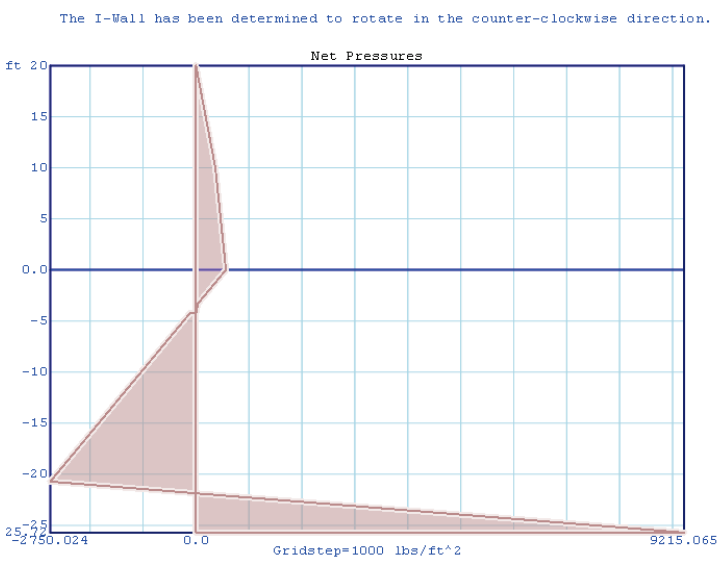

(a)

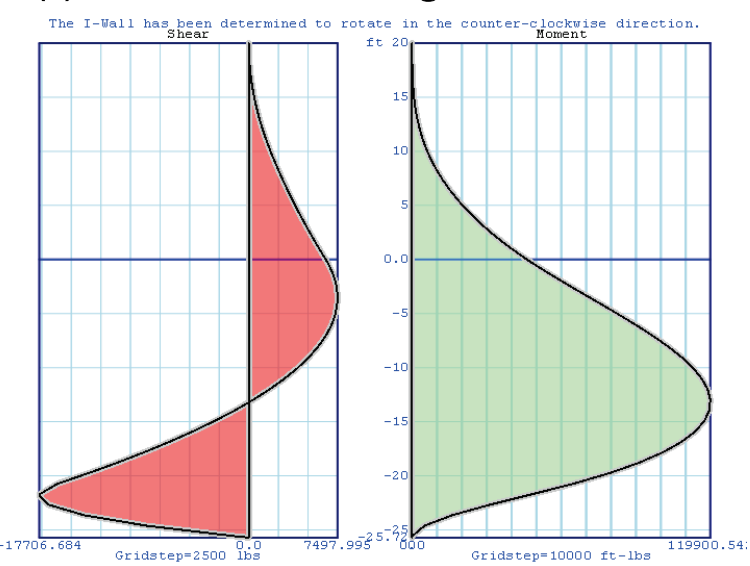

(b)

\subsubsection{Example 4.6.4 sheet-pile wall penetrating sand (Bowles)}

This example problem uses the same sheet-pile conditions as problem 4.6.3, but requires the Factor of Safety for passive earth pressures of 1.5 for

1 Thus, only one mobilized net passive pressure value in Figure 4.78a is used to construct the Figure 4.79a net pressure diagram; corresponding to the sheet-pile tip elevation at el -25.72. The majority of the net pressure diagram is derived from the Figure 4.78a net active pressure diagram. 
determining the depth of embedment of sheet-pile walls in granular soil. The Corps_I-Wall adaptation to Bowles (1968) calculations required the passive Factor of Safety to be set to 1.6333 because Bowles applied the Factor of Safety to $\varphi^{\prime}$ (i.e., $\varphi^{\prime}$ mob $\left.=30 / 1.5\right)$ rather than as $\tan \left(\varphi^{\prime}\right.$ mob $)=$ $\tan \left(\varphi^{\prime}\right) / 1.5$.

This analysis resulted in a computed mobilized active earth pressure coefficient $\left(K_{a}\right)$ of 0.3333 and a mobilized passive pressure coefficient $\left(K_{p}\right)$ of 1.9999. Figure 4.80 displays the results obtained from this analysis with the sheet-pile tip elevation computed at $-34.5 \mathrm{ft}$ for a calculated depth of embedment of $34.5 \mathrm{ft}$. The final results for this Example 4.6.4 has been recorded as check problem 2 in Table 4.14.

Figure 4.80. Corps_I-Wall output information.

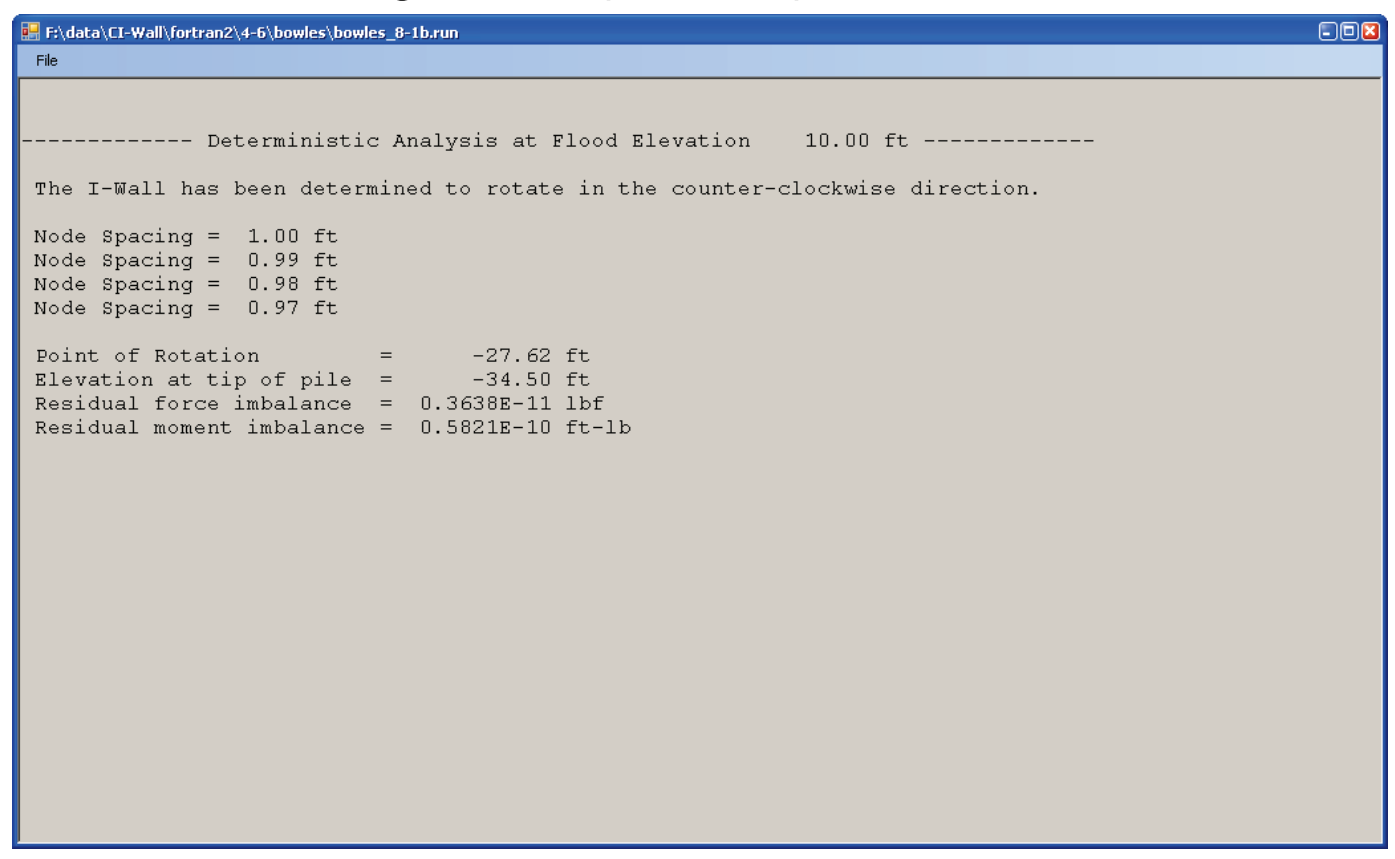

\subsubsection{Sheet-pile wall penetrating saturated clay (Bowles)}

To determine the depth of embedment of sheet-pile walls of retained sand and saturated clay, the following two problems (Bowles 1968) of examples 4.6.5 and 4.6.6 are examined. These two problems differ with the assignment of the Factor of Safety for both active and passive earth pressures of 1.0 (Section 4.6.4.1) and of 1.5 (Section 4.6.4.2). Table 4.14 shows the material properties for the problems. 


\subsubsection{Example 4.6.5 sheet-pile wall penetrating saturated clay (Bowles)}

To determine the depth of embedment of a sheet-pile wall in two-layered soil, Figure 4.81 is examined. This example problem (Bowles 1968) represents the case of saturated clay soil below the dredge line on both sides of the sheet-pile wall with retained sandy soil above the dredge line on the landside (RHS) of the sheet-pile wall. The sand layer has an effective stress definition with no cohesion and an effective angle of internal friction $\left(\varphi^{\prime}\right)$ of $30 \mathrm{deg}$. The sand is assigned a moist unit weight of $110 \mathrm{pcf}$ and a saturated unit weight of $122.4 \mathrm{pcf}$. The clay soil layer has a total stress definition with $\varphi=0$ and cohesion (c) of 1,200 psf. Hydrostatic water pressures are at el $10 \mathrm{ft}$ on both sides of the sheet-pile wall. For this design, the required factors of safety on the active and passive pressures are assigned 1.o. An effective angle of wall to soil interface friction $\left(\delta^{\prime}\right)$ of o deg as well as the Coulomb coefficient method of analysis (for $K_{a}$ and $K_{p}$ ) form the basis for this example ${ }^{1}$. Hydraulic fracturing is not included as an option for this analysis. The Corps_I-Wall representation of this problem is presented by the schematic of Figure 4.82. This figure outlines and describes the geometry and structural parameters for the sheet-pile wall.

This analysis resulted in a computed mobilized active earth pressure coefficient $\left(K_{a}\right)$ of 0.3333 and a mobilized passive pressure coefficient $\left(K_{p}\right)$ of 3.0 for the sandy soil on the retained side above the dredge line. For the clay layers (with $\varphi=0$ ) below the dredge line, $K_{a}$ and $K_{p}$ obtained values of 1.o. Figure 4.83 displays the results obtained from this analysis with the sheet-pile tip elevation computed at $-10.04 \mathrm{ft}$ for a calculated depth of embedment of $10.04 \mathrm{ft}$. The final results for this Example 4.6.5 has been recorded as check problem 3 in Table 4.14.

The distribution of both active and passive earth pressures for both sides of the sheet-pile wall is illustrated in Figures 4.84a and 4.84b. The hydrostatic water pressures acting on both sides of the sheet-pile wall are shown in Figure 4.85a with the resultant net water pressure calculated by taking the difference between the water pressures acting on both sides of The wall. In the case when the piezometric surface elevation is equal to the surface water elevation, no resultant net water pressure will be calculated as shown in Figure 4.85b.

1 For no soil-to-I-Wall interface friction (i.e., $\delta=0 \mathrm{deg}$ ), the Coulomb solution for both active and passive earth pressure coefficients corresponds to the Rankine earth pressure coefficients. 
Figure 4.81. Sheet-pile wall penetrating saturated clay.

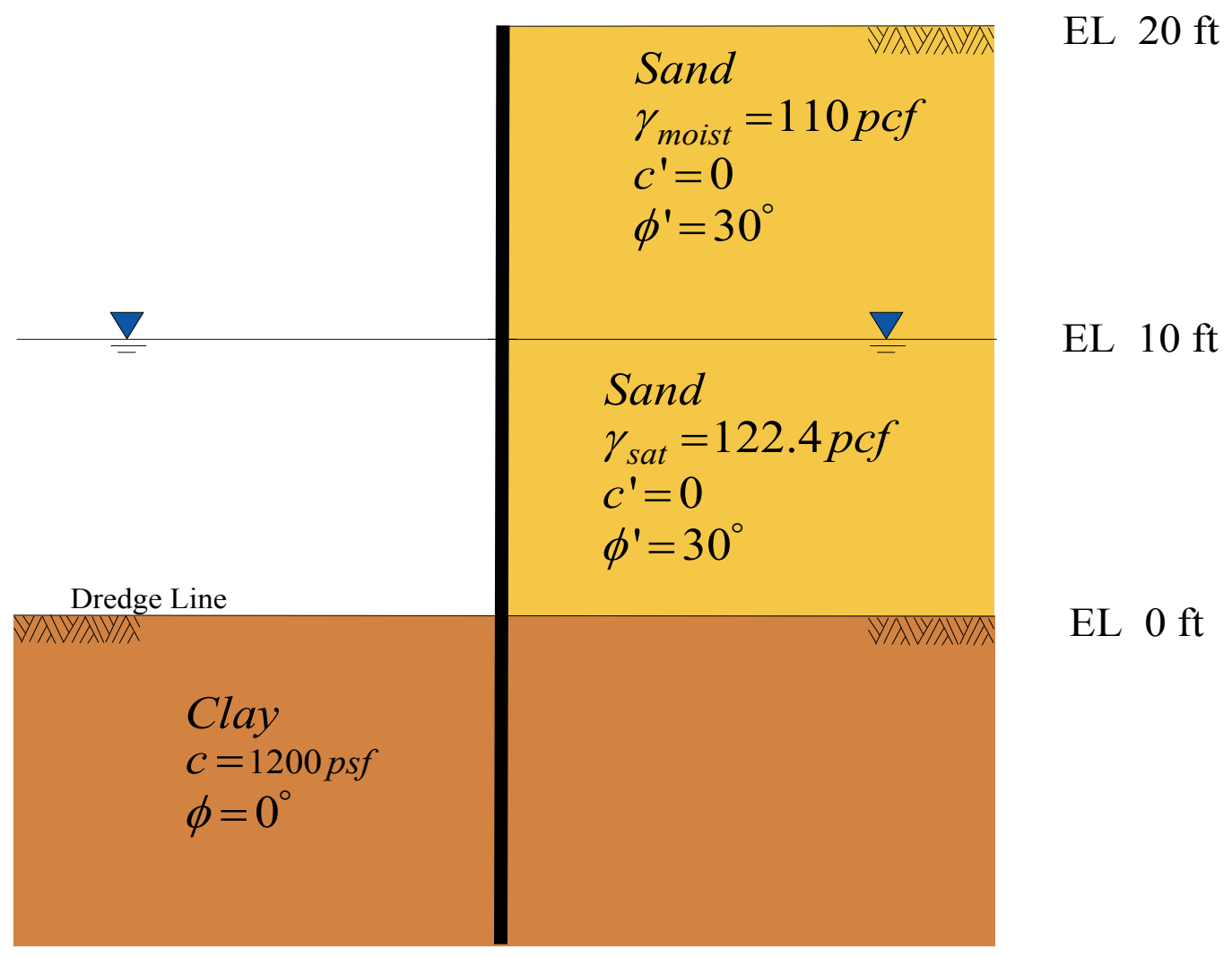

Figure 4.82. Corps_I-Wall schematic of sheet-pile wall in sand and saturated clay site.

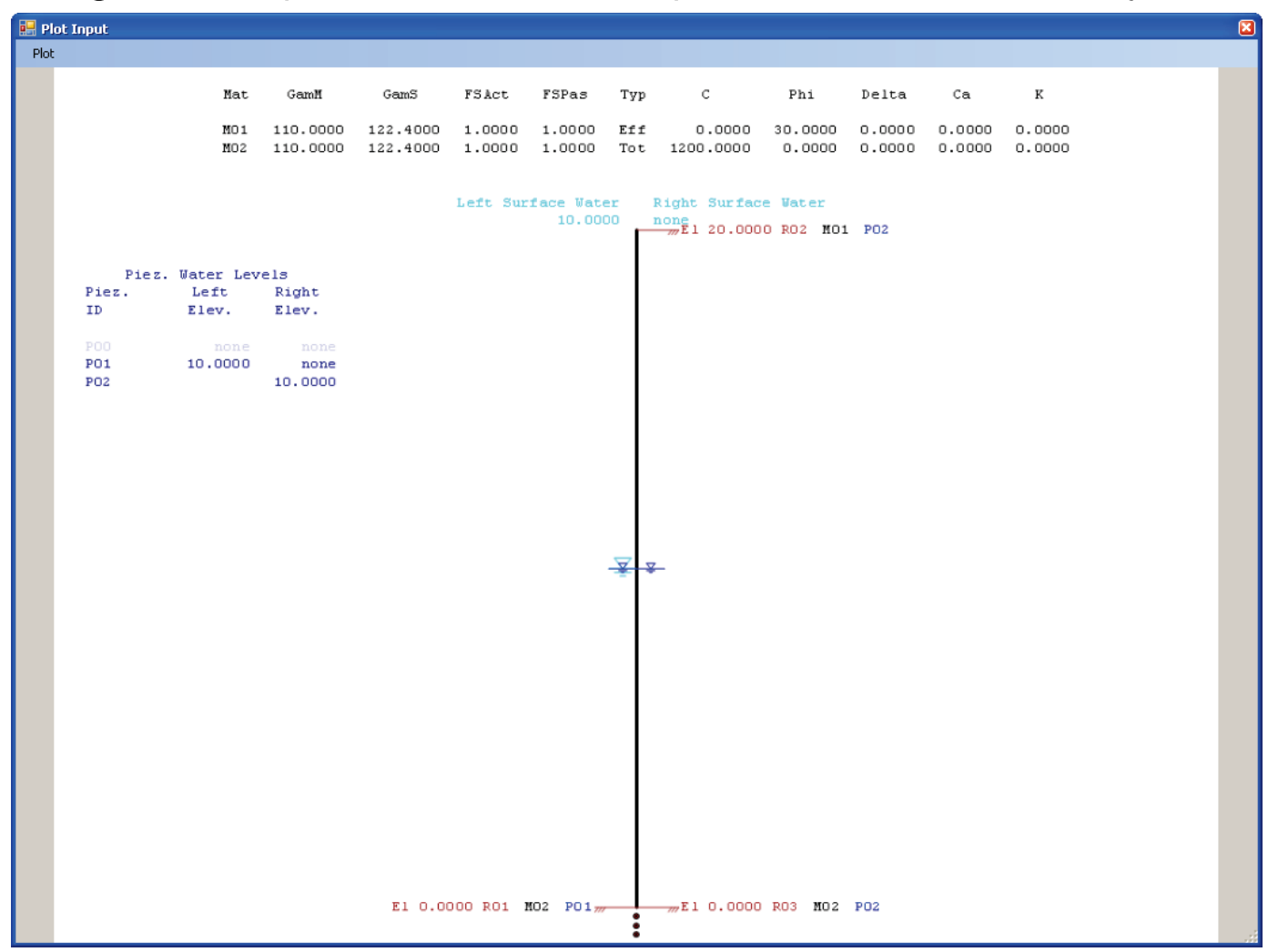


Figure 4.83. Corps_I-Wall output information.

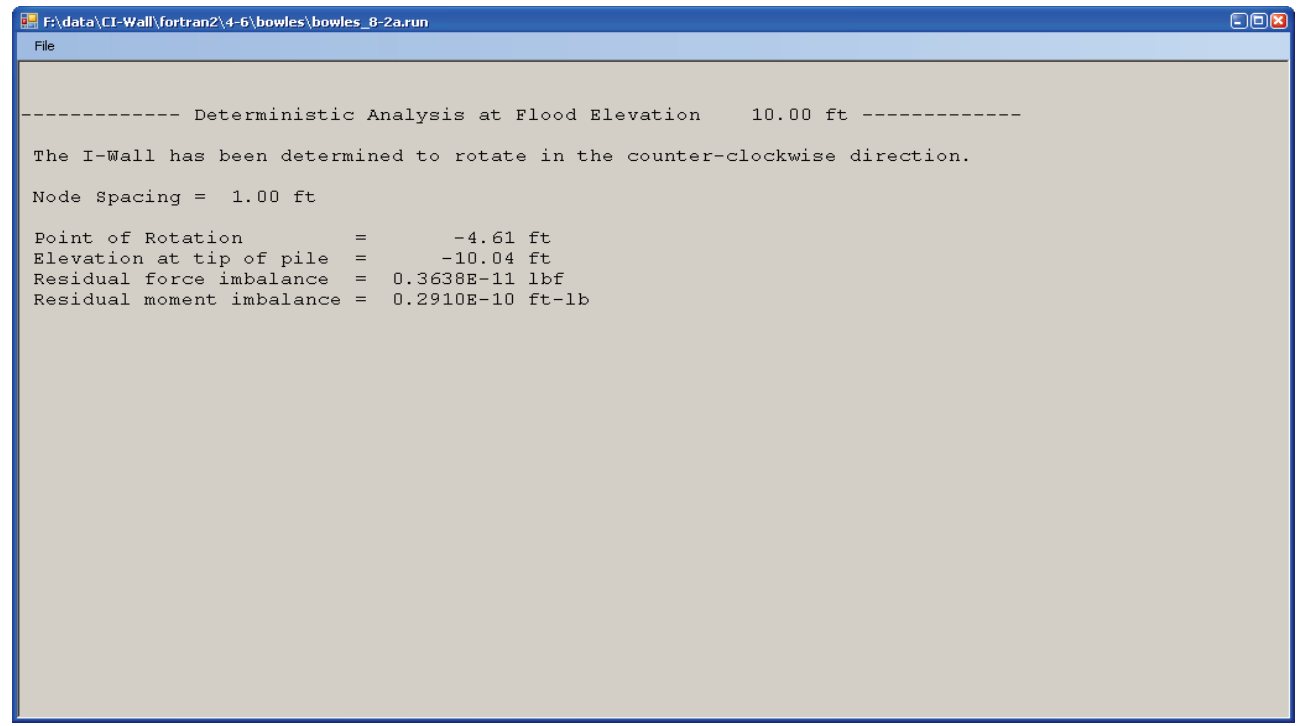

Figure 4.84. Active and passive earth pressures. (a) Left-hand side. (b) Right-hand side.

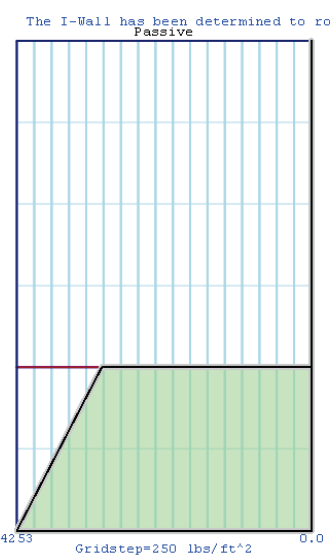

Gridstep $=250 \quad 1 b s / f t^{\wedge} 2$

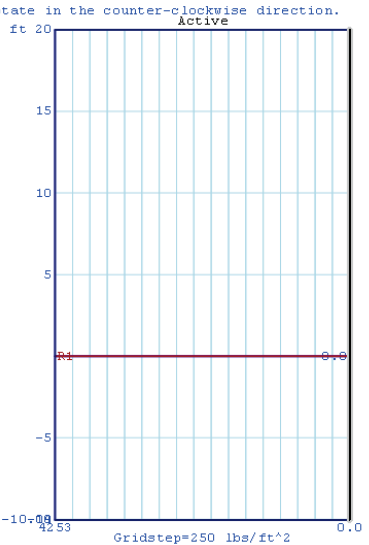

(a)
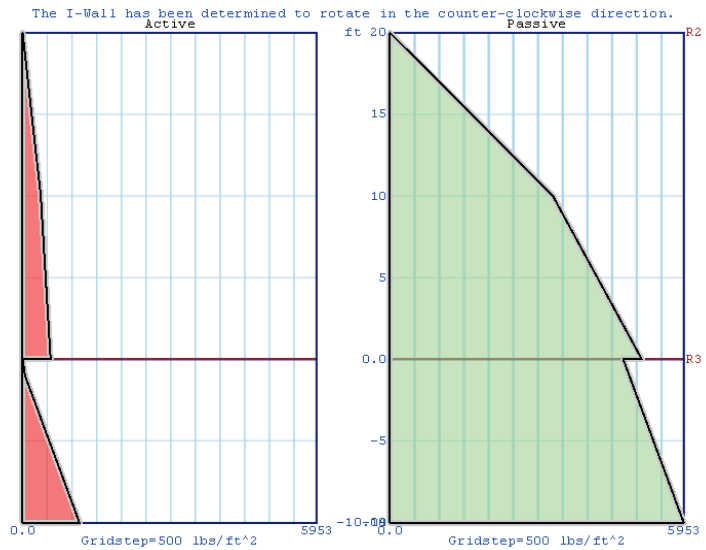

(b)

Figure 4.85. Hydrostatic water pressures. (a) Left- and right-hand side. (b) Net water pressures.

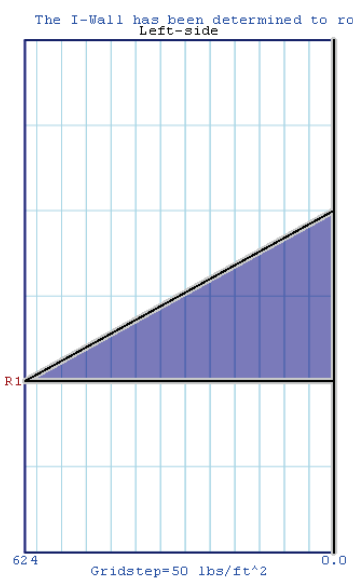

Gridstep $=50 \quad 1 b s / \mathrm{ft}^{\wedge} 2$

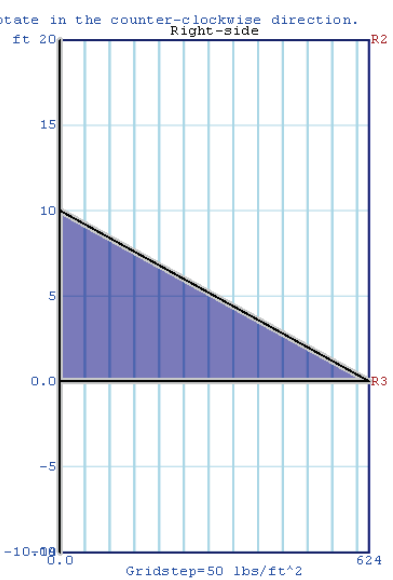

(a)

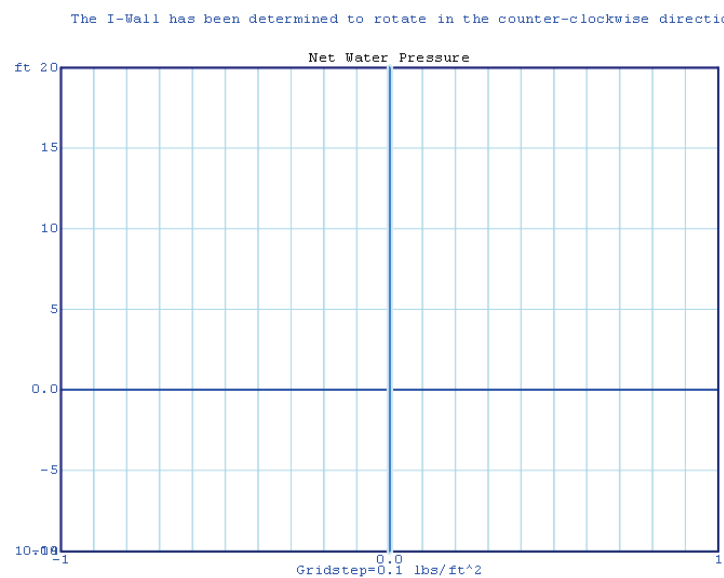

(b) 
From these pressures (Figures 4.84 and 4.85), the net active and net passive pressures are constructed and presented in Figure 4.86a. For the applied earth loading, the I-Wall will rotate counterclockwise. The net active pressure is determined from the difference between the RHS-mobilized active earth pressure and the LHS-mobilized passive earth pressure (i.e., acting on the other side of the wall) with the addition of any net water pressures (none in this case) and any external horizontal net pressures and overburden pressures (if any). The net passive pressure is determined by the difference between the RHS-mobilized passive earth pressure and the LHS-mobilized active earth pressure acting on the other side of the sheetpile wall with the addition of any net water pressures and any external horizontal net pressures and overburden pressures (if any).

Figure 4.86. Net pressures. (a) Net active and net passive pressures. (b) Net pressure diagram.

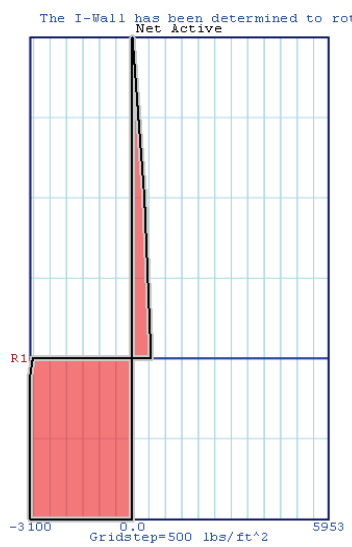

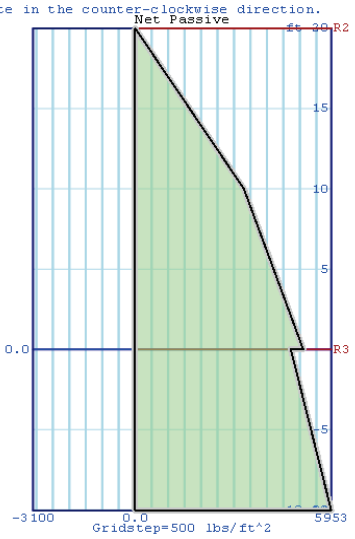

(a)

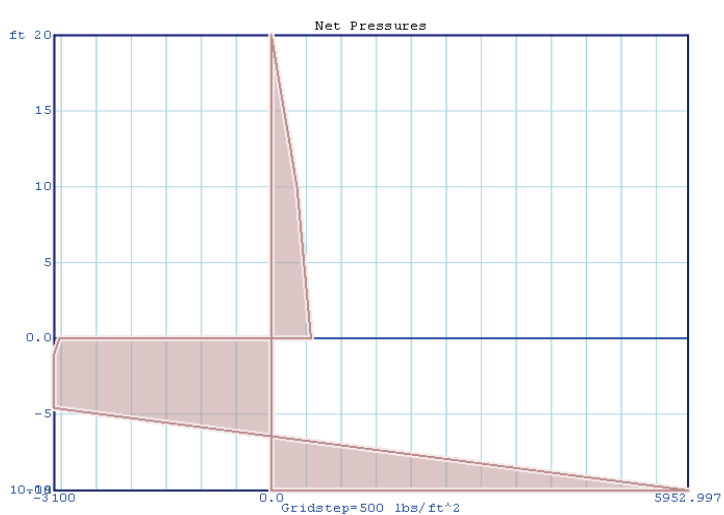

(b)

The net pressure diagram of Figure 4.86b is derived from the replication of all values of the net active pressures, from the top of the wall until the elevation at the point of rotation (el $-4.61 \mathrm{ft}$ ), at which instant the remaining values of the net pressure diagram is assumed to be linear with elevation between (1) the net active pressure value at the elevation of the point of rotation and (2) the net mobilized passive pressure at the elevation of the approximated sheet-pile tip (i.e., el $-10.04 \mathrm{ft}$ ). ${ }^{1}$ Figure $4.86 \mathrm{~b}$ is an illustration of how the net pressure diagram was formed. The final results are illustrated in Figure 4.87 by the presentation of the net pressure diagram Figures $4.87 \mathrm{a}$ (replica of Figure $4.86 \mathrm{~b}$ ) and $4.87 \mathrm{~b}$, the shear and moment diagrams. Figure $4.87 \mathrm{~b}$ shows that the maximum bending moment occurs at the zero crossing of the shear diagram.

1 Only one mobilized net passive pressure value in Figure 4.86a is used to construct the Figure 4.86b net pressure diagram, corresponding to the sheet-pile tip elevation at el $-10.04 \mathrm{ft}$. The majority of the net pressure diagram is derived from the Figure 4.86a net active pressure diagram. 
Figure 4.87. (a) Net pressure diagram. (b) Shear and moment diagram.

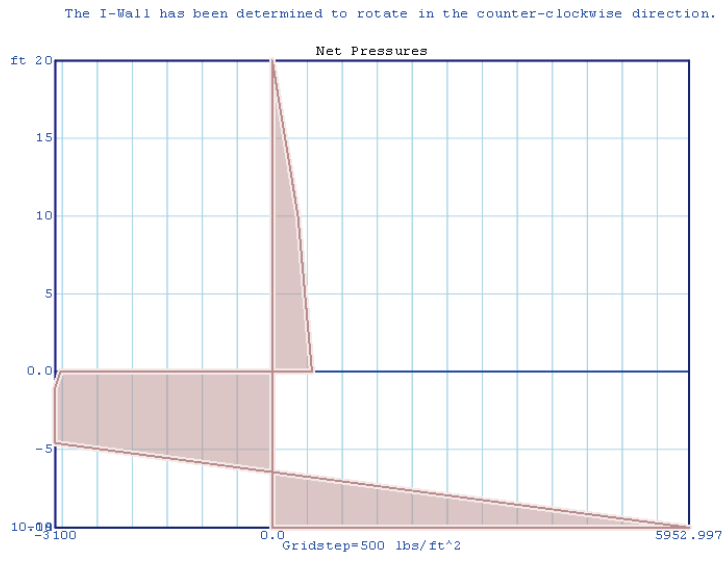

(a)

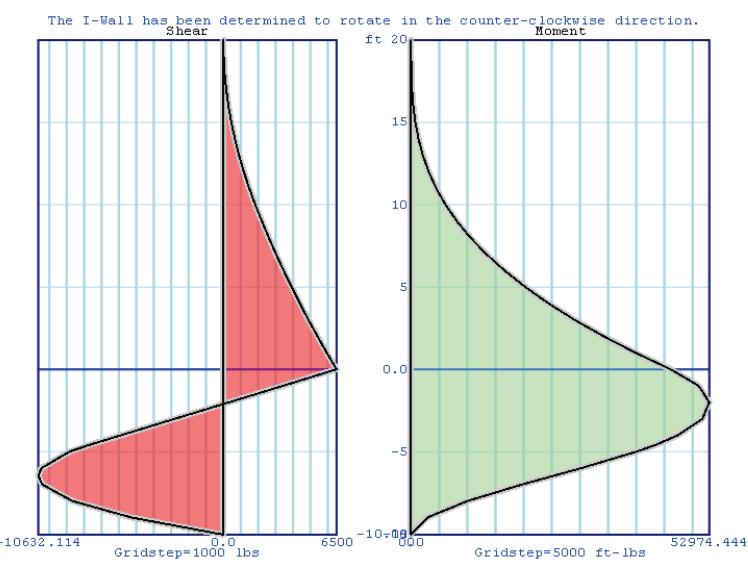

(b)

\subsubsection{Example 4.6.6 sheet-pile wall penetrating saturated clay (Bowles)}

This example problem uses the same sheet-pile conditions as problem 4.6.5, but uses the Factor of Safety of 1.5 instead of 1.0 for both the active earth pressure and passive earth pressure for determining the depth of embedment of sheet-pile walls in granular soil.

This analysis resulted in a computed mobilized active earth pressure coefficient $\left(K_{a}\right)$ of 0.3333 and a mobilized passive pressure coefficient $\left(K_{p}\right)$ of 3.0. Figure 4.88 displays the results obtained from this analysis with the sheet-pile tip elevation computed at $\mathbf{- 1 5 . 1 4} \mathrm{ft}$ for a calculated depth of embedment of $15.14 \mathrm{ft}$. This depth of embedment required an increase of approximately $5 \mathrm{ft}$ when the Factor of Safety was increased from 1.0 to 1.5.

The final results for this Example 4.6.6 has been recorded as check problem 4 in Table 4.14 .

The Corps_I-Wall computed results for point of rotation and sheet-pile tip agree with those from Bowles for the four sheet-pile walls analyzed. 
Figure 4.88. Corps_I-Wall output information.

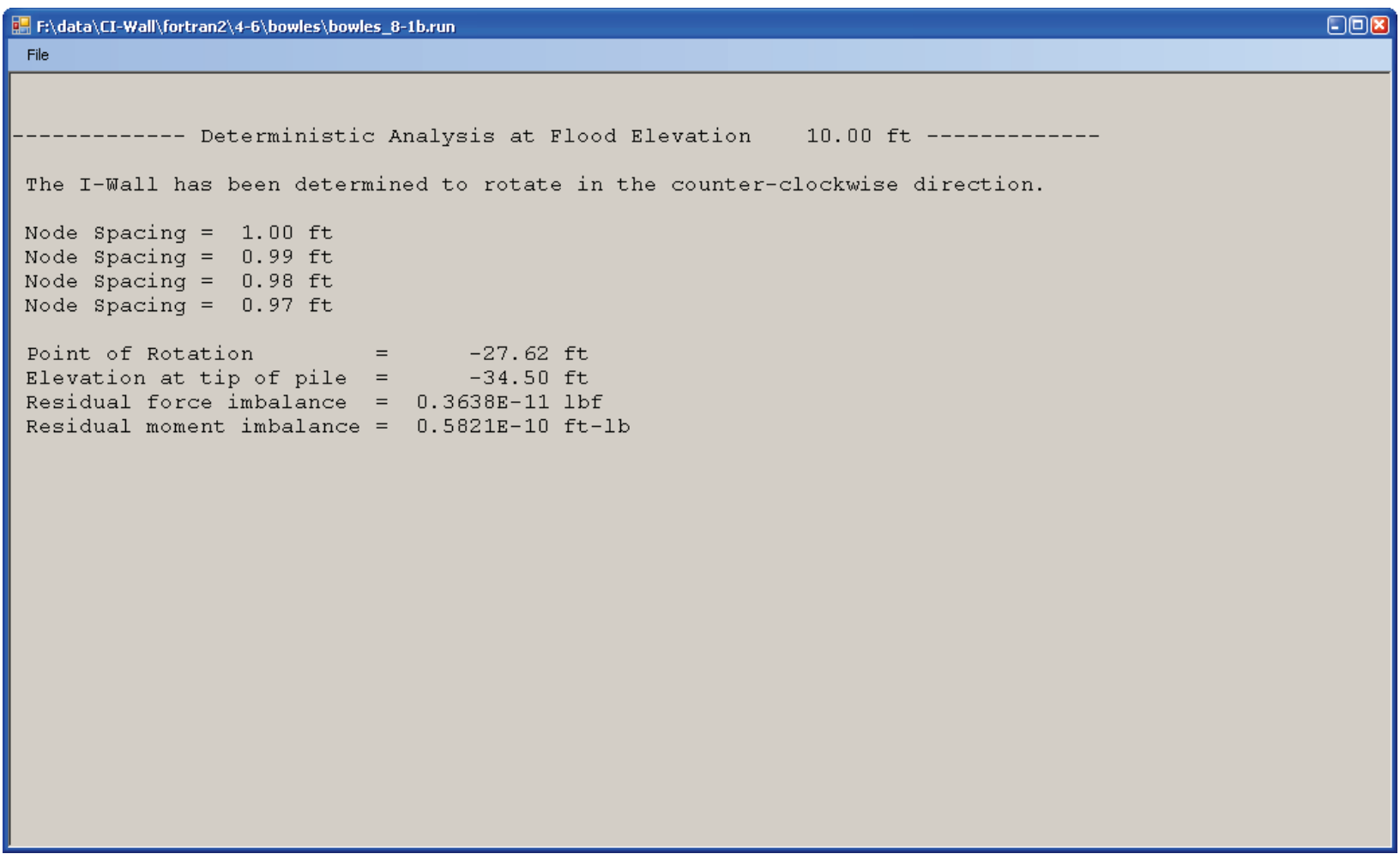

\subsubsection{Example 4.6.7 sheet-pile wall penetrating clay (Pace et al. 2012)}

To determine the depth of embedment of sheet-pile walls in clay soil, Figure 4.89 is examined. This example problem, taken from Pace et al. (2012), represents a total stress method of analysis where pore pressures are not calculated within the soil. The cohesive soil $(\varphi=0$ and $\delta=0)$ has undrained shear strength $\left(S_{u}\right)$ and adhesion $\left(C_{a}\right)$ parameters. The clay layers are assigned a moist unit weight of $110 \mathrm{pcf}$ and a saturated unit weight of $110 \mathrm{pcf}$. Flood side surface water is at $9 \mathrm{ft}$. Hydrostatic water pressures are at el $-15 \mathrm{ft}$ on both sides of the sheet-pile wall. For this design, the required Factor of Safety for active earth pressures is 1.0 and the Factor of Safety for passive earth pressures is assigned 1.5. The Coulomb coefficient method of analysis (for $K_{a}$ and $K_{p}$ ) as well as hydraulic fracturing forms the basis for this analysis ${ }^{1}$.

\footnotetext{
1 For no soil-to-I-Wall interface friction (i.e., $\delta=0$ deg), the Coulomb solution for both active and passive earth pressure coefficients corresponds to the Rankine earth pressure coefficients.
} 
Figure 4.89. Sheet-pile wall penetrating clay.

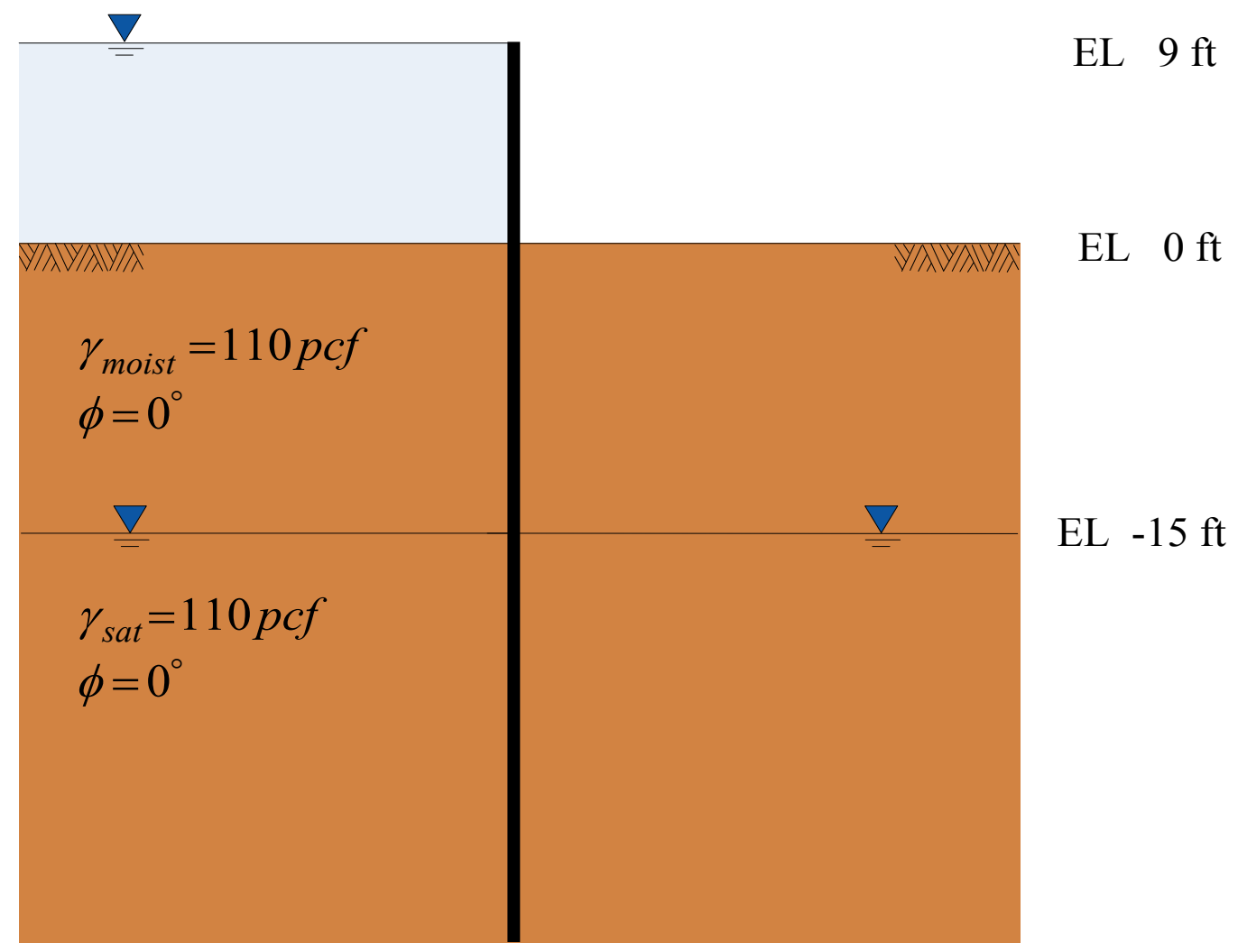

The Corps_I-Wall representation of this problem with $S_{u}=300$ psf and $C_{a}=0 \mathrm{psf}$ is presented by the schematic of Figure 4.90. This figure outlines and describes the geometry and structural parameters for the sheet-pile wall.

For clay soil (with $\varphi=0$ ), this analysis resulted in computed mobilized earth pressure coefficients $K_{a}$ and $K_{p}$ of 1.o. Figure 4.91 displays the results obtained from this analysis with the sheet-pile tip elevation computed at $-29.85 \mathrm{ft}$ for a calculated depth of embedment of $29.85 \mathrm{ft}$. The depth of gap is computed to be $12.61 \mathrm{ft}$. The final results for this Example 4.6.7 has been recorded as check problem 2 in Table 4.14.

The distribution of active and passive earth pressures for both sides of the sheet-pile wall is illustrated in Figures 4.92a and 4.92b. The hydrostatic water pressures acting on both sides of the sheet-pile wall are shown in Figure 4.93a with the resultant net water pressure calculated by taking the difference between the water pressures acting on both sides of the wall. Note that the active earth pressure in Figure 4.92a only develops at the gap depth of $12.61 \mathrm{ft}$ and hydrostatic water pressure are applied within the gap below the ground surface as shown in Figure 4.93a and at the resultant net water pressure of Figure 4.93b. 
Figure 4.90. Corps_I-Wall schematic of sheet-pile wall in clay site.

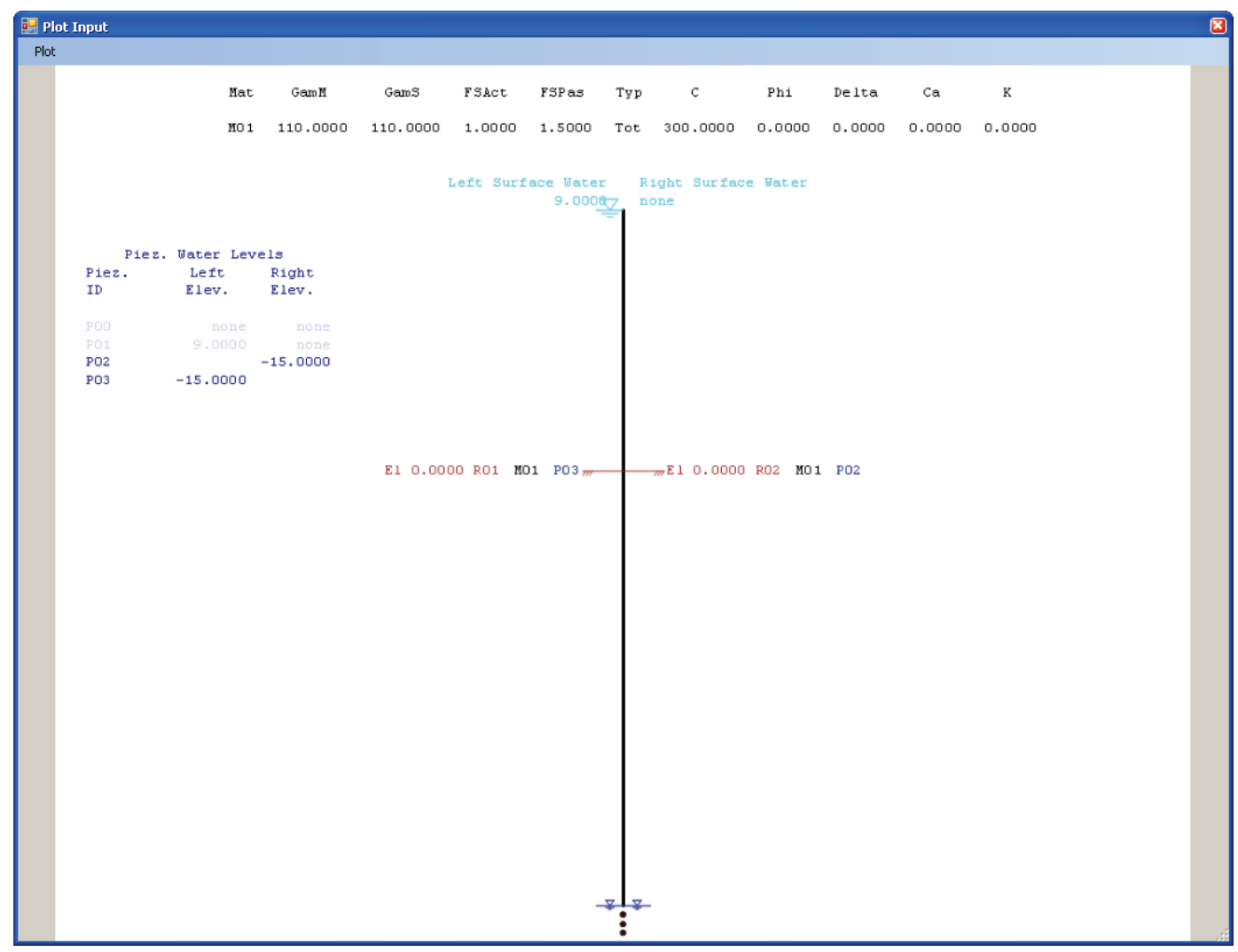

Figure 4.91. Corps_I-Wall output information.

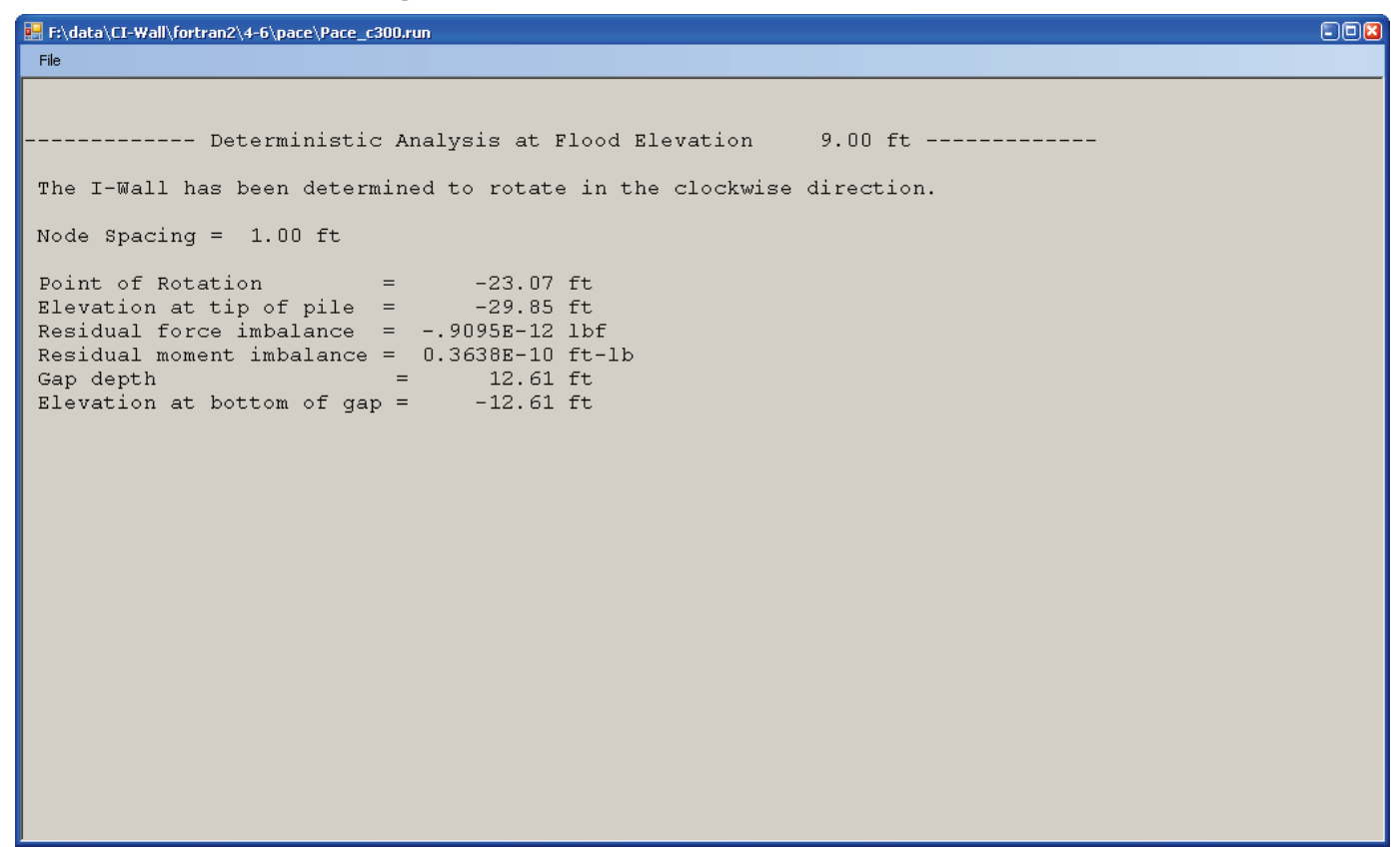


Figure 4.92. Active and passive earth pressures. (a) Left-hand side. (b) Right-hand side.

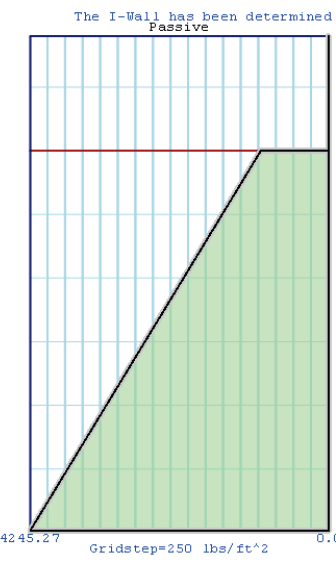

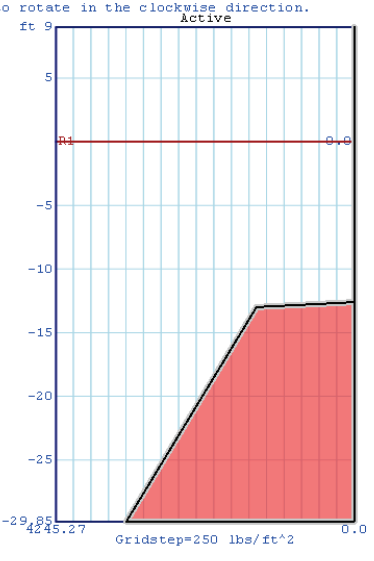

(a)
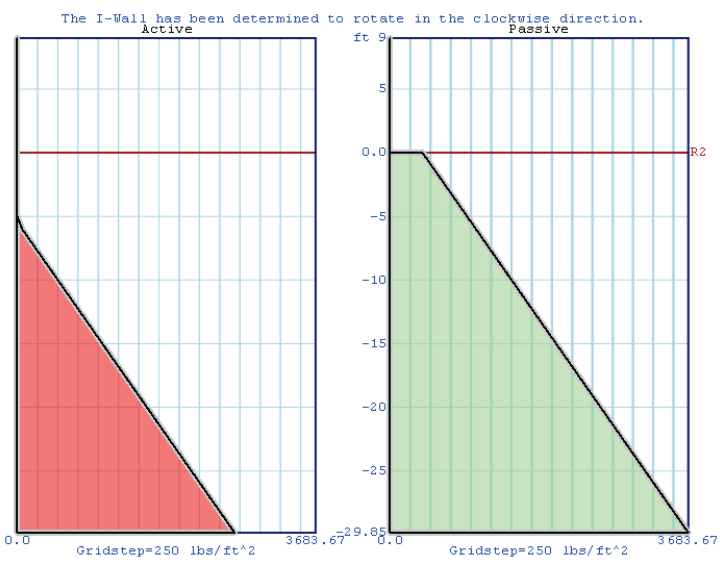

(b)

Figure 4.93. Hydrostatic water pressures. (a) Left- and right-hand side.(b) Net water pressures.
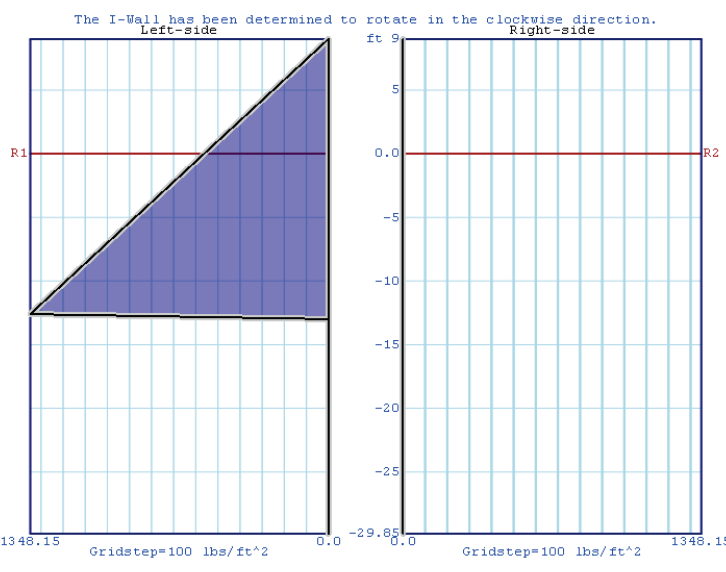

(a)

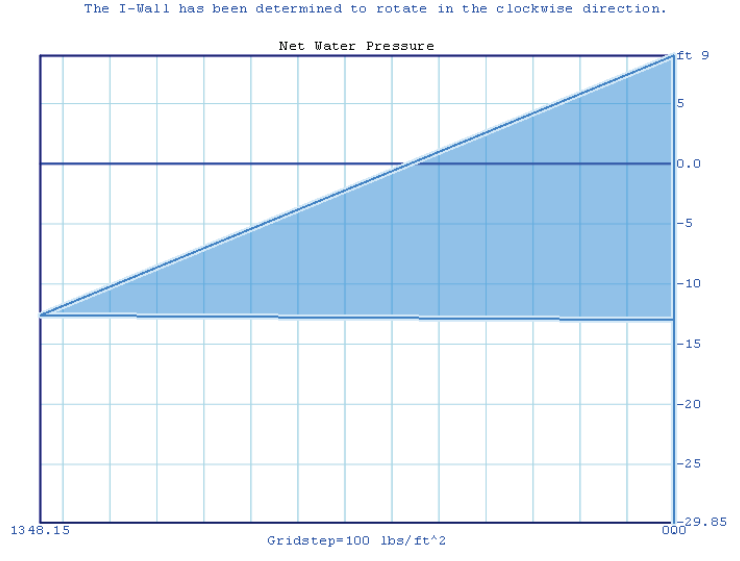

(b)

From these pressures (Figures 4.92 and 4.93), the net active and net passive pressures are constructed and presented in Figure 4.94a. For the applied flood loading, the I-Wall will rotate counterclockwise. The net active pressure is determined from the difference between the RHSmobilized active earth pressure and the LHS-mobilized passive earth pressure (i.e., acting on the other side of the wall) with the addition of any net water pressures and any external horizontal net pressures and overburden pressures (if any). The net passive pressure is determined by the difference between the RHS-mobilized passive earth pressure and the LHS- mobilized active earth pressure acting on the other side of the sheetpile wall with the addition of any net water pressures and any external horizontal net pressures and overburden pressures (if any). 
Figure 4.94. Net pressures (a) Net active and net passive pressures. (b) Net pressure diagram.

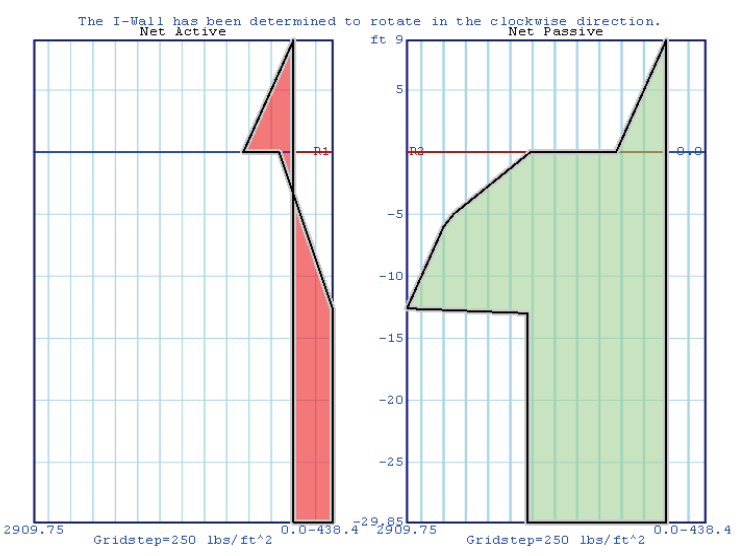

(a)

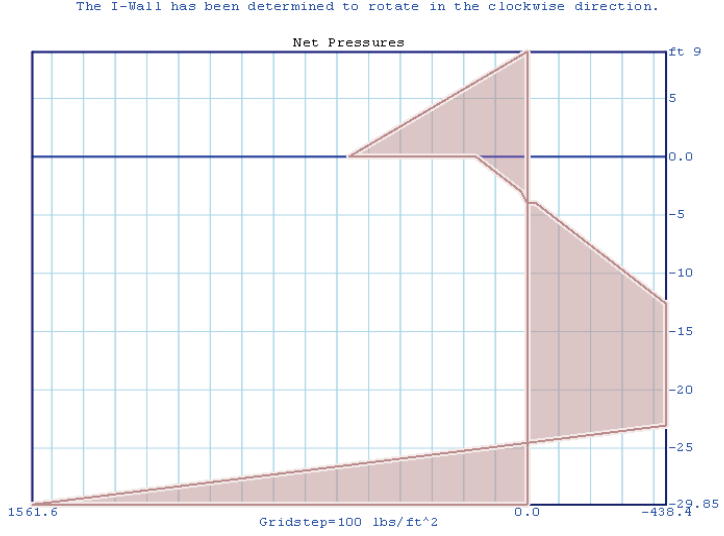

(b)

The net pressure diagram of Figure 4.94b is derived from the replication of all values of the net active pressures, from the top of the wall until the elevation at the point of rotation (el $-23.07 \mathrm{ft}$ ), at which instant the remaining values of the net pressure diagram are assumed to be linear with elevation between (1) the net active pressure value at the elevation of the point of rotation and (2) the net mobilized passive pressure at the elevation of the approximated sheet-pile tip (i.e., el -29.85 ft). ${ }^{1}$ Figure $4.94 \mathrm{~b}$ is an illustration of how the net pressure diagram was formed. The final results are illustrated in Figure 4.95 by the presentation of the net pressure diagram, Figure 4.95a (replica of Figure 4.94b) and Figure 4.95b, the shear and moment diagrams. Figure 4.95b shows that the maximum bending moment occurs at the zero crossing of the shear diagram.

Figure 4.95. (a) Net pressure diagram. (b) Shear and moment diagram.

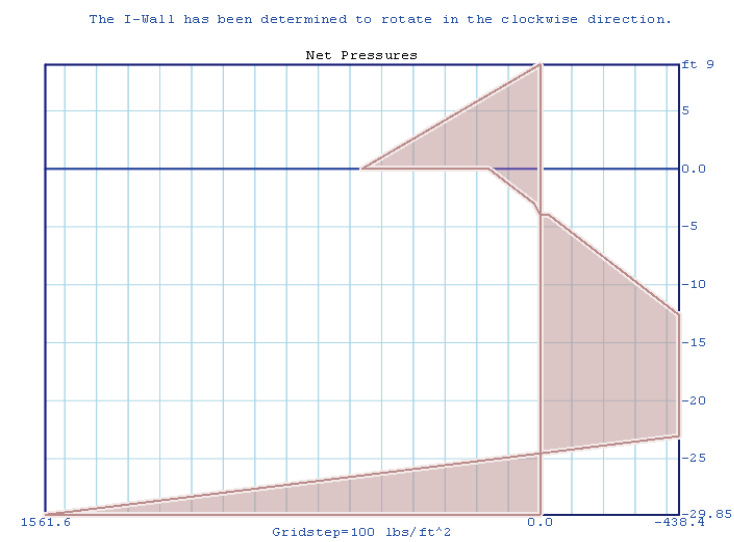

(a)

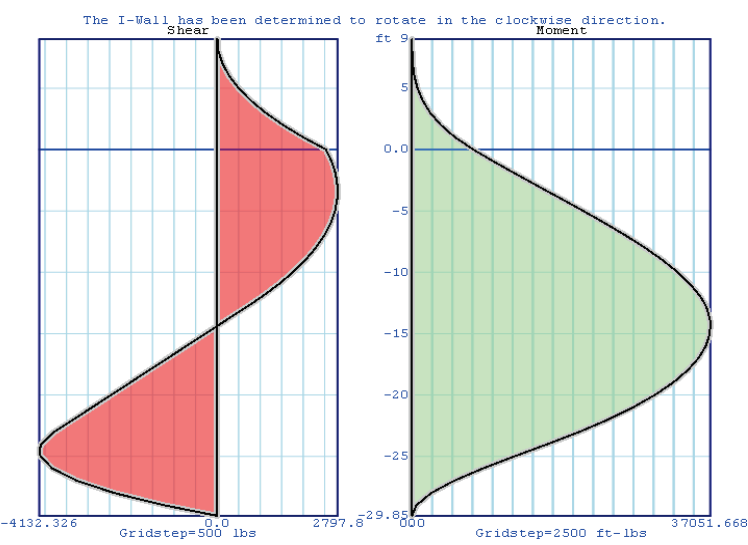

(b)

1 Thus, only one mobilized net passive pressure value in Figure 4.894.94a is used to construct Figure 4.894.94b net pressure diagram; corresponding to the sheet-pile tip elevation at el $-29.85 \mathrm{ft}$. The majority of the net pressure diagram is derived from Figure 4.894.94a net active pressure diagram. 
Corps_I-Wall designs were further performed for different material properties of $S_{u}$ and $C_{a}$. All the various example problems and final results have been recorded as check problems 1 through 5 in Table 4.15

Table 4.15. Comparisons of I-Wall results contained within Pace (2012).

\begin{tabular}{|c|c|c|c|c|c|c|c|c|}
\hline \multirow{2}{*}{$\begin{array}{l}\text { Check } \\
\text { Problem }\end{array}$} & \multirow{2}{*}{$\begin{array}{l}S_{u} \\
(p s f)\end{array}$} & \multirow{2}{*}{$\begin{array}{l}\mathrm{Ca}_{a} \\
\text { (psf) }\end{array}$} & \multicolumn{2}{|c|}{$\begin{array}{l}\text { Point of Rotation EL } \\
\text { (ft) }\end{array}$} & \multicolumn{2}{|c|}{$\begin{array}{l}\text { Gap Depth } \\
\text { (ft) }\end{array}$} & \multicolumn{2}{|c|}{$\begin{array}{l}\text { Depth of Embedment } \\
(\mathrm{ft})\end{array}$} \\
\hline & & & Reference & Corps_I-Wall & Reference & Corps_I-Wall & Reference & Corps_I-Wall \\
\hline 1 & 200 & 0 & - & -76.32 & 8.4 & 8.4 & No solution & 83.10 \\
\hline 2 & 300 & 0 & - & -23.07 & 12.6 & 12.61 & 29.85 & 29.85 \\
\hline 3 & 300 & 240 & - & -16.31 & 16.91 & 16.31 & No solution & 22.20 \\
\hline 4 & 300 & 15 & - & -22.21 & 12.92 & 12.92 & 28.98 & 28.96 \\
\hline 5 & 400 & 0 & - & -16.38 & 16.8 & 16.38 & No solution & 22.33 \\
\hline
\end{tabular}

Reference is Pace et al. (2012) Complete Soil-Structure Interaction (SSI) Analyses of I-Walls embedded in level ground during flood loading, Table 4.3. Corps_I-Wall has an active Factor of Safety of 1.0 and a passive Factor of Safety of 1.5. $\gamma_{\text {moist }}$ and $\gamma_{\text {sat }}$ are the moist and saturated unit weights of the soil, respectively, with both given the same value of $110 \mathrm{pcf} . \gamma^{\prime}$ is equal to the buoyant unit weight of the soil. Point of rotation elevations were not recorded for CWALSHT results. All problems are in English units.

Check problem 2 was previously evaluated as Example 4.6.7 with depth of embedment of the sheet-pile wall equal to $29.85 \mathrm{ft}$ and a gap depth of $12.61 \mathrm{ft}$. Both analyses had similar results. Check problem 4 was also evaluated with similar results for the two analyses. As shown on Table 4.15, CWALSHT solutions were not possible for check problems 1, 3, and 5 .

Corps_I-Wall produced results for these problems with the sheet-pile wall rotating in a clockwise direction. For check problem 3, Corps_I-Wall produced a 16.31-ft gap depth that is less than the CWALSHT computed value of $16.91 \mathrm{ft}$. This gap depth at $16.31 \mathrm{ft}$ was set to the point of rotation elevation of $-16.31 \mathrm{ft}$. This situation will occur in a Corps_I-Wall analysis when the gap depth progresses to the point of rotation (see Appendix B for more details). Below the point of rotation, higher than active earth pressures will develop and this type of response has been seen to arrest the gap in Corps_I-Wall analyses as well as in complete SSI analyses (Pace et al. 2012). Corps_I-Wall hydraulic fracture calculations for check problem 5 also produced a gap depth that was less than calculated because the gap depth extended below the point of rotation.

Check problem 2 showed a depth of embedment of $29.85 \mathrm{ft}$ with $S_{u}=$ $300 \mathrm{psf}$ and an adhesion of $C_{a}=0$. For check problem $1\left(S_{u}=200 \mathrm{psf}\right)$, the wall depth increased to $83.10 \mathrm{ft}$ and for check problem 5 ( $S_{u}=400 \mathrm{psf}$ ), the wall depth decreased to $22.33 \mathrm{ft}$. The undrained shear strength affects the depth of sheet-pile embedment. Inclusion of a small amount of adhe- 
sion of $C_{a}=15 \mathrm{psf}$ resulted in a reduction in the calculated depth of embedment, from $29.85 \mathrm{ft}$ (check problem 2) to $28.96 \mathrm{ft}$ (check problem 4). Check problem 3 incorporates a larger amount of adhesion $\left(C_{a}=\right.$ $0.8 * \mathrm{c}=240 \mathrm{psf}$ ), which results in a deeper gap depth as compared with the no adhesion condition of check problem 2: $16.31 \mathrm{ft}$ versus $12.61 \mathrm{ft}$. A change in the specified $C_{a}$ value from o to $240 \mathrm{psf}$ results in a calculated Corps-I-Wall depth of embedment of $22.2 \mathrm{ft}$ versus $29.85 \mathrm{ft}$, a 7.65 - $\mathrm{ft}$ shallower sheet-pile tip.

The suite of five analyses summarized in Table 4.15 verifies Corps_I_Wall software and demonstrate the robustness of its engineering solution process.

\subsection{Example 4.7 Analysis mode of operation for a clay site, an extension of Example 4.3}

A total stress definition with the angle of internal friction of ( $\varphi$ ) of o deg and two material layers, where the upper clay layer possesses an undrained shear strength (c) of 300 psf and the lower clay layer has $\mathrm{c}=400 \mathrm{psf}$. This I-Wall system has an exposed height of $10 \mathrm{ft}$. The water surfaces on both sides of the I-Wall are at $10 \mathrm{ft}$ on the flood side and $\mathrm{oft}$ on the landside. The flood side corresponds to the LHS of the I-Wall. In this scenario, the I-Wall will rotate clockwise during flood loading. The clay layers have a level ground surface on both sides of the I-Wall at el $0.0 \mathrm{ft}$ with saturated unit weight of $122 \mathrm{pcf}$ and at el $-10 \mathrm{ft}$ with saturated unit weight of $124 \mathrm{pcf}$. A user-specified sheet-pile tip elevation of $-28.27 \mathrm{ft}$ has been assigned for this analysis of the design problem of Example 4.3.

Figure 4.96 outlines the basic geometry and structural parameters for the sheet-pile wall. The top row of text documents the input values of all parameters associated with each material type. For this problem, two materials are assigned and listed as Mo1 and Mo2; the "M" signifies the material type and is followed by the ID, which starts from "01." All information concerning material types are written in black text. The representation of a portion of a layer of soil on each side of the I-Wall is characterized as a region. Two soil regions are required to represent each soil layer. Regions are labeled with an " $R$ " and followed by an ID starting from "01." For this problem four regions are assigned, Region 1 and Region 2 (as Ro1 and R02), and are located on the LHS of the sheet-pile wall. Region 3 and Region 4 (as Ro3 and Ro4) are located on the RHS of the sheet-pile wall. Each region and its surface elevation are written in red text. 
Surface water on either side of the sheet-pile wall are drawn with the water symbol at its defined elevation and labeled as "Left Water Surface" and "Right Water Surface" at the top of the wall in light blue text. Piezometric surfaces are labeled as "P" and followed by an ID starting from 01. Piezometric water surfaces are listed in the upper left-hand corner and drawn with the water symbol at its defined elevation and shown in blue. Observe that piezometric surface ID, Po1, is assigned to all four regions. The LHS piezometric surface elevation is assigned as the LHS surface water elevation. Similarly, the RHS piezometric surface elevation is assigned as the RHS surface water elevation. This allows for the assignment of submerged unit weights to the four soil regions.

Figure 4.96. Corps_I-Wall schematic of sheet-pile wall in clay site.

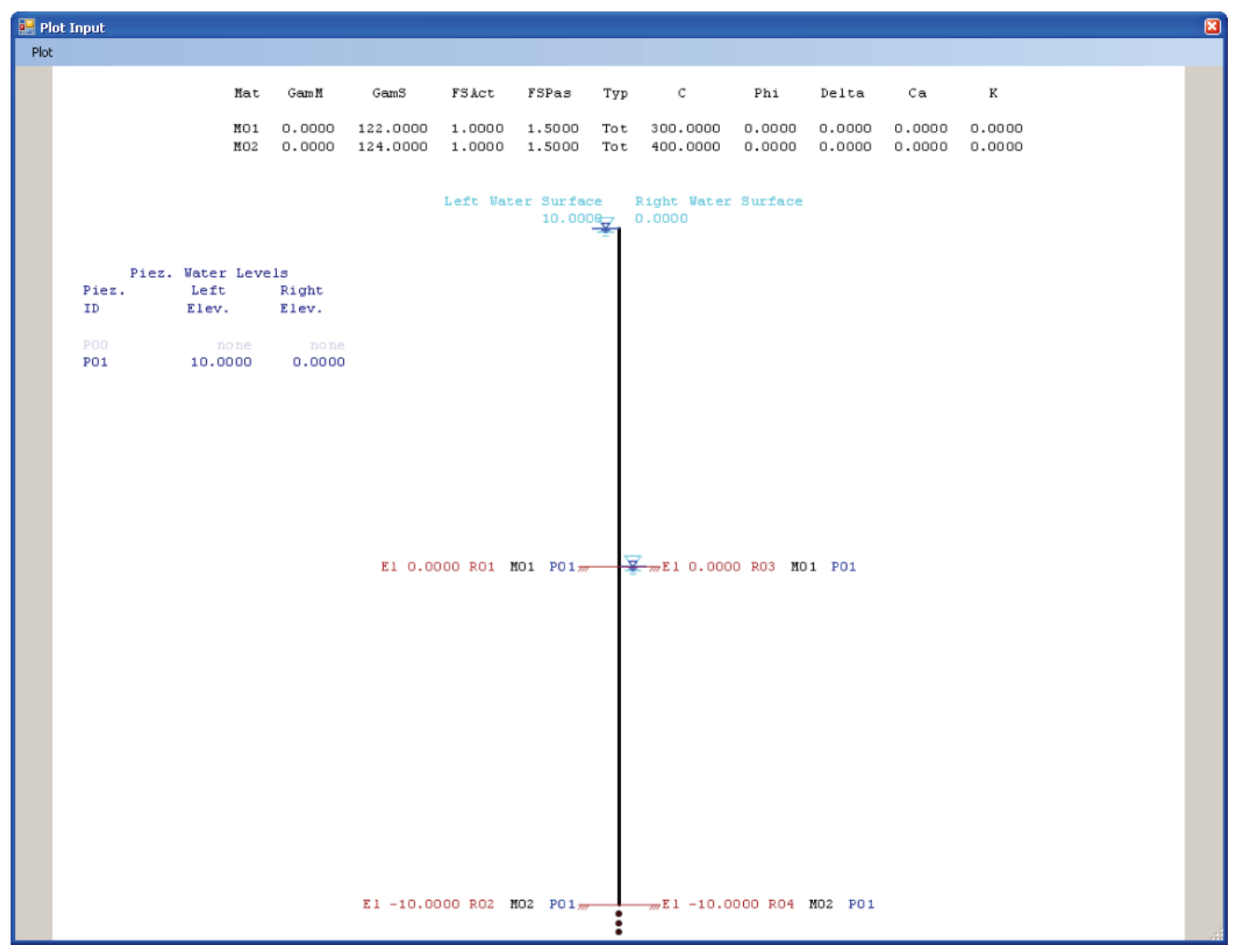

A clay site using a total stress definition with $\varphi=0$ as illustrated in Figure 4.96 is considered for the "Analysis" process where the passive Factor of Safety is successively adjusted until the calculated sheet-pile tip is approximately equal to the user-specified input within a set tolerance. Hydrostatic water pressures, as well as the Coulomb coefficient method of analysis and hydraulic fracturing, are specified in this example. 
This $\varphi=0$ analysis resulted in a computed mobilized active earth pressure coefficient $\left(K_{a}\right)$ of 1.0 and a mobilized passive pressure coefficient $\left(K_{p}\right)$ of 1.o. Both the active and passive earth pressures for both sides of the sheetpile wall are illustrated in Figure 4.97. The hydraulic fracturing procedure discussed in Appendix B estimates a gap depth of $13.3117 \mathrm{ft}$. Active earth pressures only develop at the gap depth or where the fracturing terminates; this is shown by the LHS active earth pressure diagram of Figure 4.97a. Note the change in earth pressures at el -10 ft: at the interface of Regions 1 and 2 of the passive earth pressure of Figure 4.97a and also at the interface of Regions 3 and 4 of Figure 4.97b. Please refer to Chapter 2 for a detailed description of multilayered soils. The hydrostatic water pressures acting on both sides of the sheet-pile wall are shown in Figure 4.98a.

Figure 4.97. Active and passive earth pressures for undrained shear strength. (a) Left-hand side of wall, (b) Right-hand side of wall.

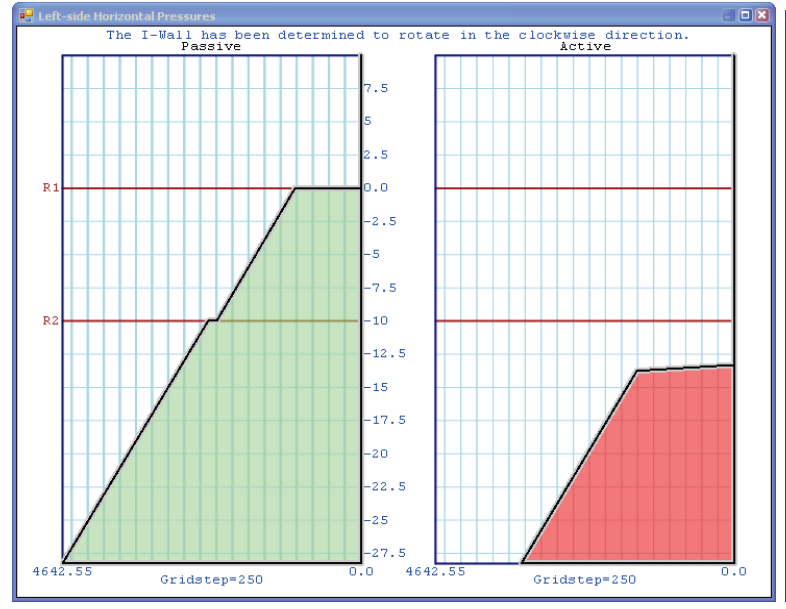

(a)

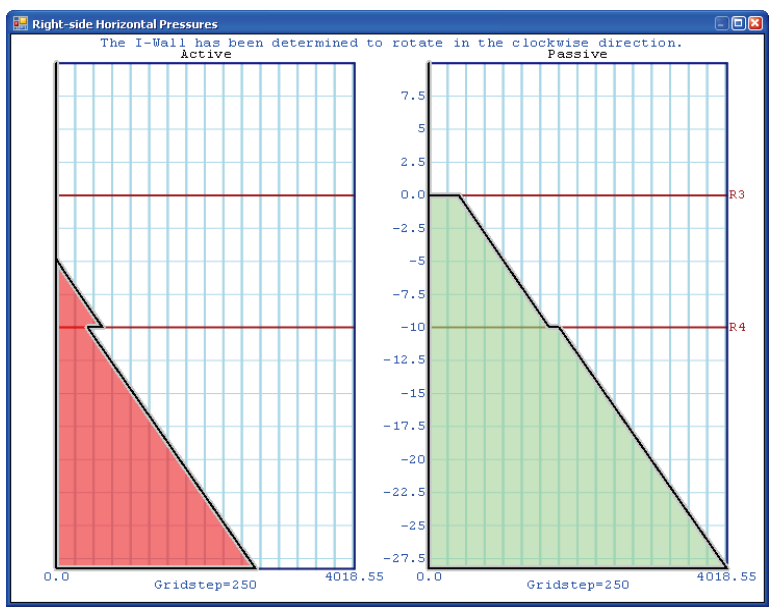

(b)

Figure 4.98. Pore water pressures. (a) Left- and right-hand side of wall. (b) Net water pressures.

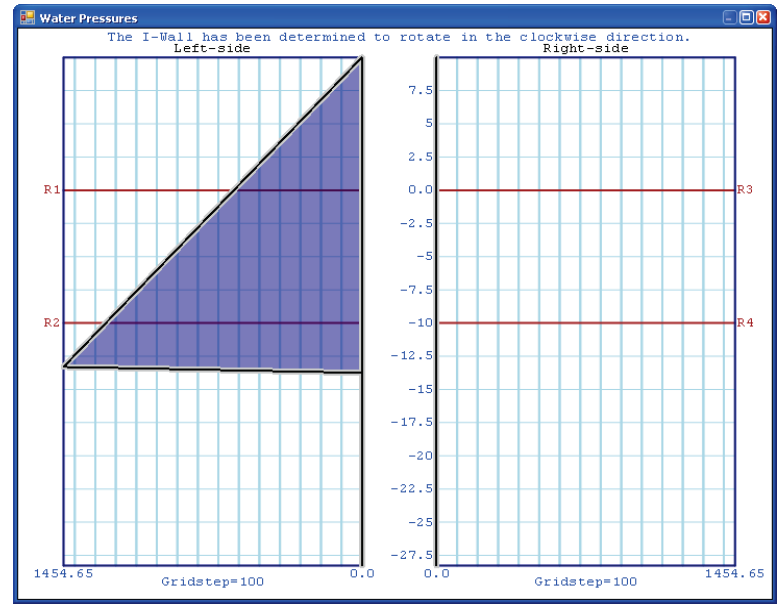

(a)

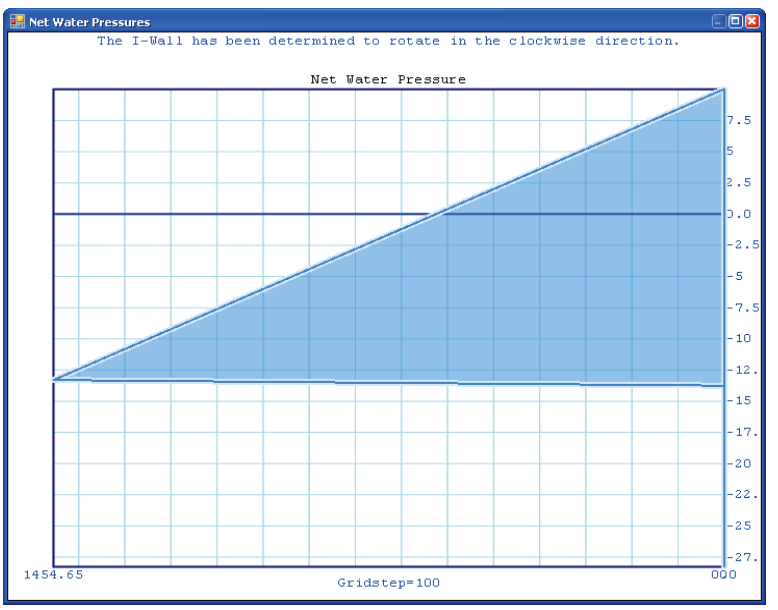

(b) 
The resultant net water pressure calculated by taking the difference between the water pressures acting on both sides of the wall (Figure 4.98a) is shown in Figure $4.98 \mathrm{~b}$. In a total stress analysis, only overburden water pressure is included in the analysis (i.e., there is no pore water pressures calculated within the soil below the gap on the LHS and below the ground surface on the RHS). Also note that boundary water pressures acting on the flood wall for this total stress clay site increase with the propagation of the fracture down to the gap depth.

From the calculated pressures of Figures 4.97 and 4.98, the net active and net passive pressures are constructed and presented in Figure 4.99a. For the prescribed LHS flood loading, the I-Wall will rotate in a clockwise direction. The net active pressure is determined from the difference between the LHS-mobilized active earth pressure and the RHS-mobilized passive earth pressure (i.e., acting on the other side of the wall) with the addition of the net water pressures and any external horizontal net pressures and overburden pressures (if any). The net passive pressure is determined by the difference between the LHS-mobilized passive earth pressure and the RHS-mobilized active earth pressure acting on the other side of the sheet-pile wall with the addition of any net water pressures and any external horizontal net pressures and overburden pressures (if any). The calculated net active and net passive pressures above the ground surface el 0.0 (and above Ro1 and Ro3) of Figure 4.99 are solely derived from the net water pressures of Figure 4.98b.

Figure 4.99. Net pressures. (a) Net active and net passive. (b) Net pressure diagram.

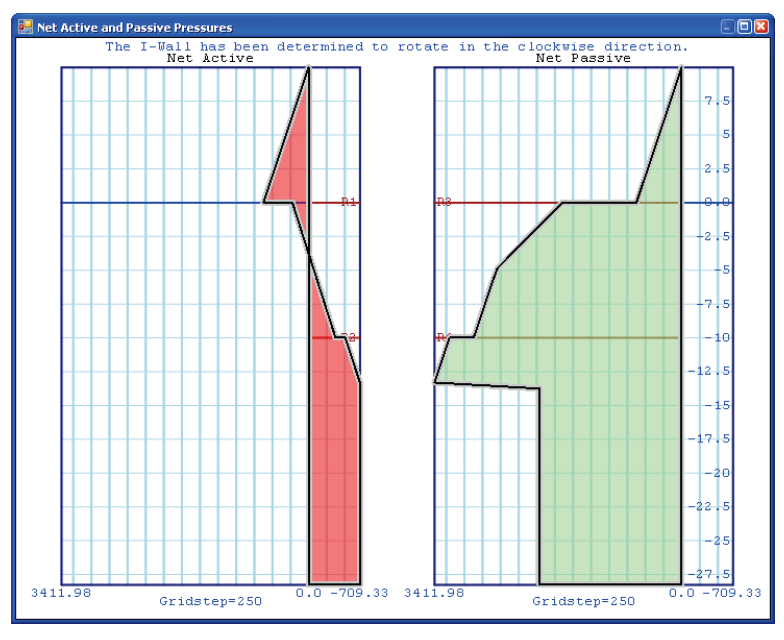

(a)

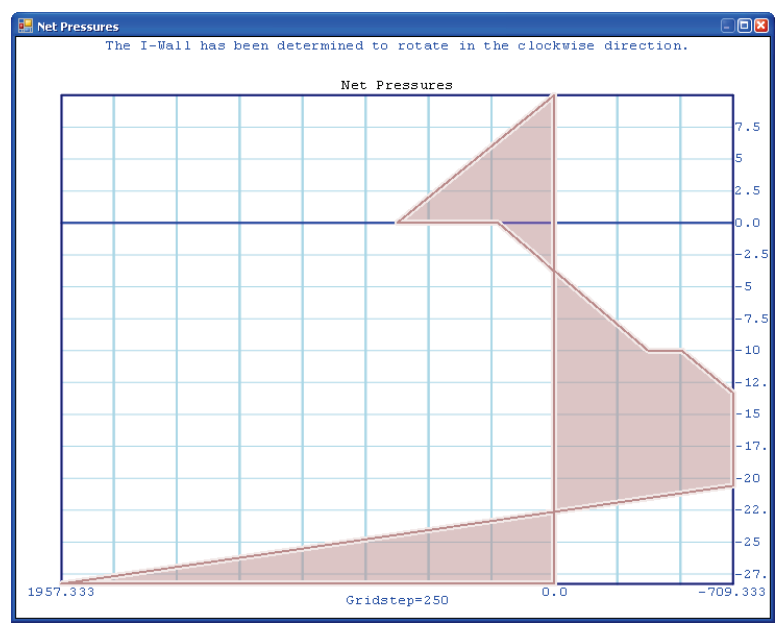

(b) 
The net pressure diagram of Figure 4.99b is derived from the replication of all values of the net active pressures, from the top of the wall until the elevation at the point of rotation (at el -20.59 ft), at which instant the remaining values of the net pressure diagram are assumed to be linear with elevation between (1) the net active pressure value at the elevation of the point of rotation and (2) the net mobilized passive pressure at the elevation of the approximated sheet-pile tip (i.e., el -28.27). ${ }^{1}$ Figure $4.99 \mathrm{~b}$ is an illustration of how the net pressure diagram was formed. Because the I-Wall has been determined to rotate in a clockwise direction, the net pressure diagram of Figure 4.99b shows that the upper zone of net active pressures plot on the LHS and the lower zone plot on the RHS. The net passive pressures plot on the LHS of the sheet-pile wall. The final results are illustrated in Figure 4.100 by the presentation of the net pressure diagram Figures 4.100a (replica of Figure 4.99b) and 4.100b, the shear and moment diagrams. Figure $4.100 \mathrm{~b}$ shows that the maximum bending moment occurs at the zero crossing of the shear diagram.

Figure 4.100. (a) Net pressure diagram. (b) Shear and moment.

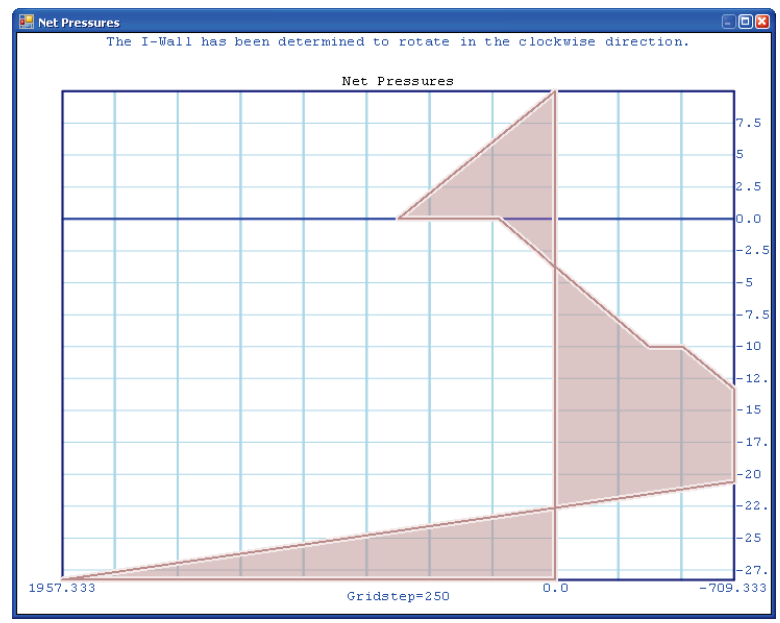

(a)

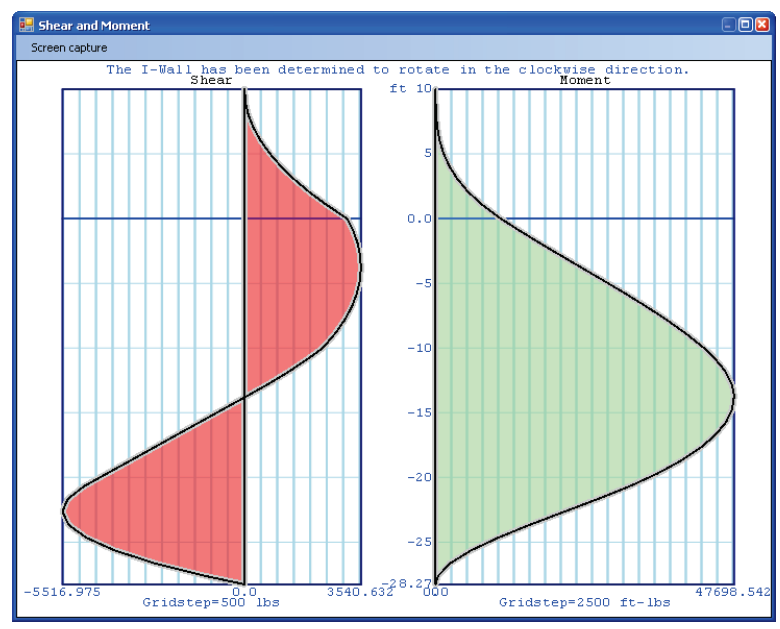

(b)

The sheet-pile tip elevation is computed to be at $-28.27 \mathrm{ft}$, which is equivalent to the value specified as input. For this analysis, the results produced a passive Factor of Safety of 1.5, which corresponds to the same passive Factor of Safety for this problem executed as the Example 4.3 design problem.

\footnotetext{
1 Only one mobilized net passive pressure value in Figure 4.99a is used to construct the Figure 4.99b net pressure diagram; corresponding to the sheet-pile tip elevation at el $-28.27 \mathrm{ft}$. The majority of the net pressure diagram is derived from the Figure 4.99a net active pressure diagram.
} 


\section{Probability Analysis and Example Problems}

\subsection{Fragility curve (i.e., system response curve)}

Within the Corps, the term system response curve (SRC) is now being introduced to describe what has been commonly referred to in the technical literature as the fragility curve; the term is also used for the hydrologic fragility assessment of rock-founded concrete gravity dams. This report uses this new Corps terminology. Tekie and Ellingwood (2002) discuss the development of fragility curves used in a hydrologic fragility assessment for gravity dams. The procedure for constructing an SRC for an I-Wall with a rotational limit state is analogous.

In safety or risk assessment of sheet-pile I-Walls, limit states or probability of failure serve as a measure of system performance. The system response curve is used to predict the probably of failure, given the hydraulic hazard. Consider the example of a sheet-pile I-Wall in which the rotational limit state (i.e., $F S_{\text {rotate }} \leq 1.0$ ) is the limit state resulting in failure of the I-Wall. Introducing the names for the variables used in this report into Equation 5.1 of Tekie and Ellingwood (2002), the probability of failure, $P_{\text {failure, }}$ is given by the expression

$P_{\text {failure }}=\sum_{H_{\text {Flood }}}\left[P\left(F S_{\text {rotate }} \leq 1.0 \mid\right.\right.$ Flood $\left.=H_{\text {Flood }}\right) \cdot P\left(\right.$ Flood $\left.\left.=H_{\text {Flood }}\right)\right]$

in which Flood is a vector of random variables describing the intensity of demand (e.g., flood elevation) and other factors; $P\left(\right.$ Flood $\left.=H_{\text {Flood }}\right)$ is the hazard, which considers river floods that overtop the I-Wall or from a coastal surge and is expressed in terms of annual probability; and $P\left(F S_{\text {slide }}\right.$ $\leq 1.0 \mid$ Flood $=H_{\text {Flood }}$ ) is the conditional probability of I-Wall rotational structural failure, given that Flood $=H_{F l o o d}$, as obtained for the Figure 5.1 system response curve developed using the engineering procedure and corresponding PC-based software discussed in this report. Expressing the limit state probability as in Equation 5.1 allows the overall risk to be deconstructed into its significant contributors. 
Figure 5.1. System response curve for rotational limit state.

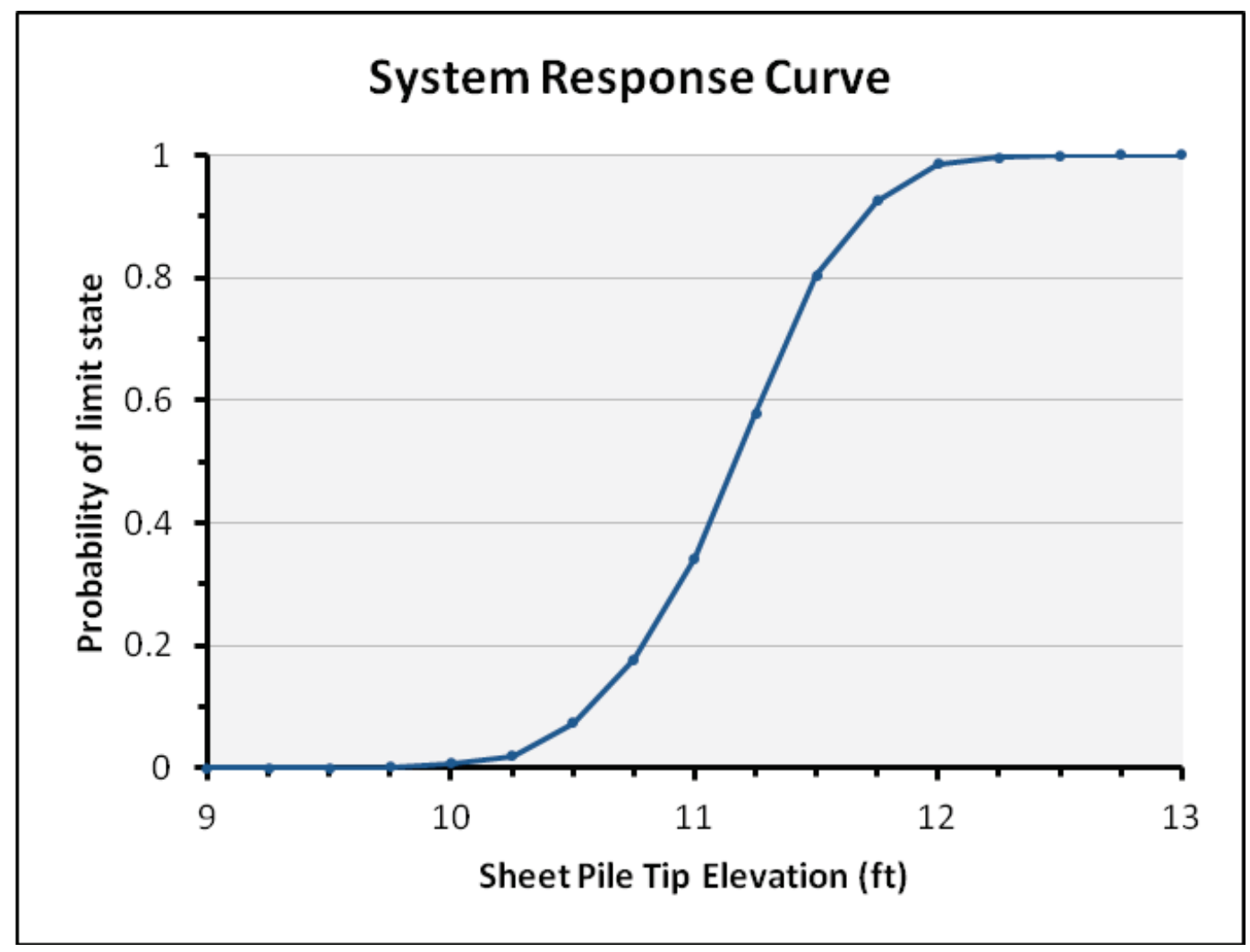

In Chapter 6 of Tekie and Ellingwood (2002), they observed that a fully coupled probabilistic safety assessment combines the probabilistic/ statistical definitions of hazard (i.e., demand) and system response curve (i.e., capacity) and leads to a point or interval estimate of failure state probability for the limit state associated with structure failure, depending on how the epistemic uncertainties are incorporated in the analysis. The hazard is described by the complementary cumulative distribution function (CCDF) and the system response curve is defined by the cumulative distribution function (CDF). Tekie and Ellingwood observed that the probability of failure can be expressed as the convolution of the hazard function and the system response curve in their Equation 6.1, an integral form of Equation 5.1. Additionally, modeling (epistemic) uncertainties propagated through the analysis of the hazard and fragility will give rise to a distribution for the probability of failure, $P_{\text {failure }}$, as depicted in Figure 6.1 in Tekie and Ellingwood (2002). Therefore, if the mean hazard and mean system response curve are used, they observed that the convolution integral (their Equation 6.1) yields a point estimate for the limit state probability. Citing previous research published by Ellingwood, they conclude that this point estimate does not necessarily yield the mean of $P_{\text {failure }}$, as the uncertainties 
are not propagated in the same way but this estimate is usually close to the mean of $P_{\text {failure. }}$

\subsection{Random variables for each soil layer ( $\varphi, c, \delta, C a)$ and correlation between variables}

The shear strength parameters of soil are derived from the Mohr-Coulomb relationship between cohesion (c) and the angle of internal friction $(\varphi)$. These two variables, as well as the soil adhesion (Ca) and the angle of soilwall interface friction $(\delta)$, have been determined to behave inherently random and are, therefore, considered as uncertain variables in this report. These variables are represented by their mean $(\mu)$ and standard deviation ( $\sigma$ ) and representative coefficient of variation (COV), along with their correlation $(\rho)$ relationship.

Correlation measures the relationship between two variables. Two variables are positively correlated when one variable increases as the other increases or one variable decreases as the other decreases. They are inversely correlated; as one variable increases, the other decreases or conversely when one variable decreases, the other increases. The value for the correlation $\rho$ between two variables ranges from -1.0 to 1.0. A $\rho$ value set equal to o implies that the variables have no correlation, but it does not mean that they are independent from each other. However, independent variables possess zero correlation. Considering Mohr-Coulomb shear strength parameters $\{c, \varphi\}$, a strong correlation is represented by values ranging from ( \pm 0.7 to \pm 0.99 ), moderately correlated values range from $( \pm 0.7$ to \pm 0.3$)$ and values closer to 0.0 represent weak correlations. These correlation strength definitions have been used only as guidelines for this report.

When non-zero correlations are specified between two random variables, a rank correlation method is used by the software to determine the correlation between the two variables, which results in a rank correlation matrix. For n-variables, a symmetric n-by-n correlation matrix will be applied. Correlation between variables should be specified for every pair of random variables. When more than two random variables are defined and one variable is correlated to two or more variables, there could be the possibility of the introduction of an adjusted rank correlation matrix. This adjusted correlation matrix will contain correlation coefficients that have been modified from the original matrix for numerical stability. 
Table 5.1 represents the correlation relationship in some examples contained within this report between $\mathrm{c}$ and $\varphi$ as being inversely correlated, between $\mathrm{c}$ and $\mathrm{Ca}$ as positively correlated, and between $\varphi$ and $\delta$ as also positively correlated. Correlations can also be assigned among pairs of variables from different soil layers. It is anticipated that actual project data will be used to establish the statistics for each of the variables and the correlation between variables. From these statistical properties, each variable can be characterized by either a normal, log-normal or uniform probability distribution function, and the variables generated for the number of simulations requested will vary between $o$ and $\mu+3 \sigma$. The lower limit of a value of zero is specified in the distribution so as to eliminate the possibility of negative values being generated for c, $\varphi, \delta$, or Ca variables during Latin hypercube simulations. These variables can also be represented with distributions that allow user defined limits. The bounded normal, bounded log-normal and triangular functions require lower and upper bounds in addition to the aforementioned statistical parameters of $\mu, \sigma$, and COV.

Table 5.1. Correlations used among variables in some example problems.

\begin{tabular}{|c|c|c|c|c|}
\hline & Cohesion & $\begin{array}{l}\text { Angle of } \\
\text { Internal } \\
\text { Friction }\end{array}$ & $\begin{array}{l}\text { Soil-to-Sheet-pile Wall } \\
\text { Interface Friction Angle }\end{array}$ & Adhesion \\
\hline & (c) & $(\varphi)$ & (ठ) & (Ca) \\
\hline \multirow{3}{*}{$\begin{array}{l}\text { Correlation } \\
\text { between } \\
\text { two variables }\end{array}$} & $x$ & & & $x$ \\
\hline & & $x$ & $x$ & \\
\hline & $-x$ & $-x$ & & \\
\hline
\end{tabular}

Note: $x$ signifies that the variables are positively correlated, and $-x$ signifies the variables are inversely correlated

Total stress (c, $\varphi, \delta$, or $\mathrm{Ca})$ or effective stress $\left(\mathrm{c}^{\prime}, \varphi^{\prime}, \delta^{\prime}\right.$, or $\left.\mathrm{Ca}^{\prime}\right)$ based shear strength parameters may be used in the analyses.

As discussed in Cherubini (2000); for drained tests, Harr (1987) indicates correlation between effective cohesion $\mathrm{c}^{\prime}$ and friction angle $\varphi^{\prime}$. In particular, the results of Wolff (1985) (correlation coefficient $\rho=-0.47$ ); Yucemen et al. (1973) (-0.24 $\leq \rho \geq-0.49)$; and Lumb (1970) (-0.37 $\leq \rho \geq$ -0.70) are cited. Cherubini (1997) found a value of $\rho=-0.61$ for drained triaxial tests on Blue Matera clays. 
According to Cherubini (2000), Table 5.2 shows the range of most frequent values of the coefficient of variation of some geotechnical soil properties (Becker 1996; Cherubini 1997).

Table 5.2. Ranges of the most frequent values of coefficients of variation.

\begin{tabular}{|l|l|}
\hline Unit Weight & $\begin{array}{l}\text { Range (\%) } \\
\mathbf{3}-\mathbf{1 0}\end{array}$ \\
\hline Effective friction angle $\varphi^{\prime}$ & \\
\hline Clay & $10-50$ \\
\hline Silt & $5-25$ \\
\hline Sand & $5-15$ \\
\hline Effective cohesion c' & $10-70$ \\
\hline
\end{tabular}

\subsection{Finding the system response curve for an analysis of an existing I-Wall}

The analysis of an I-Wall considers the estimation of the passive Factor of Safety of an existing wall. A probabilistic assessment for the passive Factor of Safety of less than or equal to 1 for a predetermined number of simulations and specified flood elevation is used to define a point on the system response curve. It is recommended to initially experiment with 100 simulations. After completion of analyzing the 100 simulations at the various flood elevations, the system response curve should be found.

An important feature on locating the system response curve is to establish flood elevations that will cover the region that contains the system response curve, as shown in Figure 5.2c. If flood elevations are defined too low at the I-Wall, the result would cause an underestimation of the system response curve and completely miss the curve as in Figure 5.2a. This similar result could occur for an overestimation of higher flood elevations that are close to or above the top of the wall, as in Figure 5.2b. Vertical red arrows of Figure 5.2 indicate flood elevations. When the system response curve requires only a few flood elevations to define its shape (e.g., narrow at 1 -ft increments), it might be necessary to decrease flood elevations by 0.5 - $\mathrm{ft}$ or 0.25 - $\mathrm{ft}$ increments, which increases the number of flood elevations to better visualize the entire region. 
Figure 5.2. System response curve. (a) underestimated flood elevations for SRC, (b) overestimated flood elevations for SRC, (c) located region of SRC curve.

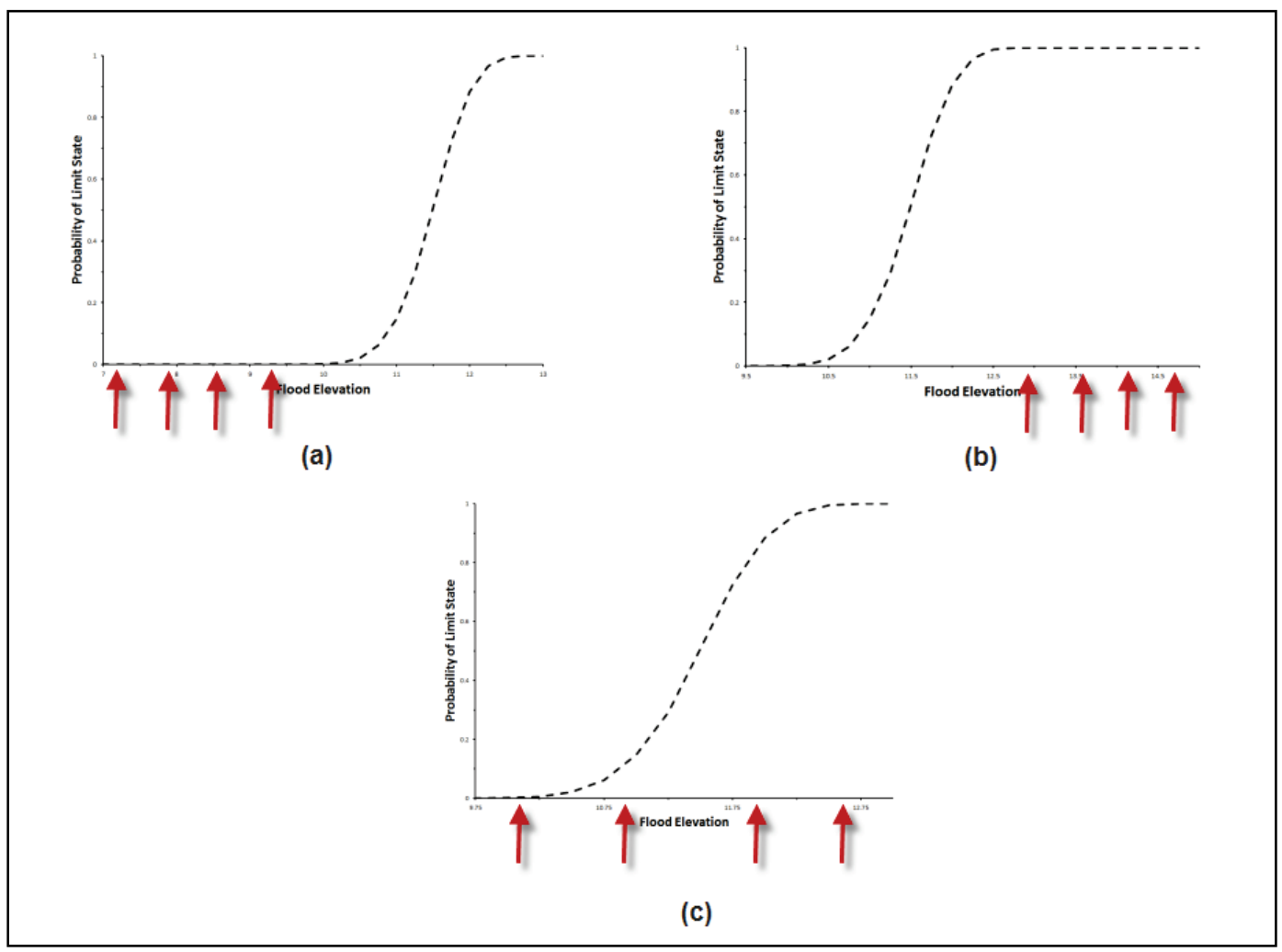

\subsection{Accuracy and timing}

Accuracy and timing influence the frequent decision reached as to the number of simulations required for a probabilistic analysis that results in the construction of a cumulative distribution function or system response curve. Initially, from numerous observations of simulating either a new design of a sheet-pile wall or the analysis of an existing I-Wall (Sections 5.5 through 5.7); the number of simulations applied was established to be around 1, ooo simulations while retaining the most accuracy. However, in some of these cases, the resulting execution times were excessively lengthy and considered inadequate. Further investigation provided insight necessary to decrease execution times. It has been determined that the most timeconsuming executions resulted from the probabilistic analysis of an existing I-Wall with effective stress-based earth pressure calculations and with seepage. Various scenarios were examined for the improvement of execution times for the above conditions: (1) a consistent relationship between the number of simulations and the execution time. When the number of simulations was decreased, the execution time decreased. Therefore, many simulations were studied that would produce the best 
acceptable accuracy while minimizing the execution time; (2) the sheet- pile base elevation. The designated sheet-pile base elevation is initially set at $-125 \mathrm{ft}$. All estimated pressures and resultant forces are calculated from the top of the wall down to the sheet-pile tip elevation by an iterative solution that approximates the depth of embedment. It was decided that decreasing the initial sheet-pile base elevation (about one half and depending on soil classifications) would lower execution times by reducing the number of computations along the shorter wall; (3) change the minimum node spacing. The computational procedure, as discussed in Appendix A, determines pressures and forces estimated from nodal points along the wall. If numerical convergence is not achieved with the initial nodal point spacing (set to $1 \mathrm{ft}$ ), the node spacing will be decreased by the node spacing interval, and this process is repeated until the minimum node spacing is reached and the program stops. Decreasing the minimum node spacing (to 0.8 or 0.9) most often allows the program to stop computations when $F S_{\text {passive }}$ is well below 1.o.

The following three sections describe results and show the minimum number of simulations selected for each example problem. Section 5.5 describes the design of an I-Wall with effective stress-base shear strength parameters, seepage and hydraulic fracturing. Section 5.6 describes the analysis of an I-Wall with total stress-based shear strength parameters, hydrostatic water pressures and hydraulic fracturing. Section 5.7 also describes the analysis of an I-Wall, but with effective stress based shear strength parameters, seepage, and hydraulic fracturing. These two analysis sections each develop a system response curve.

\subsection{Example 5.1 sand site with hydraulic fracturing criteria and seepage, an extension of design problem 4.1.5}

An effective stress definition of an I-Wall founded in sand with $\mathrm{c}^{\prime}=0$ is illustrated by a design problem in order to determine the depth of embedment (d) of the sheet-pile wall. This example problem applies an active Factor of Safety of 1.0 for computing active earth pressures and a passive safety factor of 1.5 for computing passive earth pressures of all soil layers. This I-Wall system has an exposed height of $10 \mathrm{ft}$. Water surfaces at both sides of the I-Wall are at $40 \mathrm{ft}$ on the flood side or RHS and $34 \mathrm{ft}$ on the landside or LHS. The homogeneous soil layer of sand has a level ground surface on both sides of the I-Wall at $30 \mathrm{ft}$ with saturated unit weight of $122.4 \mathrm{pcf}$, the effective angle of internal friction of $30 \mathrm{deg}$ and the effective angle of soil-to-sheet-pile wall interface friction of $15 \mathrm{deg}$. This 
analysis also considers homogeneous seepage. Figure 5.3 outlines the basic geometry and structural parameters for the sheet-pile wall.

The top row of text listed in Figure 5.3 documents the input values of all parameters associated with each material type. For this problem, there is only one material assigned for the sand site and recorded as "Mo1," the "M" signifies the material type and is followed by the ID, which starts from "01." All information concerning material types are written in black text. The representation of a soil structure is characterized by a region. Regions are labeled with an "R" and followed by an ID starting from "01." For this problem two regions are assigned; Region 1 (as Ro1), is located on the LHS of the sheet-pile wall and Region 2 (as Ro2) is located on the RHS of the sheet-pile wall. Each region and its surface elevation are written in red text.

Figure 5.3. Corps_I-Wall schematic of sheet-pile wall in sand site.

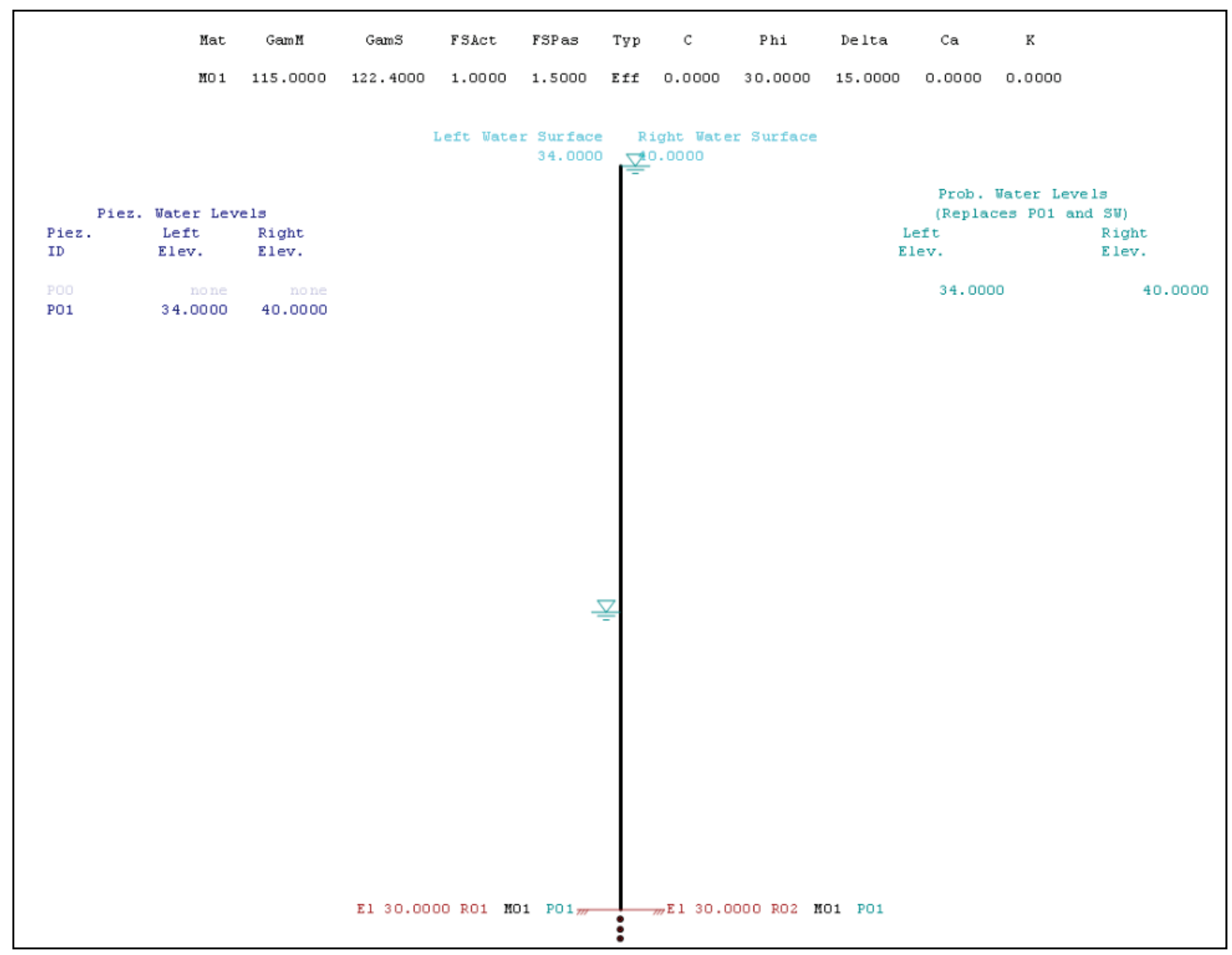


Water elevations are listed in the upper left-hand corner and shown in dark blue. Water levels are labeled at the soil interface of any specified region with an initial character "P" and followed by an ID. Label "Poo" is one that signifies that there is no water associated with a soil layer or region, and the soil is partially saturated. If all soil regions are fully saturated, "Poo" will be grayed out in Figure 5.3. Surface water on either side of the sheet-pile wall is represented by water symbols at its defined elevation along the sheet-pile wall and labeled as "P01." If there is no surface water associated with any soil region, "Po1" will be grayed out.

For the probabilistic analysis considered in this section, the mean of the effective angle of internal friction and the mean of the effective angle of wall-soil interface friction were defined previously as $30 \mathrm{deg}$ and $15 \mathrm{deg}$ respectively. The standard deviation, $\mathrm{COV}$, the correlation coefficient, and probability distribution function provided for these two variables are shown in Figure 5.4. Note that the two variables are strongly correlated with a correlation coefficient of 0.9. Only one flood elevation will be considered for the probabilistic analysis of the design depth of embedment. Upon completion, a histogram detailing the frequency of sheet-pile tip elevations together with its cumulative distribution at each flood level are reported.

Figure 5.4. Statistical parameters of sandy soil.

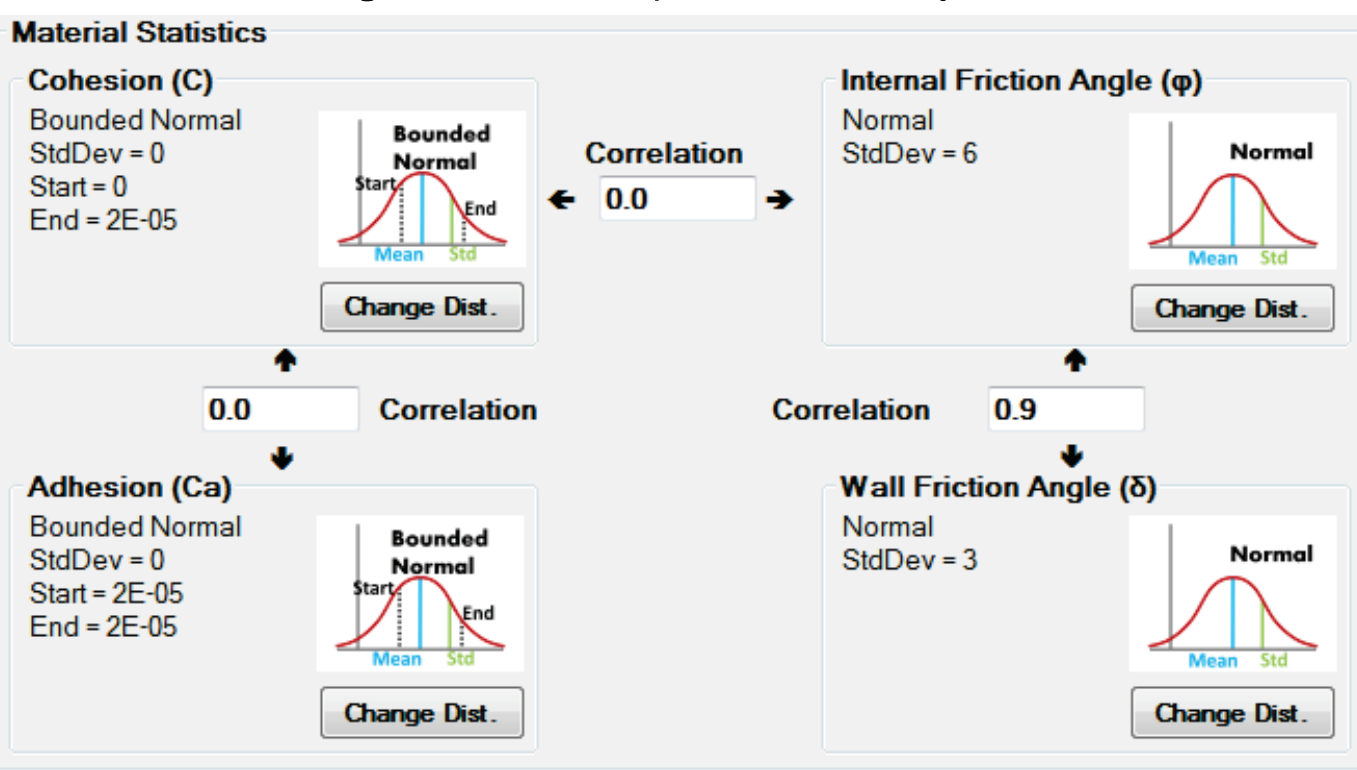




\subsubsection{Evaluation of results from the deterministic solution with flood elevation pairs of $40 \mathrm{ft}$ and $34 \mathrm{ft}$}

An effective stress definition with $\varphi^{\prime}=30 \mathrm{deg}, \delta^{\prime}=15 \mathrm{deg}$, and $\mathrm{c}^{\prime}=\mathrm{O}$ as illustrated in Figure 5.4 is considered for the evaluation of the depth of embedment. Homogeneous soil, homogeneous seepage by the line of seepage method, as well as the passive earth pressure coefficient obtained from the logarithmic spiral procedure, form the basis for this example.

At the flood elevation of $40 \mathrm{ft}$, a deterministic analysis is first evaluated to estimate the design depth of embedment and various output parameters. This analysis resulted in a mobilized active earth pressure coefficient $\left(K_{a}\right)$ of 0.3014 and a mobilized passive pressure coefficient $\left(K_{p}\right)$ of $2.7645 \cdot{ }^{1}$ The distribution of active and mobilized passive earth pressures for both sides of the sheet-pile wall is illustrated in Figures $5.5 \mathrm{a}$ and $5.5 \mathrm{~b}$.

Figure 5.5. Active and passive earth pressures. (a) left hand side of wall, (b) right hand side of wall.

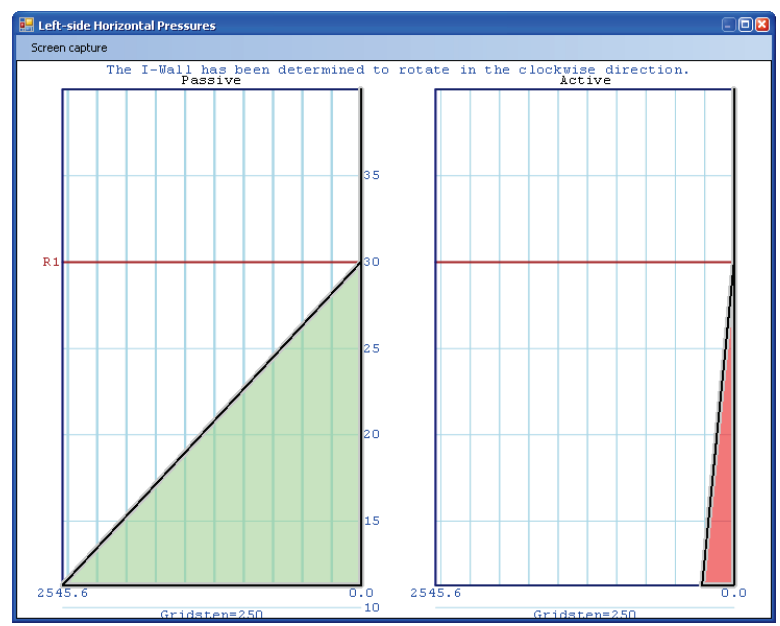

(a)

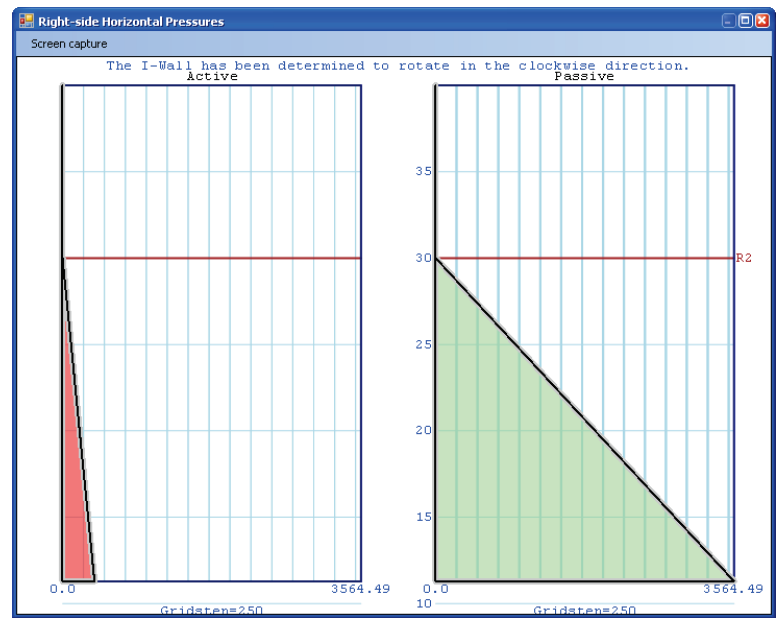

(b)

The resulting water pressures acting on both sides of the sheet-pile wall are shown in Figure 5.6a. The resultant net water pressure calculated by taking the difference between sum of the water pressures (Figure 5.6a) and seepage on both sides of the wall is shown in Figure 5.6b.

\footnotetext{
${ }^{1}$ Recall that an FS passive value equal to 1.5 was specified in this example, consistent with EM 1110-22504 (HQUSACE 1994).
} 
Figure 5.6. Pore water pressures. (a) left and right hand side of wall, (b) net water pressures.

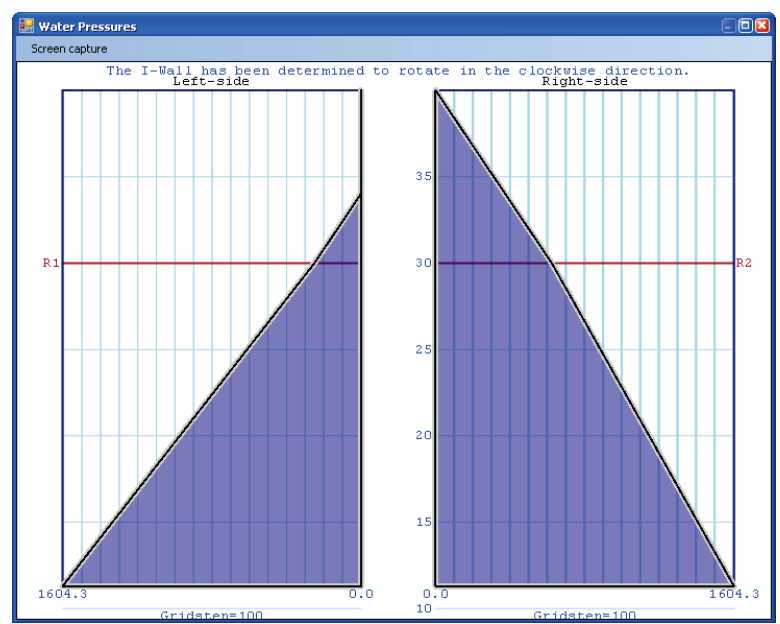

(a)

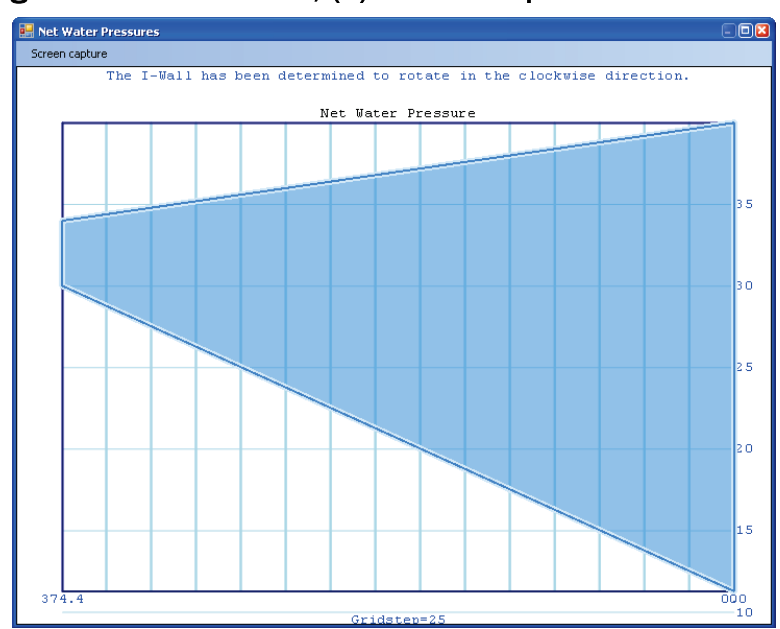

(b)

Homogeneous seepage occurring in this problem is clearly shown by the net water pressure diagram of Figure 5.6b. The net water pressure diagram of Figure 5.6b is first calculated from the RHS hydrostatic water pressures from el $40 \mathrm{ft}$ to el $34 \mathrm{ft}$, resulting in the uppermost triangular distribution. Between el $34 \mathrm{ft}$ and el $30 \mathrm{ft}$, the increase in water pressure on both sides of the wall is the same, resulting in a uniform net pressure diagram between these two elevations. At the ground surface elevation of $30 \mathrm{ft}$, which coincides with a gap depth elevation of $30 \mathrm{ft}$ (i.e., a zero gap depth because $c^{\prime}$ is equal to zero), homogeneous seepage calculations are initiated from RHS ground surface (on the high head side) down the RHS to the sheet-pile tip and then up the LHS of the sheet pile, concluding at the LHS ground surface. Corps_I-Wall line of seepage computations result in a net water pressure of 0.0 psf at the sheet-pile tip elevation of $11.29 \mathrm{ft}$.

From these pressures (Figures 5.5 and 5.6), both the net active and net passive pressures are constructed and presented in Figure 5.7a. For the applied flood loading, the I-Wall will rotate counterclockwise. The net active pressure is determined from the difference between the RHS- mobilized active earth pressure and the LHS-mobilized passive earth pressure (i.e., acting on the other side of the wall) with the addition of any net water pressures and any external horizontal net pressures and overburden pressures (if any). The net passive pressure is determined by the difference between the RHS-mobilized passive earth pressure and the LHS- mobilized active earth pressure acting on the other side of the sheet-pile wall with the addition of any net water pressures and any external horizontal net pressures and overburden pressures (if any). The net pressure diagram of 
Figure $5.7 \mathrm{~b}$ is derived from the replication of all values of the net active pressures, from the top of the wall until the elevation at the point of rotation (el $16.24 \mathrm{ft}$ ), at which instant the remaining values of the net pressure diagram is assumed to be linear with elevation between (1) the net active pressure value at the elevation of the point of rotation and (2) the net mobilized passive pressure at the elevation of the approximated sheet-pile tip (i.e., el 11.29). ${ }^{1}$ Figure $5.7 \mathrm{~b}$ is an illustration of how the net pressure diagram was formed. The final results are illustrated in Figure 5.8 by the presentation of the net pressure diagram Figures 5.8a (replica of Figure $5.7 \mathrm{~b}$ ) and $5.8 \mathrm{~b}$, the shear and moment diagrams. Figure $5.8 \mathrm{~b}$ shows that the maximum bending moment occurs at the zero crossing of the shear diagram. The sheet-pile tip elevation of the deterministic analysis is computed at $11.29 \mathrm{ft}$ with a depth of embedment of $18.71 \mathrm{ft}$.

Figure 5.7. Net pressures. (a) Net active and net passive. (b) Net pressure diagram.

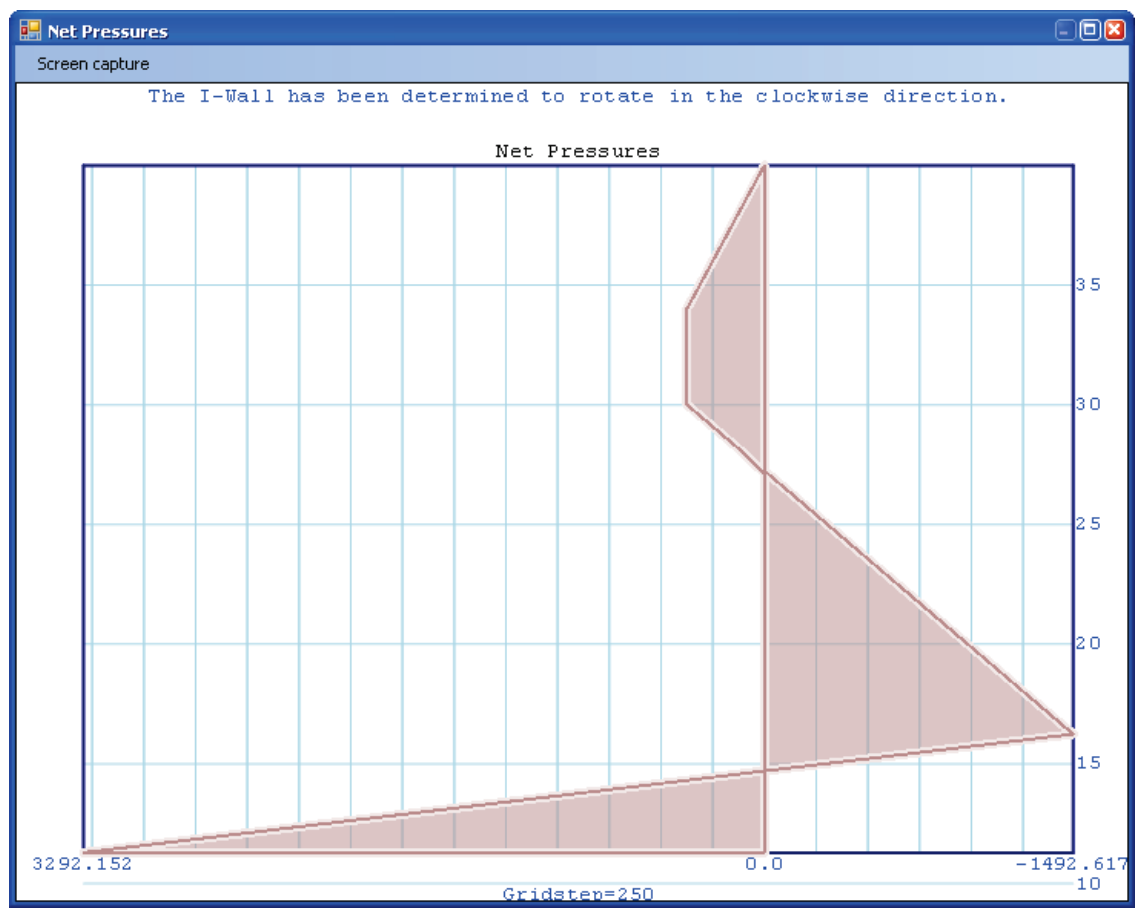

(a)

(b)

1 Only one mobilized net passive pressure value in Figure 5.7a is used to construct the Figure 5.7b net pressure diagram; corresponding to the sheet-pile tip elevation at el 11.29 . The majority of the net pressure diagram is derived from the Figure 5.7a net active pressure diagram. 
Figure 5.8. (a) Net pressure diagram. (b) Shear and moment.

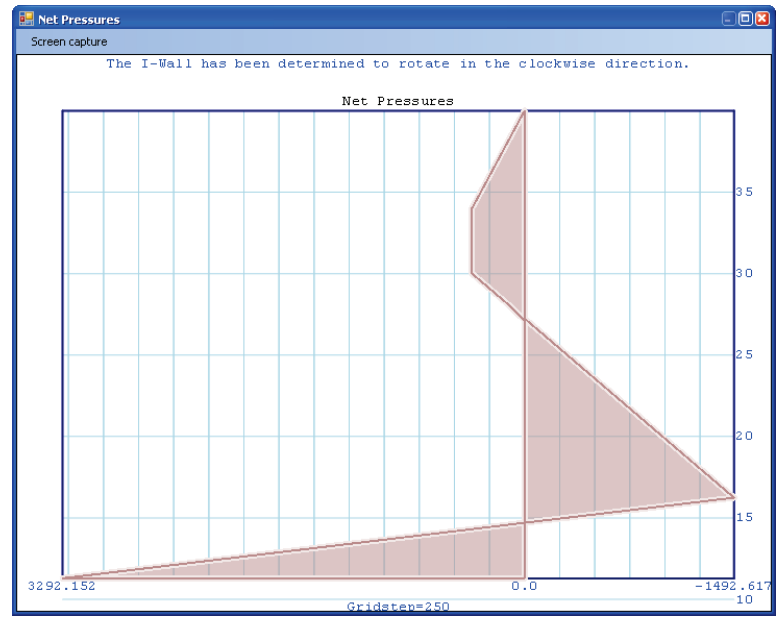

(a)

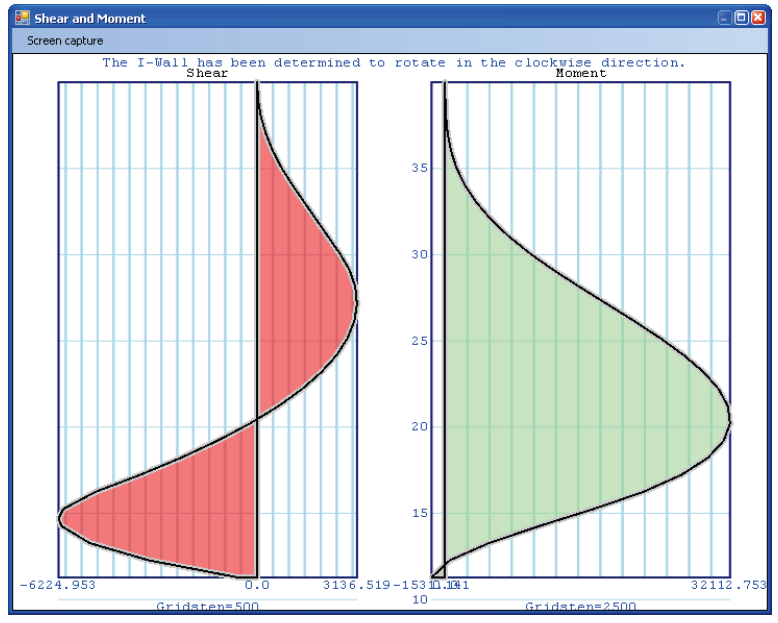

(b)

This problem required using homogeneous seepage in the analysis. An input hydraulic conductivity for sand (i.e., $3.28 \mathrm{e}-5 \mathrm{ft} / \mathrm{sec}$ ) was entered and the constant seepage gradient was calculated to be 0.1603 . Figure 5.9 provides an interpretation of the line of seepage method by a graph of the total head along the seepage path. The LHS graph or Figure 5.9a, shows a schematic of seepage, which starts at the ground surface of R2 at the RHS of the wall (i.e., flood side), continues down to the sheet-pile tip at $11.29 \mathrm{ft}$., then continues upward toward the ground surface of R1 at the LHS (i.e., landside) of the sheet-pile wall. The second graph or Figure 5.9b displays total head values of flow along the distance travelled. Head loss occurs as water seeps from el $40.0 \mathrm{ft}$ at the RHS to the ground surface at $30.0 \mathrm{ft}$ (vertical green line). The seepage slope (also referred to as the seepage gradient) of 0.16 starts at el $30 \mathrm{ft}$; continues past the sheet-pile tip of $11.29 \mathrm{ft}$ (vertical black line) and terminates at the LHS ground surface at el $30 \mathrm{ft}$ (vertical red line) where it merges with the surface water at el $34 \mathrm{ft}$ on the LHS of the wall. Further details on the line of seepage method are given in Appendix C. 
Figure 5.9. Line of seepage. (a) Direction of flow. (b) Head loss along distance of flow.

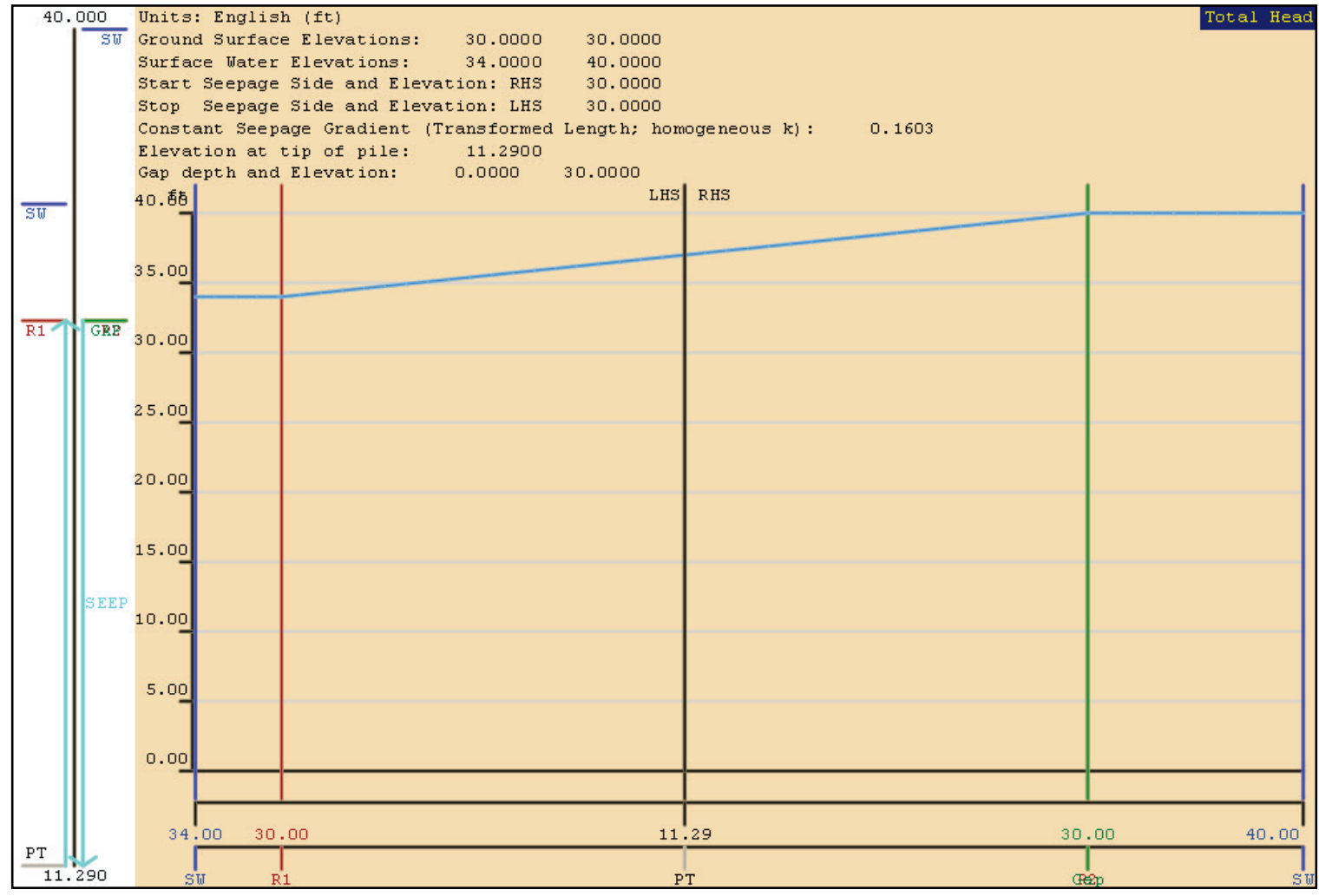

(a)

(b)

\subsubsection{Evaluation of results for 1,000 simulations with seepage}

This section discusses the computed results of the probabilistic version of the Example 5.1 design problem for 1,000 simulations. Only one flood level is tested and set at $40 \mathrm{ft}$. Section 5.4.1 discussed the results for the deterministic solution part of this problem. For the probabilistic analysis, the $\mathrm{COV}$ was set at 20 percent of the mean for both variables $\left(\varphi^{\prime}\right.$ and $\left.\delta^{\prime}\right)$ with a correlation coefficient of 0.9 between the two variables. This characterized the variables as having a strong correlation with some dispersion. The normal distribution was selected and bounded between 0 and $\mu+3 \sigma$, which is the default when bounds are not specified. Results from sheet-pile wall design with 1,000 simulations for the estimation of the sheet-pile tip led to a histogram of frequency at intervals of equally spaced sheet-pile tip elevations.

For the 1,000 simulation output recorded in the run file of Figure 5.10, designs for the calculated sheet-pile tip elevations ranged from -11 ft to $19 \mathrm{ft}$. The mean sheet-pile tip elevation was determined at $10.81 \mathrm{ft}$ with the standard deviation of $3.61 \mathrm{ft}$. Figure 5.11a shows the histogram and its 
relative cumulative distribution function in Figure 5.11b for this analysis. From Figure 5.11, the histogram shows the largest fractional frequency of sheet-pile tip elevation at $12 \mathrm{ft}$, which is deeper than the $11.29 \mathrm{ft}$ for the deterministic analysis that results from the applied dispersion of this analysis.

The 1,00o designs were also evaluated for depth of embedment parameter, and statistical parameters were calculated and produced in Figure 5.12. The mean of the depth of embedment was $19.20 \mathrm{ft}$, with the standard deviation of $3.61 \mathrm{ft}$ and $\mathrm{COV}$ of approximately 0.18 ; values ranged from a minimum of $12 \mathrm{ft}$ to a maximum of $40 \mathrm{ft}$. Figure 5.12a shows the histogram and its related cumulative distribution function in Figure 5.12b for the depth of embedment variable. From Figure 5.12, the histogram shows the largest fractional frequency of 0.27 at the depth of embedment of $18 \mathrm{ft}$.

\subsubsection{Comparisons of results for $100,300,600$ and 1,000 simulations}

This subsection discusses the results of Example 5.1 for 100, 300, 600 and 1,000 simulations. As discussed in the previous Section 5.5.2, the probabilistic design of a sheet-pile wall resulted in histograms and probability distribution functions for 1,000 simulations. Further simulations of 100, 300, 600 and 1,000 were conducted with results shown in Figure 5.13. Sheet-pile tip elevations ranged from $-8 \mathrm{ft}$ to $17 \mathrm{ft}$ for all four simulations.

Figure 5.10. Output runtime file.

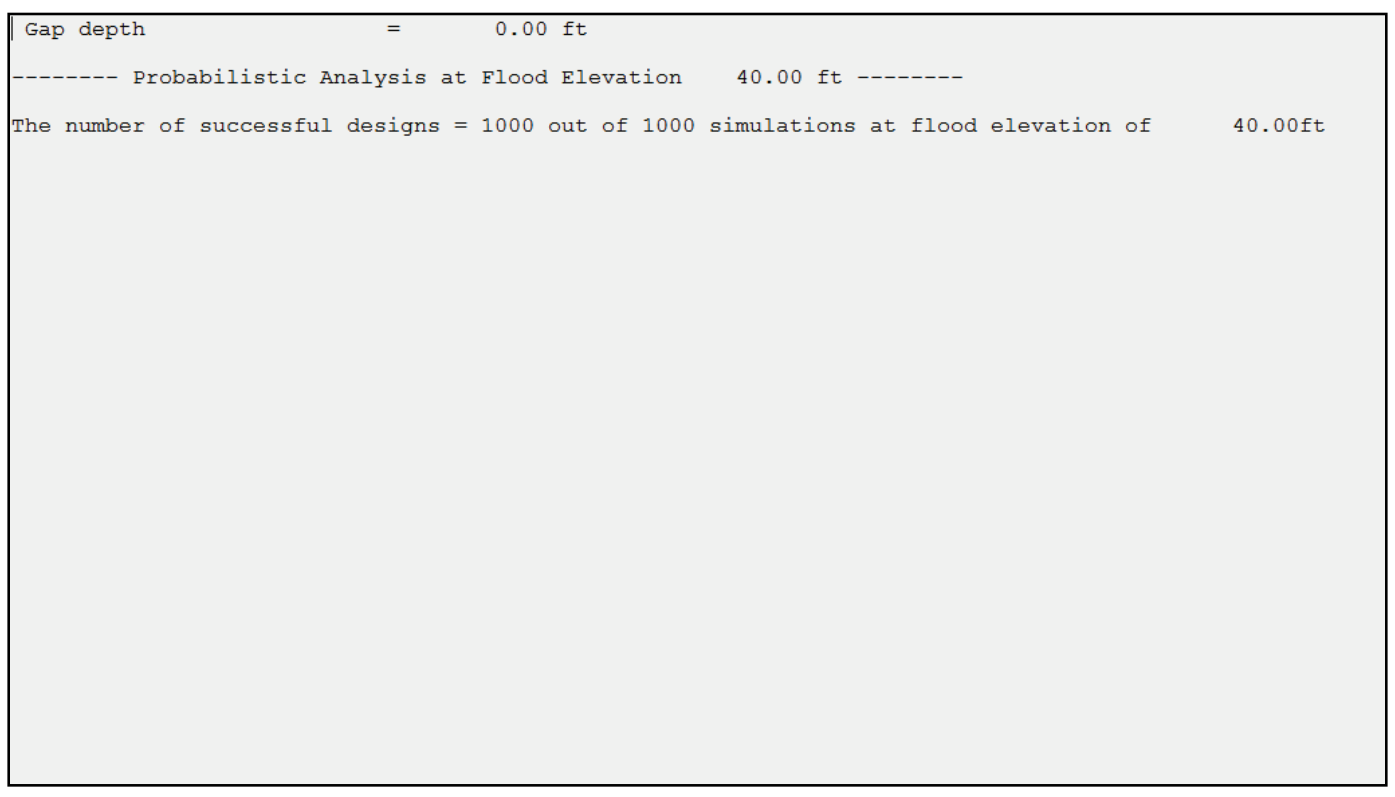


Figure 5.11. Design probabilistic analysis of sheet-pile tip with 1,000 simulations (a) Histogram. (b) Cumulative distribution function.

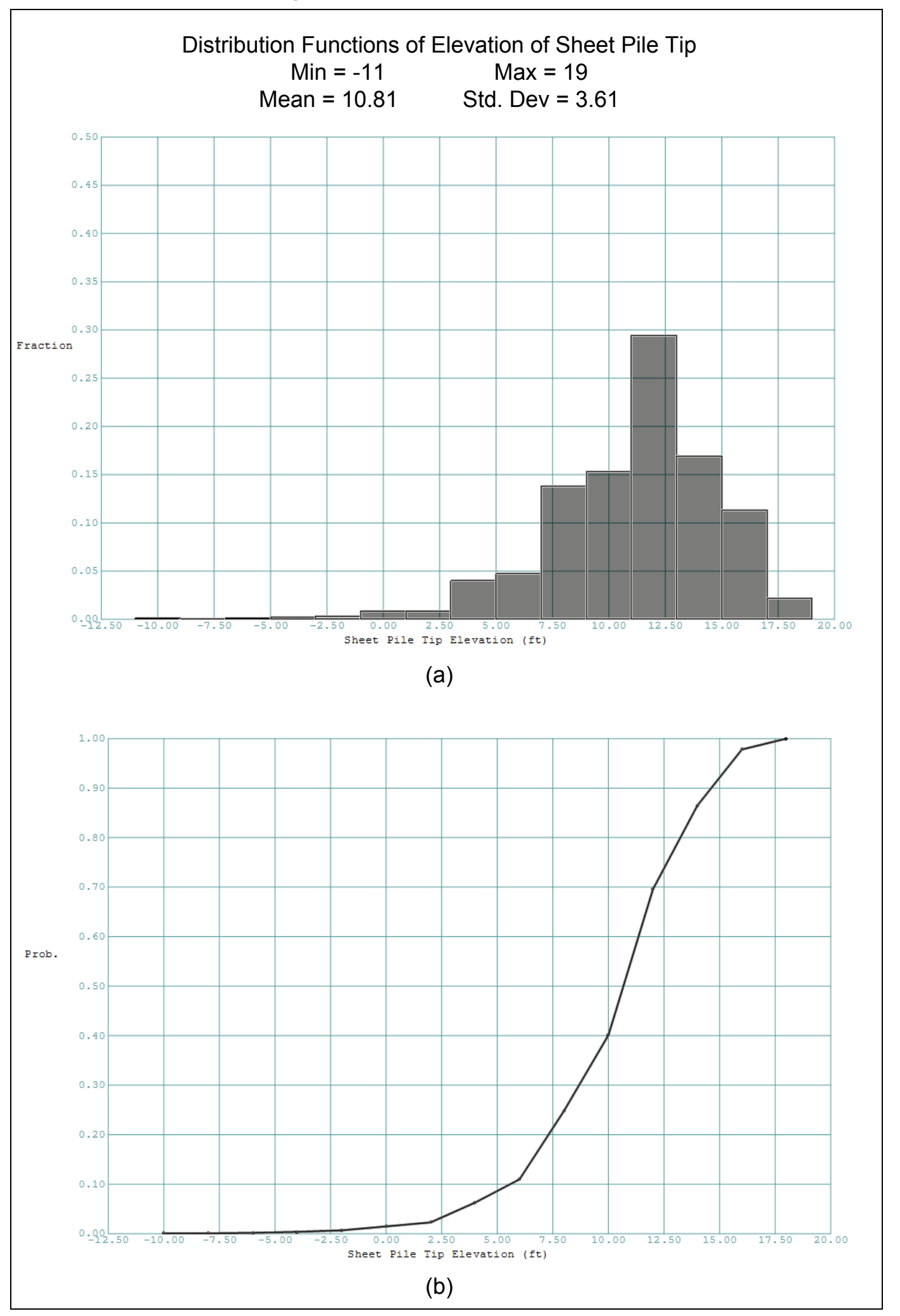


Figure 5.12. Design probabilistic analysis of depth of embedment with 1,000 simulations (a) Histogram. (b) Cumulative distribution function.

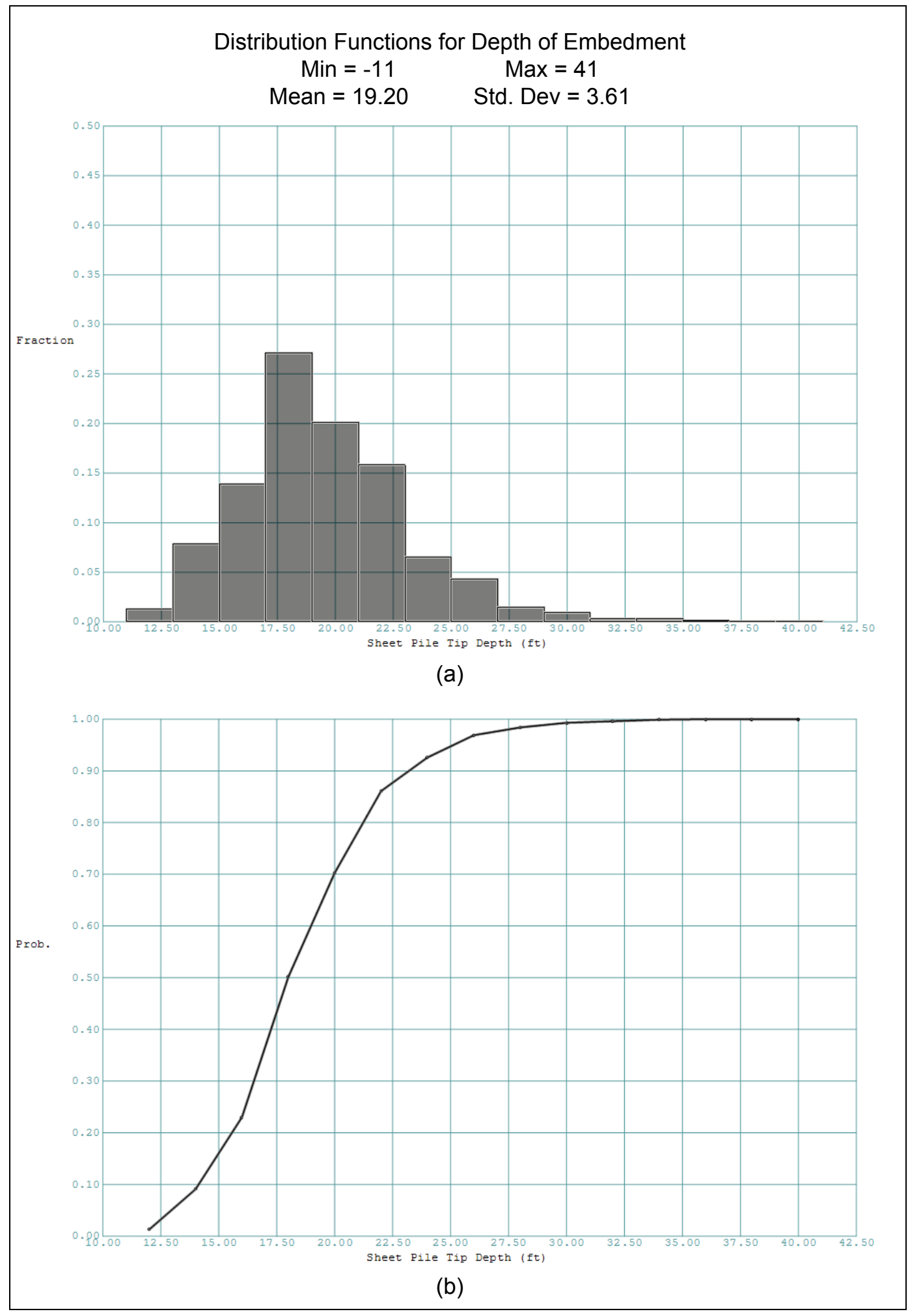


Figure 5.13. Cumulative distribution function for sheet-pile tip elevation for $100,300,600$ and 1,000 simulations.

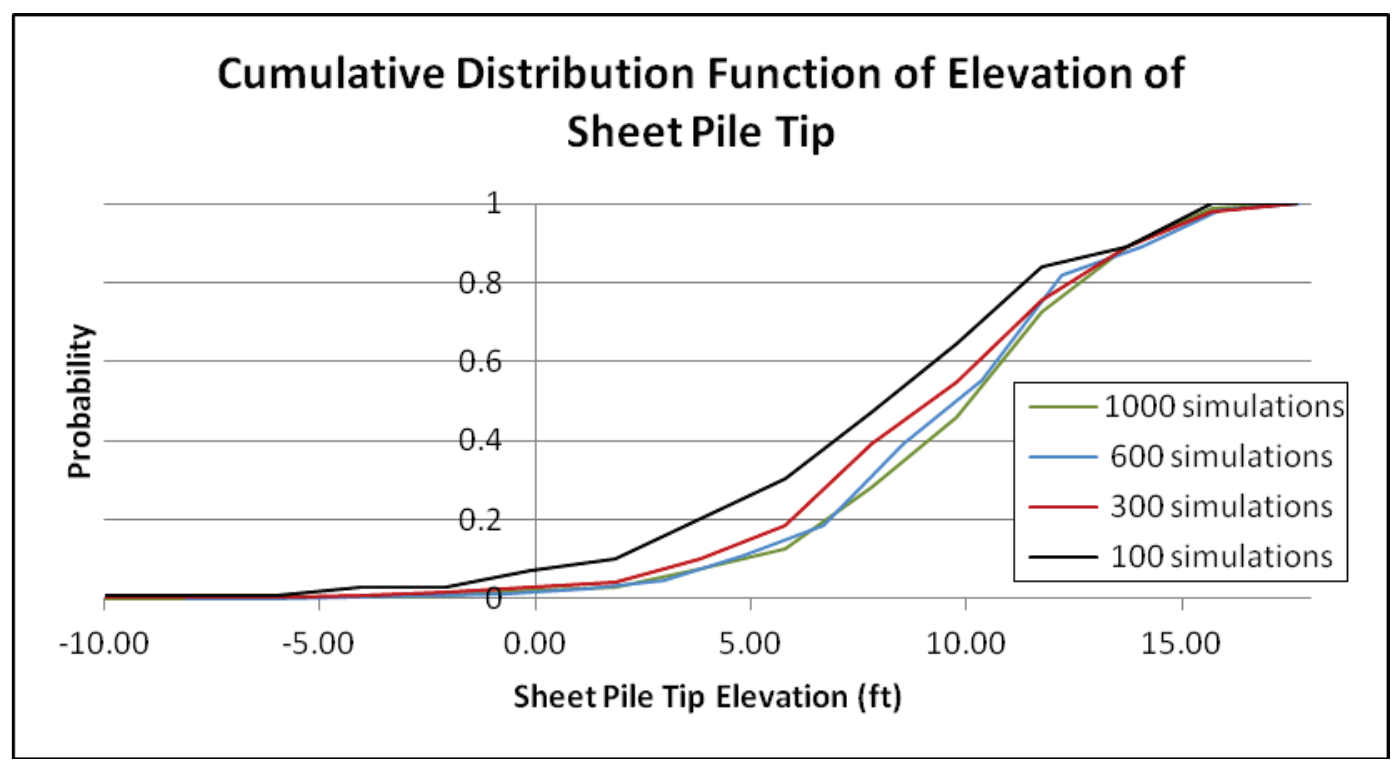

Observe in Figure 5.13 the near perfect agreement in cumulative distribution function curves for the 1,000 and 600 simulation results. This suggests convergence is achieved for 600 or more simulations in this design problem. A comparison of the curve for the 300 simulation analysis to the 600 and 1,000 simulation curves indicates that 300 simulations will provide adequate results for this design.

Execution times were also recorded for various simulations. These timings as well as normalized values as recorded with the timing for 300 simulations are tabulated in Table 5.3. Data from the table show that there is a near linear relationship between timing and number of simulations.

Table 5.3. Execution times for 100, 300, 600 and 1,000 simulations.

\begin{tabular}{|c|l|l|}
\hline Number of Simulations & Execution Time (hh:mm) & Normalized \\
\hline 100 & $0: 01$ & 0.5 \\
\hline 300 & $0: 02$ & 1 \\
\hline 600 & $0: 05$ & 0.1 \\
\hline 1,000 & $0: 09$ & 0.18 \\
\hline
\end{tabular}

\subsection{Example 5.2 system response curve (clay site with hydraulic fracturing, an extension of Example 4.7)}

A total stress definition with $\varphi=0$ and an undrained shear strength $(\mathrm{Su})$ is illustrated using a series of total stress analysis problems in order to 
calculate a uniform passive Factor of Safety $\left(F S_{p}\right)$ of an existing sheet-pile wall in an Analysis mode. A global active Factor of Safety of 1.0 for computing active earth pressures is applied to all soil layers. This I-Wall system has an exposed height of $10 \mathrm{ft}$. The four regions consist of two soil or material layers with level ground interfaces on both sides of the I-Wall at $\mathrm{oft}$ and $-10 \mathrm{ft}$. The saturated unit weight of $122 \mathrm{pcf}$ and cohesion of $300 \mathrm{psf}$ for the upper soil layer and a saturated unit weight of $124 \mathrm{pcf}$ and cohesion of $400 \mathrm{psf}$ for the lower soil layer have been assigned. The water surfaces at both sides of the I-Wall are at $13 \mathrm{ft}$ on the flood side (LHS) and at $\mathrm{o} \mathrm{ft}$ on the landside. Figure 5.14 outlines the geometry and soil parameters of the sheet-pile wall. This analysis procedure was specified given a predesigned wall tip el -28.2679 ft. This sheet-pile tip elevation was established in the Example 4.7 deterministic analysis of Chapter 4.

Figure 5.14. Schematic of sheet-pile wall in clay site for various water levels.

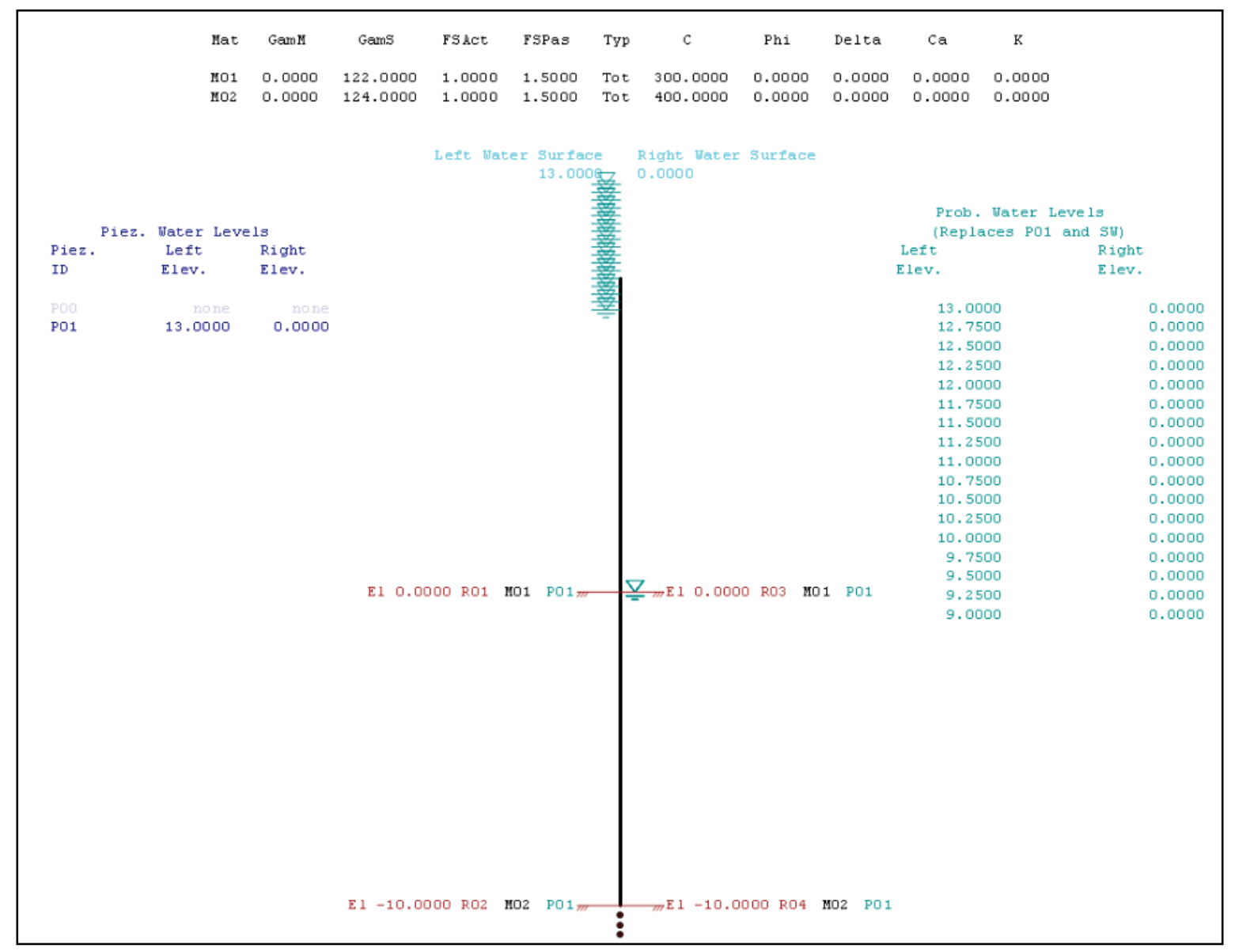

The top row of text listed in Figure 5.14 documents the input values of all parameters associated with each material type. For this problem, there are two materials assigned for the clay site and recorded as Mo1 and Mo2, the 
"M" signifies the material type and is followed by the ID which starts from "01." All information concerning material types are written in black text. The representation of a soil structure is characterized by a region. Regions are labeled with an "R" and followed by an ID starting from "01." For this problem four regions are assigned, Region 1 and Region 2 (as Ro1 and Ro2) are located on the LHS of the sheet-pile wall and Region 3 and Region 4 (as Ro3 and Ro4) are located on the RHS of the sheet-pile wall. Each region and its surface elevation are written in red text.

Hydrostatic water elevations are listed in the upper left-hand corner and shown in dark blue. Water levels are labeled at the soil interface of any specified region with an initial character "P" and followed by an ID. Label "Poo" is one that signifies that there is no water associated with a soil layer or region and the soil is partially saturated. If all soil regions are fully saturated, "Poo" will be grayed out in Figure 5.14. Surface water on either side of the sheet-pile wall is represented by water symbols at its defined elevation along the sheet-pile wall and labeled as "Po1." If there is no surface water associated with any soil region, "Po1" will be grayed out.

For the probabilistic analysis, the mean of the cohesion is listed in the table of Figure 5.15 for the materials designated as Mo1 and Mo2. A standard deviation of $300 \mathrm{psf}, \mathrm{COV}$ of 0.1 , and bounded normal probability distribution function are specified for the material Mo1 cohesion, as shown in Figure 5.15. A standard deviation of 400 psf, COV of 0.1, and bounded normal probability distribution function are specified for the material Mo2 cohesion. The cohesions of the two layers are considered independent variables, so there is zero correlation specified between them.

Figure 5.15. Statistical parameters for the clayey soil of material M01.

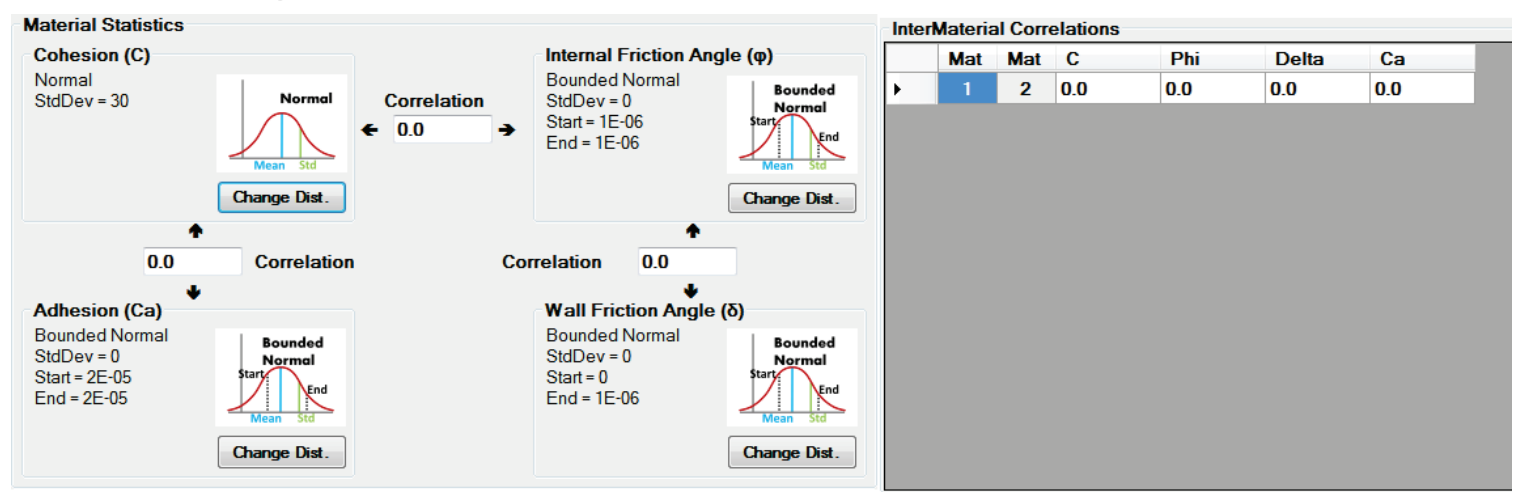


This problem was analyzed for flood elevations ranging from $9 \mathrm{ft}$ to $13 \mathrm{ft}$ with a constant landside water elevation of $\mathrm{oft}$. These flood elevations were tested initially with 1 - $\mathrm{ft}$ increments totaling 5 water levels and later at 0.25 -ft increments for a total of 17 water levels. Four different tests were conducted by varying the number of simulations of 100, 300, 1,000 and 2,000 simulations for all 17 water levels.

\subsection{Comparisons of results for $100,300,1,000$ and 2,000 simulations}

This subsection discusses the analyzed results of Example 5.1 for 100, 300, 1,000 and 2,000 simulations. Flood levels were set at 0.25 -ft increments with maximum flood elevation at $13 \mathrm{ft}$ and a minimum flood elevation at $9 \mathrm{ft}$. A total of 17 pairs of flood levels are considered for each of the flood level simulation groups. At the RHS of the I-Wall, a constant water elevation at $\mathrm{o} \mathrm{ft}$ is assigned.

This analysis resulted with a system response curve of an I-Wall rotational limit state for 100, 300, 1,000 and 2,000 simulations and 17 flood levels. Table 5.4 lists the probabilities for the rotational limit state for each of the simulation groups tabulated at all 17 flood elevations.

Figure 5.16 plots the system response curve (SRC) using the results from the Table 5.4, 1,000 simulation analysis (blue curve). Each point on the Figure 5.16 SRC gives the probability of the rotational limit state of the I-Wall, using 1,000 simulations at each flood level to define each point. The probability for a rotational limit state is shown with a value of 0.0 at 10-ft flood elevation and reaching a maximum value of 1.0 at $12.75 \mathrm{ft}$. The curves in Figure 5.16 show a difference between the different flood levels chosen for 1,000 simulations. The 1 -ft increments in flood elevations (green line) from $9 \mathrm{ft}$ to $13 \mathrm{ft}$ of Figure 5.16 only provided 5 data points and therefore could not fully describe the system response curve. A much finer flood level increment of $0.25 \mathrm{ft}$ was used for the 1,000 simulations and a more refined SRC was produced and represented by the blue curve. 
Table 5.4. Probabilities of a rotational limit state for $100,300,1,000$ and 2,000 number of simulations.

\begin{tabular}{|l|l|l|l|l|}
\hline \multirow{2}{*}{ Flood Elevation (ft) } & \multicolumn{5}{|c|}{ Number of Simulations } \\
\cline { 2 - 5 } & 100 & 300 & 1,000 & 2.000 \\
\hline 13.00 & 1.00 & 1.00 & 1.00 & 1.00 \\
\hline 12.75 & 1.00 & 1.00 & 1.00 & 1.00 \\
\hline 12.50 & 0.97 & 1.00 & 0.99 & 1.00 \\
\hline 12.25 & 0.92 & 0.96 & 0.97 & 0.97 \\
\hline 12.00 & 0.88 & 0.86 & 0.86 & 0.88 \\
\hline 11.75 & 0.72 & 0.68 & 0.69 & 0.73 \\
\hline 11.50 & 0.44 & 0.45 & 0.46 & 0.51 \\
\hline 11.25 & 0.32 & 0.28 & 0.28 & 0.29 \\
\hline 11.00 & 0.16 & 0.12 & 0.14 & 0.15 \\
\hline 10.75 & 0.05 & 0.06 & 0.06 & 0.06 \\
\hline 10.50 & 0.02 & 0.02 & 0.02 & 0.02 \\
\hline 10.25 & 0.00 & 0.01 & 0.00 & 0.01 \\
\hline 10.00 & 0.01 & 0.00 & 0.00 & 0.00 \\
\hline 9.75 & 0.00 & 0.00 & 0.00 & 0.00 \\
\hline 9.50 & 0.00 & 0.00 & 0.00 & 0.00 \\
\hline 9.25 & 0.00 & 0.00 & 0.00 & 0.00 \\
\hline 9.00 & 0.00 & 0.00 & 0.00 & 0.00 \\
\hline & & & & \\
\hline
\end{tabular}

Figure 5.16. System response curve for 1,000 simulations at $0.25-\mathrm{ft}$ and 1 -ft flood increments.

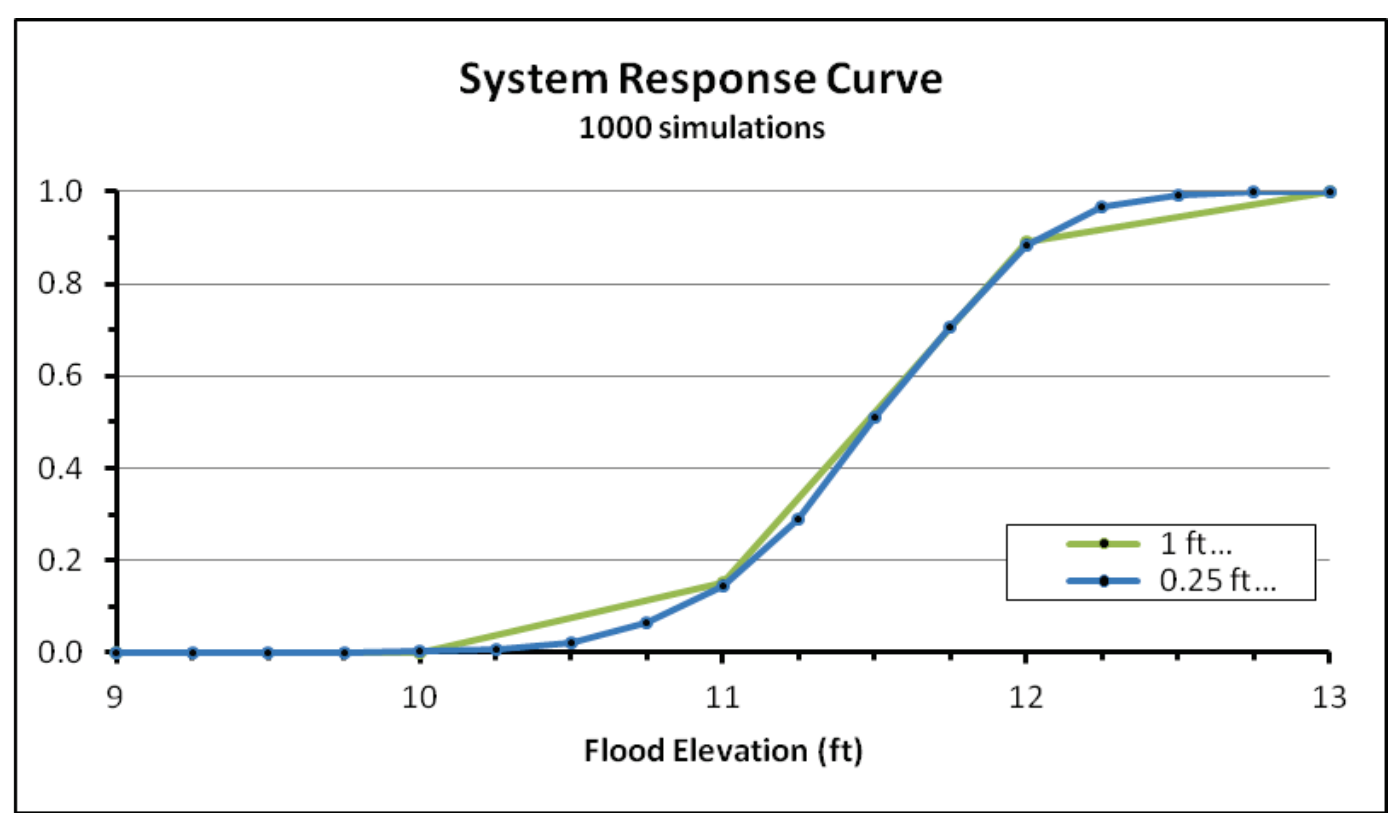


Figure 5.17 presents all four Table 5.4 SRC curves representing the 100, $300,1,000$ and 2,000 simulations for 17 flood levels. A close inspection of this figure and the data contained in Table 5.4 show that the response curve from the 2,000 simulations are close to the results for the response curves of 1,000 and 300 simulations. Comparison between the Table 5.4 data for the 300 and 2,000 simulation results at flood el $11.50 \mathrm{ft}$ and at el $11.75 \mathrm{ft}$ demonstrates a difference between the probability for rotational limit state values for both the 300 and 2,000 simulation results of 0.45 and 0.51 , and 0.68 and 0.73 , respectively. The fact that the 2,000 simulation results are in agreement with the 300 simulation results demonstrate that 300 simulations are ideal for the calculation of the system response curve with close accuracy and much better execution times as compared to the 1, OOO simulations.

Figure 5.17. System response curve for $100,300,1,000$ and 2,000 simulations at 0.25 -ft increments.

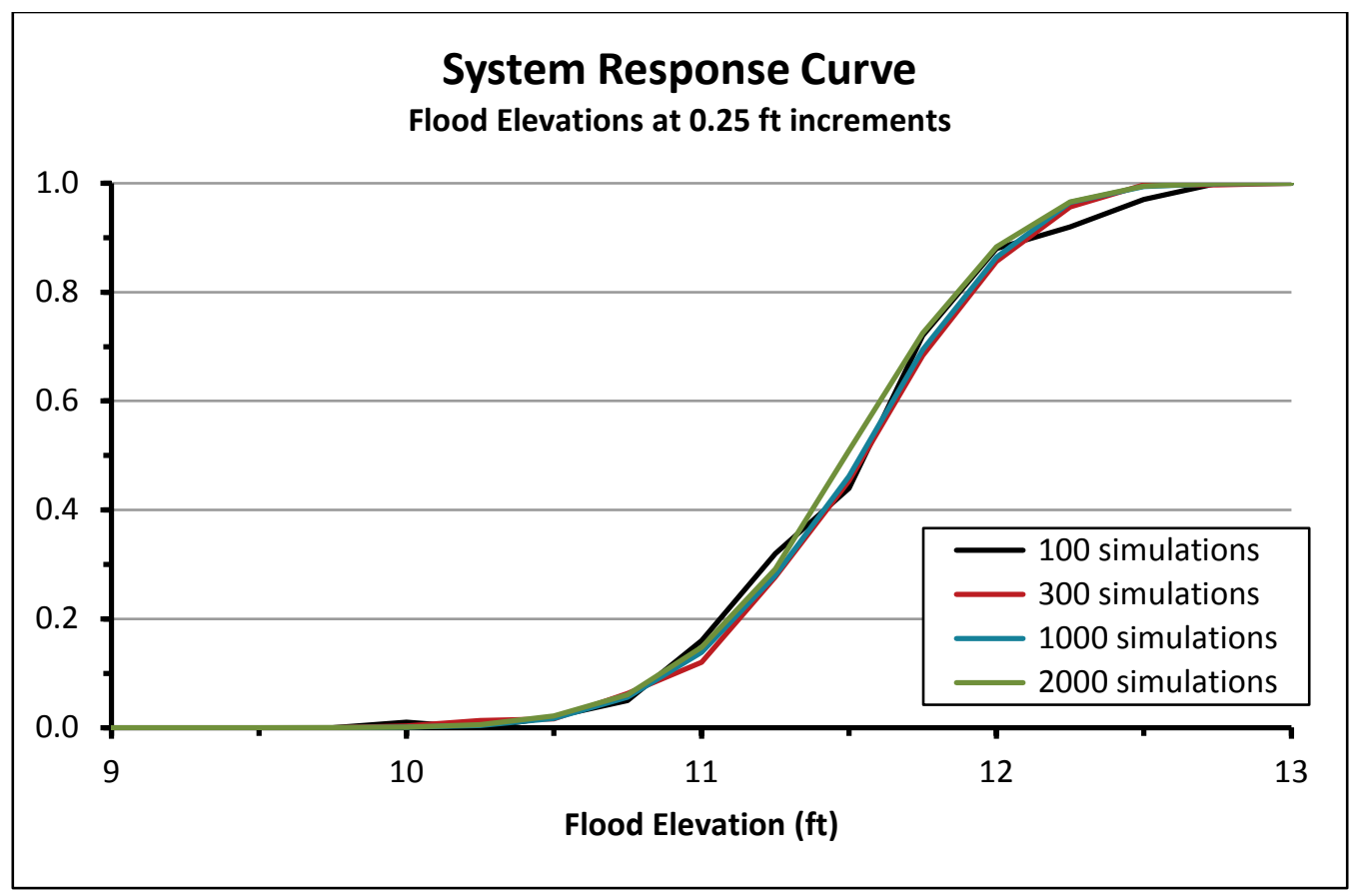

Execution times were recorded for the various simulations. These timings as well as normalized values with the timing for 300 simulations are tabulated in Table 5.5. Data from the table show that there is a linear relationship between the timings and number of simulations. 
Table 5.5. Execution times for $100,300,1,000$ and 2,000 number of simulations.

\begin{tabular}{|c|l|l|}
\hline Number of Simulations & Execution Time $(\mathrm{hh}: \mathrm{mm})$ & Normalized \\
\hline 100 & $0: 17$ & 0.34 \\
\hline 300 & $0: 50$ & 1.00 \\
\hline 1,000 & $2: 46$ & 3.32 \\
\hline 2000 & $5: 24$ & 6.48 \\
\hline
\end{tabular}

\subsection{Example 5.3 system response curve (two-layer sand site with hydraulic fracturing criteria and seepage)}

An effective stress definition of an I-Wall founded in a two-layer sand site (i.e., two regions on either side of I-Wall) with $\mathrm{c}^{\prime}=\mathrm{o}$ is illustrated using a series of effective stress-based analyses in order to calculate a uniform passive Factor of Safety (FSp) of an existing sheet-pile wall in an Analysis mode. A global active Factor of Safety of 1.0 for computing active earth pressures is applied to all soil layers. This I-Wall system has an exposed height of $10 \mathrm{ft}$. The four regions consist of two soil or material layers with level ground interfaces on both sides of the I-Wall at o ft and -10 ft. All soil layers are assigned a saturated unit weight of 122.0 pcf. The upper soil layers have an effective angle of internal friction of $30 \mathrm{deg}$ and the effective angle of soil-to-sheet-pile wall interface friction of $15 \mathrm{deg}$. The lower soil layers have been assigned an effective angle of internal friction of $35 \mathrm{deg}$ and the effective angle of soil-to-sheet-pile wall interface friction of $17.5 \mathrm{deg}$. Surface water on both sides of the I-Wall are at el $15 \mathrm{ft}$ on the flood side or LHS and at el o ft on the landside or RHS. This analysis also considers homogeneous seepage. Figure 5.18 outlines the geometry and soil parameters for the sheet-pile wall. For the analysis of an existing sheet-pile wall, the sheet-pile tip el was predesigned for $-13 \mathrm{ft}$.

The top row of text listed in Figure 5.18 documents the input values of all parameters associated with each material type. For this problem, there are two materials assigned for the clay site and recorded as Mo1 and Mo2, the "M" signifies the material type and is followed by the ID, which starts from "o1." All information concerning material types are written in black text. The representation of a soil structure is characterized by a region. Regions are labeled with an "R" and followed by an ID starting from "01." For this problem four regions are assigned: Region 1 and Region 2 (as Ro1 and Ro2), are located on the LHS of the sheet-pile wall and Region 3 and Region 4 (as Ro3 and Ro4) are located on the RHS of the sheet-pile wall. Each region and its surface elevation are written in red text. 
Figure 5.18. Schematic of sheet-pile wall in sandy site for 12 flood levels.

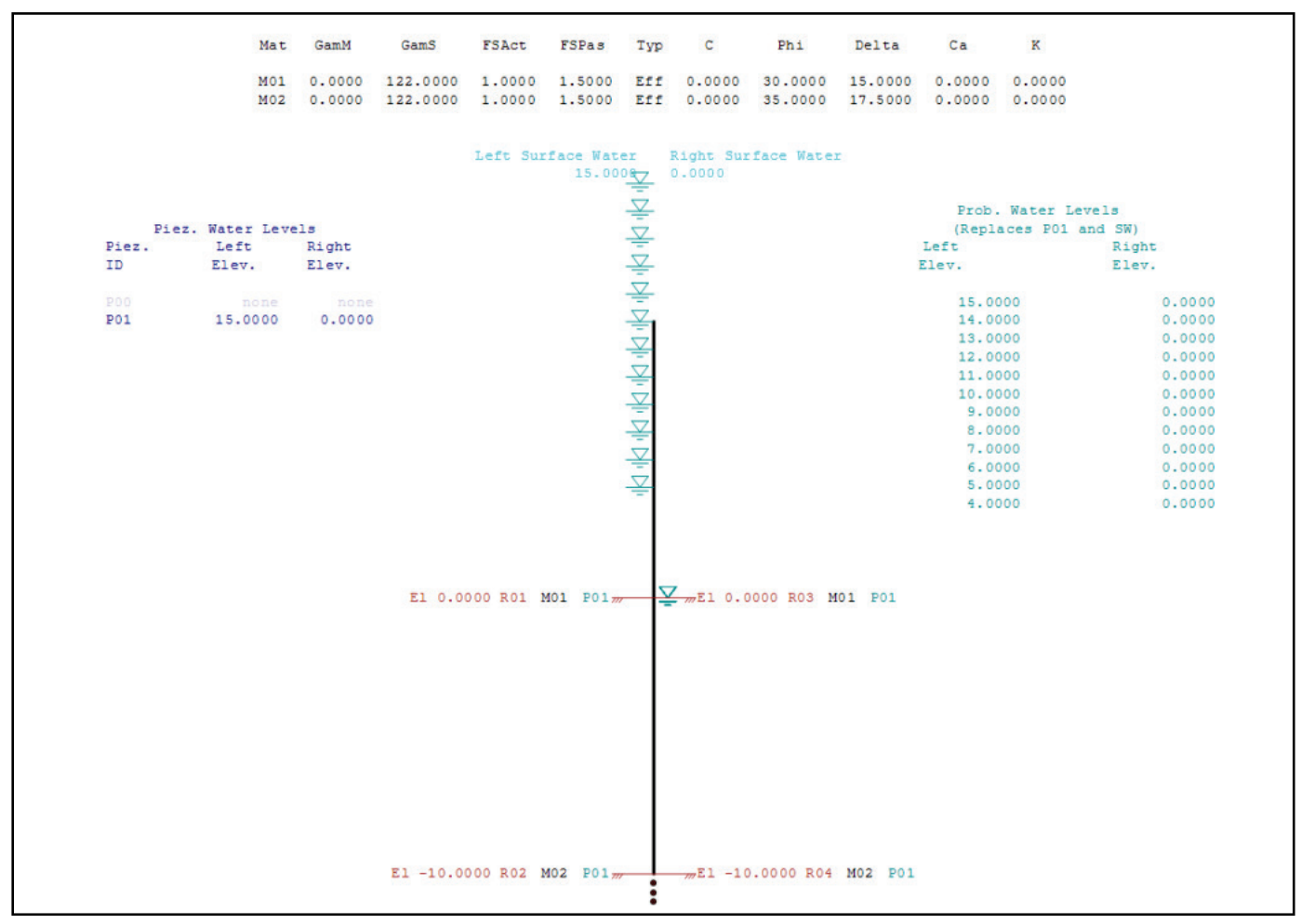

Water elevations are listed in the upper left-hand corner and shown in dark blue. Water levels are labeled at the soil interface of any specified region with an initial character "P" and followed by an ID. Label "Poo" is one that signifies that there is no water associated with a soil layer or region and the soil is partially saturated. If all soil regions are fully saturated, "Poo" will be grayed out in Figure 5.18. Surface water on either side of the sheet-pile wall is represented by water symbols at its defined elevation along the sheet-pile wall and labeled as "Po1." If there is no surface water associated with any soil region, "Po1" will be grayed out.

For the probabilistic analysis considered in this section, the mean effective angle of internal friction $\left(\varphi^{\prime}\right)$ and the mean effective angle of wall-soil interface friction $\left(\delta^{\prime}\right)$ were defined previously as $30 \mathrm{deg}$ and $15 \mathrm{deg}$ for material Mo1, and 35 deg and 17.5 deg for material Mo2, respectively, and listed in the table of Figure 5.18. A standard deviation of $6 \mathrm{deg}$, COV of 0.2and normal probability distribution function is specified for the effective angle of internal friction, as shown in Figure 5.19. A standard deviation of $3 \mathrm{deg}$, COV of 0.2 and normal probability distribution function is specified for the effective angle of wall-soil interface friction. Note that these two variables are strongly correlated with a correlation coefficient of 0.7 , and the intermaterial correlations of $\varphi^{\prime}$ and $\delta^{\prime}$ of materials Mo1 and Mo2 have been 
assigned values of 0.6 as shown in Figure 5.19. Section 5.2 discusses random variables and the correlation between them.

This example was analyzed for flood elevations ranging from $4 \mathrm{ft}$ to $15 \mathrm{ft}$ on the RHS with constant landside water elevation of o ft on the LHS of the I-Wall. These flood elevations were tested with $1-\mathrm{ft}$ increments for a total of 12 water levels. Four different tests were conducted by varying the number of simulations of 100, 300, 500 and 1,000 simulations for all 12 water levels.

Figure 5.19. Statistical parameters for the sandy soils.

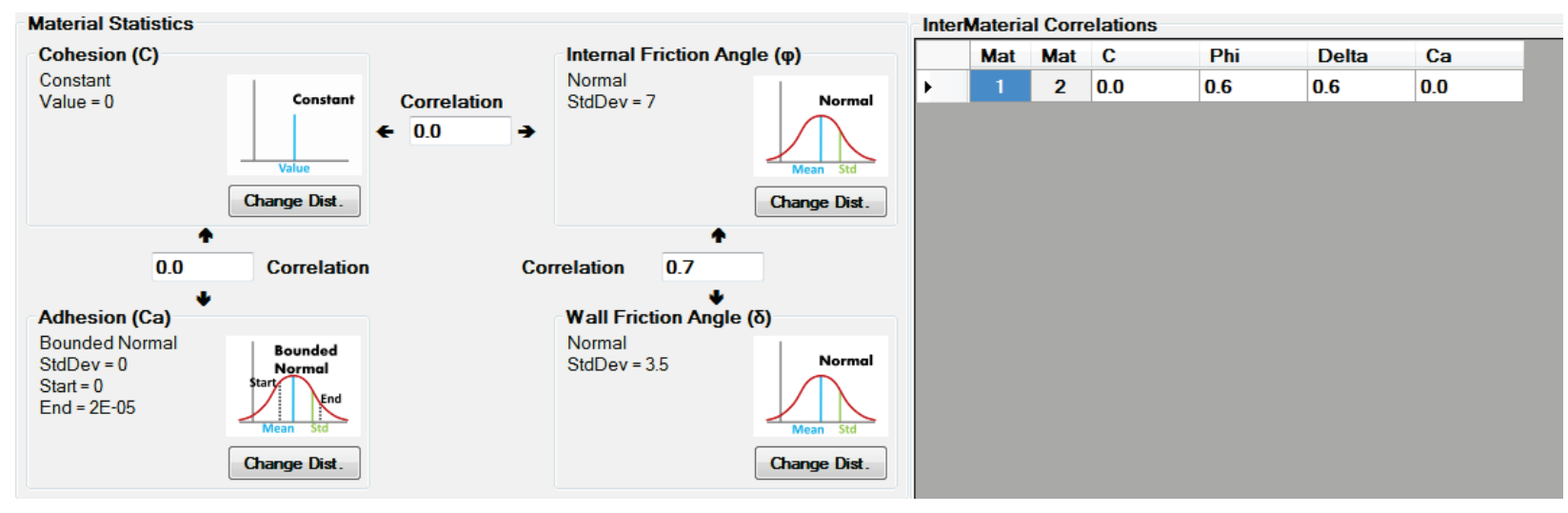

\subsection{Comparisons of results for $100,300,500$ and 1,000 simulations}

This subsection discusses the analyzed results of Example 5.2 for 100, 300, 500 and 1,000 simulations. Flood levels on the LHS are set at 1-ft increments with maximum flood elevation at $15 \mathrm{ft}$ and a minimum flood elevation at $4 \mathrm{ft}$. A total of 12 pairs of flood levels are considered for each of the flood level simulation groups. At the RHS of the I-Wall, a constant water elevation at $\mathrm{o} f t$ is assigned.

This analysis resulted with a system response curve of an I-Wall rotational limit state with 100, 300, 500 and 1,000 simulations and 12 flood levels. Table 5.6 lists the probabilities for the rotational limit state for each of the simulations tabulated at all 12 flood elevations.

Figure 5.20 plots the four Table 5.6 system response curves (SRC). Each point on the gray Figure 5.20 system response curve (SRC) gives the probability of rotational limit state of the I-Wall, using 1,000 simulations to define each point. The probability for a rotational limit state is shown with values approximately $\mathrm{o}$ at 4 - $\mathrm{ft}$ flood elevation and reaching a maximum 
value of 1.0 at $14 \mathrm{ft}$. The black, red and blue Figure 5.20 SRC curves correspond to 100, 300 and 500 simulations, respectively, of 12 flood levels. A close inspection of this figure and the data contained in Table 5.6 show that responses from all simulations are close to the results for the 1,000 simulations. Table 5.6 numerically shows minimal changes, however Figure 5.20 shows the response of the 100 simulations does not provide a smooth curve, which implies more than 100 simulations are needed.

Recognizing that this is the most computational intensive problem, the evaluation of a probabilistic, analysis of rotational stability of the I-Wall, effective stress-based earth pressure calculations, and seepage, the determination of the number of simulations essential to produce the best acceptable accuracy is a necessity while minimizing run time for the evaluation of the system response curve. From this reasoning, the 300-simulation SRC curve was determined to provide good accuracy as well as a smoother SRC.

Execution times have been recorded for the various simulations. These timings as well as normalized values with the timing for 300 simulations are tabulated in Table 5.7. Data from the table show that there is a near linear relationship between execution times and number of simulations.

Table 5.6. Probabilities of a rotational stability for $100,300,500$ and 1,000 simulations.

\begin{tabular}{|l|l|l|l|l|}
\hline \multirow{2}{*}{ Flood Elevation (ft) } & \multicolumn{4}{|c|}{ Number of Simulations } \\
\cline { 2 - 5 } & 1,000 & 500 & 300 & 100 \\
\hline 15 & 1.00 & 1.00 & 1.00 & 1.00 \\
\hline 14 & 1.00 & 1.00 & 1.00 & 1.00 \\
\hline 13 & 0.99 & 0.99 & 0.99 & 0.99 \\
\hline 12 & 0.99 & 0.98 & 0.99 & 0.98 \\
\hline 11 & 0.96 & 0.96 & 0.95 & 0.98 \\
\hline 10 & 0.91 & 0.91 & 0.92 & 0.90 \\
\hline 9 & 0.81 & 0.81 & 0.81 & 0.83 \\
\hline 8 & 0.63 & 0.62 & 0.65 & 0.65 \\
\hline 7 & 0.38 & 0.38 & 0.38 & 0.35 \\
\hline 6 & 0.15 & 0.16 & 0.17 & 0.15 \\
\hline 5 & 0.04 & 0.03 & 0.05 & 0.03 \\
\hline 4 & 0.01 & 0.01 & 0.01 & 0.01 \\
\hline
\end{tabular}


Figure 5.20. System response curve for $100,300,500$ and 1,000 simulations.

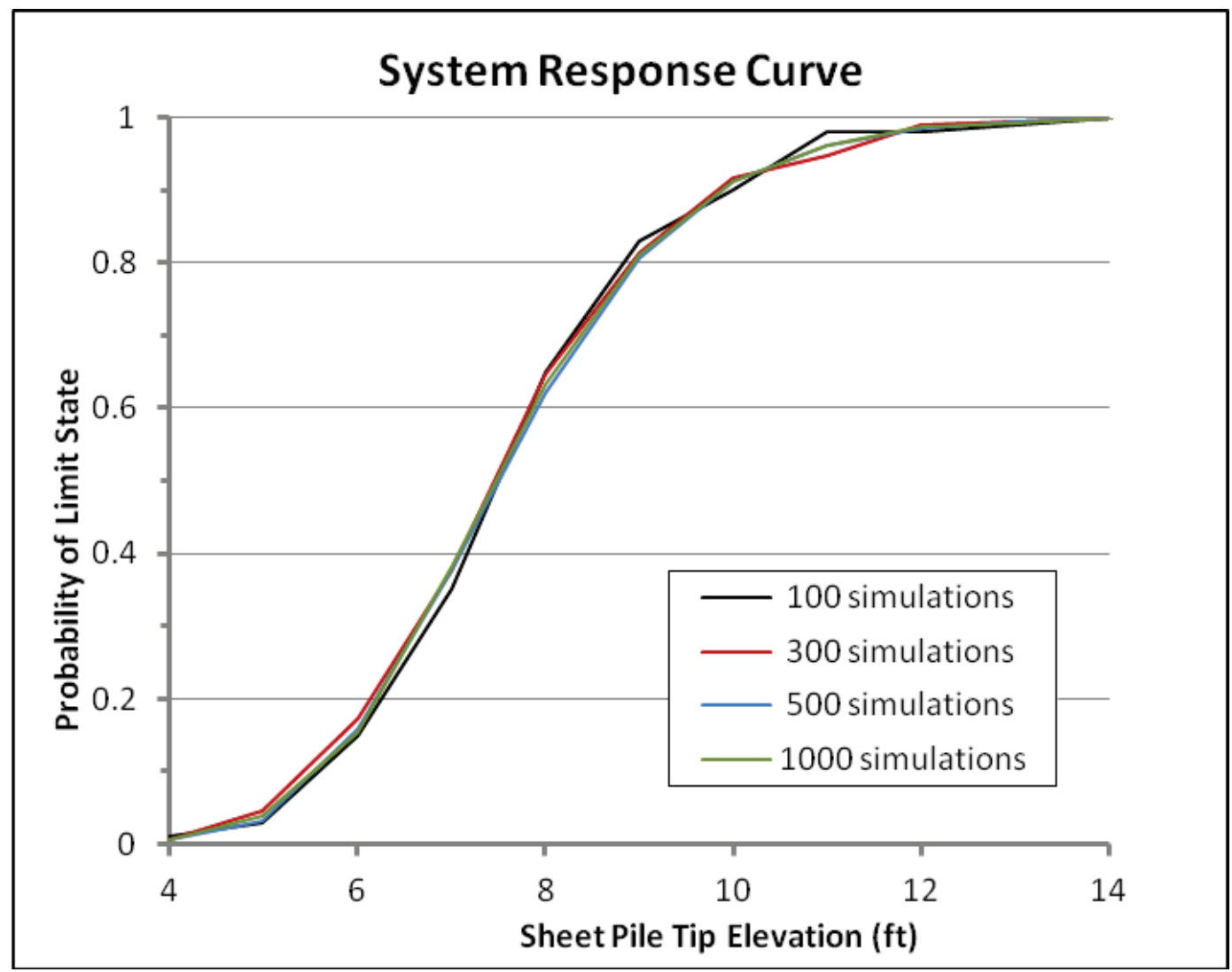

Table 5.7. Execution times for $100,300,500$ and 1,000 simulations.

\begin{tabular}{|c|l|l|}
\hline Number of Simulations & Execution Time (hh:mm) & Normalized \\
\hline 100 & $1: 41$ & 0.34 \\
\hline 300 & $5: 01$ & 1.00 \\
\hline 500 & $8: 28$ & 1.69 \\
\hline 1,000 & $15: 52$ & 3.16 \\
\hline
\end{tabular}




\section{Summary and Conclusions}

\subsection{Summary}

This report summarizes an engineering method and its implementation in the software Corps_I-Wall Version 1.o used for the design of new I-Walls or the analysis of existing I-Walls in floodplains (i.e., I-Walls embedded in level ground) during flood loading. Horizontally stratified soil layers are assumed in Version 1.0.

Other Corps_I-Wall software capabilities for engineering analysis include: total stress and/or effective stress earth pressure calculations; Rankine, Coulomb, or logarithmic spiral-based earth pressure coefficients; hydrostatic pore water pressure computed from surface water(s) and/or piezometric surface(s) within each of the layered soil regions; pore water pressures computed from line of seepage, steady-state seepage in soil regions with different hydraulic conductivities; gap initiation and propagation using hydraulic fracturing criterion; and boundary pressures (e.g., for wave loading of coastal structures) and/or surface surcharge.

Probabilistic analysis is a primary component of Corps_I-Wall for new and existing I-Wall analysis. The probabilistic analysis capabilities for the design of new I-Walls results in a statistical characterization of sheet-pile tip embedment. The probabilistic analysis capabilities for analyzing existing I-Walls include the construction of a system response curve (a.k.a., fragility curve), which gives the probability of rotational instability as a function of flood elevation.

Examples are provided to highlight each of these capabilities. Select examples from geotechnical literature and an ERDC technical report were used to validate the basic engineering methodologies as they were implemented in the software. A user's manual for using the GUI and executing CI-Wall is also included.

\subsection{Conclusions}

The I-Wall Analysis R\&D effort summarized in this report provides the USACE districts with essential tools in analyzing and evaluating I-Wall 
performance in riverine, fluvial, and coastal environments. The validated engineering methodologies and graphical user interface implemented in software provide ease of use for the modeling and evaluation of these soilstructure interactions. Advanced techniques are included to address hydraulic fracturing, seepage, etc. 


\section{References}

Bardet, Jean-Pierre. 1997. Experimental soil mechanics. Upper Saddle River, NJ: Prentice Hall.

Becker, D.E. 1996. Eighteenth Canadian geotechnical colloquium: Limit states design for foundations. Part II. Development for the National Building Code of Canada. Canadian Geotechnical Journal 33: 984-1007.

Bowles, Joseph E. 1968. Foundation analysis and design. New York: McGraw-Hill Book Company.

Bullock, G., C. Obhrai, G.Wolters, G.Müller, H.Peregrine, and H.Bredmose. 2004. Characteristics and design implications of breaking wave impacts. Proc. Int. Conf. Coastal Engineering, Lisbon.

Caquot, A., and J.Kerisel. 1948. Tables for the calculation of passive pressure, active pressure and bearing capacity of foundations. Gauthier-Villars, Paris.

Cedergren, Harry R. 1977. Seepage, drainage and flownets. $2^{\text {nd }}$ edition. New York:.John Wiley and Sons.

Cherubini, C. 2000. Reliability evaluation of shallow foundation bearing capacity on c', $\phi$ ' soils. Canadian Geotechnical Journal 37(1): 264-269.

Cherubini, C. 1997. Data and considerations on the variability of geotechnical properties of soils. In Proceedings of the International Conference on Safety and Reliability (ESREL) 97, Lisbon. 2:1583-1591.

Clough, G.W., and J.M. Duncan. 1991. Earth pressures. In Foundation engineering handbook. $2^{\text {nd }}$ edition: 223-235. Ed: Hsai-Yang Fang and van Nostrand. New York: Reinhold.

Chen, W.F. and X.L. Liu. 1990. Limit analysis in soil mechanics. Developments in geotechnical engineering 52. New York: Elsevier Science Publishers, Amsterdam.

Das, Braja M. 2007. Principles of foundation engineering. $6^{\text {th }}$ edition. Canada Ltd.:Nelson/Thomson.

Dawkins, W. P. 1991. Users guide: Computer program for design and analysis of sheetpile walls by classical methods (cwalsht) including Rowe's moment reduction. Instruction Report ITL-91-1. Vicksburg, MS: U.S. Army Engineer Waterways Experiment Station.

Department of the Navy. 1982. Foundations and earth structures. Design manual, DM 7.2. Alexandria, VA: Naval Facilities Engineering Command.

Duncan, J. Michael and Stephen G. Wright. 2005. Soil strength and slope stability. Hoboken, NY: John Wiley and Sons, Inc. 
Ebeling, R. M., and E. E. Morrison. 1992. The seismic design of waterfront retaining structures. WES Technical Report ITL-92-11. Vicksburg, MS: U.S. Army Engineer Waterways Experiment Station.

Goda, Y. 1974. New wave pressure formulae for composite breakwater. Proceedings of the $14^{\text {th }}$ Conference on Coastal Engineering, Copenhagen, Denmark.

Harr, M.E. 1987. Reliability-based design in civil engineering. New York: McGraw-Hill Book Company.

Headquarters, U.S. Army Corps of Engineers.1989. Retaining and flood walls. EM 11102-2502. Washington, DC: Headquarters, U.S. Army Corps of Engineers.

Headquarters, U.S. Army Corps of Engineers. 1990. Retaining and flood walls. ETL 11102-322. Washington, DC: Headquarters, U.S. Army Corps of Engineers.

Headquarters, U.S. Army Corps of Engineers. 1994. Design of sheet pile walls. EM 11102-2504. Washington, DC: Headquarters, U.S. Army Corps of Engineers.

Headquarters, U.S. Army Corps of Engineers. 2002. Coastal engineering manual. EM 1110-2-1100, Part VI. Washington DC: Headquarters, U.S. Army Corps of Engineers.

Headquarters, U.S. Army Corps of Engineers. 2011a. Engineering and design of I_Walls. Engineering Circular 1110-2-6066. Washington, DC: Headquarters, U.S. Army Corps of Engineers.

Headquarters, U.S. Army Corps of Engineers. 2011b. Evaluation of I-Walls. ETL 1110-2575. Washington, DC: Headquarters, U.S. Army Corps of Engineers.

Independent Panel to Review Cause of Teton Dam Failure.1976. Report to U.S. Department of the Interior and the state of Idaho on failure of Teton Dam. Washington, DC: Superintendent of Documents, U.S. Government Printing Office.

Interagency Performance Evaluation Task Force. 2007. The performance- levees and floodwall. Performance Evaluation of the New Orleans and Southeast Louisiana Hurricane Protection System. Final Report of the Interagency Performance Evaluation Task Force. Vol V. Washington, DC: Interagency Performance Evaluation Task Force. https://ipet.wes.army.mil/

Jumikis, Alfreds R. 1984. Soil mechanics. Malabar, FL.: Robert E. Krieger Publishing Company, Inc.

Kerisel, J., and E. Absi. 1990. Active and passive earth pressure tables. $3^{\text {rd }}$ edition. A.A. Balkema/Rotterdam/Brookfield.

Lumb, P. 1970. Safety factors and the probability distribution of soil strength. Canadian Geotechnical Journal 7: 225-242.

Nobari, E. S., K. L. Lee, and J. M. Duncan. 1973. Hydraulic fracturing in zoned earth and rockfill dams. WES Contract Report S-73-2. Vicksburg, MS: U.S. Army Engineer Waterways Experiment Station. 
Pace, M. C., K. Abraham, and R. M. Ebeling. 2012. Complete soil-structure interaction (SSI) analyses of I-walls embedded in level ground during flood loading. ERDC/ITL TR-12-4. Vicksburg, MS: U.S. Army Engineer Research and Development Center.

Sherard, J. L. 1973. Embankment dam cracking. Embankment dam engineering-the Casagrande volume. New York: John Wiley and Sons.

Sherard, J. L. 1986. Hydraulic fracturing in embankment dams. ASCE Journal of Geotechnical Engineering 112 (10) 905-927.

Sokolovskii, V.V. 1965. Static of granular media. New York: Pergamon Press.

Spangler, M. G., and R. L. Handy. 1973. Soil engineering. $3^{\text {rd }}$ edition. New York: Educational Publishers.

Tekie, P. B., and B. R. Ellingwood. 2002. Fragility analysis of concrete gravity dams. ERDC/ITL TR-02-6. Vicksburg, MS: U.S. Army Engineer Research and Development Center.

Terzaghi, K. 1943. Theoretical soil mechanics. New York: John Wiley and Sons.

Terzaghi, K. and Ralph B.Peck. 1948. Soil mechanics in engineering practice. New York: John Wiley and Sons.

Terzaghi, K., R. B. Peck, and G. Mesri. 1996. Soil mechanics in engineering practice. $3^{\text {rd }}$ edition. New York: Wiley-Interscience.

U.S. Army Engineer Waterways Experiment Station. 1984. Shore protection manual. $4^{\text {th }}$ ed., 2 Vol. Washington DC: U.S. Government Printing Office.

Weggel, J.R. 1972. Maximum breaker height. Journal of the Waterways, Harbors and Coastal Engineering Division 98(WW4): 529-548.

Widjaja, H., J. M. Duncan, and H. B.Seed. 1984. Scale and time effects in hydraulic fracturing. Miscellaneous Paper GL-84-10. Vicksburg, MS: U.S. Army Engineer Waterways Experiment Station.

Wolff, T.H. 1985. Analysis and design of embankment dam slopes: A probabilistic approach. Ph.D. diss., Purdue University, Lafayette, Ind.

Yucemen, M.S., W.H. Tang, and A.H.S. Ang. 1973. A probabilistic study of safety and design of earth slopes. Civil Engineering Studies, Structural Research Series 402, University of Illinois, Urbana. 


\section{Appendix A: Iterative Solution to Compute the Design Depth Using a Force and a Moment Imbalance Based Computational Procedure}

The design of an I-Wall/sheet-pile wall to resist flood loading requires the determination of (1) its depth of embedment and (2) its point of rotation (PR). This appendix highlights key aspects of the numerical procedure implemented in Corps_I-Wall by means of an application of Corps_I-Wall to the design illustrated of the Figure A1 cantilever I-Wall. ${ }^{1}$

Figure A1. Cantilever retaining wall. (a) Two layered soil site. (b) Counterclockwise rotation of the cantilever sheet-pile wall about its point of rotation (PR).

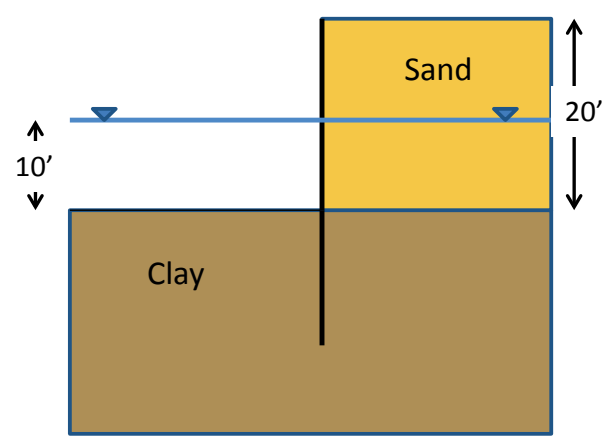

(a)

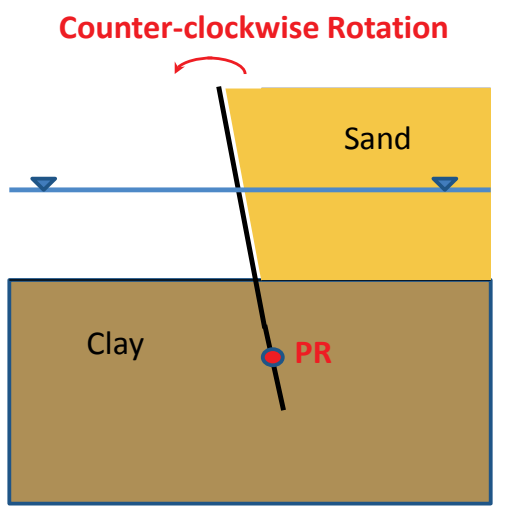

(b)

This design problem was first presented in Bowles (1968) and referred to as Example 8-2b. A closed-form solution was used by Bowles to determine its depth of embedment and PR. A comparison will be made between Corps_I-Wall numerical procedure results and those from the Bowles (1968) solution.

The Figure A1 design problem consists of a cantilever sheet-pile wall retaining sand that overlays a clay layer. The clay possesses undrained shear strength, $S_{u}$, equal to $1,200 \mathrm{psf}$. The cantilever wall retains a 20 -ft-high sand layer with an effective angle of internal friction, $\varphi^{\prime}$, equal to $30 \mathrm{deg}$ and an

\footnotetext{
${ }^{1}$ The design mode method of analysis is specified in this Corps_I-Wall analysis.
} 
effective interface friction, $\delta^{\prime}$, between the sheet-pile wall and the retained sand equal to zero degrees. A pool of water exists on the LHS of the cantilever wall to a height of $10 \mathrm{ft}$ above the top of the clay layer. A hydrostatic water table exists mid-height within the retained sand layer. Moist and saturated unit weights assigned in this problem are $110 \mathrm{pcf}$ and 122.4 pcf, respectively. The unit weight of water is $62.4 \mathrm{pcf}$. User-specified values of factors of safety are applied to the soil shear strengths when calculating both the active and passive earth pressures acting on the portion of the I-Wall/sheet-pile wall embedded within the soil foundation. Table A1 lists the factors of safety assigned to the layers of sand and clay.

Table A1. Factors of Safety assigned to the sand and clay layers.

\begin{tabular}{|l|l|l|}
\hline Soil Material & FSactive & FS passive \\
\hline Sand & 1.0 & 1.5 \\
\hline Clay & $1.5^{1}$ & 1.5 \\
\hline
\end{tabular}

1 This user assigned FS active value of 1.5 in the clay is not consistent with Corps' design criteria. This value is required so that the Corps_I-Wall results match the problem analyzed by Bowles (1968).

Two equations are required to solve for the two unknowns (i.e., depth of embedment and PR). At the precise value for depth of embedment and for the precise PR elevation, the summation of horizontal forces and summation of moments acting on vertical I-Wall/sheet-pile wall are in static equilibrium,

$$
\begin{aligned}
& \sum F_{h}=0 \\
& \sum M=0
\end{aligned}
$$

When imprecise values for depth of embedment and PR are assumed, the summation of horizontal forces and/or moments is nonzero.

A region-based (soil layer) geometry definition is implemented within Corps_I-Wall. This feature easily accommodates a multilayereded soil site. However, an iterative solution scheme is now required to perform these two sets of calculations (i.e., summation of externally applied forces and moments of Equations A1 and A2). 
Points along the center line of the vertical I-Wall/sheet-pile wall are discretized at approximately every foot ${ }^{1}$ into nodal points and then connected sequentially by a series of vertical I-Wall/sheet-pile wall line elements. The (1) net water pressures and (2) net soil pressure distributions acting normal to the vertical I-Wall/sheet-pile wall line model, along with (3) the net external horizontal forces (or pressures), and (4) horizontal forces due to external overburden pressures acting on an elevation behind the sheet-pile wall, are each converted into equivalent nodal point forces using the one-third/one-sixth relationship discussed in Chapter 2. These computations are performed for each trial depth of embedment and for each trial PR value.

The iterative solution developed for Corps_I-Wall is a numerical method for determining the (1) depth of embedment and (2) PR values corresponding to static equilibrium (i.e., satisfying Equations A1 and A2). The solution procedure starts with the assignment of a shallow trial depth of embedment and a shallow trial PR value. For this first trial depth of embedment and first shallow trial PR, the summations of horizontal forces as well as the summation of moments are computed along the sheet-pile wall. A large force and moment imbalance are usually achieved. For the second computation, the (first) trial depth of embedment is maintained while the trial PR point is moved down a foot, the net forces are reformulated (because the PR value has changed) and the summation of horizontal forces and the summation of moments are computed along the sheet-pile wall. The PR point is then moved down another foot if there is a force and moment imbalance. The process is repeated until the trial PR is lowered and there is a change in sign for the force and moment imbalance. When the trial PR has moved $1 \mathrm{ft}$ above the tip of the sheet pile and there is still no change in sign, then the trial depth of embedment is increased by $1 \mathrm{ft}$ and the entire computational process is repeated, starting over with a shallow trial $P R$ value.

The trial PR value and trial depth of embedment are considered to be converged in the calculations when both Equations A1 and A2 are satisfied or have small, non-zero values.

The following subsections further discuss details regarding the numerical procedures being used when applied to the Figure A1 cantilever wall

\footnotetext{
1 The nodal point discretization may be revised in subsequent versions.
} 
problem. Although the summation of forces and summation of moment computations are done concurrently within Corps_I-Wall, the results from the summation of horizontal force calculations are discussed first. This is followed by a discussion of moment imbalance for these same pair of variables. Further details on arriving at the resulting design depth of embedment are discussed last.

\section{A.1 Summation of horizontal forces}

A trial depth of embedment is first established; $9 \mathrm{ft}$. Next the elevation for the first, shallow, trial value for PR is assigned; $-2 \mathrm{ft}$. The total horizontal force at each nodal point elevation (at approximately $1-\mathrm{ft}$ increments), acting normal to the sheet-pile wall is accumulated down to the trial depth of embedment of $9 \mathrm{ft}$. This results in an imbalance of nearly 15,00o lb per $\mathrm{ft}$ run of wall. The trial value for PR is next lowered by $1 \mathrm{ft}$ to $-3 \mathrm{ft}$. The imbalance in horizontal forces reduces to approximately $12,000 \mathrm{lb}$ per $\mathrm{ft}$ run of wall. This resultant summation of horizontal forces is now compared to the value of the previous resultant summation of horizontal forces for the previous trial depth of embedment. If there is a sign change, this means that the summation of horizontal forces has reached a value that is close to zero and this iteration has converged. The dark blue line of data in Figure A2 shows that there was no sign change (in force imbalance) until the trial PR value was lowered to $-7 \mathrm{ft}$ ( $2 \mathrm{ft}$ above the trial sheet-pile tip depth). When this change in sign is achieved, the incremental increase in trial PR values is terminated for the trial depth of embedment (that has been held constant).

This process of varying trial PR values is repeated for the next trial depth of embedment, $10 \mathrm{ft}$. The results are added to Figure A2 (orange line of data).

Trial depths of embedment equal to $11,12,13,14$, and $15 \mathrm{ft}$ are also examined with these results added to Figure A2. A total of seven trial depths of embedment were investigated. Table A2 lists the elevations that straddle the zero summation of horizontal force crossings for the trial PR values for each trial depth of embedment. Each label in this table signifies the trial depth of embedment (in feet). ${ }^{1}$

\footnotetext{
1 It is anticipated that this aspect of the numerical procedure and size of the elements will be refined in subsequent versions.
} 
Figure A2. Schematic of summation of horizontal forces versus trial points of rotation.

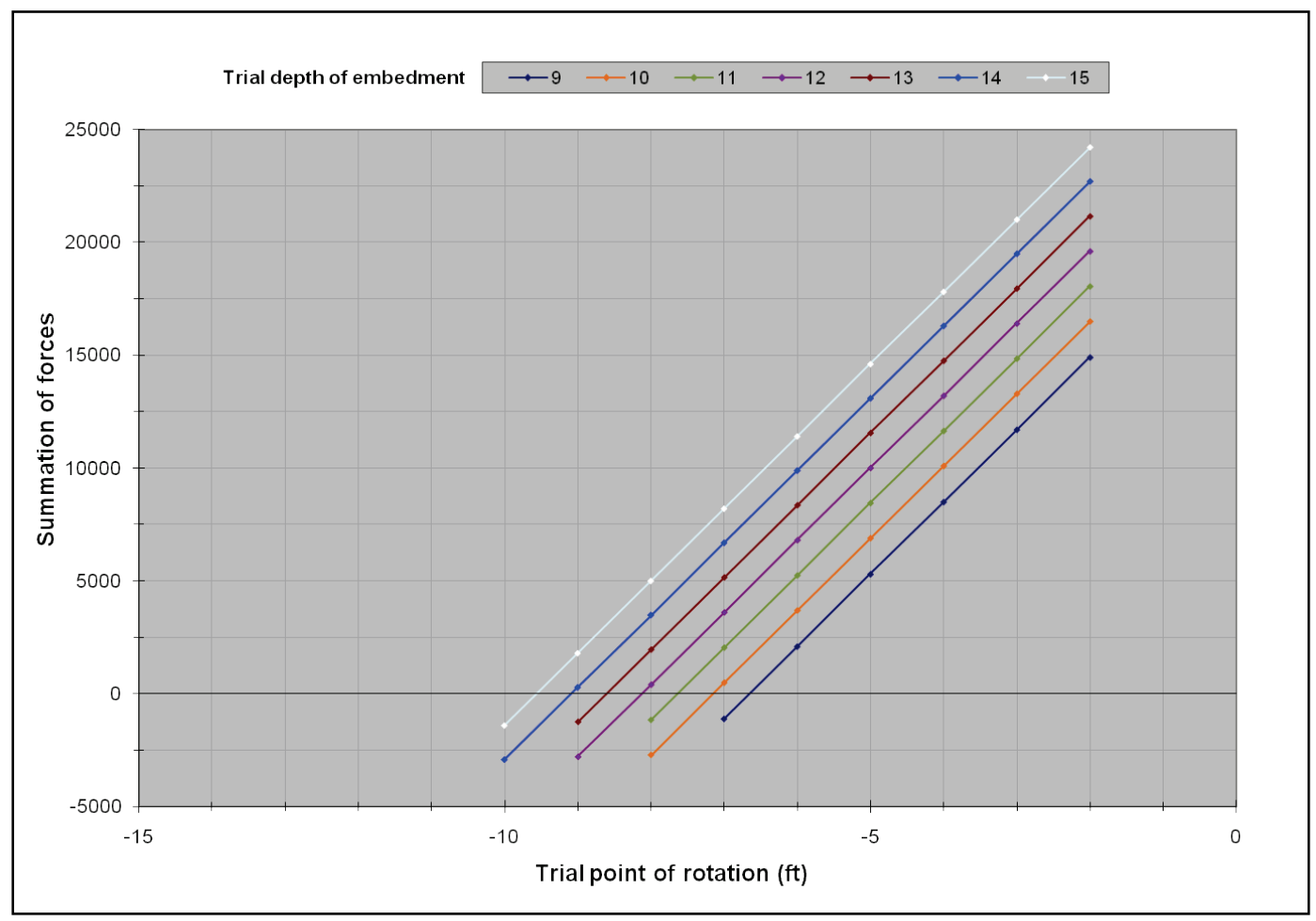

Table A2. PR elevations bounding the zero crossings for summation of forces.

\begin{tabular}{|c|l|l|}
\hline Label & Current Elevation $(\mathrm{ft})$ & Previous Elevation $(\mathrm{ft})$ \\
\hline 9 & -7 & -6 \\
\hline 10 & -8 & -7 \\
\hline 11 & -8 & -7 \\
\hline 12 & -9 & -8 \\
\hline 13 & -9 & -8 \\
\hline 14 & -10 & -9 \\
\hline 15 & -10 & -9 \\
\hline
\end{tabular}

\section{A.2 Summation of moments}

For the trial depth of embedment and the trial PR value, the summation of moment computation is made immediately following the summation of forces computation discussed in the previous A.1 section. The summation of moments is calculated as the product of the total horizontal force at each nodal point elevation (at approximately 1 -ft increments) on the sheet-pile wall and its lever arm, about the trial point of rotation, and accumulated down to the trial depth elevation. During this stage of analyses, after the moment imbalance has been made for two consecutive trial PR values, the resultant summation of moments is compared to the value of the previous PR's resultant summation of moments. If there is a sign change, this 
signifies that the summation of moments has reached a value that is close to zero and that this iteration has converged. Figure A3 shows the results of the same seven iterations of Figure A2 for trial depths of 9 thru $15 \mathrm{ft}$ at $1-\mathrm{ft}$ increments. Each trial PR curve in Figure A3 starts at elevation - $2 \mathrm{ft}$ and proceeds down to $1 \mathrm{ft}$ above its trial depth of embedment. This figure shows that all of the seven curves have crossed the summation of moments (imbalance) line. These PR values that bound the cross-over PR value for each of the seven trial depths of embedment (from 9 to $15 \mathrm{ft}$ ) have been tabulated and are listed in Table A3. Each label in this table signifies the trial depth of embedment (in feet).

Figure A3. Schematic of summation of moments versus trial points of rotation.

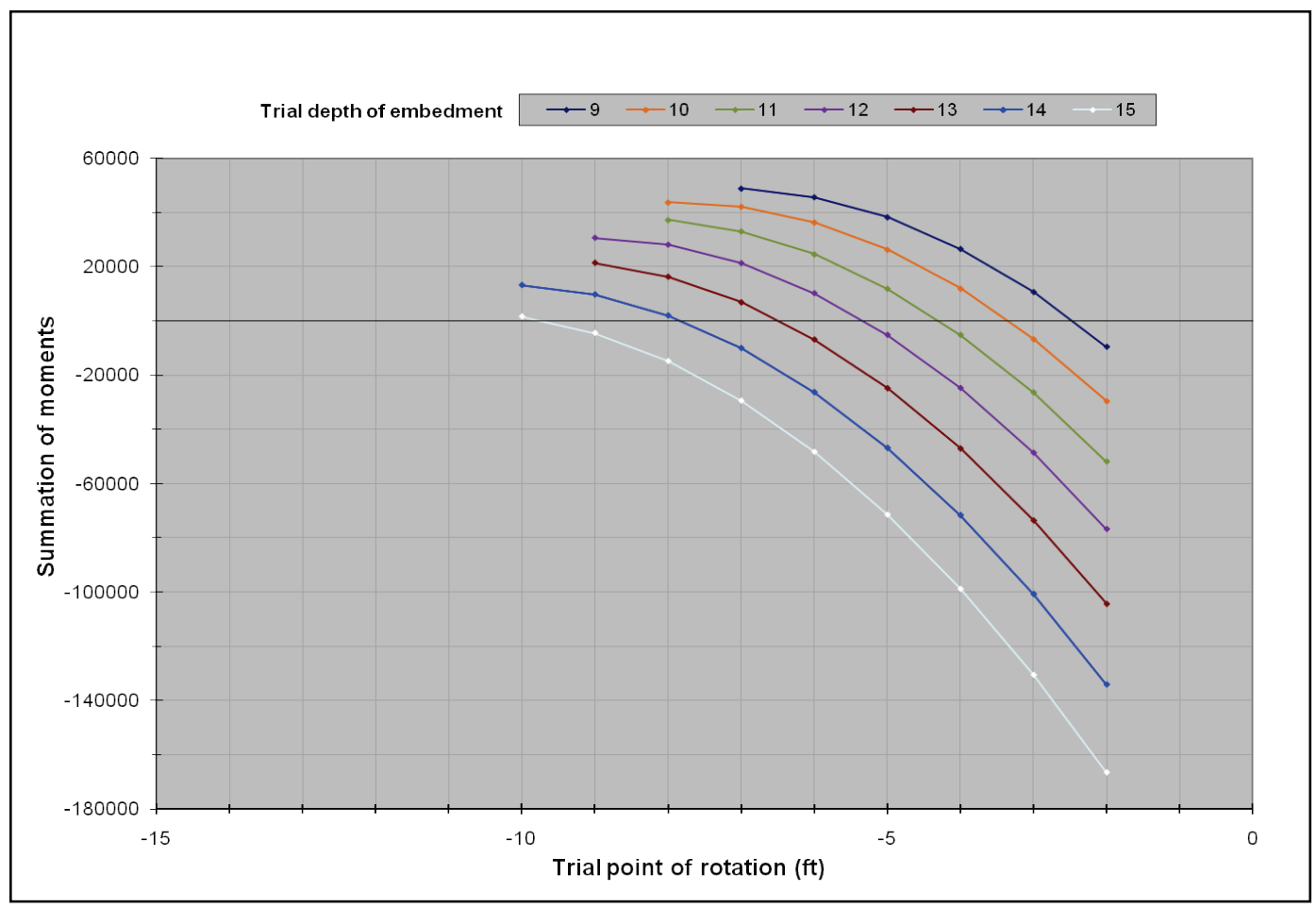

Table A3. PR elevations bounding the zero crossings for summation of moments.

\begin{tabular}{|c|l|l|}
\hline Label & Current Elevation & Previous Elevation \\
\hline 9 & -3 & -2 \\
\hline 10 & -4 & -3 \\
\hline 11 & -5 & -4 \\
\hline 12 & -6 & -5 \\
\hline 13 & -7 & -6 \\
\hline 14 & -8 & -7 \\
\hline 15 & -10 & -9 \\
\hline
\end{tabular}




\section{A.3 Resulting design depth of embedment}

The resulting design depth of embedment is arrived at when (1) the summation of forces converge at a trial PR value, and (2) the summation of moments also converge at the same trial PR value for the same iteration. Corps_I-Wall achieves this in two stages: (1) an approximation is made for the trial depth of embedment and for a pair of trial PR values in the first stage of analysis, and (2) the next stage of analysis uses the Stage 1 data as initial conditions to a Newton-Raphson procedure that performs a refined search within close proximity of this resulting Stage 1 set of data.

Stage 1: For force equilibrium, as shown in Table A2, for a trial depth of embedment equal to $15 \mathrm{ft}$ (i.e., label 15 in Table A2) or the light blue curve in Figure A2, the PR convergence occurs between els -9 $\mathrm{ft}$ (1805 lbf) and $-10 \mathrm{ft}(-13.95 \mathrm{lbf})$. For moment equilibrium, for a trial depth of embedment equal to $15 \mathrm{ft}$ (i.e., label 15 in Table A3) or the light blue curve in Figure A3, the PR convergence occurs between els of $-9 \mathrm{ft}(-4543.89 \mathrm{ft}-\mathrm{lb})$ and $-10 \mathrm{ft}$ (1527.78 ft-lb). This signifies that both Equations A1 and A2 have been satisfied for the design depth of approximately $15 \mathrm{ft}$ and a point of rotation between $9 \mathrm{ft}$ and $10 \mathrm{ft}$. This completes Stage 1 evaluation.

Note: If the two conditions of Equations A1 and A2 are not met for the same trial depth of embedment, the iterative procedure will restart at the top of the sheet-pile wall and the initial node spacing of 1 - $\mathrm{ft}$ increments used to determine elevations for computations on the sheet-pile wall will be changed by the node spacing increment factor or 0.01-ft increments. ${ }^{1}$ Therefore, the nodal point spacing will decrease from 1.0 to 0.99 and static equilibrium will be tested from Stage 1 evaluations. This process continues until static equilibrium is satisfied or the minimum node spacing of 0.7 is reached.

Stage 2: Once the resulting Stage 1 set of data is defined (coming close to satisfying static equilibrium), the trial depth of embedment and trial PR are presented as initial values to a Newton-Raphson procedure (contained within Corps_I-Wall), which will then numerically approximate the resultant depth of embedment and PR values by iteratively solving the expressions of Equations A1 and A2. The computed point of rotation is

\footnotetext{
1 It is anticipated that this numerical procedure and size of the elements will be refined in subsequent versions.
} 
equal to $-9.5391 \mathrm{ft}$ and the computed elevation at tip of pile is equal to $-14.9472 \mathrm{ft}$.

Bowles (1968) solved a closed-form equation for the depth of embedment and obtained a value of $15.1 \mathrm{ft}$. The Corps_I-Wall numerical solution agrees with Bowles. 


\section{Appendix B: Gap Initiation and Propagation Criteria and Formulation}

Hydraulic fracturing criteria is applied to determine if a gap develops and if it propagates down the soil-to-sheet-pile/I-Wall interface. ${ }^{1}$ In this procedure of analysis, the horizontal earth pressure ${ }^{2}$ (i.e., a value of total horizontal stress) at the ground surface soil-to-sheet-pile/I-Wall interface on the flood side of the wall, is compared to the hydrostatic water pressure developing at the top of the ground surface due to the presence of the (specified) flood pool elevation. If the hydrostatic water pressure of the flood pool (i.e., the demand) exceeds the total horizontal stress (i.e., the capacity) at the ground surface, a gap will initiate at the ground surface along the soil-to-sheet-pile/I-Wall interface. 3 After hydraulic fracturing commences (at the ground surface), the next or lower element of soil interface at the soil-to-sheet-pile/I-Wall interface 4 is then checked for hydraulic fracturing. Its total horizontal stress is compared to a hydrostatic water pressure for the same flood pool elevation that initiated/propagated a gap above the top of this current soil interface element in question. If the hydrostatic water pressure of the flood pool exceeds the total horizontal stress at this soil interface element, a gap will propagate through this element and down to the next soil interface element along the soil-to-sheetpile/I-Wall interface. This check for gap propagation proceeds until a depth is reached for which the total horizontal stress at the soil-to-sheetpile/I-Wall interface (i.e., capacity) exceeds the hydrostatic water pressure of the flood pool (i.e., demand) at this same elevation, and thus, the gap is arrested by the horizontal total stresses contained within that soil element.

\footnotetext{
1 This hydraulic fracturing type of analysis was first adapted in 2005 by the primary author of this report when analyzing gap initiation/propagation responses of New Orleans I-Walls during hurricane Katrina (Volume V of IPET 2007), from the procedure used to estimate the potential for "crack" formation in earthen dams (e.g., Nobari et al. 1973; Widjaja et al. 1984; Sherard 1973, 1986; and the Independent Panel to Review Cause of Teton Dam Failure 1976) and in particular, in a clay core.

2 In many instances the total horizontal earth pressure is based on the horizontal component of the "mobilized active" earth pressure at the ground surface. However, under circumstances involving a shallow point of rotation for the sheet-pile/I-Wall, the horizontal component of the "mobilized passive" earth pressure at the ground surface may be used. This issue will be discussed in detail within this appendix.

3 Zero tensile strength capability is assumed along this soil-to-sheet-pile/I-Wall interface.

4 This initial Corps_I-Wall, Version 1.0, checks for gap propagation using trial gap depths progressing in 1-ft increments along the soil-to-sheet-pile/I-Wall interface. Should convergence not be achieved, the gap prorogation process starts over using a $0.01-\mathrm{ft}$ increment. It is anticipated that this numerical procedure and size of the soil interface elements will be refined in subsequent versions.
} 
The formulation used in Corps_I-Wall, Version 1.o, assumes the sheetpile/I-Wall rotates as a rigid body about some point along the embedded portion of the I-Wall. This point falls somewhere between the ground surface and the tip of the sheet-pile and is designated as the PR. The value for PR is an unknown variable for both the Design mode as well as for the Analysis mode in Corps_I-Wall.

The first two sections, B.1 and B.2, describe the gap formulation and propagation criteria when the PR is below the trial soil-to-sheetpile/I-Wall soil interface element. The last two sections, B.3 and B.4, describe the formulation used when the PR is at or above the trial soil-tosheet-pile/I-Wall interface gap depth.

A gap is developed and/or determined to propagate by a total stress gap criteria, such that,

$$
\begin{array}{cl}
\text { Gap Propagation Criteria: } & \text { Demand }>\text { Capacity } \\
\text { Demand: } & \gamma_{w} * \text { Dept }_{w} \\
\text { Capacity: } & \sigma_{h}
\end{array}
$$

where

$$
\begin{aligned}
\gamma_{w}= & \text { unit weight of water } \\
\text { Depth }_{w}= & \text { depth of water to the center of the trial soil-to-sheet-pile/ } \\
& \text { I-Wall soil interface element } \\
\sigma_{h}= & \text { mobilized total stress for defining the horizontal earth } \\
& \text { pressure at the center of the trial soil-to-sheet-pile/I-Wall soil } \\
& \text { interface element gap depth }
\end{aligned}
$$

Note: Mobilized parameters are stated throughout this appendix and are assigned (via soil material properties) to each soil layer on either side of the I-Wall. The user-defined values for the active Factor of Safety and for the passive Factor of Safety define the level of mobilization, as discussed in Chapter 2.

\section{B.1 Total stress gap criteria}

Total stress gap criteria are based on the assumptions that (1) the Wall rotates (away) from the flood side and (2) PR is below the current gap 
depth. Figure B1 provides the schematic for the total stress gap criteria for this counterclockwise I-Wall rotation situation.

Figure B1. Schematic of gap propagation for a soil element above the point of rotation with a mobilized total horizontal earth pressure definition.

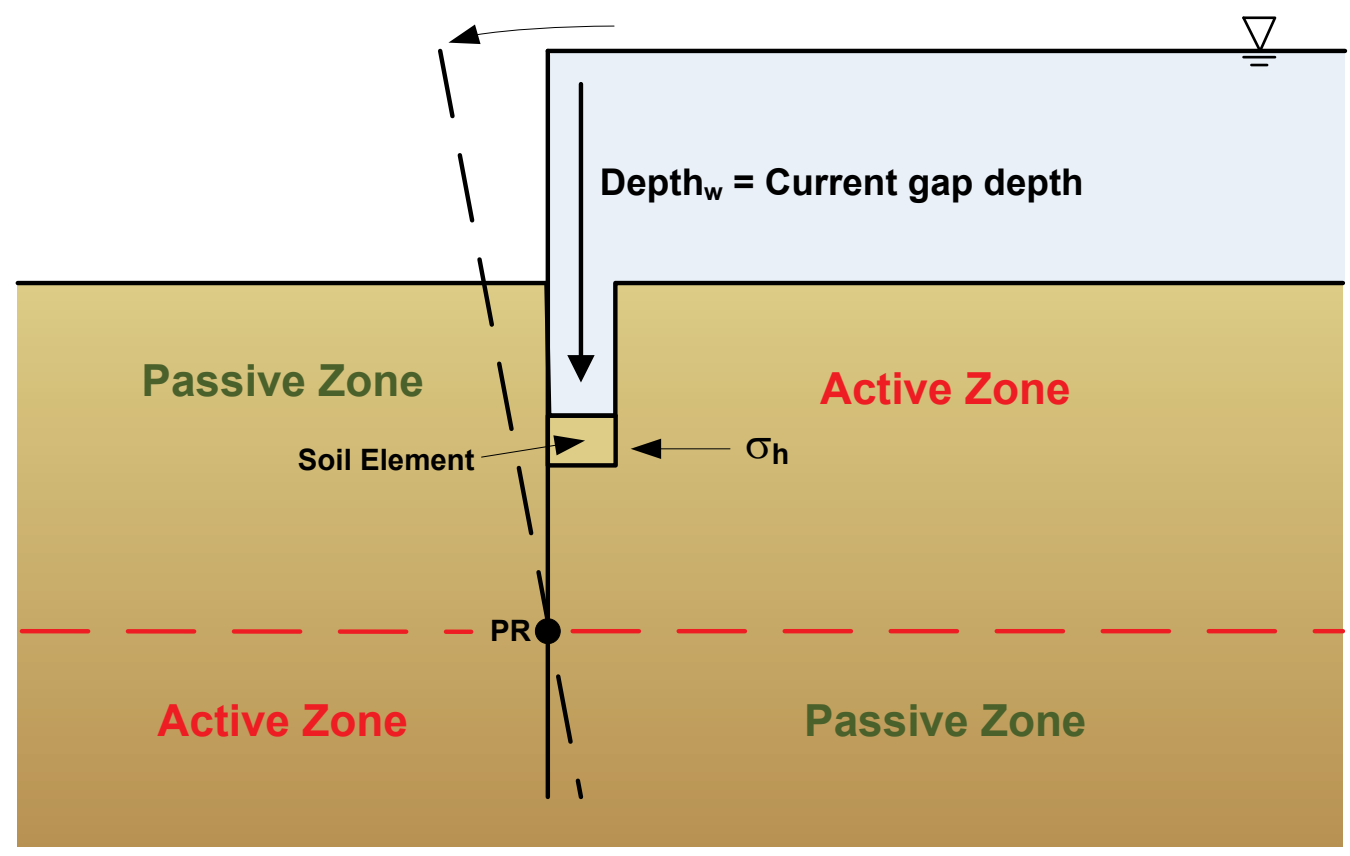

In soils, the analytical solution for the active earth pressure equation is given as

$$
\sigma_{a}=\sigma_{v} K_{a}-2 c_{a} \sqrt{K_{a}}
$$

where $\sigma_{a}$, is the mobilized active earth pressure, $\sigma_{v}$, is the overburden pressure, $c_{a}$, the mobilized active cohesion(total stress), and $K_{a}$, the mobilized active earth pressure coefficient, which can be calculated using one of the earth pressure relationships given in Chapter 2. The mobilized horizontal active stress or horizontal component of the mobilized active earth pressure is expressed as

$$
\sigma_{h}=\sigma_{a} \cos \left(\delta_{a}\right)
$$

with $\delta_{a}$ defined as the mobilized active wall friction angle (total stress). From the two assumptions and Equations B1 through B3, the gap initiation and propagation criteria are 


$$
\gamma_{w} * \operatorname{Depth}_{w}>\sigma_{h}
$$

This can be expanded using Equations $\mathrm{B}_{4}$ and $\mathrm{B}_{5}$ to obtain the total stress gap initiation and propagation criteria of

$$
\gamma_{w} * \operatorname{Depth}_{w}>\left[\sigma_{v} K_{a}-2 c_{a} \sqrt{K_{a}}\right] \cos \left(\delta_{a}\right)
$$

\section{B.2 Total stress gap criteria but using effective stress and pore water pressure to define total stress}

Total stress gap criteria is based on the assumptions that (1) the wall rotates (away) from the flood side and (2) the point of rotation is below the current gap depth. Figure B2 provides the schematic for the total stress gap criteria but using effective stress and pore water pressure to define total stress for this counterclockwise I-Wall rotation situation.

Figure B2. Schematic of gap propagation for a soil element above the point of rotation with a mobilized effective horizontal earth pressure definition.

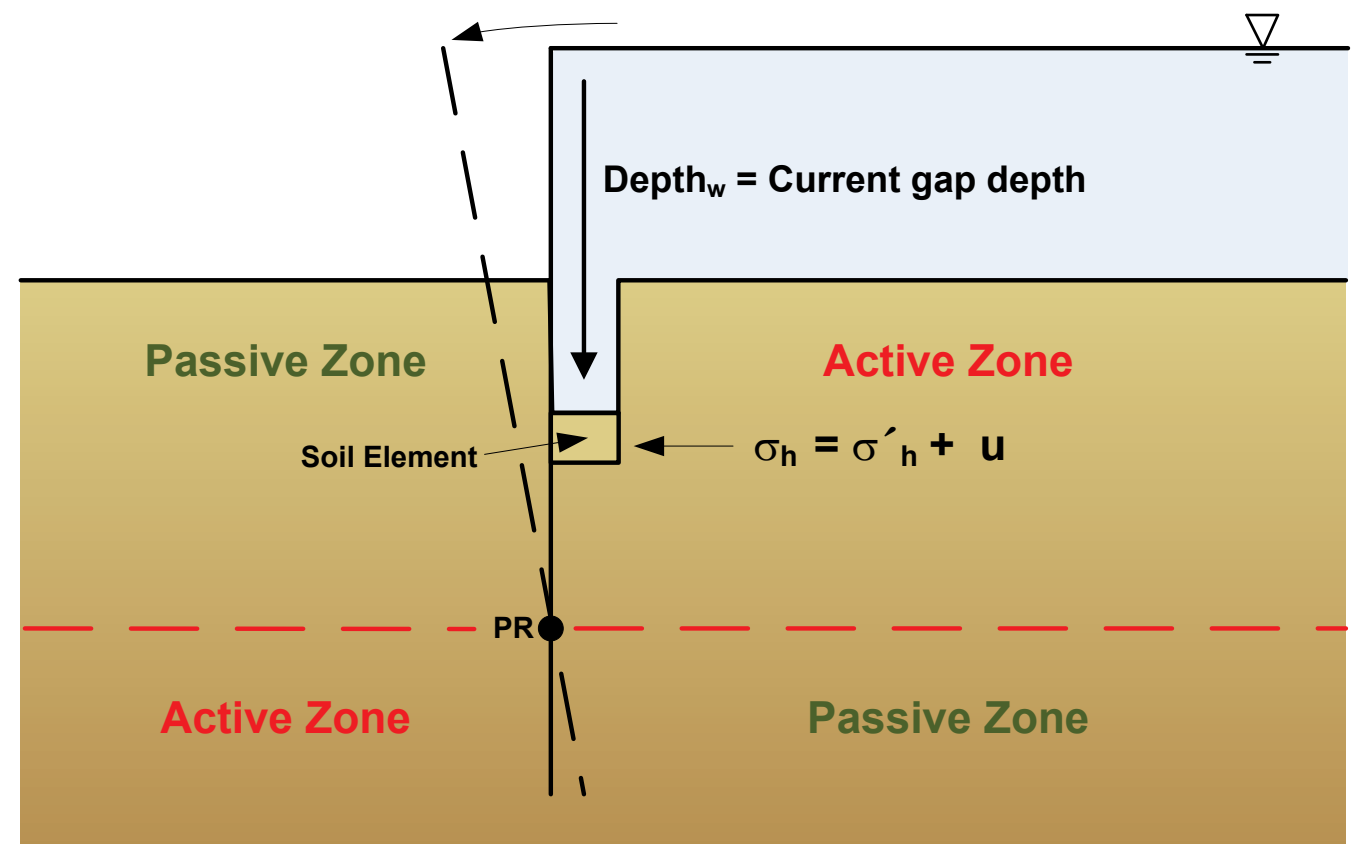

In soils, the analytical solution for gap propagation criteria is based on total stress, given previously as

$$
\gamma_{w} * \operatorname{Depth}_{w}>\sigma_{h}
$$


The mobilized total stress, $\sigma_{h}$, and the overburden pressure, $\sigma_{v}$, may both be rewritten for an effective stress analysis, respectively as,

$$
\begin{aligned}
& \sigma_{h}=\sigma_{h}^{\prime}+u \\
& \sigma_{v}=\sigma_{v}^{\prime}+u
\end{aligned}
$$

where $\sigma_{h}^{\prime}$ is the effective mobilized horizontal stress, and $u$, the pore water pressure in soil. The mobilized effective horizontal stress is given as

$$
\sigma_{h}^{\prime}=\sigma_{a}^{\prime} \cos \left(\delta_{a}^{\prime}\right)
$$

with $\sigma_{a}^{\prime}$ as the effective mobilized active earth pressure, $\delta_{a}^{\prime}$, the effective mobilized active wall friction angle. The effective mobilized active earth pressure equation can be defined as follows

$$
\sigma_{a}^{\prime}=\sigma_{v}^{\prime} K_{a}-2 c_{a}^{\prime} \sqrt{K_{a}}
$$

with $c_{a}^{\prime}$ defined as the effective mobilized active cohesion. Equations B10 and $\mathrm{B} 11$ can be substituted into Equations B6 and B8 to produce the following equation

$$
\gamma_{w} * \operatorname{Depth}_{w}>\sigma_{a}^{\prime} \cos \left(\delta_{a}^{\prime}\right)+u
$$

that can be further expanded with Equations B9 and B11 as

$$
\gamma_{w} * \operatorname{Depth}_{w}>\left[\left(\sigma_{v}-u\right) K_{a}-2 c_{a}^{\prime} \sqrt{K_{a}}\right] \cos \left(\delta_{a}^{\prime}\right)+u
$$

This equation expresses the total stress gap initiation and propagation criteria with a mobilized effective horizontal earth pressure definition.

\section{B.3 Total stress gap criteria with shallow point of rotation}

The total stress gap criteria in this section is based on the assumptions that (1) the wall rotates (away) from the flood side and (2) the point of rotation is at (or above) the current gap depth. Figure B3 illustrates this condition for this counterclockwise I-Wall rotation situation. 
Figure B3. Schematic of gap propagation for a soil element located at the point of rotation with a mobilized total horizontal earth pressure definition.

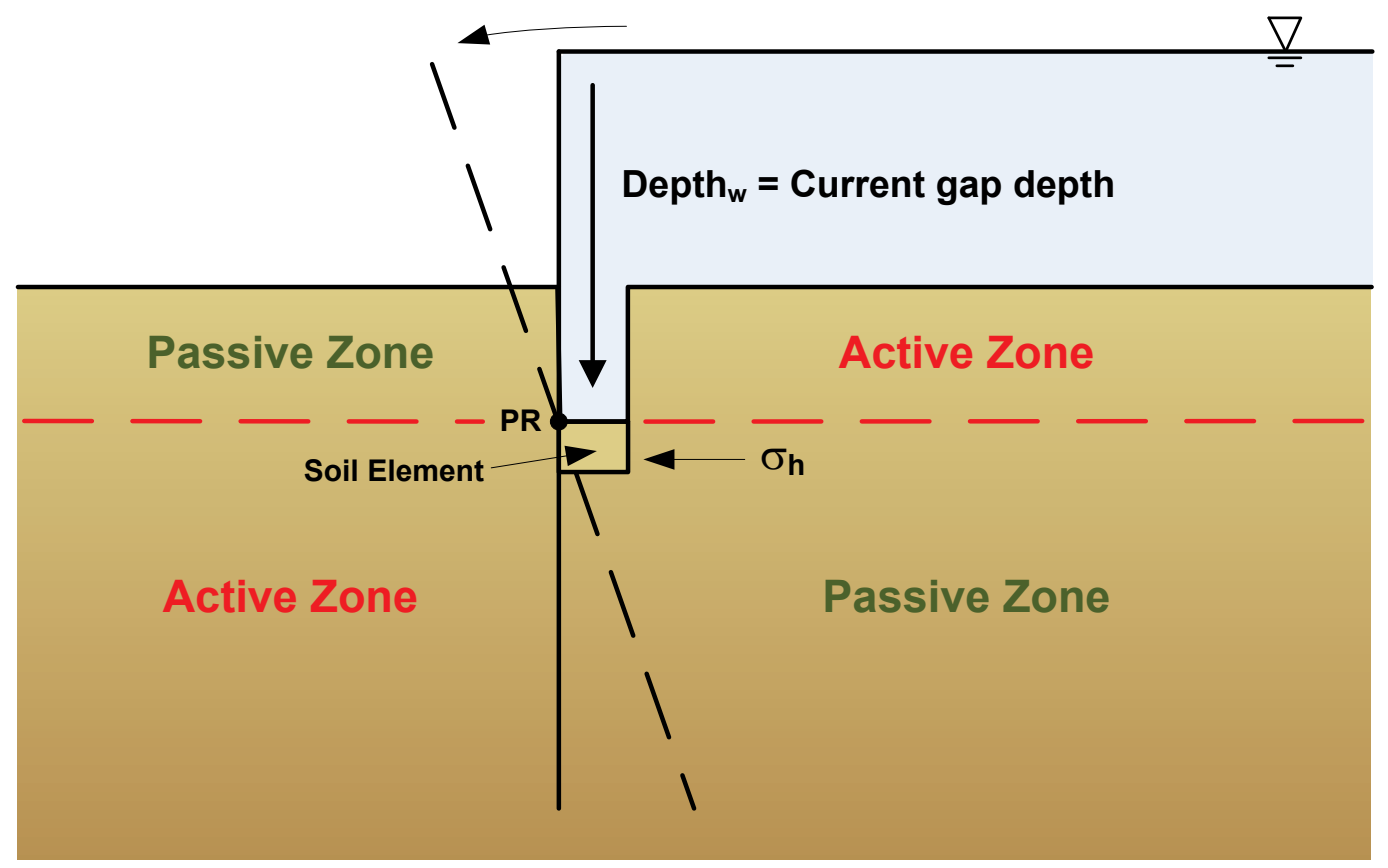

For the soil element just below the PR and on the flood side of the wall, the horizontal stress, located in the passive zone of Figure B3, is expressed as the mobilized passive earth pressure equation and given as

$$
\sigma_{h}=\sigma_{p} \cos \left(\delta_{p}\right)
$$

with $\sigma_{p}$, the mobilized passive earth pressure, and $\delta_{p}$, the mobilized passive wall friction angle. The mobilized passive earth pressure can be expressed as

$$
\sigma_{p}=\sigma_{v} K_{p}+2 c_{p} \sqrt{K_{p}}
$$

where $c_{p}$, the mobilized passive cohesion, and $K_{p}$, the mobilized lateral passive earth pressure, which can be calculated using the earth pressure relationships given in Chapter 2. From Equations B1 through B3, there is

$$
\gamma_{w} * \operatorname{Depth}_{w}>\sigma_{h}
$$

that can be further expanded using Equations B14 and B15 for the total stress gap criteria with a shallow point of rotation

$$
\gamma_{w} * \operatorname{Depth}_{w}>\left[\sigma_{v} K_{p}+2 c_{p} \sqrt{K_{p}}\right] \cos \left(\delta_{p}\right)
$$


This equation expresses the total stress gap initiation and propagation criteria.

\section{B.4 Total stress gap criteria but using effective stress and pore water pressure to define total stress and with shallow point of rotation}

The total stress gap criteria in this section is based on the assumptions that (1) the wall rotates (away) from the flood side and (2) the point of rotation is at (or above) the current gap depth. Figure B4 provides the schematic for the total stress gap criteria but using effective stress and pore water pressure to define total stress for this counterclockwise I-Wall rotation situation.

Figure B4. Schematic of gap propagation for a soil element located above the point of rotation with a mobilized effective horizontal earth pressure definition.

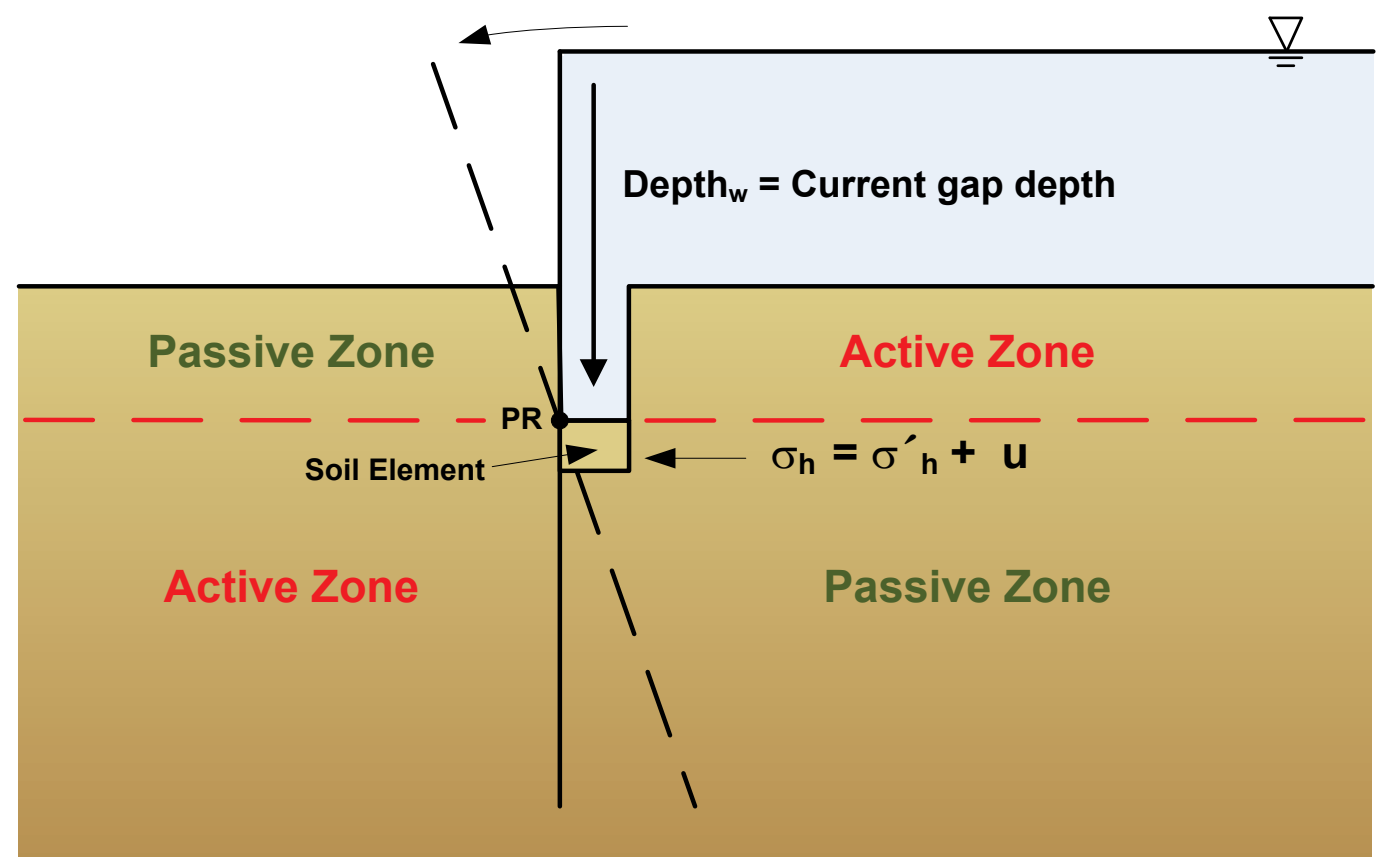

For the soil element just below the PR and on the flood side of the wall, the horizontal stress, located in the passive zone of Figure B4, is expressed as the mobilized passive earth pressure equation and given as

$$
\sigma_{h}^{\prime}=\sigma_{p}^{\prime} \cos \left(\cos \delta_{p}^{\prime}\right)
$$


with $\sigma_{p}^{\prime}$ is the effective mobilized passive earth pressure, $\delta_{p}^{\prime}$, the effective mobilized passive wall friction angle. The effective mobilized passive earth pressure equation can be defined as follows

$$
\sigma_{p}^{\prime}=\sigma_{v}^{\prime} K_{p}+2 c_{p}^{\prime} \sqrt{K_{p}}
$$

with $c_{p}^{\prime}$ defined as the effective mobilized passive cohesion. Equations B17 and $\mathrm{B} 18$ can be substituted into Equations B1 and B8 to produce the following equation

$$
\gamma_{w} * \operatorname{Depth}_{w}>\sigma_{p}^{\prime} \cos \left(\delta_{p}^{\prime}\right)+u
$$

that can be further expanded with Equations B9 and B18 as

$$
\gamma_{w} * \operatorname{Depth}_{w}>\left[\left(\sigma_{v}-u\right) K_{p}+2 c_{p}^{\prime} \sqrt{K_{p}}\right] \cos \left(\delta_{p}^{\prime}\right)+u
$$

This equation expresses the total stress gap initiation and propagation criteria with a mobilized effective horizontal earth pressure definition. 


\section{Appendix C: Line of Seepage Formulation}

This appendix discusses the line of seepage (a.k.a., line of creep) associated with the one-dimensional (1-D) seepage analysis where the seepage gradient and length of seepage are to be determined first. The results from this simplified method of analysis are then used for determining water pressures acting within the soil adjacent to the vertical sheet-pile wall and acting normal to both flood side and landside embedded faces of this sheet-pile wall. Water will travel from high (total) head to low (total) head, dictating the direction of flow within the soil regions. Consequently, the side with the highest (total) head dictates that the direction of flow is downward along that side of the sheet-pile wall. A vertical streamline, or flow line, is assumed within the soil and adjacent to the sheet-pile wall. Water flows within the soil, downward and parallel to the face of the vertical sheet-pile wall, to the sheet-pile tip. At the sheetpile tip the vertical flow of water reverses direction (by $180 \mathrm{deg}$ ) and travels up through the soil along the opposite face of the sheet-pile wall.

The transformation procedure for the line of seepage method is for 1-D flow along a singular path consisting of 1-D flow paths connected in series from one soil region on into the next soil region that are aligned along the two faces of the vertical sheet-pile wall. These flow paths contained within the soil regions may possess different values for the saturated hydraulic conductivity of the soil. For example, Figure $\mathrm{C} 1$ contains three unique soil regions. Each flow line contained within each soil region has a unique value for its saturated hydraulic conductivity, $K$. The hydraulic conductivity of the soil Regions 2 and $3\left(K_{2}\right.$ and $\left.K_{3}\right)$ are greater than the hydraulic conductivity of the soil contained within soil Region $1\left(K_{1}\right)$. Consequently, a transformation is required in order to first construct a 1-D flow regime down one face of the sheet-pile wall and up the opposite face of the sheet-pile wall in order to represent a 1-D flow regime of constant hydraulic conductivity of value $K_{1}$. 
Figure C1. I-Wall. (a) Potential slip plane within soil regions with different saturated hydraulic conductivities ( $K$ ) (lengths not to scale). (b) Constant slope in total head with distance along the transformed length of line of seepage. (c) Variation of slope in total head with distance along the three soil regions of the line of seepage.

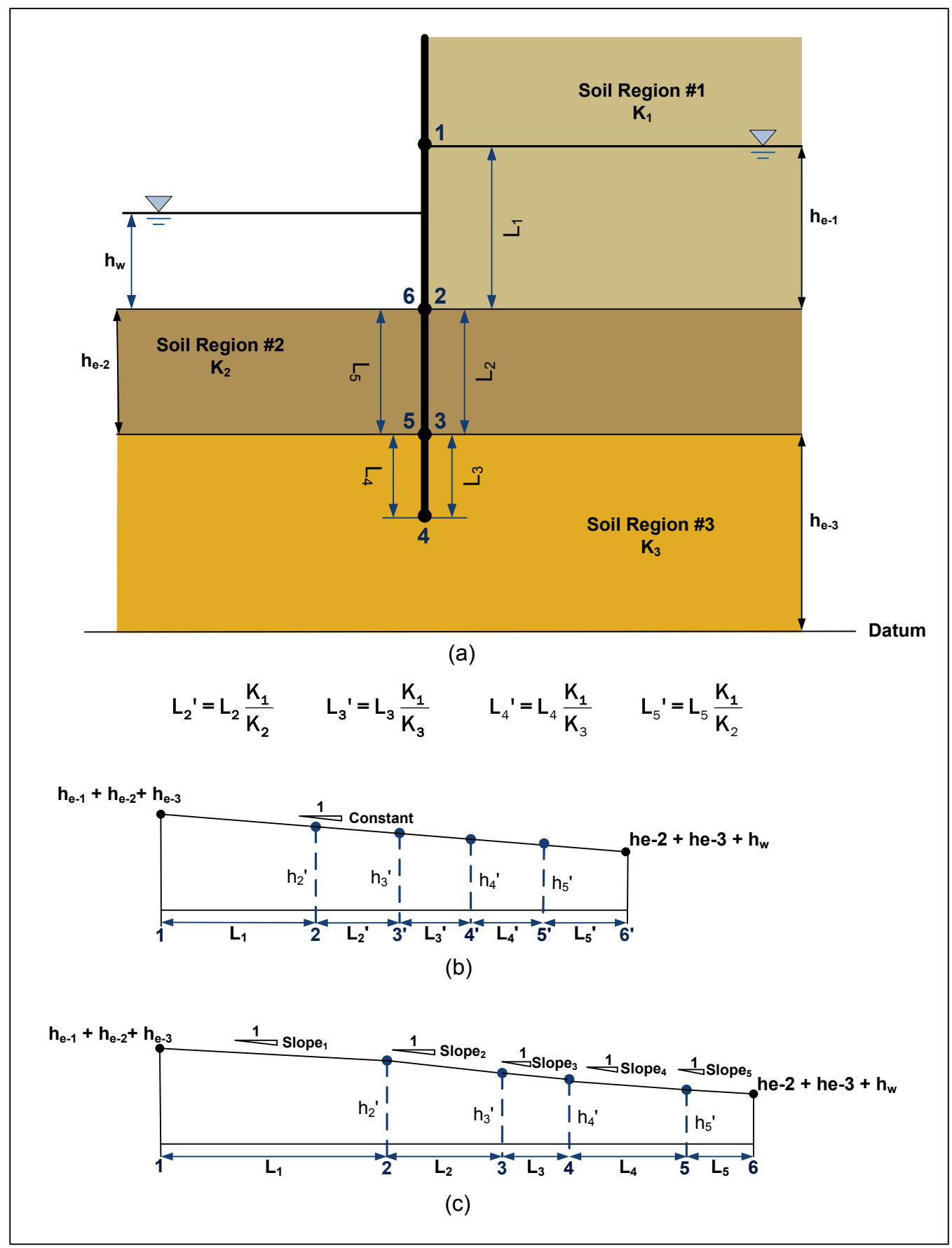

The transformed horizontal axis is first constructed (Figure $\mathrm{C} 1 \mathrm{~b}$ ) using the four transformed lengths $L_{2}{ }^{\prime}, L_{3}{ }^{\prime}, L_{4}{ }^{\prime}$ and $L_{5}{ }^{\prime}$ by means of the equations given in the figure. Note that starting with the second 1-D flow path (of transformed length $L_{2}{ }^{\prime}$ ), there is a transformed position denoting the end 
of 1-D flow path 2, designated as point 3'. With $K_{2}$ being greater than $K_{1}$, the length of the transformed segment $L_{2}{ }^{\prime}$ is less than the physical distance from points 2 to 3 (i.e., the height of soil Region 2), $L_{2}$. Similarly for 1-D flow Path 3 of transformed length $L_{3}{ }^{\prime}$, its starting and ending transformed points are 3 ' and 4' respectively. With $K_{3}$ being greater than $K_{1}$, the length of the transformed segment $L_{3}$ ' is less than the physical distance from points 3 to $4, L_{3}$. Because the transformed 1-D line of flow model now possesses a constant hydraulic conductivity of value $K_{1}$, the variation in head along the transformed section is linear (see Figure 12-1 in Spangler and Handy 1973). There exists a linear variation in total head from point 1 to point 6 ' as denoted in Figure $\mathrm{C} 1 \mathrm{~b}$ for this transformed 1-D line of flow. The gradient is defined as equal to the change in total head (from point 1 to point 6 ') divided by the total length of the transformed 1-D line of flow $\left(=L_{1}+L_{2}{ }^{\prime}+L_{3}{ }^{\prime}+L_{4}{ }^{\prime}+L_{5}{ }^{\prime}\right)$. Also at points 2 and $3^{\prime}$ of Figure C1b the value for total head is obtained and is designated as $h_{2}{ }^{\prime}$ and $h_{3}{ }^{\prime}$, respectively, computed by taking advantage of the key feature of the Figure C1b, which shows that the hydraulic gradient is a known constant. The total head values $h_{2}{ }^{\prime}$ and $h_{3}{ }^{\prime}$ are easily obtained from a linear relationship in total head with distance between Figure C1b points 1 and 6'.

Figure C1c shows the original axis for the problem using the actual dimensions (labeled $\mathrm{L}_{1}, \mathrm{~L}_{2}, \mathrm{~L}_{3}, \mathrm{~L}_{4}$ and $\mathrm{L}_{5}$ ) for the entire length of the 1-D streamline contained within the three soil regions and along the front and back vertical faces of the embedded sheet-pile wall. The values for total head are mapped from points 1, 2, 3', 4', 5' and 6' from Figure C1b, to points 1, 2, 3, 4, 5 and 6 in Figure C1c. Observe that the transformed Figure $\mathrm{C} 1 \mathrm{~b}$ is required to compute the head values $h_{2}{ }^{\prime}, h_{3}{ }^{\prime}, h_{4}{ }^{\prime}$, and $h_{5}{ }^{\prime}$ at the transformed position points of $2,3^{\prime}, 4^{\prime}, 5^{\prime}$ respectively. Figure C1c shows these computed heads of $h_{2}{ }^{\prime}, h_{3}{ }^{\prime}, h_{4}{ }^{\prime}, h_{5}{ }^{\prime}$ are mapped back to their actual position points $2,3,4$, and 5 , respectively.

Further observe in Figure C1c that the slope of total head with distance along each of the five 1-D flow line segments is no longer a single constant, as is the case for the transformed horizontal axis plot of the Figure $\mathrm{C} 1 \mathrm{~b}$ streamline segments. Here, the hydraulic gradient is constant along the segment of the 1-D flow line contained within each of the five Figure C1b soil regions. Recall that each 1-D flow line possesses a different value for saturated hydraulic conductivity, resulting in a different hydraulic gradient for each of the five Figure C1c streamline segments. 
Values of water pressures at points 1, 2, 3, 4, 5, and 6 of Figure C1c are calculated using Bernoulli's equation by determining the total head at each point. For point 1, the total head value is established by the boundary condition stipulated at point 1 in Figure C1, with a value of $h_{e-1}+h_{e-2}+h_{e-3 .}{ }^{1}$. Additionally, according to Bernoulli's equation the total head $\left(h_{1}\right)$ at this point can be expressed as,

$$
h_{1}=h p_{1}+h v_{1}+h e_{1}
$$

where $h p_{1}$ is the pressure head, $h v_{1}$ is the velocity head, and $h e_{1}$ is the elevation head at point 1 . Because groundwater seeps and flows slowly for finer materials than open-pored, coarse gravels, the velocity head is considered negligible. Total head is frequently calculated as the sum of the pressure head and elevation head, and the pressure head is the difference between the total head and the elevation head.

$$
h p_{1}=h_{1}-h e_{1}
$$

Once the pressure head at point 1 is determined, it can be multiplied by the unit weight of water to determine the pore water pressure $\left(u_{1}\right)$

$$
u_{1}=h p_{1} * \gamma_{w}
$$

Equations $\mathrm{C}_{2}$ and $\mathrm{C}_{3}$ are used to compute the pressure head for points along the line of seepage and the pore water pressure at that point based on the pressure head. Figure C1c provides the total head value for all points along the line of seepage.

\footnotetext{
${ }^{1} \mathrm{~A}$ similar boundary condition exists at point 6 as stipulated in Figure $\mathrm{C} 1$; the total head $\mathrm{h}_{6}$ is equal to $h_{w}+h_{e-2}+h_{e-3}$.
} 


\section{Appendix D: Description of Corps_I-Wall ASCII Input Data File (project.in)}

The prefix of the input filename, project, is a user-defined base filename that may include a drive letter, a path and name of a project.

The exclamation mark '!' at the first column signifies the start of a comment. (maximum number of characters is 256 per comment line).

A blank line is acceptable.

Descriptive code words are in bold and are three characters in length. They are followed by their relevant parameters. All are separated by spaces.

Some of these codes are optional and do not need to be specified. The mandatory codes for an analysis are nnd, nrg, mod, mth, wal, gmw, mat, nod, wat, reg, ndr, inc, tol. Codes nnd, nrg should be placed at the beginning of the file. Code words preceded by “*”denote multiple entries possible for the same code word.

nrg $\operatorname{lrg}, \mathrm{rrg}$

$\operatorname{lrg}$

$\operatorname{rrg}$

nnd nnodes

*nod node_id, $X, Y$

node id

$\mathrm{X}$

$\mathrm{Y}$
Number of regions at each side of wall.

Number of regions at the left side of wall.

Number of regions at the right side of wall.

Number of nodes.

$\mathrm{X}$-, Y-coordinates of nodes defining regions

id of node

X-coordinate of node

Y-coordinate of node 
mod mode, unit

mode

unit

mth method, method_type

method

method_type
Modes of operation and unit system

=1, Design mode

$=2$,Analysis mode

$=1$, English unit

$=2$, metric

Method for the determination of earth pressure distributions

$=1$, coefficient method

$=2$, wedge solution

if (method=1)

Coefficient methods for determining earth pressures

$=1$, Rankine

$=2$, Coulomb

$=3$, logarithmic spiral - passive

if (method=2)

Wedge solution for determining earth pressures

$=1$, Sweep search method

$=2$,Fixed search method

Position at top of I-Wall

X-coordinate of I-Wall. (=o at center)

wall_top
Elevation at the top of the I-Wall. 
wlt wall_tip, tol_d,

wall_tip

tol_d
Wall tip elevation, tolerance, and maximum number of iterations for an analysis. $($ mode $=2)$

Elevation at the base of the I-Wall for an analysis

tolerance for estimating the passive factor of safety. decimal fraction of the depth of embedment (default $=0.05$ )

Number of sections delineating the I-Wall. The first value given for "wall_ele" is the wall_ele, modulus_elasticity, moment_inertia

wall_id wall_ele modulus_elasticity

moment_inertia

gmw gamma_w

dbg

sim num_sims, num_bins, num_pools elevation at the top of the Wall.

Section of I-Wall

Elevation at the top of the I-Wall section

Modulus of elasticity (psi -- kPascals) of the I-Wall section

Moment of inertia (in. ${ }^{4}-\mathrm{cm}^{4}$ ) of the I-Wall section

Unit weight of water

Debug output file

Simulation variables for a probabilistic analysis. 

num_sims
num_bins
num_pools
Number of simulations ( $>$ 3000)
Number of bins for a histogram (> 3)
Number of pools in order to establish a system response curve with passive factors of safety $<=1.0$ (Analysis mode only).

*fld $s w l, s w r$

swl

Swr

*pmv sim_vars

sim_vars

*mat material_id, gamma_moist, gamma_saturated, FS_active, FS_passive, $C, \varphi, \delta, \beta, \mathrm{C}_{a}, K$, stress
Surface water elevations and sometimes used for piezometric elevations. The number of "fld" entries should match the num_pools (see sim section for probabilistic analysis). For a deterministic analysis, there will only be one entry.

surface water elevation on the left side of wall

surface water elevation on the right side of wall

Probabilistic material variables

The four variables evaluated are $\mathrm{C}$, $\varphi, \delta, \mathrm{Ca}$, respectively are provided for each material. Please see Appendix C for detailed description. Each of the four variables will have a value of o or 1 .

(ex. 1100 )

$=1$, probabilistic

$=0$, deterministic

Material properties 


$\begin{array}{ll}\text { material_id } & \text { Material number } \\ \text { gamma_moist } & \text { Moist unit weight of soil } \\ \text { gamma_saturated } & \text { Saturated unit weight of soil } \\ & \begin{array}{l}\text { Active Factor of Safety associated with } \\ \text { this material }\end{array} \\ \text { FS_passive } & \begin{array}{l}\text { Passive Factor of Safety associated with } \\ \text { this material }\end{array} \\ \text { C } & \text { cohesion } \\ \varphi & \text { internal friction angle (deg) } \\ \delta & \text { wall friction angle (deg) } \\ \beta & \text { surface angle to the horizontal (deg) } \\ \text { Ca } & \text { wall/soil adhesion } \\ \text { K } & \begin{array}{l}\text { Saturated hydraulic conductivity (ft/sec in } \\ \text { English units or m/sec in metric units) }\end{array} \\ \text { stress } & \begin{array}{l}\text { Method to define strength parameters } \\ =1\end{array} \\ =2 & \text { S-Case (effective stress) }\end{array}$

*wat water_id, $\quad$ piezometric surface or surface water w_nodes, $w \_i d\left(1: n \_n o d e s\right)$

water_id water ID w_nodes the number of nodes identifying the water surface for this region 
w_id(1:n_nodes)

region_id

side

water_type

side,

water_type,

water_id, all the nodes associated with this piezometric surface or water surface. These nodes are listed from the Xcoordinate nearest the I-Wall to the $\mathrm{X}$-coordinate farthest away from the I-Wall (1 to w_nodes). The coordinates for the w_id's can be found in the "nod" section.

Region located on either side of wall material_id

$=1$ left side of wall

$=2$ right side of wall

Piezometric water surface associated with this region (for pore water pressure calculations only ${ }^{1}$.

$=0$, No water ${ }^{2}$

$=1$, Surface water 3

$=2$, Piezometric surface 4

${ }^{1}$ For each nodal elevation the pore water pressure, $u$, acting at that node is computed as $u=$ $\gamma_{w} *$ (piezometric elevation - nodal elevation).

2 For water_type $=0$, the pore water pressure $u=0.0$.

3 The piezometric elevation for water_type $=1$ is obtained from the fld data line. In a probabilistic analysis this piezometric elevation may be made to vary over a series of elevation values by specifying multiple fld data lines.

4 The piezometric elevation for water_type $=2$ is obtained from the wat data lines for elevation of the node corresponding to the water_id index designated for the region in question. In a probabilistic analysis this piezometric elevation does not vary. 
water_id

material_id
Water ID associated with this region $=0$, No water source

$>0$, Water surface or piezometric surface

Material number associated with this region

nodes defining the region

*rgn region_id,

$n \_$nodes, n_id(1:n_nodes)

region_id

global region number

n_nodes

the number of nodes defining this region

n_id(1:n_nodes)

all nodes defining this region. The coordinates for the node_id's can be found in the "nod" section. These nodes are listed in the counter-clockwise direction ( 1 to $n \_$nodes) from the $\mathrm{X}$-coordinate nearest to the I-Wall.

gap gap_type

gap_type

spg spg_type,

spg_type
Gap initiation and propagion

$=1 \quad$ Hydraulic fracture

Seepage effects. Current version allows only one water source on each side of wall. $=3$, line of seepage (Homogeneous or heterogeneous soils $)^{1}$

\footnotetext{
1 Pore water pressures are computed within the soil regime using the line of seepage method (Appendix C) to first compute total heads at each node along the I-Wall/sheet-pile wall, combined with the application of Bernoulli's equation to compute the pressure head $h_{p}$. Bernoulli's equation states that the total head $H$ is equal to the elevation head, $h_{e}$, plus the pressure head, $h_{p}$, i.e., $H=h_{e}+h_{p}$. The nodal elevation provides for the value for $h_{e}$. Using the computed value for $h_{p}$, the pore water pressure $u$ is equal to $\gamma_{w} h_{p}$.
} 
*hll horz_line_load_el, horz_line_load

horz_line_load_el

horz_line_load
Horizontal line load. Positive load acts to the left of the wall.

elevation of the $i^{\text {th }}$ horz_line_load

horizontal load of the $i^{\text {th }}$ line load normal to the wall (plf)

Horizontal distributed load. Positive load acts to the left of the wall

number of nodes comprising the $\mathrm{i}^{\text {th }}$ horizontal

horz_dist_load_el distribution elevation of the $\mathrm{j}^{\text {th }}$ node of the $\mathrm{i}^{\text {th }}$ horizontal distribution horz_dist_load

the magnitude of the $j^{\text {th }}$ node of the $i^{\text {th }}$ horizontal distribution (psf)

Surface surcharge vertical line loads applied to the ground surface. Positive loads act vertically downward.

location of the $\mathrm{i}^{\text {th }}$ vertical line load

$=1$, Left side of wall

$=2$, Right side of wall

vert_line_load_x $\quad x$-position of the $i^{\text {th }}$ vertical line load

vert_line_load_mag positive magnitude of the $i^{\text {th }}$ vertical line load (plf) 
*vul vert_uniform_load_side, Vertical distributed uniform load. Only vert_uniform_load_mag one or none on each side of wall.

vert_uniform_load_side location of the vertical uniform load $=1$, Left side of wall

$=2$, Right side of wall

vert_uniform_load_mag positive magnitude of the uniform load (psf)

*vsl vert_strip_side, vert_strip_x1, vert_strip_x2, vert_strip_mag

vert_strip_side

vert_strip_x1

vert_strip_x2

vert_strip_mag

*vrl vert_ramp_side, vert_ramp_x1, vert_ramp_x2, vert_ramp_mag
Surface surcharge vertical distributed strip loads applied to the ground surface. Positive loads act vertically downward.

location of the $\mathrm{i}^{\text {th }}$ vertical strip load $=1$, Left side of wall

$=2$, Right side of wall

$\mathrm{x}$-position ar the start of the $\mathrm{i}^{\text {th }}$ strip load

$\mathrm{x}$-position at the end of the $\mathrm{i}^{\text {th }}$ vertical strip load

magnitude of the $i^{\text {th }}$ uniform strip load. (psf)

vertical distributed ramp loads. Only one or none on each side of wall. 
vert_ramp_side

vert_ramp_x1

vert_ramp_x2

vert_ramp_mag

*vtl vert_tri_side, vert_tri_x1, vert_tri_x2, vert_tri_x3, vert_tri_mag

vert_tri_side

vert_tri_x1

vert_tri_x2

vert_tri_x3

vert_tri_mag location of the vertical ramp load

$=1$, Left side of wall

$=2$, Right side of wall

$\mathrm{x}$-position at the start of the ramp

$\mathrm{x}$-position at the end of the ramp. (this point is the start of uniform loading).

positive magnitude at uniform loading. (psf)

vertical distributed triangular loads.

Positive loads act vertically downward. location of the $i^{\text {th }}$ vertical triangular load $=1$, Left side of wall

$=2$, Right side of wall

$\mathrm{x}$-position at the start of the $\mathrm{i}^{\text {th }}$ triangular load.

$\mathrm{x}$-position at the peak of the $\mathrm{i}^{\text {th }}$ triangular load.

$\mathrm{x}$-position at the end of the $\mathrm{i}^{\text {th }}$ vertical triangular load.

magnitude of the $i^{\text {th }}$ vertical triangular load. (psf)

vertical trapezoidal loads

*vdl vert_trap_side, vert_trap_x1, vert_trap_mag1, vert_trap_x2, vert_trap_mag2 

vert_trap_side location of the $i^{\text {th }}$ vertical trapezoidal load $=1$, Left side of wall
$=2$, Right side of wall
vert_trap_x1
vert_trap_mag1
vert_trap_x2
vert_trap_mag2
$\mathrm{x}$-position at the start of the $\mathrm{i}^{\text {th }}$ trapezoid magnitude at the start of the $\mathrm{i}^{\text {th }}$ trapezoidal distribution (psf)
$\mathrm{x}$-position at the end of the $\mathrm{i}^{\text {th }}$ trapezoidal distribution
magnitude at the end of the $\mathrm{i}^{\text {th }}$ trapezoidal distribution (psf)

inc init_node_spacing, length defining the distance separating node_inc, min_node_spacing init_node_spacing node_inc min_node_spacing

tol tol_m

itr max_iter each I-Wall node initial node spacing (default $=1 \mathrm{ft} ; 0.30480 \mathrm{~m}$ )

node spacing increment. Decimal fraction of the initial node spacing (default $=0.01$ )

minimum node spacing. Decimal fraction of the initial node spacing (default $=0.7$ )

For the case when there is ample resistance within a soil berm on the landside to withstand load, the tolerance used for the sum of moment calculations (default $=10 \mathrm{ft}-\mathrm{lb} ; 13.55818 \mathrm{~N}-\mathrm{m}$ )

maximum number of iterations executed for an analysis $(=100)$ 


\section{Appendix E: Description of Corps_I-Wall ASCII Output Data Files}

The prefix of all the output filenames is a user-defined base filename that may include a drive letter, a path and name of a project. A three-digit numeric field preceding the dot of a filename identifies the flood level for this particular analysis. The following sections discuss the various output files, with the prefix appended before each output filename.

\section{E.1 prefix_001.out}

The first line of the file lists information regarding the left-hand side of the I-Wall. The first value of this line is the number of text lines that follow. The second value represents the number of data lines following the text lines. The rest of the line lists the flood elevation.

As part of the data, the value of the first data character is defined. Values in this file are tabulated with the wall elevation, the water pressure, the soil vertical stress, the mobilized active stress, the mobilized passive stress, the mobilized horizontal active stress and mobilized horizontal passive stress. Data are recorded at every nodal point elevation of the I-Wall.

Following the data lines, the next line starts the same set of data for the RHS of the I-Wall. The following is an example code of the first several lines of this file.

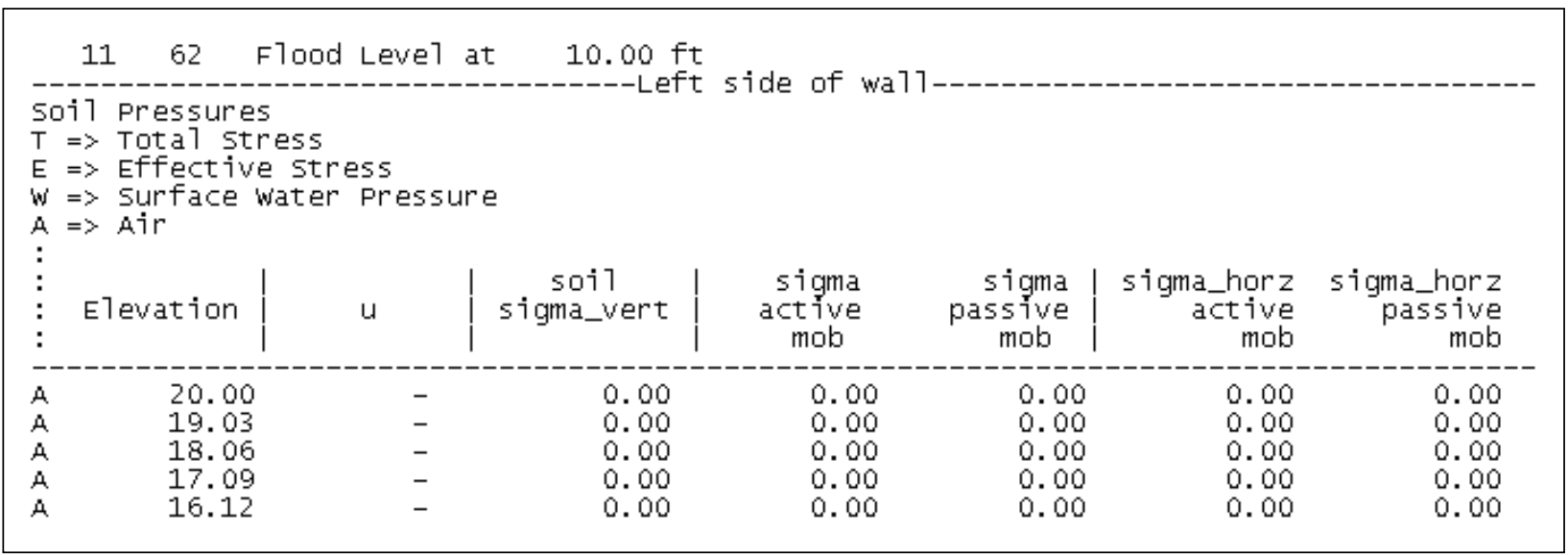




\section{E.2 prefix_net_pressures_001.out}

The first line lists relevant information of this file. The first value of this line is the number of text lines that follow. The second value represents the number of data lines following the text lines. The rest of the line lists the flood elevation.

Data values in this file record the node ID, the wall elevation, the net water pressure, the net active earth pressure, and the net passive earth pressure. The net water pressure is the difference between the water pressure of the side that the wall will rotate from and the other side water pressure. The net active pressure is calculated by taking the difference between the horizontal active stress and the horizontal passive stress and adding the net water pressure and net horizontal and net vertical external pressures. The following is an example of the first seven lines of this file.

$\begin{array}{cccrr}1220 & \text { Flood Level at } 14.00 \mathrm{ft} \\ \text { node wall_ele net_u net_a net_p } & & \\ 1 & 20.00 & 0.00 & 0.00 & 0.00 \\ 2 & 19.76 & 0.00 & 8.62 & 56.83 \\ 3 & 19.52 & 0.00 & 17.24 & 113.66 \\ 4 & 19.28 & 0.00 & 25.86 & 170.49 \\ 5 & 19.04 & 0.00 & 34.48 & 227.33\end{array}$

\section{E.3 prefix_internal_shear_moment_001.out}

The first line lists relevant information of this file. The first value of this line is the number of text lines that follow. The second value represents the number of data lines following the text lines. The rest of the line lists the flood elevation.

Data values in this file record the wall elevation, the shear force, the bending moment and the overall net pressure. Net is defined as the sum of horizontal pressures acting on both sides of the I-Wall. The following is an example of the first six lines of this file. 
163 Flood Level at $10.00 \mathrm{ft}$

Elevation Shear Moment Net Pressure

$\begin{array}{lrcc}20.000000 & 5.749944 & 0.000000 & 0.000000 \\ 19.030000 & 17.249833 & 5.577446 & 35.566667 \\ 18.060000 & 68.999333 & 44.619569 & 71.133333 \\ 17.090000 & 155.248500 & 150.591045 & 106.700000 \\ 16.120000 & 275.997333 & 356.956551 & 142.266667\end{array}$

\section{E.4 prefix.run}

This file displays runtime information such as the direction that the wall will rotate. The node spacing and the sum of forces and sum of moment calculations at each estimated point of rotation and depth of embedment. The final calculated PR and elevation at tip of pile is recorded at the end of this file. The following is an example of the first several lines of this file.

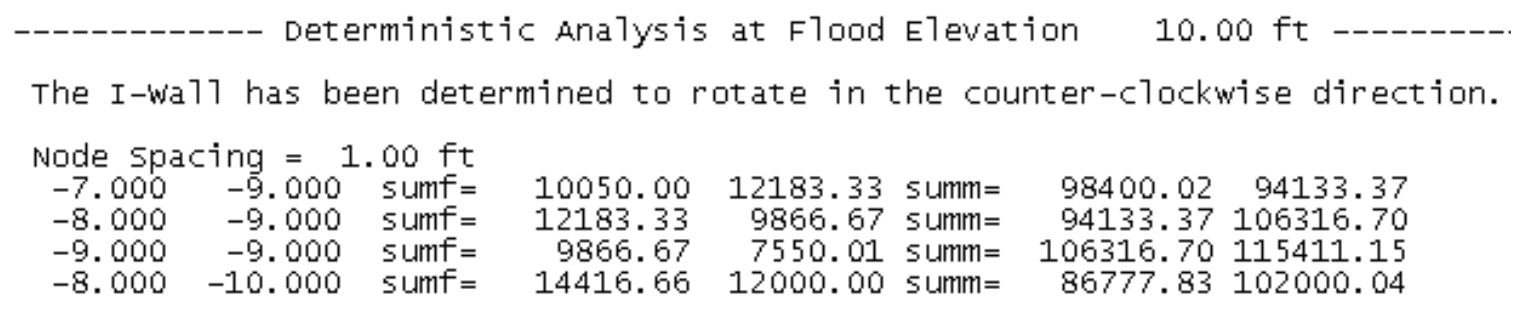

\section{E.5 prefix.inp}

This file is an echo to the input file as described in Appendix D: Description of Corps_I-Wall ASCII Input Data File (project.in).

\section{E.6 prefix_001._pdf}

This file displays statistical information of the probability density function for the depth of embedment variable. The first line gives information on the number of proceeding text lines with the second value representing the number of data lines following the text lines. Data values for the histogram representing the probability density function are the ID, the midpoint of each bin and the frequencies occurring at this bin. Recall that the number of bins is a user input. The following is a sample of this file. 


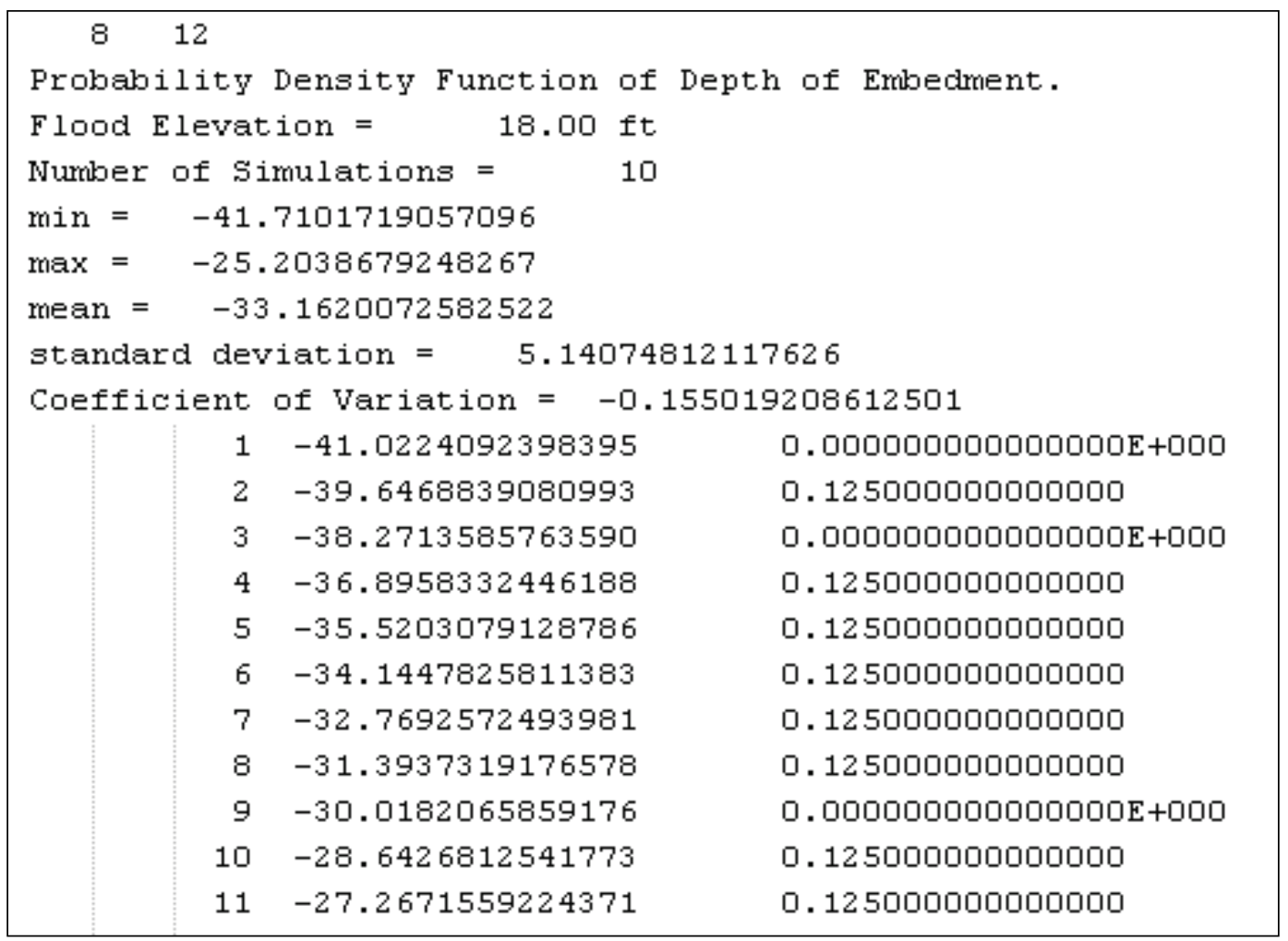

\section{E.7 prefix_001._cdf}

This file displays statistical information of the cumulative distribution function (CDF) for the depth of embedment variable. The first line gives information on the number of proceeding text lines with the second value representing the number of data lines following the text lines. Data values representing this CDF are the ID, the midpoint of each bin and the probability that the variable will have a value less than or equal to the midpoint of the bin. Recall that the number of bins is a user input. The following is a sample of this file. 


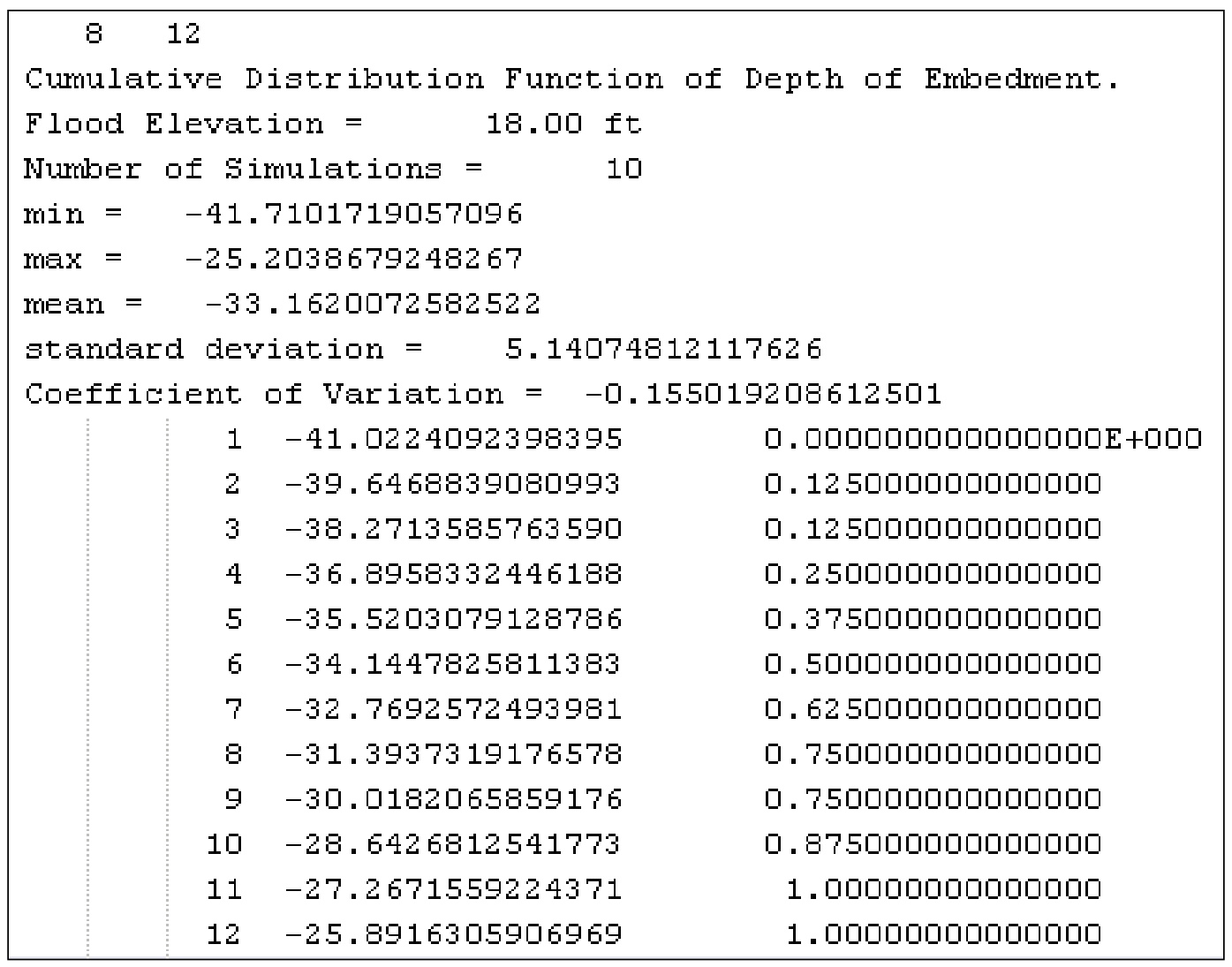

\section{E.8 prefix.src}

This file displays the SRC for an Analysis mode study. For each pool, the probability of a sliding limit state is given. The first line of the file gives information on the number of proceeding text lines with the second value representing the number of data lines following the text lines. Data values representing this SRC are the ID, the pool elevation and the probability of the sliding limit state. The following is a sample of this file.

$1 \quad 12$

System Response Curve

$\begin{array}{lll}1 & 18.0 & 0.6 \\ 2 & 17.0 & 0.584 \\ 3 & 16.0 & 0.503 \\ 4 & 15.0 & 0.411 \\ 5 & 14.0 & 0.347 \\ 6 & 13.0 & 0.127\end{array}$




\section{Appendix F: Calculation of Coastal Surge Pressures Acting on an I-Wall for a Breaking Wave}

This appendix summarizes material adapted from a Memorandum for Record created in August 2008 by Dr. Kevin Abraham and Dr. Robert Ebeling of ERDC for support of the development of Engineering Circular 1110-2-6066 Engineering and Design of I-Walls (April 2011)(HQUSACE 2011a).

\section{F.1 Background}

The purpose of this example was to investigate the feasibility of using an I-Wall type retaining wall and levee system to provide protection against flood and wave loading in a coastal environment using simplified limit equilibrium procedures. Typical coastal structural systems consist of seawalls, anchored bulkheads, and revetments (Shore protection manual 1984). These systems are generally more robust and stiffer systems than a more flexible I-Wall section. ${ }^{1}$

First, a literature review was performed in an attempt to develop a database of projects that used I-walls for flood protection in a coastal environment. Regrettably, this literature review did not find a published example of an I-Wall and levee system in a coastal environment that provided flood and wave protection. However, a generic I-Wall system was developed based on general design guidance in Engineering Manuals 2502 and 2504, Retaining and flood walls, and Design of sheet-pile walls, respectively. This example outlines the process of using simplified limit equilibrium procedures and simplified methods of computing wave load to perform an initial design for an I-Wall in a coastal environment. The simplified Minikin method (for computing breaking wave loads on vertical walls) was used in the analysis/design of this I-Wall system. If a new IWall is being investigated, then it is recommended that the design of this I-Wall be performed using an incremental approach. First, obtain an initial I-Wall design that satisfies the Corps' criteria without any applied

\footnotetext{
1 The moment resistance of a sheet-pile wall can be enhanced with the addition of a landside soil berm that acts to counter the effects of surge loading during wave action sea side of the wall.
} 
wave loading. Second, a series of analyses is performed in an effort to obtain an I-Wall initial design with an applied wave loading that would satisfy the Corps' design criteria (given in EM 1110-2-2504 (HQUSACE 1994)). Lastly, a parametric study may need to be performed in an effort to modify the elevation of the ground surface on the landside of the wall. This ground surface is needed in an I-Wall design that satisfies the Corps' criteria and soil profile consistent with a typical buttress levee when the I-Wall is subjected to significant surge loading during wave action.

\section{F.2 Initial I-Wall design without wave loading}

Typical cantilevered I-wall systems have an exposed height above the dredge level of $15 \mathrm{ft}$ or less, so a profile with an exposed height of $15 \mathrm{ft}$ was selected. The soil for this example is a medium dense sand with a unit weight $\left(\gamma_{\text {moist }}=\gamma_{\mathrm{sat}}=135 \mathrm{pcf}\right)$ and shear strength parameters of $\varphi^{\prime}=35 \mathrm{deg}$ and $c^{\prime}=0$. Coastal protection structures, such as seawall and anchored bulkheads, could have soil surface elevations on the landside at or near the top of the wall. Additionally, these structures are often designed with a stick-up height (exposed height of the wall above the design wave) of a minimum of $2 \mathrm{ft}$. With respect to wave heights, Hurricane Katrina generated surges $5 \mathrm{ft}$ to $7 \mathrm{ft}$ above what was assumed in the I-Wall design (IPET report Vol. 1) at some locations on the eastern side of New Orleans. Figure F1 shows a general profile that was used as a basis for the 2008 preliminary designs. Note that the 2008 initial design calculations did not include a complete breaking wave loading applied pressure definition as per the simplified Minikin method.

In 2008, the only sheet-pile wall analysis/design software available to the Corps was CWALSHT. After numerous attempts, Drs. Abraham and Ebeling determined that CWALSHT is not able to accommodate the modeling of breaking wave loading using the simplified Minikin method. Consequently, a series of preliminary cantilever design calculations were performed in 2008 using CWALSHT to compute depths of penetration $(d)$ for an overly simplified, incomplete, and unconservative representation of wave load-induced pressures applied sea side of the sheet-pile wall in order to gain insight into the range in required depth(s) of sheet-pile wall penetration. The design calculations complied with Corps design criteria of applying a Factor of Safety 1.0 to the shear strength of the soil used to compute active soil pressures and applying a Factor of Safety of 1.5 to the shear strength of the soil used to the compute passive soil pressures below the dredge level. Table F1 shows results of computed depths of penetration 
(d) for varied soil surface elevations on the landside without wave loads being applied and an average still-water level (SWL) of elevation $8 \mathrm{ft}$. These results were used in subsequent analysis to determine if otherwise reasonable cantilever designs (including the wave load) were feasible.

Figure F1. Profile used in the initial I-Wall design calculations, including a soil buttress on the landside using an overly simplified and incomplete representation of wave load-induced, pseudo-static pressures.

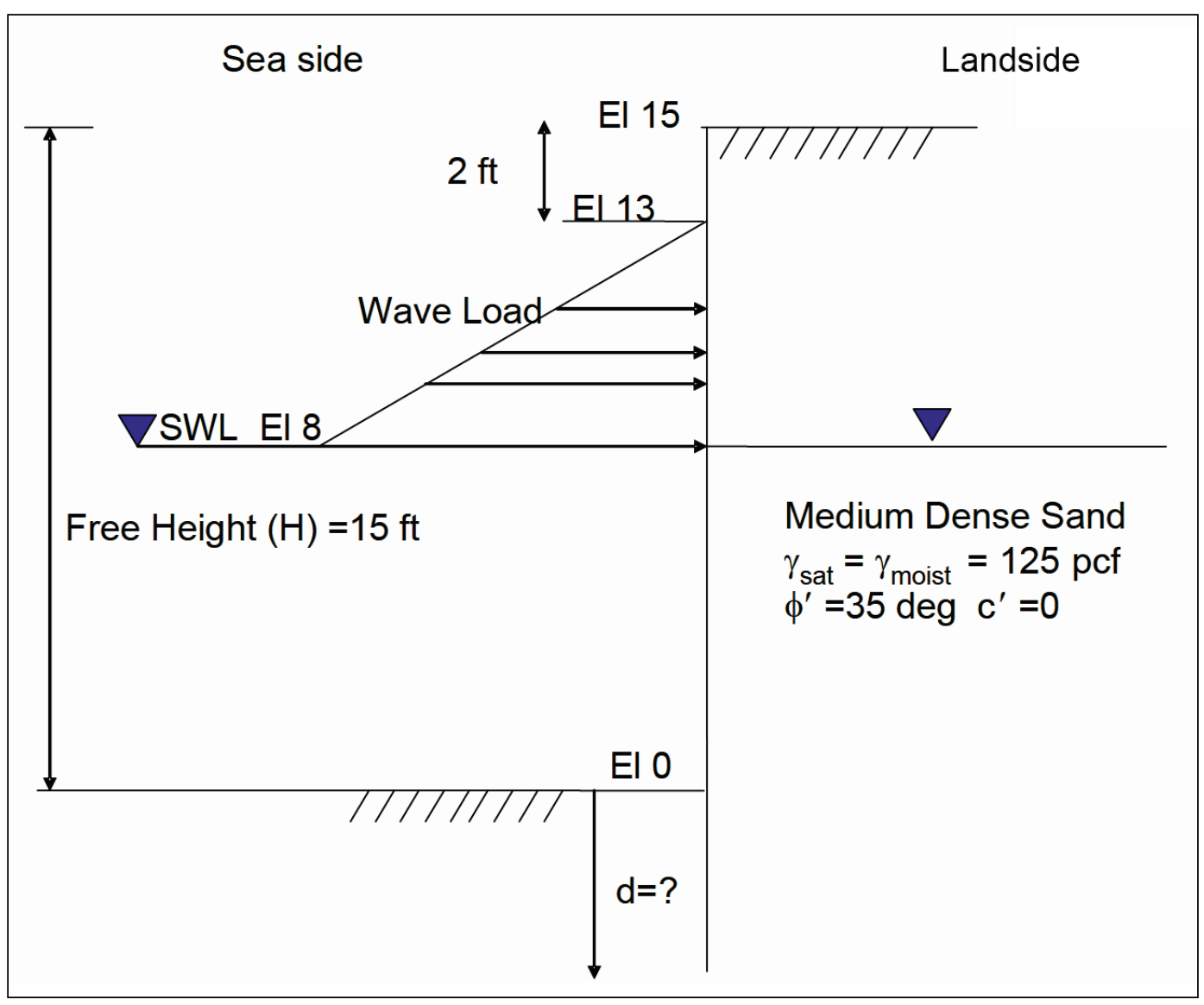

Table F1. Summary of 2008 preliminary I-Wall analysis results for varied heights of soil surface on the landside for an overly simplified and incomplete representation of wave loadinduced pressures.

\begin{tabular}{|l|l|l|}
\hline $\begin{array}{l}\text { Protected Side Surface EL } \\
(\mathrm{ft})\end{array}$ & $\begin{array}{l}\text { Free Height }(\boldsymbol{H}) \\
(\mathrm{ft})\end{array}$ & $\begin{array}{l}\text { Design Depth of Penetration }(\boldsymbol{d}) \\
(\mathrm{ft})\end{array}$ \\
\hline 0 & 15 & NA \\
\hline 5 & 15 & 5 \\
\hline 9 & 15 & 10 \\
\hline 12 & 15 & 15 \\
\hline 15 & 15 & 20 \\
\hline
\end{tabular}


The following subsection outlines the calculations associated with the simplified Minikin method for computing breaking wave loads on vertical walls.

\section{F.3 I-Wall design including wave loading}

There are two general groups of procedures for computing wave impact loads on vertical walls in a coastal environment: the method used by Goda for nonbreaking waves (Coastal engineering manual 2002) and the Minikin method for breaking waves (Shore protection manual 1984 and Dr. Steven Hughes', presentation, "Wave Forces on Vertical Wall", at the $20^{\text {th }}$ Annual National Conference on Beach Preservation Technology). ${ }^{1}$ In Dr. Hughes' presentation, he made two comments that pertain to the discussion in this report: (1) The Minikin method was not included in the Coastal engineering manual because some experts think it gives excessively high forces, and (2) Bullock et al. (2004) found that Minikin estimates of $p_{\max }$ (maximum pressure) matched full-scale tests results. The Minikin method of computing breaking wave impact loads on vertical walls was used in this example.

The simplified procedure used to estimate the effects of wave loading in a sheet-pile wall design is as follows:

Step 1. Compute $p_{\mathrm{m}}$ by the Minikin method.

The maximum pressure $\left(p_{\mathrm{m}}\right)$ is assumed to act on the elevation corresponding to the SWL and is given by Equation F1. (Refer to Figure 1). In this example the SWL is at el $8 \mathrm{ft}$.

$$
p_{m}=101 w \frac{H_{b}}{L_{b}} \frac{d_{s}}{D}\left(D+d_{s}\right)
$$

where $w$ is the specific weight of water, $H_{b}$ is the breaking wave height, $L_{D}$ is the local wavelength of water of depth $D$, and $d_{s}$ is depth to the toe of the wall. The distribution of the dynamic breaking wave pressure is shown in

\footnotetext{
${ }_{1}^{1}$ A 1988 Coastal Engineering Technical Note on Breaking wave forces on walls (CETN-III-38, 3/88) observed that "as the Minikin method is based on the shock pressure caused by breaking waves, the resulting forces and structure designs analyzed by using this procedure are generally considered to be conservative. It also stated that a less conservative method recommended by Goda (1974) is an alternative procedure for breaking wave force determination. The rationale of using the Goda method for design analysis is that the duration of the impulsive breaking wave is relatively brief, on the order of tenth or hundredth of a second, and the effect of this force on the stability of massive concrete wall structures may be rather insignificant."
} 
Figure F2. As shown in Figure F2, the pressure distribution consists of three components: (1) hydrostatic, (2) dynamic, and (3) a combined total. Note that Equation F1 only computes the dynamic component of the pressure diagram.

Figure F2. Minikin breaking wave pressure diagram (after Figure 7-99; Shore protection manual 1984).

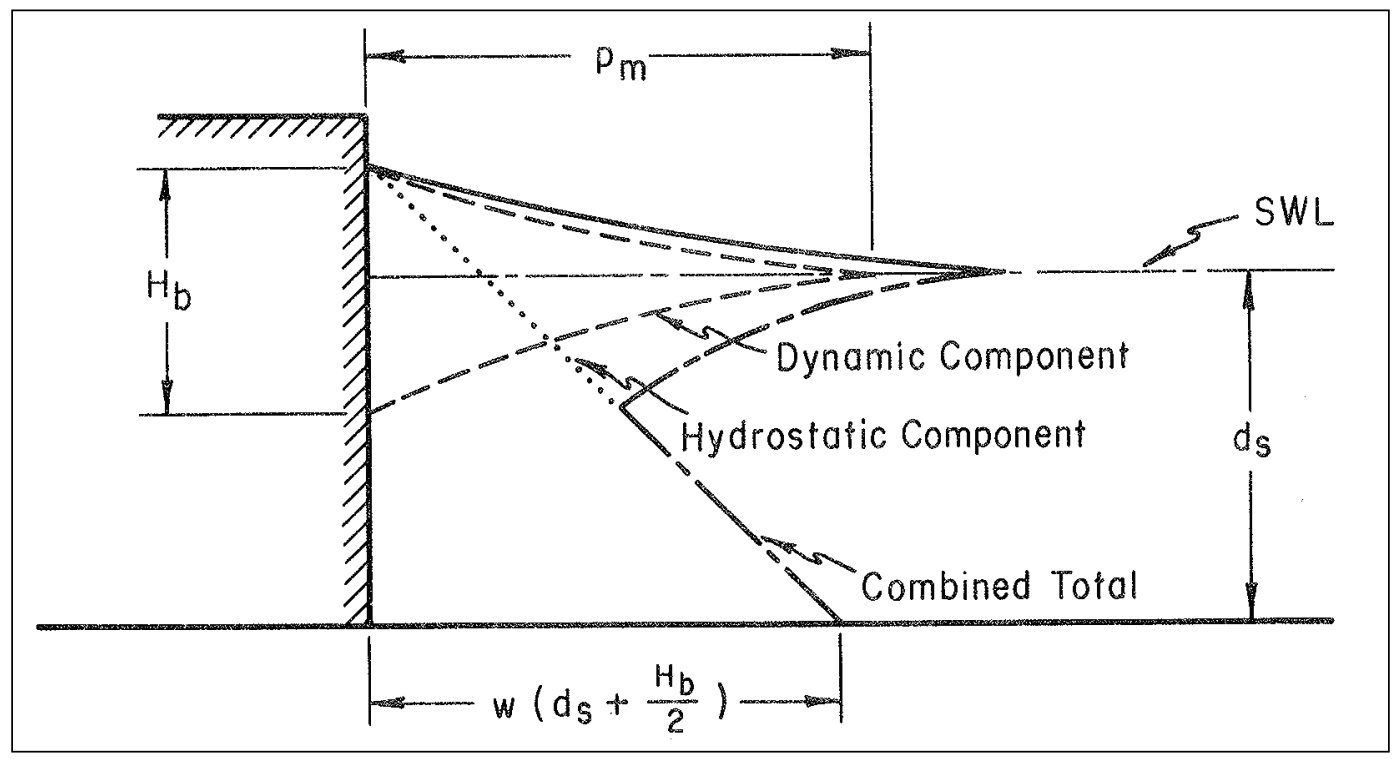

a. Compute the breaking wave height $\left(H_{b}\right)$

Assume $d_{s}=8 \mathrm{ft}$ (depth to dredge level) (Refer to Figure F1) and wave period

$\mathrm{T}=10 \mathrm{sec}$. Note: This wave period corresponds to typical maximum value (Shore protection manual 1984). The near shore slope value $m$ assumed to be zero.

Next compute the relative depth at structure using Equation F2.

$$
\frac{d_{s}}{g T^{2}}=\frac{8 f t}{32.2 \frac{f t}{\sec } 10^{2}}=0.0025
$$

Using this value and Figure F3 with $\mathrm{m}=\mathrm{o}$ gives

$$
\begin{gathered}
\frac{H_{b}}{d_{s}} \approx 0.78 \\
H_{b} \approx 6.24 f t
\end{gathered}
$$


Figure F3. Dimensionless design breaker height versus relative depth at the wall (after Figure 7-4 Shore protection manual1984 and Weggel 1972).

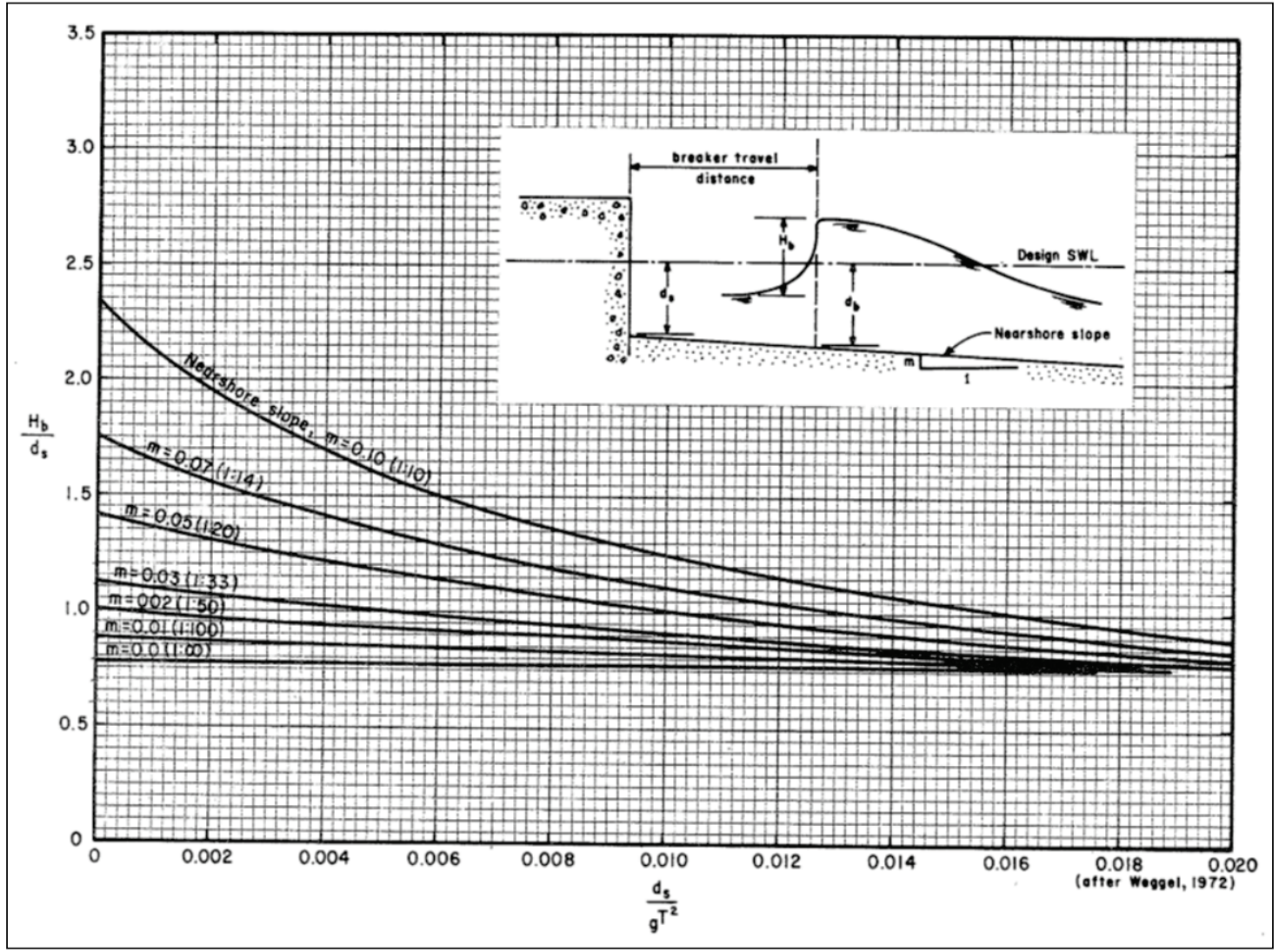

b. Compute local wavelength in water depth $(D)$

First, compute the wavelength in deep water $\left(L_{o}\right)$ for $(T=10 \mathrm{sec})$ using Equation F3.

$$
L_{o}=\frac{g T^{2}}{2 \pi}=\frac{32 * 10^{2}}{2 \pi}=512.48 \mathrm{ft}
$$

Then

$\frac{d}{L_{o}}=\frac{8}{512.48}=0.0156 ;$ where $d=d_{s}$

Using Table C1, Appendix C of the Shore protection manual (1984) and the computed value of $\frac{d}{L_{o}}$ gives $\frac{d}{L} \approx 0.050684$ where $L$ is wavelength at depth $d$

$$
L_{d}=\frac{8 f t}{0.050648} \approx 158 f t
$$


c. Compute the water depth, $D$, one wavelength seaward of the vertical wall using Equation F4.

$$
D=d_{s}+L_{d} m ; \text { since } \mathrm{m}=0
$$

$D=d_{s}=8 \mathrm{ft}$

Therefore: $\frac{D}{L_{o}}=\frac{d}{L_{o}}$ and $\mathrm{Ld}=\mathrm{LD}$

$$
\begin{gathered}
p_{m}=101 w \frac{H_{b}}{L_{D}} \frac{d_{s}}{D}\left(D+d_{s}\right) \\
p_{m}=101 * 64 \frac{l b}{f t^{3}} \frac{6.24 f t}{158 f t} \frac{8 f t}{8 f t}(8 f t+8 f t) \\
p_{m} \approx 4,084.6 \frac{l b}{f t^{2}}
\end{gathered}
$$

Using Figure $\mathrm{F}_{4}$, the curve for $m=$ oand Equation 2, an approximate value for $p_{m}$ can be computed

$$
\frac{d_{S}}{g T^{2}}=\frac{8 f t}{32.2 \frac{f t^{2}}{\sec } 10^{2}}=0.0025
$$

The ratio of $\frac{p_{m}}{w H_{b}} \approx 10.2 w$ is the specific weight of water, $H_{b}$ is the breaking wave height, $p_{m}=10.2 * 64 \frac{l b}{f t^{3}} * 6.24 \mathrm{ft} \approx 4,073.5 \frac{l b}{f t^{2}} p_{m} \approx 4,073.5 \frac{l b}{f t^{2}}$; is approximately equal to $p_{m}$ computed using Equation F1 $\left(4,084.6 \frac{l b}{f t^{2}}\right)$.

Recall this computed wave load pressure distribution acting over breaking wave height $H_{b}$, designated "Dynamic Component" in Figure F2 with a maximum magnitude $p_{m}$, is then incorporated in an initial sheet-pile wall design calculation as an applied horizontal distributed load on the wall. Note that in the sheet-pile wall analysis, the elevation of the SWL specified on the coastal side of the sheet-pile wall increases from SWL to the elevation of this surge wave (depicted in Figure F1). Again, this simplified pressure load approximates the dynamic wave effects on the I-Wall. In 2008, calculations were attempted using the Figure 1 overly simplified approximation for a surge wave loading using CWALSHT. These attempts were deemed unsuccessful. This experience contributed to requests being made by district engineers for the development of new I-Wall analysis software that is capable of analyzing coastal sheet-pile walls using the simplified Minikin method for computing breaking wave loads on vertical walls. 
Figure F4. Dimensionless Minikin wave pressure and force (after Figure 7-100; Shore protection manual 1984).

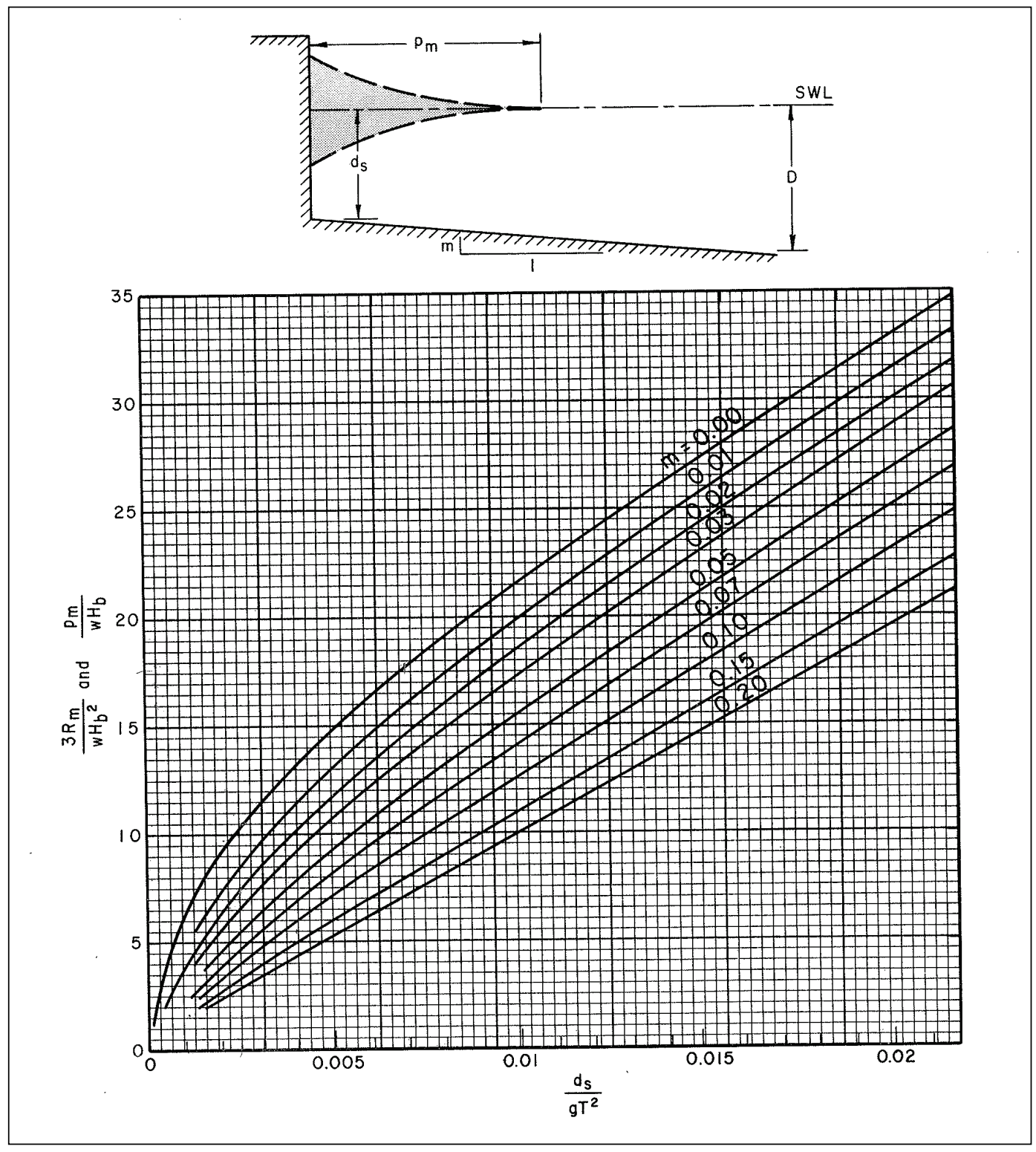




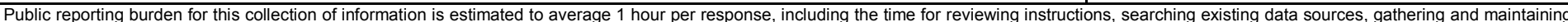

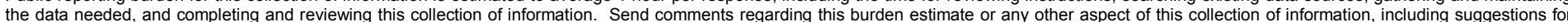

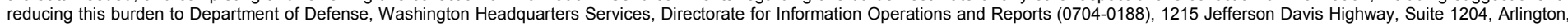

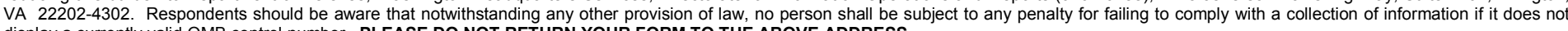
display a currently valid OMB control number. PLEASE DO NOT RETURN YOUR FORM TO THE ABOVE ADDRESS.
1. REPORT DATE (DD-MM-YYYY) 2. REPORT TYPE
July 2016 Final report

3. DATES COVERED (From - To)

\section{TITLE AND SUBTITLE}

5a. CONTRACT NUMBER

Analysis of a Floodplain I-Wall Embedded in Horizontally Stratified Soil Layers During

Flood Events Using Corps I-Wall Software Version 1.0

\section{5b. GRANT NUMBER}

5c. PROGRAM ELEMENT NUMBER

Civil Works Program Element 031398

6. AUTHOR(S)

5d. PROJECT NUMBER

454633 FY16 Talbot-Flood and Coastal

Robert M. Ebeling, Moira T. Fong, and Barry C. White

5e. TASK NUMBER

A1170-FY16 "Wall-Analysis"

5f. WORK UNIT NUMBER

\section{PERFORMING ORGANIZATION NAME(S) AND ADDRESS(ES)}

8. PERFORMING ORGANIZATION REPORT NUMBER

U.S. Army Engineer Research and Development Center

Information Technology Laboratory

ERDC/ITL TR-16-3

3909 Halls Ferry Road

Vicksburg, MS 39180-6199

9. SPONSORING / MONITORING AGENCY NAME(S) AND ADDRESS(ES)

10. SPONSOR/MONITOR'S ACRONYM(S)

Headquarters, U.S. Army Corps of Engineers

$441 \mathrm{G}$ Street NW

Washington, DC 20314-1000

11. SPONSOR/MONITOR'S REPORT NUMBER(S)

\section{DISTRIBUTION / AVAILABILITY STATEMENT}

Approved for public release; distribution is unlimited.

\section{SUPPLEMENTARY NOTES}

\section{ABSTRACT}

The purpose of the I-Wall Analysis R\&D effort is to provide the U.S. Army Corps of Engineers (USACE) districts with essential tools in analyzing and evaluating I-Wall performance in riverine, fluvial, and coastal environments. This report summarizes an engineering methodology and its implementation in the software Corps_I-Wall, Version 1.0. The program is referred to as CI-Wall. This initial version of Corps_I-Wall is used for the design of new I-Walls or the analysis of existing I-Walls in floodplains (i.e., I-Walls embedded in level ground) during flood loading. Horizontally stratified soil layers are assumed for Version 1.0. Other CI-Wall software engineering analysis capabilities include: total stress and/or effective stress earth pressure calculations; Rankine, Coulomb, or logarithmic spiral based earth pressure coefficients; hydrostatic pore water pressure computed from surface water(s) and/or piezometric surface(s) within each of the layered soil regions; pore water pressures computed from line of seepage, steady-state seepage in soil regions with different hydraulic conductivities; gap initiation and propagation using hydraulic fracturing criterion; and boundary pressures (e.g., for wave loading of coastal structures) and/or surface surcharge. Probabilistic analysis is a primary component of Corps_I-Wall for new and existing I-Wall analysis. The probabilistic analysis capabilities for the design of new I-Walls results in a statistical characterization of sheet-pile tip embedment. The probabilistic analysis capabilities for analyzing existing I-Walls include the construction of a system response curve (a.k.a., fragility curve), which gives the probability of rotational instability as a function of flood elevation. Examples are provided to highlight each of these capabilities and a user's manual for using the graphical user interface for CI-Wall is included.

15. SUBJECT TERMS (see reverse)

16. SECURITY CLASSIFICATION OF:

a. REPORT

\section{b. ABSTRACT}

17. LIMITATION OF ABSTRACT
18. NUMBER OF PAGES

324 19a. NAME OF RESPONSIBLE PERSON

19b. TELEPHONE NUMBER (include area code) 
15. SUBJECT TERMS (concluded)

I-Wall

Floodwall

Corps I-Wall

CI-Wall

Hydraulic fracturing

System response curve

Fragility analysis

Flood and Coastal Storm Damage Reduction Program

Infrastructure resiliency and reliability 